55Th CONGRESS, \} HOUSE OF REPRESENTATIVES. \{ DOOUMENT $2 d$ Session.

DEPARTMENT OF THE INTERIOR

\title{
WATER-SUPPLY
}

AND

IRRIGATION PAPERS

$-\quad{ }^{\circ o r ~ r u x}$

UNITED STATES GEOLOGICAL SURVEY

No. 11

RIVER HEIGH'TS, 1896.-DAVIS

WASHINGTON

GOVERNMENT PRINTING OFFICB

1898 
UNITED STATES GEOLOGICAL SURVEY CHARLES D. WALCOTT, DIRECTOR

\section{RIVER HEIGHTS FOR 1896}

BY

ARTHUR POWELL DAVIS

WASHINGTON

GOVERNMENT PRINTING OFFICE

1897 


\section{CONTENTS.}

Daily gage heights observed during 1896, arranged in geographic order by drainage basins........................................................

Current meters rated in 1896 7

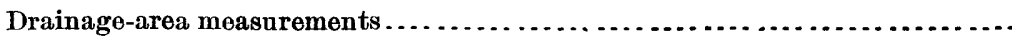




\title{
LETTER OF TRANSMITTAL.
}

\author{
DEPARTMENT OF THE INTERIOR, \\ United States Geological Survey, \\ DIVISION OF HYDROGRAPHY, \\ Washington, June 1, $189 \%$.
}

SIR: I have the honor to transmit herewith a series of tables pertaining mainly to river heights, prepared by the Division of Hydrography of this Survey, and to recommend that it be published in the series of papers "in relation to the gauging of streams and to the methods of utilizing the water resources," the printing of which was authorized in the act making appropriations for sundry civil expenses of the Government for the fiscal year ending June 30, 1897, approved June 11, 1896.

Very respectfully,

F. H. NEWELL, Hydrographer in Charge.

Hon. Charles D. Walcott, Director United States Geological Survey. 6 


\section{RIVER HEIGHTS FOR 1896.}

By Arthur P. Davis.

\section{DAILY GAGE HEIGHTS OBSERVED DURING 1896.}

The series of tables here presented pertain mainly to the river heights observed and recorded during 1896 by persons employed by or cooperating with the Division of Hydrography of this Survey. There are also included a few tables obtained from the Weather Burean through the courtesy of Hon. Willis L. Moore, chief of that Bureau. These give the heights of water at points near localities where measurements have been made by hydrographers of this Survey, and are of value in computations of discharge. A few similar tables have been obtained through officers of the Engineer Corps of the United States Army, from observations made in connection with investigations pertaining to the improvement of navigation. There are appended tables of evaporation, seepage, meter ratings, and other similar details to which reference is made by hydrographers of this Survey.

These tables pertain to the progress report of stream measurements for 1896, prepared by the Division of Hydrography and printed in Part IV of the Eighteenth Annual Report of the Survey. They might properly have been included in the progress report, but since they relate to minor details and have a comparatively limited application, it has been thought best, from considerations of economy and from the necessity of reducing the size of the annual report, to include these in a separate publication of more limited distribution. On the other hand, these data should be printed and thus made available for the use of hydrographers, not only those connected with this Survey, but others occupied in similar examinations throughout the country. 
Patapsco River at Woodstock, Maryland.

\begin{tabular}{|c|c|c|c|c|c|c|c|c|c|c|c|}
\hline Day. & Ang. & Sept. & Oct. & Nov. & Dec. & Day. & Aug. & Sept. & Oct. & Noy. & Dec. \\
\hline 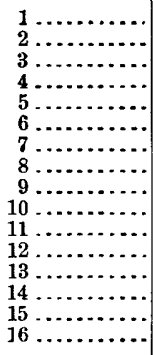 & \begin{tabular}{|l|}
$\ldots \ldots \ldots$ \\
$\ldots \ldots \ldots$ \\
$\ldots \ldots \ldots$ \\
$\cdots \ldots .$. \\
$\cdots 3.60$ \\
3.70 \\
3.60 \\
3.50 \\
3.80 \\
3.60 \\
3.70 \\
3.85 \\
3.85 \\
3.75 \\
3.70
\end{tabular} & $\begin{array}{l}3.55 \\
3.50 \\
3.45 \\
3.50 \\
3.65 \\
3.85 \\
3.85 \\
3.60 \\
3.55 \\
3.50 \\
3.50 \\
3.50 \\
3.45 \\
3.55 \\
3.55 \\
3.65\end{array}$ & $\begin{array}{l}3.85 \\
\text { 3. } 65 \\
3.60 \\
3.60 \\
3.65 \\
\text { 3. } 65 \\
3.65 \\
3.50 \\
3.70 \\
3.65 \\
3.70 \\
3.85 \\
3.95 \\
3.80 \\
3.75 \\
3.65\end{array}$ & $\begin{array}{l}3.70 \\
3.70 \\
3.70 \\
3.75 \\
3.90 \\
4.10 \\
3.95 \\
3.85 \\
4.10 \\
4.00 \\
3.85 \\
3.80 \\
3.80 \\
3.80 \\
3.80 \\
4.00\end{array}$ & $\begin{array}{l}4.05 \\
4.00 \\
3.95 \\
\text { 3. } 75 \\
3.75 \\
3.85 \\
3.90 \\
3.70 \\
3.75 \\
3.75 \\
3.70 \\
3.70 \\
3.70 \\
3.75 \\
3.65 \\
3.65\end{array}$ & 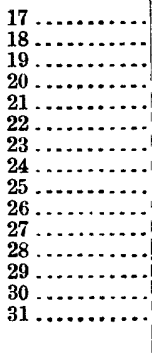 & $\begin{array}{l}3.70 \\
3.70 \\
3.70 \\
3.65 \\
3.55 \\
3.35 \\
3.40 \\
3.65 \\
3.65 \\
3.55 \\
3.70 \\
3.50 \\
3.40 \\
3.55 \\
3.35\end{array}$ & $\begin{array}{l}\text { 3. } 60 \\
3.60 \\
3.60 \\
\text { 3. } 60 \\
3.70 \\
3.20 \\
3.60 \\
3.50 \\
3.55 \\
3.50 \\
3.55 \\
3.50 \\
3.60 \\
3.40 \\
\ldots . . .\end{array}$ & $\begin{array}{l}3.70 \\
3.70 \\
3.65 \\
3.65 \\
3.70 \\
3.70 \\
3.70 \\
3.65 \\
3.75 \\
3.80 \\
3.70 \\
3.70 \\
3.70 \\
3.70 \\
3.70\end{array}$ & \begin{tabular}{|c|}
$\ldots \ldots .$. \\
$\ldots 3.90$ \\
3.70 \\
3.65 \\
3.70 \\
3.75 \\
3.70 \\
3.65 \\
3.50 \\
3.65 \\
3.65 \\
4.90 \\
4.20 \\
$\ldots \ldots .$.
\end{tabular} & $\begin{array}{l}3.65 \\
3.70 \\
3.65 \\
3.45 \\
3.65 \\
3.65 \\
3.65 \\
3.60 \\
3.50 \\
3.65 \\
3.65 \\
3.85 \\
3.85 \\
3.50 \\
3.75\end{array}$ \\
\hline
\end{tabular}

Patuxent River at Laurel, Maryland.

\begin{tabular}{|c|c|c|c|c|c|c|c|c|c|c|c|}
\hline Day. & Aug. & Sept. & Oet. & Nov. & Dec. & Day. & Ang. & Sept. & Oct. & Nov. & Dec. \\
\hline 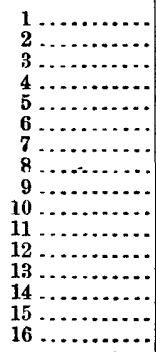 & $\begin{array}{l}\cdots \\
4.30 \\
4.30 \\
4.10 \\
4.20 \\
4.00 \\
4.00 \\
3.50 \\
3.90 \\
4.30 \\
4.10 \\
4.10 \\
4.30 \\
4.50 \\
3.60\end{array}$ & $\begin{array}{l}4.20 \\
4.00 \\
4.00 \\
3.50 \\
4.20 \\
4.50 \\
4.00 \\
3.30 \\
3.30 \\
4.30 \\
2.30 \\
4.50 \\
2.20 \\
2.90 \\
4.50 \\
4.70\end{array}$ & $\begin{array}{l}4.75 \\
4.30 \\
3.80 \\
3.20 \\
3.95 \\
3.75 \\
3.80 \\
3.75 \\
3.75 \\
3.80 \\
3.10 \\
3.90 \\
3.80 \\
3.90 \\
3.80 \\
3.80\end{array}$ & $\begin{array}{l}3.15 \\
4.35 \\
3.05 \\
4.40 \\
4.60 \\
4.65 \\
4.80 \\
3.25 \\
4.80 \\
4.45 \\
4.70 \\
4.50 \\
4.60 \\
4.45 \\
3.55 \\
4.60\end{array}$ & $\begin{array}{l}4.75 \\
4.85 \\
4.65 \\
4.70 \\
4.75 \\
3.95 \\
4.85 \\
4.75 \\
4.75 \\
4.70 \\
4.55 \\
4.65 \\
3.85 \\
4.60 \\
4.65 \\
4.60\end{array}$ & 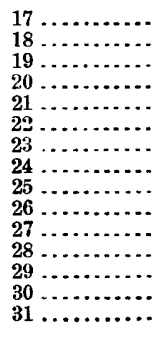 & $\begin{array}{l}4.60 \\
4.00 \\
4.10 \\
4.60 \\
4.60 \\
4.40 \\
3.60 \\
4.40 \\
4.60 \\
4.60 \\
4.50 \\
4.50 \\
4.40 \\
2.70 \\
4.70\end{array}$ & $\begin{array}{l}4.50 \\
3.75 \\
4.35 \\
5.10 \\
3.90 \\
3.75 \\
3.65 \\
3.80 \\
3.70 \\
3.80 \\
3.05 \\
3.70 \\
3.70 \\
4.85 \\
\ldots \ldots . .\end{array}$ & $\begin{array}{l}3.80 \\
3.10 \\
3.85 \\
3.90 \\
4.45 \\
4.30 \\
4.45 \\
4.40 \\
3.25 \\
4.60 \\
4.55 \\
4.15 \\
3.95 \\
4.10 \\
3.95\end{array}$ & $\begin{array}{c}4.50 \\
4.40 \\
4.50 \\
4.50 \\
4.50 \\
3.00 \\
4.65 \\
4.30 \\
4.30 \\
3.15 \\
4.55 \\
4.20 \\
5.50 \\
5.00 \\
\ldots . . .\end{array}$ & $\begin{array}{l}4.40 \\
4.55 \\
4.40 \\
3.20 \\
4.60 \\
4.50 \\
4.30 \\
3.85 \\
4.15 \\
4.25 \\
4.05 \\
4.25 \\
4.70 \\
4.65 \\
4.90\end{array}$ \\
\hline
\end{tabular}

Potomac River at Cumberland, Maryland.

\begin{tabular}{|c|c|c|c|c|c|c|c|c|c|c|c|c|}
\hline Day. & Jan. & Feb. & Mar. & Apr. & May. & J une. & July. & Aug. & Sept. & Oct. & Nov. & Dec. \\
\hline & 2.90 & 4. 60 & 3.90 & 5.00 . & 4. 50 & 3.60 & 3.10 & 3.80 & 2. 60 & 5.50 & 3. 10 & 3. 70 \\
\hline & 2.90 & 4. 80 & 3. 70 & 4. 80 & 4. 50 & 3.50 & 3. 10 & 3.80 & 2.70 & 4.50 & $\overline{3} .10$ & 3.50 \\
\hline 3. & 3.00 & 4.00 & 3.00 & 4.40 & 4.50 & 3.40 & 3.10 & 3.80 & 2.70 & 4. 50 & 3. 10 & 3. 40 \\
\hline & 2.90 & 3. 80 & 3.30 & 4.20 & 4. 50 & 3.50 & 3.10 & 3.70 & 2.70 & 3. 50 & 3. 20 & 3. 30 \\
\hline & 2.90 & 3.80 & 3.10 & 3.80 & 3. 40 & 3. 30 & 3.10 & 3.30 & 2.70 & 3.40 & 3. 20 & 3. 30 \\
\hline & 2.90 & 3.80 & 3.10 & 3.80 & 3. 30 & 3. 30 & 2. 90 & 3.30 & 2.80 & 3.30 & 5. 50 & 3.30 \\
\hline & 2.90 & 4.50 & 3. 10 & 3. 70 & 3. 10 & 3. 80 & 2.90 & 3.20 & 2. 90 & 3. 10 & 4. 50 & 3. 30 \\
\hline 8. & 2.90 & 3. 90 & 3. 60 & 3. 70 & 3. 00 & 3. 70 & 2.90 & 3. 20 & 2. 90 & 3. 10 & 3. 80 & 3. 20 \\
\hline 9. & 2. 90 & 3.00 & 3. 40 & 3.70 & 3.00 & 3.40 & 3.10 & 3.40 & 2. 90 & 3. 10 & 3. 80 & 3.30 \\
\hline 10. & 2.90 & 3.40 & 3. 30 & 3.70 & 3.00 & 3.40 & 3. 70 & 3.40 & 2. 90 & 3.00 & 3. 70 & 3. 30 \\
\hline 11. & 2.90 & 3. 10 & 3. 30 & 3. 70 & 2. 90 & 3.40 & 3. 40 & 3.30 & 2. 80 & 3.00 & 3. 60 & 3.40 \\
\hline 12. & 3.00 & 3. 30 & 3.40 & 4. 30 & 2.90 & 3.40 & 3. 30 & 3.30 & 2. 70 & 3.10 & 3. 50 & 3. 60 \\
\hline 13. & 3.00 & 4. 90 & 3. 10 & 4. 30 & 3.50 & 3.20 & 3.10 & 3.10 & 2.90 & 3.10 & อิ. 50 & 3. 40 \\
\hline 14. & 3.00 & 4. 10 & 3.10 & 4. 10 & 3.50 & 3.20 & 3.00 & 3.60 & 3. 00 & 3.00 & 3.40 & 3. 30 \\
\hline 15. & 3.00 & 3.80 & 3. 10 & 3.90 & 3. 30 & 3.30 & 3.00 & 3.50 & 3.10 & 3. 00 & 3. 20 & 3. 30 \\
\hline 16. & 3. 00 & 3. 80 & 3.20 & 3. 80 & 3. 20 & 3. 30 & 3. 00 & 3.30 & 3.50 & 3. 00 & 3. 10 & 3. 30 \\
\hline 17 & 3.00 & 3. 50 & 3.30 & 3.80 & 3. 10 & 3. 70 & 3. 00 & 3. 10 & 3. 00 & 3.00 & 3. 10 & 3. 30 \\
\hline 18. & 3. 00 & 3. 50 & 3.30 & 3. 70 & 3.40 & 4. 10 & 3.30 & 3.00 & 3. 00 & 3.00 & 3. 20 & 3. 30 \\
\hline 19. & 2.90 & 3. 20 & 3.50 & 3.50 & 3.50 & 3.70 & 3. 10 & 2.90 & 3. 00 & 2. 90 & 3. 30 & 3. 30 \\
\hline 20. & 2.90 & 3.20 & 3.60 & 3. 50 & 3. 80 & 3. 60 & 3.10 & 2. 90 & 2. 90 & 2. 90 & 3. 30 & 3.30 \\
\hline 21 . & 3.00 & 3.20 & 3.70 & 3. 50 & 3. 80 & 3. 30 & 3. 30 & 3.00 & 3.00 & 3.10 & 3. 20 & 3. 20 \\
\hline 22 . & 3.00 & 3. 20 & 3.70 & 3.50 & 3.60 & 3. 30 & 4.00 & 3.00 & 3. 10 & 3. 10 & 3. 30 & 3. 20 \\
\hline 23. & 3.00 & 3.30 & 4. 00 & 3.30 & 3. 40 & 3.20 & 4. 70 & 3.00 & 3. 10 & 3.10 & 3. 30 & 3. 20 \\
\hline 24 & 3.10 & 3. 10 & 3. 90 & 3.40 & 3.40 & 3.50 & 4.60 & 3. 20 & 3. 00 & 3. 30 & 3. 40 & 3. 20 \\
\hline 25. & 3.50 & 3. 30 & 3. 70 & 3. 50 & 3. 50 & 3.50 & 10.00 & 3.00 & 3. 00 & 3.70 & 3. 30 & 3. 20 \\
\hline 26. & 3. 30 & 3. 30 & 4.00 & 3.50 & 3.50 & 3. 60 & 6.50 & 2. 90 & 3. 00 & 3. 50 & 3. 30 & 3. 20 \\
\hline & 3.10 & 3.30 & 5.00 & 3.50 & 4. 00 & 3.50 & 4.70 & 2.90 & 3. 00 & 3. 20 & 3. 20 & 3.10 \\
\hline & 3.00 & 3. 20 & 4. 50 & 3. 50 & 3. 70 & 3. 30 & 4. 90 & 2.90 & 2.90 & 3.20 & 3. 20 & 3. 10 \\
\hline & 3.00 & 3.70 & 5.80 & 3. 50 & 4. 10 & 3. 30 & 4. 50 & 2.90 & 2. 90 & 3. 10 & 3.90 & 3. 10 \\
\hline 30. & 3.70 & ..... & 8.00 & 3.40 & 4. 00 & 3. 20 & 4. 50 & 2.90 & 10.00 & 3. 10 & 4.00 & 3. 10 \\
\hline 31. & 4. 20 & $\ldots .$. & 6. 00 & $\ldots .$. & 3.80 & -. & 4.50 & $\ldots \ldots$ & $\ldots \ldots$ & 3.10 & $\ldots$ & 3.20 \\
\hline
\end{tabular}


North River at Port Republic, Tirginia.

\begin{tabular}{|c|c|c|c|c|c|c|c|c|c|c|c|c|}
\hline Day. & Jan. & Feb. & Mar. & Apr. & May. & June. & July. & Aug. & Sept. & Oct. & Nor. & Dec. \\
\hline & 3.00 & 2.50 & 2. 60 & 5. 40 & 2.32 & 2. 42 & 2.80 & 2.43 & 2.00 & 7.50 & 2. 35 & 4.40 \\
\hline & 2. 70 & 2.50 & 2.60 & 5.00 & 2.52 & 2. 32 & 2.70 & 2.43 & 1. 95 & 4.50 & 2.35 & 3.50 \\
\hline & 2.50 & 2.80 & 2.60 & 4. 60 & 2.52 & 2.32 & 2. 70 & 2.55 & 1.90 & 4.00 & 2. 35 & 3.30 \\
\hline & 2. 40 & 3.80 & 2.60 & 4. 20 & 3.32 & 2.22 & 2.60 & 2.45 & 1.90 & 3.50 & 2.35 & 3.30 \\
\hline & 2.40 & 3.80 & 2. 60 & 4.00 & 3. 32 & 220 & 2.60 & 2.40 & 1.90 & 3. 30 & 4. 70 & 3. 25 \\
\hline & 2.40 & 5.00 & 2.50 & 3. 60 & 3. 32 & 2.20 & 2. 80 & 2.40 & 1. 85 & 3.20 & 6.00 & 3.10 \\
\hline & 2.40 & 5. 70 & 2.50 & 3. 50 & 3. 12 & 4.50 & 2.80 & 2. 30 & 185 & 3. 10 & 4. 30 & 3. 05 \\
\hline & 2. 30 & 4. 50 & 2. 50 & 3.50 & 2. 92 & 3.00 & 2.80 & 2. 30 & 1. 80 & 3. 00 & 3.60 & 2. 90 \\
\hline 9. & 2.30 & 4. 10 & 2.50 & 3. 40 & 2. 72 & 2.60 & 7. 50 & 2. 30 & 1. 80 & 2.90 & 3. 30 & 2.80 \\
\hline 10. & 2. 30 & 3. 70 & 2.50 & 3. 30 & 2.72 & 2.50 & 5.30 & 2.25 & 1. 75 & 2.80 & 3.30 & 2. 70 \\
\hline 11. & 2. 30 & 3.50 & 2. 40 & 3.30 & 2.72 & 2.30 & 3. 90 & 2.20 & 1.70 & 2.65 & 3. 20 & 2.65 \\
\hline 12 . & 2.30 & 3. 30 & 2.40 & 3. 20 & 2.62 & 2. 30 & 3. 60 & 2.20 & 1. 70 & 2.55 & 3. 00 & 2. 60 \\
\hline 13. & 2.30 & 3.30 & 2. 40 & 3. 20 & 2. 52 & 2. 20 & 3. 30 & 2. 20 & 1.70 & 2.50 & 2.80 & 2. 55 \\
\hline 14. & 2.20 & 5. 20 & 2. 40 & 3.00 & 5. 82 & 2.20 & 3.00 & 3.60 & 1. 70 & 2.50 & 2. 80 & 2. 55 \\
\hline 15. & 2.20 & 4. 70 & 2.60 & 3.00 & 3.82 & 2.20 & 3. 30 & 2.70 & 2.00 & 2. 50 & 2. 70 & 2.50 \\
\hline 16. & 2.10 & 3.80 & 2.60 & (a) & 3. 22 & 2.20 & 3.00 & 2.70 & 1. 90 & 2.50 & 2.70 & 2. 50 \\
\hline 17. & 2.10 & 3.60 & 2.80 & $\ldots$. & 3. 02 & 2. 50 & 3.10 & 3.00 & 1.80 & 2.50 & 2.65 & 2.45 \\
\hline 18 & 2. 00 & 3.10 & 3.20 & $\ldots \ldots$ & 2.82 & 2.40 & 2.70 & 2.40 & 1.80 & 2.45 & 2.65 & 2.45 \\
\hline & 2. 00 & 3.00 & 4. 80 & $\ldots \ldots$ & 2. 62 & 2.40 & 2.70 & 2. 30 & 1. 80 & 2. 45 & 2. 60 & 2.45 \\
\hline 20 & 3. 00 & 2.70 & 7.20 & & 2.62 & 3.30 & 2.70 & 2.25 & 1.70 & 2.40 & 2.60 & 2.45 \\
\hline 21 . & 2.00 & 2.70 & 5. 00 & & 2.62 & 3. 00 & 2.60 & 2.25 & 1. 70 & 2.40 & 2. 55 & 2.45 \\
\hline 22. & 2.00 & 2.70 & 4.80 & & 2.62 & 2.90 & 2.50 & 2.25 & 1. 70 & 2.35 & 2. 50 & 2.45 \\
\hline 23. & 2.10 & 2. 70 & 4.00 & & 2. 62 & 2.50 & 2.50 & 2.20 & 1.70 & 2. 35 & 2.50 & 2. 45 \\
\hline 24. & 6.15 & 2. 70 & 3.80 & & 2.52 & 2.40 & 2.50 & 2. 20 & 1. 65 & 2. 35 & 2.45 & 2. 45 \\
\hline 25 . & 5. 30 & 2.70 & 3.50 & & 2. 52 & 2.40 & 3.00 & 2.40 & 1. 65 & 2.35 & 2. 40 & 2. 40 \\
\hline 26. & 4.00 & 2.60 & 3. 50 & & 2.52 & 3.20 & 2.70 & 2.20 & 1.65 & 2. 35 & 2.40 & 2.40 \\
\hline & 3.30 & 2.60 & 3.50 & & 2.52 & 3.00 & 2.70 & 2. 20 & 1. 65 & & 2.35 & 2. 40 \\
\hline & 3.00 & 2.60 & 3.50 & $b 2.22$ & 2.42 & 3.00 & 2.60 & 2. 15 & 1.65 & 2. 35 & 2. 35 & 2. 40 \\
\hline & 2. 80 & 2.60 & 4. 40 & 2.22 & 2.42 & 2.90 & 2. 60 & 2.15 & T. 21 & 2. 35 & 3.00 & 2.40 \\
\hline & 2.60 & $\ldots$. & 6.50 & 2. 22 & 2.42 & 2.90 & 2.50 & 2.10 & 18.00 & 2.35 & 5.50 & 2. 40 \\
\hline 31 & 2.50 & $\ldots .$. & 5. 90 & ...... & 2.42 & ..... & 2.45 & 2.10 & $\ldots$. & 2.35 & $\ldots \ldots$ & 2. 35 \\
\hline
\end{tabular}

$a$ Gage carried away.

$b$ New gage.

South River at Port Republic, Virginia.

\begin{tabular}{|c|c|c|c|c|c|c|c|c|c|c|c|c|}
\hline Day. & Jan. & Feb. & Mar. & Apr. & May. & June. & July. & Aug. & Sept. & Oet. & Nov. & Dec. \\
\hline & 1. 80 & 2.00 & 2.00 & 3.20 & 1. 70 & 1.60 & 1.80 & 1.50 & 1.40 & 5.00 & 1.60 & 2.70 \\
\hline & 1.80 & 2.00 & 2.00 & 3.00 & 1. 70 & 1.50 & 1.80 & 1. 50 & 1. 40 & 3.30 & 1.60 & 2.55 \\
\hline & 1.80 & 2.30 & 2.00 & 2.80 & 2. 20 & 1.50 & 1.70 & 1,50 & 1.40 & 3.00 & 1.60 & 2.40 \\
\hline & 1.70 & 2.50 & 2.00 & 2.70 & 2.20 & 1. 50 & 1. 60 & 1. 50 & 1.40 & 2.75 & 1.60 & 2.40 \\
\hline & 1.60 & 2.50 & 2.00 & 2.50 & 2.20 & 1. 50 & 1. 60 & 1.50 & $\begin{array}{l}1.40 \\
1.40\end{array}$ & 2.40 & 4. 10 & 2.35 \\
\hline & 1.50 & 3.15 & 1.90 & 2.50 & 2.20 & 1.50 & 1. 70 & 1.45 & 1.40 & 2.30 & 3,50 & 2.10 \\
\hline & 1.40 & 3.60 & 1.90 & 2.40 & 2.10 & 2.50 & 1. 70 & 1.45 & 1.40 & 2.30 & 3. 00 & 2,10 \\
\hline 8. & $\begin{array}{l}1.40 \\
1.40\end{array}$ & 3.10 & 1.90 & 2.30 & 2.00 & 2.50 & 1.70 & 1.40 & 1. 40 & 2.20 & 2.60 & 2.00 \\
\hline 9. & 1.30 & 3.10 & 1.90 & 2.30 & 1.90 & $\cdot 2.50$ & 4.80 & 1.40 & 1.40 & 2.10 & 2.40 & 1.90 \\
\hline 10. & 1. 30 & 2.70 & 1.90 & 2.20 & 1. 90 & 2.00 & 3.20 & 1. 40 & 1.40 & 2.00 & 2.30 & 1.80 \\
\hline 11 . & 1.30 & 2.50 & 1.85 & 2.20 & 1. 90 & 1.80 & 2.70 & 1. 40 & 1.40 & 2.00 & 2.15 & 1.70 \\
\hline 12. & 1. 30 & 2.30 & 1.85 & 2.20 & 1. 80 & 1. 80 & 2.50 & 1. 40 & 1.40 & 1.95 & 2. 15 & 1.60 \\
\hline 13. & 1. 30 & 2.30 & 1. 85 & 2.20 & 1. 70 & 1. 70 & 2.40 & 1. 40 & 1.35 & 1. 95 & 2. 10 & 1.50 \\
\hline 14. & 1.20 & 2.80 & 1. 85 & 2. 15 & 3.00 & J. 60 & 2.30 & 200 & 1. 35 & 1.90 & 2. 10 & 1.50 \\
\hline 15. & 1.20 & 2.70 & 2.00 & 2.15 & 2.00 & 1. 60 & 2.30 & 1. 60 & 1. 35 & 1.85 & 2.00 & 1.50 \\
\hline 16. & 1.20 & 2.50 & 2.00 & 2.10 & 1. 80 & 1. 60 & 2.00 & 1. 60 & 1. 35 & 1.85 & 2.00 & 1.45 \\
\hline 17. & 1. 20 & 2.40 & 2.20 & 2.00 & 180 & 2.50 & 2.00 & 1. 60 & 1. 35 & 1.85 & 1. 90 & 1. 45 \\
\hline 18. & 1. 20 & 2.30 & 2.20 & 2. 00 & 1.70 & 2. 20 & 1.70 & 1.50 & 1.35 & 1. 85 & 1.90 & 1.45 \\
\hline 19 & 1.20 & 2. 30 & 2. 80 & 1. 90 & 1. 60 & 2.20 & 1. 80 & 1.50 & 1.35 & 1.85 & 1.85 & 1,45 \\
\hline 20. & 1.20 & 2.20 & 4. 30 & 1. 90 & 1.60 & 2.50 & 1. 80 & 1. 50 & 1. 35 & 1.80 & 1.85 & 1. 45 \\
\hline 21 . & 1. 20 & 2.20 & 3.00 & 1.90 & 1.60 & 2.00 & 1. 70 & 1. 50 & 1.35 & 1. 75 & 1.80 & 1.45 \\
\hline 23 & 1. 20 & 2.20 & 2.60 & 1.80 & 1. 60 & 2. 00 & 1.70 & 1. 50 & 1.30 & 1. 70 & 1. 80 & 1.45 \\
\hline 23. & 1.30 & 2.10 & 2. 60 & 1.80 & 1. 60 & 1.80 & 1.70 & 1. 50 & 1.30 & 1.65 & 1.80 & 1.45 \\
\hline 24. & 4. 90 & 2.10 & 2. 50 & 1. 80 & 1. 60 & 1. 70 & 1.70 & 1. 50 & 1. 25 & 1,65 & 1.80 & 1. 45 \\
\hline 25. & 3. 60 & 2.10 & 2.50 & 2.00 & 1.60 & 1.70 & 1. 70 & 1. 65 & 1.25 & 1.65 & 1. 75 & 1.45 \\
\hline & 3.00 & 2.00 & 2. 40 & 2.00 & 1. 60 & 2.00 & 1. 70 & 1.50 & 1. 25 & 1.65 & 1. 70 & 1. 45 \\
\hline & 2. 70 & 2.00 & 2. 40 & 1. 90 & 1.60 & 2. 00 & 1. 70 & 1.50 & 1. 25 & & 1. 65 & 1.45 \\
\hline & 2.40 & 2.00 & 2.40 & 1. 80 & 1.60 & 2. 00 & 1. 70 & 1.50 & 1.25 & 1.60 & 1. 65 & 1.45 \\
\hline & 2. 30 & 2.00 & 2.50 & 1.75 & 1.60 & 1. 90 & 1. 60 & 1. 50 & 9.69 & 1.60 & 2.00 & 1.45 \\
\hline & ०.2. & ..... & 3.30 & 1. 70 & 1.60 & 1.90 & 1.60 & 1.45 & 17.00 & 1. 60 & 3.10 & 1.45 \\
\hline & 2.10 & . & 3. 20 & $\ldots . .$. & 1.60 & $\begin{array}{c}1.80 \\
\ldots \ldots\end{array}$ & 1.50 & 1.45 & $\begin{array}{l}11.00 \\
\ldots . . .\end{array}$ & 1. 60 & ....... & 1.45 \\
\hline
\end{tabular}


Shenandoah River at Millville, West Virginia.

\begin{tabular}{|c|c|c|c|c|c|c|c|c|c|c|c|c|}
\hline Day. & Jan. & Feb. & Mar. & Apr. & May. & June. & July. & Aug. & Sept. & Oct. & Nov. & Dec. \\
\hline 0 & & & & & & & & & & & & \\
\hline 1. & 1. 30 & 1.40 & 2.60 & 4. 60 & 1.40 & 0.90 & 1.60 & 1. 30 & 0.70 & 19.72 & 0.90 & 2.60 \\
\hline 2. & 1.90 & 1.60 & 2. 40 & 4. 20 & 1.40 & .90 & 1.60 & 1. 20 & .60 & 10.00 & .90 & 3.80 \\
\hline & 1.80 & 1.80 & 2.30 & 3.80 & 1.50 & .90 & 1.50 & 1. 10 & 60 & 5. 60 & .90 & 3. 10 \\
\hline 4. & 1. 80 & 2.00 & 2.00 & 3.60 & 1. 70 & .90 & 1.50 & 1. 00 & .60 & 4. 00 & 90 & 2. 60 \\
\hline 5. & 1. 70 & 2.60 & 1.70 & 3. 20 & 1.80 & .90 & 1. 50 & 1. 00 & 61 & 3.40 & 1.00 & 2. 00 \\
\hline 6. & 1. 60 & 2.90 & 1. 50 & 2.80 & 1. 90 & 80 & 1.50 & 1. 00 & 60 & 2.80 & 1. 10 & 2.00 \\
\hline 7. & 1.60 & 3.80 & 1. 40 & 2.60 & 2.40 & 1.00 & 1.60 & 1. 10 & .50 & 2.10 & 3.20 & 2. 00 \\
\hline & 1. 60 & 5. 60 & 1. 50 & 2.60 & 2.20 & 1. 40 & 1.60 & 1. 10 & .50 & 2.00 & 2.70 & 2. 00 \\
\hline & 1. 40 & 4.10 & 1. 50 & 2.00 & 2.00 & 1.90 & 3.15 & 1.00 & .50 & 1.90 & 2.40 & 2.00 \\
\hline 10. & 1. 30 & 3.70 & 1.50 & 2.00 & 2.00 & 2.70 & 4.65 & 1. 00 & .50 & 1.80 & 2. 10 & 2. 00 \\
\hline 11. & 1. 30 & 3. 20 & 1. 50 & 2.00 & 1. 90 & 2. 80 & 3.60 & 1.00 & .60 & 1. 80 & 1. 90 & 1.90 \\
\hline 12. & 1.20 & 2.90 & 1. 40 & 2.00 & 1. 70 & 2.40 & 2.50 & 1. 40 & .60 & 1. 60 & 1.90 & 1. 90 \\
\hline 13. & 1. 20 & 2. 50 & 1. 40 & 2.00 & 1.70 & 1.60 & 2.20 & 1. 60 & .60 & 1. 40 & 1. 90 & 1. 80 \\
\hline 14. & 1.00 & 2. 40 & 1. 40 & 1. 90 & 1. 60 & 1. 60 & 2. 20 & 1. 50 & .60 & 1. 40 & 1.90 & 1. 80 \\
\hline 15. & 1. 00 & 3.80 & 1.50 & 1. 70 & 2.40 & 1. 90 & 2. 20 & 1. 40 & .60 & 1. 40 & 1.90 & 1.70 \\
\hline 16. & 1.00 & 3.50 & 1. 50 & 1. 70 & 3.00 & 1. 90 & 2. 20 & 1.40 & 60 & 1. 40 & 1. 80 & 1. 70 \\
\hline 17. & 1.00 & 3.00 & 1. 60 & 1. 60 & 2.50 & 1. 80 & 2.10 & 1. 70 & 60 & 1. 30 & 1. 70 & 1.70 \\
\hline 18. & 1.00 & 2.70 & 3.00 & 1.60 & 2.40 & 1.90 & 2.00 & 1. 90 & 60 & 1.30 & 1.60 & 1. 70 \\
\hline 19. & 1.00 & 2.50 & 4. 20 & 1.60 & 2.40 & 2.20 & 2.00 & 1.30 & 60 & 1.30 & 1. 60 & 1. 70 \\
\hline 20. & 1.00 & 2.40 & 4. 60 & 1.50 & 2.20 & 2.00 & 1. 80 & 1. 20 & .80 & 1.20 & 1. 50 & 1. 60 \\
\hline $21 \ldots \ldots$ & .90 & 2.40 & 5. 50 & 1.50 & 1.80 & 1.90 & 1.60 & 1. 20 & .80 & 1.10 & 1.50 & 1.50 \\
\hline 22 . & .90 & 2. 30 & 4. 60 & 1.50 & 1.60 & 2.00 & 1.50 & 1.20 & .80 & 1.10 & 1. 50 & 1. 50 \\
\hline 23. & 90 & 2.20 & 4.00 & 1.50 & 1.50 & 2.20 & 4.10 & 1.00 & .70 & 1.00 & 1. 50 & 1. 40 \\
\hline 24 . & 2.40 & 2.20 & 3.50 & 1.50 & 1.60 & 2.00 & 3.00 & 1.00 & .60 & 1.00 & 1.50 & 1. 40 \\
\hline 25 & 5. 10 & 1. 80 & 3.00 & 1. 50 & 1. 50 & 1.90 & 3. 30 & 1.00 & 60 & 1.00 & 1. 40 & 1. 60 \\
\hline 26. & 5.30 & 1. 70 & 2.90 & 1.40 & 1.50 & 1.80 & 2.60 & .90 & .60 & 1.00 & 1.40 & 1. 60 \\
\hline 27. & 4.35 & 1.60 & 2. 80 & 1. 40 & 1. 50 & 1.80 & 2.00 & .90 & .60 & 1.00 & 1.40 & 1.60 \\
\hline & 3. 35 & 1.50 & 2. 70 & 1.40 & 1. 40 & 2. 20 & 1.70 & .80 & 60 & 1.00 & 1. 40 & 1.80 \\
\hline & 2.00 & 2.00 & 2. 70 & 1. 40 & 1. 40 & 2.30 & 1.50 & .80 & .60 & 1.00 & 1.40 & 1. 80 \\
\hline & 1. 90 & . . . . & 2.60 & 1. 40 & 1.40 & 1.90 & 1. 40 & .80 & 5.75 & 1.00 & 1. 60 & 1. 80 \\
\hline 31. & 1,70 & $\ldots$ & 4.60 & ....... & 1.10 & $\ldots$ & 1. 30 & .80 & .... & .90 & $\ldots \ldots$ & 1. 80 \\
\hline
\end{tabular}

Potomae River at Point of Rocks, Maryland.

\begin{tabular}{|c|c|c|c|c|c|c|c|c|c|c|}
\hline Day. & Jan. & Feb. & May. & June. & July. & Aug. & Sept. & Oet. & Nov. & Dec. \\
\hline 1. & 1. 30 & 1. 30 & & 1.10 & 1.60 & 2. 00 & 0.50 & 21.85 & 0.80 & 2. 00 \\
\hline 2 & 1.20 & 1. 20 & & .80 & 1.40 & 2. 20 & .50 & 12.05 & .80 & 3.00 \\
\hline 3. & 1.30 & 1. 30 & & .60 & 1. 20 & 2.50 & .40 & 6.30 & .70 & 2.80 \\
\hline $4 .$. & 1. 40 & 1.80 & $\ldots$ & .50 & 1.10 & 2.00 & .40 & 4.00 & .70 & 2.30 \\
\hline 5. & .40 & 2.80 & & .40 & 1.00 & 1. 80 & .40 & 3.00 & 80 & 2.00 \\
\hline $6 \ldots$ & 1.40 & 3.50 & 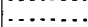 & .30 & 1. 30 & 1.60 & .50 & 2.40 & 6.00 & 1. 80 \\
\hline $7 \ldots$ & 1.30 & 6.80 & $\cdots$ & .60 & 1.20 & 1. 40 & 30 & 2.10 & 6.00 & 1. 70 \\
\hline $8 \ldots$ & 1.30 & 7.00 & . & .60 & 1.60 & 1.20 & 30 & 1.80 & 5. 10 & 1.70 \\
\hline $9 \ldots$ & 1.30 & 5.00 & & 1.60 & 1.90 & 1. 10 & .30 & 1.50 & 3.80 & 1.60 \\
\hline $10 \ldots$ & 1. 30 & 3.80 & $\ldots$ & 2.00 & 2.50 & 1.20 & .30 & 1. 30 & 3.00 & 1.50 \\
\hline $11 \ldots$ & 1. 00 & 3.90 & 0.80 & 2.20 & 4. 00 & 1. 10 & .40 & 1. 20 & 2.50 & 1. 70 \\
\hline $12 \ldots$ & .80 & 2.80 & .70 & 1. 70 & 3.80 & 1.00 & .30 & 1. 10 & 2.20 & 1. 70 \\
\hline 13. & .70 & 2.40 & .60 & 1.40 & 2.70 & 1.00 & .30 & 1.10 & 1.90 & $1.6 \mathrm{v}$ \\
\hline 14. & .60 & 2.30 & 60 & 1. 10 & 2.30 & 1.10 & .30 & 1.00 & 1. 70 & 1. 50 \\
\hline $15 \ldots$ & .50 & 2.70 & .50 & 1.00 & 2. 30 & 1. 10 & .30 & 1. 00 & 1. 50 & 1. 40 \\
\hline 16. & .50 & 3.90 & 1.60 & 1. 10 & 1.00 & 1. 10 & .80 & 1.00 & 1.50 & 1. 30 \\
\hline 17. & .50 & 3.50 & 1. 10 & 1.20 & 1. 50 & 1.20 & .50 & 1.00 & 1.40 & 1. 20 \\
\hline $18 . \ldots$ & .40 & 2.90 & 1.00 & 3.40 & 1. 40 & 1.00 & 40 & 1.00 & 1.30 & 1. 10 \\
\hline $19 \ldots$ & .30 & 2.30 & .80 & 3.20 & 1. 40 & .80 & .40 & .90 & 1. 20 & 1.00 \\
\hline $20 \ldots$ & .30 & 1.30 & 60 & 2.70 & 1. 30 & .70 & 70 & .40 & 1.10 & 1.00 \\
\hline $21 \ldots$ & .30 & .90 & .60 & 2.20 & 1.20 & .60 & .40 & .80 & 1.00 & 1.00 \\
\hline 22. & .50 & 1.10 & .70 & 1.80 & 1.10 & .60 & .50 & .80 & 1.00 & 100 \\
\hline $23 \ldots$ & .60 & 1.50 & 1.00 & 1. 80 & 1. 20 & .60 & .40 & .80 & 1.00 & 1.00 \\
\hline 24. & 2. 30 & 1.50 & 1. 00 & 1.80 & 1.60 & .60 & .40 & .70 & 1.00 & 1.00 \\
\hline $25 \ldots$ & 4.90 & 1.60 & .90 & 1. 60 & 3.30 & .60 & .30 & .70 & .90 & .90 \\
\hline 26 . & 4.80 & 1. 40 & .80 & 1. 70 & 8.70 & .50 & .40 & .90 & .90 & 1.10 \\
\hline $27 \ldots$ & 3. 70 & 1.30 & .70 & ' 1.90 & 6.00 & .50 & .40 & 1. 00 & .90 & 1.00 \\
\hline $28 \ldots$ & 2.90 & 1.20 & 60 & 2.30 & 3. 30 & .50 & 30 & .90 & 1. 00 & .70 \\
\hline & 2.10 & 1.80 & .80 & 2.00 & 2.70 & .60 & .40 & .90 & 1.00 & .70 \\
\hline & 1.60 & ....... & 1.00 & 1.80 & 2.80 & .60 & 5.30 & .80 & 1.20 & .80 \\
\hline $31 .$. & 1. 40 & & 1. 60 & ........ & 2. 40 & .50 & ..... & .80 & ..... & .90 \\
\hline
\end{tabular}


Monocacy River at Frederick, Maryland.

\begin{tabular}{|c|c|c|c|c|c|c|c|c|c|c|c|}
\hline Day. & Ang. & Sept. & Oet. & Nov. & Dec. & Day. & Aug. & Sept. & Oct. & Nov. & Dec. \\
\hline 1. & • & 3.60 & 4.80 & 3.90 & 4.80 & 17 & 3.80 & 3. 90 & 4.00 & 4. 20 & 4.00 \\
\hline 2. & . & 3. 60 & 4.40 & 4.00 & 4. 50 & 18 & 4. 40 & 3. 90 & 3. 90 & 4. 20 & 4. 00 \\
\hline & & 3. 60 & 4. 10 & 4. 00 & 4. 50 & ..... & 4. 15 & 4. 00 & 3. 90 & 4. 20 & 4.00 \\
\hline & 4.10 & 3.60 & 4. 00 & 4. 00 & 4. 30 & 20. & 3. 90 & 4. 00 & 3. 80 & 4. 20 & 4.00 \\
\hline & 4. 10 & 3.60 & 3.90 & 5. 45 & 4. 30 & $21 \ldots$ & 3. 90 & 4. 00 & 3.90 & 4.20 & 4.00 \\
\hline & 4.00 & 3.70 & 3. 90 & 7. 50 & 4. 10 & 22 & 3.90 & 3.90 & 3. 90 & 4.00 & 3. 90 \\
\hline & 4. 00 . & 3.80 & 3.90 & 5.00 & 4. 10 & 23. & 3. 80 & 3.90 & 3. 90 & 4. 00 & 3. 90 \\
\hline & 3. 90 & 3.90 & 3. 90 & 4. 50 & 4. 10 & 24. & 3. 70 & 3.80 & 3. 90 & 4. 00 & 4. 10 \\
\hline & 3.90 & 3.90 & 3. 90 & 4.50 & 4. 20 & 25 & 3.70 & 3. 90 & 3. 90 & 4. 00 & 4. 20 \\
\hline 10. & 3.90 & 3.80 & 3.90 & 4. 80 & 4. 20 & 26 & 3.90 & 3. 80 & 3. 90 & 4. 00 & 4. 10 \\
\hline & 3.80 & 3. 70 & 3.80 & 4. 50 & 4. 30 & 27 & 3.90 & 3. 80 & 3. 90 & 4. 00 & 4. 10 \\
\hline 12. & 3.90 & 3. 70 & 3.80 & 4. 50 & 4. 20 & 28. & 3.80 & 3. 80 & 3.90 & 4.00 & 4. 10 \\
\hline 13. & 3.90 & 3. 70 & 3. 90 & 4. 30 & 4. 10 & 29 & 3. 70 & 3.80 & 3.90 & 4.35 & 4. 00 \\
\hline 14. & 3.90 & 3.70 & 4.20 & 4. 30 & 4. 10 & 30 & 3. 60 & 5.00 & 3. 90 & 5. 20 & 4.00 \\
\hline 15. & 3. 90 & 3.70 & 4. 10 & 4. 20 & 4. 10 & $31 \ldots \ldots \ldots$ & 3. 70 & $\ldots \ldots$ & 3.90 & ...... & 4. 20 \\
\hline 16. & 3.80 & $a 3.75$ & 4. 10 & 4. 20 & 4. 10 & & & & & & \\
\hline
\end{tabular}

$a$ New gage.

North River at Glasgow, Virginia.

\begin{tabular}{|c|c|c|c|c|c|c|c|c|c|c|c|c|}
\hline Day. & Jan. & Feb. & Mar. & Apr. & May. & June. & July. & Aug. & Sept. & Oet. & Nov. & Dec. \\
\hline $1 \ldots$ & 2.50 & 1.80 & 1.90 & 5. $2 b$ & & 1.40 & 1.50 & 1.55 & 1,42 & 5.03 & $1.5 \mathrm{4}$ & 4. 13 \\
\hline 2. & 2.05 & 1. 50 & 1. 80 & $\ldots$. & N. & 1. 33 & 1.36 & 3.35 & 1. 38 & 3.70 & 1. 60 & 3. 42 \\
\hline 3. & 1.85 & 3.50 & 1.80 & 3.00 & . & 1. 25 & 1. 32 & 2.24 & 1. 40 & 2.90 & 1.60 & 2.50 \\
\hline & (a) & 2.80 & 1.76 & $\ldots .$. & $\ldots$ & 1. 30 & 1. 50 & 1. 90 & 1. 46 & 2.70 & 1. 60 & 2.50 \\
\hline & 1. 33 & $(a)$ & 1. 72 & $\ldots$ & 2.33 & 1. 28 & 1.60 & 1.76 & 1.35 & 2.60 & 4.75 & \\
\hline & 1.35 & 4. 20 & 1. 68 & 2.76 & 2.12 & 1. 23 & 1.40 & 1. 54 & 1. 35 & 2. 40 & 4. 85 & 2. 40 \\
\hline & (a) & 4.15 & 1. 63 & 2. 60 & 1.97 & 1. 23 & 3.22 & 1.62 & 1. 38 & 2. 30 & 3. 35 & 2. 30 \\
\hline & 1. 37 & 4. 35 & 1. 62 & 2.40 & ]. 86 & 1.24 & $(\alpha)$ & 1. 50 & 1. 35 & 2. 15 & 2. 90 & 2. 20 \\
\hline 9. & 1. 40 & $\ldots \ldots$ & 1.62 & 2.37 & 1.84 & 2.20 & 8.45 & 1. 44 & 1.33 & 2.08 & 2. 65 & 2. 15 \\
\hline 10. & 1.30 & 3.85 & 1. 60 & ..... & 1.84 & 1. 95 & 4.45 & 1. 36 & 1. 30 & 2. 00 & 2. 42 & 2. 11 \\
\hline 11. & 1.25 & 2. 60 & $(a)$ & & $\ldots$. & 1. 60 & 3.50 & 1. 32 & 1.28 & 1.96 & 2.30 & 2. 01 \\
\hline 12. & 1.22 & 2. 50 & 1. 66 & & & 1. 42 & & 1. 30 & 1. 26 & 1.90 & 2. 21 & 1. 94 \\
\hline 13. & 1.20 & 2.40 & 1. 60 & & 3. 40 & 1. 35 & 2. 78 & 1.50 & $\ldots$. & 1.90 & 2. 22 & \\
\hline 14. & 1.20 & 4. 40 & 1.58 & 2. 02 & 2.50 & 1.96 & 2.40 & $\cdots$ & ... & 1.88 & 2.11 & 1. 90 \\
\hline 15. & 1.18 & 4.00 & 1.60 & & 2.25 & 1. 55 & 2. 26 & 2.00 & $\ldots$. & 1.82 & 2.02 & 1.90 \\
\hline 16. & 1.15 & 3.05 & 2.85 & 1.94 & 1.82 & 1.50 & 2. 20 & 1. 72 & $\ldots$ & 1.82 & 2.05 & 1.84 \\
\hline 17. & 1. 12 & 2. 50 & 4.93 & & . . . . & ]. 40 & 2.52 & & 1.00 & 1.80 & 2.01 & 1.80 \\
\hline 18. & 1.12 & 2.25 & 3.65 & 1.90 & ... & 1. 50 & 2. 00 & 1.72 & 1,00 & 1.75 & 2. 02 & 1.80 \\
\hline 19. & 1.15 & 2. 24 & 4. 20 & 1.87 & $\ldots$ & 1. 62 & 1.82 & 1. 60 & 1.00 & 1. 71 & 2.00 & 1.78 \\
\hline 20. & 1. 19 & 2.14 & 5.25 & ..... & 2.08 & 1.96 & 1. 75 & 1.52 & .98 & 1. 70 & 2. 01 & 1. 75 \\
\hline 21. & 1.17 & 2. 05 & 4. 00 & & 2.00 & (a) & 1.72 & 1.48 & .95 & 1. 71 & 1.95 & 1.70 \\
\hline 22. & 1.12 & 1.95 & 3.12 & 1.80 & 1.74 & 1. 46 & 1.65 & 1. 40 & .95 & 1. 68 & $\cdots$ & 1. 70 \\
\hline 23. & (a) & 1. 90 & $(a)$ & $\ldots$ & 1.82 & 2. 08 & 2.25 & 2. 37 & .96 & 1. 70 & $\cdots$ & 1.65 \\
\hline 24. & 4. 95 & 1.86 & 2.90 & 1.77 & 1.61 & 1. 47 & 1. 83 & 1.54 & .90 & 1. 70 & $\ldots$ & 1. 60 \\
\hline & 3. 76 & 1. 80 & 2. 80 & $\ldots$ & 1. 60 & 1. 47 & 1.80 & 2.25 & .90 & 1. 63 & 1. 67 & 1.58 \\
\hline 26. & 2. 88 & 1.75 & 2. 77 & 1. 75 & 1.61 & 2. 26 & 1.67 & 1. 82 & .93 & 1. 67 & $\ldots \ldots$ & 1.56 \\
\hline & 2.42 & 1.70 & 2. 61 & ..... & 1.59 & 2. 58 & 1.60 & 1. 70 & .91 & 1. 60 & $\cdots$ & 1.56 \\
\hline 28. & 2.20 & 1.70 & 2.51 & $\ldots$ & 1.63 & 2.00 & 1.50 & 1.60 & .90 & 1.63 & $\ldots$ & 1.60 \\
\hline & 2.00 & I. 90 & 3. 05 & $\ldots$ & 1.61 & 1.74 & 1. 50 & 1.50 & 4.04 & 1.63 & 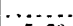 & 1. 60 \\
\hline & 1.90 & $\ldots .$. & 6. 72 & $\ldots$ & 1. 56 & 1.61 & 1.60 & & 16.09 & 1. 62 & 5.60 & 1. 50 \\
\hline 31. & 1.80 & & 5. 41 & & 1.45 & ....... & 1.90 & 1.42 & ...... & 1.62 & $\ldots \ldots$ & 1.55 \\
\hline
\end{tabular}

$a$ No observation.

Roanoke River at Raanoke, Virginia.

\begin{tabular}{|c|c|c|c|c|c|c|c|c|c|c|c|c|c|}
\hline Day. & July. & Aug. & Sept. & Oet. & Nov. & Dec. & Day. & July. & Aug. & Sept. & Oct. & Nor. & Dec. \\
\hline $\begin{array}{r}1 \ldots \ldots \\
2 \ldots \ldots \\
3 \ldots \ldots \\
4 \ldots \ldots \\
5 \ldots \ldots \\
6 \ldots \ldots \\
7 \ldots \ldots \\
8 \ldots \ldots \\
9 \ldots \ldots \\
10 \ldots \ldots \\
11 \ldots \ldots \\
12 \ldots \ldots \\
13 \ldots \ldots \\
14 \ldots \ldots \\
15 \ldots \ldots \\
16 \ldots \ldots\end{array}$ & 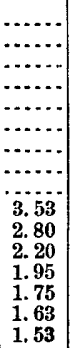 & $\begin{array}{l}0.80 \\
.90 \\
.90 \\
.90 \\
.90 \\
.85 \\
.70 \\
.70 \\
.70 \\
.70 \\
.90 \\
.80 \\
.70 \\
.75 \\
.80 \\
.74\end{array}$ & $\begin{array}{r}0.70 \\
.65 \\
.60 \\
.72 \\
.78 \\
.80 \\
.75 \\
.70 \\
.70 \\
.55 \\
.50 \\
.60 \\
.50 \\
.55 \\
.52 \\
.65\end{array}$ & $\begin{array}{r}1.95 \\
1.70 \\
1.40 \\
1.10 \\
1.00 \\
.95 \\
.90 \\
.85 \\
.85 \\
.80 \\
.80 \\
.75 \\
.80 \\
.85 \\
.80 \\
.75\end{array}$ & $\begin{array}{r}0.75 \\
.75 \\
.75 \\
.75 \\
2.60 \\
2.10 \\
1.95 \\
1.95 \\
1.90 \\
1.85 \\
1.82 \\
1.80 \\
1.70 \\
1.65 \\
1.50 \\
1.45\end{array}$ & $\begin{array}{r}2.00 \\
1.90 \\
1.90 \\
.85 \\
.82 \\
.82 \\
.80 \\
.80 \\
.78 \\
.78 \\
.76 \\
.75 \\
.75 \\
.75 \\
.75 \\
.75\end{array}$ & $\begin{array}{l}17 \ldots \ldots \\
18 \ldots \ldots \\
19 \ldots \ldots \\
20 \ldots \ldots \\
21 \ldots \ldots \\
22 \ldots \ldots \\
23 \ldots \ldots \\
24 \ldots \ldots \\
25 \ldots \ldots \\
26 \ldots \ldots \\
27 \ldots \ldots \\
28 \ldots \ldots \\
29 \ldots \ldots \\
30 \ldots \ldots \\
31 \ldots \ldots\end{array}$ & $\begin{array}{l}1.55 \\
1.50 \\
1.45 \\
1.40 \\
1.40 \\
1.38 \\
1.50 \\
1.33 \\
1.30 \\
1.25 \\
1.20 \\
1.20 \\
1.18 \\
.75 \\
.85\end{array}$ & $\begin{array}{r}0.80 \\
.69 \\
.62 \\
.62 \\
.60 \\
.65 \\
.75 \\
1.03 \\
.99 \\
.81 \\
.75 \\
.75 \\
.75 \\
.60 \\
.75\end{array}$ & $\begin{array}{r}0.60 \\
.59 \\
.50 \\
.55 \\
.60 \\
.55 \\
.50 \\
.50 \\
.45 \\
.50 \\
.50 \\
.55 \\
1.33 \\
3.40 \\
\ldots . . .\end{array}$ & $\begin{array}{l}0.75 \\
.75 \\
.75 \\
.75 \\
.75 \\
.75 \\
.75 \\
.75 \\
.75 \\
.75 \\
.75 \\
.75 \\
.75 \\
.75 \\
.75\end{array}$ & $\begin{array}{r}1.40 \\
1.30 \\
1.22 \\
1.10 \\
1.00 \\
.90 \\
.85 \\
85 \\
.80 \\
.75 \\
1.00 \\
1.30 \\
2.80 \\
2.45 \\
\ldots . . .\end{array}$ & $\begin{array}{r}0.90 \\
.95 \\
.90 \\
.85 \\
.85 \\
.85 \\
.82 \\
.82 \\
.80 \\
.80 \\
.80 \\
.80 \\
.80 \\
.75 \\
.75\end{array}$ \\
\hline
\end{tabular}


James River at Buchanan, Virginia.

\begin{tabular}{|c|c|c|c|c|c|c|c|c|c|c|c|c|}
\hline Day. & Jan. & Feb. & Mar. & Apr. & May. & June. & July. & Aug. & Sept. & Oet. & Nov. & Dec. \\
\hline 1. & $\cdots$ & 1.30 & 1. 30 & 7.85 & -0.60 & 0.60 & 0.60 & 1.00 & 0.10 & 5. 30 & -0.20 & 4.40 \\
\hline & $\cdots$ & 1.30 & 1.30 & 7.65 & -.60 & .60 & .40 & 1.50 & .00 & 2.60 & -.20 & 3.15 \\
\hline 3. & 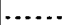 & 3.25 & 1.30 & 5. 05 & +1.30 & 50 & .30 & 1.30 & -.10 & 1.65 & -.20 & 2. 40 \\
\hline & & 5. 60 & 1.30 & 3.85 & 1.55 & .50 & .30 & 1.05 & -10 & 1.25 & -.20 & 1.85 \\
\hline & $\ldots \ldots$ & 3.90 & 1.30 & 2.90 & 1.05 & .35 & .30 & .90 & -.20 & .85 & +4.10 & 1.65 \\
\hline 6. & & 3.80 & 1.30 & 2.40 & .70 & .10 & .30 & .50 & -.20 & .65 & 7.20 & 1. 50 \\
\hline 7. & & 6.05 & 1.30 & 2.15 & .70 & 10 & .85 & .35 & -.20 & .50 & 3. 20 & 1. 40 \\
\hline 8. - & & 4. 35 & 1. 30 & 1.85 & .60 & 90 & 1. 00 & .40 & -.20 & .50 & 2. 35 & 1.25 \\
\hline $9 .$. & $\ldots$ & 3. 60 & 1. 30 & 1. 65 & .50 & 1. 30 & 10.25 & .25 & -.30 & .40 & 1.70 & 1.20 \\
\hline $10 \ldots$ & $\ldots .$. & 3.50 & 1. 30 & 1.35 & .30 & 1. 10 & 6.85 & .90 & -.30 & .30 & 1.40 & 1.10 \\
\hline $11 \ldots \ldots \ldots \ldots \ldots$ & $\ldots \ldots$ & 2.95 & 1.30 & 1.30 & .30 & 1.05 & 3.55 & .55 & -.30 & .30 & 1.15 & 1.00 \\
\hline $12 \ldots .$. & $\ldots \ldots$ & 2.30 & 1.30 & .50 & 20 & .70 & 2. 40 & .20 & -.30 & .15 & .90 & .90 \\
\hline $13 \ldots$. & ...... & 2.05 & 1.60 & .40 & 20 & .45 & 2.45 & .10 & -.30 & .10 & .80 & .90 \\
\hline $14 \ldots$ & $\ldots \ldots$ & 3.90 & 1.60 & 15 & 10 & 35 & 1.90 & .10 & -.30 & 10 & 80 & .80 \\
\hline 15 . & ..... & 4. 30 & 1.60 & 10 & 10 & .30 & 1.40 & .45 & -.30 & .10 & .80 & .70 \\
\hline 16. & & 3. 30 & 1.60 & 10 & 10 & .30 & 1.10 & .60 & -.30 & .10 & .70 & .60 \\
\hline 17. & & 2.90 & 5. 95 & .10 & .00 & .30 & 1.00 & .25 & -.30 & .10 & .70 & .50 \\
\hline 18. & & 2.30 & 5.45 & -.45 & .00 & .30 & .90 & .15 & -.30 & .10 & .60 & .50 \\
\hline 19. & ..... & 2. 00 & 4.50 & -.20 & .00 & .35 & .80 & .10 & -.30 & .00 & .60 & .50 \\
\hline 20 . & $\ldots .$. & 2.00 & 6.25 & +.05 & .00 & .70 & .55 & .10 & -.30 & .00 & .50 & .50 \\
\hline 21. & & 2.00 & 5.05 & .10 & .85 & .65 & .40 & -10 & --.30 & .00 & .50 & .40 \\
\hline 22. & & 2.00 & 3.80 & .10 & 1.15 & .45 & .40 & +.50 & -.30 & -.10 & .40 & .40 \\
\hline 23. & .. & 2.00 & 3.25 & .10 & .80 & .30 & .75 & .45 & -.30 & -.10 & .40 & .40 \\
\hline 24. & $\ldots \ldots$ & 1. 60 & 3.75 & .10 & .90 & .20 & .55 & .20 & -.30 & -.20 & .40 & .40 \\
\hline 25 & 4. 80 & 1.35 & 3. 90 & .10 & .90 & .20 & .40 & .20 & -.30 & -.20 & .40 & .40 \\
\hline 26 & 3.40 & 1.30 & 3.55 & $\ldots .30$ & .75 & 1.80 & .30 & .35 & -.30 & -.20 & .40 & .30 \\
\hline 27. & 2. 60 & 1. 30 & 3.40 & -.60 & .65 & 2.05 & .20 & .20 & -.30 & -.20 & 40 & .30 \\
\hline 28 & 2.00 & 1.30 & 3.40 & -.60 & .75 & 1.45 & .20 & .20 & -.30 & -.20 & .30 & .20 \\
\hline 29 & 1.65 & 1. 30 & 3.55 & -60 & .70 & 1. 15 & .20 & .20 & -.30 & -20 & 1. 30 & .20 \\
\hline 30. & 1.50 & $\ldots .$. & 9.45 & -60 & .60 & 1.85 & .70 & .20 & 12.30 & -.20 & 5. 35 & .20 \\
\hline 31. & 1. 30 & $\ldots .$. & 8.80 & $\ldots .$. & 60 & $\cdots$ & 70 & .10 & ....... & $\ldots$ & ....... & .20 \\
\hline
\end{tabular}

Dan River at Clarksville, Virginia.

\begin{tabular}{|c|c|c|c|c|c|c|c|c|c|c|c|}
\hline Das.. & Feb. & Mar. & Apr. & May. & June. & July. & Aug. & Sept. & Oet. & Nov. & Dec. \\
\hline & & & 9. 47 & 2.62 & 1.22 & 1.02 & 0.92 & 0.42 & 7.92 & 0.97 & 3.53 \\
\hline $2 \ldots$ & $\ldots \ldots$ & & 10.37 & 3.67 & 1. 17 & .92 & .87 & .40 & 8.97 & 1.02 & 4.82 \\
\hline $3 \ldots$ & $\ldots$ & . & 9.52 & 4.42 & 1.07 & .87 & .82 & .52 & 3.47 & 1.04 & 3.65 \\
\hline $4 \ldots$ & $\ldots . .$. & $\ldots . .$. & 5. 77 & 3.47 & .97 & .82 & 77 & 1.37 & 1. 72 & 1.47 & 2.55 \\
\hline & $\ldots$ & $\therefore$ & 3.99 & 2.38 & 1. 47 & .87 & .75 & 1. 52 & 1. 47 & 2.62 & 2.17 \\
\hline & & & 3.37 & 2.26 & 2.12 & 1. 02 & .72 & .97 & 1.32 & 6.92 & 2.05 \\
\hline 7. & 8.95 & $\ldots$ & 3.32 & 2.12 & 1. 97 & 4. 02 & .70 & 2.97 & 1.25 & 4. 97 & 1.97 \\
\hline 8. & 7.42 & & 2.97 & 1.97 & 1.47 & 6.02 & .67 & 1.82 & 1.17 & 2.47 & 1. 89 \\
\hline & 5.75 & ..... & 2.77 & 1.82 & 1. 47 & 9.12 & .65 & 1. 47 & 1.07 & 2.17 & 1.80 \\
\hline $10 \ldots$ & 5. 42 & ...... & 2.52 & 1. 72 & 1.97 & 13. 97 & .65 & .97 & 1.02 & 1. 77 & 1. 72 \\
\hline $11 \ldots$ & 4. 40 & $\ldots .$. & 2.51 & 1. 67 & 1.82 & 14. 07 & .87 & .82 & 1.02 & 1.57 & 1.62 \\
\hline $12 \ldots$ & 3.42 & ...... & 2.50 & 1. 62 & 1. 72 & 8.87 & 1. 65 & .62 & .97 & 1. 47 & 1. 56 \\
\hline $13 \ldots$ & 2.92 & ...... & 2.45 & 1. 57 & 1.67 & 5. 62 & 1. 77 & .57 & .95 & 1.57 & 1.51 \\
\hline $14 \ldots$ & 3.70 & ..... & 2.22 & 1. 54 & 1.62 & 3.22 & 1.92 & .55 & .95 & 1.52 & 1. 43 \\
\hline $15 \ldots$ & 3.47 & 1.90 & 2.17 & 1.47 & 1.62 & 2.67 & 1.97 & 57 & 1.02 & 1. 50 & 1. 53 \\
\hline $16 \ldots$ & 3. 32 & 1.87 & 2.12 & 1.02 & 1.60 & 2.47 & 2. 12 & .57 & .97 & 1. 48 & 1.72 \\
\hline 17 . & 2.97 & 2.45 & 2.07 & .97 & 1.57 & 2. 39 & 1. 47 & 62 & .96 & 1. 48 & 2.15 \\
\hline 18. & 2.40 & 2.68 & 2.02 & 1.02 & 1.47 & 2.27 & .97 & 59 & .92 & 1.25 & 1.97 \\
\hline 19. & 2.22 & 2.80 & 1.97 & 1.12 & 1.37 & 2.17 & 72 & .57 & .87 & 1. 22 & 1.72 \\
\hline 20 . & 2.07 & 4. 09 & 1. 92 & 162 & 1. 32 & 2.03 & .67 & 67 & .87 & 1.19 & $1.6 \overline{5}$ \\
\hline 21 & 1.92 & 4. 52 & 1.85 & 2. 18 & 1.47 & 1.85 & .65 & .92 & .85 & 1.16 & 1. 59 \\
\hline 22 . & 1.77 & 3.32 & 1.90 & 3. 07 & 1.57 & 1. 79 & .62 & 1.47 & .82 & 1.16 & 1.52 \\
\hline 23. & 1.97 & 2.72 & 1.90 & 4. 37 & 1.72 & 1. 70 & .62 & 1. 75 & .89 & 1.15 & 1.45 \\
\hline 24. & 2.10 & 2.49 & 1.82 & 3.17 & 2.47 & 1.57 & .62 & .72 & .92 & 1.14 & 1. 39 \\
\hline 25 . & 2.17 & 2.72 & 1.90 & 2.17 & 3. 02 & 1. 42 & .59 & 57 & .97 & 1.13 & 1.35 \\
\hline 26 . & 2.07 & 2.80 & 2. 47 & 1.50 & 3.53 & 1.35 & .67 & .52 & .94 & 1. 12 & 1.31 \\
\hline 27 & 1.96 & 2.47 & 2.40 & 2.32 & 3.67 & 1. 17 & .77 & 47 & .92 & 1. 11 & 1.29 \\
\hline 28. & 1. 99 & 2.27 & 2.00 & 2.97 & 2.67 & 1. 12 & .62 & .45 & .92 & 1.10 & 1.27 \\
\hline 29 . & 2. 17 & 2. 27 & 1. 90 & 2.32 & 2. 02 & 1.07 & .57 & 1.72 & .92 & 1.32 & 1.24 \\
\hline & $\ldots .$. & 3. 39 & 1. 77 & 1.82 & 1.52 & 1.02 & .47 & 5.47 & 91 & 2.02 & 1.22 \\
\hline 31 & $\ldots$ & 7.89 & $\cdots$ & 1.27 & $\ldots$ & .97 & & & & ....... & 1.22 \\
\hline
\end{tabular}


Staunton River at Clarksville, Virginia.

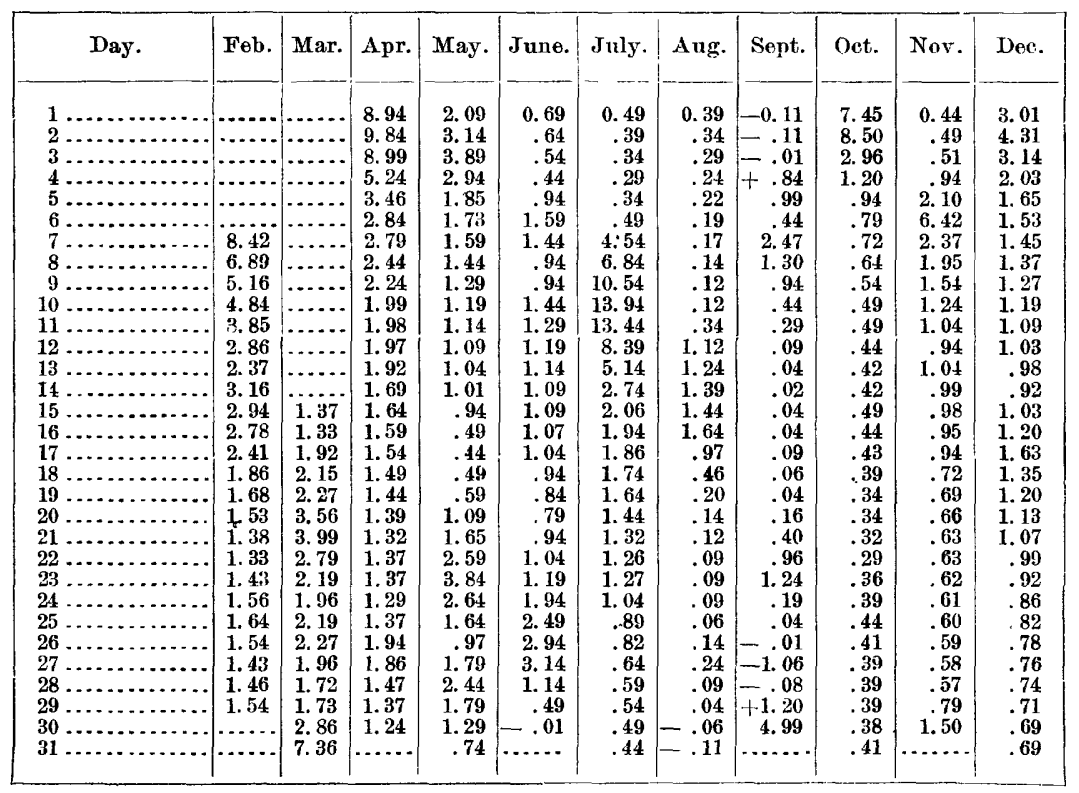

Roanoke River' at Clarksville, Virginia, for 1893.

\begin{tabular}{|c|c|c|c|c|c|c|c|c|c|c|c|c|}
\hline Day. & Jan. & Feb. & Mar. & Apr. & May. & June. & July. & Aug. & Sept. & Oet. & Nov. & Dec. \\
\hline 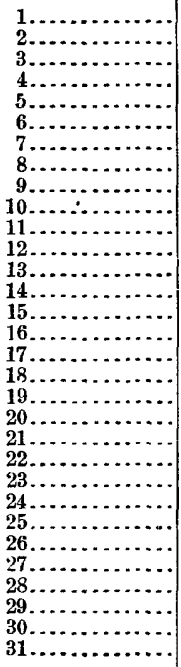 & \begin{tabular}{r}
3.1 \\
3.9 \\
2.7 \\
2.0 \\
1.5 \\
1.1 \\
1.1 \\
1.0 \\
1.0 \\
1.0 \\
1.0 \\
1.0 \\
1.0 \\
1.0 \\
1.0 \\
1.0 \\
2.5 \\
3.0 \\
$\ldots \ldots \ldots$ \\
$\ldots \ldots \ldots$ \\
\hdashline$\ldots \ldots$ \\
$\ldots \ldots \ldots$ \\
$\ldots \ldots \ldots$ \\
$\ldots \ldots \ldots$ \\
$\cdots \ldots \ldots$ \\
$\ldots \ldots \ldots$ \\
$\ldots \ldots \ldots$ \\
$\ldots \ldots \ldots$ \\
$\ldots \ldots \ldots$
\end{tabular} & 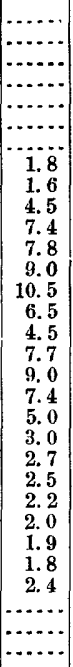 & $\begin{array}{l}2.6 \\
2.3 \\
3.0 \\
3.2 \\
3.3 \\
2.5 \\
2.2 \\
2.0 \\
2.2 \\
2.0 \\
2.0 \\
1.9 \\
1.8 \\
2.0 \\
1.8 \\
1.6 \\
1.5 \\
1.5 \\
1.4 \\
1.4 \\
1.4 \\
1.4 \\
1.3 \\
1.3 \\
1.3 \\
1.3 \\
1.3 \\
1.2 \\
1.2 \\
1.2 \\
1.2\end{array}$ & $\begin{array}{r}1.0 \\
1.0 \\
1.0 \\
1.0 \\
1.4 \\
1.3 \\
1.2 \\
1.1 \\
1.0 \\
1.0 \\
1.0 \\
1.0 \\
1.0 \\
1.3 \\
1.3 \\
1.1 \\
1.1 \\
1.0 \\
1.0 \\
1.0 \\
.9 \\
.9 \\
.9 \\
.9 \\
.9 \\
1.0 \\
2.7 \\
2.8 \\
2.3 \\
2.0 \\
\ldots . . .\end{array}$ & $\begin{array}{r}1.6 \\
1.5 \\
9.0 \\
13.0 \\
12.5 \\
4.5 \\
4.2 \\
2.8 \\
2.5 \\
2.2 \\
2.0 \\
1.7 \\
1.6 \\
1.5 \\
1.8 \\
3.0 \\
1.9 \\
1.5 \\
1.3 \\
1.1 \\
1.1 \\
1.1 \\
1.1 \\
1.1 \\
1.0 \\
1.0 \\
1.0 \\
1.9 \\
2.8 \\
2.1 \\
1.6\end{array}$ & $\begin{array}{r}1.4 \\
1.7 \\
1.5 \\
1.5 \\
1.5 \\
1.5 \\
1.6 \\
1.4 \\
1.0 \\
.9 \\
.8 \\
.7 \\
.9 \\
2.2 \\
4.4 \\
3.0 \\
2.1 \\
1.9 \\
1.7 \\
1.2 \\
1.0 \\
1.0 \\
1.6 \\
1.1 \\
.9 \\
.8 \\
.8 \\
.7 \\
1.3 \\
2.6 \\
\ldots . . .\end{array}$ & $\begin{array}{r}2.0 \\
1.9 \\
2.0 \\
1.6 \\
1.5 \\
1.0 \\
.7 \\
.6 \\
.8 \\
1.0 \\
.6 \\
.5 \\
.4 \\
.4 \\
.4 \\
.4 \\
.3 \\
.5 \\
.5 \\
.3 \\
.8 \\
.5 \\
.4 \\
.3 \\
.2 \\
.1 \\
.1 \\
.1 \\
0.0 \\
.1 \\
+.5\end{array}$ & $\begin{array}{r}1.5 \\
1.7 \\
2.0 \\
2.8 \\
2.0 \\
.8 \\
.5 \\
.4 \\
.2 \\
.1 \\
.1 \\
.1 \\
.3 \\
.3 \\
.2 \\
.1 \\
.0 \\
.0 \\
.1 \\
.0 \\
-.1 \\
-.3 \\
.0 \\
+2.5 \\
1.0 \\
.7 \\
.6 \\
5.0 \\
9.0 \\
11.0 \\
7.6\end{array}$ & $\begin{array}{r}9.0 \\
11.3 \\
9.0 \\
3.8 \\
2.8 \\
1.9 \\
1.5 \\
1.5 \\
1.4 \\
3.0 \\
6.5 \\
9.0 \\
11.0 \\
13.5 \\
11.1 \\
3.5 \\
2.5 \\
2.1 \\
1.9 \\
1.7 \\
1.6 \\
1.4 \\
1.4 \\
1.3 \\
1.2 \\
4.0 \\
3.5 \\
2.6 \\
1.8 \\
1.5 \\
\ldots . . .\end{array}$ & $\begin{array}{r}2.2 \\
2.8 \\
2.5 \\
7.7 \\
8.0 \\
4.5 \\
2.6 \\
2.2 \\
1.9 \\
1.7 \\
1.6 \\
1.5 \\
6.0 \\
8.8 \\
8.7 \\
4.0 \\
2.8 \\
2.3 \\
2.0 \\
1.8 \\
7.3 \\
9.9 \\
10.1 \\
10.5 \\
5.8 \\
3.8 \\
3.3 \\
3.0 \\
2.7 \\
2.4 \\
2.3\end{array}$ & $\begin{array}{r}2.1 \\
2.0 \\
1.9 \\
1.9 \\
1.9 \\
1.8 \\
1.8 \\
2.0 \\
1.9 \\
1.8 \\
1.8 \\
1.7 \\
1.6 \\
1.7 \\
2.0 \\
2.0 \\
1.8 \\
1.6 \\
1.6 \\
1.5 \\
1.7 \\
1.8 \\
1.8 \\
1.6 \\
1.5 \\
1.5 \\
1.6 \\
3.2 \\
3.0 \\
2.2 \\
2 . . .\end{array}$ & $\begin{array}{l}1.9 \\
1.8 \\
2.0 \\
2.5 \\
2.4 \\
2.4 \\
2.3 \\
2.3 \\
2.2 \\
2.0 \\
1.9 \\
1.8 \\
1.7 \\
1.6 \\
1.6 \\
3.6 \\
3.5 \\
2.4 \\
2.1 \\
1.9 \\
1.8 \\
1.6 \\
1.6 \\
1.5 \\
1.5 \\
1.5 \\
1.4 \\
1.4 \\
1.4 \\
2.1 \\
2.2\end{array}$ \\
\hline
\end{tabular}

1 Record kept by United States Weather Bureau. 
Roanoke River ${ }^{\prime}$ at Clarksville, Virginia, for 1894.

\begin{tabular}{|c|c|c|c|c|c|c|c|c|}
\hline Day. & Jan. & Feb. & Mar. & Apr. & May. & June. & July. & Aug. \\
\hline 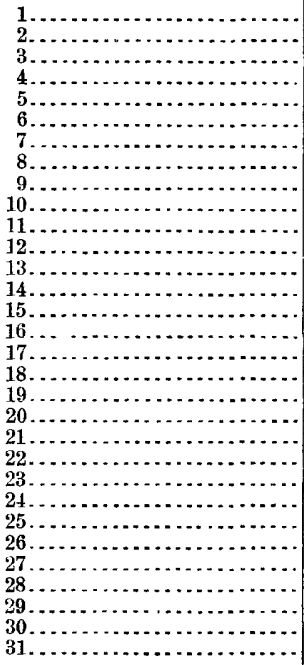 & $\begin{array}{l}1.8 \\
1.7 \\
1.6 \\
1.5 \\
1.5 \\
1.8 \\
3.2 \\
3.1 \\
2.8 \\
2.7 \\
3.3 \\
3.0 \\
2.5 \\
2.1 \\
2.0 \\
1.9 \\
1.8 \\
1.7 \\
1.6 \\
1.6 \\
1.6 \\
1.7 \\
1.7 \\
1.7 \\
1.6 \\
1.7 \\
1.7 \\
1.9 \\
4.0 \\
4.1 \\
3.3\end{array}$ & \begin{tabular}{|r|}
2.4 \\
2.0 \\
4.4 \\
7.0 \\
6.9 \\
3.4 \\
3.0 \\
3.2 \\
3.5 \\
3.4 \\
2.6 \\
4.0 \\
6.2 \\
6.2 \\
5.6 \\
4.5 \\
3.5 \\
2.9 \\
2.7 \\
2.4 \\
2.9 \\
3.1 \\
2.8 \\
2.4 \\
2.4 \\
2.8 \\
4.3 \\
4.7 \\
$\ldots \ldots \ldots$ \\
$\ldots \ldots .$. \\
$\ldots \ldots .$.
\end{tabular} & $\begin{array}{l}5.4 \\
5.8 \\
5.4 \\
4.0 \\
3.2 \\
3.0 \\
2.8 \\
2.6 \\
2.4 \\
2.3 \\
2.2 \\
2.1 \\
2.0 \\
1.9 \\
1.8 \\
1.8 \\
2.0 \\
2.1 \\
2.1 \\
1.9 \\
1.9 \\
1.9 \\
1.8 \\
1.9 \\
2.0 \\
1.9 \\
1.7 \\
1.6 \\
1.7 \\
1.7 \\
1.6\end{array}$ & $\begin{array}{r}1.7 \\
1.7 \\
1.7 \\
1.6 \\
1.5 \\
1.5 \\
1.4 \\
1.4 \\
1.4 \\
2.0 \\
2.3 \\
2.0 \\
1.7 \\
1.5 \\
1.5 \\
1.4 \\
1.4 \\
1.4 \\
1.4 \\
1.4 \\
2.0 \\
1.8 \\
1.5 \\
1.4 \\
1.3 \\
1.3 \\
1.2 \\
1.2 \\
1.4 \\
1.3 \\
\ldots . . .\end{array}$ & $\begin{array}{l}1.3 \\
1.3 \\
1.3 \\
2.0 \\
3.1 \\
3.4 \\
2.2 \\
1.6 \\
1.4 \\
1.3 \\
1.2 \\
1.3 \\
1.3 \\
1.2 \\
1.1 \\
1.1 \\
1.1 \\
1.3 \\
2.0 \\
4.6 \\
2.4 \\
1.7 \\
1.5 \\
1.5 \\
1.4 \\
1.2 \\
1.1 \\
1.1 \\
1.1 \\
1.1 \\
1.2\end{array}$ & $\begin{array}{r}1.1 \\
1.0 \\
.9 \\
.8 \\
.8 \\
.8 \\
.8 \\
1.0 \\
1.0 \\
.8 \\
.7 \\
.7 \\
.6 \\
.6 \\
.6 \\
.5 \\
.5 \\
.6 \\
.7 \\
.9 \\
.8 \\
.7 \\
.6 \\
.6 \\
.6 \\
.5 \\
.5 \\
.6 \\
.7 \\
.9 \\
. . . \\
\end{array}$ & $\begin{array}{r}1.1 \\
2.1 \\
2.0 \\
1.8 \\
1.0 \\
.9 \\
1.2 \\
1.1 \\
.6 \\
.4 \\
.4 \\
.3 \\
.3 \\
.2 \\
.2 \\
.2 \\
.2 \\
0.0 \\
-.2 \\
.1 \\
+.3 \\
.9 \\
2.0 \\
1.5 \\
.9 \\
.7 \\
.6 \\
.5 \\
.5 \\
1.1 \\
1.3\end{array}$ & $\begin{array}{r}1.3 \\
1.5 \\
1.7 \\
1.3 \\
1.2 \\
.7 \\
.5 \\
.3 \\
.3 \\
.5 \\
.7 \\
1.1 \\
1.0 \\
.8 \\
1.1 \\
.9 \\
.8 \\
.7 \\
.5 \\
.4 \\
.3 \\
.2 \\
.1 \\
.1 \\
.1 \\
1.6 \\
1.4 \\
.7 \\
.5 \\
.4 \\
.3\end{array}$ \\
\hline
\end{tabular}

Roanoke River ${ }^{\perp}$ at Clarksville, Virginia, for 1895.

\begin{tabular}{|c|c|c|c|c|c|c|c|c|c|c|c|c|c|}
\hline Day. & Mar. & Apr. & May. & June. & July. & Ang. & Day. & Mar. & Apr. & Mas. & June. & July. & Aug. \\
\hline 1. & 4,0 & 1.7 & 5.9 & $1 . .5$ & 2. 5 & 0.8 & 17. & 2.5 & 3.0 & 2.0 & 1.3 & 1.6 & 1. 0 \\
\hline & 6.7 & 1. 8 & 4.7 & 1.4 & 3.5 & .7 & 18. & 2.2 & 3.5 & 2.7 & 1.2 & 1.1 & 1.4 \\
\hline 3. & 9.0 & 2.0 & 3.5 & 1.3 & 2.7 & .7 & 19. & 2.1 & 2.8 & 2.1 & 1.1 & .8 & 1.3 \\
\hline 4. & 4.4 & 1.8 & 3.0 & 1.2 & 7 & 6 & $20 \ldots$ & 4.0 & 2.0 & 2.2 & 1.1 & 8 & 1.0 \\
\hline 5. & 3.1 & 1.5 & 2.5 & 1.4 & 1.1 & 6 & 21 . & 5.9 & 1.9 & 3.0 & .9 & .9 & 1.4 \\
\hline $6 \ldots \ldots$ & 2.5 & 1.5 & 2.2 & $1 . \overline{7}$ & .9 & .9 & 22. & 4. 3 & 1.8 & 4. 2 & .9 & .9 & 1.2 \\
\hline 7. & 3.2 & 3.9 & 2.4 & 2.0 & .9 & 1.0 & $23 \ldots$ & 3.5 & 1. 7 & 3.0 & .9 & 1. 3 & 1. 0 \\
\hline 8. & 4. 7 & 8. 7 & 2.7 & 1.3 & .9 & .9 & $24 \ldots$ & 3.1 & 1.6 & 2.5 & 9 & 1.5 & .7 \\
\hline 9 & 3.3 & 9.6 & 2.1 & 1.2 & 1.0 & .9 & 25 . & 2.6 & 1.5 & 2.9 & 1. 0 & 3.3 & .6 \\
\hline 10. & 2.5 & 4. 5 & 1.9 & 1.2 & 1.1 & 1.4 & 26. & 2.5 & 1.5 & 5.0 & 1.2 & 4.4. & .6 \\
\hline 11. & 2. 6 & 2.9 & 1.8 & 1.4 & 1.2 & 1.0 & 27. & 2.3 & 7.4 & 4. 0 & 20 & 2.5 & .7 \\
\hline 12. & 2.7 & 2.6 & 2.6 & 1. 6 & 1.0 & .8 & 28. & 2.1 & 6.5 & 2.5 & 1. 8 & 1.8 & .6 \\
\hline 13. & 3.1 & 2.5 & 2. 2 & 1.7 & .9 & .9 & 29. & 1.9 & 7.7 & 2.1 & 1.5 & 1.0 & 6 \\
\hline $14 \ldots$ & 3. 4 & 2. 3 & 1.9 & 1.8 & 9 & .8 & 30. & 1.7 & 7.9 & 1. 8 & 1. 2 & .9 & .5 \\
\hline 15. & 3.2 & 2.1 & 1. 6 & 1.6 & 1.1 & .7 & $31 \ldots$ & 1.6 & $\ldots \ldots$ & 1.7 & $\ldots . .$. & .9 & .5 \\
\hline $16 .$. & 2.9 & 2.0 & 1.5 & 1.4 & 2.4 & .6 & & & & & & & \\
\hline
\end{tabular}

1 Record kept by United States Weather Burean. 
Roanoke River ${ }^{1}$ at Clarksville, Virginia, for 1896.

\begin{tabular}{|c|c|c|c|c|c|c|c|}
\hline Day. & Feb. & Mar. & Apr. & May. & June. & July. & Aug. \\
\hline 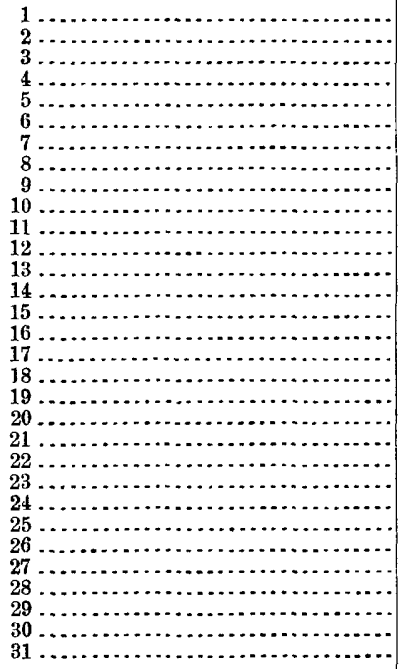 & 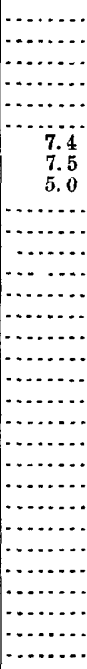 & $\begin{array}{l}2.0 \\
1.5 \\
1.3 \\
1.2 \\
1.1 \\
1.0 \\
1.0 \\
1.0 \\
1.0 \\
1.0 \\
1.0 \\
1.5 \\
1.4 \\
1.3 \\
1.2 \\
1.6 \\
1.7 \\
2.0 \\
3.0 \\
3.9 \\
2.7 \\
2.0 \\
1.8 \\
1.9 \\
1.9 \\
1.9 \\
1.8 \\
1.8 \\
2.5 \\
4.0 \\
7.8\end{array}$ & $\begin{array}{l}9.0 \\
9.1 \\
4.5 \\
3.3 \\
2.5 \\
2.3 \\
2.0 \\
1.9 \\
1.8 \\
1.8 \\
1.6 \\
1.5 \\
1.4 \\
1.4 \\
1.4 \\
1.4 \\
1.3 \\
1.3 \\
1.3 \\
1.3 \\
1.2 \\
1.2 \\
1.2 \\
1.2 \\
2.0 \\
1.8 \\
1.4 \\
1.2 \\
1.1 \\
4.8 \\
. .2\end{array}$ & $\begin{array}{r}3.8 \\
3.7 \\
3.3 \\
2.5 \\
1.8 \\
1.5 \\
1.3 \\
1.2 \\
1.1 \\
1.0 \\
1.0 \\
.9 \\
.9 \\
.8 \\
.8 \\
.8 \\
.8 \\
.8 \\
.8 \\
2.7 \\
3.5 \\
2.9 \\
2.5 \\
1.9 \\
1.5 \\
1.8 \\
1.6 \\
1.5 \\
1.5 \\
1.5 \\
1.5\end{array}$ & $\begin{array}{r}1.3 \\
1.0 \\
1.9 \\
1.2 \\
2.0 \\
1.5 \\
1.4 \\
1.3 \\
1.8 \\
1.5 \\
1.3 \\
1.2 \\
1.5 \\
1.3 \\
1.2 \\
1.3 \\
1.5 \\
1.4 \\
1.2 \\
1.2 \\
1.1 \\
1.8 \\
1.6 \\
1.5 \\
1.9 \\
3.0 \\
2.6 \\
2.4 \\
2.1 \\
1.3 \\
\ldots . . .\end{array}$ & $\begin{array}{r}0.8 \\
.8 \\
.7 \\
.7 \\
2.7 \\
4.3 \\
5.0 \\
8.0 \\
12.5 \\
13.8 \\
11.0 \\
5.5 \\
4.0 \\
2.7 \\
2.4 \\
2.2 \\
2.5 \\
1.8 \\
1.4 \\
1.3 \\
1.2 \\
1.2 \\
1.0 \\
1.0 \\
1.0 \\
.9 \\
.9 \\
.8 \\
.8 \\
.8 \\
.8\end{array}$ & $\begin{array}{r}0.7 \\
.7 \\
.7 \\
.6 \\
.6 \\
.6 \\
.5 \\
.5 \\
.5 \\
.4 \\
1.4 \\
.7 \\
.6 \\
.5 \\
.5 \\
2.0 \\
1.0 \\
.9 \\
.7 \\
.5 \\
.5 \\
.5 \\
.5 \\
.5 \\
.5 \\
.4 \\
.4 \\
.4 \\
.4 \\
.4 \\
.4\end{array}$ \\
\hline
\end{tabular}

Roanoke River at Neal, North Carolina.

\begin{tabular}{|c|c|c|c|c|c|c|c|c|c|c|c|c|c|}
\hline Day. & $J u I y$. & Aug. & Sept. & Oet. & Nov. & Dec. & Day. & July. & Ang. & Sept. & Oet. & Nov. & Dec. \\
\hline 1 & & 3.56 & 1.65 & 2.80 & 2. 60 & 5.40 & 17. & . & 3.50 & 4. 30 & 3.60 & 6.20 & 13.30 \\
\hline & & 3.90 & 1.30 & $a 21.80$ & 2.50 & 15.85 & 18. & & 2.70 & 4. 64 & 3.40 & 5. 80 & 13. 30 \\
\hline & & 4. 15 & 1.00 & 23.00 & 2. 40 & 19.70 & 19. & & 5.30 & 3.50 & 3. 10 & 5.12 & 11.70 \\
\hline & & 3. 70 & 1.00 & 25.10 & 2.40 & 17.70 & 20. & & 5.20 & 2.50 & 2.90 & 4.70 & 10.00 \\
\hline 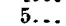 & & 3.40 & 1.24 & 24.20 & 2.90 & 14.80 & 21 . & & $3: 90$ & 2.80 & 2.70 & 4. 40 & 8.80 \\
\hline 0 & & 3.00 & 1.30 & 20.80 & 3.10 & 12.50 & 22 . & & 2.90 & 2.60 & 2.50 & 4.20 & 7. 80 \\
\hline & & 2.90 & 1.30 & 17.00 & 17.00 & 11.00 & 23. & & 2.40 & 2.80 & 2.40 & 4.00 & 7.00 \\
\hline & & 2.80 & 5.05 & 13.70 & 20.30 & 10.30 & 24 & & 2.00 & 3. 30 & 2.50 & 3. 80 & 6. 40 \\
\hline 9 & & 2. 50 & 8. 90 & 11. 10 & 18.50 & 9.90 & 25 & & 2. 03 & 3.30 & 2.60 & 3.70 & 5. 90 \\
\hline 10. & $\ldots$ & 2.20 & 7.30 & 8.90 & 14.90 & 9.20 & 26 & & 2.25 & 2. 60 & 2. 60 & 3.60 & 5. 40 \\
\hline 11 & & 2.10 & 5. 00 & 7.10 & 11.80 & 8.40 & 27 & 7.05 & 2.50 & 8.10 & 2. 70 & 3.60 & 5.00 \\
\hline 12. & $\ldots$ & 2.30 & ธ். 30 & 5. 60 & 9.70 & 7. 60 & 28 & 5.09 & 2.20 & 1. 80 & 2.70 & 3.50 & 4. 60 \\
\hline 13. & & 2.90 & 2.20 & 4. 50 & 8.20 & 6. 90 & 29 & 4. 08 & 2.90 & 1. 60 & 2.80 & 3.50 & 4. 10 \\
\hline 14. & & 5.20 & 1.80 & 4.20 & 7. 10 & 6. 30 & 30. & 4.24 & 2.70 & 1.90 & 2.72 & 3.80 & 3.90 \\
\hline 15 & & 5.60 & 1. 56 & 4.10 & 6.30 & 6.00 & 31.. & 3.80 & 2. 20 & ...... & 2.60 & ...... & 4.00 \\
\hline 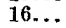 & & 4.60 & 2. 10 & 3.80 & 6.10 & 8. 30 & & & & & & & \\
\hline
\end{tabular}

a Rise began about $10 \mathrm{a}$. $\mathrm{m}$. and continued at rate of about 2 feet per hour for some time.

Tar River at Tarboro, North Carolina.

\begin{tabular}{|c|c|c|c|c|c|c|c|c|c|c|c|c|c|}
\hline Day. & July. & Aug. & Sept. & Oet. & Nor. & Dec. & Day. & July. & Aug. & Sept. & Oet. & Nov. & Dec. \\
\hline & & 1.30 & 1.00 & 2. 82 & 0.89 & 3.87 & 17. & & 1.25 & 4.50 & 1.98 & 2.45 & 12.73 \\
\hline & & 1.00 & .60 & 3.55 & .96 & 4. 34 & 18 & & 1.28 & 4. 35 & 1.50 & 1.95 & 13.82 \\
\hline & & .90 & .45 & 4.20 & 1.20 & 4.85 & 19 . & & 1.00 & 4. 20 & 1.00 & 1.63 & 14.50 \\
\hline & & 1.20 & .80 & 2.95 & 1.05 & 4. 90 & 20. & $\ldots$ & $\begin{array}{r}.73 \\
\end{array}$ & 4.60 & 1.15 & 1. 61 & 13.50 \\
\hline & & 1. 46 & 51 & 2. 18 & 1.07 & 4.50 & 21 . & $\cdots$ & .50 & 3.52 & .90 & 1. 50 & 12.20 \\
\hline & & 3. 48 & .30 & 1. 90 & 2.15 & 4. 70 & 22 . & & .65 & 2.10 & .31 & 1.42 & 10.40 \\
\hline & & 2.87 & .35 & 1.50 & 2.76 & 5.00 & 23. & & .62 & 5.85 & .69 & 1. 30 & 8.20 \\
\hline & & 1.95 & 60 & 1.20 & 3.72 & 5. 80 & 24 & $\ldots$ & .65 & 7.30 & .85 & 1.44 & 6.44 \\
\hline & & 1.45 & .35 & 1.05 & 3.13 & 6. 50 & & ... & .70 & 6.40 & 1.28 & 1. 12 & 5. 40 \\
\hline 10. & & 1.00 & 23 & .85 & 2.59 & 6. 72 & 26 & 3. 56 & 1.20 & 4.60 & 1.12 & 1.05 & 4.75 \\
\hline 11. & & .82 & .20 & .90 & 2. 10 & 6.10 & 27 & 3.00 & 1. 30 & 3.60 & 1.32 & 1.02 & 4. 20 \\
\hline 12 & & .50 & 22 & .81 & 1. 71 & 5.33 & 28 & 2.68 & 1. 40 & 2.85 & 1.00 & 1.00 & 4.05 \\
\hline 13 . & & 1.78 & 12 & 1.05 & 2.62 & 4. 64 & & 2. 25 & 1.60 & 2.58 & .89 & 1.02 & 3.80 \\
\hline . & & 1.92 & 10 & 1.42 & 4.05 & 4.07 & 30 : & 2.00 & 1. 48 & 2. 72 & .80 & 1.45 & 3.50 \\
\hline & & 1.85 & .18 & 1.70 & 3. 20 & 3. 98 & 31. & 1. 76 & 1.10 & ..... & .58 & ...... & 3. 20 \\
\hline & & 1.53 & 3.45 & 2.00 & 2. 62 & 9. 75 & & & & & & & \\
\hline
\end{tabular}

1 Record kept by United States Weather Burean. 
Neuse River at Selma, North Carolina.

\begin{tabular}{|c|c|c|c|c|c|c|c|c|c|c|c|c|c|}
\hline Day. & July. & Ang. & Sept. & Oet. & Nor. & Dec. & Day. & July. & Aug. & Sept. & Oct. & Nov. & Dec. \\
\hline 1. & & 2.00 & & & & & & & & & & & \\
\hline & & $\begin{array}{l}2.00 \\
2.00\end{array}$ & $\begin{array}{r}0.30 \\
.30\end{array}$ & $\begin{array}{l}7.00 \\
7.20\end{array}$ & .60 & 3. 50 & 18 & & $\begin{array}{r}0.90 \\
.90\end{array}$ & $\begin{array}{l}0.00 \\
.05\end{array}$ & $\begin{array}{r}.50 \\
.40\end{array}$ & $\begin{array}{l}0.70 \\
.70\end{array}$ & $\begin{array}{l}9.00 \\
8.60\end{array}$ \\
\hline & & 2.00 & .35 & 2.30 & .60 & 3.00 & 19 & & 1.10 & .00 & .40 & 60 & 5.50 \\
\hline & & 1.90 & .40 & .90 & .40 & 2.80 & 20 & & 1.00 & .00 & .30 & .60 & 4.60 \\
\hline & & 1.20 & .30 & .50 & 1. 20 & 2.60 & 21. & & 1. 00 & i. 04 & .40 & 60 & $\begin{array}{l}3.50 \\
3.50\end{array}$ \\
\hline 6. & & 1.00 & .30 & .50 & 4.80 & 2.50 & .22 . & & .50 & .04 & .60 & 50 & 3.00 \\
\hline & & 1.00 & 30 & .30 & 4.40 & 3.00 & 23 . & & 40 & 5.04 & .90 & 50 & 2.80 \\
\hline & $\cdots$ & 1.00 & 60 & .20 & 2.30 & 4.00 & 24 & & 50 & 1.07 & .70 & .50 & 2.00 \\
\hline & 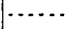 & .90 & .50 & .20 & 1. 50 & 3.80 & 25 & & 2.10 & .07 & .50 & .50 & 1.90 \\
\hline 10. & $\ldots$ & 5. 05 & .30 & .10 & 1. 20 & 3.50 & 26. & $\mid \ldots \ldots$. & 2.00 & .07 & .40 & 50 & 1.80 \\
\hline 11. & & 5.50 & $\cdot 20$ & .20 & .80 & 2.60 & & & 1.10 & .40 & . 30 & 50 & 1.50 \\
\hline 13. & & $\begin{array}{l}5.50 \\
2,20\end{array}$ & .20 & $\begin{array}{r}.50 \\
70\end{array}$ & $\begin{array}{r}80 \\
1.30\end{array}$ & 2. 0 & 28 & & 1.00 & .30 & .30 & .50 & 1.50 \\
\hline $14 \ldots$ & $\cdots$ & 1.50 & .01 & $\begin{array}{r}.70 \\
170\end{array}$ & $\begin{array}{l}1.30 \\
1.20\end{array}$ & $\begin{array}{l}2.00 \\
2.00\end{array}$ & 30. & $\begin{array}{l}1.10 \\
1.00\end{array}$ & $\begin{array}{l}1.00 \\
.50\end{array}$ & $\begin{array}{r}.10 \\
1.50\end{array}$ & $\begin{array}{l}.30 \\
.40\end{array}$ & $\begin{array}{l}.50 \\
.70\end{array}$ & $\begin{array}{l}1.40 \\
1.30\end{array}$ \\
\hline 15 & & 1.20 & .01 & 1.00 & .90 & 3.00 & 31... & 1.00 & .30 & & .40 & & 1.20 \\
\hline & & .80 & .01 & .60 & .80 & 7.30 & & & & & & & \\
\hline
\end{tabular}

Cape Fear River at Fayetteville, North Carolina.

\begin{tabular}{|c|c|c|c|c|c|c|c|c|c|c|c|c|}
\hline Day. & Jan. & Feb. & Mar. & Apr. & May. & June. & July. & Aug. & Sept. & Oet. & Nov. & Dec. \\
\hline & 18.00 & 6.50 & 9.00 & 7.50 & 3. 70 & 4.00 & 7.00 & 3.30 & 1.30 & 31.00 & 2.50 & 6.00 \\
\hline & 18.90 & 6.50 & 10.40 & 9.50 & 5. 00 & 3.20 & 5.30 & 4. 30 & 1.10 & 26.80 & $2.70^{\circ}$ & 15.40 \\
\hline & 11.40 & 7.90 & 9. 30 & 22.50 & 8.00 & 2.70 & 4. 00 & 4.00 & 1.00 & 19.50 & 2.70 & 14.00 \\
\hline & 8.00 & 27.70 & 7. 60 & 19.40 & 12.80 & 4.00 & 3.50 & 3.30 & .80 & 12.90 & 3.00 & 12.40 \\
\hline & 6.50 & 31.00 & 6.80 & 13.00 & 10.40 & 19.00 & 3.50 & 3.00 & 1.50 & 7.80 & 4. 30 & 10.40 \\
\hline & 5.70 & 30.00 & 6.00 & 9.80 & 8.30 & 20.00 & 5.90 & 3.00 & 3.00 & 4. 90 & 17.00 & 9.50 \\
\hline & 4. 60 & 45.40 & 5.80 & 8.00 & 6.00 & 14.00 & 4.40 & 2.80 & 8.50 & 4. 20 & 19.00 & 9.60 \\
\hline & 4. 40 & 48.00 & E. 80 & 6.80 & 4.50 & 10.00 & 15.00 & 2.60 & 9.50 & 3.60 & 14.30 & 13.50 \\
\hline 9. & 4.60 & 45.00 & 5.40 & 6.00 & 4.40 & 8.00 & 34.80 & 2.30 & 5. 50 & 3.20 & 10.40 & 14.50 \\
\hline 10. & 4.60 & 42.40 & 5.40 & 5. 40 & 3.90 & 6. 20 & 47.50 & 2.00 & 4.00 & 3.00 & 7.70 & 13.60 \\
\hline 11. & 4.70 & 36.00 & 5.50 & 5.20 & 3.50 & 6.00 & 49. 50 & 2.00 & 3. 30 & 2.70 & 6.00 & 11.70 \\
\hline 12. & 4.40 & 30.40 & 7.80 & 5.50 & 3.20 & 6.00 & 47.00 & 1.90 & 2.50 & 2. 70 & 4. 80 & 9.50 \\
\hline 13. & 4.20 & 19.00 & 11.00 & 5.50 & 2.80 & 5.00 & 41.00 & 2.70 & 2.00 & 2.70 & 4. 60 & 8. 20 \\
\hline 14. & 4.00 & 16.20 & 10.00 & 5.00 & 2.80 & 7.00 & 30.30 & 4.00 & 1.50 & 3. 00 & 4.50 & 7.00 \\
\hline 15. & 4.10 & 17. 40 & 8. 60 & 4.90 & 2.60 & 10.90 & 19.00 & 3. 40 & 1. 40 & 3.30 & 4. 50 & 7.50 \\
\hline 16. & 4.00 & 16.00 & 7. 20 & 5.00 & 2.50 & 8.50 & 14.40 & 3. 70 & 1.20 & 3.20 & 4.80 & 9.40 \\
\hline 17. & 5.00 & 13.40 & 7.50 & 4.90 & 2.50 & 5.70 & 9.50 & 3.70 & 1.00 & 3.00 & 4.20 & 14.00 \\
\hline 18. & 9.00 & 11. 60 & 8.00 & 4.70 & 2.10 & 4.60 & 9.30 & 3.00 & 1.50 & 2.90 & 4.00 & 12.30 \\
\hline 19. & 6.00 & 10.50 & 7.80 & 4.50 & 2,40 & 6. 00 & 9.00 & 2. 60 & 1.90 & 2.60 & 4.00 & 9.00 \\
\hline 20. & 1.40 & 10.60 & 8.50 & 4.20 & 2. 30 & 5.30 & 8.70 & 2.50 & 3.00 & 2.40 & 3.80 & 9.00 \\
\hline & 15.00 & 9.80 & 10.50 & 4.00 & 3.50 & 6. 40 & 8.90 & 2.50 & 2.50 & 2. 10 & 3.60 & 8. 20 \\
\hline & 22.20 & 9.00 & 9.60 & 3.80 & 4. 10 & 5.00 & 8.30 & 2.10 & 1.50 & 2.20 & 3.50 & 7.00 \\
\hline 23. & 20.00 & 8.00 & 7. 80 & 3.60 & 8.17 & 4. 30 & 7. 90 & 2.70 & 6.00 & 2. 30 & 3.20 & 6.90 \\
\hline & 15.00 & 7.80 & 6.80 & 3.50 & 11.40 & 4.50 & 7.20 & 2.00 & 6.80 & 2.70 & 3.10 & 6.30 \\
\hline & 15.60 & 7.80 & 6.50 & 4. 00 & 17.40 & 6.40 & 7.50 & 2.40 & 5.00 & 4. 40 & 3.00 & 5. 40 \\
\hline & 11.00 & 7.70 & 6.60 & 4. 60 & 12.00 & 7.80 & 7.00 & 4.00 & 3.90 & 4. 10 & 3.00 & 4. 60 \\
\hline & 26.20 & 7.40 & 7.00 & 7.00 & 9.50 & 8.60 & 5.20 & 3. 70 & 3.20 & 3. 50 & 3.00 & 5.00 \\
\hline & 22.00 & 7.30 & 6.30 & 5.50 & 8.00 & 6. 00 & 4. 30 & 2.70 & 2.90 & 3. 00 & 3.30 & 4. 90 \\
\hline & 29.40 & 7. 90 & 6. 20 & 4. 90 & 7.20 & 10.80 & 3.70 & 2.00 & 3.50 & 2.80 & 3. 20 & 4. 80 \\
\hline & 18.10 & & 5.80 & 4. 10 & 6.00 & 8.90 & 3.60 & 1.90 & 8.00 & 2.50 & 3.50 & 4.90 \\
\hline & 17.00 & ....... & 5.30 & $\ldots . .$. & 5.00 & $\ldots . .$. & 3. 40 & 1. 40 & ....... & 2.50 & ...... & 5.00 \\
\hline
\end{tabular}

Yadkin River at Norwood, North Carolina.

\begin{tabular}{|c|c|c|c|c|c|c|c|c|c|c|c|c|c|c|}
\hline Day. & Sept. & Oct. & Nov. & Dec. & Day. & Sept. & Oet. & Nov. & Dec. & Day. & Sept. & Oet. & Nov. & Dec. \\
\hline 1. & 1.00 & 7. 40 & 1.30 & 1.50 & $12 \ldots$ & 1.20 & 1.20 & 1.70 & 1.70 & 23. & 1. 20 & 1. 20 & 1.40 & 1.80 \\
\hline 2. & 1.00 & 3. 40 & 1. 30 & 2. 70 & $13 \ldots$ & 1. 20 & 1. 20 & 2.20 & 1.30 & 24 & 1.30 & 1. 20 & 1.40 & 1.80 \\
\hline & 1.00 & 2. 30 & 1.30 & 2.70 & $14 \ldots$ & 1. 20 & 1.20 & 1.30 & 1. 60 & 25 & 1.20 & 1. 30 & 1.40 & 1.70 \\
\hline & .90 & 1.80 & 1.40 & 2.30 & & 1.30 & 1. 30 & 1. 90 & 1. 90 & 26. & 1.20 & 1. 30 & 1.40 & 1.70 \\
\hline & .90 & 1.60 & 3.10 & 2. 30 & $16 \ldots$ & 1.20 & 1. 30 & 1.70 & 2.50 & 27 & 1.10 & 1. 30 & 1.40 & 1. 70 . \\
\hline & 2.50 & 1.50 & 6.00 & 2.20 & 17. & 1.30 & 1. 20 & 1.60 & 3.80 & $28 \ldots$ & 1.00 & 1.30 & 1.40 & 1.70 \\
\hline & 3.30 & 1.40 & 3.40 & 2.30 & $18 \ldots$ & 1. 30 & 1.20 & 1. 50 & 2.90 & $29 \ldots$ & 1.20 & 1.20 & 1.50 & 1.60 \\
\hline 8. & 2.10 & 1. 40 & 2.50 & 2.40 & & 1.20 & 1. 20 & 1.50 & 2. 30 & $30 \ldots$ & 1. 30 & 1. 20 & 1.70 & 1.60 \\
\hline 9. & 1.65 & 1. 30 & 2.20 & 2.20 & & 1. 20 & 1. 10 & 1. 50 & 2.10 & $31 \ldots$ & ..... & 1. 20 & $\ldots \ldots$ & 1.70 \\
\hline 10. & 1. 45 & 1. 30 & 2.10 & 2.10 & & 1. 10 & 1. 10 & 1. 50 & 2.00 & $=$ & ...... & $\ldots .$. & ...... & $\ldots \ldots$ \\
\hline 11 & 1.30 & 1.20 & 1.90 & 1.90 & $22 \ldots$ & t. 10 . & 1. 10 & 1.50 & 1.90 & & & & & $\cdots$ \\
\hline
\end{tabular}


Fadkin River at Salisbury, North Carolina.

\begin{tabular}{|c|c|c|c|c|c|c|c|c|c|c|c|c|}
\hline Day. & Jan. & Feb. & Mar. & Apr. & May. & June. & July. & Aug. & Sept. & Oet: & Nov. & Dec. \\
\hline & 3.60 & 2.00 & 2. 50 & 2.50 & 1.80 & 1,50 & 1.80 & 2. 00 & 1.40 & $8.40^{\circ}$ & 1. 68 & 6.70 \\
\hline & 3. 00 & 2.00 & 2.20 & 8.00 & 1.80 & 1.50 & 1.60 & 1.90 & 1. 40 & 3.70 . & 1.70 & 4.10 \\
\hline 3. & 2.40 & 5. 70 & 2.70 & 7.30 & 2.10 & 1.80 & 1. 60 & 1.90 & 1.40 & 2.20 & 1.72 & 3.70 \\
\hline 4. & 2.20 & 6.70 & 1.80 & 4.00 & 2.90 & 3. 90 & 2.60 & 1.70 & 1. 40 & 2.50 & ....... & 2.80 \\
\hline 5 & 1.80 & 4.90 & 2.00 & 3.10 & 2.30 & 3. 70 & 2.70 & 1.70 & 1. 50 & 1.80 & 5.80 & 2.50 \\
\hline & 1.60 & 5. 30 & 2.00 & 2.60 & 2.20 & 2. 50 & 2. 80 & 1. 60 & 4.50 & 1. 70 & 6. 78 & 2. 55 \\
\hline & 1. 60 & 7.00 & 1.90 & 2. 50 & 2.00 & 2.20 & 4. 50 & 1.60 & 2. 70 & 1.70 & 3.60 & 2.53 \\
\hline & 1.90 & 5. 00 & 1. 90 & 2. 40 & 1.90 & 2.00 & 4. 90 & 1.60 & 2.20 & 1.60 & 3. 50 & 2.52 \\
\hline 9 . & 3. 70 & 5. 00 & 1.90 & 2.20 & 1.90 & 2.00 & 12.90 & 1.50 & 1. 90 & 1.60 & 2.50 & 2.30 \\
\hline 10. & 3.60 & 4. 70 & 1.90 & 2.20 & 1.90 & 1.90 & $a 15.00$ & 2. 00 & 1. 60 & 1. 60 & 2.30 & 2.27 \\
\hline 11. & 3.40 & 3. 60 & 2.00 & 2.20 & 1. 90 & 2. 20 & 10.00 & 2.00 & 1. 50 & 1. 60 & 2.20 & 2.26 \\
\hline 12. & 2,60 & 3. 00 & 2.20 & 2.10 & 1.90 & 1. 80 & 9.80 & 1.90 & 1.50 & 1.62 & 2.10 & 2.26 \\
\hline 13. & 2.10 & 2.70 & 2.40 & 2.00 & 1.80 & 1. 80 & 7.90 & 1. 80 & 2.00 & 1. 66 & 2.15 & 2.25 \\
\hline 14. & 1. 70 & 3. 30 & 2.10 & 2. 00 & 1. 70 & 1. 80 & 4. 90 & 1. 60 & 1. 75 & 1.64 & 2.00 & 2. 23 \\
\hline 15. & 1. 70 & 3. 10 & 2.00 & 2.00 & 1.70 & 1. 70 & 4.00 & 1. 60 & 1. 60 & 1. 70 & 2.00 & ‥ 30 \\
\hline 16. & 1.70 & 2. 80 & 1.80 & 2.00 & 1.70 & 1. 60 & 3. 70 & 2. 50 & 1. 50 & 1. 70 & 2. 00 & 5.00 \\
\hline 17. & 1.90 & 2.50 & 2. 00 & 2.00 & 1. 70 & 1.60 & 2.80 & 2. 20 & 1.60 & 1.68 & 1.98 & 3. 55 \\
\hline 18. & 2. 20 & 2.30 & 2.00 & 2.00 & 1.70 & 1.80 & 2. 40 & 2. 90 & 1. 60 & 1.65 & 1.95 & 3. 40 \\
\hline 19. & 2. 10 & 2. 20 & 1.90 & 2. 10 & 1. 60 & 1. 80 & 3. 40 & 2. 70 & 1. 60 & 1. 50 & 1. 92 & 2.95 \\
\hline 20. & 2.00 & 2.10 & 2.20 & 2.00 & 1.60 & 1.80 & 2. 20 & 2.50 & 1.50 & 1.50 & 1.90 & 3.90 \\
\hline 21. & 1.90 & 2.00 & 2. 60 & 2.00 & 1.50 & 1. 70 & 2. 20 & 1.90 & 1. 50 & 1.50 & 1.90 & 3.70 \\
\hline 22 . & 1. 80 & 1. 80 & 2.20 & 2.00 & 1. 80 & 1. 70 & 2. 30 & 1. 90 & 1. 50 & 1. 48 & 1. 90 & 3. 33 \\
\hline 23 . & 2. 50 & 2. 10 & 2.10 & 2. 00 & 1.80 & $1.6 v$ & 2.50 & 1. 50 & 1. 70 & 1. 48 & 1. 89 & 2. 30 \\
\hline 24 & 4. 30 & 2.20 & 2. 10 & 1. 90 & 1. 80 & 1.80 & 2.30 & 1. 50 & 1.60 & 1.60 & 1. 88 & 2.20 \\
\hline & 3.70 & 2.10 & 2,50 & 1. 90 & 1.70 & 2. 10 & 2.20 & 1. 50 & 1.50 & 1.76 & 1.88 & 2. 13 \\
\hline & 3. 20 & 2.10 & 2.40 & 2. 00 & 2. 00 & 2.80 & 2.00 & 1. 60 & 1.50 & 1.67 & 1.68 & 2.12 \\
\hline & 2. 60 & 2.10 & 2.20 & 2.00 & 2.00 & 2.60 & 1.90 & 1. 60 & 1. 46 & 1.65 & 1. 90 & 2. 10 \\
\hline & 2.40 & 2.20 & 2. 10 & 1. 90 & 1. 80 & 2. 20 & 1. 90 & 1.50 & 1.45 & 1. 65 & 1: 90 & 2.00 \\
\hline & 2.20 & 2.30 & 2.00 & 1.90 & 1. 70 & 2.10 & 2.20 & 1. 50 & 1. 50 & 1.65 & 2.70 & 2.00 \\
\hline 30. & 2.10 & ...... & 2. 10 & 1. 90 & 1. 70 & 1.90 & 1.90 & 1.50 & 8.60 & 1. 65 & 6. 30 & 2. 08 \\
\hline & 2.00 & ..... & 2. 60 & $\ldots$. & 1.60 & ....... & 1.90 & 1.40 & ....... & 1. 68 & & 2.07 \\
\hline
\end{tabular}

a July 10 river rose 14.90 above low-water mark.

Catawba River at Rockhill, South Carolina.

\begin{tabular}{|c|c|c|c|c|c|c|c|c|c|c|c|c|}
\hline Day. & Jan. & Feb. & Mar. & Apr. & May. & June. & July. & Aug. & Sept. & Oet. & Nov. & Dec. \\
\hline 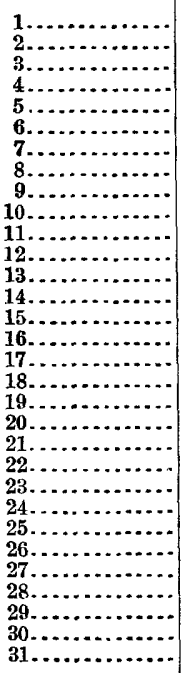 & $\begin{array}{l}3.30 \\
2.90 \\
2.40 \\
2.20 \\
2.00 \\
1.90 \\
1.80 \\
1.80 \\
2.00 \\
1.90 \\
1.90 \\
1.90 \\
1.80 \\
1.80 \\
1.80 \\
1.80 \\
2.10 \\
2.40 \\
2.20 \\
2.00 \\
2.00 \\
1.90 \\
2.60 \\
3.80 \\
4.50 \\
3.80 \\
2.90 \\
2.60 \\
2.40 \\
2.20 \\
2.20\end{array}$ & $\begin{array}{l}2.10 \\
2.10 \\
3.60 \\
4.90 \\
3.50 \\
6.25 \\
6.60 \\
4.90 \\
5.70 \\
5.30 \\
4.00 \\
3.20 \\
2.80 \\
2.90 \\
2.80 \\
2.70 \\
2.60 \\
2.40 \\
2.40 \\
2.30 \\
2.30 \\
2.20 \\
2.10 \\
2.10 \\
2.20 \\
2.10 \\
2.10 \\
2.10 \\
2.30 \\
\cdots \ldots \\
\cdots \ldots . .\end{array}$ & $\begin{array}{l}2.30 \\
2.20 \\
2.10 \\
2.10 \\
2.00 \\
2.00 \\
2.00 \\
2.00 \\
1.90 \\
1.90 \\
1.90 \\
2.10 \\
2.10 \\
2.00 \\
2.00 \\
1.90 \\
1.90 \\
1.90 \\
1.90 \\
2.10 \\
2.00 \\
2.10 \\
2.00 \\
2.00 \\
2.00 \\
2.20 \\
2.10 \\
2.00 \\
2.00 \\
1.90 \\
2.00\end{array}$ & \begin{tabular}{|l|}
2.10 \\
2.70 \\
5.80 \\
3.50 \\
2.80 \\
2.50 \\
2.30 \\
2.20 \\
2.10 \\
2.10 \\
2.00 \\
2.00 \\
2.00 \\
1.90 \\
1.90 \\
1.90 \\
1.90 \\
1.90 \\
1.80 \\
1.80 \\
1.80 \\
1.80 \\
1.80 \\
1.80 \\
1.80 \\
1.80 \\
1.80 \\
1.80 \\
1.70 \\
1.70 \\
$\ldots . .$.
\end{tabular} & $\begin{array}{l}1.70 \\
1.80 \\
3.80 \\
4.30 \\
2.90 \\
2.50 \\
2.30 \\
2.10 \\
2.00 \\
2.00 \\
1.90 \\
1.80 \\
1.80 \\
1.80 \\
1.70 \\
1.70 \\
1.70 \\
1.70 \\
1.70 \\
1.70 \\
1.60 \\
1.60 \\
1.60 \\
1.60 \\
2.60 \\
1.90 \\
1.80 \\
1.90 \\
1.80 \\
1.70 \\
1.60\end{array}$ & $\begin{array}{l}1.50 \\
1.90 \\
2.20 \\
2.50 \\
3.20 \\
2.60 \\
2.10 \\
1.90 \\
1.80 \\
2.90 \\
1.80 \\
1.70 \\
1.70 \\
1.60 \\
1.60 \\
1.60 \\
1.60 \\
1.60 \\
1.80 \\
2.00 \\
2.00 \\
1.90 \\
2.10 \\
1.90 \\
1.90 \\
2.10 \\
2.00 \\
2.00 \\
2.00 \\
1.90 \\
\cdots . . .\end{array}$ & \begin{tabular}{|r|}
1.70 \\
1.60 \\
2.00 \\
2.60 \\
2.00 \\
4.50 \\
5.20 \\
7.37 \\
12.22 \\
15.20 \\
10.65 \\
7.90 \\
6.30 \\
4.40 \\
3.40 \\
3.10 \\
2.80 \\
2.60 \\
2.40 \\
2.70 \\
2.50 \\
2.50 \\
2.20 \\
2.30 \\
2.20 \\
2.10 \\
2.10 \\
2.40 \\
2.40 \\
2.30 \\
2.10 \\
\end{tabular} & $\begin{array}{l}2.00 \\
1.90 \\
2.00 \\
1.90 \\
1.90 \\
2.00 \\
1.90 \\
1.80 \\
1.80 \\
1.70 \\
1.70 \\
1.70 \\
1.80 \\
1.70 \\
3.10 \\
2.00 \\
1.90 \\
2.00 \\
2.00 \\
1.80 \\
1.70 \\
1.60 \\
1.60 \\
1.60 \\
1.60 \\
1.60 \\
1.60 \\
1.60 \\
1.60 \\
1.50 \\
1.50\end{array}$ & $\begin{array}{l}1.50 \\
1.50 \\
1.50 \\
1.50 \\
2.10 \\
2.10 \\
2.90 \\
2.20 \\
1.90 \\
1.70 \\
1.60 \\
1.60 \\
1.50 \\
2.00 \\
1.70 \\
1.60 \\
1.60 \\
1.60 \\
1.60 \\
1.60 \\
1.50 \\
1.60 \\
1.60 \\
1.60 \\
1.60 \\
1.60 \\
1.50 \\
1.50 \\
1.60 \\
4.30 \\
\cdots . . .\end{array}$ & $\begin{array}{l}3.90 \\
2.60 \\
2.00 \\
1.70 \\
1.60 \\
1.60 \\
1.60 \\
1.50 \\
1.50 \\
1.50 \\
1.60 \\
1.50 \\
1.50 \\
1.50 \\
1.60 \\
1.60 \\
1.60 \\
1.50 \\
1.50 \\
1.50 \\
1.50 \\
1.50 \\
1.50 \\
1.60 \\
1.60 \\
1.60 \\
1.60 \\
1.60 \\
1.50 \\
1.50 \\
1.60\end{array}$ & $\begin{array}{r}1.60 \\
1.70 \\
1.70 \\
1.70 \\
3.30 \\
5.40 \\
3.90 \\
2.60 \\
2.40 \\
2.20 \\
2.00 \\
1.90 \\
2.50 \\
2.20 \\
2.20 \\
2.10 \\
1.90 \\
1.80 \\
1.80 \\
1.80 \\
1.80 \\
1.70 \\
1.70 \\
1.70 \\
1.70 \\
1.70 \\
1.70 \\
1.70 \\
1.90 \\
2.20 \\
\cdots . . . .\end{array}$ & $\begin{array}{l}5.50 \\
4.30 \\
3.10 \\
2.60 \\
2.40 \\
2.30 \\
2.40 \\
2.30 \\
2.20 \\
2.10 \\
2.00 \\
1.90 \\
1.90 \\
1.90 \\
1.90 \\
3.21 \\
3.40 \\
2.70 \\
2.80 \\
2.20 \\
2.10 \\
2.00 \\
2.00 \\
1.90 \\
1.80 \\
1.80 \\
1.80 \\
1.80 \\
1.80 \\
1.80 \\
1.80\end{array}$ \\
\hline
\end{tabular}

IRR $11-2$ 
Catawba River at Catawba, North Carolina.

\begin{tabular}{|c|c|c|c|c|c|c|c|c|c|c|c|c|c|}
\hline Day. & July. & Aug. & Sept. & Oct. & Nov. & Dec. & Day. & July. & Aug. & Sept. & Oet. & Nov. & Dec. \\
\hline $\begin{array}{r}3 \ldots \ldots \\
4 \ldots \ldots \\
5 \ldots \ldots \\
6 \ldots \ldots \\
7 \ldots \ldots \\
8 \ldots \ldots \\
10 \ldots \ldots \\
11 \ldots \ldots \\
12 \ldots \ldots \\
13 \ldots \ldots \\
14 \ldots \ldots \\
15 \ldots \ldots \\
16 \ldots . . .\end{array}$ & \begin{tabular}{l}
$\ldots \ldots \ldots$ \\
$\cdots \ldots \ldots$ \\
\hdashline 3.05 \\
2.95 \\
4.32 \\
3.82 \\
5.75 \\
5.00 \\
7.72 \\
7.48 \\
5.95 \\
5.25 \\
4.38 \\
3.58 \\
3.32
\end{tabular} & $\begin{array}{l}2.25 \\
2.25 \\
2.22 \\
2.28 \\
2.15 \\
2.05 \\
1.98 \\
1.92 \\
1.92 \\
1.95 \\
2.02 \\
1.98 \\
1.92 \\
1.88 \\
2.02 \\
2.15\end{array}$ & $\begin{array}{l}1.65 \\
1.85 \\
1.48 \\
1.45 \\
3.32 \\
2.32 \\
2.32 \\
1.72 \\
1.62 \\
1.52 \\
1.52 \\
1.72 \\
1.95 \\
1.68 \\
1.62 \\
1.62\end{array}$ & $\begin{array}{l}2.45 \\
2.25 \\
1.92 \\
1.65 \\
1.52 \\
1.58 \\
1.58 \\
1.55 \\
1.52 \\
1.52 \\
1.52 \\
1.55 \\
1.55 \\
1.68 \\
1.62 \\
1.52\end{array}$ & $\begin{array}{l}1.68 \\
1.65 \\
1.58 \\
1.88 \\
7.38 \\
3.92 \\
3.35 \\
2.32 \\
2.12 \\
1.95 \\
1.88 \\
1.88 \\
1.85 \\
1.82 \\
1.92 \\
1.92\end{array}$ & $\begin{array}{l}5.58 \\
5.32 \\
3.32 \\
2.65 \\
2.65 \\
2.32 \\
2.32 \\
2.18 \\
2.12 \\
2.10 \\
1.98 \\
1.98 \\
1.98 \\
1.95 \\
3.78 \\
3.68\end{array}$ & $\begin{array}{l}17 \ldots \ldots \\
18 \ldots \ldots \\
19 \ldots \ldots \\
20 \ldots \ldots \\
21 \ldots \ldots \\
22 \ldots \ldots \\
23 \ldots \ldots \\
24 \ldots \ldots \\
25 \ldots \ldots \\
26 \ldots \ldots \\
27 \ldots \ldots \\
28 \ldots \ldots \\
29 \ldots \ldots \\
30 \ldots \ldots \\
31 \ldots \ldots\end{array}$ & $\begin{array}{l}2.98 \\
2.92 \\
2.78 \\
2.75 \\
2.62 \\
2.72 \\
2.72 \\
2.62 \\
2.52 \\
2.48 \\
2.42 \\
2.92 \\
2.48 \\
2.35 \\
2.28\end{array}$ & $\begin{array}{l}2.08 \\
1.88 \\
1.82 \\
1.68 \\
1.68 \\
1.62 \\
1.65 \\
1.68 \\
1.65 \\
1.62 \\
1.62 \\
1.52 \\
1.52 \\
1.65 \\
1.85\end{array}$ & \begin{tabular}{|l|}
1.82 \\
1.62 \\
1.52 \\
1.58 \\
1.52 \\
1.55 \\
1.52 \\
1.52 \\
1.52 \\
1.48 \\
1.48 \\
1.52 \\
1.55 \\
4.02 \\
$\ldots \ldots .$.
\end{tabular} & $\begin{array}{l}1.48 \\
1.48 \\
1.48 \\
1.48 \\
1.58 \\
1.50 \\
1.52 \\
1.58 \\
1.58 \\
1.52 \\
1.52 \\
1.52 \\
1.55 \\
1.55 \\
1.68\end{array}$ & $\begin{array}{l}1.92 \\
1.92 \\
1.92 \\
1.93 \\
1.92 \\
1.88 \\
1.85 \\
1.85 \\
1.78 \\
1.75 \\
1.72 \\
1.72 \\
2.98 \\
7.42 \\
\ldots \ldots .\end{array}$ & $\begin{array}{l}3.28 \\
2.95 \\
2.95 \\
2.42 \\
2.22 \\
2.05 \\
2.00 \\
2.12 \\
1.98 \\
1.95 \\
1.85 \\
1.90 \\
1.90 \\
1.90 \\
1.90\end{array}$ \\
\hline
\end{tabular}

Broad River at Gaffney, South Carolina.

\begin{tabular}{|c|c|c|c|c|c|c|c|c|c|c|c|c|c|}
\hline Day. & July. & Aug. & Sept. & Oet. & Nov. & Dec. & Day. & July. & Aug. & Sept. & Oet. & Nov. & Dec. \\
\hline $\begin{array}{r}1 \ldots \\
2 \ldots \\
3 \ldots \\
4 \ldots \\
5 \ldots \\
6 \ldots \\
7 \ldots \\
8 \ldots \\
9 \ldots \\
10 \ldots \\
11 \ldots \\
12 \ldots \\
13 \ldots \\
14 \ldots \\
15 \ldots \\
16 \ldots\end{array}$ & $\begin{array}{r} \\
2.52 \\
5.88 \\
1.60 \\
.83 \\
.81 \\
.78 \\
.83\end{array}$ & $\begin{array}{r}0.43 \\
.41 \\
.60 \\
.67 \\
.38 \\
.28 \\
.32 \\
.30 \\
.18 \\
.30 \\
.25 \\
.33 \\
.22 \\
.48 \\
.50 \\
.36\end{array}$ & $\begin{array}{r}0.10 \\
.11 \\
.84 \\
.46 \\
.63 \\
.49 \\
.40 \\
.41 \\
.40 \\
.14 \\
.20 \\
.82 \\
.29 \\
.20 \\
.23 \\
.20\end{array}$ & $\begin{array}{r}0.81 \\
.44 \\
.38 \\
.30 \\
.22 \\
.35 \\
.31 \\
.30 \\
.25 \\
.30 \\
.22 \\
.15 \\
.31 \\
.35 \\
.37 \\
.30\end{array}$ & $\begin{array}{r}0.47 \\
.28 \\
.40 \\
.40 \\
1.50 \\
5.82 \\
2.40 \\
1.98 \\
.92 \\
.68 \\
.62 \\
.31 \\
.75 \\
1.04 \\
1.84 \\
1.75\end{array}$ & $\begin{array}{r}2.63 \\
1.60 \\
1.02 \\
.92 \\
1.50 \\
1.11 \\
1.15 \\
1.10 \\
1.30 \\
.91 \\
.85 \\
.83 \\
.77 \\
.99 \\
1.15 \\
2.05\end{array}$ & $\begin{array}{l}17 \ldots \\
18 \ldots \\
19 \ldots \\
20 \ldots \\
21 \ldots \\
22 \ldots \\
23 \ldots \\
24 \ldots \\
25 \ldots \\
26 \ldots \\
27 \ldots \\
28 \ldots \\
29 \ldots \\
30 \ldots \\
31 \ldots\end{array}$ & $\begin{array}{r}0.59 \\
.53 \\
.52 \\
.51 \\
.50 \\
.50 \\
.49 \\
.50 \\
.48 \\
.55 \\
.53 \\
1.64 \\
.78 \\
.62 \\
.48\end{array}$ & $\begin{array}{r}0.68 \\
.49 \\
.30 \\
.22 \\
.28 \\
.23 \\
.20 \\
.18 \\
.21 \\
.24 \\
.17 \\
.18 \\
.20 \\
.10 \\
.14\end{array}$ & $\begin{array}{r}0.30 \\
.35 \\
.23 \\
.10 \\
.10 \\
.30 \\
.25 \\
.18 \\
.14 \\
.19 \\
.12 \\
.05 \\
.19 \\
1.64 \\
\ldots . . .\end{array}$ & $\begin{array}{r}0.29 \\
.23 \\
.20 \\
.25 \\
.29 \\
.27 \\
.29 \\
.28 \\
.32 \\
.28 \\
.31 \\
.35 \\
.29 \\
.31 \\
.30\end{array}$ & $\begin{array}{r}0.65 \\
.59 \\
.53 \\
.72 \\
.79 \\
.60 \\
.65 \\
.71 \\
.69 \\
.66 \\
.68 \\
.69 \\
.45 \\
1.70 \\
. . .2 .\end{array}$ & $\begin{array}{r}1.45 \\
1.21 \\
1.09 \\
1.12 \\
1.11 \\
1.05 \\
.99 \\
.94 \\
.91 \\
1.01 \\
.89 \\
.93 \\
.95 \\
.92 \\
.89\end{array}$ \\
\hline
\end{tabular}

Broad River at Alston, South Carolina.

\begin{tabular}{|c|c|c|c|c|c|c|c|c|c|c|c|c|c|}
\hline Day. & July. & Aug. & Sept. & oct. & Nov. & Dec. & Day. & July. & Ang. & Sept. & Oet. & Nov. & Dec. \\
\hline $\begin{array}{r}1 \ldots \ldots \\
2 \ldots \ldots \\
3 \ldots \ldots \\
4 \ldots \ldots \\
5 \ldots \ldots \\
6 \ldots \ldots \\
7 \ldots \ldots \\
8 \ldots \ldots \\
9 \ldots \ldots \\
10 \ldots \ldots \\
11 \ldots \ldots \\
12 \ldots \ldots \\
13 \ldots \ldots \\
14 \ldots \ldots \\
15 \ldots \ldots \\
16 \ldots \ldots\end{array}$ & \begin{tabular}{r}
$\ldots . .$. \\
\hdashline 2.21 \\
2.17 \\
2.87 \\
2.64 \\
8.20 \\
13.39 \\
16.24 \\
17.26 \\
13.59 \\
13.39 \\
9.92 \\
6.55 \\
5.05 \\
4.20
\end{tabular} & $\begin{array}{l}2.61 \\
3.02 \\
3.03 \\
2.95 \\
2.86 \\
2.61 \\
2.51 \\
2.43 \\
2.30 \\
2.03 \\
1.91 \\
2.87 \\
2.07 \\
2.92 \\
2.71 \\
3.31\end{array}$ & $\begin{array}{l}1.50 \\
1.85 \\
1.93 \\
2.73 \\
4.75 \\
4.93 \\
3.89 \\
3.21 \\
2.52 \\
2.18 \\
2.13 \\
2.15 \\
2.36 \\
2.51 \\
1.96 \\
2.15\end{array}$ & $\begin{array}{l}8.35 \\
3.95 \\
3.45 \\
2.52 \\
2.21 \\
1.90 \\
1.85 \\
2.15 \\
2.10 \\
2.00 \\
2.05 \\
1.81 \\
1.72 \\
2.02 \\
2.20 \\
2.40\end{array}$ & $\begin{array}{r}2.71 \\
2.63 \\
2.20 \\
2.40 \\
9.47 \\
13.38 \\
10.33 \\
7.20 \\
4.11 \\
3.56 \\
2.73 \\
3.15 \\
5.11 \\
4.78 \\
3.85 \\
3.31\end{array}$ & $\begin{array}{l}8.46 \\
7.80 \\
6.55 \\
6.00 \\
5.33 \\
5.21 \\
5.84 \\
6.77 \\
6.35 \\
5.61 \\
4.40 \\
3.96 \\
3.64 \\
3.70 \\
3.40 \\
6.30\end{array}$ & $\begin{array}{l}17 \ldots \ldots \\
18 \ldots \ldots \\
19 \ldots \ldots \\
20 \ldots \ldots \\
21 \ldots \ldots \\
22 \ldots \ldots \\
23 \ldots \ldots \\
24 \ldots \ldots \\
25 \ldots \ldots \\
26 \ldots \ldots \\
27 \ldots \ldots \\
28 \ldots \ldots \\
29 \ldots \ldots \\
30 \ldots \ldots \\
31 \ldots \ldots\end{array}$ & $\begin{array}{l}4.83 \\
4.03 \\
3.87 \\
5.00 \\
5.42 \\
4.06 \\
3.84 \\
3.30 \\
3.14 \\
2.90 \\
2.87 \\
2.79 \\
4.77 \\
3.40 \\
2.90\end{array}$ & $\begin{array}{l}2.13 \\
2.86 \\
2.52 \\
2.35 \\
3.67 \\
2.81 \\
1.98 \\
1.95 \\
2.05 \\
2.01 \\
2.15 \\
2.19 \\
2.44 \\
2.15 \\
1.65\end{array}$ & $\begin{array}{l}1.99 \\
1.09 \\
2.05 \\
2.01 \\
1.93 \\
2.78 \\
4.63 \\
2.68 \\
2.51 \\
1.94 \\
1.85 \\
1.85 \\
2.11 \\
8.78 \\
\ldots . . .\end{array}$ & $\begin{array}{l}2.60 \\
1.96 \\
1.95 \\
1.75 \\
1.90 \\
2.02 \\
2.03 \\
2.40 \\
2.64 \\
2.49 \\
2.07 \\
2.25 \\
2.20 \\
2.10 \\
2.26\end{array}$ & $\begin{array}{l}3.07 \\
2.89 \\
2.73 \\
2.74 \\
2.76 \\
2.60 \\
2.69 \\
2.56 \\
2.72 \\
2.66 \\
2.62 \\
2.70 \\
2.75 \\
4.26 \\
. . . .\end{array}$ & $\begin{array}{l}6.01 \\
4.39 \\
4.06 \\
3.80 \\
3.55 \\
3.50 \\
3.45 \\
3.40 \\
3.25 \\
3.15 \\
3.04 \\
3.06 \\
3.09 \\
3.18 \\
3.07\end{array}$ \\
\hline
\end{tabular}


Saluda River at Waterloo, South Carolina.

\begin{tabular}{|c|c|c|c|c|c|c|c|c|c|c|c|}
\hline Day. & Ang. & Sept. & Oet. & Nov. & Dec. & Day. & Aug. & Sept. & Oet. & Nov. & Dec. \\
\hline 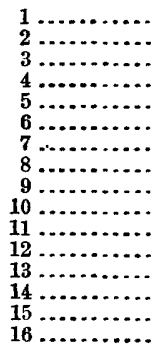 & $\mid \begin{array}{l}\ldots \ldots \\
\ldots \ldots \\
\ldots \ldots \\
\ldots \ldots \\
\ldots \ldots \\
\cdots \ldots \\
\cdots \\
\cdots\end{array}$ & $\begin{array}{l}2.90 \\
3.50 \\
2.80 \\
1.90 \\
3.50 \\
4.15 \\
3.80 \\
4.00 \\
3.60 \\
3.30 \\
3.30 \\
3.90 \\
3.00 \\
2.30 \\
3.65 \\
3.25\end{array}$ & $\begin{array}{l}4.00 \\
3.50 \\
3.75 \\
3.70 \\
2.40 \\
3.70 \\
3.65 \\
3.65 \\
3.45 \\
3.10 \\
3.00 \\
2.20 \\
3.45 \\
3.60 \\
3.60 \\
3.30\end{array}$ & $\begin{array}{r}3.70 \\
2.60 \\
4.00 \\
10.80 \\
14.50 \\
14.50 \\
11.35 \\
5.60 \\
5.10 \\
4.90 \\
4.70 \\
4.50 \\
6.30 \\
6.20 \\
4.85 \\
4.70\end{array}$ & $\begin{array}{l}8.30 \\
(a) \\
(a) \\
(a) \\
5.50 \\
5.90 \\
5.15 \\
5.85 \\
5.80 \\
5.20 \\
4.90 \\
4.75 \\
3.90 \\
4.30 \\
8.40 \\
7.80\end{array}$ & 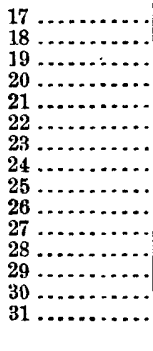 & $\begin{array}{l}\ldots \ldots \\
\ldots \ldots \ldots \\
\ldots \ldots \\
\ldots \ldots \\
\ldots \ldots \\
\ldots \ldots \ldots \\
\cdots \ldots \\
\ldots \ldots \\
\ldots \ldots \\
\ldots \ldots \\
\ldots \ldots \\
\ldots \ldots \\
\ldots \ldots \\
3.20 \\
2.20\end{array}$ & $\begin{array}{l}3.15 \\
3.15 \\
3.70 \\
3.00 \\
2.20 \\
4.25 \\
4.35 \\
4.00 \\
3.30 \\
3.20 \\
2.90 \\
2.30 \\
5.40 \\
5.35 \\
\ldots \ldots \ldots\end{array}$ & $\begin{array}{l}2.80 \\
3.15 \\
2.20 \\
3.20 \\
3.15 \\
3.00 \\
3.60 \\
4.10 \\
3.90 \\
2.45 \\
3.80 \\
3.80 \\
3.55 \\
3.70 \\
4.30\end{array}$ & $\begin{array}{l}4.65 \\
4.55 \\
4.50 \\
4.45 \\
4.45 \\
3.80 \\
3.80 \\
4.30 \\
4.35 \\
4.25 \\
4.30 \\
4.25 \\
5.20 \\
7.55 \\
\ldots . . .\end{array}$ & $\begin{array}{l}5.55 \\
4.75 \\
5.15 \\
4.75 \\
4.35 \\
4.65 \\
4.00 \\
4.55 \\
3.85 \\
3.20 \\
2.85 \\
3.80 \\
4.35 \\
4.40 \\
4.35\end{array}$ \\
\hline
\end{tabular}

$a$ Ice on river; no readings December 2,3 , and 4 .

Savannah River near Calhoun Falls, South Carolina.

\begin{tabular}{|c|c|c|c|c|c|c|c|c|c|c|c|}
\hline Day. & Aug. & Sept. & Oct. & Nov. & Dec. & Day. & Aug. & Sept. & Oct. & Nov. & Dec. \\
\hline 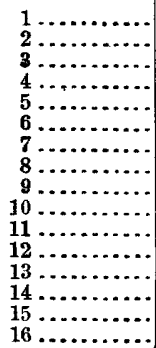 & $\begin{array}{l}\ldots \ldots \\
\cdots \ldots \ldots \\
\cdots \ldots . \\
2.40 \\
2.30 \\
2.15 \\
2.10 \\
2.00 \\
2.05 \\
2.10 \\
2.05 \\
2.00 \\
1.95 \\
2.80 \\
3.10 \\
2.30\end{array}$ & $\begin{array}{l}2.00 \\
1.90 \\
1.85 \\
1.80 \\
1.95 \\
3.85 \\
3.00 \\
2.40 \\
2.40 \\
2.25 \\
2.20 \\
2.30 \\
2.15 \\
2.10 \\
2.05 \\
2.00\end{array}$ & $\begin{array}{l}2.00 \\
1.95 \\
1.90 \\
1.95 \\
1.90 \\
1.85 \\
1.90 \\
1.75 \\
1.70 \\
1.70 \\
2.00 \\
2.25 \\
2.50 \\
2.40 \\
2.15 \\
2.00\end{array}$ & $\begin{array}{l}2.20 \\
2.15 \\
2.05 \\
3.00 \\
5.65 \\
7.15 \\
4.75 \\
3.00 \\
2.60 \\
2.45 \\
2.30 \\
2.20 \\
5.60 \\
4.10 \\
3.60 \\
3.25\end{array}$ & $\begin{array}{l}5.60 \\
5.00 \\
4.95 \\
5.15 \\
5.00 \\
4.05 \\
3.50 \\
3.75 \\
3.05 \\
3.20 \\
2.85 \\
2.60 \\
2.45 \\
2.55 \\
3.85 \\
3.20\end{array}$ & 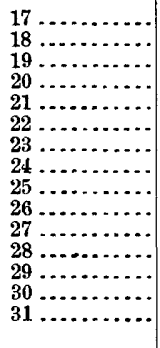 & $\begin{array}{l}2.10 \\
2.05 \\
2.05 \\
2.00 \\
1.90 \\
1.85 \\
1.80 \\
1.75 \\
1.75 \\
2.00 \\
2.45 \\
2.00 \\
1.95 \\
1.90 \\
1.85\end{array}$ & $\begin{array}{l}2.00 \\
1.95 \\
1.90 \\
1.85 \\
1.80 \\
1.70 \\
2.50 \\
2.40 \\
2.35 \\
2.25 \\
2.00 \\
1.90 \\
1.95 \\
2.00 \\
\ldots \ldots\end{array}$ & $\begin{array}{l}1.95 \\
1.90 \\
1.85 \\
1.80 \\
1.80 \\
1.75 \\
1.75 \\
2.15 \\
2.05 \\
2.00 \\
1.95 \\
1.90 \\
1.85 \\
1.95 \\
2.10\end{array}$ & $\begin{array}{c}3.00 \\
2.90 \\
2.65 \\
2.40 \\
2.25 \\
2.35 \\
2.30 \\
2.30 \\
2.30 \\
2.25 \\
2.25 \\
2.20 \\
2.30 \\
2.95 \\
\ldots \ldots . .\end{array}$ & $\begin{array}{l}3.40 \\
3.10 \\
3.00 \\
2.85 \\
2.80 \\
2.75 \\
2.65 \\
2.60 \\
2.55 \\
2.50 \\
2.45 \\
2.40 \\
2.40 \\
2.40 \\
2.35\end{array}$ \\
\hline
\end{tabular}

Oconee River ${ }^{1}$ at Dublin, Georgia, for 1894.

\begin{tabular}{|c|c|c|c|c|c|c|c|c|c|c|c|c|}
\hline Day. & Jan. & Feb. & Mar. & Apr. & Мау. & June. & July. & Aug. & Sept. & Oct. & Nov. & Dec. \\
\hline 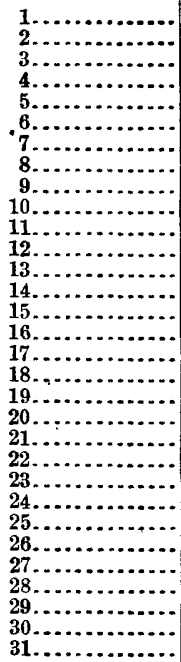 & $\begin{array}{l}3.6 \\
3.6 \\
3.0 \\
2.6 \\
1.9 \\
1.8 \\
2.0 \\
2.0 \\
2.6 \\
3.8 \\
4.4 \\
\text { 5.0 } \\
6.0 \\
6.6 \\
6.9 \\
6.5 \\
4.9 \\
5.0 \\
4.9 \\
4.3 \\
3.5 \\
3.0 \\
3.2 \\
3.8 \\
3.7 \\
3.5 \\
4.0 \\
3.9 \\
3.4 \\
3.0 \\
2.9\end{array}$ & \begin{tabular}{r}
2.8 \\
2.5 \\
2.3 \\
2.3 \\
2.5 \\
2.7 \\
2.9 \\
2.7 \\
2.3 \\
2.2 \\
2.2 \\
4.0 \\
6.6 \\
7.8 \\
9.7 \\
11.3 \\
12.3 \\
13.7 \\
14.9 \\
14.3 \\
12.7 \\
10.3 \\
8.4 \\
7.8 \\
8.0 \\
9.6 \\
10.2 \\
10.8 \\
\hdashline$\ldots .$. \\
$\cdots \ldots .$. \\
$\cdots \cdots$
\end{tabular} & $\begin{array}{r}11.2 \\
11.8 \\
12.4 \\
12.8 \\
13.0 \\
12.7 \\
11.0 \\
8.0 \\
6.5 \\
6.0 \\
5.5 \\
5.5 \\
7.5 \\
8.6 \\
8.9 \\
8.6 \\
6.9 \\
6.4 \\
6.4 \\
6.3 \\
5.9 \\
5.0 \\
4.4 \\
\text { 4. } \\
5.1 \\
6.2 \\
6.9 \\
6.0 \\
5.3 \\
4.5 \\
4.0\end{array}$ & $\begin{array}{r}3.8 \\
3.5 \\
3.4 \\
3.4 \\
3.5 \\
3.8 \\
3.8 \\
3.7 \\
3.1 \\
2.9 \\
3.0 \\
4.0 \\
4.8 \\
4.1 \\
3.3 \\
2.9 \\
2.6 \\
2.5 \\
2.5 \\
2.8 \\
4.5 \\
5.0 \\
4.9 \\
4.0 \\
3.0 \\
2.5 \\
2.3 \\
2.0 \\
1.9 \\
1.9 \\
\ldots \ldots . .\end{array}$ & $\begin{array}{r}1.9 \\
1.8 \\
1.8 \\
1.5 \\
1.4 \\
1.3 \\
1.1 \\
1.0 \\
.9 \\
.9 \\
.9 \\
1.5 \\
1.1 \\
.9 \\
.9 \\
1.5 \\
2.9 \\
2.5 \\
2.0 \\
1.6 \\
1.9 \\
2.0 \\
1.5 \\
1.2 \\
1.0 \\
1.0 \\
.9 \\
.6 \\
.5 \\
.5 \\
.4\end{array}$ & $\begin{array}{r}0.2 \\
.1 \\
.0 \\
.1 \\
.1 \\
.2 \\
.2 \\
.3 \\
.4 \\
.4 \\
.4 \\
.5 \\
.6 \\
.7 \\
.7 \\
.7 \\
.7 \\
.6 \\
.3 \\
.4 \\
.2 \\
.1 \\
.4 \\
.8 \\
.4 \\
1.0 \\
2.9 \\
1.5 \\
.9 \\
1.5 \\
. . .5\end{array}$ & $\begin{array}{r}1.4 \\
.6 \\
.6 \\
1.0 \\
.8 \\
.5 \\
1.1 \\
.9 \\
.3 \\
1.4 \\
4.9 \\
4.8 \\
3.3 \\
1.8 \\
.9 \\
.8 \\
.7 \\
.4 \\
1.1 \\
.3 \\
3.7 \\
5.0 \\
4.4 \\
3.8 \\
4.4 \\
4.6 \\
3.8 \\
3.4 \\
4.4 \\
3.4 \\
3.0\end{array}$ & $\begin{array}{r}5.0 \\
6.8 \\
7.5 \\
6.5 \\
5.0 \\
8.0 \\
9.4 \\
10.0 \\
11.0 \\
13.2 \\
14.9 \\
13.6 \\
7.8 \\
4.0 \\
3.8 \\
2.0 \\
1.4 \\
1.4 \\
4.9 \\
6.0 \\
5.1 \\
3.3 \\
2.9 \\
2.3 \\
2.1 \\
2.8 \\
4.4 \\
4.2 \\
4.8 \\
4.2 \\
5.0\end{array}$ & $\begin{array}{r}4.9 \\
3.9 \\
2.9 \\
1.4 \\
1.0 \\
1.3 \\
1.4 \\
1.1 \\
1.8 \\
1.8 \\
1.4 \\
1.4 \\
1.3 \\
1.0 \\
.9 \\
.8 \\
.6 \\
.9 \\
2.2 \\
4.5 \\
6.1 \\
6.6 \\
4.7 \\
2.1 \\
1.4 \\
1.0 \\
1.0 \\
1.0 \\
1.0 \\
1.0 \\
. .2\end{array}$ & $\begin{array}{r}1.0 \\
.8 \\
.6 \\
.4 \\
.2 \\
.1 \\
.2 \\
.2 \\
1.1 \\
6.5 \\
8.6 \\
9.8 \\
12.5 \\
14.8 \\
13.8 \\
8.4 \\
3.4 \\
2.6 \\
2.0 \\
1.8 \\
1.6 \\
1.4 \\
1.1 \\
1.0 \\
1.0 \\
1.0 \\
1.0 \\
.9 \\
.9 \\
.8 \\
.8\end{array}$ & $\begin{array}{r}2.0 \\
2.4 \\
3.0 \\
6.1 \\
7.6 \\
6.0 \\
3.6 \\
2.7 \\
2.0 \\
1.7 \\
1.5 \\
1.4 \\
1.3 \\
1.3 \\
1.4 \\
1.6 \\
1.8 \\
1.8 \\
1.6 \\
1.6 \\
1.4 \\
1.5 \\
1.6 \\
1.5 \\
1.7 \\
1.7 \\
1.8 \\
1.7 \\
1.4 \\
1.4 \\
\ldots . . .6\end{array}$ & $\begin{array}{r}1.3 \\
1.2 \\
1.1 \\
1.0 \\
2.0 \\
2.4 \\
2.4 \\
2.1 \\
1.9 \\
1.7 \\
2.2 \\
3.4 \\
6.0 \\
8.1 \\
9.5 \\
11.4 \\
12.9 \\
13.0 \\
10.5 \\
5.4 \\
3.8 \\
3.2 \\
2.9 \\
2.5 \\
2.3 \\
2.2 \\
2.7 \\
3.9 \\
5.0 \\
5.3 \\
4.3\end{array}$ \\
\hline
\end{tabular}

1 Record from United States Weather Bureau. 
Oconee River ${ }^{1}$ at Dublin, Georgia, for 1895.

\begin{tabular}{|c|c|c|c|c|c|c|c|c|c|c|}
\hline Day. & Jan. & Feb. & Mar. & Apr. & May. & June. & July. & Aug. & Sept. & Oet. \\
\hline 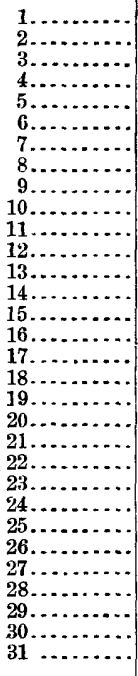 & $\begin{array}{r}3.4 \\
3.2 \\
3.2 \\
3.5 \\
3.8 \\
3.7 \\
3.4 \\
3.0 \\
3.0 \\
7.7 \\
10.4 \\
11.9 \\
15.5 \\
18.0 \\
17.9 \\
16.9 \\
15.5 \\
13.4 \\
10.1 \\
7.4 \\
6.1 \\
5.4 \\
5.0 \\
4.9 \\
4.7 \\
7.8 \\
10.5 \\
10.7 \\
11.8 \\
13.2 \\
14.0\end{array}$ & $\begin{array}{r}14.2 \\
14.5 \\
14.8 \\
14.2 \\
13.4 \\
12.1 \\
11.3 \\
10.0 \\
9.2 \\
8.7 \\
7.8 \\
7.1 \\
7.8 \\
7.9 \\
7.9 \\
7.3 \\
7.4 \\
7.5 \\
7.7 \\
8.1 \\
8.5 \\
8.3 \\
8.0 \\
7.7 \\
7.4 \\
6.8 \\
6.1 \\
5.7 \\
\ldots \ldots . . \\
\ldots \ldots . . . \\
\ldots \ldots . .\end{array}$ & $\begin{array}{r}5.4 \\
5.2 \\
5.0 \\
6.8 \\
7.8 \\
8.3 \\
8.5 \\
8.6 \\
9.9 \\
10.9 \\
12.0 \\
13.1 \\
13.1 \\
13.1 \\
15.4 \\
17.9 \\
20.2 \\
22.0 \\
23.4 \\
22.9 \\
21.6 \\
19.6 \\
17.2 \\
15.5 \\
14.0 \\
12.6 \\
10.9 \\
9.0 \\
7.5 \\
6.8 \\
5.6\end{array}$ & $\begin{array}{r}5.2 \\
5.0 \\
5.2 \\
5.8 \\
5.7 \\
5.2 \\
4.6 \\
5.8 \\
8.4 \\
9.2 \\
10.3 \\
10.1 \\
10.2 \\
8.8 \\
6.0 \\
4.8 \\
4.8 \\
6.1 \\
7.4 \\
8.5 \\
9.8 \\
10.4 \\
9.7 \\
7.4 \\
5.5 \\
5.0 \\
5.0 \\
6.6 \\
7.8 \\
8.8 \\
\ldots \ldots . . \\
\end{array}$ & $\begin{array}{r}9.6 \\
10.3 \\
10.5 \\
8.7 \\
6.6 \\
5.6 \\
5.2 \\
5.2 \\
4.9 \\
4.7 \\
6.4 \\
6.1 \\
6.0 \\
5.4 \\
4.4 \\
3.8 \\
3.4 \\
3.1 \\
3.3 \\
3.2 \\
3.5 \\
3.7 \\
4.7 \\
4.7 \\
4.8 \\
5.6 \\
6.7 \\
7.8 \\
8.4 \\
9.2 \\
9.0\end{array}$ & $\begin{array}{c}6.8 \\
4.4 \\
3.6 \\
3.0 \\
2.8 \\
2.6 \\
2.4 \\
2.4 \\
3.0 \\
2.3 \\
2.1 \\
2.0 \\
1.9 \\
1.9 \\
2.3 \\
3.0 \\
2.6 \\
3.8 \\
5.7 \\
4.8 \\
2.9 \\
2.2 \\
1.9 \\
1.9 \\
1.8 \\
1.7 \\
1.6 \\
1.6 \\
2.5 \\
2.0 \\
\ldots \ldots . . \\
. .9\end{array}$ & $\begin{array}{r}2.5 \\
5.0 \\
6.5 \\
6.9 \\
6.4 \\
5.0 \\
3.8 \\
4.3 \\
7.4 \\
8.7 \\
9.5 \\
10.5 \\
11.4 \\
11.5 \\
7.6 \\
4.0 \\
3.2 \\
2.7 \\
2.4 \\
2.9 \\
2.5 \\
2.2 \\
2.0 \\
1.8 \\
1.7 \\
1.5 \\
1.4 \\
1.3 \\
1.2 \\
1.2 \\
1.0\end{array}$ & $\begin{array}{r}0.9 \\
.7 \\
.6 \\
.8 \\
6.5 \\
6.6 \\
4.0 \\
2.7 \\
3.5 \\
3.8 \\
2.9 \\
2.3 \\
2.0 \\
2.1 \\
3.3 \\
5.3 \\
6.1 \\
7.0 \\
7.5 \\
8.2 \\
9.5 \\
11.0 \\
12.0 \\
11.7 \\
9.7 \\
5.5 \\
3.3 \\
3.3 \\
3.9 \\
3.0 \\
2.4\end{array}$ & $\begin{array}{r}2.3 \\
2.9 \\
2.5 \\
2.4 \\
5.7 \\
6.5 \\
7.0 \\
7.3 \\
8.3 \\
9.2 \\
7.5 \\
4.3 \\
4.8 \\
4.3 \\
3.2 \\
2.4 \\
2.2 \\
1.8 \\
1.8 \\
1.5 \\
1.4 \\
1.2 \\
1.0 \\
.9 \\
.8 \\
.6 \\
.5 \\
.4 \\
.3 \\
.3 \\
. . .7\end{array}$ & $\begin{array}{l}0.3 \\
.3 \\
.2 \\
.1 \\
.1 \\
.0 \\
.0 \\
.1 \\
.1 \\
.1 \\
.5 \\
.4 \\
.3 \\
.3 \\
.7 \\
.6 \\
.5 \\
.4 \\
.4 \\
.3 \\
.3 \\
.3 \\
.1 \\
.1 \\
.1 \\
.0 \\
.0 \\
.0 \\
.0 \\
.1 \\
.1\end{array}$ \\
\hline
\end{tabular}

Oconee River ${ }^{1}$ at Dublin, Georgia, for 1896.

[No record was kept at this station from May to October, 1896. Capt. J. D. Roberts, in charge of the steamers Henry and Gipsy, states that from the 15 th of September to the 15th of October, 1896, oceurred the lowest water in six years, the gage at Dublin going down to -1.20 and remaining at about that level for nearly thirty days. This iuformation is valuable and authentic.]

\begin{tabular}{|c|c|c|c|c|c|c|c|}
\hline Day. & Jan. & Feb. & Mar. & Apr. & Oct. & & Dec. \\
\hline 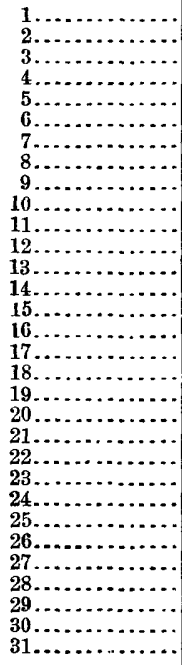 & $\begin{array}{r}4.1 \\
5.0 \\
4.7 \\
3.6 \\
2.8 \\
2.2 \\
1.9 \\
1.9 \\
2.7 \\
4.0 \\
4.0 \\
3.5 \\
3.0 \\
2.5 \\
2.3 \\
2.0 \\
3.5 \\
6.7 \\
7.5 \\
8.0 \\
8.0 \\
6.4 \\
5.0 \\
6.9 \\
8.3 \\
8.9 \\
10.0 \\
11.4 \\
12.3 \\
12.0 \\
10.9\end{array}$ & $\begin{array}{r}6.0 \\
4.5 \\
4.4 \\
6.5 \\
6.4 \\
8.4 \\
11.0 \\
11.6 \\
14.8 \\
17.0 \\
16.8 \\
16.5 \\
15.9 \\
15.0 \\
13.4 \\
11.2 \\
9.6 \\
8.7 \\
6.7 \\
5.8 \\
5.2 \\
4.8 \\
4.6 \\
4.2 \\
4.1 \\
4.0 \\
4.0 \\
3.9 \\
5.0 \\
\ldots \ldots \\
\cdots \ldots \ldots\end{array}$ & $\begin{array}{l}6.6 \\
6.8 \\
6.4 \\
5.4 \\
5.0 \\
4.0 \\
3.8 \\
4.6 \\
6.4 \\
6.2 \\
5.8 \\
6.7 \\
7.3 \\
7.6 \\
7.0 \\
5.8 \\
5.2 \\
4.8 \\
4.6 \\
4.4 \\
4.7 \\
4.6 \\
4.3 \\
4.1 \\
3.9 \\
4.5 \\
4.4 \\
4.2 \\
3.9 \\
3.7 \\
3.3\end{array}$ & $\begin{array}{r}3.0 \\
3.8 \\
5.5 \\
5.8 \\
5.0 \\
4.4 \\
3.5 \\
3.0 \\
2.8 \\
2.7 \\
2.6 \\
2.4 \\
2.3 \\
2.2 \\
2.1 \\
2.0 \\
1.9 \\
1.8 \\
1.7 \\
1.7 \\
1.6 \\
1.4 \\
1.2 \\
1.0 \\
.9 \\
.9 \\
1.1 \\
1.1 \\
1.5 \\
1.8 \\
\ldots . . .\end{array}$ & 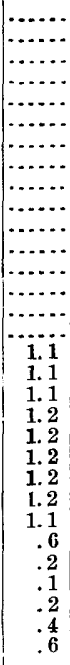 & $\begin{array}{r}0.5 \\
.1 \\
.4 \\
1.1 \\
5.4 \\
7.7 \\
19.3 \\
10.5 \\
0.2 \\
7.5 \\
3.4 \\
2.5 \\
2.5 \\
3.6 \\
3.7 \\
3.9 \\
3.5 \\
2.5 \\
1.7 \\
1.5 \\
1.3 \\
1.1 \\
1.0 \\
.9 \\
.8 \\
.6 \\
.5 \\
.6 \\
.6 \\
.6 \\
\ldots . .5\end{array}$ & $\begin{array}{r}3.2 \\
6.7 \\
9.5 \\
10.7 \\
11.4 . \\
12.8 \\
13.1 \\
12.4 \\
10.1 \\
7.3 \\
6.5 \\
5.6 \\
4.5 \\
4.1 \\
4.1 \\
4.0 \\
8.0 \\
8.9 \\
9.7 \\
8.1 \\
5.3 \\
4.1 \\
3.7 \\
3.2 \\
3.0 \\
2.8 \\
2.5 \\
2.3 \\
2.3 \\
2.2 \\
2.2\end{array}$ \\
\hline
\end{tabular}

1 Record from United States Weather Bureau. 
Oconee River at Cary, Georgia.

\begin{tabular}{|c|c|c|c|c|c|c|c|c|c|c|c|}
\hline Day. & Nov. & Dec. & Day. & Nov. & Dec. & Day. & Nov. & Dec. & Day. & Nov. & Dec. \\
\hline $\begin{array}{l}1 \ldots . \\
2 \ldots \\
3 \ldots \\
4 \ldots \\
5 \ldots \\
6 \ldots \\
7 \ldots . \\
8 \ldots\end{array}$ & $\begin{array}{l}2.10 \\
1.90 \\
1.70 \\
2.10 \\
2.70 \\
2.30 \\
2.20 \\
1.80\end{array}$ & $\begin{array}{l}5.10 \\
4.80 \\
4.40 \\
4.20 \\
3.70 \\
3.40 \\
3.20 \\
3.00\end{array}$ & $\begin{array}{c}9 \ldots \\
10 \ldots \\
11 \ldots \\
12 \ldots \\
13 \ldots \\
14 \ldots \\
15 \ldots \\
16 \ldots\end{array}$ & $\begin{array}{l}1.80 \\
1.80 \\
1.80 \\
1.90 \\
1.80 \\
1.90 \\
1.90 \\
2.00\end{array}$ & $\begin{array}{l}2.90 \\
2.70 \\
2.60 \\
2.50 \\
2.40 \\
2.30 \\
4.00 \\
2.80\end{array}$ & $\begin{array}{l}17 \ldots \\
18 \ldots \\
19 \ldots \\
20 \ldots \\
21 \ldots \\
22 \ldots \\
23 \ldots \\
24 \ldots\end{array}$ & $\because$ & $\begin{array}{l}2.40 \\
2.20 \\
2.40 \\
2.30 \\
2.20 \\
2.20 \\
2.10 \\
2.00\end{array}$ & $\begin{array}{l}25 \ldots \\
26 \ldots \\
27 \ldots \\
28 \ldots \\
29 . \ldots \\
30 \ldots \\
31 \ldots\end{array}$ & $\begin{array}{r}1.90 \\
1.90 \\
1.80 \\
1.90 \\
2.90 \\
3.90 \\
\ldots . . .\end{array}$ & $\begin{array}{r}2.00 \\
2.10 \\
2.00 \\
1.90 \\
2.10 \\
2.00 \\
2.00\end{array}$ \\
\hline
\end{tabular}

Oemulgee River ${ }^{1}$ at Macon, Georgia, for 1893.

\begin{tabular}{|c|c|c|c|c|c|c|c|c|c|c|c|c|}
\hline Day. & Jan. & Feb. & Mar. & Apr. & May. & June. & Jaly. & Aug. & Sept. & Oet. & Nov. & Dec. \\
\hline 1. & & 5.30 & 12.70 & $2.40^{\circ}$ & 1.60 & 2.60 & 1.00 & 1. 40 & 9. 30 & 1. 30 & 1.70 & 2. 50 \\
\hline 2 . & $\ldots$ & 4. 80 & 10,00 & 2.30 & 1.40 & 2.40 & 1. 10 & 1.80 & 8. 70 & 1. 30 & 1. 60 & 3. 00 \\
\hline 3. & & 3.90 & 7.80 & 2.20 & 1. 80 & 5.40 & 1. 10 & 2.20 - & 5.50 & 1.50 & 1. 60 & 2. 80 \\
\hline 4... & & 3.10 & 12. 70 & 2.10 & 6. 70 & 6.80 & 1.80 & 1.80 & 3.00 & 8.00 & 1. 60 & 2.70 \\
\hline $5 \ldots$ & & 2.80 & 12.20 & 2.10 & 3. 40 & 6.70 & 1.00 & 2. 00 & 2. 80 & 5. 00 & 1. 60 & 2. 50 \\
\hline & & 3.40 & 9.50 & 2.30 & 3.10 & 5. 10 & 1.00 & 6.20 & 2.40 & 3. 50 & 1. 60 & 3. 20 \\
\hline & & 2. 30 & 7.10 & 2.50 & 7.20 & 5. 50 & 1.00 & 13.40 & 2. 40 & 3.00 & 1. 50 & 2. 00 \\
\hline & & 1.80 & 6.50 & 2.20 & 4. 90 & 9.70 & 1.00 & 9.50 & 2. 30 & 2.80 & 1. 50 & 1.80 \\
\hline 9 . & & 1.60 & 4. 70 & 2.00 & 4. 90 & 7.00 & 1.80 & 5.00 & 3.00 & 2. 70 & 1.50 & 1.70 \\
\hline 10. & & 1.30 & 8. 90 & 1.80 & 3.40 & 3. 40 & 1.20 & 3.20 & 4.00 & 2. 60 & 1. 50 & 160 \\
\hline 11. & & 2.80 & 7.80 & 1.70 & 2.90 & 2.50 & 1. 20 & 2.00 & 4.10 & 2.60 & 1.50 & 1. 50 \\
\hline 12. & & 4.60 & 5.80 & 1.70 & 2.20 & 2.20 & 1. 20 & 1.80 & 5.40 & 2.50 & 1.50 & 1.50 \\
\hline 13. & & 14.60 & 5. 00 & 1.60 & 1.80 & 2.00 & 1.10 & 1. 70 & 9. 40 & 2. 50 & 1.50 & 1. 50 \\
\hline 14. & & 13.00 & 4.10 & 1. 60 & 1.60 & 3.00 & 1.10 & 1. 70 & 10.90 & 2.80 & 1.50 & 1.50 \\
\hline 15. & & 11.20 & 3.50 & 1. 60 & 1. 30 & 3.00 & 1.10 & 4. 20 & 6. 80 & 2.70 & 1.50 & 1. 50 \\
\hline 16. & & 11.00 & 2.60 & 1.70 & 1. 20 & 3.00 & .90 & 4.00 & 4.00 & 2.50 & 1.50 & 1.51 \\
\hline 17. & & 13.40 & 3.00 & 1.60 & 1.50 & 2.00 & .30 & 2.00 & 3.50 & 2.50 & 1.50 & 3.00 \\
\hline 18. & & 12.00 & 2.40 & 1. 40 & 1. 20 & 1.80 & 1.00 & 1.90 & 2.00 & 2. 50 & 1.50 & 2. 80 \\
\hline 19. & & 11.30 & 2. 20 & 1. 20 & 1.20 & 3.00 & 1. 40 & 1. 70 & 2.00 & 2. 40 & 150 & 3.50 \\
\hline 20 . & & 10.60 & 2.10 & 2.50 & 1.00 & 2. 60 & 1.60 & 1.60 & 1.60 & 2.20 & 1. 50 & 3.20 \\
\hline 21. & 2.70 & 6.80 & $\ldots$ & 9.00 & .80 & 2.30 & 1.70 & 1.60 & 1.60 & 2.10 & 2. 00 & 3. 10 \\
\hline 22. & 2.80 & 4. 80 & .. & 7.40 & .70 & 2. 40 & 5. 40 & 1.40 & 1. 50 & 2.10 & 2.80 & 3.00 \\
\hline 23. & 2.80 & 3. 30 & & 4. 30 & .60 & 2.20 & 2. 40 & 1.30 & 1.50 & 2.00 & 3.00 & 2. 80 \\
\hline & 2.60 & 5.40 & 2.90 & 2.90 & .70 & 2. 20 & 2. 20 & 1. 30 & 1.50 & 2. 00 & $2 . \times 0$ & 2.60 \\
\hline & 4. 60 & 4.00 & 3. 70 & 2.20 & .60 & 2. 20 & 2.00 & 1. 20 & 1. 40 & 2.00 & 2. 60 & 2. 40 \\
\hline & 5.00 & 3.60 & 5. 30 & 1.80 & .60 & 1. 00 & 1.80 & 1.20 & 1. 40 & 2. 00 & 2.40 & 3. 20 \\
\hline & 4.70 & 3.50 & 4.00 & 1.80 & .70 & 1.00 & 1. 80 & 1.10 & 1. 60 & 2. 00 & 2. 30 & 2. 00 \\
\hline 28 & 5. 30 & 11.90 & 3.10 & 1. 70 & .40 & 1.00 & 2.00 & 1.20 & 1. 50 & 1.80 & 2.10 & 1. 90 \\
\hline 29 & 5.80 & $\ldots$. & 2.80 & 1.60 & .20 & 1.10 & 1.40 & 1.40 & 1. 40 & 1. 70 & 2.00 & 1. 80 \\
\hline $30 \ldots$ & 6. 30 & ...... & 2.60 & 1.60 & 6. 10 & 1.00 & 1.30 & 1.40 & 1. 40 & 1.70 & 2.00 & 2.50 \\
\hline 31. & 6.60 & $\cdots$ & 2.50 & $\ldots . .$. & 5.60 & 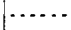 & 1.20 & 2.00 & $\ldots \ldots$ & 1.70 & $\ldots \ldots$ & 3,40 \\
\hline
\end{tabular}

1 Record from United States Weather Burean. 
Ocmulgee River ${ }^{1}$ at Macon, Georgia, for 1894.

\begin{tabular}{|c|c|c|c|c|c|c|c|c|c|c|c|c|}
\hline Day. & Jan. & Feb. & Mar. & Apr. & May. & June. & July. & Ang. & Sept. & Oet. & Nov. & Dec. \\
\hline $1 .$. & 3.20 & 2. 30 & 10.40 & 3.00 & 1.80 & 1. 20 & 1.80 & 7.00 & 3.40 & .90 & 5.00 & ]. 00 \\
\hline 2. & 3.00 & 2.00 & 9.10 & 3. 00 & 1.70 & 1.20 & 1.60 & 6. 00 & 3.20 & .90 & 5. 60 & 1.00 \\
\hline 3... & 2.80 & 2.00 & 8.60 & 2.50 & 1.50 & 1. 20 & 1.40 & 5. 80 & 3.00 & .90 & 7.00 & .90 \\
\hline $4 .$. & 2.60 & 3.00 & 8.00 & 2.50 & 1.50 & 1.20 & 1.30 & 4. 80 & 2. 80 & .80 & 6.50 & 1. 00 \\
\hline 5. & 2.30 & 2.80 & 4. 90 & 3.00 & 1.50 & 1.20 & 1. 20 & 9.00 & 2.60 & .80 & 6.00 & 1. 50 \\
\hline 6. & 2.00 & 2.60 & 4.20 & 2.80 & 1.50 & 1. 10 & 2.50 & 14.20 & 3.00 & .80 & 5.00 & 1.50 \\
\hline & 1.80 & 2.40 & 3.90 & 2.80 & 1. 50 & 1. 10 & 3.00 & 12.20 & 4.00 & .80 & 4.00 & 1. 40 \\
\hline 8. & 1. 60 & 2.30 & 3.80 & 2.50 & 1.50 & 1.00 & 2.80 & 9.60 & 6.00 & .90 & 3.00 & 1.30 \\
\hline & 3. 70 & 2. 20 & 3.70 & 2.50 & 1.50 & 1.00 & 2.60 & 6. 90 & 5. 50 & 11.00 & 2.80 & 1. 30 \\
\hline 10. & 3. 30 & 2.10 & 3.50 & 2.50 & 1. 30 & 1.00 & 3.00 & 5.40 & 5.00 & 15.10 & 2.40 & 2. 00 \\
\hline 11. & 4.50 & 3.00 & 3.20 & 5. 60 & 1. 30 & .90 & 4.00 & 4.00 & 4. 80 & 8.00 & 2.30 & 4. 00 \\
\hline 12. & 6.00 & 7.20 & 7.10 & 5. 20 & 1.30 & .90 & 3.80 & 4.00 & 4. 60 & 6. 00 & 2. 10 & 9.80 \\
\hline 13. & 6. 80 & 10.00 & 7.90 & 5. 00 & 1.20 & .90 & 3.40 & 3.80 & 5.00 & 4. 50 & 2.00 & 13.20 \\
\hline 14. & 5. 50 & 11.00 & 6.00 & 4. 60 & 1. 50 & 1,00 & 3.20 & 3.60 & 5. 80 & 4. 30 & 2.00 & 10.30 \\
\hline 15. & 3.20 & 13. 70 & 4. 00 & 4.00 & 3.00 & .90 & 3.00 & 3.40 & 4. 60 & 4. 10 & 1. 90 & 8. 40 \\
\hline 16. & 3.80 & 9.80 & 3.50 & 3.80 & 2.50 & .90 & 2.80 & 3.10 & 4.30 & 3.90 & 1. 80 & 7. 20 \\
\hline $17 .$. & 4.20 & 7.50 & 4.00 & 3.50 & 2.00 & .90 & 3.00 & 3.00 & 4. 20 & 3.60 & 1. 70 & 3. 00 \\
\hline 18. & 3.30 & 6.20 & 6.00 & 3.00 & 1.70 & 1.00 & 3.00 & 3.00 & 5.00 & 3.40 & 1. 70 & 2.50 \\
\hline 19. & 3.00 & 5.70 & 5.00 & 2.80 & 2.50 & .90 & 7. 60 & 4.00 & 5.30 & 3.20 & 1. 50 & 2. 40 \\
\hline 20. & 2.80 & 5.20 & 4. 60 & 6. 50 & 2.30 & .90 & 6.80 & 3.00 & 11.30 & 3.10 & 1. 50 & 2. 20 \\
\hline 21. & 3.00 & 4.90 & 4.30 & 6. 00 & 2.00 & .80 & 7. 90 & 4.50 & 5. 50 & 3.00 & 1.50 & 2.10 \\
\hline 22. & 3. 20 & 5,10 & 4.00 & 5.80 & 1.70 & 2.00 & 4.00 & 4.00 & 3.00 & 2.80 & 1. 50 & 2. $\dot{0} 0$ \\
\hline 23. & 3.00 & 5. 20 & 3.80 & 5.50 & 1. 50 & 2.50 & 3. 00 & 3.80 & 2. 80 & 2.60 & 1.50 & 2. 00 \\
\hline 24. & 3.60 & 5.60 & 3.50 & 4.00 & 1.30 & 2.60 & 3. 00 & 3.60 & 2. 60 & 2.40 & 1. 40 & 1.90 \\
\hline 25. & 3.20 & 6.00 & 3.20 & 3.70 & 1. 30 & 3.00 & 2.60 & 5.00 & 2.70 & 2.30 & 1. 30 & 1. 80 \\
\hline 26. & 3. 30 & 8.90 & 6.00 & 3. 50 & 1.30 & 2. 50 & 2.30 & 4.80 & 2. 30 & 2. 10 & 1. 30 & 1. 70 \\
\hline 27. & 3.20 & 10.40 & 5.60 & 3.20 & 1.30 & 2.00 & 3.00 & 460 & 2. 20 & 2.00 & 1.20 & 3.00 \\
\hline 28. & 3.00 & 9.50 & 4. 00 & 2.90 & 1.30 & I. 80 & 4. 00 & 4.20 & 2.10 & 1.80 & 1.20 & 4.00 \\
\hline & 2.80 & ...... & 3.50 & 2.00 & 1. 30 & 1.60 & 3. 70 & 4. 00 & 1.00 & 1. 70 & 1.10 & 3. 90 \\
\hline & 2.70 & & 3.20 & 2.00 & 1. 30 & 2.00 & 4.00 & 3.80 & 1.00 & 1.60 & 1.00 & 3. 70 \\
\hline & 2.50 & $\ldots \ldots$ & 3.00 & ...... & 1. 20 & ..... & 7.20 & 3.60 & $\ldots$. & 4.00 & ..... & 3. 50 \\
\hline
\end{tabular}

Ocmulgee River ${ }^{1}$ at Macon, Georgia, for 1895.

\begin{tabular}{|c|c|c|c|c|c|c|c|c|c|c|c|c|}
\hline Day. & Tan. & Feb. & Mar. & Apr. & May. & June. & July. & Aug. & Sept. & Oet. & Nov. & Dec. \\
\hline & 3. 50 & 6.00 & 3.00 & 4.60 & 6.00 & 3.80 & 10.90 & 280 & 4.40 & $a 200$ & 0.50 & 0.50 \\
\hline & $\begin{array}{l}0.50 \\
3.40\end{array}$ & $\begin{array}{l}0.00 \\
6.00\end{array}$ & $\begin{array}{l}3.00 \\
4.00\end{array}$ & $\begin{array}{l}4.00 \\
4.50\end{array}$ & $\begin{array}{l}0.50 \\
5.50\end{array}$ & 3.70 & 10.00 & $\begin{array}{l}2.80 \\
2.80\end{array}$ & $\begin{array}{l}4.40 \\
4.20\end{array}$ & $\begin{array}{r}a .00 \\
1.80\end{array}$ & $\begin{array}{r}.77 \\
\end{array}$ & $\begin{array}{r}.50 \\
.50\end{array}$ \\
\hline 3. & 3.20 & 5. 80 & 10.80 & 4. 30 & 4. 00 & 3. 60 & $\begin{array}{r}7.80 \\
7.80\end{array}$ & 2.80 & 4.00 & 1.60 & .85 & .65 \\
\hline & 3. 00 & 5. 30 & 10,00 & 4. 20 & 3.80 & 3.50 & 11.80 & 4.00 & 5. 00 & 1. 40 & 67 & 62 \\
\hline & 3.00 & 5.10 & 6.00 & 4.00 & 3. 70 & 3.50 & 10.00 & 6.00 & 4. 80 & 1. 40 & 55 & .54 \\
\hline & 2.80 & 4. 00 & 4.00 & 4.00 & 3. 60 & 3.40 & 9.50 & 5. 80 & 4. 60 & 1.20 & 45 & .51 \\
\hline & 2.70 & 4. 00 & 5.70 & 3. 90 & 3.60 & 3.30 & 9.00 & 5. 60 & 4. 30 & 1.00 & 36 & $\because 44$ \\
\hline & 2.60 & 3.90 & 10.90 & 11. 20 & 3.60 & 3. 20 & 13.40 & 5. 40 & 4.20 & 1.00 & 47 & .40 \\
\hline 9. & 3.00 & 3. 70 & 8. 50 & 11.00 & 3. 60 & 3. 20 & 10.00 & 5. 30 & 4.00 & 1.00 & 55 & .46 \\
\hline 10. & 15.00 & 3.60 & 6.00 & 8. 00 & 5.00 & 3.00 & 7.00 & 5. 00 & 3.90 & .80 & 63 & $4 \overline{5}$ \\
\hline 11. & 14.50 & 3.50 & 4.00 & 7.00 & 5.00 & 3. 00 & 5.00 & 4. 90 & 5. 10 & .60 & 65 & 2.50 \\
\hline 12. & 11. 50 & 3.50 & 4. 00 & 6.50 & 5.50 & 2.90 & 4.00 & 4. 70 & 4. 80 & .70 & 60 & 2. 29 \\
\hline 13. & 7.00 & 4.00 & 6.00 & 6.00 & 5. 30 & 2.90 & 3. 90 & 4.50 & 4.50 & .65 & 77 & 1. 51 \\
\hline 14. & 6.00 & 4. 20 & 14.50 & 5.80 & 5.00 & 3. 00 & 3. 70 & 4.60 & 4. 30 & .40 & 94 & 1. 11 \\
\hline 15. & 5. 00 & 4.70 & 15.80 & 5. 30 & 4.80 & 4.00 & 3.50 & 7.00 & 4. 10 & .30 & .72 & 1.01 \\
\hline 16. & 3.00 & 4.50 & 16. 40 & 5.00 & 4.60 & 4.00 & 3.40 & 9.70 & 4.00 & .20 & 65 & 1. 72 \\
\hline 17. & 2.90 & 4. 20 & 18. 40 & 4.80 & 4. 20 & 4. 50 & 3.30 & 12.20 & 3.90 & .20 & 57 & .58 \\
\hline 18. & 2.70 & 4. 10 & 16. 80 & 12. 50 & 5.00 & 4. 30 & 3. 20 & 13.00 & 3.70 & .17 & 55 & 64 \\
\hline 19. & 2. 60 & 4.00 & 12.00 & 10.00 & 4. 20 & 4.00 & 3.30 & 12.00 & 3. 60 & .20 & 50 & 61 \\
\hline 20. & 2.50 & 3.90 & 9.00 & 8.00 & 4. 00 & 3. 90 & 3. 60 & 9.00 & 3.50 & .18 & 50 & 59 \\
\hline & 2. 20 & 3. 60 & 11. 00 & 7.00 & 3.80 & 3.80 & 3.50 & 6.00 & 3.50 & .25 & 50 & 2.02 \\
\hline 22. & 2.10 & 3.50 & 9.00 & 6.00 & 4. 00 & 3.70 & 3. 40 & 10.00 & 3.50 & .22 & 50 & 3. 10 \\
\hline 23 . & 2.00 & 350 & 8.00 & 6. 50 & 4. 50 & 4. 00 & 3. 30 & 9.00 & 3. 40 & .21 & 50 & 2. 68 \\
\hline 24 . & 2.00 & 3.40 & 7.00 & 6.00 & 5.00 & 3. 90 & 3.30 & 6.00 & 3.40 & .21 & 50 & 1. 70 \\
\hline & 2.00 & 3. 30 & 6.00 & 4. 80 & 5.00 & 3. 80 & 3. 20 & 4. 50 & 3. 30 & .17 & 49 & 1. 48 \\
\hline & 12.50 & 3. 20 & 5. 80 & 4.70 & 7.00 & 3. 70 & 3. 10 & 4.00 & 3. 20 & .19 & 49 & 1.01 \\
\hline & 8.00 & 3.20 & 5.50 & 8.10 & 12.50 & 3. 50 & 3.00 & 4.00 & 3.20 & 18 & 47 & 1.00 \\
\hline & 7.00 & 3.10 & 5.40 & 10.90 & 7.00 & 3. 40 & 3.00 & 5. 00 & 3.20 & .18 & 43 & 1.20 \\
\hline & 11.00 & ..... & 5.30 & 10.00 & 5.00 & 3.20 & 2.90 & 4.00 & 3. 10 & 17 & 55 & 1.30 \\
\hline & 11.00 & $\ldots .$. & 5.00 & 7.00 & 4. 00 & ...... & 2.90 & 4. 00 & 3.00 & 22 & 54 & 1. 35 \\
\hline 31. & 7.00 & $\cdots$ & 4. 80 & $\ldots . . .$. & 3.90 & ....... & 2. 90 & 4.50 & $\ldots \ldots$ & .50 & & 4. 46 \\
\hline
\end{tabular}

1 Record from United States Weather Bureau.

a From October 1 to 23 , estimated by B. M. Hall. 
Ocmulgee River at Macon, Georgia, for 1896.

\begin{tabular}{|c|c|c|c|c|c|c|c|c|c|c|c|c|}
\hline Day. & Jan. & Feb. & Mar. & Apr. & May. & June. & July. & Aug. & Sept. & Oet. & Nov. & Dec. \\
\hline 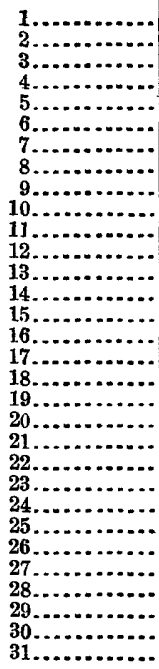 & $\begin{array}{r}4.81 \\
3.50 \\
2.20 \\
1.70 \\
1.42 \\
1.08 \\
.96 \\
1.72 \\
2.83 \\
2.77 \\
2.10 \\
1.60 \\
1.50 \\
1.20 \\
2.00 \\
2.50 \\
7.20 \\
5.00 \\
4.50 \\
4.00 \\
3.90 \\
3.70 \\
9.40 \\
13.80 \\
12.00 \\
9.30 \\
7.00 \\
5.80 \\
5.30 \\
4.80 \\
3.20\end{array}$ & $\begin{array}{r}3.00 \\
2.90 \\
3.20 \\
3.00 \\
2.90 \\
13.50 \\
10.70 \\
7.50 \\
13.10 \\
11.30 \\
8.70 \\
7.00 \\
6.30 \\
6.50 \\
5.00 \\
4.80 \\
4.20 \\
3.40 \\
3.20 \\
3.00 \\
2.90 \\
2.70 \\
2.60 \\
2.50 \\
2.48 \\
2.40 \\
2.30 \\
3.30 \\
2.80 \\
\ldots \ldots . . \\
\ldots . . .\end{array}$ & $\begin{array}{l}\text { a2. } 50 \\
2.20 \\
2.10 \\
2.00 \\
2.00 \\
1.90 \\
4.00 \\
6.00 \\
5.00 \\
4.00 \\
5.00 \\
7.20 \\
6.50 \\
6.20 \\
6.00 \\
6.00 \\
5.50 \\
5.30 \\
5.00 \\
4.90 \\
4.70 \\
4.50 \\
4.20 \\
5.00 \\
5.00 \\
4.80 \\
4.70 \\
4.60 \\
4.40 \\
4.20 \\
4.10\end{array}$ & $\begin{array}{r}3.10 \\
3.00 \\
4.60 \\
3.40 \\
2.90 \\
1.80 \\
1.60 \\
1.10 \\
1.10 \\
1.40 \\
1.30 \\
1.10 \\
1.20 \\
1.20 \\
1.13 \\
1.12 \\
1.09 \\
1.05 \\
.98 \\
.94 \\
.86 \\
.76 \\
.71 \\
.63 \\
.63 \\
1.02 \\
2.90 \\
2.32 \\
1.36 \\
1.02 \\
\ldots . . .\end{array}$ & $\begin{array}{r}0.89 \\
.75 \\
.63 \\
.90 \\
2.12 \\
2.73 \\
1.97 \\
1.62 \\
.86 \\
.61 \\
.38 \\
.30 \\
.19 \\
.11 \\
.09 \\
.07 \\
.05 \\
.03 \\
-.05 \\
-.10 \\
-.10 \\
-.15 \\
-.05 \\
.56 \\
.50 \\
.78 \\
.52 \\
.34 \\
.17 \\
.12 \\
.12\end{array}$ & $\begin{array}{r}0.08 \\
-.11 \\
.56 \\
.85 \\
1.92 \\
1.52 \\
.96 \\
.73 \\
.25 \\
.01 \\
-.05 \\
-.10 \\
-.17 \\
.29 \\
.32 \\
.20 \\
.25 \\
.25 \\
.30 \\
.40 \\
.47 \\
.56 \\
.70 \\
.50 \\
.30 \\
-.20 \\
-.35 \\
-.65 \\
-.75 \\
-.85 \\
\ldots . . . .\end{array}$ & $\begin{array}{r}-0.90 \\
-1.00 \\
+.10 \\
.15 \\
2.00 \\
4.00 \\
5.30 \\
11.00 \\
20.00 \\
19.40 \\
15.00 \\
10.20 \\
8.20 \\
7.10 \\
7.00 \\
6.20 \\
16.00 \\
18.20 \\
13.00 \\
7.05 \\
3.80 \\
3.20 \\
3.00 \\
2.90 \\
2.85 \\
2.70 \\
2.40 \\
2.10 \\
1.92 \\
1.66 \\
1.41\end{array}$ & $\begin{array}{r}1.02 \\
1.50 \\
1.82 \\
2.22 \\
2.62 \\
3.00 \\
3.05 \\
2.78 \\
2.41 \\
1.88 \\
1.48 \\
.40 \\
.20 \\
.13 \\
.25 \\
1.08 \\
.50 \\
.60 \\
.38 \\
.20 \\
.11 \\
.08 \\
-.06 \\
-.05 \\
-.00 \\
-.03 \\
-.04 \\
-.07 \\
-.07 \\
-.09 \\
-.12\end{array}$ & $\begin{array}{r}0.11 \\
.12 \\
.11 \\
.28 \\
.19 \\
.19 \\
.19 \\
.12 \\
.08 \\
.04 \\
.01 \\
-.01 \\
-.31 \\
-.45 \\
-.80 \\
-.68 \\
-.78 \\
-.80 \\
-.82 \\
-.91 \\
-.90 \\
-.82 \\
-.41 \\
-.48 \\
-.61 \\
-.72 \\
-.78 \\
-.83 \\
-.91 \\
-.80 \\
\ldots . . . \\
.92\end{array}$ & $\begin{array}{r}-0.82 \\
-.86 \\
-.88 \\
-.81 \\
-.75 \\
-.79 \\
-.78 \\
-.82 \\
-.82 \\
-.82 \\
-.73 \\
-.62 \\
-.75 \\
-.73 \\
-.65 \\
-.77 \\
-.80 \\
-.83 \\
-.85 \\
-.88 \\
-.89 \\
-.90 \\
-.73 \\
-.40 \\
-.52 \\
-.25 \\
-.08 \\
-.20 \\
-.32 \\
-.23 \\
-.18\end{array}$ & $\begin{array}{r}-0.14 \\
-.08 \\
10.02 \\
10.00 \\
14.20 \\
14.40 \\
8.80 \\
5.40 \\
3.22 \\
2.25 \\
1.50 \\
1.18 \\
10.00 \\
8.10 \\
5.32 \\
1.53 \\
1.14 \\
.97 \\
.85 \\
.63 \\
.70 \\
.62 \\
.58 \\
.58 \\
.51 \\
.51 \\
.50 \\
.47 \\
.44 \\
.32 \\
\ldots . . .\end{array}$ & $\begin{array}{r}9.50 \\
11.08 \\
12.60 \\
10.20 \\
8.00 \\
6.15 \\
4.52 \\
3.62 \\
3.00 \\
2.42 \\
1.98 \\
1.58 \\
1.26 \\
1.12 \\
11.70 \\
6.00 \\
4.62 \\
3.94 \\
2.85 \\
2.38 \\
2.00 \\
1.90 \\
1.76 \\
1.38 \\
1.18 \\
1.11 \\
.96 \\
.90 \\
.83 \\
.78 \\
.70\end{array}$ \\
\hline
\end{tabular}

$a$ Gage heights from March 1 to April 14 supplied from Weather Bureau records.

Chattahoochee River at Oakdale, Georgia, for 1895.

\begin{tabular}{|c|c|c|c|c|c|c|c|c|c|c|c|}
\hline Day. & Oct. & Nov. & Dec. & Day. & Oot. & Nov. & Dec. & Day. & Oct. & Nov. & Dec. \\
\hline $\begin{array}{r}1 . . \\
2 . . \\
3 . . \\
4 . . \\
5 . . \\
6 . . \\
7 \ldots \\
8 \ldots \\
9 . . \\
10 . . \\
11 . .\end{array}$ & 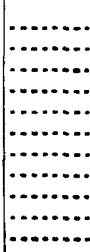 & $\begin{array}{r}0.75 \\
1.70 \\
1.00 \\
.60 \\
.50 \\
.55 \\
.45 \\
.40 \\
.60 \\
.80 \\
1.00\end{array}$ & $\begin{array}{r}0.50 \\
.50 \\
.55 \\
.60 \\
.60 \\
.50 \\
.45 \\
.40 \\
.40 \\
.60 \\
1.30\end{array}$ & $\begin{array}{l}12 \ldots \\
13 \ldots \\
14 \ldots \\
15 \ldots \\
16 \ldots \\
17 \ldots \\
18 \ldots \\
19 \ldots \\
20 \ldots \\
21 \ldots \\
22 \ldots\end{array}$ & \begin{tabular}{l}
$\cdots \ldots . .$. \\
\hdashline 0.30 \\
0.40 \\
.40 \\
.35 \\
.30 \\
.25 \\
.30 \\
.20 \\
.25
\end{tabular} & $\begin{array}{l}1.35 \\
1.00 \\
.75 \\
.60 \\
.60 \\
.55 \\
.60 \\
.50 \\
.55 \\
.50 \\
.50\end{array}$ & $\begin{array}{r}1.10 \\
1.00 \\
.65 \\
.60 \\
.55 \\
.55 \\
.50 \\
.40 \\
.50 \\
.65 \\
1.00\end{array}$ & $\begin{array}{l}23 \ldots \\
24 \ldots \\
25 \ldots \\
26 \ldots \\
27 \ldots \\
28 \ldots \\
29 \ldots \\
30 \ldots \\
31 \ldots\end{array}$ & $\begin{array}{l}0.20 \\
.30 \\
.25 \\
.20 \\
.25 \\
.25 \\
.30 \\
.50 \\
.50\end{array}$ & $\begin{array}{c}0.50 \\
.50 \\
.50 \\
.55 \\
.55 \\
.70 \\
.75 \\
.45 \\
. .2 \\
\end{array}$ & $\begin{array}{r}2.00 \\
1.20 \\
1.00 \\
.55 \\
.80 \\
.75 \\
.70 \\
2.00 \\
2.95\end{array}$ \\
\hline
\end{tabular}


Chattahoochee River at Oakdale, Georgia, for 1896.

\begin{tabular}{|c|c|c|c|c|c|c|c|c|c|c|c|c|}
\hline Day. & Jan. & Feb. & Mar. & Apr. & May. & June. & July. & Ang. & Sept. & Oct. & Nov. & Dec. \\
\hline & 3. 20 & 1.70 & 1.40 & 1. 40 & 0.75 & 0.25 & 0.35 & 0.45 & -0.40 & 0.20 & 0.50 & 0.65 \\
\hline & 2.00 & 1.55 & 1.35 & 1.70 & $\begin{array}{r}.75 \\
.75\end{array}$ & 1.40 & $\begin{array}{r}.00 \\
.40\end{array}$ & .50 & -.60 & .10 & $\begin{array}{r}.50 \\
.50\end{array}$ & 1.20 \\
\hline & 1.00 & 1.75 & 1.40 & 2.75 & 75 & 1.45 & -.45 & 1.40 & -.60 & .05 & .65 & 1.70 \\
\hline & 1. 20 & 2.10 & 1.45 & 1.85 & 70 & 2.10 & +.15 & 1. 70 & -.60 & -.20 & 1. 13 & 3.20 \\
\hline 5. & 1.10 & 2.20 & 1.40 & 1.35 & 65 & 1.60 & $\begin{array}{r}7 \\
.20\end{array}$ & .75 & -.60 & -.30 & 1. 60 & 2. 30 \\
\hline & 1.00 & 2.80 & 1.35 & 1. 30 & 3.15 & 1.15 & -.15 & .40 & -.60 & -.35 & 1. 35 & 1.30 \\
\hline & .80 & 3.70 & 1.95 & 1.10 & 1. 70 & .60 & +2.00 & .20 & -.60 & -.40 & 1.20 & 1.15 \\
\hline & .95 & 4. 30 & 1.75 & 1.15 & 1.20 & .40 & 12.25 & .15 & -.55 & -.50 & 1. 10 & 1.00 \\
\hline 9. & 1.20 & 5.80 & 1.55 & 1.05 & .90 & .70 & 17.70 & .15 & 一. . 55 & -.50 & 1.00 & 1. 00 \\
\hline 10. & 1.25 & 6. 55 & 1.50 & 1.05 & .75 & .70 & 18.45 & 10 & -.50 & -.50 & 1.05 & 1.00 \\
\hline 11. & 1.00 & 4. 30 & 1. 75 & 1.05 & .65 & .65 & 4.75 & .05 & -.50 & -.60 & 1.05 & .90 \\
\hline 12. & .85 & 3.30 & 1.85 & 1.00 & 60 & .50 & 3. 25 & .05 & -.55 & -.60 & 1.60 & .70 \\
\hline 13. & .80 & 2.80 & 1.55 & 1.00 & .50 & .30 & 3. 10 & .30 & -.60 & -.55 & 3. 80 & .60 \\
\hline 14. & .75 & 2.95 & 1.35 & .90 & .50 & .20 & 2. 90 & .25 & -.60 & -.55 & 4. 60 & 65 \\
\hline 15. & .70 & 4. 10 & 1. 20 & .90 & .40 & -.05 & 1. 95 & .05 & -.60 & -.55 & 2.60 & .70 \\
\hline 16. & .85 & 2.90 & ]. 15 & .90 & .30 & +.05 & 3. 00 & .10 & -.65 & -.55 & 1. 50 & 65 \\
\hline 17. & 2. 40 & 2.55 & 1.10 & .85 & 30 & -.05 & 4. 40 & 10 & -.65 & -.55 & 1.00 & .65 \\
\hline & 2.35 & 2.20 & 1. 35 & .85 & .25 & +.10 & 2.70 & .10 & -.65 & -.55 & .80 & .65 \\
\hline 19. & 2. 10 & 2.10 & 1.70 & .85 & .25 & .15 & 1. 75 & -.10 & -.65 & -.55 & .60 & .65 \\
\hline 20. & 1.50 & 1.80 & 1. 65 & .85 & 20 & 55 & 1. 90 & -.25 & -.65 & -.55 & .50 & .65 \\
\hline 21. & 1.25 & 1. 60 & 1.45 & .75 & .15 & 1. 60 & 1. 65 & -.30 & -.50 & -.55 & .40 & .60 \\
\hline 22. & 1.80 & 1. 45 & 1.35 & .75 & .20 & .45 & 1.90 & -.30 & -.15 & -.50 & .40 & .55 \\
\hline 23. & 6. 30 & 1.55 & 1.40 & .65 & .20 & .50 & 2.45 & -.30 & +.40 & -.23 & .30 & .50 \\
\hline 24 & 9.80 & 1. 60 & 1.45 & .70 & 1.10 & .30 & 2.40 & -.30 & .60 & -.05 & .25 & .50 \\
\hline 25. & 9.95 & 1.50 & 1.40 & .65 & 1. 55 & .20 & 1. 50 & +1.00 & -.10 & +.05 & .20 & .45 \\
\hline 26. & 5.10 & 1. 40 & 1. 35 & .65 & .65 & -.10 & 1. 30 & -.10 & -.40 & .10 & .20 & .45 \\
\hline 27. & 3. 55 & 1. 35 & 1.25 & 1.40 & .85 & +.45 & .90 & -.30 & -.40 & .15 & .20 & .40 \\
\hline 28. & 2. 65 & 1.30 & 1.20 & 1. 70 & 65 & -.10 & .80 & -.20 & +.20 & .25 & .20 & .35 \\
\hline 29 & 2. 30 & 1.55 & 1.15 & 1.15 & .95 & -.20 & .80 & -.30 & .45 & .90 & .20 & .35 \\
\hline 30. & 2.10 & $\ldots \ldots$ & 1. 20 & .85 & .10 & -.25 & .80 & -.35 & 30 & .6 & .20 & .30 \\
\hline 31. & 1.80 & . & 1.20 & $\ldots$ & .35 & $\cdots$ & .50 & -.40 & ...... & .50 & ...... & .25 \\
\hline
\end{tabular}

Chattahoochee River at Westpoint, Georgia, for 1896.

\begin{tabular}{|c|c|c|c|c|c|c|c|c|c|c|c|}
\hline Day. & Aug. & Sept. & Oet. & Nov. & Dec. & Day. & Ang. & Sept. & Oet. & Nov. & Dec. \\
\hline 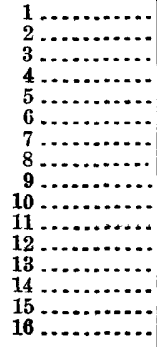 & $\begin{array}{l}2.70 \\
3.90 \\
4.50 \\
\text { 6. } 00 \\
\text { 5. } 50 \\
5.00 \\
3.65 \\
3.20 \\
2.75 \\
2.60 \\
2.20 \\
2.00 \\
1.85 \\
1.70 \\
1.60 \\
1.60\end{array}$ & $\begin{array}{l}1.30 \\
1.20 \\
1.10 . \\
1.05 \\
1.00 \\
1.00 \\
1.00 \\
1.05 \\
1.10 \\
1.05 \\
.95 \\
.85 \\
.85 \\
.90 \\
.90 \\
.85\end{array}$ & $\begin{array}{l}4.10 \\
4.00 \\
3.00 \\
2.60 \\
2.40 \\
2.00 \\
1.90 \\
1.50 \\
1.30 \\
1.25 \\
1.25 \\
1.20 \\
1.15 \\
1.15 \\
1.15 \\
1.15\end{array}$ & $\begin{array}{l}1.70 \\
2.00 \\
3.25 \\
8.00 \\
9.20 \\
7.60 \\
5.50 \\
4.30 \\
3.45 \\
2.80 \\
2.00 \\
2.15 \\
6.30 \\
5.00 \\
4.50 \\
3.30\end{array}$ & $\begin{array}{l}4.20 \\
4.00 \\
3.75 \\
3.60 \\
3.40 \\
3.20 \\
3.10 \\
3.10 \\
3.05 \\
3.00 \\
2.90 \\
2.80 \\
2.65 \\
2.50 \\
3.00 \\
3.10\end{array}$ & 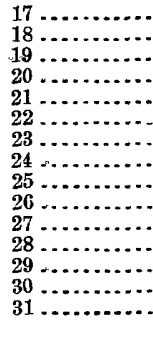 & $\begin{array}{l}1.60 \\
1.55 \\
1.50 \\
1.45 \\
1.40 \\
1.40 \\
1.30 \\
1.20 \\
3.00 \\
2.00 \\
1.80 \\
1.75 \\
1.60 \\
1.50 \\
1.40\end{array}$ & $\begin{array}{r}0.85 \\
.80 \\
.80 \\
.80 \\
.80 \\
3.30 \\
3.00 \\
2.50 \\
2.00 \\
1.70 \\
1.60 \\
1.40 \\
3.60 \\
4.20 \\
\cdots \cdots\end{array}$ & $\begin{array}{l}1.10 \\
1.10 \\
1.10 \\
1.10 \\
1.15 \\
1.10 \\
1.50 \\
1.75 \\
1.75 \\
1.70 \\
1.65 \\
1.60 \\
1.50 \\
1.50 \\
1.45\end{array}$ & $\begin{array}{l}3.00 \\
2.60 \\
2.55 \\
2.40 \\
2.25 \\
2.25 \\
2.20 \\
2.20 \\
2.90 \\
1.90 \\
1.80 \\
2.00 \\
4.90 \\
4.30 \\
\cdots \cdots\end{array}$ & $\begin{array}{l}3.00 \\
3.00 \\
2.90 \\
2.80 \\
2.70 \\
2.60 \\
2.40 \\
2.20 \\
2.15 \\
2.10 \\
2.10 \\
2.05 \\
2.00 \\
1.95 \\
1.90\end{array}$ \\
\hline
\end{tabular}


Etowah River ${ }^{1}$ at Canton, Georgia, for 1892.

\begin{tabular}{|c|c|c|c|c|c|c|c|c|c|c|}
\hline Day. & Mar. & Apr. & May. & June. & July. & Aug. & Sept. & Oet. & Nov. & Déc. \\
\hline 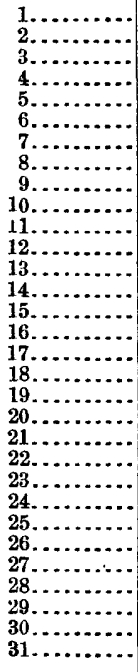 & 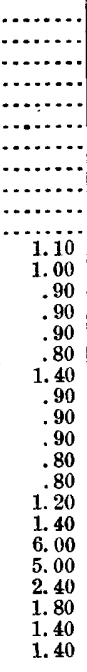 & $\begin{array}{r}1.00 \\
1.00 \\
1.00 \\
1.00 \\
1.00 \\
9.00 \\
17.80 \\
11.80 \\
4.00 \\
3.00 \\
2.00 \\
2.00 \\
2.20 \\
2.20 \\
2.20 \\
2.20 \\
2.10 \\
1.80 \\
1.70 \\
5.40 \\
2.30 \\
1.90 \\
2.20 \\
2.00 \\
2.00 \\
1.50 \\
1.40 \\
1.10 \\
1.00 \\
1.00 \\
\ldots . .2 \\
2 . .\end{array}$ & $\begin{array}{r}1.00 \\
1.00 \\
1.00 \\
1.00 \\
1.00 \\
1.00 \\
1.00 \\
1.00 \\
1.00 \\
1.00 \\
1.50 \\
1.10 \\
1.00 \\
.90 \\
.80 \\
.80 \\
.90 \\
1.20 \\
1.80 \\
1.00 \\
.90 \\
.90 \\
1.00 \\
.90 \\
.90 \\
.80 \\
.80 \\
.80 \\
.80 \\
.80 \\
.80\end{array}$ & $\begin{array}{r}0.80 \\
.80 \\
2.00 \\
3.80 \\
1.00 \\
1.50 \\
.80 \\
.80 \\
.60 \\
.60 \\
.60 \\
.60 \\
.60 \\
.50 \\
.40 \\
.40 \\
.40 \\
.40 \\
.60 \\
1.80 \\
1.40 \\
1.00 \\
1.00 \\
.80 \\
.70 \\
1.70 \\
1.00 \\
2.00 \\
1.00 \\
1.00 \\
\cdots . . .\end{array}$ & $\begin{array}{r}1.00 \\
.80 \\
.70 \\
.70 \\
2.00 \\
2.00 \\
1.50 \\
1.00 \\
.80 \\
2.40 \\
5.20 \\
5.60 \\
3.00 \\
1.80 \\
1.80 \\
1.00 \\
.80 \\
.60 \\
.50 \\
.60 \\
.50 \\
.70 \\
.70 \\
.60 \\
.60 \\
.60 \\
.50 \\
.40 \\
.40 \\
.30 \\
.30\end{array}$ & $\begin{array}{r}0.30 \\
.30 \\
.30 \\
.30 \\
.30 \\
.30 \\
.30 \\
.20 \\
.20 \\
.20 \\
.20 \\
.20 \\
.20 \\
.20 \\
.20 \\
.10 \\
.20 \\
.80 \\
.60 \\
.60 \\
.60 \\
.70 \\
.90 \\
1.50 \\
1.50 \\
1.00 \\
1.00 \\
.80 \\
.80 \\
.60 \\
.60\end{array}$ & $\begin{array}{r}0.80 \\
.60 \\
.40 \\
.30 \\
.20 \\
.10 \\
.10 \\
.10 \\
.10 \\
.10 \\
.10 \\
.10 \\
.20 \\
1.00 \\
.80 \\
.60 \\
.50 \\
.40 \\
.30 \\
.20 \\
.20 \\
.20 \\
.20 \\
.20 \\
.10 \\
.10 \\
.10 \\
.10 \\
.10 \\
.10 \\
\ldots . .\end{array}$ & $\begin{array}{l}0.10 \\
.00 \\
.00 \\
.00 \\
.00 \\
.00 \\
.00 \\
.00 \\
.00 \\
.00 \\
.00 \\
.00 \\
.00 \\
.00 \\
.00 \\
.00 \\
.00 \\
.00 \\
.00 \\
.00 \\
.00 \\
.00 \\
.00 \\
.00 \\
.00 \\
.00 \\
.10 \\
.10 \\
.10 \\
.10 \\
.10\end{array}$ & $\begin{array}{r}0.10 \\
.10 \\
.10 \\
.10 \\
.10 \\
.10 \\
.10 \\
.20 \\
1.00 \\
3.00 \\
1.00 \\
.50 \\
.40 \\
.30 \\
2.00 \\
1.00 \\
.50 \\
2.00 \\
1.00 \\
.80 \\
.60 \\
.40 \\
.40 \\
.30 \\
.20 \\
.20 \\
.20 \\
.30 \\
.20 \\
.20 \\
\ldots . .\end{array}$ & $\begin{array}{r}0.20 \\
.20 \\
.20 \\
.20 \\
.20 \\
.10 \\
.10 \\
.10 \\
.10 \\
.10 \\
.10 \\
.10 \\
.10 \\
.10 \\
.10 \\
.10 \\
1.00 \\
.60 \\
.40 \\
2.00 \\
1.50 \\
1.00 \\
.50 \\
.40 \\
.40 \\
.40 \\
.40 \\
.40 \\
.40 \\
.40 \\
.40\end{array}$ \\
\hline
\end{tabular}

Etowah River ${ }^{2}$ at Canton, Georgia, for 1993.

\begin{tabular}{|c|c|c|c|c|c|c|c|c|c|c|c|c|}
\hline Day. & Jan. & Feb. & Mar. & Apr. & May. & June. & July. & Aug. & Sept. & Oet. & Nov. & Dec. \\
\hline 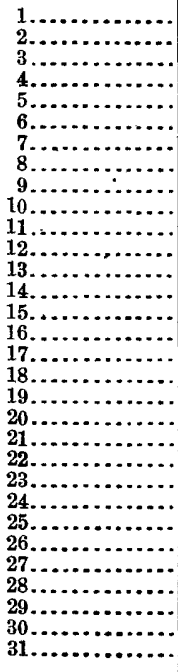 & $\begin{array}{r}0.30 \\
1.00 \\
.60 \\
.50 \\
.40 \\
.30 \\
.30 \\
.30 \\
.30 \\
.30 \\
.30 \\
.30 \\
.40 \\
.40 \\
.40 \\
.30 \\
.30 \\
.30 \\
.50 \\
.50 \\
.40 \\
.40 \\
.40 \\
.40 \\
.40 \\
.40 \\
.40 \\
.60 \\
1.50 \\
1.40 \\
1.00\end{array}$ & $\begin{array}{r}0.60 \\
.50 \\
.40 \\
.40 \\
.40 \\
.30 \\
.30 \\
.30 \\
.30 \\
.20 \\
2.00 \\
2.20 \\
4.20 \\
3.00 \\
3.00 \\
12.00 \\
5.00 \\
3.00 \\
2.00 \\
1.50 \\
1.40 \\
1.30 \\
1.00 \\
1.00 \\
1.00 \\
.80 \\
.80 \\
3.00 \\
\cdots \ldots \ldots \\
\cdots \ldots . \\
\cdots \ldots \\
\ldots .-\end{array}$ & $\begin{array}{l}2.00 \\
1.00 \\
1.00 \\
1.00 \\
1.00 \\
1.00 \\
1.00 \\
1.00 \\
4.80 \\
2.00 \\
1.20 \\
1.50 \\
1.30 \\
1.00 \\
1.00 \\
1.00 \\
.80 \\
.80 \\
.70 \\
.70 \\
.60 \\
.50 \\
.40 \\
.80 \\
1.40 \\
1.00 \\
1.00 \\
.80 \\
.80 \\
.80 \\
.80\end{array}$ & $\begin{array}{r}0.80 \\
.70 \\
.70 \\
.70 \\
.70 \\
.70 \\
.70 \\
.60 \\
.60 \\
.60 \\
.50 \\
.50 \\
.50 \\
.50 \\
.40 \\
.40 \\
.40 \\
.40 \\
.40 \\
.40 \\
.40 \\
.40 \\
.30 \\
.30 \\
.30 \\
.30 \\
.30 \\
.30 \\
.30 \\
.30 \\
. .2 \\
. . \\
.50\end{array}$ & $\begin{array}{r}0.30 \\
.30 \\
.90 \\
1.20 \\
.90 \\
.90 \\
2.00 \\
1.00 \\
2.00 \\
1.00 \\
.90 \\
.80 \\
.70 \\
.70 \\
.60 \\
1.00 \\
.90 \\
.80 \\
.80 \\
.70 \\
.60 \\
.50 \\
.50 \\
.40 \\
.40 \\
.40 \\
.40 \\
.40 \\
.90 \\
1.60 \\
1.00\end{array}$ & $\begin{array}{r}0.60 \\
2.00 \\
5.60 \\
2.00 \\
1.00 \\
3.00 \\
3.80 \\
2.00 \\
1.00 \\
.60 \\
.60 \\
.60 \\
.60 \\
.50 \\
.50 \\
.50 \\
.50 \\
.40 \\
.40 \\
3.00 \\
1.00 \\
.80 \\
2.60 \\
1.00 \\
.80 \\
.80 \\
.70 \\
.60 \\
.60 \\
.50 \\
. .2 .\end{array}$ & 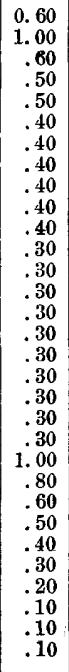 & $\begin{array}{r}0.10 \\
.50 \\
.30 \\
.20 \\
.80 \\
.60 \\
.40 \\
.30 \\
.30 \\
.30 \\
.30 \\
.30 \\
.30 \\
.30 \\
.30 \\
.30 \\
.30 \\
.20 \\
.10 \\
.10 \\
.10 \\
.10 \\
.00 \\
.00 \\
.00 \\
-.10 \\
-.10 \\
-.10 \\
-.10 \\
-.10 \\
\cdots . . \\
.2\end{array}$ & $\begin{array}{r}1.00 \\
.80 \\
.60 \\
.40 \\
.20 \\
.00 \\
.00 \\
.00 \\
.10 \\
1.00 \\
3.00 \\
4.00 \\
3.80 \\
1.50 \\
1.00 \\
.50 \\
.30 \\
.10 \\
.10 \\
.10 \\
.00 \\
.00 \\
.00 \\
.00 \\
.00 \\
.00 \\
.00 \\
-.10 \\
-.10 \\
-.10 \\
. . . .\end{array}$ & $\begin{array}{r}0.00 \\
.00 \\
.00 \\
.00 \\
-.10 \\
-.10 \\
-.10 \\
-.10 \\
-.10 \\
-.20 \\
-.20 \\
-.20 \\
-.20 \\
-.20 \\
-.20 \\
-.20 \\
-.20 \\
-.20 \\
-.20 \\
-.20 \\
-.20 \\
-.20 \\
-.20 \\
-.20 \\
-.20 \\
-.20 \\
-.20 \\
-.20 \\
-.20 \\
-.20 \\
-.20\end{array}$ & $\begin{array}{r}-0.20 \\
-.20 \\
-.20 \\
-.20 \\
-.20 \\
-.20 \\
-.20 \\
-.20 \\
-.20 \\
-.20 \\
-.20 \\
-.20 \\
-.20 \\
-.20 \\
-.20 \\
-.20 \\
-.20 \\
-.20 \\
-.20 \\
-.20 \\
-.20 \\
-.20 \\
.00 \\
.00 \\
.00 \\
.00 \\
.00 \\
.00 \\
+.20 \\
.20 \\
\cdots . . \\
\cdots\end{array}$ & $\begin{array}{l}0.10 \\
.10 \\
.10 \\
.40 \\
.40 \\
.20 \\
.20 \\
.20 \\
.20 \\
.20 \\
.20 \\
.20 \\
.10 \\
.00 \\
.00 \\
.20 \\
.20 \\
.20 \\
.20 \\
.20 \\
.10 \\
.10 \\
.10 \\
.10 \\
.10 \\
.10 \\
.10 \\
.10 \\
.10 \\
.20 \\
.20\end{array}$ \\
\hline
\end{tabular}

' From United States Weather Bureau records. 
Etowah River ${ }^{1}$ at Canton, Georgia, for 1894.

\begin{tabular}{|c|c|c|c|c|c|c|c|c|c|c|c|c|}
\hline Day. & Jan. & Feb. & Msir. & Apr. & May. & June. & July. & Ang. & Sept. & Oct. & Nov. & Dec. \\
\hline 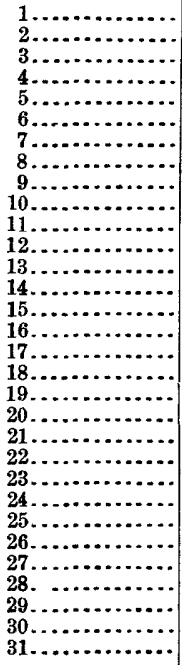 & $\begin{array}{r}0.20 \\
.20 \\
.20 \\
.20 \\
.20 \\
.20 \\
2.00 \\
1.00 \\
1.00 \\
1.80 \\
2.00 \\
1.00 \\
1.00 \\
.80 \\
1.80 \\
1.00 \\
.80 \\
.60 \\
.60 \\
.80 \\
.80 \\
.80 \\
.80 \\
.80 \\
.80 \\
.60 \\
.50 \\
.50 \\
.50 \\
.50 \\
.50\end{array}$ & $\begin{array}{r}0.50 \\
.50 \\
.50 \\
.50 \\
.50 \\
.50 \\
.50 \\
.50 \\
1.50 \\
1.00 \\
.80 \\
1.00 \\
1.00 \\
1.00 \\
1.00 \\
1.00 \\
1.00 \\
1.00 \\
1.50 \\
1.50 \\
1.00 \\
.80 \\
.80 \\
.80 \\
.80 \\
.80 \\
.80 \\
.80 \\
\cdots \cdots \\
\cdots \ldots .- \\
\ldots \ldots\end{array}$ & $\begin{array}{r}0.80 \\
.80 \\
.80 \\
.80 \\
.80 \\
.80 \\
80 \\
.80 \\
.70 \\
.60 \\
.50 \\
.50 \\
.40 \\
.40 \\
.40 \\
.40 \\
.40 \\
.40 \\
.40 \\
.40 \\
2.00 \\
1.80 \\
.80 \\
.80 \\
.80 \\
.80 \\
.80 \\
.80 \\
.80 \\
.80 \\
.80\end{array}$ & $\begin{array}{r}0.70 \\
.70 \\
.70 \\
.70 \\
.80 \\
.80 \\
.80 \\
.80 \\
.80 \\
.80 \\
1.80 \\
.80 \\
.80 \\
.80 \\
.70 \\
.60 \\
.50 \\
.50 \\
.50 \\
.80 \\
.80 \\
.80 \\
.60 \\
.60 \\
.60 \\
.60 \\
.60 \\
.60 \\
.60 \\
.60 \\
. . .6\end{array}$ & $\begin{array}{r}0.60 \\
.60 \\
.60 \\
.60 \\
.60 \\
.60 \\
.60 \\
.60 \\
.60 \\
.60 \\
.60 \\
.60 \\
.60 \\
.60 \\
.80 \\
.80 \\
.80 \\
.80 \\
.80 \\
.60 \\
.60 \\
.60 \\
.60 \\
.60 \\
.60 \\
.60 \\
.60 \\
.60 \\
.50 \\
.50 \\
.50\end{array}$ & $\begin{array}{r}0.50 \\
.50 \\
.50 \\
.50 \\
.50 \\
.50 \\
.50 \\
.40 \\
.40 \\
.30 \\
.20 \\
.20 \\
.20 \\
.20 \\
.20 \\
.20 \\
.20 \\
.90 \\
.80 \\
.60 \\
.60 \\
.60 \\
.60 \\
.60 \\
.60 \\
.60 \\
.60 \\
.60 \\
.60 \\
.60 \\
\ldots . .\end{array}$ & $\begin{array}{r}0.60 \\
.60 \\
.60 \\
.60 \\
.60 \\
.60 \\
.60 \\
.60 \\
.60 \\
.60 \\
.60 \\
.60 \\
.60 \\
.50 \\
.50 \\
.50 \\
.50 \\
1.00 \\
1.00 \\
1.00 \\
.50 \\
3.00 \\
1.00 \\
.50 \\
.50 \\
.50 \\
.50 \\
.50 \\
.50 \\
.50\end{array}$ & $\begin{array}{r}0.60 \\
.50 \\
.50 \\
1.50 \\
.50 \\
.50 \\
.50 \\
.50 \\
.50 \\
.50 \\
.50 \\
.50 \\
.50 \\
.50 \\
.50 \\
.50 \\
.40 \\
.40 \\
.40 \\
.40 \\
.40 \\
.40 \\
.40 \\
.40 \\
1.00 \\
2.40 \\
1.00 \\
.50 \\
.50 \\
.50 \\
.50\end{array}$ & $\begin{array}{r}0.50 \\
.50 \\
.50 \\
.50 \\
.50 \\
.50 \\
.50 \\
.50 \\
.50 \\
.50 \\
.50 \\
.50 \\
.50 \\
.50 \\
.50 \\
.50 \\
.50 \\
5.20 \\
1.20 \\
1.00 \\
.60 \\
.60 \\
.60 \\
.60 \\
.60 \\
.50 \\
.50 \\
.60 \\
.60 \\
.60 \\
. . . .\end{array}$ & $\begin{array}{l}0.60 \\
.60 \\
.60 \\
.60 \\
.60 \\
.60 \\
.60 \\
.60 \\
.60 \\
.60 \\
.60 \\
.60 \\
.60 \\
.60 \\
.60 \\
.60 \\
.60 \\
.60 \\
.60 \\
.60 \\
.60 \\
.60 \\
.60 \\
.60 \\
.50 \\
.50 \\
.50 \\
.50 \\
.50 \\
.50 \\
.50\end{array}$ & $\begin{array}{r}0.50 \\
.50 \\
.50 \\
.50 \\
.50 \\
.50 \\
.50 \\
.50 \\
.50 \\
.50 \\
.50 \\
.40 \\
.40 \\
.40 \\
.50 \\
.50 \\
.50 \\
.50 \\
.50 \\
.50 \\
.50 \\
.50 \\
.50 \\
.50 \\
.50 \\
.50 \\
.50 \\
.50 \\
.50 \\
.50 \\
. .5 \\
.50\end{array}$ & $\begin{array}{r}0.50 \\
.50 \\
.50 \\
.50 \\
.50 \\
.50 \\
.50 \\
.50 \\
.50 \\
1.00 \\
2.00 \\
7.50 \\
2.00 \\
1.00 \\
.60 \\
.60 \\
.60 \\
.60 \\
.60 \\
.60 \\
.60 \\
.60 \\
.60 \\
.60 \\
.60 \\
.60 \\
1.80 \\
1.80 \\
.80 \\
.80 \\
.80\end{array}$ \\
\hline
\end{tabular}

Etowah River ${ }^{1}$ at Canton, Georgia, for 1895.

\begin{tabular}{|c|c|c|c|c|c|c|c|c|c|c|c|c|}
\hline Day. & Jan. & Feb. & Mar. & Apr. & May. & June. & July. & Aug. & Sept. & Oet. & Nov. & Dec. \\
\hline & 0.80 & 1.00 & 1. 00 & 1.00 & 1. 40 & 1.00 & 2.00 & 0.60 & 0.80 & 0.40 & 0.10 & 0.30 \\
\hline & .80 & 1.00 & 1.00 & 1.00 & 1. 60 & 1.00 & 1.00 & .60 & .80 & .40 & .10 & .30 \\
\hline & .80 & 1.00 & 2.00 & 1.00 & 1.00 & .80 & 1.00 & .60 & .80 & .40 & .10 & .30 \\
\hline & .80 & 1.00 & 2.00 & 1.00 & 1. 00 & 80 & 1.00 & .60 & .80 & .40 & .10 & .30 \\
\hline & .80 & 1.00 & 2.00 & 1. 00 & 1.00 & 80 & 1.00 & .50 & .80 & .40 & .10 & .30 \\
\hline & .80 & 1.00 & 1. 00 & 1.00 & 1.00 & 2.00 & .80 & .50 & .80 & .30 & .00 & .30 \\
\hline & .80 & 1. 00 & 1.00 & 1. 50 & 1.00 & 1.00 & 1.00 & .50 & 80 & .30 & .00 & .30 \\
\hline & .80 & 1. 00 & 1.00 & 4.50 & 1.00 & 1.00 & 2.50 & 2.00 & 80 & .30 & .00 & .30 \\
\hline 9. & 7.60 & 1.00 & 1.00 & 2.00 & 1.00 & 1. 00 & 2.00 & .80 & .80 & .30 & 00 & .40 \\
\hline 10. & 15.50 & 1.00 & 1.00 & 1.60 & 1.00 & 1.00 & 1.00 & .80 & .80 & .30 & .00 & .50 \\
\hline 11. & 9.80 & 1.00 & 1.00 & 1.40 & 1.00 & .80 & 1.00 & .80 & 80 & .30 & .00 & .50 \\
\hline 12. & 3.00 & 1.00 & 1.00 & 1.40 & 1.00 & 1.00 & .80 & .80 & 80 & .30 & 60 & .50 \\
\hline 13 . & 1.50 & 1.00 & 2.00 & 1.20 & .80 & 1.00 & .80 & .80 & 80 & .30 & .60 & .50 \\
\hline $14 .$. & 1.00 & 1.00 & 3.50 & 1. 20 & .80 & 1.00 & .80 & 2.00 & 80 & .30 & .50 & .50 \\
\hline & 1.00 & 1.00 & 4. 00 & 1.20 & .80 & .80 & .80 & 1.00 & .80 & .30 & .50 & 50 \\
\hline 16. & 1.20 & 1. 00 & 6.00 & 1. 20 & .80 & 80 & .80 & 3.60 & .80 & .30 & .50 & .50 \\
\hline 17 & 1.80 & 1.00 & 3. 00 & 6.00 & 80 & 1.00 & .70 & 10.50 & .80 & .30 & .50 & .50 \\
\hline & 1.00 & 1.00 & 2. 00 & 3.00 & .80 & .80 & .90 & 2.50 & .80 & .30 & .50 & .50 \\
\hline 19. & 1.00 & 1. 00 & 1. 60 & 1. 50 & .80 & .80 & .60 & 1.50 & .80 & .20 & .50 & .50 \\
\hline & 1.00 & 1.00 & 2. 40 & 1.50 & .70 & .80 & .60 & 1.00 & .80 & .20 & .40 & .50 \\
\hline & 2.00 & 1.00 & 5. 60 & 1.40 & .70 & .80 & .60 & 1.00 & .70 & .20 & .40 & 1. 00 \\
\hline 22 & 2.00 & 1.00 & 2.80 & 1.40 & .70 & .80 & .60 & 1.00 & .70 & .20 & .40 & 1. 00 \\
\hline 23. & 1.00 & 1.00 & 2.00 & 1.40 & 1.00 & .80 & 60 & 1. 00 & 60 & .20 & 40 & .60 \\
\hline 24. & 1.00 & 1.00 & 2.00 & 1. 30 & 1.00 & 80 & .60 & 1.00 & 60 & .20 & 40 & .60 \\
\hline 25. & 1.00 & 1.00 & 2.00 & 1.20 & 2.80 & 80 & 1.00 & 1.00 & 60 & .10 & .40 & 60 \\
\hline 26. & 2.00 & 1.00 & 1. 80 & 1. 20 & 2.80 & 80 & .80 & .80 & 80 & .10 & 40 & .60 \\
\hline & 1.00 & 1.00 & 1. 40 & 1.60 & 3.00 & 70 & .80 & .80 & .60 & .10 & 40 & .60 \\
\hline & 1.00 & 1.00 & 1.00 & 1.00 & 2. 00 & 70 & .60 & .80 & .60 & .10 & .40 & .60 \\
\hline 29. & 2.00 & $\ldots .$. & 1. 00 & 1.40 & 2.00 & 70 & .60 & .80 & 60 & .10 & .40 & .60 \\
\hline $30 \ldots$ & 1.00 & ...... & 1.00 & 1.40 & 1.60 & 2.00 & .60 & .80 & .50 & .10 & .40 & .60 \\
\hline 31. & 1. 00 & $\ldots .$. & 1. 00 & $\ldots \ldots$ & 1.00 & $\ldots .$. & .60 & .80 & $\ldots$ & .10 . & $\ldots$ & .60 \\
\hline
\end{tabular}

IFrom United States Weather Bureau recorde. 
Etowah River at Canton, Georgia, for 1896.

\begin{tabular}{|c|c|c|c|c|c|c|c|}
\hline Day. & Jan. & Feb. & Mar. & Sept. & Oet. & Nov. & Dec. \\
\hline 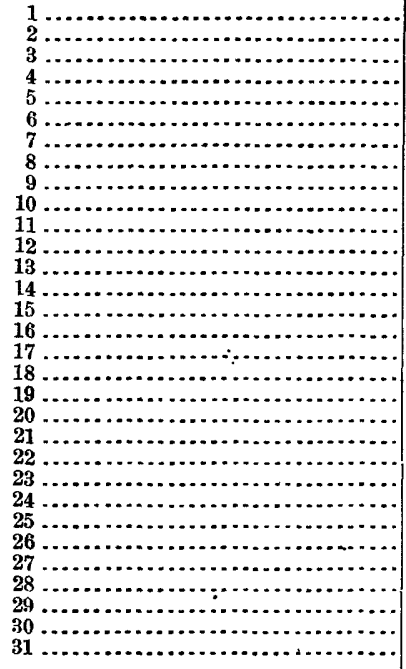 & $\begin{array}{r}0.60 \\
60 \\
.60 \\
.60 \\
.60 \\
.60 \\
.50 \\
.70 \\
.70 \\
.70 \\
.70 \\
.70 \\
.70 \\
.70 \\
.70 \\
.70 \\
1.00 \\
.80 \\
.80 \\
.80 \\
.80 \\
1.00 \\
3.80 \\
5.80 \\
3.00 \\
2.00 \\
2.00 \\
2.00 \\
1.80 \\
1.80 \\
1.80\end{array}$ & $\begin{array}{r}1.80 \\
1.80 \\
1.80 \\
1.80 \\
1.80 \\
2.00 \\
3.00 \\
3.00 \\
3.50 \\
2.00 \\
1.80 \\
1.80 \\
1.80 \\
1.00 \\
1.00 \\
1.00 \\
1.00 \\
1.00 \\
1.00 \\
1.00 \\
1.00 \\
.80 \\
.80 \\
.80 \\
.80 \\
.60 \\
.60 \\
.60 \\
.60 \\
\ldots \ldots \\
\ldots . . .\end{array}$ & $\begin{array}{r}0.60 \\
.50 \\
.50 \\
.50 \\
.50 \\
.50 \\
.80 \\
.80 \\
.80 \\
.80 \\
.80 \\
.80 \\
.80 \\
.80 \\
.70 \\
.80 \\
.80 \\
.70 \\
1.09 \\
1.00 \\
1.00 \\
.80 \\
.80 \\
.80 \\
.60 \\
.60 \\
.60 \\
.60 \\
.60 \\
.60 \\
a 1.00\end{array}$ & 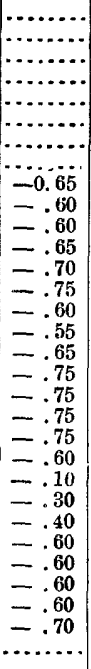 & $\begin{array}{r}0.00 \\
-.20 \\
-.30 \\
-.30 \\
-.40 \\
-.40 \\
-.50 \\
-.50 \\
-.50 \\
-.80 \\
-.30 \\
-.40 \\
-.20 \\
-.30 \\
-.40 \\
-.40 \\
-.40 \\
-.40 \\
-.40 \\
-.50 \\
-.50 \\
-.50 \\
-.50 \\
.00 \\
-.10 \\
-.10 \\
-.10 \\
+1.12 \\
1.10 \\
1.00 \\
.00\end{array}$ & $\begin{array}{r}0.00 \\
-.10 \\
-.10 \\
.00 \\
+2.80 \\
.80 \\
.60 \\
.60 \\
.40 \\
.40 \\
.40 \\
.90 \\
3.60 \\
1.00 \\
.70 \\
.70 \\
.40 \\
.20 \\
.00 \\
.00 \\
.00 \\
.00 \\
.00 \\
.00 \\
.00 \\
.00 \\
.00 \\
.00 \\
.00 \\
1.00 \\
1.40\end{array}$ & $\begin{array}{r}1.00 \\
1.00 \\
.60 \\
.30 \\
.30 \\
.20 \\
.10 \\
.10 \\
.10 \\
.10 \\
.10 \\
.00 \\
.00 \\
.00 \\
.20 \\
.40 \\
.20 \\
.00 \\
.00 \\
.00 \\
.00 \\
.00 \\
.00 \\
.00 \\
.00 \\
.00 \\
.00 \\
-.10 \\
-.10 \\
-.10 \\
-.10\end{array}$ \\
\hline
\end{tabular}

$a$ Weather Bureau discontinued observations March 31, 1896 ; Geological Survey began observations September 9, 1896.

Coosawattee River at Carters, Georgia.

\begin{tabular}{|c|c|c|c|c|c|c|c|c|c|c|c|}
\hline Day. & Aug. & Sept. & Oet. & Nov. & Dec. & Day. & A.ng. & Sept. & Oet. & Nov. & Dec. \\
\hline 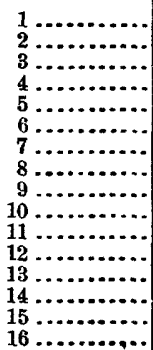 & $\because$ & $\begin{array}{l}0.75 \\
.75 \\
.75 \\
.70 \\
.70 \\
.65 \\
.65 \\
.60 \\
.60 \\
.65 \\
.70 \\
.65 \\
.60 \\
.55 \\
.55 \\
.55\end{array}$ & $\begin{array}{l}1.25 \\
1.10 \\
1.00 \\
.95 \\
.95 \\
.90 \\
.80 \\
.70 \\
.60 \\
.50 \\
.50 \\
.80 \\
.90 \\
.80 \\
.75 \\
.70\end{array}$ & $\begin{array}{r}0.80 \\
.85 \\
1.00 \\
1.05 \\
3.10 \\
1.25 \\
1.00 \\
1.00 \\
.90 \\
.90 \\
6.05 \\
3.50 \\
2.60 \\
1.40 \\
1.00 \\
.90\end{array}$ & $\begin{array}{l}2.50 \\
2.25 \\
2.00 \\
2.00 \\
1.90 \\
1.80 \\
1.80 \\
1.75 \\
1.65 \\
1.60 \\
1.50 \\
1.40 \\
1.40 \\
1.35 \\
2.50 \\
2.50\end{array}$ & 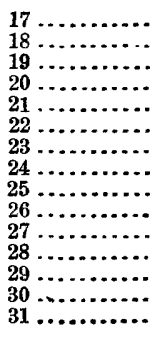 & $\begin{array}{l}0.95 \\
.90 \\
.85 \\
.80 \\
.80 \\
.80 \\
.75 \\
.95 \\
.95 \\
.95 \\
.90 \\
.90 \\
.85 \\
.85 \\
.80\end{array}$ & $\begin{array}{r}0.50 \\
.50 \\
.45 \\
.50 \\
.50 \\
.55 \\
.65 \\
.75 \\
.65 \\
.60 \\
.60 \\
.55 \\
1.60 \\
1.40 \\
\ldots . . .\end{array}$ & $\begin{array}{r}0.65 \\
.60 \\
.60 \\
.55 \\
.55 \\
.60 \\
.80 \\
1.30 \\
.95 \\
.60 \\
.60 \\
.70 \\
1.25 \\
.90 \\
.80\end{array}$ & $\begin{array}{r}0.95 \\
.90 \\
.90 \\
.90 \\
.85 \\
.85 \\
.85 \\
.90 \\
1.00 \\
1.00 \\
.95 \\
1.25 \\
1.25 \\
3.50 \\
\cdots \ldots . .\end{array}$ & $\begin{array}{l}2.35 \\
2.20 \\
2.05 \\
2.00 \\
1.85 \\
1.85 \\
1.80 \\
1.70 \\
1.60 \\
1.50 \\
1.50 \\
1.45 \\
1.40 \\
1.40 \\
1.35\end{array}$ \\
\hline
\end{tabular}


Oostanaula River at Resaca, Georgia, for 1892.

\begin{tabular}{|c|c|c|c|c|c|c|c|c|c|}
\hline Day. & Apr. & May. & June. & July. & Aug. & Sept. & Oct. & Nor. & Dec. \\
\hline 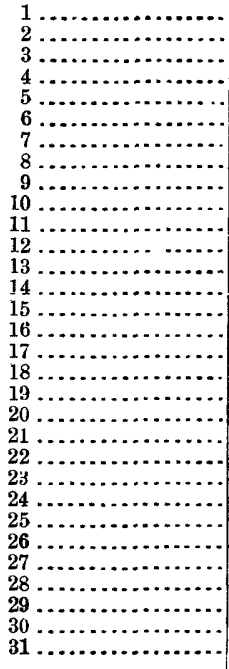 & $\begin{array}{r}6.00 \\
5.60 \\
5.30 \\
5.10 \\
5.00 \\
\mathbf{1 7 . 2 0} \\
28.70 \\
32.00 \\
30.70 \\
28.10 \\
24.40 \\
18.30 \\
10.20 \\
8.10 \\
8.70 \\
7.70 \\
7.00 \\
6.90 \\
6.70 \\
8.40 \\
8.00 \\
6.90 \\
7.50 \\
6.90 \\
6.30 \\
6.00 \\
5.70 \\
\mathbf{5 . 6 0} \\
\mathbf{5 . 8 0} \\
\mathbf{6 . 6 0} \\
. . . .\end{array}$ & $\begin{array}{l}\text { 5. } 80 \\
5.50 \\
5.30 \\
5.10 \\
5.00 \\
4.80 \\
4.70 \\
4.70 \\
4.60 \\
4.60 \\
5.50 \\
5.60 \\
4.80 \\
4.50 \\
4.40 \\
4.20 \\
4.20 \\
4.20 \\
6.50 \\
6.00 \\
5.10 \\
5.10 \\
5.30 \\
4.70 \\
4.40 \\
4.20 \\
4.10 \\
4.00 \\
4.00 \\
3.90 \\
3.90\end{array}$ & $\begin{array}{r}3.80 \\
3.90 \\
4.60 \\
10.70 \\
7.50 \\
6.60 \\
5.70 \\
5.00 \\
5.60 \\
6.70 \\
5.60 \\
5.00 \\
4.30 \\
4.10 \\
3.80 \\
3.70 \\
3.60 \\
3.60 \\
3.60 \\
4.30 \\
5.20 \\
6.50 \\
5.40 \\
4.50 \\
\mathbf{4 . 5 0} \\
\mathbf{5 . 3 0} \\
\mathbf{4 . 6 0} \\
7.70 \\
\mathbf{5 . 6 0} \\
4.40 \\
\ldots . . .\end{array}$ & $\begin{array}{r}4.00 \\
4.00 \\
3.70 \\
3.60 \\
4.20 \\
9.80 \\
8.70 \\
7.30 \\
5.40 \\
9.20 \\
13.70 \\
13.50 \\
12.50 \\
10.10 \\
8.50 \\
7.50 \\
6.30 \\
6.00 \\
5.20 \\
6.80 \\
5.70 \\
5.50 \\
5.110 \\
4.60 \\
4.30 \\
4.20 \\
4.30 \\
3.80 \\
3.70 \\
3.70 \\
3.60\end{array}$ & $\begin{array}{l}3.50 \\
3.50 \\
4.00 \\
3.70 \\
3.30 \\
3.00 \\
3.10 \\
3.00 \\
3.00 \\
3.40 \\
3.70 \\
3.20 \\
3.90 \\
3.20 \\
3.00 \\
2.90 \\
2.80 \\
3.10 \\
3.30 \\
3.20 \\
3.30 \\
3.10 \\
7.10 \\
9.40 \\
6.00 \\
4.60 \\
4.80 \\
4.40 \\
4.00 \\
3.90 \\
4.60\end{array}$ & $\begin{array}{r}4.60 \\
3.60 \\
3.00 \\
3.00 \\
2.90 \\
3.00 \\
3.00 \\
2.80 \\
2.70 \\
2.70 \\
2.60 \\
2.50 \\
2.50 \\
3.10 \\
2.90 \\
2.70 \\
2.50 \\
2.50 \\
2.50 \\
2.60 \\
2.90 \\
5.00 \\
5.20 \\
3.20 \\
2.91 \\
2.70 \\
2.60 \\
2.50 \\
2.40 \\
2.30 \\
\cdots\end{array}$ & $\begin{array}{l}230 \\
2.20 \\
2.20 \\
2.20 \\
2.20 \\
2.20 \\
1.90 \\
1.90 \\
2.30 \\
2.20 \\
2.10 \\
2.10 \\
2.10 \\
2.10 \\
2.10 \\
2.10 \\
2.100 \\
2.00 \\
2.00 \\
1.90 \\
1.90 \\
1.90 \\
1.90 \\
1.90 \\
1.90 \\
1.90 \\
1.90 \\
1.90 \\
1.90 \\
1.90 \\
1.90\end{array}$ & $\begin{array}{r}1.90 \\
1.90 \\
2.20 \\
3.60 \\
4.00 \\
3.00 \\
2.40 \\
3.60 \\
4.60 \\
11.40 \\
10.00 \\
6.90 \\
4.30 \\
3.70 \\
6.60 \\
7.80 \\
6.20 \\
5.90 \\
7.20 \\
5.70 \\
4.50 \\
4.10 \\
3.90 \\
3.70 \\
3.50 \\
3.30 \\
3.20 \\
3.30 \\
3.90 \\
3.70 \\
\ldots . .2\end{array}$ & $\begin{array}{r}2.10 \\
2.10 \\
2.20 \\
2.20 \\
2.20 \\
2.20 \\
2.20 \\
2.20 \\
2.20 \\
2.20 \\
2.20 \\
2.20 \\
2.20 \\
1.90 \\
2.20 \\
2.60 \\
3.00 \\
5.60 \\
6.00 \\
7.00 \\
10.00 \\
8.00 \\
5.60 \\
4.00 \\
3.60 \\
3.40 \\
3.00 \\
2.90 \\
2.60 \\
2.50 \\
2.30\end{array}$ \\
\hline
\end{tabular}

Oostanaula River at Resaca, Georgia, for 1893.

\begin{tabular}{|c|c|c|c|c|c|c|c|c|c|c|c|c|}
\hline Day. & Jan. & Feb. & Mar. & Apr. & May. & June. & July. & Aug. & Sept. & Oct. & Nov. & Dec. \\
\hline 1. & 3.70 & 4.60 & 7.80 & 4.30 & 3.70 & 4. 40 & 3.20 & 2.40 & 2. 30 & 1.70 & 1.40 & 1.90 \\
\hline & 4.80 & 4. 30 & 6.30 & 4. 20 & 3.60 & 7.20 & 3.20 & 2.40 & 3.60 & 1.70 & 1. 40 & 1. 80 \\
\hline 3. & 4.70 & 4.00 & 5. 60 & 4.00 & 7.00 & 7.99 & 3.80 & 2.50 & 2. 30 & 1.70 & 1.50 & 1. 80 \\
\hline 4. & 4.40 & 4. 10 & 6.60 & 4.00 & 14.50 & 7.20 & 3.70 & 2.40 & 2.00 & 1.70 & $1.60^{\circ}$ & 4.00 \\
\hline 5. & 4.00 & 4.00 & 7.20 & 4. 20 & 13.50 & 5.00 & 3,30 & 4.80 & 1.90 & 2.00 & 1.60 & 3.80 \\
\hline 6. & 3.70 & 4.00 & 6.00 & 4.80 & 13.40 & 7.90 & 3.00 & 3.20 & 1.80 & 1.80 & 1.60 & 3.00 \\
\hline 7. & 3.60 & 4. 00 & 5. 50 & 5. 10 & 8.70 & 15. 10 & 3.00 & 3.50 & 1.70 & 1.70 & 1. 50 & 2.40 \\
\hline & 3.50 & 4. 20 & 5. 20 & 4.40 & 7.20 & 12.20 & 2.90 & 3. 20 & 1.80 & 1. 70 & 1. 50 & 2.00 \\
\hline 9 & 3.40 & 4.00 & 10.60 & 4. 10 & 6.20 & 7.70 & 3.30 & 2.90 & 1.90 & 1. 70 & I. 50 & 1. 90 \\
\hline 10. & 3.40 & 3.80 & 13.30 & 4.00 & 5. 90 & 6.00 & 3.00 & 2.50 & 2.10 & 1. 60 & 1. 50 & 1. 90 \\
\hline 11. & 3.00 & 9.00 & 9.00 & 3.90 & 5. 30 & 5.20 & 2.90 & 2.30 & 9.90 & 1. 60 & 1.50 & 1.90 \\
\hline 12. & 3.00 & 13.00 & 8. 20 & 3.90 & 4. 80 & 4. 70 & 2.80 & 2. 20 & 4.40 & ¿. 60 & 1. 50 & 1.80 \\
\hline 13. & 3.30 & 14.10 & 7.80 & 3. 80 & 4.50 & 4. 40 & 2. 70 & 2.30 & 5.60 & 1.60 & 1.50 & 1.80 \\
\hline 14. & 3.00 & 15.50 & 6.70 & 5.00 & 4.40 & 4. 30 & 2.60 & 2.80 & 5. 20 & 1. 60 & 1.50 & 1.80 \\
\hline 15. & 3. 00 & 12.20 & 6.00 & 7.86 & 4. 20 & 4. 10 & 2.50 & 3.20 & 4.40 & 1. 60 & 1. 50 & 1.80 \\
\hline 16. & 3.00 & 18.90 & 5.40 & 6.40 & 4.70 & 4. 00 & 2. 70 & 2.80 & 3.20 & 1.70 & 1.50 & 1.90 \\
\hline 17. & 3.00 & 20.90 & 5.20 & 4. 60 & 5. 30 & 3.90 & 2.50 & 2.60 & 2.50 & 1. 60 & 1. 50 & 2.20 \\
\hline 18. & 3.00 & 20.10 & 5.10 & 4. 30 & 4. 80 & 3.80 & 2.50 & 2.30 & 2.30 & 1.50 & I. 50 & 2.80 \\
\hline $19 \ldots$ & 3.00 & 18.30 & 5.00 & 4.10 & 4.40 & 3.80 & 2.40 & 2.20 & 2.10 & 1. 60 & P. 50 & 2. 50 \\
\hline 20 . & 3.00 & 16. 80 & 4.80 & 4. 40 & 4. 00 & 3.90 & 2.60 & 2.10 & 2. 10 & 1.60 & 1.50 & 2. 20 \\
\hline 21. & 3.00 & 13.60 & 4.60 & 5. 50 & 3. 80 & 4. 80 & 2.60 & 2.00 & 2.00 & 1.60 & 1.60 & 2. 00 \\
\hline 22 & 3.00 & 7.90 & 4.50 & 5.10 & 3.70 & 4. 50 & 3.60 & 1.90 & 1.90 & 1. 80 & 2. 10 & 1.90 \\
\hline 23 . & 3.00 & 7. 10 & 4.50 & 4. 30 & 3.60 & 6.70 & 3.20 & 1.90 & 1.90 & 1. 70 & 2.00 & 1. 80 \\
\hline 24. & 3.50 & 6.40 & 4. 70 & 4. 00 & 3. 60 & 5. 50 & 2.60 & 1.90 & 1. 80 & 1. 60 & 1. 80 & 1.80 \\
\hline & 4. 40 & 5.90 & 7.70 & 3.80 & 3.50 & 4. 20 & 2.40 & 1.80 & 1.80 & 1.60 & 1. 70 & 1.70 \\
\hline 26. & 4. 80 & 5. 50 & 6.30 & 3. 90 & 3. 40 & 3.90 & 2.30 & 1.70 . & 1.80 & 1.60 & 1. 70 & 1.60 \\
\hline 27. & 4. 80 & 5. 20 & 5. 00 & 4. 00 & 3. 30 & 3.80 & 2.30 & 1.70 & 1. 80 & 1. 60 & 1.70 & 1.60 \\
\hline & 4. 50 & 6. 90 & 4. 70 & 4.20 & 3.70 & 3. 70 & 2.30 & 1. 80 & 1. 80 & 1. 50 & 1. 70 & 1. 60 \\
\hline & 5. 20 & $\ldots . .$. & 4.50 & 4. 30 & 4.90 & 3.40 & 2.30 & 1.80 & 1.70 & 1.50 & 2. 20 & 1.60 \\
\hline & 5. 60 & & 4. 80 & 3. 80 & 7.90 & 3.20 & 2.20 & 1.70 & 1.70 & 1. 50 & 2.00 & 2. 60 \\
\hline $31 \ldots$ & 5.10 & $\ldots$ & 4.50 & $\ldots$ & 6.70 & ....... & 2.30 & 1.60 & ....... & 1. 40 & ..... & 3.90 \\
\hline
\end{tabular}


Oostanaula River at Resaca, Georgia, for 1894.

\begin{tabular}{|c|c|c|c|c|c|c|c|c|c|c|c|c|}
\hline Day. & Jan. & Feb. & Mar. & Apr. & May. & June. & July. & Aug. & Sept. & Oct. & Nov. & Dec. \\
\hline & 3. 40 & 3.20 & 8. 10 & 3.70 & 2.90 & 2. 40 & 1. 70 & 2.10 & 1.90 & 1.00 & 2.10 & 1.30 \\
\hline 2. & 2.90 & 3.00 & 7.60 & 3.80 & 2.80 & 2.30 & 1.90 & 2.00 & 1.80 & 1.00 & 1. 90 & 1. 30 \\
\hline 3. & 2.50 & 3.20 & 6.80 & 4.60 & 2.80 & 2. 20 & 1. 80 & 2.10 & 1.70 & 1.00 & 1. 70 & 1. 30 \\
\hline 4. & 3. 30 & 7.90 & 5.80 & 4.30 & 2.90 & 2.10 & 1. 70 & 1.80 & 1.50 & 1.00 & 1. 60 & 1. 30 \\
\hline 5 & 2.20 & 9.90 & 5.20 & 4.40 & 2.90 & 2.00 & 1. 60 & 2.80 & 1. 40 & 1.00 & 1. 50 & 1. 30 \\
\hline & 2.30 & 10.10 & 4.70 & 4.30 & 2.80 & 2.00 & 1.50 & 2.10 & 1. 40 & 1.00 . & 1.30 & 1. 30 \\
\hline & 2.90 & 6.50 & 4. 80 & 3. 90 & 2.70 & 2.00 & 1. 50 & 1.80 & 1.30 & 1.00 & 1. 20 & 1.30 \\
\hline & 6.00 & 4.80 & 5. 20 & 3. 60 & 2.60 & 2.00 & 2.00 & 1.60 & 1.30 & 1.00 & 1.10 & 1. 30 \\
\hline & 4. 60 & 6. 30 & 4.60 & 3.50 & 3.10 & 1. 90 & 1. 70 & 1.50 & 1. 70 & 1.10 & 1. 10 & 1. 30 \\
\hline 10. & 5. 70 & 9. 30 & 4.00 & 3.90 & 3.10 & 1.90 & 1. 60 & 1.50 & 1. 90 & 1.10 & 1. 10 & 1.80 \\
\hline 11. & 7.00 & 7. 00 & 3.90 & 6. 90 & 3. 10 & 1. 90 & 1.50 & 1.50 & 1. 60 & 1.10 & 1. 10 & 4. 10 \\
\hline 12. & 6.50 & 5.20 & 5.30 & 6.40 & 2. 90 & 1. 80 & 1.50 & 1. 50 & 1. 50 & 1.10 & 1. 10 & 11.00 \\
\hline 13. & 5. 10 & 9.60 & 8.10 & 5.00 & 4. 40 & 1. 80 & 1. 40 & 1.50 & 1.40 & 1.10 & 1.00 & 13.80 \\
\hline 14. & 4. 10 & 8.20 & 7.60 & 4.40 & 5. 00 & 1.80 & 1. 30 & 1. 50 & 1.40 & 1.10 & 1. 30 & 9.20 \\
\hline 15. & 3.60 & 6.50 & 5.00 & 4.10 & 6. 10 & 1.80 & 1. 30 & 1.40 & 1. 30 & 1.00 & 1. 30 & 4.50 \\
\hline 16. & 5. 90 & 6. 20 & 4.80 & 3.90 & 4.50 & ]. 80 & 1. 30 & 1.60 & 1. 30 & 1.00 & 1.30 & 3.40 \\
\hline 17. & 6.80 & 5. 30 & 5.90 & 3.70 & 3.50 & 1.70 & 1. 90 & 1.50 & 1.20 & 1.00 & 1.20 & 3.00 \\
\hline 18. & 5.30 & 4.90 & 5.40 & 3.60 & 3. 20 & 1.90 & 2.70 & 1.50 & 2. 10 & 1.00 & 1.20 & 3.00 \\
\hline 19 & 4. 30 & 6. 30 & 4. 60 & 3.60 & 3. 10 & 2.10 & 2.50 & 1. 80 & 3.70 & .90 & 1.20 & 2.70 \\
\hline 20 & 3. 80 & 6.70 & 4. 30 & 4.20 & 3.90 & 2.70 & 2.70 & 2.40 & 2.00 & .90 & 1.20 & 2.40 \\
\hline 21. & 3.60 & 6.10 & 7.20 & 4. 60 & 3,30 & 2.20 & 2.90 & 2.20 & J. 80 & 90 & 1.20 & 2.30 \\
\hline 22 . & 4. 70 & 5.50 & 10.00 & 4.00 & 3. 20 & 2.10 & 3.00 & 2.00 & 1. 60 & .80 & 1.20 & 2.20 \\
\hline 23. & 4. 60 & 5.00 & 7.30 & 3.50 & 3. 20 & 2.10 & 3.50 & 2.10 & 1.50 & .80 & 1.20 & 2.10 \\
\hline & 3.90 & 4. 60 & 5. 80 & 3.40 & 3. 20 & 2.00 & 2.50 & 1. 90 & 1. 40 & .80 & 1. 30 & 2.10 \\
\hline & 3. 90 & 4.40 & 5.20 & 3.30 & 3. 10 & 1. 90 & 2. 10 & 1.90 & 1.20 & .80 & 1.90 & 2.00 \\
\hline 26 & 4. 30 & 5. 60 & 4. 80 & 3.20 & 2.80 & 2.50 & 2.00 & 2.80 & 1. 20 & .80 & 1. 60 & 2. 10 \\
\hline & 3.80 & 6.60 & 4. 30 & 3.00 & 2.70 & 2. 60 & 1. 90 & 3.40 & 1. 10 & .80 & 1.40 & 13.20 \\
\hline & 3.50 & 7.70 & 4. 00 & 3.20 & 2.60 & 2.50 & 2.30 & 2.60 & 1.10 & .80 & 1.30 & 12.60 \\
\hline & 3. 10 & ..... & 3.90 & 3.00 & 2.50 & 2.00 & 2.60 & 4.60 & 1. 00 & .80 & 1.30 & 8. 70 \\
\hline & 3.20 & ....... & 3.90 & 3.00 & 2.50 & 1.80 & 2.40 & 3.20 & 1.00 & 1.20 & 1. 30 & 4.20 \\
\hline 31 & 3. 20 & $\ldots \ldots$ & 3. 80 & & 2.40 & ...... & 2.20 & 2.30 & ... 0 & 1.70 & $\ldots$ & 3.90 \\
\hline
\end{tabular}

Oostanaula River at Resaca, Georgia, for 1895.

\begin{tabular}{|c|c|c|c|c|c|c|c|c|c|c|c|c|}
\hline Day. & Jan. & Feb. & Mar. & Apr. & May. & June. & July. & Ang. & Sept. & Oet. & Nov. & Dec. \\
\hline & 3.50 & 7. 00 & 4. 70 & 5.30 & 5.20 & 4. 10 & 8.00 & 2.60 & 2.90 & 1.90 & 3.50 & 2.10 \\
\hline & 3. 30 & 6.90 & 4. 70 & 5.20 & 4.70 & 4. 00 & 5.20 & 2.40 & 2.80 & 1.90 & 3.00 & 2.10 \\
\hline 3. & 3. 40 & 10. 20 & 12.30 & 5. 30 & 4. 60 & 3.80 & 4.90 & 2. 30 & 2.80 & 1.90 & 2. 70 & 2.30 \\
\hline & 3.40 & 8.90 & 12.00 & 5.00 & 4.30 & 3.70 & 4. 70 & 2.20 & 2.80 & 1.90 & 2. 20 & 2.30 \\
\hline & 3.40 & 7.20 & 9.30 & 4.70 & 4. 20 & 3.70 & 4.00 & 2.20 & 2.80 & 1.90 & 2. 20 & 2. 20 \\
\hline & 3.40 & 6.30 & 6.50 & 4. 60 & 4.30 & 6.80 & 3.90 & 2. 20 & 2.80 & 1.90 & 2.20 & 2. 10 \\
\hline & 3.80 & 6.40 & 5.80 & 5. 60 & 5.30 & 4.80 & 3.90 & 2.40 & 2.70 & 1.90 & 2. 10 & 2. 10 \\
\hline & 8.80 & 7.30 & 5.70 & 15.30 & 6.00 & 3.90 & 8.00 & 5. 40 & 2. 60 & 1. 90 & 2.10 & 210 \\
\hline 9. & 20.20 & 5.20 & 6.00 & 13.30 & 7.70 & 3. 70 & 8.10 & 3. 80 & 2. 60 & 2.10 & 2.10 & 2. $30^{\circ}$ \\
\hline 10. & 23.20 & 5.00 & 5.20 & 10.00 & 7.70 & 3.50 & 7.30 & 3.40 & 2.60 & 2.00 & 2. 20 & 3.00 \\
\hline 11. & 25.80 & 5.00 & 4.90 & 6.70 & 6.00 & 3. 40 & 4.90 & 2.80 & 2.60 & 1.90 & 4.20 & 2.90 \\
\hline 12. & 26.00 & 4.90 & 5.20 & 5. 50 & 8.70 & 3.60 & 4. 20 & 4. 30 & 2.60 & 2.00 & 3.90 & 2. 80 \\
\hline 13. & 22.70 & 4. 80 & 6.00 & 5.50 & 9.00 & 3.80 & 4.00 & 3.70 & 2.60 & 2.00 & 3.20 & 2. 50 \\
\hline 14. & 14.90 & 4.70 & 13.20 & 5.20 & 7.10 & 3.50 & 3.80 & 3.60 & 2. 50 & 2. 00 & 2. 70 & 2. 40 \\
\hline 15. & 6.50 & 4.70 & 13.80 & 5. 20 & 6.00 & 3.40 & 3.60 & 3.20 & 2.40 & 1. 90 & 2. 40 & 2.30 \\
\hline 16. & 6.30 & 4.70 & 12.40 & 6. 00 & 5. 20 & 3,40 & 3. 70 & 3. 40 & 2.30 & 1.90 & 2. 40 & 2. 30 \\
\hline 17. & 8.10 & 4. 70 & 11.50 & 7.80 & 4.80 & 6.90 & 4. 00 & 9.20 & 2.50 & 1.90 & 2.30 & 2. 20 \\
\hline 18. & 7.10 & 4.40 & 8.50 & 6. 30 & 4.70 & 5.70 & 3.60 & 7.50 & 3. 10 & 1. 80 & 2.20 & 2.20 \\
\hline 19. & 6.00 & 4. 40 & 7.20 & 5.70 & 4.60 & 4. 20 & 3. 20 & 5. 60 & 2. 70 & 1.80 & 2.20 & 2.20 \\
\hline 20. & 5.50 & 5.10 & 12.60 & 5. 20 & 4.40 & 3. 70 & 3. 00 & 5. 40 & 2.50 & 1.80 & 2. 10 & 2. 20 \\
\hline 21. & 13.40 & 6.20 & 19.80 & 4.90 & 4.20 & 3.50 & 3.00 & 4.50 & 2.30 & 1.70 & 2. 20 & 3. 50 \\
\hline 22 . & 12.80 & 6.60 & 19.90 & 4.80 & 4. 20 & 3.80 & 3.00 & 6.30 & 2.20 & 1.70 & 2.10 & 5.20 \\
\hline 23. & 9. 90 & 6.20 & 17.80 & 4. 70 & 4.10 & 3.70 & 2.90 & 4.60 & 2.10 & 1.70 & 2.10 & 4.40 \\
\hline 24 & 7. 70 & 5.50 & 13.20 & 4.50 & 3.90 & 3.20 & 2.80 & 4.00 & 2.10 & 1.70 & 2. 10 & 3. 50 \\
\hline 25. & 6.40 & 5.00 & 7.70 & 4. 50 & 5. 30 & 3. 10 & 2.70 & 3. 80 & 2.10 & 1. 70 & 2. 10 & 3. 10 \\
\hline 26. & 7.50 & 4.80 & 7.50 & 5.30 & 6.10 & 3. 00 & 3.20 & 3.60 & 2.10 & 1.70 & 2.10 & 3.10 \\
\hline 27. & 7.60 & 4. 70 & 6.70 & 5.70 & 8.60 & 2. 90 & 3.20 & 3. 30 & 2.00 & 1.70 & 2. 10 & $3.6 \mathrm{i}$ \\
\hline 28. & 6.30 & 4. 70 & 6.30 & 6.30 & 7.90 & 2.80 & 2.90 & 4.80 & 2.00 & 1. 80 & 2.10 & 4. 60 \\
\hline & 8.00 & ..... & 5.90 & 5.80 & 5.50 & 2.80 & 2.80 & 3.70 & 2. 00 & 2.50 & 2. 10 & 4. 20 \\
\hline & 8.90 & 0. & 5.60 & & 4. 80 & 4.20 & 2.70 & 3. 30 & 1.90 & 2. 10 & 2. 10 & 3. 70 \\
\hline & 8.20 & ...... & $\ldots . .$. & $\cdots$ & 4.30 & $\ldots \ldots$ & 2.70 & 3.00 & ....... & 2. 20 & $\cdots+$ & 6.20 \\
\hline
\end{tabular}


Oostanaula River at Resaca, Georgia, for 1896.

\begin{tabular}{|c|c|c|c|c|c|c|c|c|c|}
\hline Day. & Jan. & Feb. & Mar. & Apr. & Aug. & Sept. & Oct. & Nov. & Dec. \\
\hline 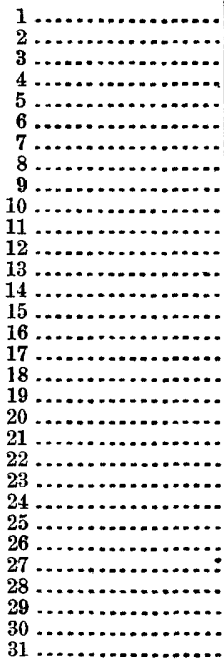 & $\begin{array}{r}\text { 5. } 50 \\
4.60 \\
4.20 \\
3.80 \\
\text { 3. } 40 \\
3.00 \\
2.90 \\
3.30 \\
4.00 \\
4.00 \\
3.50 \\
3.40 \\
3.30 \\
3.10 \\
\text { 3. } 00 \\
2.90 \\
3.70 \\
4.00 \\
\text { 3. } 80 \\
3.40 \\
3.20 \\
\text { 3. 40 } \\
\text { 5. } 70 \\
10.80 \\
11.50 \\
10.00 \\
6.20 \\
5.20 \\
4.70 \\
4.30 \\
4.00\end{array}$ & $\begin{array}{r}\text { 3. } 90 \\
\text { 3. } 90 \\
4.60 \\
4.80 \\
4.50 \\
10.70 \\
16.70 \\
15.50 \\
15.90 \\
14.10 \\
9.30 \\
6.90 \\
5.00 \\
9.10 \\
9.70 \\
7.80 \\
6.40 \\
5.70 \\
5.30 \\
5.00 \\
4.70 \\
4.30 \\
4.10 \\
4.00 \\
4.00 \\
3.90 \\
3.80 \\
3.70 \\
3.70 \\
\ldots \ldots . . \\
\ldots \ldots . .\end{array}$ & $\begin{array}{l}\text { 3. } 70 \\
3.70 \\
3.70 \\
3.60 \\
3.50 \\
3.40 \\
3.80 \\
4.20 \\
3.90 \\
3.60 \\
3.40 \\
4.50 \\
4.90 \\
4.00 \\
3.70 \\
3.60 \\
6.50 \\
8.40 \\
6.60 \\
7.80 \\
7.10 \\
5.80 \\
5.20 \\
4.90 \\
5.30 \\
5.00 \\
4.70 \\
4.40 \\
4.20 \\
4.00 \\
4.60\end{array}$ & $\begin{array}{r}4.80 \\
11.80 \\
11.60 \\
11.70 \\
6.60 \\
5.40 \\
5.00 \\
4.90 \\
4.80 \\
4.30 \\
4.20 \\
4.00 \\
3.80 \\
3.70 \\
3.60 \\
3.60 \\
3.60 \\
3.50 \\
3.50 \\
3.40 \\
3.30 \\
3.20 \\
3.20 \\
4.20 \\
3.70 \\
3.30 \\
3.10 \\
3.40 \\
3.40 \\
3.30 \\
3.10\end{array}$ & $\begin{array}{l}2.10 \\
2.30 \\
2.65 \\
2.40 \\
2.15 \\
2.00 \\
1.90 \\
1.80 \\
1.75 \\
1.75 \\
1.65 \\
1.65 \\
1.70 \\
1.60 \\
1.60 \\
1.70 \\
1.70 \\
1.70 \\
1.50 \\
1.35 \\
1.30 \\
1.25 \\
1.20 \\
1.50 \\
3.20 \\
2.80 \\
1.95 \\
1.70 \\
1.50 \\
1.40 \\
1.30\end{array}$ & $\begin{array}{r}1.20 \\
1.25 \\
1.20 \\
1.25 \\
1.15 \\
1.15 \\
1.30 \\
1.10 \\
1.10 \\
1.05 \\
1.00 \\
1.00 \\
1.50 \\
1.25 \\
1.10 \\
1.05 \\
1.00 \\
1.00 \\
.95 \\
.90 \\
. .85 \\
1.95 \\
1.55 \\
1.25 \\
1.20 \\
1.10 \\
1.10 \\
1.70 \\
8.35 \\
\ldots \ldots \\
\ldots \ldots . . .\end{array}$ & 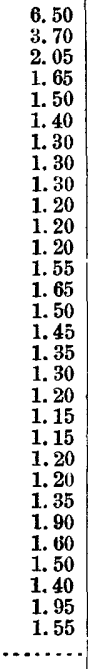 & $\begin{array}{r}1.50 \\
1.40 \\
1.40 \\
1.35 \\
1.90 \\
3.15 \\
2.10 \\
2.00 \\
2.10 \\
1.85 \\
1.70 \\
3.00 \\
13.65 \\
11.35 \\
11.10 \\
4.25 \\
3.30 \\
3.00 \\
2.70 \\
2.60 \\
2.50 \\
2.35 \\
2.40 \\
2.35 \\
2.25 \\
2.20 \\
2.10 \\
2.15 \\
3.80 \\
8.70 \\
\ldots \ldots . . .\end{array}$ & $\begin{array}{l}\text { 9. } 25 \\
6.70 \\
\text { 4. } 65 \\
3.90 \\
\text { 3. } 50 \\
3.20 \\
3.00 \\
2.90 \\
3.00 \\
3.50 \\
3.30 \\
3.10 \\
2.90 \\
2.80 \\
4.20 \\
3.90 \\
3.30 \\
3.00 \\
3.00 \\
2.90 \\
2.80 \\
2.60 \\
2.55 \\
2.55 \\
2.40 \\
2.30 \\
2.25 \\
2.20 \\
2.20 \\
2.20 \\
2.20\end{array}$ \\
\hline
\end{tabular}

Coosa River at Riverside, Alabama.

\begin{tabular}{|c|c|c|c|c|c|c|c|c|c|c|c|c|c|c|}
\hline Day. & Sept. & Oet. & Nov. & Dec. & Day. & Sept. & Det. & Nov. & Dec. & Day. & Sept. & Oct. & Nov. & Dec. \\
\hline $\begin{array}{r}1 \ldots \\
2 \ldots \\
3: \\
4 \ldots \\
5 . \\
6 . \\
7 . \\
8 \ldots \\
9 . \\
10 . \\
11 .\end{array}$ & 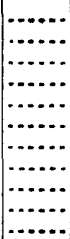 & $\begin{array}{l}0.60 \\
1.75 \\
3.10 \\
2.75 \\
2.00 \\
1.50 \\
1.20 \\
.85 \\
.70 \\
.60 \\
.65\end{array}$ & $\begin{array}{l}1.10 \\
1.40 \\
1.20 \\
1.10 \\
1.05 \\
1.10 \\
1.20 \\
2.55 \\
2.30 \\
1.90 \\
1.30\end{array}$ & $\begin{array}{l}1.30 \\
2.10 \\
4.38 \\
3.80 \\
3.20 \\
2.50 \\
2.20 \\
1.90 \\
1.70 \\
1.60 \\
1.55\end{array}$ & $\begin{array}{l}12 . . \\
13 . . \\
14 \ldots \\
15 . . \\
16 \ldots \\
17 \ldots \\
18 . . \\
19 . . \\
20 . . \\
21 \ldots\end{array}$ & $\mid \begin{array}{l}\ldots \ldots \\
\ldots \ldots \\
\ldots \ldots \\
\ldots \ldots \\
\ldots \ldots \\
\cdots \ldots \\
\ldots \ldots \\
\ldots \ldots \\
\ldots \ldots \\
\ldots\end{array}$ & $\begin{array}{r}0.60 \\
.60 \\
.60 \\
.55 \\
.55 \\
.55 \\
.65 \\
.80 \\
.85 \\
.75\end{array}$ & $\begin{array}{l}1.60 \\
2.25 \\
2.70 \\
4.00 \\
5.20 \\
4.70 \\
4.20 \\
3.20 \\
2.30 \\
1.50\end{array}$ & $\begin{array}{l}1.55 \\
1.60 \\
1.60 \\
1.80 \\
2.00 \\
2.10 \\
2.20 \\
2.00 \\
1.80 \\
1.70\end{array}$ & $\begin{array}{l}22 . . \\
23 . . \\
24 . . \\
25 \ldots \\
26 . . \\
27 . . \\
28 . . \\
29 \ldots \\
30 \ldots \\
31 . .\end{array}$ & $\begin{array}{r}\ldots \ldots \\
\ldots \ldots \\
\ldots \ldots \\
\ldots \ldots . \\
\cdots \\
0.45 \\
.45 \\
.45 \\
.50 \\
\cdots \ldots .\end{array}$ & $\begin{array}{r}0.70 \\
.60 \\
.55 \\
.60 \\
.70 \\
.80 \\
.85 \\
.90 \\
.95 \\
.85\end{array}$ & $\begin{array}{c}1.40 \\
1.35 \\
1.30 \\
1.25 \\
1.20 \\
1.15 \\
1.15 \\
1.10 \\
1.20 \\
\ldots \ldots . .\end{array}$ & $\begin{array}{l}1.50 \\
1.45 \\
1.40 \\
1.35 \\
1.30 \\
1.25 \\
1.20 \\
1.10 \\
1.10 \\
1.10\end{array}$ \\
\hline
\end{tabular}


Lock No. 4.-This point is about 3 miles above Riverside, Alabama, and no water comes into the river between these points except Blue Spring Branch, which does not furnish a cubic foot of water per second. The measurements made from the bridge of the Southern Railway at Riverside, Alabama, may therefore be used in estimating the values of gage heights observed at Lock No. 4.

A record has been kept at this point by the United States Engineer Corps, which is given below.

Coosa River at Lock No. 4, near Lincoln, Alabama, for 1890.

\begin{tabular}{|c|c|c|c|c|c|c|c|c|c|c|c|}
\hline Day. & Feb. & Mar. & Apr. & May. & June. & July. & Aug. & Sept. & Oct. & Nov. & Dec. \\
\hline 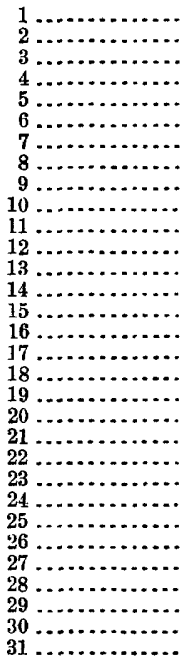 & \begin{tabular}{|r|}
8.5 \\
8.5 \\
7.6 \\
6.4 \\
5.1 \\
4.5 \\
4.1 \\
5.4 \\
7.1 \\
7.8 \\
7.9 \\
7.5 \\
6.6 \\
5.7 \\
5.4 \\
5.5 \\
5.6 \\
5.4 \\
4.7 \\
4.3 \\
3.9 \\
3.6 \\
3.5 \\
5.2 \\
11.6 \\
16.4 \\
18.6 \\
$\cdots \ldots .$. \\
$\cdots \ldots .$. \\
$\cdots \ldots .$. \\
$\ldots \ldots$
\end{tabular} & $\begin{array}{r}20.9 \\
20.2 \\
19.1 \\
18.2 \\
17.7 \\
17.9 \\
17.5 \\
16.7 \\
13.9 \\
10.3 \\
7.8 \\
6.5 \\
5.9 \\
6.8 \\
8.7 \\
9.2 \\
8.7 \\
7.9 \\
7.1 \\
8.5 \\
10.5 \\
11.4 \\
14.4 \\
14.2 \\
14.0 \\
13.4 \\
12.1 \\
10.0 \\
7.9 \\
6.4 \\
6.0\end{array}$ & $\begin{array}{r}17.1 \\
7.6 \\
6.8 \\
10.4 \\
1.5 \\
2.1 \\
11.8 \\
0.2 \\
8.0 \\
6.8 \\
6.0 \\
5.5 \\
5.1 \\
4.7 \\
4.3 \\
4.1 \\
5.1 \\
5.6 \\
5.8 \\
6.3 \\
5.7 \\
5.0 \\
4.3 \\
3.9 \\
3.8 \\
3.7 \\
3.8 \\
3.8 \\
3.8 \\
3.7 \\
\ldots . . .\end{array}$ & $\begin{array}{l}3.7 \\
3.4 \\
3.2 \\
3.2 \\
3.2 \\
3.2 \\
3.3 \\
3.3 \\
3.2 \\
3.1 \\
3.0 \\
2.8 \\
2.7 \\
2.7 \\
2.7 \\
3.2 \\
3.7 \\
4.2 \\
4.0 \\
4.0 \\
3.9 \\
3.6 \\
4.0 \\
4.1 \\
3.6 \\
3.1 \\
3.1 \\
3.4 \\
3.4 \\
5.1 \\
4.0\end{array}$ & $\begin{array}{r}3.1 \\
2.7 \\
2.5 \\
2.4 \\
2.2 \\
2.3 \\
2.3 \\
2.2 \\
2.2 \\
2.4 \\
2.8 \\
3.9 \\
3.7 \\
3.8 \\
2.5 \\
2.2 \\
2.2 \\
2.1 \\
2.0 \\
1.9 \\
2.3 \\
2.1 \\
1.9 \\
1.8 \\
1.8 \\
1.7 \\
1.5 \\
1.5 \\
\ldots \ldots . . \\
\ldots \ldots . .\end{array}$ & $\begin{array}{r}1.4 \\
1.4 \\
1.3 \\
1.3 \\
1.3 \\
1.3 \\
1.3 \\
1.2 \\
1.1 \\
1.1 \\
1.0 \\
1.0 \\
1.0 \\
1.0 \\
.9 \\
.9 \\
1.2 \\
1.4 \\
1.4 \\
1.2 \\
1.1 \\
1.1 \\
1.1 \\
1.1 \\
1.2 \\
1.5 \\
3.2 \\
5.2 \\
4.3 \\
3.1 \\
2.8\end{array}$ & $\begin{array}{r}2.6 \\
2.0 \\
1.8 \\
1.8 \\
2.3 \\
2.3 \\
2.9 \\
3.0 \\
2.7 \\
2.6 \\
2.9 \\
2.5 \\
2.7 \\
2.7 \\
2.0 \\
1.6 \\
1.9 \\
2.2 \\
1.9 \\
1.6 \\
1.4 \\
1.2 \\
1.0 \\
.9 \\
.9 \\
.9 \\
.9 \\
1.1 \\
1.7 \\
2.5 \\
\ldots . . .\end{array}$ & $\begin{array}{r}3.4 \\
3.1 \\
2.3 \\
1.6 \\
1.2 \\
1.0 \\
1.0 \\
1.0 \\
1.2 \\
1.2 \\
1.3 \\
1.6 \\
1.4 \\
1.1 \\
1.0 \\
1.0 \\
1.4 \\
1.4 \\
1.1 \\
.9 \\
.9 \\
.9 \\
1.1 \\
1.9 \\
5.1 \\
6.1 \\
6.0 \\
4.5 \\
4.0 \\
3.4 \\
\ldots . . .\end{array}$ & $\begin{array}{r}3.5 \\
4.6 \\
4.9 \\
5.9 \\
6.3 \\
5.4 \\
4.2 \\
3.9 \\
4.0 \\
3.9 \\
3.9 \\
3.4 \\
2.8 \\
2.4 \\
2.2 \\
2.2 \\
2.6 \\
3.2 \\
4.7 \\
4.9 \\
3.8 \\
3.6 \\
8.9 \\
11.0 \\
11.5 \\
10.9 \\
8.9 \\
6.6 \\
5.0 \\
4.0 \\
3.6\end{array}$ & $\begin{array}{l}3.2 \\
2.9 \\
2.7 \\
2.5 \\
2.4 \\
2.3 \\
2.2 \\
2.1 \\
2.0 \\
1.9 \\
1.8 \\
1.7 \\
1.7 \\
1.7 \\
1.6 \\
1.5 \\
1.5 \\
1.6 \\
1.7 \\
1.7 \\
1.7 \\
1.7 \\
1.5 \\
1.4 \\
1.3 \\
1.3 \\
1.3 \\
1.3 \\
1.2 \\
1.2 \\
\ldots . . .\end{array}$ & $\begin{array}{l}1.2 \\
1.2 \\
1.2 \\
1.2 \\
1.2 \\
1.3 \\
2.6 \\
3.3 \\
5.0 \\
6.4 \\
6.2 \\
5.0 \\
4.8 \\
2.9 \\
2.5 \\
2.3 \\
2.0 \\
1.9 \\
1.9 \\
2.0 \\
2.0 \\
1.9 \\
1.8 \\
1.7 \\
1.7 \\
2.7 \\
4.6 \\
5.0 \\
5.4 \\
5.3 \\
4.4\end{array}$ \\
\hline
\end{tabular}

Coosa River at Lock No. 4, for 1891.

\begin{tabular}{|c|c|c|c|c|c|c|c|c|c|c|c|c|}
\hline Day. & Jan. & Feb. & Mar. & Apr. & May. & June. & July. & Aug. & Sept. & Oct. & Nov. & Dec. \\
\hline $\begin{array}{l}1 \\
2 \ldots \\
3 \\
4 \\
5 \\
6 \\
7 \\
8\end{array} \ldots \ldots$ & $\begin{array}{r}3.6 \\
5.4 \\
6.6 \\
7.5 \\
7.6 \\
6.7 \\
5.2 \\
4.1 \\
3.6 \\
3.4 \\
3.7 \\
4.9 \\
6.5 \\
6.5 \\
6.6 \\
5.7 \\
5.4 \\
5.4 \\
5.6 \\
5.3 \\
4.9 \\
6.8 \\
8.3 \\
9.3 \\
10.3 \\
10.1 \\
9.5 \\
8.5 \\
9.0 \\
11.0 \\
11.4\end{array}$ & $\begin{array}{l}13.7 \\
13.8 \\
14.4 \\
15.1 \\
14.9 \\
14.5 \\
16.3 \\
16.7 \\
16.0 \\
19.0 \\
18.8 \\
18.3 \\
19.2 \\
19.4 \\
19.0 \\
18.4 \\
18.1 \\
17.6 \\
16.9 \\
16.2 \\
16.2 \\
16.0 \\
14.9 \\
14.7 \\
14.7 \\
14.4 \\
13.7 \\
11.9 \\
\ldots \ldots . . \\
\ldots \ldots . . \\
\ldots \ldots .\end{array}$ & $\begin{array}{r}10.0 \\
8.3 \\
7.5 \\
7.0 \\
6.7 \\
9.1 \\
12.7 \\
16.2 \\
18.9 \\
18.9 \\
18.3 \\
18.2 \\
18.1 \\
18.0 \\
17.8 \\
17.6 \\
17.4 \\
16.4 \\
15.1 \\
11.3 \\
8.1 \\
7.1 \\
6.7 \\
6.4 \\
6.2 \\
6.3 \\
8.1 \\
8.8 \\
9.3 \\
8.9 \\
10.4\end{array}$ & $\begin{array}{r}12.2 \\
13.5 \\
13.8 \\
13.5 \\
12.3 \\
9.9 \\
8.0 \\
7.0 \\
6.5 \\
6.0 \\
5.8 \\
5.7 \\
5.6 \\
5.5 \\
5.2 \\
5.2 \\
5.1 \\
4.9 \\
4.9 \\
4.9 \\
5.1 \\
4.7 \\
4.5 \\
5.0 \\
6.7 \\
6.7 \\
6.0 \\
5.2 \\
4.6 \\
4.1 \\
\ldots . . .\end{array}$ & $\begin{array}{l}3.9 \\
3.6 \\
3.5 \\
3.5 \\
3.4 \\
3.3 \\
3.1 \\
3.0 \\
2.9 \\
2.8 \\
2.8 \\
2.8 \\
2.8 \\
2.7 \\
2.6 \\
2.6 \\
2.8 \\
2.7 \\
2.7 \\
2.8 \\
2.8 \\
3.1 \\
3.6 \\
2.8 \\
2.6 \\
2.6 \\
2.9 \\
3.0 \\
3.2 \\
3.2 \\
3.0\end{array}$ & \begin{tabular}{|l|}
2.8 \\
2.4 \\
2.2 \\
2.2 \\
2.0 \\
2.0 \\
2.0 \\
2.1 \\
2.4 \\
6.6 \\
5.6 \\
3.9 \\
3.3 \\
3.9 \\
3.5 \\
3.1 \\
2.8 \\
2.8 \\
2.9 \\
3.0 \\
3.8 \\
4.4 \\
3.4 \\
3.0 \\
3.0 \\
2.8 \\
2.4 \\
2.0 \\
1.8 \\
1.7 \\
$\ldots . .$.
\end{tabular} & $\begin{array}{l}1.6 \\
1.5 \\
1.5 \\
1.5 \\
1.4 \\
1.4 \\
1.3 \\
2.0 \\
2.5 \\
2.6 \\
3.8 \\
4.0 \\
3.6 \\
2.8 \\
2.2 \\
1.7 \\
1.5 \\
1.5 \\
1.6 \\
1.5 \\
1.8 \\
2.4 \\
2.0 \\
1.8 \\
1.7 \\
1.9 \\
2.0 \\
1.9 \\
2.3 \\
3.2 \\
2.9\end{array}$ & $\begin{array}{r}8.6 \\
10.8 \\
11.7 \\
11.3 \\
9.9 \\
7.0 \\
6.2 \\
4.7 \\
3.4 \\
2.7 \\
2.4 \\
2.2 \\
2.2 \\
2.1 \\
2.0 \\
1.9 \\
1.7 \\
1.6 \\
1.6 \\
1.6 \\
1.7 \\
1.6 \\
1.7 \\
1.5 \\
1.6 \\
1.7 \\
2.4 \\
2.0 \\
1.5 \\
1.5 \\
1.5\end{array}$ & $\begin{array}{r}1.5 \\
1.3 \\
1.3 \\
1.4 \\
1.6 \\
2.8 \\
3.8 \\
3.0 \\
1.9 \\
1.6 \\
2.1 \\
1.6 \\
1.4 \\
1.6 \\
2.7 \\
2.6 \\
2.1 \\
1.6 \\
1.3 \\
1.0 \\
.9 \\
.8 \\
.7 \\
.7 \\
.6 \\
.6 \\
.7 \\
.5 \\
.5 \\
.5\end{array}$ & $\begin{array}{l}0.4 \\
.4 \\
.5 \\
.4 \\
.3 \\
.3 \\
.4 \\
.3 \\
.3 \\
.3 \\
.4 \\
.5 \\
.4 \\
.3 \\
.3 \\
.3 \\
.3 \\
.3 \\
.3 \\
.2 \\
.2 \\
.2 \\
.1 \\
.1 \\
.1 \\
.1 \\
.1 \\
.1 \\
.1 \\
.1 \\
.1\end{array}$ & $\begin{array}{r}0.1 \\
.1 \\
.1 \\
.1 \\
.1 \\
.1 \\
.1 \\
.1 \\
.4 \\
.3 \\
.4 \\
.6 \\
1.7 \\
1.0 \\
.7 \\
.6 \\
.5 \\
.5 \\
.5 \\
.5 \\
1.0 \\
1.1 \\
1.1 \\
3.3 \\
3.4 \\
3.2 \\
2.1 \\
2.0 \\
1.7 \\
\ldots . .\end{array}$ & $\begin{array}{l}1.5 \\
1.3 \\
1.0 \\
1.0 \\
2.4 \\
3.3 \\
5.1 \\
5.8 \\
6.4 \\
5.9 \\
4.4 \\
3.0 \\
3.0 \\
2.5 \\
2.0 \\
3.2 \\
4.6 \\
5.4 \\
4.8 \\
3.8 \\
3.2 \\
2.4 . \\
2.1 \\
2.2 \\
2.0 \\
3.0 \\
3.5 \\
4.0 \\
4.1 \\
4.0 \\
3.8\end{array}$ \\
\hline
\end{tabular}


Coosa River ${ }^{1}$ at Lock No. 4, for 1892.

\begin{tabular}{|c|c|c|c|c|c|c|c|c|c|c|c|c|}
\hline Day. & Jan. & Feb. & Mar. & Apr. & May. & June. & July. & Ang. & Sept. & Oet. & Nov. & Dec. \\
\hline & 3.3 & 4.3 & 3.9 & 6.8 & 4.9 & 2.3 & 4.3 & 2.0 & 2.8 & 1. 2 & 0.4 & 3. 2 \\
\hline & 4.3 & $\begin{array}{l}4.0 \\
4.1\end{array}$ & $\begin{array}{l}3.8 \\
3.8\end{array}$ & $\begin{array}{l}\text { 5. } 9 \\
\text { 5. }\end{array}$ & 4.8 & 2.1 & $\begin{array}{l}4.3 \\
3.4\end{array}$ & 1. 9 & 2.4 & 1.2 & $\begin{array}{r}0.4 \\
.4\end{array}$ & 2.8 \\
\hline 3. & 5.8 & 4.0 & 3.6 & 5.5 & 4. 6 & 2.5 & 2.5 & 1.8 & 2.2 & 12 & .3 & 2.4 \\
\hline 4. & 6.0 & 3.8 & 3.5 & 5.0 & 4.2 & 2.8 & 2.1 & 1.8 & 1.8 & 1.0 & 4 & 2. 4 \\
\hline $5 \ldots \ldots$ & 5. 2 & 3.8 & 3.3 & 4.7 & 3.8 & 3.5 & 2.0 & -1.7 & 1. 5 & .9 & 5 & 2. 7 \\
\hline $6 \ldots .$. & 4.6 & 3.7 & 3.2 & 6.6 & 3.6 & 5. 1 & 2.1 & 1.7 & 1.4 & .9 & .5 & 2.4 \\
\hline 7. & 4.2 & 3.7 & 4.0 & 20.8 & 3.5 & 5. 5 & 2.1 & 1.6 & 1.3 & .8 & 1. 0 & 2.4 \\
\hline 8 & 4.6 & 3.9 & 5.8 & 23.7 & 3.3 & 4.7 & 3.3 & 1.5 & 1.2 & .8 & 1.0 & 2.8 \\
\hline 9. & 5.0 & 4.6 & 8.0 & 23.4 & 3.3 & 3.8 & 5.6 & 1.5 & 1.2 & .7 & 1.0 & 2.3 \\
\hline 10. & 5. 5 & 4.8 & 9.8 & 22.5 & 3.3 & 3.6 & 6.4 & 1.4 & 1.1 & 7 & 1.2 & 2.2 \\
\hline 11. & 5.8 & 5.0 & 10.4 & 22.3 & 3.5 & 3.5 & 6.3 & 1. 4 & 1.0 & .7 & 3.2 & 2.9 \\
\hline 12. & 7.8 & 4. 7 & 9.5 & 22.7 & 3.6 & 3.0 & 8.7 & 1. 6 & .9 & .8 & 6.9 & 2.5 \\
\hline 13. & 16.3 & 4.2 & 7.2 & 23.0 & 4.0 & 3.4 & 10.6 & 1. 9 & 1.0 & .8 & 6.9 & 2. 4 \\
\hline 14. & 19.3 & 3.8 & 5.8 & 22.6 & 4.0 & 3.0 & 10. 9 & 1. 7 & .9 & .8 & 4. 7 & 2.1 \\
\hline 15. & 19.7 & 3.8 & 4.9 & 21.6 & 3.6 & $2 . \overline{5}$ & 11. 3 & 1. 6 & .9 & .7 & 3. 9 & 2. 4 \\
\hline $16 \ldots$ & 19.1 & 3.9 & 4.5 & 20.0 & 3. 3 & 2.2 & 9. 4 & 1. 5 & 1.0 & .8 & 3.1 & 2.8 \\
\hline 17. & 18.8 & 3.9 & 4. 2 & 18.4 & 3.0 & 2.0 & 7.5 & 1. 5 & 3.3 & .8 & & 3.1 \\
\hline 18. & 19.6 & 4.0 & 4.9 & 14.4 & 2.8 & 1. 9 & 6.0 & 1. 5 & 1.9 & .8 & 6.0 & 4. 4 \\
\hline 19. & 21.5 & 4. 1 & 5.9 & 8.5 & 3.5 & 1.8 & 5.5 & 1. 4 & 1.3 & .7 & 4. 8 & 5.5 \\
\hline $20 \ldots$ & 23.1 & 3.9 & 6.1 & 6.1 & 3.3 & 1. 9 & 8.1 & 1. 9 & 1. 3 & .5 & 4. 0 & 6.9 \\
\hline 21 & 23.4 & 4.2 & 5.9 & 6.0 & 3. 7 & 2.1 & 6.4 & 2.1 & 1.9 & .5 & 4. 2 & 8.9 \\
\hline 22. & 22.8 & 5.3 & 5.4 & 8.3 & 4.4 & 2.4 & 6.3 & 2.3 & 3.2 & .5 & 3.9 & 9.2 \\
\hline 23 & 22.1 & 7.2 & 5.2 & 8.4 & 3.8 & 4.4 & 5.3 & 2.4 & 5.5 & .5 & 3.2 & 9.5 \\
\hline 24 . & 21.3 & 8.4 & 7.1 & 7.1 & 3.4 & 3.9 & 5.3 & $\ldots$ & 5. 8 & .5 & 2.6 & 8.1 \\
\hline $25 \ldots$ & 20.0 & 8.0 & 7.8 & 6.1 & 3.3 & 3.9 & 4.6 & 5. 4 & 4.1 & .5 & 2.2 & 6. 3 \\
\hline 26. & 18.9 & 6.6 & 9.4 & 5.8 & 3.3 & 3.3 & 3.8 & 7.0 & 3.3 & .4 & 1.9 & 5. 1 \\
\hline 27. & 16.6 & 5.0 & 10.8 & 5.3 & 2.9 & 4.0 & 3.3 & 6.8 & 2. 3 & .5 & 1.8 & 4. 2 \\
\hline 28. & 11.0 & 4. 6 & 11.4 & 4.8 & 2.8 & 4.1 & 3.0 & 5.1 & 1.8 & .5 & 1.7 & 3.7 \\
\hline & 6.7 & 4. 3 & 11.8 & 4.6 & 2.7 & 4. 2 & 2.8 & 4.2 & 1.5 & .5 & 1.9 & 3.3 \\
\hline & 5.4 & $\ldots$ & 11.1 & 4. 8 & 2.6 & 4.5 & 2.5 & 3.7 & 1.3 & .4 & 3.3 & 2.9 \\
\hline & 4.5 & & 8.9 & ...... & 2.5 & .... & 2.1 & 3.2 & ...... & .4 & ....... & 2.6 \\
\hline
\end{tabular}

Coosa River ${ }^{1}$ at Lock No. 4, for 1893.

\begin{tabular}{|c|c|c|c|c|c|c|c|c|c|c|c|c|}
\hline Day. & Jan. & Feb. & Mar. & Apr. & May. & June. & July. & Ang. & Sept. & Oct. & Nov. & Dec. \\
\hline $1 .$. & 2.7 & 5.6 & 7.1 & 3.6 & 4.5 & 5.4 & 1.9 & 1.0 & .2 & 0.3 & -0.1 & 0.3 \\
\hline $2 \ldots$ & 2.8 & 5. 0 & 7.9 & 3.5 & 5. 2 & 7.4 & 1.8 & .9 & .2 & .3 & -.1 & .3 \\
\hline $3 .$. & 3.1 & 4.4 & 8.1 & 3.3 & 9.7 & 7.9 & 1.7 & 1.1 & .5 & .3 & -.1 & .9 \\
\hline $4 .$. & $3 . \overline{1}$ & 3.8 & 7. 9 & 3.2 & 14.4 & 8.0 & 1.7 & 1.4 & 1.2 & .3 & -.1 & 1.0 \\
\hline 5. & 3.3 & 3.5 & 8.4 & 3.4 & 15.1 & 8.0 & 1.6 & 1.4 & 1.7 & .2 & .0 & 1. 2 \\
\hline 6. & 3.0 & 3.3 & 8.2 & 3.5 & 12.5 & 7.3 & 1.8 & 1.8 & 1.1 & .2 & 0 & 1. 3 \\
\hline $7 .$. & 2.8 & 3.1 & 7.6 & 3.7 & 12.5 & 7.3 & 1.6 & 1.6 & 1.6 & .2 & .0 & 1.5 \\
\hline 8. & 2.5 & 3.1 & 6.6 & 3.8 & 10. 5 & 9. 3 & 1.5 & 1. 5 & .5 & .3 & .0 & 1.2 \\
\hline 9. & 2.2 & .3 .1 & 7.7 & 4.0 & 9.8 & 10.4 & 1.5 & 1.5 & .5 & .3 & .0 & .7 \\
\hline $10 .$. & 2.1 & 3.0 & 8.8 & 3.8 & 7.9 & 10.2 & 1.4 & 1.4 & .4 & .3 & 0 & .6 \\
\hline $11 \ldots . .$. & 1.9 & 3.8 & 10.1 & 3.3 & 6. 4 & 7.9 & 1.5 & 1.5 & .5 & .1 & -.1 & .4 \\
\hline $12 \ldots \ldots \ldots \ldots \ldots$ & 2.1 & 6.5 & 10.5 & 3.0 & 5.6 & 5.6 & 1.4 & 1.4 & .8 & .1 & 0 & .3 \\
\hline $13 \ldots \ldots$ & 2.0 & 9.2 & 9.8 & 2.9 & 4.9 & 4.2 & 1.4 & 1.0 & 1.5 & .1 & 0 & .3 \\
\hline $14 \ldots \ldots \ldots \ldots$ & 2.0 & 11.3 & 8.0 & 3.3 & 4.4 & 3.6 & 1.3 & 1.4 & 2.7 & .1 & .0 & .3 \\
\hline $15 \ldots \ldots$ & 2.3 & 12.1 & 6.9 & 4.0 & 3.9 & 3.1 & 1.2 & 1.3 & 3.3 & .0 & & .3 \\
\hline $16 \ldots \ldots$ & 2.6 & 20.8 & 5.9 & 4. 4 & 3.7 & 3.0 & 1.3 & 1.1 & 3.5 & 0 & +.1 & .5 \\
\hline 17. & 2.4 & 20.6 & 5.3 & 4. 4 & 3.5 & 2.9 & 1.2 & 1.2 & 3.0 & 0 & .0 & 6 \\
\hline 18. & 2.1 & 19.5 & 4.8 & 4.3 & 3.2 & 2.6 & 1.1 & 1.3 & 2.1 & .0 & .0 & 6 \\
\hline 19. & 2.1 & 18. 4 & 4.5 & 3.9 & 3.1 & 2.5 & 1.0 & 1.4 & 1.5 & .0 & 0 & 9 \\
\hline 20 . & 2.0 & 17.8 & 4. 2 & 3.3 & 3.0 & 2.9 & .9 & 1.1 & 1.0 & .0 & 0 & 7 \\
\hline 21. & 2. 0 & 17. 3 & 4. 0 & 3.6 & 2.8 & 2.8 & 1.0 & 1.0 & .7 & .0 & .1 & 7 \\
\hline 22. & 2.0 & 16.8 & 3.8 & 3.8 & 2.6 & 2.4 & .9 & .8 & .6 & .0 & .1 & .6 \\
\hline$\ldots$. & 2.0 & 15.9 & 3.7 & 3.7 & 2.5 & 3.0 & .9 & .5 & .5 & .0 & .1 & .4 \\
\hline $24 \ldots$ & 2.1 & 14.0 & 3.9 & 3.6 & 2.3 & 3.1 & .9 & .4 & .4 & .0 & 2 & .3 \\
\hline $25 \ldots$ & $2 . \overline{8}$ & 9.4 & 5.9 & 3.3 & 2.2 & 2.9 & 1.3 & .3 & .3 & .0 & 2 & 3 \\
\hline 26 . & 3.7 & 6.5 & 6.0 & 3.1 & 2. 1 & 3.8 & 1.5 & .3 & .3 & .0 & .1 & .2 \\
\hline 27 & 4. 7 & 5.5 & 5.8 & 4.8 & 2. 0 & 3.7 & 1.2 & .4 & .4 & .0 & & .1 \\
\hline 28. & 5.5 & 6.2 & 5.4 & 6.4 & 2.0 & 3.4 & .8 & .2 & .2 & .0 & & 1 \\
\hline 29 . & 5.8 & ....... & 4.2 & 5.9 & 3.0 & 2.0 & .7 & .1 & .1 & .0 & & .9 \\
\hline & 5. 8 & $\ldots \ldots$ & 4. 0 & 5.4 & 5.4 & 1. 9 & .7 & .2 & .2 & .0 & & .2 \\
\hline 31 & 5.7 & & 3.7 & $\ldots . .$. & 6.0 & .... & 1.0 & .2 & $\cdots$ & .0 & & $\cdots$ \\
\hline
\end{tabular}

I Record from United States Engineer Office. 
Coosa River ${ }^{1}$ at Lock No. 4, for 1894.

\begin{tabular}{|c|c|c|c|c|c|c|c|c|c|c|c|c|}
\hline Day. & Jan. & Feb. & Mar. & Apr. & May. & June. & July. & Aug. & Sept. & Oct. & Nov. & Dec. \\
\hline 1. & 2.3 & 2.1 & 7.4 & 2.8 & 1.7 & 0.7 & 0.7 & 0.9 & 1.4 & 0.1 & -0.1 & 0.0 \\
\hline 2. & 1.5 & 2.0 & 7.6 & 3.4 & 1.7 & .7 & $\begin{array}{r}.5 \\
.5\end{array}$ & 1.0 & $\begin{array}{l}1.4 \\
1.4\end{array}$ & -.1 & -.2 & -.1 \\
\hline 3. & 1.3 & 2.0 & 7.6 & 1.3 & 1. 6 & 6 & .3 & 1.0 & 1.0 & -.1 &.$- \overline{1}$ & -.2 \\
\hline $4 .$. & 1.3 & 2.0 & 6.9 & 3.9 & 1. 6 & .6 & .3 & 1. 0 & .8 & -.3 & & -.1 \\
\hline & 1.2 & 2.8 & 6.0 & 3.7 & 1. 7 & .5 & .3 & .7 & .5 & -.4 & +.1 & -.2 \\
\hline & 1.2 & 4.1 & 5.2 & 3.9 & 1.8 & .5 & 3 & .7 & .2 & $-\div 3$ & & -.2 \\
\hline 7. & 2.5 & 5.0 & 4.6 & 2.7 & 1.7 & .4 & 1.0 & 1.2 & .1 & -.2 & -.1 & -.3 \\
\hline 8. & 4. 1 & 5.2 & 4.2 & .... & 1. 4 & .4 & 1.0 & 1.2 & .1 & -.2 & -.1 & -.1 \\
\hline 9. & 4.1 & 6.0 & 3.9 & & 1.4 & .4 & .8 & .8 & .1 & -.2 & -.2 & .0 \\
\hline 10. & 7. 6 & 6.2 & 3.7 & & 1. 4 & .3 & .8 & .5 & .1 & -.1 & -.3 & +.1 \\
\hline 11. & 9. 3 & 6. 3 & 3.4 & $\ldots$ & 1.5 & .3 & 1.3 & .3 & .0 & -.3 & -.3 & .3 \\
\hline 12. & 9.3 & 6.8 & $3 . \hat{9}$ & & 2.2 & .2 & 1.1 & .2 & .3 & -.3 & -.3 & 1.5 \\
\hline 13. & 8.1 & 8.3 & 5.6 & & 1.9 & .2 & .5 & .2 & .4 & -.2 & -.4 & 6.0 \\
\hline 14.. & 6.8 & 8.9 & 5.6 & & 1.9 & .2 & .3 & .2 & .7 & -.1 & -.3 & 9.6 \\
\hline 15 . & 5.4 & 9.1 & 5.1 & 4. 1 & 2.0 & .1 & .2 & .1 & .5 & -.1 & -.2 & 10.0 \\
\hline 16. & $5 . \hat{1}$ & 8.3 & 5.1 & 3.1 & 2.4 & .1 & .1 & .1 & .5 & -.1 & -.2 & 8. 0 \\
\hline 17. & 4.9 & 6.7 & 5.9 & 2.8 & 2.8 & .1 & .1 & .1 & .4 & -.1 & -.1 & 5. 0 \\
\hline 18. & 4.8 & 5.8 & 5.3 & 2.8 & 3.0 & 3 & .1 & .2 & .4 & -.2 & -.1 & 2.9 \\
\hline 19. & 4.9 & 5.7 & 4.8 & 2.6 & 2.4 & .3 & .2 & .1 & 1.4 & -.3 & .0 & 2.0 \\
\hline 20 . & 4.5 & 5.9 & 4.6 & 3.1 & 1. 7 & .7 & 6 & .6 & .3 .9 & -.4 & .0 & 1.6 \\
\hline & 3.9 & 6.5 & 7.1 & 2.8 & 1.4 & .8 & 1.6 & .5 & 3.1 & -.4 & $\therefore$ & 1.4 \\
\hline & 4.0 & 6.6 & 8.7 & 3. 0 & 1.4 & .9 & 1.4 & .2 & 2.9 & -.3 & .0 & 1. 3 \\
\hline & 4. 4 & 6.6 & 9.2 & 3. 0 & 1.4 & 1.0 & 1. 2 & .3 & 1.3 & -.4 & .0 & 1.1 \\
\hline 24 & 4.1 & 6.3 & 8.3 & 2.9 & 1.2 & .9 & 1.4 & 1.9 & .8 & -.4 & .0 & .8 \\
\hline 25. & 3.9 & 5.2 & 7.3 & 2.5 & 1.2 & 1.2 & 1.8 & 2. 6 & .5 & -.3 & & .7 \\
\hline & 3. 5 & $5 . \overline{5}$ & 5.8 & 2.1 & 1.2 & 2.4 & 2.1 & 2.0 & .3 & -.3 & & 1.0 \\
\hline 27 . & 3.1 & 5.9 & 4.7 & 2. 0 & 1.2 & 2.4 & 1.4 & 1.8 & .2 & -.3 & +.1 & 4.9 \\
\hline 28. & 2.8 & 5.6 & 4.0 & 2.0 & 1.2 & 1.2 & .9 & 1.5 & .2 & -.3 & & 6.9 \\
\hline & 2.7 & ..... & 3.6 & 1.7 & .9 & 1. 0 & 1.9 & 3.7 & .1 & -.4 & .2 & 8.4 \\
\hline & 2.5 & ....... & 3. 3 & 1.7 & .9 & .8 & 1.3 & 2.4 & .2 & -.1 & & 8.5 \\
\hline 31 . & 2.2 & $\ldots \ldots$ & 3.0 & & .8 & & .9 & 1. 6 & &.$- \overline{1}$ & & 6.8 \\
\hline
\end{tabular}

Coosa River ${ }^{1}$ at Lock No. 4, for 1895.

\begin{tabular}{|c|c|c|c|c|c|c|c|c|c|c|c|c|}
\hline Day. & Jan. & Feb. & Mar. & Apr. & May. & June. & July. & Ang. & Sept. & Oet. & Nov. & Dec. \\
\hline & 4.5 & 8.4 & 4.4 & 5.6 & 5.2 & 4.8 & 2.2 & 1.6 & 3.3 & 0.8 & 0.9 & 0.9 \\
\hline 2. & 3. 0 & 8.0 & 6.1 & 5.4 & 5.2 & $\begin{array}{l}\text { 4.0 } \\
\text { 4. } 0\end{array}$ & 2.6 & 1. 5 & 3.1 & $\begin{array}{r}.0 \\
.8\end{array}$ & 1. 0 & $\begin{array}{r}0.8 \\
.9\end{array}$ \\
\hline 3. & 2.5 & 9.0 & 8.2 & 5. 0 & 4.8 & 3.8 & 4.0 & 1.5 & 2.7 & .8 & 1. 1 & .9 \\
\hline 4. & 2.3 & 9.2 & 9.4 & 4.8 & 4.4 & 3.6 & 4.8 & 1.5 & 2.6 & .8 & 1.4 & .8 \\
\hline 5. & 2.3 & 9.4 & 9.8 & 4.6 & 4.8 & 3.4 & 5.4 & 1.4 & 4. 1 & .8 & 1.6 & .8 \\
\hline 6. & 2.4 & 8.6 & 9.0 & 4.2 & 4. 4 & 3.4 & 4. 8 & 1.3 & 3.1 & .8 & 1.2 & .8 \\
\hline 7.. & 2.5 & 8. 0 & 8. 0 & 5.4 & 4.4 & 3.4 & 3.2 & 1.3 & 2.8 & .8 & 1.0 & .9 \\
\hline 8. & 5.4 & 7. 0 & 7.5 & 9.6 & 5. 0 & 4.0 & 3. 0 & 1.3 & 2.8 & .9 & .9 & .9 \\
\hline 9. & $12 . \overline{4}$ & 6. 6 & 6.2 & 11.6 & 5. 7 & 4.6 & 2.8 & 1.3 & 2.8 & .8 & .8 & .8 \\
\hline $10 \ldots$ & 15.7 & 8.8 & 5.8 & 12.0 & 5.8 & 3.8 & 2.6 & 1.4 & 2.5 & .8 & 9 & .9 \\
\hline 11. & 16.1 & 7.8 & 5. 4 & 11.2 & 6.0 & 3.4 & 2.4 & 1.5 & 2.1 & .7 & 1.0 & .9 \\
\hline 12. & 15.6 & 5. 0 & 5.4 & 9.4 & 5. 6 & 3.0 & 2.8 & 1.4 & 2.0 & .9 & 1. 1 & .9 \\
\hline 13. & 15.7 & 5.0 & 5. 4 & 7.2 & 5. 0 & 3.0 & 2.2 & 1.2 & 1.8 & .9 & 1. 4 & 1.2 \\
\hline 14. & 15.3 & 5.2 & 8.0 & 5.8 & 5. 0 & 3.0 & 3.5 & 2.9 & 1.7 & .8 & 1.9 & 1. 2 \\
\hline $15 .$. & 15.1 & 4.6 & 11.6 & 5.2 & 5.2 & 3.6 & 3. 3 & 3. 0 & 1.7 & .8 & 2.0 & 1. 2 \\
\hline 16. & 15. 0 & 4.4 & 15.6 & 5.0 & 5.0 & 3.4 & 3.4 & 2.7 & 1.6 & .8 & 1.6 & 1. 1 \\
\hline 17. & 15. 0 & 4.2 & 16.4 & 5.6 & 4.4 & 3.2 & 3.3 & 3. 1 & 1.5 & .8 & 1.3 & 1.0 \\
\hline 18. & 12.4 & 4.4 & $13 . \overline{6}$ & 6.2 & 4.0 & 3. 0 & 3. 1 & 4.0 & 1.5 & .7 & 1.1 & 1. 0 \\
\hline 19. & 8.9 & 4.4 & 14.6 & 8.2 & 4.0 & 3.0 & 2.8 & 7.5 & 1.7 & .7 & 1. 0 & .9 \\
\hline 20. & 6.9 & 4.6 & 13.4 & 8.8 & 3. 8 & 3.6 & 2.6 & 8.3 & 1.7 & .7 & 1.0 & .9 \\
\hline 21 . & 6.8 & 5.0 & 14. 2 & 7.6 & 3.6 & 4.0 & 2.4 & 6.5 & 1. 7 & .6 & .9 & 1.1 \\
\hline 22 . & 10.3 & 5.6 & 14. 4 & 6.0 & 3. 4 & 3.2 & 2.3 & 7.9 & 1.6 & .6 & .9 & 1.2 \\
\hline 23. & 11.5 & 5.8 & 14.6 & 5.4 & 3.0 & 3.4 & 2.3 & 7.7 & 1. 4 & .6 & .8 & 1. 5 \\
\hline 24 . & 11.3 & 5.8 & 14. 0 & 4.8 & 3. 2 & 3.0 & 2. 0 & 7.0 & 1.3 & .6 & .8 & 1.8 \\
\hline 25. & 10.0 & 5.8 & 13.6 & 4.6 & 3.4 & 2.8 & 2.1 & 5.6 & 1.2 & .6 & .8 & 2.5 \\
\hline & 9.8 & 5.2 & 13. 0 & 4.4 & 5.0 & 2.6 & 2.0 & 4.2 & 1.1 & .6 & .8 & 2.5 \\
\hline & 9.4 & 5.0 & 9.8 & 4.4 & 6.4 & 2.4 & 1.8 & 3.4 & 1. 1 & .6 & .9 & 2.5 \\
\hline 28 . & 9.0 & 4.6 & 7.4 & 5. 0 & 9. 0 & 2.8 & 1.8 & 3.2 & 1. 0 & .7 & .8 & 2.5 \\
\hline & 9.4 & $\cdots$ & 6.2 & 5.6 & 9.0 & 2.2 & 1.9 & 3.2 & 1.0 & .7 & .8 & 2.5 \\
\hline & 9.2 & . & 6. 0 & 5.6 & 7.8 & 2.0 & 1.8 & 4. 0 & .9 & .7 & .8 & 2.5 \\
\hline 31. & 9.0 & & 5.4 & & 6.0 & & 1.7 & 3.4 & ..... & .8 & & 2.8 \\
\hline
\end{tabular}

I Record from United States Engineer Uffice,

IRR $11-3$ 
Coosa River ${ }^{\mathrm{I}}$ at Lock No. 4, for 1896.

\begin{tabular}{|c|c|c|c|c|c|c|c|c|c|c|c|c|}
\hline Day. & Jan. & Feb. & Mar. & Apr. & May & June. & July. & Aug. & Sept. & Oct. & Nov. & Dec. \\
\hline 1. & 4.0 & 3.8 & 3.4 & 3.5 & 2.5 & 0.1 & 0.6 & 1.3 & 0.6 & 0.2 & 0.7 & 1.5 \\
\hline $2 .$. & 4.1 & 3.8 & 3.3 & 5.2 & 2.4 & .1 & .5 & 1.1 & .5 & 2.0 & 1.2 & 2.8 \\
\hline 3.. & 4.1 & 3.4 & 3. 0 & 7.0 & 2.2 & .1 & .5 & 1.2 & .3 & 3.5 & 1.0 & 4.7 \\
\hline 4.. & 3.5 & 3.5 & 2.8 & 8.5 & 2. 1 & .1 & .5 & 1.4 & .2 & 3. 0 & .6 & 5. 6 \\
\hline 5. & 3.5 & 3.5 & 2.8 & 7.3 & 2.8 & .2 & .4 & 1.4 & .2 & 2.9 & .5 & 3.6 \\
\hline 6. & 2.6 & 6.5 & 2.7 & 7.3 & 2.9 & .2 & .5 & 1.4 & .1 & 3.1 & .5 & 2.7 \\
\hline $7 .$. & 2.1 & 10.0 & 3. 0 & 6.8 & 2.9 & .1 & 1.0 & $\begin{array}{l}1.4 \\
1.2\end{array}$ & 1 & .6 & .5 & 2. 2 \\
\hline 8. & 2.0 & 11. 8 & 3.1 & 5.0 & 2. 7 & .1 & 2.7 & 1.0 & .1 & .5 & 2.5 & 1.9 \\
\hline & 1.8 & 13. 7 & 3.0 & 4.1 & 2.4 & .1 & 4.7 & .9 & .1 & .4 & 2.5 & 1. 7 \\
\hline 10. & 1.9 & 13.4 & $\begin{array}{l}.0 .0 \\
2.9\end{array}$ & $\begin{array}{l}7.1 \\
3.6\end{array}$ & 2.1 & 1 & 6.2 & .7 & .0 & .3 & 1.7 & 1.6 \\
\hline 11. & 2.1 & 13.0 & 3.4 & 3.6 & 1.8 & .2 & 7.5 & .5 & .0 & .3 & 1.1 & 1. 5 \\
\hline & 2.4 & 11.8 & 4.7 & 3.6 & 1.8 & .2 & 7. 0 & .5 & .0 & .2 & 1.3 & 1. 4 \\
\hline 13. & 2.3 & 10.0 & 5.2 & 3.0 & 1.5 & .1 & 4.8 & .5 & .0 & .2 & 2. 2 & 1. 5 \\
\hline 14. & 2.0 & 8.7 & 4.8 & 2.9 & 1. 4 & .1 & 2.8 & .5 & .1 & .2 & 2. 6 & 1. 5 \\
\hline 15 & 1.8 & 7.8 & 4. 4 & 2.7 & 1. 3 & .1 & 2.6 & 1. 0 & 0 & .2 & 4.4 & 1.6 \\
\hline 16. & 1.8 & 7.6 & 4.1 & 2.5 & 1.2 & .1 & 2.4 & .6 & .0 & .2 & 6. 0 & 1. 4 \\
\hline & 2.8 & 7.6 & 5.0 & 2.5 & 1.2 & 1 & 2.8 & .5 & 0 & .1 & 5.4 & 1.7 \\
\hline 18. & 3.4 & 6.8 & 5.8 & 2.4 & 1. 2 & .1 & 2.8 & .5 & 0 & 1 & 4.4 & 2.0 \\
\hline 19 & 4.1 & 6.0 & 6.8 & 2.4 & 1.1 & .1 & 3. 3 & .5 & .0 & .2 & 3. 8 & 2. 0 \\
\hline & 4.0 & 5.2 & 7.7 & 2.2 & 1.1 & 1 & 3.9 & .5 & .1 & .2 & 1. & 1.7 \\
\hline 21. & 3.6 & 4.5 & 8.0 & 2.1 & 1.1 & 1 & 3.4 & .4 & .1 & .2 & 1.4 & 1.4 \\
\hline & 4.4 & 4.1 & 7.4 & 2.0 & 1.0 & .1 & 2.6 & .4 & .0 & .2 & 1.3 & 1.8 \\
\hline 23. & 6.9 & 3.9 & 6.5 & 2.0 & 1. 1 & 1 & 2.2 & 4 & .0 & .1 & 1. 1 & 1. 3 \\
\hline 24 . & 9.0 & 3.5 & 5.7 & 2.0 & 1.4 & .1 & 2. 0 & 4 & .0 & .1 & 1. 0 & 1.3 \\
\hline & 9.0 & 3.3 & 5.2 & 2. 0 & .9 & .1 & 1.9 & .5 & .0 & .1 & .9 & 1.1 \\
\hline 26 . & 10.0 & 3.2 & 4. 8 & 2.2 & 1.2 & 1 & 2.0 & 4 & .1 & 1 & .9 & 1.0 \\
\hline 27 . & 10.0 & 3.0 & 4.3 & $2 . \overline{5}$ & 1.5 & $\cdots$ & 2.0 & .5 & .1 & .2 & .9 & 1.0 \\
\hline 28. & 8. 7 & 3. 0 & 4.0 & 2.7 & 1.5 & & 1.9 & 7 &. & .2 & .9 & .9 \\
\hline 29. & 7.8 & 3. 0 & 4.0 & 2.4 & 1.3 & -- & 1.5 & 9 & .1 & 5 & .9 & .9 \\
\hline & 5.2 & & 3. 6 & 2.2 & 1.5 & ..... & 1.3 & .8 & .2 & .5 & 1.3 & .8 \\
\hline 31. & 4.4 & . & 3.4 & 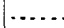 & 1.4 & & 1.2 & .7 & 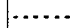 & .5 & $\cdots$ & .8 \\
\hline
\end{tabular}

I Record from United States Engineer Office.

Lock No. 5.-This is a site for a proposed lock to improve the navigation of the Coosa River. It is about 6 miles below the crossing of the Southern Railway. at Riverside, Alabama. Blue Eye Creek and Choccoloceo Creek tlow in to the river between Riverside and Lock No. 5 . The record of gage heights furnished by the United States Engineer Corps is presented below:

Coosa River at Lock No. 5, Georgia, for 1892.

\begin{tabular}{|c|c|c|c|c|c|c|c|c|c|c|c|c|}
\hline Day. & Jan. & Feb. & Mar. & Apr. & May. & June. & July. & Aug. & Sept. & Oet. & Nov. & Deo. \\
\hline 1. & 2.0 & 2.9 & 2.5 & 4.9 & 3.0 & 1.3 & 2,6 & 1.0 & 1.5 & 0.8 & 0.2 & 1.9 \\
\hline & 2.8 & 2.7 & 2.4 & 4.0 & 2.9 & 1.3 & $\begin{array}{l}2.7 \\
\text { 2. }\end{array}$ & 1.0 & 1.5 & .7 & .2 & 1. 6 \\
\hline & 3. 3 & 2.5 & 2.3 & 3.6 & 2.8 & 1.5 & 1.5 & 1.0 & 1.3 & .5 & .2 & 1. 4 \\
\hline & 3.7 & 2.4 & 2.1 & 3.3 & 2.8 & 1.6 & 1.2 & .9 & 1.0 & .5 & .3 & 1. 3 \\
\hline $\mathbf{5}$ & 3.8 & 2.3 & 2.0 & 3.0 & 2.5 & 1.6 & 1. 1 & 1.0 & 1.0 & .5 & .4 & 1. 1 \\
\hline 6. & 3.3 & 2.2 & 1.9 & 3.0 & 2.2 & 2.6 & 1.1 & 1.0 & .8 & .5 & .3 & 1.5 \\
\hline 7 & 2.5 & 2.1 & 2.1 & 16. 8 & 2.1 & 3.1 & 1.0 & .9 & .8 & .4 & .5 & 1.4 \\
\hline & 2.2 & 2.5 & 3.4 & 19.4 & 1.9 & 2.9 & & .8 & .8 & .4 & .8 & 1. 5 \\
\hline 9. & 2.5 & 3.1 & 5.4 & 18. 2 & 2.1 & 2. 3 & 3.4 & .8 & .7 & .4 & 2.0 & 1. 9 \\
\hline 10. & 3.5 & 3.0 & 6.7 & 17. 2 & 2.2 & 2.2 & 4.3 & .8 & .7 & .4 & 1.8 & 2.0 \\
\hline 11.. & 3. 8 & 3.2 & 7.2 & 17.1 & 2.3 & 2.0 & 4.0 & .9 & .6 & .4 & 3.5 & 1. 5 \\
\hline 12. & 4.4 & 3.0 & 6.7 & 17. 4 & 2.4 & 1.8 & 5.7 & .9 & .6 & .4 & 4.5 & 1.6 \\
\hline 13. & 13.0 & 2.9 & 5.0 & 17.1 & 2.5 & 1.5 & 7.3 & 1.0 & .5 & .4 & 3.8 & 1. 3 \\
\hline 14. & 16.5 & 2.3 & 3. 7 & 16.8 & 2. 3 & 2.0 & 7.6 & .9 & .5 & .4 & 2. 7 & 1.2 \\
\hline 15 & 158 & 2.3 & 3.0 & 16. 1 & 2.1 & 1.4 & 8.0 & .9 & .5 & .4 & 2. & 1. 6 \\
\hline 16. & 14.8 & 2.4 & 2.7 & 15.0 & 2.0 & 1.2 & 7.0 & .8 & 6 & .4 & 1.9 & 1.8 \\
\hline 17. & 14. 2 & 2.4 & 2.6 & 13.6 & 1.9 & 1.1 & 5.1 & .8 & 1.0 & .4 & & 2.1 \\
\hline & 14.4 & 2.5 & 2.4 & 9.5 & 1.9 & 1.1 & 4.0 & .9 & 6.0 & .4 & 1. & 2.4 \\
\hline 19. & 16. 7 & 2.5 & 3.8 & 6.3 & 2.1 & 1.1 & 3.5 & .8 & 1.3 & .3 & 1. 7 & 3.4 \\
\hline 20 . & 18.4 & 2.4 & 4.0 & 4.1 & 2.0 & 1.1 & & .8 & 1.7 & .3 & 2.1 & 4.4 \\
\hline & 18.4 & 2.7 & 3.9 & 3.7 & 2.2 & 1.3 & 4.5 & .9 & 2.0 & .3 & 2. & 6 \\
\hline 22. & 17.3 & 3.4 & 3.5 & 5.7 & 2.4 & 2.3 & 3.5 & .9 & 2.5 & .3 & 1.7 & 6.6 \\
\hline & 16.5 & 4.6 & 3.1 & 5.7 & 2.3 & 2.5 & 3.4 & 1.3 & 3.8 & .3 & 1.7 & 6.4 \\
\hline 24. & 15.7 & 5.6 & 4.9 & 4.9 & 2.0 & 2.3 & 3.6 & 1.7 & 3.8 & 3 & 1.5 & 5.5 \\
\hline & 14.6 & 5.4 & 5.7 & 4.0 & 1.9 & & 3.2 & 3.4 & 2.4 & .3 & 1.3 & 4.5 \\
\hline 26 & 13.8 & $\begin{array}{l}4.5 \\
4.5\end{array}$ & 7. 2 & 3. 7 & 2.0 & 2. & 3.0 & 4.3 & 2.0 & .3 & 1. & 3.1 \\
\hline 27. & 12.2 & 3.5 & 8.7 & 3.3 & 1.7 & 2.2 & 2.8 & 4.7 & 1.4 & 2 & 1.2 & 2.7 \\
\hline & 7.5 & 3. 1 & 8.7 & 3.0 & 1.5 & 2.4 & 2.3 & 3. 5 & 1.2 & .2 & 1. 1 & 2.4 \\
\hline & 4.5 & $\begin{array}{l}.1 \\
2.7\end{array}$ & 8.6 & $\begin{array}{l}\text {.0 } \\
2.8\end{array}$ & 1.5 & 2.4 & 1. & 2.7 & .9 & .2 & 1. & 1.9 \\
\hline 30. & 3. 4 & . & 8.0 & 3.0 & 1.5 & 2.7 & 1.3 & 2.1 & .8 & .2 & 1. 8 & 1. 6 \\
\hline & 3. 0 & 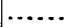 & 6.9 & & 1.5 & & 1.1 & 2.0 & & .2 & & 1.5 \\
\hline
\end{tabular}


Coosa River at Lock No. 5, Georgia, for 1893.

\begin{tabular}{|c|c|c|c|c|c|c|c|c|c|c|c|c|}
\hline Day. & Jan. & Feb. & Mạ. & Apr. & May. & June. & July. & Aug. & Sept. & Oet. & Nov. & Dec. \\
\hline $1 .$. & $\cdots$ & 3. 4 & 5.0 & 2.1 & 2.8 & 4. 7 & 1.1 & 0.5 & 0.2 & 0.3 & 0.0 & 0.2 \\
\hline $2 \ldots$ & $\ldots . .$. & 3.0 & 5.1 & 2.1 & 3.5 & 5.5 & 1.0 & .5 & .3 & .3 & .0 & .3 \\
\hline$\ldots . .$. & ..... & 2.6 & 5.6 & 2.0 & 6. 3 & 5.9 & 1. 0 & 5 & .3 & 3 & 0 & 3 \\
\hline$\ldots .$. & $\ldots .$. & 2.4 & 5.6 & 1.9 & 9.9 & 5.2 & 1.0 & 6 & 5 & 3 & 0 & 3 \\
\hline … & $\ldots . .$. & 2,1 & 6.1 & 2.1 & 11.5 & 5.1 & 1.0 & .8 & 9 & 3 & 0 & 5 \\
\hline$\ldots \ldots$ & $\ldots .$. & 2.0 & 5.9 & 2.2 & 10.6 & 5.0 & 1.0 & 1.1 & 7 & 3 & 1 & 6 \\
\hline $7 \ldots$ & ...... & 1. 8 & 4.6 & 2.3 & 19.1 & 5.0 & 1. 0 & 1.4 & .4 & 2 & 1 & 1. 0 \\
\hline $8 \ldots \ldots \ldots$ & 1.5 & 1.8 & 4.6 & 2.3 & 7.6 & 6.2 & .9 & 1.7 & 3 & .2 & 1 & 1. 0 \\
\hline $9 \ldots \ldots \ldots \ldots \ldots$ & 1.3 & 1.8 & 5.7 & 2.3 & 6.3 & 7.2 & .8 & 1.8 & 3 & .2 & .1 & .8 \\
\hline $10 \ldots \ldots \ldots \ldots \ldots$ & 1.2 & 1.9 & 6.4 & 2.2 & 5.7 & 7.2 & .8 & 1.2 & 3 & 2 & .1 & 8 \\
\hline $11 \ldots \ldots \ldots \ldots \ldots$ & 1,2 & 2. 3 & 7. 3 & 2.0 & 4. 6 & 4. 9 & .7 & .8 & 5 & .1 & 1 & .5 \\
\hline $12 \ldots \ldots \ldots \ldots$ & 1. 2 & 4.7 & 7.5 & 1.8 & 3.7 & 3.9 & .7 & .8 & 9 & .1 & .1 & .3 \\
\hline 13. & 1.1 & 7.1 & 6.4 & 1.8 & 3. 2 & 2.7 & .7 & 8 & 1. 6 & .1 & .1 & .3 \\
\hline $14 .$. & 1.2 & 8.0 & 5. 6 & 2.0 & 2.7 & 2.2 & .6 & 1.0 & 1.9 & .1 & .1 & .3 \\
\hline $15 .$. & 1.2 & 12.3 & 4. 6 & 2.2 & 2. 6 & 2.0 & .6 & 1.0 & 1.9 & .1 & .1 & .3 \\
\hline 16. & 1. 3 & 18.1 & 4.0 & 2.6 & 2.3 & 1.7 & .6 & .8 & 2.0 & .1 & .1 & .3 \\
\hline 17. & 1. 5 & 17. 4 & 3. 4 & 2.7 & 2. 1 & 1.6 & .6 & .8 & 1.8 & .1 & .1 & .3 \\
\hline $18 \ldots$ & 1.5 & 15. 3 & 3.1 & 2.7 & 2.0 & 1.6 & .6 & .9 & 1.6 & .1 & .1 & .3 \\
\hline 19. & 1. 9 & 14.0 & 2.9 & 2.7 & 2.0 & 1.5 & .5 & - & 1.0 & .1 & .1 & .3 \\
\hline$\ldots \ldots$. & 1. 7 & 13.7 & 2.6 & 2.2 & 2.0 & 1. 4 & .5 & .7 & .7 & .1 & .1 & .4 \\
\hline $21 \ldots \ldots \ldots \ldots \ldots$ & 1.6 & 12.9 & 2.5 & 2.3 & 1. 8 & 1.4 & .5 & 6 & .5 & .1 & .1 & .4 \\
\hline $22 \ldots$ & 1.5 & 12.4 & 2. 4 & 2.4 & 1. 7 & 1. 5 & .5 & .4 & 4 & .1 & .2 & .5 \\
\hline $23 \ldots$ & 1.1 & 10.4 & 2. 3 & ..... & 1.6 & 1.5 & .5 & .3 & .4 & .1 & .2 & .4 \\
\hline $24 \ldots$ & 1.3 & 9.7 & 2. 3 & ...... & 1.4 & 1. 9 & .5 & .3 & .4 & .0 & .2 & .4 \\
\hline $25 .$. & 1.3 & 7.5 & 3.8 & ...... & $1 . \overline{3}$ & 1.8 & .5 & $\mathbf{3}$ & 3 & 0 & .2 & 4 \\
\hline 26 .. & 1. 7 & 4. 4 & 3. 9 & $\ldots .$. & 1. 3 & 1.9 & .5 & .2 & 3 & .0 & .2 & .3 \\
\hline $27 .$. & 2.0 & 3.6 & 3.7 & $\ldots . .$. & 1. 2 & 2.7 & .5 & .3 & .3 & .0 & .2 & 3 \\
\hline 28. & 2.9 & 4.5 & 3.4 & $\ldots . .$. & 2.8 & 2.1 & .5 & .2 & .2 & .0 & .3 & 2 \\
\hline & 3.9 & $\ldots$. & 2.8 & $\ldots$ & 3.4 & 1.5 & .6 & .2 & .2 & .0 & .3 & .2 \\
\hline & 3.9 & & 2.4 & 3.6 & 3.5 & 1.2 & 0 & .2 & .2 & .0 & .2 & .5 \\
\hline & 3.8 & ... & 2.3 & $\ldots . .$. & 3.5 & $\ldots .$. & & .2 & $\cdots$ & .0 & $\cdots$ & 1.1 \\
\hline
\end{tabular}

Coosa River at Lock No. 5, Georgia, for 1894.

\begin{tabular}{|c|c|c|c|c|c|c|c|c|c|c|c|c|}
\hline Day. & Jan. & Feb. & Mar. & Apr. & May. & June. & July. & Aug. & Sept. & Oct. & Nov. & Dec. \\
\hline 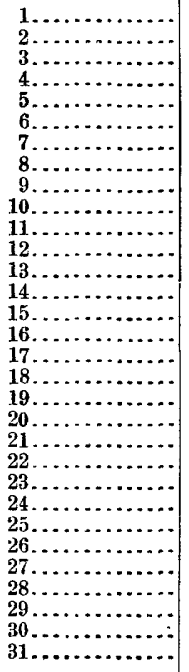 & $\begin{array}{r}1.4 \\
1.1 \\
1.1 \\
1.0 \\
.9 \\
.8 \\
1.5 \\
2.9 \\
2.8 \\
5.7 \\
7.4 \\
7.5 \\
6.0 \\
4.6 \\
3.6 \\
3.6 \\
3.3 \\
3.3 \\
3.3 \\
2.9 \\
2.5 \\
2.4 \\
2.6 \\
2.5 \\
2.4 \\
2.1 \\
1.9 \\
1.7 \\
1.6 \\
1.5 \\
1.4\end{array}$ & \begin{tabular}{|l|}
1.3 \\
1.2 \\
1.2 \\
1.3 \\
1.7 \\
2.3 \\
3.1 \\
3.2 \\
3.7 \\
4.3 \\
4.2 \\
4.7 \\
6.1 \\
6.3 \\
6.4 \\
5.7 \\
4.6 \\
4.3 \\
3.6 \\
3.7 \\
4.3 \\
4.6 \\
4.1 \\
3.4 \\
3.5 \\
4.0 \\
4.0 \\
4.4 \\
$\ldots \ldots .$. \\
$\cdots \ldots$ \\
$\cdots \cdots$
\end{tabular} & $\begin{array}{l}5.0 \\
5.3 \\
5.2 \\
4.5 \\
4.0 \\
3.4 \\
3.0 \\
2.7 \\
2.6 \\
2.5 \\
2.1 \\
3.2 \\
3.5 \\
3.8 \\
3.3 \\
3.4 \\
4.5 \\
3.9 \\
3.2 \\
3.0 \\
5.3 \\
6.3 \\
6.9 \\
6.3 \\
5.3 \\
4.0 \\
3.2 \\
2.7 \\
2.3 \\
2.1 \\
1.9\end{array}$ & $\begin{array}{l}1.8 \\
2.0 \\
2.8 \\
2.6 \\
2.2 \\
2.3 \\
2.4 \\
2.2 \\
2.0 \\
2.5 \\
3.3 \\
3.1 \\
3.0 \\
3.1 \\
2.7 \\
2.3 \\
2.0 \\
1.7 \\
1.6 \\
2.0 \\
1.9 \\
1.9 \\
2.0 \\
1.8 \\
1.6 \\
1.4 \\
1.3 \\
1.2 \\
1.2 \\
1.1 \\
\ldots \ldots . . \\
\ldots\end{array}$ & $\begin{array}{r}1.1 \\
1.0 \\
1.0 \\
1.0 \\
1.0 \\
1.0 \\
1.0 \\
1.0 \\
.8 \\
.8 \\
.8 \\
1.7 \\
1.3 \\
1.3 \\
1.3 \\
1.5 \\
1.7 \\
1.8 \\
1.5 \\
1.5 \\
1.0 \\
1.0 \\
1.0 \\
.9 \\
.8 \\
.8 \\
.8 \\
.7 \\
.7 \\
.6 \\
.5\end{array}$ & $\begin{array}{r}0.5 \\
.5 \\
.5 \\
.5 \\
.4 \\
.4 \\
.4 \\
.3 \\
.3 \\
.3 \\
.3 \\
.2 \\
.2 \\
.2 \\
.2 \\
.2 \\
.1 \\
.2 \\
.3 \\
.4 \\
.5 \\
.6 \\
.6 \\
.5 \\
.8 \\
1.5 \\
1.4 \\
1.0 \\
.7 \\
.5 \\
. . . .\end{array}$ & $\begin{array}{r}0.5 \\
.4 \\
.3 \\
.3 \\
.2 \\
.2 \\
.6 \\
.7 \\
.7 \\
.8 \\
.9 \\
.6 \\
.4 \\
.3 \\
.3 \\
.3 \\
.2 \\
.2 \\
.2 \\
.4 \\
.8 \\
.9 \\
.7 \\
.8 \\
.9 \\
1.1 \\
.9 \\
.5 \\
1.2 \\
1.0 \\
.6\end{array}$ & $\begin{array}{r}0.5 \\
.5 \\
.6 \\
.7 \\
.6 \\
.5 \\
.5 \\
.8 \\
.6 \\
.5 \\
.4 \\
.2 \\
.2 \\
.2 \\
.2 \\
.1 \\
.1 \\
.1 \\
.1 \\
.1 \\
.1 \\
.3 \\
.3 \\
1.7 \\
1.5 \\
1.7 \\
1.2 \\
.7 \\
2.2 \\
1.6 \\
1.2 \\
\end{array}$ & $\begin{array}{r}0.8 \\
1.0 \\
.8 \\
.5 \\
.3 \\
.2 \\
.2 \\
1.3 \\
.2 \\
.3 \\
.3 \\
.6 \\
.8 \\
.7 \\
.5 \\
.3 \\
.3 \\
.8 \\
1.0 \\
1.7 \\
2.3 \\
1.8 \\
1.0 \\
.6 \\
.3 \\
.3 \\
.2 \\
.2 \\
.1 \\
.1 \\
\ldots . . \\
.0\end{array}$ & $\begin{array}{l}0.0 \\
.0 \\
.1 \\
.2 \\
.2 \\
.2 \\
.2 \\
.2 \\
.2 \\
.2 \\
.2 \\
.2 \\
.2 \\
.2 \\
.2 \\
.2 \\
.2 \\
.2 \\
.2 \\
.2 \\
.3 \\
.3 \\
.3 \\
.3 \\
.3 \\
.3 \\
.3 \\
.2 \\
.2 \\
.2 \\
.2\end{array}$ & $\begin{array}{r}0.2 \\
.2 \\
.2 \\
.1 \\
.1 \\
.1 \\
.1 \\
.1 \\
.1 \\
.1 \\
.0 \\
.0 \\
.0 \\
.0 \\
.0 \\
.0 \\
.0 \\
.1 \\
.1 \\
.1 \\
.1 \\
.1 \\
.1 \\
.1 \\
.1 \\
.2 \\
.2 \\
.3 \\
.2 \\
.2 \\
\ldots . .\end{array}$ & $\begin{array}{r}0.2 \\
.1 \\
.1 \\
.1 \\
.1 \\
.1 \\
.1 \\
.1 \\
.1 \\
.3 \\
.5 \\
2.4 \\
3.8 \\
6.7 \\
7.0 \\
5.7 \\
3.6 \\
1.9 \\
1.2 \\
1.0 \\
.9 \\
.8 \\
.6 \\
.5 \\
.5 \\
.6 \\
3.2 \\
4.5 \\
5.5 \\
5.7 \\
4.6\end{array}$ \\
\hline
\end{tabular}


Coosa Riverl'at Lock No. 5, Georgia, for 1895.

\begin{tabular}{|c|c|c|c|c|c|c|c|c|c|c|c|c|}
\hline Day. & Jan. & Feb. & Mar. & Apr. & May. & June. & Jaly. & Aug. & Sept. & Oet. & Nov. & Dec. \\
\hline 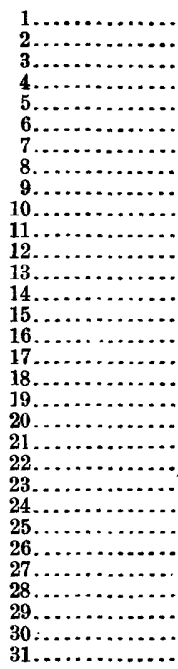 & $\begin{array}{r}3.0 \\
1.9 \\
1.5 \\
1.4 \\
1.4 \\
1.4 \\
1.4 \\
3.9 \\
10.5 \\
12.9 \\
12.6 \\
12.0 \\
11.4 \\
11.3 \\
11.3 \\
11.3 \\
11.4 \\
9.5 \\
6.5 \\
4.8 \\
4.4 \\
6.9 \\
8.1 \\
7.0 \\
6.8 \\
7.1 \\
6.8 \\
6.5 \\
6.7 \\
6.5 \\
6.3\end{array}$ & \begin{tabular}{|l}
5.8 \\
5.6 \\
6.3 \\
6.4 \\
6.5 \\
6.0 \\
5.2 \\
4.8 \\
4.4 \\
4.0 \\
3.5 \\
3.0 \\
2.9 \\
2.8 \\
2.5 \\
2.3 \\
2.2 \\
2.2 \\
2.2 \\
2.5 \\
2.9 \\
3.2 \\
3.4 \\
3.5 \\
3.1 \\
2.7 \\
2.5 \\
2.3 \\
$\ldots \ldots \ldots$ \\
$\ldots \ldots \ldots$ \\
$\ldots \ldots .$.
\end{tabular} & $\begin{array}{r}2.2 \\
3.5 \\
5.8 \\
6.8 \\
7.0 \\
6.4 \\
5.5 \\
4.6 \\
4.0 \\
3.5 \\
3.3 \\
3.3 \\
3.3 \\
5.1 \\
8.5 \\
13.8 \\
13.6 \\
12.5 \\
11.3 \\
10.3 \\
10.8 \\
10.7 \\
10.9 \\
10.6 \\
10.0 \\
9.0 \\
7.2 \\
5.1 \\
4.0 \\
3.6 \\
3.3\end{array}$ & $\begin{array}{l}3.1 \\
2.9 \\
2.7 \\
2.5 \\
2.4 \\
2.3 \\
2.1 \\
6.8 \\
8.5 \\
8.7 \\
8.0 \\
6.9 \\
5.0 \\
3.6 \\
3.0 \\
2.8 \\
3.4 \\
4.0 \\
5.5 \\
6.1 \\
5.1 \\
3.8 \\
3.1 \\
2.7 \\
2.5 \\
2.3 \\
2.4 \\
2.6 \\
3.3 \\
3.8 \\
\ldots \ldots . .\end{array}$ & $\begin{array}{l}3.0 \\
2.8 \\
2.7 \\
3.8 \\
2.8 \\
2.5 \\
2.4 \\
2.9 \\
3.5 \\
3.5 \\
3.7 \\
3.7 \\
3.5 \\
2.9 \\
3.0 \\
2.9 \\
2.4 \\
2.0 \\
1.9 \\
1.7 \\
1.7 \\
1.6 \\
1.5 \\
1.4 \\
1.5 \\
2.0 \\
3.6 \\
5.6 \\
6.2 \\
5.3 \\
3.8\end{array}$ & $\begin{array}{r}2.7 \\
2.1 \\
1.9 \\
1.6 \\
1.5 \\
1.5 \\
1.6 \\
1.9 \\
2.3 \\
1.9 \\
1.3 \\
1.3 \\
1.4 \\
1.4 \\
1.4 \\
1.3 \\
1.3 \\
1.3 \\
1.4 \\
1.5 \\
2.4 \\
1.7 \\
1.7 \\
1.1 \\
1.1 \\
1.2 \\
1.1 \\
1.0 \\
1.9 \\
1.9 \\
\ldots . . .\end{array}$ & $\begin{array}{r}1.9 \\
2.2 \\
3.4 \\
4.2 \\
4.5 \\
5.7 \\
2.9 \\
2.6 \\
4.1 \\
5.4 \\
6.0 \\
5.2 \\
3.4 \\
3.4 \\
1.9 \\
2.0 \\
1.8 \\
1.5 \\
1.4 \\
1.2 \\
1.2 \\
1.0 \\
1.0 \\
1.0 \\
1.0 \\
.9 \\
.8 \\
.8 \\
.8 \\
.8 \\
.7\end{array}$ & $\begin{array}{l}0.6 \\
.6 \\
.6 \\
.5 \\
.4 \\
.4 \\
.4 \\
.5 \\
.5 \\
.6 \\
1.0 \\
1.1 \\
1.0 \\
1.3 \\
1.5 \\
1.3 \\
1.5 \\
2.0 \\
4.0 \\
5.1 \\
4.0 \\
5.1 \\
5.2 \\
4.6 \\
3.5 \\
2.4 \\
1.8 \\
1.6 \\
1.6 \\
2.1 \\
1.9\end{array}$ & $\begin{array}{r}1.6 \\
1.5 \\
1.4 \\
1.3 \\
2.1 \\
1.6 \\
1.4 \\
1.3 \\
1.2 \\
.9 \\
.9 \\
.8 \\
.8 \\
.7 \\
.6 \\
.6 \\
.6 \\
.6 \\
.7 \\
.8 \\
.7 \\
.6 \\
.5 \\
.5 \\
.4 \\
.3 \\
.3 \\
.3 \\
.3 \\
.3 \\
. . .6\end{array}$ & $\begin{array}{l}0.3 \\
.3 \\
.2 \\
.2 \\
.2 \\
2 \\
.2 \\
.2 \\
.2 \\
.2 \\
.2 \\
.2 \\
.2 \\
.2 \\
.2 \\
.2 \\
.1 \\
.1 \\
.1 \\
.1 \\
.1 \\
.1 \\
.1 \\
.1 \\
.1 \\
.1 \\
.1 \\
.1 \\
.1 \\
.1 \\
.1\end{array}$ & $\begin{array}{l}0.1 \\
.1 \\
.1 \\
.4 \\
.7 \\
.6 \\
.5 \\
.3 \\
.2 \\
.2 \\
.5 \\
.6 \\
.7 \\
.7 \\
.9 \\
.7 \\
.6 \\
.5 \\
.5 \\
.4 \\
.3 \\
.3 \\
.3 \\
.3 \\
.3 \\
.3 \\
.3 \\
.3 \\
.3 \\
.3 \\
.3\end{array}$ & $\begin{array}{r}0.3 \\
.3 \\
.3 \\
.3 \\
.3 \\
.3 \\
.3 \\
.2 \\
.2 \\
.2 \\
.2 \\
.2 \\
.3 \\
.5 \\
.5 \\
.5 \\
.5 \\
.5 \\
.5 \\
.5 \\
.5 \\
.6 \\
.7 \\
.8 \\
.8 \\
1.1 \\
1.2 \\
1.2 \\
1.3 \\
1.4 \\
1.6\end{array}$ \\
\hline
\end{tabular}

Coosa River ${ }^{1}$ at Lock No. 5, Georgia, for 1896.

\begin{tabular}{|c|c|c|c|c|c|c|c|c|c|c|c|c|}
\hline Day. & Jan. & Feb. & Mar. & Apr. & May. & June. & July. & Aug. & Sept. & Oet. & Nov. & Dec. \\
\hline 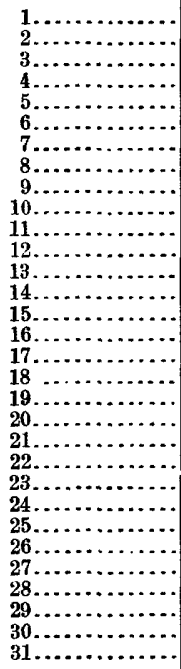 & $\begin{array}{l}2.0 \\
2.1 \\
1.9 \\
1.6 \\
1.4 \\
1.1 \\
1.1 \\
1.0 \\
1.0 \\
.9 \\
1.0 \\
1.1 \\
1.1 \\
.9 \\
.9 \\
.9 \\
1.5 \\
1.8 \\
1.8 \\
2.2 \\
1.9 \\
2.3 \\
4.6 \\
6.0 \\
6.7 \\
7.1 \\
6.7 \\
5.5 \\
4.3 \\
3.1 \\
2.4\end{array}$ & $\begin{array}{r}2.1 \\
1.9 \\
1.7 \\
1.7 \\
1.6 \\
3.5 \\
7.1 \\
8.8 \\
10.2 \\
9.9 \\
9.4 \\
8.6 \\
7.2 \\
5.7 \\
5.0 \\
4.9 \\
4.7 \\
4.1 \\
3.5 \\
2.9 \\
2.5 \\
2.2 \\
2.0 \\
1.9 \\
1.8 \\
1.7 \\
1.5 \\
1.6 \\
1.6 \\
\cdots \ldots . . \\
\ldots \ldots . .\end{array}$ & $\begin{array}{l}1.8 \\
1.7 \\
1.5 \\
1.5 \\
1.5 \\
1.4 \\
1.5 \\
1.7 \\
1.6 \\
1.5 \\
2.1 \\
2.6 \\
3.0 \\
2.7 \\
2.4 \\
1.2 \\
2.7 \\
3.3 \\
3.7 \\
4.8 \\
5.2 \\
4.8 \\
4.0 \\
3.4 \\
3.0 \\
2.6 \\
2.4 \\
2.2 \\
2.0 \\
1.9 \\
1.8\end{array}$ & $\begin{array}{r}1.7 \\
2.6 \\
4.0 \\
5.2 \\
5.5 \\
4.5 \\
3.8 \\
2.8 \\
2.2 \\
1.9 \\
1.8 \\
1.7 \\
1.6 \\
1.5 \\
1.4 \\
1.3 \\
1.2 \\
1.1 \\
1.1 \\
1.1 \\
1.0 \\
.9 \\
.9 \\
.9 \\
.9 \\
.9 \\
1.1 \\
1.3 \\
1.1 \\
1.0 \\
\ldots . . .\end{array}$ & $\begin{array}{r}1.1 \\
1.1 \\
1.0 \\
1.0 \\
1.3 \\
1.4 \\
1.3 \\
1.2 \\
1.0 \\
.9 \\
.8 \\
.7 \\
.6 \\
.6 \\
.5 \\
.4 \\
.4 \\
.4 \\
.4 \\
.3 \\
.3 \\
.3 \\
.3 \\
.4 \\
.4 \\
.5 \\
.6 \\
.6 \\
.6 \\
.5 \\
.6\end{array}$ & $\begin{array}{r}0.7 \\
.7 \\
.6 \\
.8 \\
.9 \\
.9 \\
.8 \\
.7 \\
.6 \\
.7 \\
1.0 \\
.8 \\
.7 \\
.6 \\
.6 \\
.5 \\
.4 \\
.3 \\
.3 \\
.3 \\
.3 \\
.3 \\
.5 \\
.6 \\
.5 \\
.2 \\
.2 \\
.2 \\
.2 \\
.2 \\
\ldots . . \\
.5\end{array}$ & $\begin{array}{r}0.1 \\
.1 \\
.1 \\
.1 \\
\ldots . . . \\
\ldots \ldots . . \\
\ldots \ldots . . \\
\ldots \ldots . . \\
\ldots \ldots . . \\
\ldots . .2 \\
4.2 \\
2.7 \\
1.5 \\
1.0 \\
1.0 \\
1.4 \\
1.4 \\
1.5 \\
1.9 \\
1.6 \\
1.3 \\
1.0 \\
.9 \\
.9 \\
.9 \\
.9 \\
.8 \\
.6 \\
.5 \\
.4 \\
.9\end{array}$ & $\begin{array}{l}0.4 \\
.4 \\
.4 \\
.5 \\
.5 \\
.5 \\
.4 \\
.3 \\
.2 \\
.1 \\
.1 \\
.1 \\
.1 \\
.1 \\
.1 \\
.1 \\
.1 \\
.1 \\
.1 \\
.1 \\
.1 \\
.1 \\
.1 \\
.0 \\
.1 \\
.1 \\
.1 \\
.1 \\
.1 \\
.1 \\
.1\end{array}$ & $\begin{array}{r}0.1 \\
.1 \\
.1 \\
.2 \\
.2 \\
.2 \\
.2 \\
.2 \\
.3 \\
.3 \\
.3 \\
.3 \\
.3 \\
.3 \\
.3 \\
.3 \\
.3 \\
.3 \\
.3 \\
.3 \\
.3 \\
.1 \\
.2 \\
.3 \\
.3 \\
.3 \\
.3 \\
.3 \\
.3 \\
.3 \\
. . . .\end{array}$ & $\begin{array}{r}0.3 \\
.6 \\
1.0 \\
1.3 \\
.8 \\
.4 \\
.2 \\
.1 \\
.1 \\
.1 \\
.1 \\
.2 \\
.2 \\
.2 \\
.2 \\
.2 \\
.2 \\
.2 \\
.2 \\
.2 \\
.2 \\
.2 \\
.2 \\
.2 \\
.2 \\
.2 \\
.2 \\
.2 \\
.1 \\
.1 \\
.1\end{array}$ & $\begin{array}{r}0.1 \\
.5 \\
.3 \\
.2 \\
.1 \\
.1 \\
.1 \\
\ldots . . \\
1.0 \\
.7 \\
.4 \\
.4 \\
.8 \\
1.0 \\
2.0 \\
3.3 \\
3.0 \\
2.3 \\
1.3 \\
.8 \\
.6 \\
.4 \\
.4 \\
.4 \\
.3 \\
.2 \\
.2 \\
.3 \\
.3 \\
.4 \\
. . . .\end{array}$ & $\begin{array}{l}0.6 \\
1.1 \\
2.3 \\
2.3 \\
1.8 \\
1.2 \\
.9 \\
.8 \\
.7 \\
.6 \\
.5 \\
.5 \\
.4 \\
.4 \\
.6 \\
.5 \\
.6 \\
.7 \\
.8 \\
.6 \\
.5 \\
.4 \\
.4 \\
.3 \\
.3 \\
.3 \\
.2 \\
.2 \\
.2 \\
.2 \\
.2\end{array}$ \\
\hline
\end{tabular}

I Record from United States Engineer Office. 
Black Warrior River ${ }^{1}$ at Tuscaloosa, Alabama, for 1889.

\begin{tabular}{|c|c|c|c|c|c|c|c|c|c|c|c|c|}
\hline Iay. & Jan. & Feb. & Mar. & Apr. & May. & June. & July. & Aug. & Sept. & Oet. & Nov. & Dec. \\
\hline & 15.00 & 23.50 & 18.80 & 8.50 & 8.80 & 2.30 & 2.40 & 3. 70 & 0.75 & 2.40 & 0.90 & 6.00 \\
\hline & 19.50 & 20.50 & 16.50 & 9.80 & 14.50 & 2. 90 & 3. 10 & 3.60 & 7. 30 & 1.95 & 1.05 & 5.65 \\
\hline & 18. 00 & 17. 50 & $25.00^{t}$ & 10.30 & 13. 20 & 2.75 & 4. 40 & 3. 00 & 6.40 & 1.55 & 1.85 & 5.30 \\
\hline & 16.00 & 14.80 & 31.50 & 11. 60 & 11.00 & 2. 50 & 5. 10 & 2. 95 & 5. 30 & 1. 30 & 1.90 & 4. 80 \\
\hline & 25.00 & 13.00 & 29.40 & 10.50 & 8.50 & 2.30 & 6.25 & 2.90 & 6.20 & 1.15 & 3.85 & 4. 50 \\
\hline & 33. 00 & 11. 50 & 26.50 & 9.50 & 7.00 & 2.10 & 6.85 & 3.70 & 9. 30 & 1.05 & 3.65 & 4. 20 \\
\hline & 33. 50 & 10.40 & 23.00 & 8.40 & 5.80 & 1.90 & 6.45 & 3. 45 & 14. 90 & .90 & 3.30 & 4.00 \\
\hline & 29.50 & 9.30 & 20.10 & 7.30 & 4. 60 & 1. 70 & 5. 70 & 2.95 & 19.00 & .70 & 3.00 & 3.80 \\
\hline & 26.80 & $8: 30$ & 17. 50 & 6.80 & 4. 30 & 2.00 & 4. 75 & 2. 60 & 14.00 & 50 & 2.75 & 3. 60 \\
\hline 10. & 28.40 & 8.00 & 15. 00 & 6.40 & 4.00 & 2. 60 & 3. 85 & 2.00 & 9.90 & .40 & 2.55 & 3.40 \\
\hline 11. & 29.00 & 7.80 & 12.80 & 6. 30 & 3.80 & 2.85 & 3. 05 & 1.75 & 5. 50 & .30 & 3.65 & 3.20 \\
\hline 12. & 25.50 & 7.60 & 11.00 & 6.00 & 3. 20 & 4. 20 & 2.85 & 1.55 & 5. 25 & .30 & 4.30 & 2.95 \\
\hline 13. & 22.50 & 7.30 & 10.00 & 5.60 & 3. 00 & 3. 40 & 2.45 & 3. 85 & 4.15 & 20 & 4.15 & 2. 75 \\
\hline 14. & 19.00 & 7.00 & 9.20 & 5.50 & 3.10 & 4.00 & 2.15 & 3.60 & 3. 30 & .15 & 3. 75 & 2.70 \\
\hline & 16. 00 & 7. 30 & 8.80 & 8.00 & 3. 20 & 3.45 & 1.95 & 3. 35 & 2. 70 & .15 & 3. 50 & 2.65 \\
\hline 16 & 13.60 & 27.50 & 8.10 & 16. 80 & 3.00 & 3.25 & 4. 20 & 3.15 & 2.25 & .15 & 4. 20 & 2.50 \\
\hline & 29.00 & 49.00 & 7. 20 & 16. 70 & 2.80 & 3.05 & 7.35 & 3.80 & 2.00 & .10 & 5. 20 & 2. 30 \\
\hline 18 & 40.50 & 56.40 & 7. 10 & 14.00 & 2.50 & 2.85 & 11.35 & 3. 50 & 1.55 & 10 & 12.45 & 2.20 \\
\hline & 38.50 & 56. 60 & 11. 00 & 12.00 & 2.35 & 2.65 & 11. 50 & 3. 20 & 1.40 & .05 & 18. 90 & 2.10 \\
\hline 20 & 34. 00 & 53.00 & 12.50 & 10.00 & 2.25 & 2.45 & 8.40 & 2.90 & 1. 30 & .05 & 16. 90 & 2.05 \\
\hline 21. & 30.30 & 47. 00 & 12. 30 & 8.90 & 2.05 & 2. 25 & 4. 60 & 2.40 & 1. 20 & 05 & 14.10 & 2.00 \\
\hline & 28.10 & 41.50 & 11.80 & 8. 100 & 1.85 & 3.25 & 4. 55 & 1.90 & 1. 10 & .00 & 11.70 & 2.00 \\
\hline 23 & 26.00 & 36.50 & 10.80 & 7.00 & 1.65 & 3.45 & 4. 50 & 1.50 & 1.00 & -.10 & 9.70 & 2.00 \\
\hline & 23.10 & 32.50 & 10.00 & 6.40 & 1.50 & 3.20 & 3.70 & 1.20 & 1.00 & -.30 & 7.90 & 2. 00 \\
\hline & 21.00 & 28. 50 & 9.80 & 6.30 & 1. 30 & 3.00 & 3. 15 & +90 & .95 & -.15 & 6.60 & 1.95 \\
\hline & 20.40 & 26.50 & 11.20 & 6.80 & 1.25 & 2.40 & 3. 10 & .75 & 2.25 & +.10 & 6.30 & 1.95 . \\
\hline & 28.00 & 23.50 & 11.80 & 6.50 & 1.15 & 1. 95 & 4.55 & 1.00 & 3. 70 & .35 & 6.10 & $1.95^{\circ}$ \\
\hline & 33.50 & 21.40 & 12. 30 & 6.20 & 1.05 & 1.55 & 4.05 & .95 & 3. 50 & .35 & 5.90 & 1.90 \\
\hline & 33.80 & & 11.00 & 5.80 & .95 & 1. 25 & 4.00 & .90 & 3.25 & .40 & $5.8 v$ & 1. 90 \\
\hline & 30.00 & & 10.50 & 5.50 & 1.15 & 1. 10 & 4.00 & 1.10 & 2.60 & .90 & 6.30 & 3.00 \\
\hline & 27.00 & $\ldots$ & 9.80 & $\ldots$. & 1.10 & $\ldots . .$. & 3.80 & 1.05 & $\ldots \ldots$ & 1.00 & $\cdots$ & 4.85 \\
\hline
\end{tabular}

Black Warrior River ${ }^{1}$ at Tuscaloosa, Alabama, for 1890.

\begin{tabular}{|c|c|c|c|c|c|c|c|c|c|c|c|c|}
\hline Day. & Jan. & Feb. & Mar. & Apr. & May. & June. & July. & Aug. & Sept. & Oct. & Nov. & Dec. \\
\hline & 7.70 & 30.20 & 58.90 & 24.50 & 16. 70 & 7.75 & 1.80 & 5.00 & 8.00 & 9.30 & 3.75 & 1.00 \\
\hline & 7.20 & 26.20 & 57.40 & 34. 50 & 13.70 & 6.50 & $\begin{array}{l}1.00 \\
1.65\end{array}$ & 4.05 & $\begin{array}{l}0.80 \\
5.80\end{array}$ & 7.40 & 3.35 & 1.00 \\
\hline & 6.20 & 22.50 & 52.40 & 34. 10 & 11. 95 & 5.65 & 1.45 & 3. 25 & 4. 35 & 6.10 & 3. 10 & 1.00 \\
\hline & 5. 20 & 19.05 & 45.80 & 43. 60 & 12.75 & 5.00 & 1. 25 & 2.85 & 3. 40 & 5.10 & 2.85 & 1.00 \\
\hline & 5.20 & 16.40 & 40.85 & 45.90 & 14.65 & 4. 40 & 1.10 & 3.15 & 3.10 & 4. 40 & 2.55 & 1.00 \\
\hline & 4.80 & 13.90 & 37.15 & 44.50 & 16.50 & 4. 00 & $\begin{array}{r}.80 \\
.80\end{array}$ & 3.35 & 3.85 & 3. 80 & 2.40 & 1.00 \\
\hline & 4. 50 & 12.10 & 35.40 & 38. 70 & 15.00 & 5. 20 & .60 & 3. 75 & 4. 35 & 3. 50 & 2.25 & 1. 20 \\
\hline & 4. 55 & 44. 30 & 32.60 & 34. 00 & 13.50 & 5.60 & .50 & 4. 55 & 4. 80 & 3. 45 & 2. 15 & 10.05 \\
\hline 9. & 5. 10 & 53.95 & 30.50 & 29.95 & 11. 95 & 5.40 & .45 & 4. 30 & 2.85 & 4. 50 & 2.05 & 10.10 \\
\hline 10. & 5. 30 & 52.90 & 27.50 & 26. 70 & 10.10 & 5.25 & .30 & 4. 30 & 3.20 & 4.45 & 2.00 & 8.95 \\
\hline 11. & 5. 60 & 47.50 & 25.00 & 23.45 & 9.30 & 5. 00 & .20 & 6. 10 & 2.90 & 4. 10 & 1.95 & 7. 10 \\
\hline 12. & 5.45 & 42.20 & 23.00 & 20.45 & 8. 35 & 6.00 & .15 & 6.40 & 3.45 & 3.80 & 1. 75 & 5. 80 \\
\hline 13. & 5.30 & 37.20 & 20.75 & 17.45 & 8.35 & 6.20 & .05 & 5. 30 & 3.95 & 3.50 & 1. 65 & 4. 60 \\
\hline 14. & 5. 20 & 32.65 & 22.50 & 14.50 & 9.00 & 6. 20 & 1.05 & 4. 50 & 5. 80 & 3.20 & 1. 60 & 3. 70 \\
\hline 15. & 5.60 & 29.45 & 38.20 & 12.15 & 8. 75 & 5. 60 & 2. 55 & 3.20 & 5.55 & 2.80 & 1. 60 & 3. 30 \\
\hline 16. & 11.20 & 26.95 & 38.00 & 10.65 & 8.65 & 5.05 & 3. 10 & 2. 80 & 4. 50 & 2. 70 & 1. 55 & $\begin{array}{l}2.95 \\
\end{array}$ \\
\hline 17. & 19.30 & 23.95 & 35.80 & 10.35 & 9. 50 & 4. 35 & 3. 55 & 2.50 & 3. 55 & 2.60 & 1. 65 & 2.80 \\
\hline 18. & 21.00 & 21.45 & 32.30 & 12.95 & 8.90 & 4. 30 & 3. 65 & 2. 70 & 2.90 & 2. 40 & 1. 60 & 2.75 \\
\hline 19. & 18.40 & 18.45 & 29.00 & 13. 20 & 8. 15 & 4. 20 & 4. 15 & 2.55 & 2.45 & 2.30 & 1. 55 & 2. 20 \\
\hline 20. & 15.40 & 15.65 & 27.20 & 13.95 & 7.95 & 3. 70 & 4.75 & 2.40 & 2.10 & 2.10 & 1. 50 & 2. 00 \\
\hline 21. & 13.30 & 13.45 & 32.40 & 12.15 & 7.95 & 3. 20 & 4. 20 & 2. 20 & 1.75 & 2.00 & 1.50 & 2.00 \\
\hline 22. & 11.60 & 12.00 & 34.45 & 10.45 & 9.00 & 2.80 & 3.00 & 1.80 & 1.70 & 2.45 & 1.45 & 1. 90 \\
\hline 23. & 10,10 & 10.40 & 41. 25 & 9. 30 & 8.40 & 2. 50 & 2.10 & 1. 40 & 3.05 & 4. 90 & 1. 35 & 1.90 \\
\hline 24. & 10.70 & 9.40 & 40.25 & 10.65 & 7.65 & 2.25 & 1.75 & 1.10 & 3. 10 & 8.45 & 1.30 & 1.80 \\
\hline & 12.50 & 9.50 & 36.75 & 15. 45 & 6.95 & 2.25 & 2.75 & 1.00 & 13.05 & 10.55 & 1. 25 & 3.80 \\
\hline 26. & 13.50 & 13.50 & 33.35 & 26.50 & 6.85 & 2. 15 & 12.65 & .95 & 23. 90 & 9.60 & $\begin{array}{l}1.20 \\
1.20\end{array}$ & 2.30 \\
\hline 27. & 12.80 & 35. 20 & 29.70 & 28.45 & 9.60 & 2.55 & 13.40 & .95 & 22.65 & 8.05 & 1.15 & 6. 80 \\
\hline & 11.80 & 53. 10 & 27.45 & 26.20 & 13.20 & 2.25 & 10.50 & 5.10 & 18.00 & 6.45 & 1.10 & 9.70 \\
\hline 29. & 10.60 & . & 24.55 & 23. 35 & 13. 55 & 2.05 & 9.30 & 9.55 & 14.70 & 5.30 & 1.05 & 10.20 \\
\hline 30. & 17. 60 & $\begin{array}{ll}0 \\
\ldots\end{array}$ & 21.95 & 19.80 & 11.95 & 1.90 & 7.45 & 9.15 & 11.90 & 4. 90 & 1.00 & 9.10 \\
\hline 31 . & 31.70 & - & 18.80 & ..... & 9.25 & ...... & 6.50 & 10.45 & $\ldots$. & 4.15 & ....... & 7.30 \\
\hline
\end{tabular}

1 Record from United States Engineer Office. 
Black Warrior River ${ }^{1}$ at Tuscaloosa, Alabama, for 1891.

\begin{tabular}{|c|c|c|c|c|c|c|c|c|c|c|c|c|}
\hline Day. & Jan. & Fel. & Mar. & Apr. & May. & June. & July. & Aug. & Sept. & Oet. & Nov. & Dec. \\
\hline 1. & 6.10 & 33.20 & 24.00 & 34,00 & 5. 70 & 1.80 & 2.10 & 6. 50 & 1.00 & -0.40 & -0.80 & 3. 40 \\
\hline & 8. 60 & 39.60 & 23.50 & 39.40 & 5.50 & 1. 90 & 2.00 & 13. 00 & 1.00 & $\begin{array}{r}-0.30 \\
-.30\end{array}$ & $\begin{array}{r}-0.80 \\
-.80\end{array}$ & 3.00 \\
\hline 3. & $\begin{array}{l}0.30 \\
14.30\end{array}$ & 40.90 & $\begin{array}{l}20.00 \\
20.50\end{array}$ & $\begin{array}{l}30.40 \\
36.80\end{array}$ & 5. 30 & $\begin{array}{l}1.80 \\
1.80\end{array}$ & $\begin{array}{l}2.00 \\
1.90\end{array}$ & $\begin{array}{l}10.80 \\
14.80\end{array}$ & 1.00 & $\begin{array}{r}-.00 \\
-.20\end{array}$ & $\begin{array}{r}.80 \\
-.80\end{array}$ & $\begin{array}{l}\text { 2. } 40 \\
\text { 2. } 40\end{array}$ \\
\hline & 17.90 & 40.20 & 19.60 & $\begin{array}{l}30.50 \\
32.50\end{array}$ & 4. 90 & $\begin{array}{l}1.00 \\
1.70\end{array}$ & 1. 80 & $\begin{array}{l}14.00 \\
16.00\end{array}$ & .80 & -.20 & $\begin{array}{r}-.80 \\
-.80\end{array}$ & 6. 20 \\
\hline 5 . & 15. 20 & $\begin{array}{l}40.20 \\
39.30\end{array}$ & $\begin{array}{l}18.00 \\
17.20\end{array}$ & 28.50 & $\begin{array}{l}4.90 \\
4.50\end{array}$ & 1.50 & $\begin{array}{l}1.00 \\
1.50\end{array}$ & $\begin{array}{l}10.00 \\
13.00\end{array}$ & .70 & $\begin{array}{r}-20 \\
\ldots .20\end{array}$ & $\begin{array}{r}-.80 \\
-.80\end{array}$ & 6. 80 \\
\hline 6. & 13. 10 & 36. 30 & 29.00 & 25.00 & 4.50 & 1.40 & 1.30 & 9.50 & .60 & -.20 & $\begin{array}{r}.80 \\
-\quad .80\end{array}$ & 21.50 \\
\hline 7. & 11. 10 & 37.00 & 53.00 & 22.20 & 4.20 & 1.40 & 1.40 & 7.40 & 60 & -.40 & -.80 & 19.50 \\
\hline 8. & 10.00 & 51.50 & 58.00 & 19.00 & 4.00 & 1.50 & 2.10 & 5.80 & .60 & -.50 & -.80 & 20.00 \\
\hline 9. & 9.50 & 51.50 & 60.40 & 16.00 & 3.90 & 2.10 & 7.00 & 5. 40 & 60 & -.60 & -.80 & 20.50 \\
\hline 10. & 17.50 & 52.20 & 58. 00 & 14.20 & 3.50 & 2.80 & 10.40 & 3.70 & .60 & -.70 & $\begin{array}{r}.70 \\
+\quad .70\end{array}$ & 17.00 \\
\hline 11. & 20.90 & 53.50 & 54.00 & 17.20 & 3.20 & 3.50 & 8.20 & 3.10 & .70 & -.80 & 2.10 & 14.00 \\
\hline 12. & 26.30 & 50.50 & 48.00 & 27.00 & 3.20 & 10.20 & 6.00 & 2.70 & 1.00 & -.80 & 2.80 & 11.50 \\
\hline 13. & 30.10 & 47. 60 & 43. 00 & 26.00 & 3.10 & 10.50 & 4.30 & 2.50 & 1. 20 & -.80 & 4.40 & 9.00 \\
\hline $14 \ldots$ & 25.50 & 51.40 & 40.00 & 22.50 & 3.00 & 9.80 & 3. 20 & 3.00 & 1.20 & -.80 & 3.80 & 6. 20 \\
\hline 15. & 21.00 & 49.50 & 36.50 & 19.50 & 2.80 & 8.00 & 2.30 & 3. 40 & 1. 00 & -.60 & 2. 50 & 7.00 \\
\hline 16. & 18.50 & 46.50 & 33.20 & 17.20 & 2.60 & 6. 50 & 2.60 & 4.00 & 1. 00 & -.70 & 2. 00 & 9.00 \\
\hline 17. & 17. 10 & 44.30 & 30.00 & 15.60 & 2.50 & 5.60 & 2.60 & 3. 00 & .60 & -.70 & 1.80 & 11. 00 \\
\hline 18. & 17. 70 & 41. 00 & 28.00 & 16.40 & 2.50 & 6.30 & 2.90 & 2.60 & .20 & -.70 & 1.80 & 11.20 \\
\hline 19 & 17.10 & 37.50 & 26.40 & 14.50 & 2.50 & 7. 20 & 2.50 & 2.20 & .10 & $-.60^{\circ}$ & 1. 50 & 10.00 \\
\hline 20. & 15.60 & 35.00 & 24,00 & 13,40 & 2.50 & 7.00 & 2,20 & 2.00 & 10 & -70 & 1.30 & 8.50 \\
\hline 21. & 12. 60 & 33.50 & 21.00 & 12.00 & 2.50 & 6.00 & 1.80 & 1.50 & 10 & -.70 & 1.40 & 7.00 \\
\hline 22. & 20.20 & 39.50 & 19. 90 & 11.00 & 2.70 & 5.50 & ]. 60 & 1.50 & 10 & -60 & 3,00 & 6. 00 \\
\hline 23. & 31. 60 & 41.00 & 17. 50 & 10.00 & 3.00 & 5.20 & 1.40 & 1.40 & 10 & -.60 & 8.00 & 6.00 \\
\hline 24. & 31.80 & 39.00 & 15.00 & 9.00 & 3.00 & 4. 80 & 1.30 & 1.30 & 10 & -.50 & 12.30 & 6. 20 \\
\hline 25. & 30.10 & 36.50 & 12.50 & 8.20 & 2.80 & 4.50 & 1.40 & 1.20 & .00 & -.50 & 13.30 & 7. 50 \\
\hline 26 . & 30.70 & 33.00 & 12. 10 & 7.60 & 2.60 & 4.20 & 2.60 & 1.10 & .00 & -.50 & 10.80 & 13.20 \\
\hline 27. & 29.00 & 29.00 & 15. 00 & 8.40 & 2.50 & 4. 10 & 2.00 & 1. 10 & -.20 & -.70 & 7.20 & 31.00 \\
\hline & 26. 80 & 26.50 & 18. 20 & 8.00 & 2.40 & 3.70 & 2.20 & 1.00 & - . 20 & -80 & 5. 80 & 38.90 \\
\hline & 23.70 & $\ldots \ldots$ & 19.40 & 7. 30 & 2.30 & 2.90 & 2.00 & 1.00 & -.40 & -.80 & 4.20 & 31.20 \\
\hline & 32.70 & & 18. 00 & 6.50 & 2.10 & 2.50 & 2.10 & 1.10 & -.50 & -.80 & 3. 80 & 27.00 \\
\hline & 33.00 & . & 17.00 & ....... & 2.00 & $\ldots . .$. & 2. 40 & 1.00 & $\ldots$ & -.80 & $\ldots . .$. & 22.10 \\
\hline
\end{tabular}

Black Warrior River ${ }^{1}$ at Thealoosa, Alabama, for $189:$.

\begin{tabular}{|c|c|c|c|c|c|c|c|c|c|c|c|c|}
\hline Day. & Jan. & Feb. & Mar. & Apr. & May. & June. & July. & Alug. & Sept. & Oct. & Nov. & Dec. \\
\hline & 17.90 & 12. 20 & 10.70 & 21.80 & 12. 00 & 3.40 & 4. 60 & 4.80 & 9.30 & 3.90 & 0.40 & 5.90 \\
\hline & 15. 90 & 10.50 & 10.30 & 18.30 & 11. 20 & 3. 20 & 3. 90 & 3.20 & 7.60 & 3.50 & .40 & 5.60 \\
\hline & 18.50 & 9.40 & 9.70 & 15,50 & 9.90 & 3.50 & 3. 40 & 7.00 & 6.50 & 3.30 & 1.20 & 5.30 \\
\hline & 18.70 & 8.50 & 8.80 & 15. 20 & 8.80 & 3. 70 & 3.00 & 7.40 & 5.60 & 3. 10 & 1.60 & 4.90 \\
\hline & 16. 40 & 7.80 & 8.00 & 12.00 & 7.20 & 3. 50 & 2. 90 & 6.60 & 4. 90 & 2.80 & 1.60 & 6.30 \\
\hline & 14.60 & 7.40 & 7.80 & 11.60 & 6.50 & 4. 70 & 3.50 & 5.40 & 4. 90 & 2.50 & 1.60 & 7.50 \\
\hline & 13. 00 & 7.80 & 8.00 & 56. 30 & 5. 90 & 5. 00 & 5.20 & 4.80 & 4.80 & 2.40 & 2.00 & 7.50 \\
\hline & 11.60 & 7. 50 & 16. 50 & 63.20 & 5. 30 & 4. 90 & 11.00 & 3.90 & 6.40 & 2. 20 & 3. 90 & 12.20 \\
\hline 9. & 10.80 & 9.00 & 26.80 & 62. 20 & 5. 20 & 4. 10 & 26. 70 & 3.60 & 6. 20 & 2. 10 & 4. 60 & 15. 00 \\
\hline 10. & 10.50 & 16. 00 & 28.50 & 58.00 & 4.90 & 4. 00 & 43.50 & 3.50 & 5.40 & 2. 00 & 8.90 & 13.20 \\
\hline 11. & 25.70 & 13.00 & 26.70 & 52. 30 & 4.90 & 3.80 & 46. 20 & 3.00 & 5.10 & 2. 00 & 10.90 & 11.00 \\
\hline 12. & 34.80 & 11.30 & 22.00 & 45.40 & 4. 80 & 3.50 & 41.40 & 3.00 & 3.90 & 2. 00 & 8.30 & 9.40 \\
\hline 13. & 53.00 & 10.80 & 18.00 & 40.70 & 4. 70 & 3. 30 & 38.30 & 3.30 & 3.40 & 2. 00 & 7.60 & 8.00 \\
\hline 14. & 57.40 & 9.50 & 15.80 & 36.50 & 4.60 & 2. 80 & 37.50 & 3.10 & 3.30 & 1.90 & 5. 80 & 7. 40 \\
\hline 15. & 55. 90 & 9.00 & 13.80 & 32.80 & 4.50 & 2.50 & 34. 80 & 3.90 & 4. 00 & 1. 90 & 5.00 & 8.60 \\
\hline 16. & 51. 70 & 10.00 & 13.30 & 29.50 & 4.00 & 2.40 & 32.80 & 3. 50 & 5.10 & 1. 90 & 4.50 & 10.30 \\
\hline 17. & 45.00 & 11.80 & 11. 20 & 27.00 & 3. 70 & 00 & 41. 40 & 3.50 & 4. 90 & 1.80 & 4.00 & 13.10 \\
\hline 18. & 40.10 & 11.00 & 12.90 & 24.50 & 3.60 & 2.80 & 38.00 & 6. 00 & 4. 30 & 1. 70 & 4. 20 & 26.70 \\
\hline 19. & 36.60 & 9.90 & 24.00 & 22.40 & 4. 30 & 1. 10 & 33.00 & 9.50 & 6. 00 & 1.00 & 4. 40 & 28.40 \\
\hline 20. & 41.50 & 10.50 & 25.50 & 20.20 & 4. 60 & 2.90 & 29.00 & 11.50 & 18. 30 & 1.00 & 4. 70 & 28.40 \\
\hline 21. & 41.00 & 13.50 & 22.90 & 18.10 & 6. 50 & 3.40 & 28.30 & 9.50 & 23.90 & .90 & 5.00 & 36. 50 \\
\hline 22. & 36. 80 & 18.00 & 20.00 & 15.90 & 6. 30 & 70.80 & 29. 30 & 7. 40 & 20.90 & .90 & 4.80 & 35.80 \\
\hline 23 . & 34.40 & 23.90 & 18.30 & 13.80 & 6. 00 & 10.70 & 25.10 & 10.20 & 17.00 & .90 & 4.50 & 31.50 \\
\hline 24 & 31.00 & 21.20 & 22.00 & 12. 30 & 6.00 & 10.40 & 21.50 & 12.30 & 13. 30 & 1.00 & .4 .20 & 27.00 \\
\hline 25. & 28.50 & 18.50 & 29.00 & 11. 30 & 4.80 & 8.80 & 18.80 & 14.00 & 10.50 & 1. 00 & 380 & 23.00 \\
\hline 26. & 26.00 & 16.00 & 32.00 & 10.50 & 3.50 & 7.90 & 15.80 & 14.20 & 8.00 & .90 & 3.50 & 19.50 \\
\hline & 23. 80 & 14.00 & 35. 80 & 8.80 & 4. 70 & 7.20 & 13. 00 & 13. 20 & 6. 10 & .80 & 3.30 & 16. 00 \\
\hline & 21.50 & 12.50 & 34.00 & 7.90 & 4. 00 & 7.10 & 10.50 & 11.50 & 5. 00 & .70 & 3.20 & 13.60 \\
\hline & 19.00 & 11.50 & 30.50 & 7.70 & 3.80 & 6. 20 & 7.80 & 12.00 & 4.90 & .70 & 3.90 & 11.40 \\
\hline & 16. 80 & $\ldots \ldots$ & 26.80 & 10.50 & 3. 50 & 5.40 & 6. 20 & 11.50 & 4. 30 & .50 & 5.90 & 9.80 \\
\hline 31. & 14.00 & $\cdots$ & 23.90 & $\ldots .$. & 3. 50 & $\ldots$. & 5. 50 & 10.50 & $\ldots$ & .50 & $\ldots$. & 8. 50 \\
\hline
\end{tabular}

1 Record from United States Engineer Office. 
Black Warrior River ${ }^{1}$ at Tuscaloosa, Alabama, for 1893.

\begin{tabular}{|c|c|c|c|c|c|c|c|c|c|c|c|c|}
\hline Day. & Jan. & Feb. & Mar. & Apr. & May. & June. & July. & Aug. & Sept. & Oet. & Nov. & Dec. \\
\hline & 8.20 & 18.10 & 23.00 & 9. 40 & 24.50 & 12.50 & 2.50 & 0.60 & 0. 30 & 1. 10 & 0.40 & 1.50 \\
\hline & 8.60 & 15. 90 & 21.70 & 9.30 & 21.20 & 33.50 & 2.30 & .70 & .20 & 1.00 & .40 & 1.50 \\
\hline & 9. 80 & 14.00 & 19. 70 & 9.00 & 30.00 & 49.60 & 2.80 & 1. 00 & 10 & 1.20 & .40 & 1.80 \\
\hline & 10.70 & 12.80 & 20.50 & 8.70 & 51.20 & 46.00 & 3.60 & 1. 20 & 00 & 1.40 & .40 & 2.80 \\
\hline & 10.10 & 12.50 & 23.00 & 8.00 & 52.20 & 39.00 & 3.60 & 1. 30 & -10 & 1. 30 & .40 & 3.50 \\
\hline & 9.00 & 11.80 & 24.00 & 8.70 & 48.00 & 37. 70 & 3. 20 & 1. 20 & -.10 & 1. 20 & .40 & 4.20 \\
\hline & 8.40 & 11. 20 & 22.60 & 9.30 & 42.90 & 39.90 & 2.70 & 1. 30 & .00 & 1. 20 & 40 & 4. 10 \\
\hline & 7.90 & 10.80 & 20.10 & 9.00 & 40.40 & 39.30 & 2. 40 & 1.20 & +.20 & 1. 20 & 40 & 3.50 \\
\hline & 7.00 & 10.60 & 20.00 & 8. 30 & 37.40 & 34.00 & 2.10 & 1.00 & 1.40 & 1.00 & .40 & 3.00 \\
\hline 10. & 6.70 & 10. 20 & 22.00 & 7.60 & 34.30 & 29.20 & 1.80 & .90 & 2. 00 & .90 & 60 & 2.80 \\
\hline 11. & 6.20 & 12.00 & 21.80 & 7.00 & 30.80 & 25.10 & 1.60 & .90 & 2.10 & .80 & .60 & 2. 30 \\
\hline 12. & 6.50 & 23.90 & 20.50 & 6.40 & 27.50 & 21.20 & 1.60 & .80 & 2. 20 & .80 & .50 & 1. 90 \\
\hline $13 .$. & 7.80 & 28.30 & 19. 30 & 6.10 & 24.40 & 17.40 & 2.20 & .90 & 3. 20 & .80 & .50 & 1.70 \\
\hline 14. & 9.00 & 27.00 & 18. 10 & 5.80 & 21.90 & 14. 70 & 2. 60 & 3. 60 & 4. 30 & .70 & .60 & 1.60 \\
\hline 15. & 9.60 & 25.90 & 16.30 & 23.00 & 19.10 & 11. 60 & 2.40 & 4. 90 & 4. 30 & .70 & .60 & 1.50 \\
\hline 16. & 11.40 & 52.20 & 14.70 & 27.10 & 16.20 & 9. 60 & 2.00 & 5. 10 & 3.90 & .60 & .60 & 1.70 \\
\hline 17. & 12. 20 & 55.60 & 13. 20 & 24.00 & 14.00 & 7. 60 & 1.70 & 4. 70 & 3. 30 & .50 & .60 & 2.30 \\
\hline 18. & 11. 80 & 54.70 & 12. 20 & 20.00 & 11. 90 & 6. 70 & 1.40 & 3.70 & 2.50 & .50 & .60 & 2. 40 \\
\hline 19. & 12.00 & 51.40 & 11.30 & 16.40 & 11. 00 & 6. 50 & 1. 30 & 2.80 & 2.10 & .50 & 60 & 2. 30 \\
\hline 20 . & 12.40 & 46.50 & 10.70 & 13.90 & 8.40 & 6.90 & 1. 20 & 2. 20 & 1.80 & .50 & 60 & 2. 10 \\
\hline 21 . & 11.80 & 41.80 & 9.90 & 12.00 & 6.90 & 6.90 & 1.20 & 1. 70 & 1. 40 & .50 & .70 & 2.50 \\
\hline 22. & 10.90 & 37.90 & 9.30 & 11.20 & 6.20 & 6. 60 & 1. 30 & 1.50 & 1. 30 & .50 & 1.00 & 240 \\
\hline 23. & 11.20 & 34.50 & 8.80 & 10. 70 & 5.60 & 6.40 & 1. 60 & 1. 30 & 1. 20 & .40 & 1.00 & 2. 30 \\
\hline & 12. 90 & 31.30 & 12.30 & 9.90 & 5.10 & 5.70 & 1.70 & 1. 10 & 1.00 & .40 & 1. 10 & 2. 10 \\
\hline & 15.90 & 28.40 & 22.20 & 8.90 & 4. 80 & 4. 90 & 1.50 & 1.00 & 1.30 & .40 & 1.00 & 1.90 \\
\hline 26. & 19.20 & 25.90 & 22.50 & 7.00 & 4. 50 & 4. 40 & 1.30 & .90 & 1.20 & .40 & .90 & 1. 70 \\
\hline & 22.00 & 23.80 & 20.00 & 10. 50 & 4. 30 & 4. 00 & 1. 20 & .80 & 1. 20 & .40 & 1.00 & 1.60 \\
\hline & 23.30 & 23.00 & 17.00 & 32.50 & 5. 10 & 3.50 & .90 & .80 & 1. 10 & .40 & 1.70 & 1.50 \\
\hline & 23.10 & $\ldots$ & 14.40 & 33.90 & 6. 10 & 3. 10 & .90 & .60 & 1. 10 & .40 & 1.70 & 1.40 \\
\hline & 22.10 & $\ldots \ldots$ & 12. 40 & 29.00 & 12.90 & 2.80 & .80 & .50 & 1. 10 & .40 & 1.50 & 3. 70 \\
\hline & 20.40 & $\ldots$. & 10.90 & -....... & 14. 10 & ...... & .80 & .20 & ...... & .40 & ....... & 7.60 \\
\hline
\end{tabular}

Black Warrior River ${ }^{-1}$ at Tuscaloosa, Alabama, for 1894.

\begin{tabular}{|c|c|c|c|c|c|c|c|c|c|c|c|c|}
\hline Day. & Jan. & Feb. & Mar. & Apr. & May. & June. & July. & Ang. & Sept. & Oet. & Nov. & Deo. \\
\hline & 10.30 & 8.50 & 27.00 & 11.40 & 6.50 & 1.70 & 2.50 & 1. 20 & 5.80 & 0.55 & -0.05 & -0.05 \\
\hline & 9. 60 & 7.80 & 25.60 & 14. 10 & $\begin{array}{l}0.90 \\
5.90\end{array}$ & 1.60 & 1.80 & 1.30 & 4. 60 & .45 & -.10 & -.05 \\
\hline & 7. 90 & 7.30 & 23.60 & 23.50 & 5.90 & 1. 50 & 1.35 & 1.40 & 3.60 & .40 & -.20 & $: 00$ \\
\hline & 6.20 & 7.90 & 21.00 & 24.00 & 5.80 & 1.35 & 1. 10 & 1.95 & 2.95 & .32 & -.25 & .00 \\
\hline & 5.40 & 16.90 & 18.20 & 22. 20 & 5.50 & 1. 33 & $\begin{array}{r}.80 \\
.80\end{array}$ & 2.30 & 2.45 & .25 & -.30 & .00 \\
\hline & 6.40 & 22.60 & 15.90 & 20.60 & 5. 20 & 1. 20 & .60 & 2.40 & 2.05 & .20 & -.30 & .00 \\
\hline & 16.80 & 20.40 & 14.80 & 18.90 & 5. 10 & $\begin{array}{l}1.20 \\
1.20\end{array}$ & 1.00 & 2.00 & 1.85 & .15 & -.30 & .00 \\
\hline & 22.60 & 17.80 & 14.40 & 16.30 & 4.90 & 1.10 & 1.30 & 1.60 & 1.75 & .00 & -.30 & +.05 \\
\hline 9. & 19.70 & 17.30 & 13.80 & 14.70 & 4.50 & .95 & .70 & 1. 30 & 1.65 & -.10 & -.30 & $\quad .05$ \\
\hline 10. & 26.90 & 26.00 & 12.60 & 15.70 & 4. 10 & .90 & .70 & 1.10 & 1. 60 & -.20 & -.30 & .50 \\
\hline 11. & 34.50 & 27.80 & 11.60 & 25.00 & 3. 70 & .80 & .60 & .80 & 1. 85 & -.25 & -.30 & 2.10 \\
\hline 12. & 35.80 & 25. 40 & 11.50 & 25. 60 & 3.90 & .80 & .60 & .70 & 3. 40 & -.30 & -.30 & 5. 70 \\
\hline 13. & 30.90 & 29.90 & 16. 50 & 22.70 & 4.30 & .70 & .50 & .60 & 5. 60 & -.30 & -.30 & 10. 40 \\
\hline 14. & 25.80 & 32.10 & 17.30 & 19. 50 & 7.00 & .60 & .50 & .50 & 7.50 & -.30 & -.15 & 11.20 \\
\hline 15. & 22.20 & 29.70 & 15.80 & 16.30 & 6.20 & .60 & .60 & .35 & 5.70 & -.30 & .00 & 8. 90 \\
\hline 16. & 23.60 & 26.00 & 15.50 & 15.50 & 5. 70 & .60 & .70 & .60 & 4. 20 & -.30 & +.05 & 6.40 \\
\hline 17. & 24.80 & 22.30 & 33.10 & 16.00 & 5. 10 & .55 & .75 & .55 & 3. 25 & -.30 & .05 & 4. 90 \\
\hline 18 & 22.40 & 19. 10 & 36. 70 & 17.00 & 5.00 & .50 & .70 & .45 & 3.50 & -.30 & .05 & 3. 90 \\
\hline 19. & 19.30 & 16.80 & 33. 70 & 25.30 & 4. 70 & .60 & .70 & .40 & 3. 25 & -.30 & .00 & 3. 20 \\
\hline 20. & 15.90 & 16. 00 & 29.50 & 24.80 & 4.20 & .70 & .60 & .55 & 3. 35 & -.30 & -.10 & 2.90 \\
\hline 21 . & 14.10 & 16.90 & 27.80 & 24.00 & 3.60 & .85 & .50 & 1.00 & 4. 10 & -.30 & -.10 & 2. 60 \\
\hline 22. & 18.60 & 17.90 & 29.30 & 20.40 & 3.10 & .80 & .90 & 1. 35 & 3.60 & -.30 & -.10 & 2.20 \\
\hline 23 . & 21.30 & 17.00 & 29.60 & 17.30 & 2.80 & .95 & 1.30 & 3.80 & 2.90 & -.30 & -.10 & 2.00 \\
\hline 24. & 19. 90 & 16.10 & 28.00 & 14.20 & 2,60 & 1.10 & 1.35 & 5. 30 & 2. 20 & -.35 & .00 & 1.80 \\
\hline 25 & 17.60 & 17.30 & 27.80 & 12. 20 & 2,40 & 1.00 & 1.60 & 9. 20 & 1.75 & -.40 & .00 & 1. 70 \\
\hline 26. & 15.50 & 29. 30 & 25.30 & 10.50 & 2.30 & 1.05 & 1. 50 & 16. 00 & 1.45 & -.40 & .00 & 2.00 \\
\hline 27. & 14.00 & 31.00 & 22.10 & 9.80 & 2.10 & 1.40 & 1.40 & 20,40 & 1. 20 & -.45 & -.05 & 7.70 \\
\hline 28 . & 12,40 & 28.80 & 19. 00 & 8.70 & 2.10 & 1.70 & 1. 30 & 16.00 & 1. 05 & -.45 & -.05 & 9.70 \\
\hline & 11.10 & $\ldots \ldots$ & 16.10 & 7.90 & 2.00 & 2.35 & .20 & 11.80 & .90 & -.45 & -.05 & 8.40 \\
\hline & 10.00 & $\ldots \ldots$ & 14.30 & 7.00 & 1.90 & 2.60 & 1.10 & 8.30 & .70 & -.20 & -.05 & 7.20 \\
\hline & 9. 10 & ........ & 12.70 & $\ldots$ & 1,90 & $\ldots .$. & 1.20 & 6.80 & ...... & -.05 & $\longrightarrow .05$ & 6.50 \\
\hline
\end{tabular}

1 Record from United States Engineer Office. 
Black Warrior River ${ }^{1}$ at Tuscaloosa, Alabama, for 1835.

\begin{tabular}{|c|c|c|c|c|c|c|c|c|c|c|c|c|}
\hline Day. & Jan. & Feb. & Mar. & Apr. & May & June. & July. & Aug. & Sept. & Oet. & Nov. & Dec \\
\hline & 6. 10 & 24.10 & 8. 60 & 16.20 & 15. 20 & 7. 00 & 4. 80 & 1. 80 & 1. 90 & 0.10 & -0.04 & 0.75 \\
\hline & 5. 40 & 21.50 & 13. 20 & 14.50 & 13.00 & 5. 90 & 4. 50 & 1.50 & 1.80 & .00 & +.07 & .95 \\
\hline 3 & 6.81 & 21.40 & 35.30 & 12.60 & 11.00 & 5.10 & 5.40 & 1.30 & 1.80 & -.07 & .12 & 1.05 \\
\hline & 9.00 & 21.40 & 36. 70 & 11.00 & 9.40 & 4.60 & 7.60 & 1. 20 & 1. 70 & -.10 & .09 & 1.00 \\
\hline & 8. 80 & 19.90 & 32.40 & 10.20 & 8.30 & 4. 20 & 17.40 & 1. 20 & 1.90 & -.10 & .11 & .90 \\
\hline & 8.00 & 17.90 & 27.80 & 8. 40 & 7.40 & 6.70 & 17.70 & 1. 20 & 2.60 & -.10 & .25 & 1.30 \\
\hline & 7. 40 & 16.50 & 23.80 & 7.90 & 7.00 & 5. 70 & 18.40 & 1. 10 & 3.05 & -.10 & .31 & 1. 50 \\
\hline & 35.00 & 16.80 & 20.60 & 20.50 & 8.20 & 5. 10 & 15. 70 & 1.00 & 3.70 & .00 & .30 & 1. 45 \\
\hline 9. & 50.60 & 16.90 & 20.10 & 24.00 & 13. 50 & 4. 30 & 13. 10 & .90 & 3.40 & -.03 & .28 & 1.90 \\
\hline 10. & 49.30 & 15.80 & 19.30 & 21.20 & 19.70 & 3.80 & 11.10 & .97 & 2.90 & -.07 & 70 & 1.80 \\
\hline 11. & 45.10 & 14.60 & 17.50 & 18.00 & 23. 30 & 3.10 & 9.80 & 1.40 & 2.40 & -.10 & 1. 20 & 1. 45 \\
\hline $12 \ldots$ & 40.10 & 14.10 & 17.20 & 15. 10 & 22.00 & 2.70 & 7.90 & 1.50 & 2. 20 & -.14 & 1. 10 & 1.40 \\
\hline 13. & 35.00 & 13. 90 & 18.40 & 12.60 & 18.50 & 2. 40 & 6.50 & 1. 40 & 2. 10 & -.14 & 1.18 & 1.40 \\
\hline 14. & 29.80 & 12.80 & 24.90 & 11.00 & 15. 20 & 2.20 & 5.30 & 1. 30 & 2.00 & +.00 & 1. 35 & 1. 37 \\
\hline 15. & 25.70 & 11.60 & 37.50 & 9.50 & 12.20 & 2.00 & 5.40 & 1.10 & 1.80 & .04 & 1. 35 & 1. 33 \\
\hline $16 .$. & 23.40 & 10.50 & 47.40 & 8.60 & 9.70 & 2.00 & 5.60 & 1. 30 & 1. 60 & -.08 & 1.28 & 1.23 \\
\hline 17. & 31.20 & 9.40 & 52.00 & 10.00 & 8.00 & 2.70 & 5.90 & 1.40 & 1. 40 & -.18 & 1.20 & 1.12 \\
\hline 18. & 32.90 & 9.00 & 47.30 & 15.90 & 6.90 & 3.30 & 5.80 & 2. 00 & 1.40 & -.27 & 1.05 & 1. 05 \\
\hline 19. & 29.20 & 9.00 & 42.10 & 15. 80 & 6. 30 & 4. 20 & 5.10 & 4. 00 & 1. 30 & -.31 & .88 & 1. 02 \\
\hline 20. & 25.80 & 9.50 & 38.80 & 14.00 & 5.90 & 4. 90 & 4.60 & 4. 30 & 1.00 & -.37 & .82 & 1.57 \\
\hline 21 & 23.10 & 10.20 & 48.70 & 12.00 & 5.30 & 4.70 & 4. 00 & 3.60 & 1.20 & -.42 & .70 & 2.55 \\
\hline 22 . & 21.60 & 10.90 & 51.30 & 10.40 & 4. 80 & 4.30 & 3.60 & 2. 80 & 1.50 & -.45 & .60 & 2.60 \\
\hline 23. & 21.60 & 11.40 & 47.60 & 8.90 & 4. 30 & 4. 10 & 3.40 & 2.40 & 1.30 & -.50 & .50 & 3.03 \\
\hline 24. & 19.80 & 11. 30 & 42.10 & 8.00 & 4. 10 & 3.70 & 3.30 & 4. 30 & 1.10 & -.35 & .75 & 3.65 \\
\hline 25 & 17.40 & 10.90 & 37. 30 & 7.20 & 4. 00 & 3.40 & 3.60 & 4.90 & .80 & -.33 & .60 & 3. 31 \\
\hline 26 & 16. 70 & 10.30 & 32.80 & 7.00 & 4. 30 & 3.00 & 3.70 & 4. 00 & .60 & -.53 & .38 & 4.50 \\
\hline 27. & 20.00 & 9.70 & 29.10 & 7. 10 & 5. 60 & 4.40 & 3. 30 & 3. 20 & .35 & -.70 & .64 & 10.40 \\
\hline & 21. 20 & 9.00 & 26.10 & 12.50 & 11.00 & 5. 40 & 2. 90 & 2. 40 & 10 & -.68 & .65 & 21. 02 \\
\hline & 22.00 & $\ldots \ldots$ & 23.60 & 15.00 & 13.30 & 6.00 & 2. 40 & 2. 30 & .50 & -.67 & .65 & 16. 88 \\
\hline & 27.40 & $\ldots \ldots$ & 21.10 & 16.40 & 11. 20 & 5.40 & 2.00 & 2.10 & .30 & - & .60 & 13.10 \\
\hline & 27. 10 & $\ldots \ldots$ & 18.60 & $\ldots \ldots$ & 8.90 & $\ldots \ldots$ & 2. 10 & 2.00 & $\ldots \ldots$ & -.32 & ..... & 11. 67 \\
\hline
\end{tabular}

Black Warrior River ${ }^{1}$ at Tuscaloosa, Alabama, for 1896.

\begin{tabular}{|c|c|c|c|c|c|c|c|c|c|c|c|c|}
\hline Day. & Jan. & Feb. & Mar. & Apr. & May. & Jine. & July. & Ang. & Sept. & Oct. & Nov. & Dec. \\
\hline & 11.41 & 7. 92 & 6.91 & 10.61 & 18.24 & 4.92 & 1.94 & .53 & 0.38 & -0.52 & -0.78 & 7.70 \\
\hline & 10. 26 & 8.51 & 7. 66 & 14.78 & 33.30 & 4. 28 & 1.71 & .80 & 25 & -.62 & -.77 & 6. 32 \\
\hline & 8.90 & 21.98 & 7.41 & 23.00 & 37.18 & 3.50 & 1.44 & 1.34 & 15 & -.66 & - .42 & 4. 63 \\
\hline & 7.60 & 33.12 & 6.74 & 23.50 & 30.88 & 5.44 & 1. 48 & .89 & 05 & -.20 & -.39 & 3. 40 \\
\hline & 6.55 & 30.02 & 6. 14 & 19.85 & 26.40 & 5.24 & 1.30 & .54 & -.06 & $\begin{array}{r}.78 \\
+.78\end{array}$ & $\begin{array}{r}-33 \\
-\end{array}$ & 2.60 \\
\hline & 5.74 & 30. 75 & 6. 64 & 16. 70 & 21.93 & 4.30 & 1.15 & 42 & -.15 & & -.19 & 2. 05 \\
\hline & 5. 09 & 35.92 & 10.18 & 14. 26 & 17.88 & 3.62 & 1.32 & 50 & -.23 & 68 & -.19 & 1. 70 \\
\hline & 5.24 & 35.08 & 12.04 & 12.55 & 13.95 & 3.27 & 2.20 & 60 & -.30 & 40 & -.17 & 1. 47 \\
\hline & 5. 76 & 36.21 & 11.89 & 11.20 & 10.80 & 6.60 & 8.40 & .68 & -.37 & 09 & -.18 & 1. 21 \\
\hline 10. & 6.46 & 36.52 & 13.69 & 10.02 & 8.60 & 8.18 & 6. 98 & .64 & -.44 & -.15 & -.21 & 1.10 \\
\hline 11. & 6. 58 & 33. 65 & 12. 45 & 9.04 & 7. 11 & 18. 39 & 5.37 & .55 & -.44 & -.28 & -.30 & .89 \\
\hline 12. & 6. 25 & 29.45 & 13.97 & 8. 33 & 6.09 & 15. 13 & 3.95 & .45 & -.46 & -.28 & -.20 & .84 \\
\hline 13. & 5.79 & 25.97 & 16.15 & 7.62 & 5. 34 & 10.77 & 2.99 & .38 & -.50 & $\longrightarrow .48$ & -.22 & .80 \\
\hline 14. & 5.26 & 27. 41 & 15.35 & 7.86 & 4. 77 & 7.66 & 2.45 & .35 & -.60 & -.49 & -.11 & 75 \\
\hline 15. & 4. 82 & 33.25 & 13.56 & 11.65 & 6. 13 & 5.60 & 2. 16 & .31 & -.59 & -.46 & -.10 & 1.20 \\
\hline 16. & 4. 87 & 31.02 & 13. 86 & 13.90 & 5. 45 & 4. 33 & 2.13 & 26 & -.59 & -.43 & -.01 & 1. 25 \\
\hline 17. & 6.03 & 27.30 & 22.30 & 12.36 & 4. 65 & 4. 74 & 2. 15 & 13 & -.60 & -.60 & +.26 & 1.25 \\
\hline 18. & 8.61 & 23.65 & 27.75 & 10.67 & 3.94 & 4.20 & 2.42 & 40 & -.60 & -.63 & .46 & 1. 60 \\
\hline 19. & 9.14 & 20.09 & 29.70 & 9.03 & 3. 44 & 6.18 & 2.46 & 50 & -.60 & -.78 & 52 & 1.90 \\
\hline 20. & 8.53 & 17.00 & 37.68 & 7.81 & 3.05 & 6.00 & 2.24 & 40 & -.61 & - . 82 & 45 & 1. 70 \\
\hline 21. & 7.87 & 14.45 & 37.92 & 6.88 & 2.91 & 5.32 & 2.16 & 34 & -.64 & -.82 & 39 & 1.50 \\
\hline 22. & 885 & 12.20 & 33.55 & 6.15 & 2. 77 & 4.91 & 1. 90 & 29 & -.64 & -.84 & .38 & 1. 33 \\
\hline 23. & 22. 48 & 10.32 & 29.12 & 5.56 & 2.87 & 4. 46 & 1.71 & 22 & -.36 & -.84 & .38 & 1. 20 \\
\hline 24. & 29.26 & 9.13 & 25.85 & 5.67 & 2.90 & 4.19 & 2.06 & 1.06 & -.45 & -.78 & 37 & .98 \\
\hline 25. & 26.52 & 8.60 & 23.54 & 5.76 & 2.87 & 4. 01 & 2. 53 & .95 & -.01 & -.80 & 37 & .92 \\
\hline 26. & 22.44 & 8.35 & 21.28 & 5. 30 & 2.97 & 3.37 & 2.17 & .79 & -.64 & -.82 & 37 & .77 \\
\hline 27. & 18.55 & 7.90 & 18.72 & 5.26 & 3. 66 & 2.85 & 1. 70 & .77 & -.71 & -.82 & .08 & .71 \\
\hline 28. & 14. 92 & 7.40 & 16. 35 & 10.47 & 3. 59 & 2. 69 & 1.28 & 1.35 & -.76 & -.78 & 09 & .59 \\
\hline 29 . & 12. 14 & 7.00 & 14.30 & 16. 06 & ti. 52 & 2. 54 & 94 & 1.30 & -.67 & -.80 & .33 & .52 \\
\hline 30. & 10. 18 & $\ldots \ldots$ & 12.69 & 14.18 & 6.50 & 2.21 & 76 & .95 & -.55 & -.80 & 1.00 & .48 \\
\hline & 8.88 & $\ldots \ldots$ & 11.40 & ....... & 5.78 & ....... & 64 & .60 & & -.78 & $\therefore$ & .48 \\
\hline
\end{tabular}

Record from United States Engineer Office. 
Greenbrier River at Alderson, West Virginia.

\begin{tabular}{|c|c|c|c|c|c|c|c|c|c|c|c|c|}
\hline Day. & Jan. & Feb. & Mar. & Apr. & May. & Juno. & July. & Aug. & Sept. & Oet. & Nor. & Dec. \\
\hline & 2.45 & 2.25 & 2.50 & 7.00 & 3.05 & 2.07 & 2.47 & 4.50 & 1.63 & 6.60 & 1.67 & 4.75 \\
\hline & 3. 08 & 2. 27 & 2.82 & 6.00 & 3. 35 & 1.97 & 2.25 & 3.75 & 1.60 & 4. 02 & 1.73 & 3.85 \\
\hline & 2.65 & 4.95 & 2.70 & 5. 50 & 3.05 & 1. 90 & 2.20 & 4. 35 & 1. 55 & 3.20 & 1.70 & 3. 40 \\
\hline & 2. 25 & 6. 30 & 2.50 & 4.40 & 4.15 & 1.88 & 2.10 & 3. 55 & 1.60 & 2.80 & 1.80 & 3.05 \\
\hline & 2.45 & 4.55 & 2.35 & 3.85 & 4.60 & 1. 85 & 2. 35 & 3.05 & 1.55 & 2. 55 & 7.67 & 2. 80 \\
\hline 6. & 2.40 & 3.55 & 2.33 & 3.20 & 3.70 & 2.00 & 2.30 & 2. 72 & 1. 60 & 2. 38 & 9.50 & 2.65 \\
\hline & 1. 90 & 4.80 & 2.30 & 3.10 & 3. 23 & 2. 05 & 2.55 & 2.50 & 1.60 & 2.20 & 4. 85 & 2.60 \\
\hline 8. & 1. 93 & 4. 20 & 2. 40 & 2.95 & 2.95 & 2. 20 & a3. 08 & 2. 35 & 1. 60 & ล. 10 & 3.60 & 2.50 \\
\hline 9. & 1. 95 & 3.60 & 2. 60 & 3.00 & 2.85 & 2.20 & 6.95 & 2.25 & 1.63 & 2. 00 & 3.15 & 2. 45 \\
\hline 10. & 2.10 & 3. 40 & 2. 70 & 3.05 & 2.65 & 2.20 & 6. 40 & 2.10 & 1.64 & 1.95 & 2.85 & 2. 65 \\
\hline 11. & 2. 05 & 3. 15 & 2.60 & 3.10 & 2.45 & 2.70 & 4. 28 & 2.10 & 1.60 & 1. 90 & 2.60 & 3.10 \\
\hline 12. & 2.05 & 2.85 & 2.90 & 3.15 & 2.35 & 2. 48 & 3.47 & 2.10 & 1.57 & 1. 85 & 2.48 & 2.93 \\
\hline 13. & 2.05 & 2.20 & 3.00 & 3.05 & 2.25 & 2.20 & 3.03 & 2.00 & 1.55 & 2.20 & 2.40 & 2.78 \\
\hline 14. & 2. 30 & 5. 80 & 2.75 & 3.00 & 2.25 & 2.10 & 2.85 & 1.90 & 1. 53 & 2.15 & 2. 30 & 2.62 \\
\hline 15. & 2.20 & 5. 50 & 2.85 & 2.98 & 2.65 & 2. 00 & 2.58 & 1.95 & 1.55 & 2.13 & 2.25 & 2.60 \\
\hline 16. & 1. 70 & 4. 15 & 2. 95 & 2.95 & 2.55 & 2.05 & 2.48 & 2. 10 & 1. 95 & 2.10 & 2.15 & 3. 00 \\
\hline 17. & 1.70 & 3. 60 & 6.75 & 2. 75 & 2.30 & 2.08 & 2.42 & 2.00 & 1.75 & 2.00 & 2. 10 & 3.00 \\
\hline 18. & 1. 72 & 3,05 & 5.60 & 2. 60 & 2.10 & 2.10 & 2.40 & 1.85 & 1. 63 & 1. 95 & 2.05 & 2. 85 \\
\hline 19. & 1. 73 & 2.85 & 4. 50 & 2. 55 & 2.05 & 2. 05 & 2.35 & 1.85 & 1. 57 & 1.90 & 2. 03 & 2. 65 \\
\hline 20. & 1. 73 & 2.60 & 6. 40 & 2. 50 & 2.05 & 2.08 & 2.28 & 1.80 & 1. 75 & 1.85 & 2. 00 & 2.55 \\
\hline 21. & 1. 82 & 2. 38 & 5. 20 & 2. 40 & 2.30 & 2.05 & 2.15 & 1. 75 & 1.70 & 1. 78 & 2. 00 & 2.43 \\
\hline 22. & 1.85 & 2. 42 & 4. 50 & 2.35 & 2.40 & 1.97 & 2.10 & 1. 65 & 1. 70 & 1. 75 & 2.09 & 2.35 \\
\hline 23. & 1.90 & 2. 38 & 4. 35 & 2. 35 & 2.50 & 1.95 & 2.07 & 1. 73 & 1.64 & 1. 73 & 2. 20 & 2.25 \\
\hline 24. & 2. 65 & 2. 25 & 4. 15 & 2. 40 & 2.50 & 1. 90 & 2. 58 & 1. 93 & 1.69 & 1. 70 & 2. 22 & 2. 20 \\
\hline 25. & 4. 20 & 2. 45 & 4.15 & 3.10 & 2.60 & 1. 95 & 2. 64 & 1. 95 & 1.60 & 1. 70 & 2.20 & 1.90 \\
\hline 26 . & 3. 93 & 2.33 & 3.85 & 3.05 & 2.65 & 3. 20 & 5.38 & 1. 96 & 1. 53 & 1. 73 & 2.18 & 1. 70 \\
\hline 27. & 3. 23 & 2. 28 & 3.80 & 3. 00 & 2.55 & 3.75 & 3.85 & 1. 93 & 1.58 & 1.70 & 2.15 & 1. 50 \\
\hline 28. & 2.85 & 2. 22 & 3.70 & 2.80 & 2.45 & 3. 20 & 3.10 & 1.87 & 1.55 & 1.80 & 2.15 & 1.40 \\
\hline & 2.60 & 2.28 & 3. 15 & 2.68 & 2.42 & 3. 10 & 2.80 & 1. 70 & 1.62 & 1. 73 & 2.45 & 1. 80 \\
\hline & 2. 38 & ..... & $a 10.50$ & 2.65 & 2.28 & 2.80 & 2.55 & 1.70 & 4. 93 & 1. 70 & 5.55 & 1.95 \\
\hline 31 & 2.30 & $\ldots$ & 8.88 & ..... & 2.17 & $\ldots$. & 3.83 & 1.67 & ..... & 1. 70 & $\ldots \ldots$ & 2.05 \\
\hline
\end{tabular}

$a$ Heavy rain.

New River at Fayette, West Virginia.

\begin{tabular}{|c|c|c|c|c|c|c|c|c|c|c|c|c|}
\hline Day. & Jan. & Feb. & Mar. & Apr. & May. & June. & July. & Ang. & Sept. & Oct. & Nov. & Dec. \\
\hline & 3. 10 & 3.70 & 3.75 & 22.25 & 6.20 & 2.85 & 6.08 & 4. 60 & 1.10 & 14.90 & 1.25 & 12.80 \\
\hline & $\begin{array}{l}\text { 5. } \\
\text { 3. } 60\end{array}$ & 4.90 & $\begin{array}{l}0.10 \\
3.30\end{array}$ & $\begin{array}{l}22.20 \\
23.60\end{array}$ & $\begin{array}{l}0.20 \\
6.60\end{array}$ & $\begin{array}{l}2.00 \\
2.60\end{array}$ & $\begin{array}{l}0.00 \\
5.00\end{array}$ & $\begin{array}{l}4.00 \\
5.10\end{array}$ & $\begin{array}{l}1.10 \\
1.00\end{array}$ & $\begin{array}{r}14.80 \\
9.45\end{array}$ & $\begin{array}{l}1.20 \\
1.00\end{array}$ & $\begin{array}{l}12.00 \\
11.50\end{array}$ \\
\hline 3. & 4. 80 & 12.50 & 2.95 & 21.15 & 6.70 & 2. 75 & 4. 10 & 4.85 & .90 & 6.00 & 1.30 & 8. 08 \\
\hline & 3. 70 & 11.80 & 2.50 & 17.60 & 7.05 & 2.95 & 2.80 & 4.50 & .80 & 4.50 & 2.15 & 7.40 \\
\hline & 3. 10 & 9.65 & 2.20 & 15.40 & 7.30 & 3.55 & 3.00 & 4. 25 & .80 & 3.45 & 698 & 5.80 \\
\hline 6. & 2. 19 & 7.80 & 2.90 & 11.10 & 7.00 & 3.58 & 3.15 & 4. 00 & 1.00 & 3.10 & 17.10 & 5. 32 \\
\hline & 1. 75 & 7. 65 & 3.40 & 10.30 & 6.15 & 3.35 & 3.45 & 3.65 & 1.20 & 2.65 & 13.25 & 4.95 \\
\hline 8. & 1. 40 & 11.55 & 3.70 & 9.00 & 5.20 & 3.55 & 3.90 & 3.90 & 2.50 & 2.05 & 6.53 & 4. 40 \\
\hline 9 & 1.20 & 11.40 & 4.50 & 8.20 & 4.70 & 3.75 & 17.30 & 3.80 & 2.30 & 1. 40 & 7.65 & $\ldots$ \\
\hline 10. & 1. 90 & 8.65 & 4.00 & 7.10 & 4.55 & 4.00 & 25.20 & 3.60 & 2.00 & 1.90 & 6. 30 & \\
\hline 11 . & 3. 00 & 5. 35 & 3.35 & 6.20 & 4.30 & 3.35 & 14.50 & 3.20 & 1. 50 & 1. 65 & 4.85 & \\
\hline 12. & 2.75 & 4. 15 & 3. 70 & 6.05 & 4.05 & 3.00 & 14.00 & 2.95 & 1. 25 & 1. 70 & 3.00 & \\
\hline 13. & 2. 90 & 4. 30 & 4.15 & 5.80 & 3.65 & 3.60 & 13.05 & 2.70 & 1.20 & 2.10 & 3.20 & \\
\hline 14. & 2.40 & 7.90 & 5.00 & 5.50 & 3.30 & 3.00 & 12.00 & 2.10 & 1.15 & 2.80 & 3.50 & \\
\hline 15. & 2.60 & 10.70 & 6.00 & 5. 20 & 3.30 & 2.70 & 8. 60 & 2. 45 & .90 & 3. 30 & 3.60 & \\
\hline 16. & 2. 30 & 9.00 & 11.00 & 5.05 & 3.40 & 2.50 & 7.15 & 2.40 & 1.40 & 3.00 & 3.55 & \\
\hline 17. & 2.10 & 7. 50 & 14. 93 & 4. 85 & 3.20 & 2.40 & 6.05 & 2. 30 & 1. 30 & 2. 80 & 3.25 & \\
\hline 18. & 1.95 & 5.00 & 15. 75 & 4. 70 & 3.05 & 2.60 & 5.00 & 2.05 & 1. 23 & 1. 95 & 2.90 & \\
\hline 19. & 2. 00 & 4. 70 & 15.05 & 4.45 & 3.15 & 3.00 & 4. 10 & 1.85 & 1. 00 & 1. 10 & 2.45 & 6.30 \\
\hline 20 . & 2.30 & 4.20 & 13. 00 & 4.20 & 3.25 & 3. 20 & 4.40 & 1. 40 & .90 & .85 & 2.45 & 5. 60 \\
\hline 21. & 2.50 & 2.90 & 11.50 & 4.30 & 3.50 & 3. 40 & 5. 40 & 1.25 & .90 & .50 & 2.60 & 5.00 \\
\hline 22. & 2. 10 & 2.35 & 11.00 & 4.50 & 3.75 & 3. 75 & 5. 70 & 1.35 & .90 & .40 & 2.90 & 4. 65 \\
\hline 23 . & 2.40 & 3.30 & 10.15 & 4.70 & 3.60 & 4.00 & 4.95 & 1.85 & .80 & 50 & 3.00 & 4.05 \\
\hline 24. & 2.60 & 4.05 & 9.40 & 4.90 & 5.90 & 4. 45 & 4. 10 & 1.50 & 1.05 & 1. 45 & 2.95 & 3.70 \\
\hline 25. & 6. 00 & 4. 60 & 8.85 & 5.10 & 5.45 & 5. 15 & 4. 45 & 2. 10 & 1.10 & 1.30 & 2. 70 & 3.00 \\
\hline & 10.00 & 4.80 & 8.15 & 5.00 & 5.00 & 6. 30 & 4.47 & 2.35 & 1. 10 & 1. 20 & 2.72 & \\
\hline & 8. 10 & 4. 30 & 7.50 & 5.30 & 4.10 & 8.90 & 4.20 & 2. 70 & 1.10 & 1.00 & 2.70 & 2.60 \\
\hline & 7.55 & 4.00 & 6.60 & 5.45 & 4.00 & 7.90 & 4.30 & 2.40 & 1. 10 & .70 & 3.10 & 2. 75 \\
\hline & 6. 70 & 3. 90 & 7. 40 & 5.80 & 3.55 & 7.53 & 4. 50 & 2.15 & .70 & 1. 40 & 7.45 & 2.90 \\
\hline & 4.90 & ....... & 18. 40 & 6.00 & 3. 20 & 6.80 & 4. & 1.75 & .80 & .60 & 8.30 & 2.50 \\
\hline 31 & 4.00 & 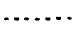 & 23.40 & . & 3.23 & ...... & 4.85 & 1.30 & $\ldots$ & 1. 30 & $\cdots$ & 2.70 \\
\hline
\end{tabular}

No observations December 9 to 17 ; wire gage broken. 
French Broad River at Asheville, North Carolina.

\begin{tabular}{|c|c|c|c|c|c|c|c|c|c|c|c|c|}
\hline Day. & Jan. & Feb. & MIar. & Apr. & May. & Jnne. & July. & Ang. & Sept. & Oet. & Nov. & Dec. \\
\hline & 3.80 & 3. 40 & & 3.80 & 2.80 & 2.90 & 2.56 & 3.20 & 2.50 & 2. 65 & 2. 75 & 5.80 \\
\hline 2. & 3.40 & 3.40 & & 4.20 & 3.20 & 2.90 & 2.55 & 3.10 & 2.63 & 2.63 & 2.65 & 4. 50 \\
\hline$\ldots$. & 3.30 & 4. 00 & $\ldots$ & 3.70 & 4.60 & 2. 90 & 2.65 & 3.25 & 2.70 & 2.60 & 2.65 & 4. 10 \\
\hline 4. & 3.20 & 3.70 & $\ldots$ & 3.45 & 4.10 & 3.13 & 2.85 & 3.10 & 2. 73 & 2. 50 & 2.85 & 3. 80 \\
\hline 5. & 3.20 & 3.60 & $\ldots$ & 3.30 & 3.90 & 2.90 & 2. 86 & 2.90 & 2.95 & 2. 45 & 6.10 & 3.80 \\
\hline 6. & 3.30 & 4.65 & $\ldots \ldots$ & 3. 27 & 3.70 & 2.90 & 4. 10 & 2.85 & 3. 20 & 2. 43 & 5. 50 & 3. 60 \\
\hline $7 \ldots$ & 3.15 & 4. 60 & & 3. 25 & 3.50 & 2.73 & 5.10 & 2. 85 & 3. 10 & 2.40 & 5. 43 & 3.50 \\
\hline $8 \ldots$ & 3.10 & 4.43 & $\ldots$ & 3.20 & 3.26 & 2.70 & 9.85 & 290 & 2.90 & 2.40 & 3. 45 & 3.40 \\
\hline $9 \ldots$ & 3.00 & 5. 45 & & 3.15 & 320 & 3.15 & 8.73 & 2.85 & 2.65 & 2.40 & 3.35 & 3.40 \\
\hline 10. & 2.75 & 4. 70 & & 3.15 & 3.07 & 2.90 & 7.00 & 3.00 & 2.53 & 2.40 & 3.25 & อ. 30 \\
\hline 11. & 2.90 & 4.20 & & 3.02 & 3.05 & 2.75 & 6.10 & 3.00 & 2.55 & 2.40 & 2. 75 & 3.25 \\
\hline 12. & 2. 90 & 3.95 & & 3.50 & 3.05 & 2.70 & 5.00 & 2.95 & 2.85 & 2. 45 & 3.50 & 3.25 \\
\hline 13. & 2.85 & 4. 85 & & 3.25 & 3.03 & 2.67 & 4. 80 & 2. 80 & 2.55 & 2.50 & 4. 25 & 3,20 \\
\hline 14. & 2.85 & 4. 45 & & 3.25 & 3.05 & 2. 65 & 4. 50 & 2.85 & 2. 55 & 2. 65 & 4. 23 & 3.20 \\
\hline 15 & 2.75 & 3.95 & & 3. 15 & 2.87 & 2. 60 & 4.30 & 2. 95 & 2.57 & 2. 55 & 4. 20 & 3.25 \\
\hline 16. & 2.80 & 3.80 & 3.50 & 2. 10 & 2. 80 & 2.60 & 4.25 & 3.25 & 2. 57 & 2. 45 & 3.85 & 3. 30 \\
\hline 17. & 2.80 & 3.70 & 3.40 & 2.95 & 2.76 & 3.15 & 4.15 & 2,95 & 2.65 & 2. 43 & 3.75 & 3.30 \\
\hline 18. & 3.40 & 3.60 & 3. 40 & 2.90 & 2.76 & 3. 30 & 4. 03 & 2.80 & 2. 53 & 2.40 & 3.63 & 3.25 \\
\hline 19. & 3.20 & 3.50 & 3.16 & 2.93 & 2.70 & 3. 39 & 3. 80 & 2. 78 & 2. 50 & 2.43 & 3.50 & 3. 10 \\
\hline 20 & 3.15 & 3.40 & 3.70 & 2.93 & 2.70 & 3.18 & 3.75 & 2.80 & 2. 40 & 2.46 & 2.95 & 3.10 \\
\hline 21. & 3.15 & 3.40 & 3. 30 & 2. 93 & 2.70 & 2.95 & 3.70 & 2. 80 & 2. 40 & 2. 45 & 2. 93 & 3.05 \\
\hline 22 . & 3.10 & 3.30 & 3. 20 & 2.95 & 2. 90 & 3.00 & 3.65 & 2.65 & 2.37 & 2. 42 & 2.90 & 3.05 \\
\hline 23 & 5.30 & 3.35 & 3. 20 & 2.95 & 3.05 & 3. 14 & 3.63 & 2. 75 & 2. 50 & 2. 45 & 2. 70 & 3.14 \\
\hline 24. & 6.50 & 3.35 & 3.30 & 2.95 & 3.10 & 3.15 & 3.60 & 2. 70 & 2.50 & 2. 45 & 2.85 & 3. 00 \\
\hline 25. & 5. 80 & 3. 30 & 3.20 & 2. 92 & 3.00 & 3. 15 & 3.55 & 2. 70 & 2.45 & 2. 45 & 2.80 & 2.95 \\
\hline 36. & 4.40 & 3.35 & 3.15 & 2.86 & 2.70 & 2. 93 & 3.35 & 2.70 & 2.45 & 2. 45 & 2. 90 & 2. 90 \\
\hline 27. & 3.95 & 3.25 & 3.10 & 2. 82 & 3.05 & 2.80 & 3. 40 & 2. 60 & 2. 35 & 2.45 & 3.10 & 2. 90 \\
\hline 28. & 3.75 & 3.35 & 3.10 & 2.83 & 2.75 & 3.05 & 3. 50 & 2. 60 & 3.00 & 2.47 & 4. 05 & 2.87 \\
\hline 29. & 3.60 & 3.30 & 3.50 & 2. 82 & 2. 75 & 2. 75 & 3. 40 & 2. 50 & 2. 70 & 2. 50 & 4.95 & 2. 85 \\
\hline 30. & 3.40 & $\ldots \ldots$ & 3.25 & 2.80 & 2. 73 & 2.63 & 3. 35 & 2. 50 & 2. 67 & 2. 50 & 6.20 & 2.83 \\
\hline 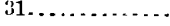 & 3.40 & $\ldots$ & 3.30 & $\ldots . .$. & 2.70 & $\ldots \ldots$ & 3.25 & 2.50 & $\ldots \ldots$ & 2.45 & $\ldots \ldots$ & 2.83 \\
\hline
\end{tabular}

Thokasegee River, three miles above Bryson, North Carolina.

\begin{tabular}{|c|c|c|c|c|c|c|c|c|c|c|c|c|c|}
\hline Dity. & July. & Aug. & Sept. & Oet. & Nov. & Dec. & Day. & July. & Aug. & Sept. & Oet. & Nov. & Dec. \\
\hline 1. & 2.19 & 2.70 & 1.86 & 2.10 & 2.00 & 4. 30 & 17. & 3.70 & 2.40 & 2.10 & 1.70 & 2.40 & 2.50 \\
\hline & 2.31 & 2.60 & 1.86 & 1.90 & 1.85 & 3.60 & 18 & 3.70 & 2.20 & 2.05 & 1. 70 & 2.30 & 2.65 \\
\hline & 2.25 & 2.56 & 2.00 & 1.85 & 1.80 & 3.30 & 19 & 3.20 & 2.10 & 2.10 & 1.70 & 2.25 & 2.50 \\
\hline & 2.30 & 2.50 & 1.90 & 1.80 & 2.60 & 3.10 & 20 . & 3.30 & 2.10 & 2.10 & 1.70 & 2.20 & $\because .20$ \\
\hline & 2.53 & 2.15 & 2.10 & 1.77 & 4.45 & 3.00 & 21 & 5.00 & 2.15 & 1.85 & 1.70 & 2.20 & 2.40 \\
\hline & 4.90 & 2. 50 & 2.50 & 1.75 & 2.80 & 2.90 & 22 & 6.00 & 2.10 & 2.80 & 1.70 & 2.15 & 2.80 \\
\hline & 4.20 & 2.70 & 2.10 & 1.72 & 2. 40 & 2.80 & 23 . & 3.97 & 2.10 & 2.20 & 1.70 & 2. 10 & 2.40 \\
\hline & 8.05 & 2.56 & 2.05 & 1.70 & 2.30 & 2. 70 & 24 & 3. 80 & 2.20 & 2.00 & 2.00 & 2. 05 & 2.20 \\
\hline 0. & 6.90 & 2.50 & 2.00 & 1.70 & 2.20 & 3.00 & 25 & 3.70 & 2.20 & 1.85 & 1. 80 & 2.00 & 2. 10 \\
\hline 10. & 4. 50 & 2.50 & 1. 95 & 1. 70 & 2.10 & 2. 70 & 26 & 3.40 & 2.70 & 1.80 & 1. 76 & 2.00 & 2.05 \\
\hline 11. & 4.00 & 2.50 & 2.10 & 1.70 & 2.00 & 2. 65 & 27 & 3.30 & 2.10 & 1.80 & 1. 70 & 3.30 & 2.30 \\
\hline 12. & 4. 30 & 2.40 & 2.00 & 2.00 & 5.00 & 2. 60 & 28 & 3.20 & 2.05 & 1. 80 & 1.70 & 3.10 & 2.20 \\
\hline 13. & 3.90 & 2.25 & 1. 98 & 1.90 & 3.30 & 2.55 & 29 & 3.03 & 2.00 & 1.80 & 1.80 & 5. 10 & 2. 30 \\
\hline $14 \ldots$. & 3. 60 & 2.50 & 1.90 & 1.90 & 2.60 & 2. 50 & 30 & 2.90 & 1.90 & 2. 30 & 2.00 & 6.50 & 2.35 \\
\hline 15 . & 3.40 & 2.25 & 2.10 & 1.85 & 2. 40 & 2.80 & & 2.80 & 1. 88 & $\ldots . .$. & 2.70 & ..... & 2.30 \\
\hline $16 \ldots$ & 4.10 & 2. 60 & 2.10 & 1. 70 & 2.60 & $\begin{array}{l}2.70 \\
2.70\end{array}$ & & & & & & & \\
\hline
\end{tabular}

Little Tennessee River at Judson, North Carolina.

\begin{tabular}{|c|c|c|c|c|c|c|c|c|c|c|c|c|c|}
\hline Day. & Julf. & Aug. & Sept. & Oct. & Nov. & Dec. & Day. & July. & Aug. & Sept. & Oet. & Nov. & Dee. \\
\hline 1. & 2.70 & 3.35 & 2.60 & 2. 90 & 3.10 & 7.70 & 17. & 4. 00 & 3.60 & 2.70 & & 3.70 & 4.53 \\
\hline 2. & 2.80 & 3.30 & 2. 60 & 2.80 & 2.90 & 6.30 & 18 & 3.65 & 3.10 & 2.70 & & 3.68 & 3.83 \\
\hline & 2.77 & 3.30 & 2.70 & 2.78 & 2.80 & 5. 30 & 19. & 3.64 & 3.00 & 2.60 & & 3.60 & 3.88 \\
\hline & 2.95 & 3. 30 & 2. 80 & 2. 68 & 2.99 & 4. 30 & 20 . & 3.70 & 3. 00 & 2.70 & -. & 3.50 & 3.80 \\
\hline & 2.85 & 3.21 & 2.90 & 2.60 & 5.70 & 4. 40 & 21. & 3.70 & 3. 20 & 2.70 & & 3.50 & 3.80 \\
\hline 0 & 3.82 & 3.19 & 2.90 & 2.53 & 4. 55 & 4. 30 & 22 & 4.10 & 3.85 & 2.67 & & 3.50 & 3.79 \\
\hline & 4.22 & 3.15 & 2.80 & 2.55 & 3. 30 & 4. 20 & 23 . & 5. 11 & 2. 83 & 2.18 & & 3.50 & 3.78 \\
\hline & 6.70 & 3.13 & 2.60 & 2.54 & 3.30 & 4. 10 & 24. & 4.28 & 2. 92 & 2.68 & & 3. 50 & 3.78 \\
\hline 9 & 8.80 & 3.19 & 2.55 & 2. 53 & 3.51 & 4. 10 & 25 & 4. 20 & 3.00 & 2.60 & 3.10 & 3.40 & 3.75 \\
\hline 10 & 5. 70 & 3. 21 & 2.53 & 2.53 & 3.50 & 4. 90 & 26. & 4.10 & 3.00 & 2.54 & 2.90 & 3.50 & 3.45 \\
\hline 11. & 8.95 & 3.12 & 2.50 & ...... & 3.40 & 4. 80 & 27. & 3.85 & 2.80 & 2.60 & 2.90 & 3.60 & 3.46 \\
\hline 12 & 4.65 & 3. 10 & 2.64 & $\ldots . .$. & 4. 30 & 3. 88 & 28. & 3.92 & 2.80 & 2.60 & 2. 80 & 3.70 & 3.54 \\
\hline 13. & 4. 50 & 3.20 & 2.63 & ...... & 5. 70 & 3. 89 & & 3. 80 & 2.90 & 2.60 & 2. 90 & 6.10 & 3.46 \\
\hline 14 & 4. 20 & 3.10 & 2.53 & ..... & 4.30 & 3. 89 & 30. & 3.56 & 2.80 & 3. 30 & 3. 10 & 7. 70 & 3. 61 \\
\hline & 3.95 & 3. 90 & 2.84 & - & 4. 20 & $\begin{array}{l}4.30 \\
4.30\end{array}$ & 31. & 3.45 & (a) & $\ldots$ & 3.00 & & 3.60 \\
\hline 16 & 4. 00 & 3. 70 & 2.75 & ...... & 3. 30 & 4.60 & & & & & & & \\
\hline
\end{tabular}


Hiwassee Riner at Murphy, North Carolina.

\begin{tabular}{|c|c|c|c|c|c|c|c|c|c|c|c|c|c|}
\hline Day. & July. & Ang. & Sept. & Oet. & Nov. & Dec. & Day. & July. & Ang. & Sept. & Oet. & Nov. & Dec. \\
\hline & 3.72 & 4,02 & 3. 65 & 3.74 & 3.66 & 5.20 & 17 & 4. 48 & 3.78 & 360 & 3.65 & 4.50 & 4. 05 \\
\hline & 3. 70 & 4. 02 & 3. 61 & 3.68 & 3. 70 & 4.70 & & 4.54 & 3. 74 & 3. 60 & 3.56 & 3. 95 & 4.00 \\
\hline & $\begin{array}{l}\text { o. } 70 \\
\text { 3. } 75\end{array}$ & 4. 11 & $\begin{array}{l}3.61 \\
3.62\end{array}$ & $\begin{array}{l}\text { 3. } 60 \\
\text { 3. }\end{array}$ & 3. 72 & 4.52 & $\begin{array}{l}10 \\
19\end{array}$ & $\begin{array}{l}4.34 \\
4.35\end{array}$ & 3.70 & 3. 58 & 3. 50 & 3. 91 & 4.00 \\
\hline & 4.00 & 3. 95 & 3. 61 & 3.60 & 4. 90 & 4. 52 & 20 . & 4.28 & 3.68 & 3. 56 & 3. 60 & 3. 90 & 4.00 \\
\hline & 3. 90 & 3.90 & 3. 61 & 3. 62 & 4.10 & 4.20 & & 4. 32 & 3. 70 & 3. 56 & 3. 60 & 3.90 & 4.00 \\
\hline & 4. 62 & 3. 90 & 3. 90 & 3. 55 & 3.90 & 4. 20 & 22 . & 4. 60 & 3.70 & 3.62 & 3. 56 & 3.85 & 4.00 \\
\hline & 4. 98 & 3. 95 & 3. 66 & 3. 60 & 3.90 & 4. 15 & 23. & 5. 10 & 3.65 & 3. 85 & 3. 60 & 3. 80 & 3. 95 \\
\hline & 6. 72 & 3. 90 & 3. 62 & 3. 51 & 3. 90 & 4.10 & 24 . & 4. 61 & 3.90 & 3.60 & 3. 90 & 3. 80 & 3. 95 \\
\hline & 6.95 & 3. 86 & 3. 60 & 3.60 & 3. 82 & 4.30 & 25 & 4. 40 & 3.80 & 3.60 & 3. 70 & 3. 80 & 3.95 \\
\hline 10 & 5. 24 & 4. 00 & 3. 60 & 3. 65 & 3. 80 & 4. 10 & 26 & 4. 30 & 3.72 & 3. 60 & 3. 62 & 3. 84 & 390 \\
\hline & 4. 75 & 3. 90 & 3. 60 & 3. 65 & 3. 76 & 4.10 & 27 & 4. 30 & 3.72 & 3. 60 & 3. 62 & 4.00 & 3. 90 \\
\hline & 4. 82 & 3. 81 & 3. 67 & 3. 65 & 7. 53 & 4. 10 & 28 & 4. 25 & 3. 70 & 3.65 & 3. 65 & 4. 00 & 3. 90 \\
\hline 13 & 4. 77 & 3.81 & 3. 56 & 3. 72 & 5. 40 & 4.05 & 29 & 4. 12 & 3.70 & 3.60 & 3. 74 & 5.03 & 3. 90 \\
\hline & 4.65 & 3. 72 & 3. 58 & 3.65 & 4. 53 & 4.02 & 30. & 4. 01 & 3.65 & 4. 20 & 3. 80 & 6.10 & 3.90 \\
\hline & 4. 42 & 3. 75 & 3. 80 & 3. 65 & 4. 30 & 4. 10 & 31 . & 4.02 & 3.65 & $\ldots \ldots$ & 3. 82 & $\ldots .$. & 3. 90 \\
\hline & 4.55 & 3.92 & 3.62 & 3. 60 & 4. 12 & 4.05 & & & & & & & \\
\hline
\end{tabular}

Tennessee River at Chattanooga, Tennessee, for 1890.

[Furnished by L. M. Pindell, observer in charge, United States Weather Burean.]

\begin{tabular}{|c|c|c|c|c|c|c|c|c|c|c|c|c|}
\hline Day. & Jan. & Feb. & Mar. & Apr. & May. & June. & July. & Aug. & Sept. & Oct. & Nov. & Dec. \\
\hline & 4. 9 & 8.0 & 40.2 & 10.0 & 7.5 & 6.1. & 3.2 & 5.8 & 7.6 & 9.4 & 6.8 & 2.4 \\
\hline 2 & 5. 2 & 7.3 & 42.5 & 9.8 & 7. 1 & 6.6 & 2.9 & 5.2 & 5.6 & 8.1 & 6.6 & 2.4 \\
\hline 3 & 5.1 & 7.3 & 41.0 & 9.4 & 6.7 & 5.3 & 2.9 & 4.7 & 5.7 & 7.7 & 5.7 & 2.3 \\
\hline $4 \ldots$ & 5.0 & 7.4 & 34.4 & 9.7 & 6.8 & 5.3 & 3.0 & 4.7 & 4.0 & 7.2 & 5. 3 & 2.3 \\
\hline $5 \ldots$ & 5. 0 & 7.2 & 23.0 & 12.2 & 7.0 & 5.2 & 3.0 & 4.8 & 3.6 & 6.4 & 5. 0 & 2.3 \\
\hline 6. & 4. 8 & 7.8 & 15.1 & 14.0 & 7.5 & 5.3 & 3.0 & 4.5 & 3.2 & 5.7 & 46 & 2. 5 \\
\hline 7. & 4.7 & 8.3 & 14.2 & 13. 6 & 8.9 & 5.2 & 3.0 & 5.2 & 3.6 & 5.2 & 4.4 & 2.8 \\
\hline 8. & 4. 6 & 11.5 & $14 . \overline{4}$ & 13. 4 & 9.1 & 4.9 & 2.9 & 5.7 & 3.2 & 5.2 & 4.3 & 2.9 \\
\hline 9 & 4. 7 & 19.3 & 12.8 & 11.9 & 8.7 & 4. 7 & 2.8 & 6.6 & 2.8 & 5.5 & 4. 0 & 7.1 \\
\hline 10. & 4.9 & 20.4 & 11. 2 & 10.5 & 8.7 & 4.5 & 2.7 & 7.5 & 2.8 & 5.3 & 3. 9 & 8.1 \\
\hline 11. & 4. 7 & 17.8 & 10.0 & 9. 6 & 8.1 & 4.5 & 2.7 & 7. 2 & 3.0 & 4.8 & 3.8 & 8.2 \\
\hline 12. & 4. 6 & 14.7 & 9.2 & 8.7 & 7.7 & 4.4 & 3.0 & 6.3 & 3.4 & 4. 6 & 3.6 & 7. 7 \\
\hline 13. & 4. 6 & 12.0 & 8.6 & 8.0 & 7. 2 & 4.0 & 2.7 & 5.8 & 3.6 & 4. 4 & 3.5 & 7.4 \\
\hline 14. & 4. 9 & 10.0 & 8.7 & 7.5 & 6. 7 & 3.9 & 2.5 & 5.2 & 4. 6 & 4.2 & 3. 4 & 6.4 \\
\hline 15. & 4. 6 & 9.8 & 9.7 & 7.1 & 6.6 & 4.0 & 2.3 & 4. 8 & 4.0 & 3. 9 & 3.3 & 3.7 \\
\hline 16. & 5.4 & 9.6 & 13.7 & 6.9 & 7.8 & 4. 1 & 2.1 & 4.2 & 4. 0 & 3. 7 & 3.2 & 4.2 \\
\hline $17 \ldots$ & 7. 2 & 9.0 & 15.1 & 7.1 & 9.3 & 4.0 & 2.0 & 3.8 & 3. 7 & 3.7 & 3.2 & 3. 9 \\
\hline $18 \ldots$ & 9.2 & 9.3 & 14.9 & 9.4 & 8.6 & 3.9 & 2.2 & 3.7 & 4.0 & 3. 7 & 3.0 & 3.9 \\
\hline $19 \ldots$ & ४. 2 & 8.5 & 13.0 & 16.6 & 7.9 & 3.7 & 4. 7 & 3.3 & 5.3 & 4.2 & 3. 2 & 4.0 \\
\hline 20. & 7.5 & 7. 8 & 11.7 & 20.4 & 8.8 & 3.6 & 4. 7 & 3.1 & 3. 7 & 4.0 & 3.1 & 4. 0 \\
\hline 21. & 7.0 & 7. 3 & 12.4 & 18. 2 & 10.7 & 3.7 & 4. 1 & 2.6 & 3.7 & 4.0 & 3.1 & 3.9 \\
\hline 22. & 0.6 & 7.1 & 14.0 & 14.3 & 11.9 & 3.8 & 3.5 & 2.5 & 5.8 & $\begin{array}{l}\text { 4. } 8 \\
\text { 3. } 8\end{array}$ & 3.1 & $\begin{array}{l}3.9 \\
\text { 3. } 9\end{array}$ \\
\hline 23. & 13.0 & 7. 2 & 20.0 & 11,3 & 11.9 & 3.7 & 3.2 & 3.8 & 3.5 & 5.3 & 2.9 & 3. 9 \\
\hline 24. & 12.3 & 7.4 & 25.5 & 9.6 & 11.6 & 3.8 & 3. 2 & 4. 0 & 3. 3 & 7.2 & 2.8 & 3.9 \\
\hline 25. & 11. 7 & 12.1 & 27.2 & 8. 7 & 9.2 & 4. 0 & 4. 1 & 3.3 & 3.4 & 8.8 & 2.8 & 4.3 \\
\hline 26. & 10.0 & 18. 7 & 26.0 & 8.4 & 7.8 & 4.0 & 5.9 & 3.8 & 4.0 & 9.2 & 2.7 & 4.6 \\
\hline 27. & 8.3 & 26.4 & 21.4 & 8.4 & 7.4 & 3.9 & 7.5 & 3.6 & 4.3 & 9.5 & 2.6 & 9.4 \\
\hline 28. & 7. 3 & 34.8 & 15.4 & -8.5 & 8.0 & 3.9 & 7.7 & 3.0 & 4. 0 & 8.6 & 2.6 & 12.5 \\
\hline 29. & 6.6 & $\ldots .$. & 13.0 & 8.4 & 8.1 & 3.5 & 7.0 & 3.8 & 3.8 & 7.6 & 2.5 & 12.9 \\
\hline $30 \ldots$ & 7.7 & . & 11. 9 & 8.1 & 7.4 & 3.1 & 6.3 & 5.4 & 5.3 & 7.1 & 2.4 & 12.4 \\
\hline $31 \ldots$ & 8.3 & . & 10.7 & ...... & 6.8 & $\ldots \ldots$ & 6.2 & 6.5 & $\ldots$. & 7. 0 & ...... & 9.3 \\
\hline
\end{tabular}


Tennessee River at Chattanooga, Tennessee, for 1891.

\begin{tabular}{|c|c|c|c|c|c|c|c|c|c|c|c|c|}
\hline Day. & Jan. & Feb. & Mar. & Apr. & May & June. & July. & Aug. & Sept. & Oct. & Nov. & Dec. \\
\hline $1 .$. & 7.7 & 9.8 & 18.6 & 15. 4 & 5.9 & 5. 8 & 3.9 & 8.3 & 5.4 & 1.8 & 1.3 & 3.7 \\
\hline 2. & 7.8 & 13.2 & 17.5 & 16.3 & 5.7 & 5.6 & 3. 8 & 10.3 & 4. 7 & 1.9 & 1.3 & 3.2 \\
\hline 3. & 9.9 & 16. 1 & 15.6 & 16. 3 & 5.6 & 5.3 & 3.6 & 15. 1 & 4. 3 & 1.9 & 1. 3 & 2.9 \\
\hline 4. & 14.1 & 19.8 & 13.3 & 15.7 & 5.5 & 4.8 & 3.6 & 16.4 & 4. 2 & 1.8 & 1.2 & 2.8 \\
\hline$\ldots . .$. & 15.5 & 22.6 & 15.4 & ]5. 1 & 5.2 & 4. 4 & 3. 6 & 12.0 & 5.1 & 1.8 & 1. 2 & 5.6 \\
\hline$\ldots \ldots$ & 15. 2 & 21. 6 & 20.0 & ]2. 6 & 5.1 & 4.1 & 3. 6 & 8.7 & 5. 1 & 1. 7 & 1.2 & 6. 1 \\
\hline 7..... & 10. 4 & 18.3 & 23.6 & 11. 6 & 4. 9 & 3.9 & 3. 4 & 6.9 & 5. 2 & 1. 7 & 1. 2 & 6.6 \\
\hline $8 \ldots \ldots$ & 8.2 & ]6. 9 & 29.1 & 10. 8 & 4. 7 & 4. 1 & 3. 4 & 5. 8 & 5. 2 & 1.6 & 1. 2 & 8.6 \\
\hline $9 \ldots \ldots$ & 7.1 & 14.5 & 34.5 & 9.8 & 4. 6 & 4. 6 & 3. 3 & 5. 1 & 5.8 & 1. 7 & 1.2 & 10.8 \\
\hline $10 \ldots \ldots \ldots \ldots \ldots$ & 6. 3 & 21.0 & 37.5 & 9.6 & 4.5 & 4. 7 & 4.5 & 4. 6 & 4.9 & 1.7 & 1. 2 & 10. 9 \\
\hline 11. & 6. 5 & 27.8 & 38.9 & 9.8 & 4.4 & 5.5 & 5.1 & 4. 4 & 4. 4 & 1. 8 & $1 . \overline{5}$ & 10.2 \\
\hline 12. & 8.9 & 34.3 & 37.6 & 9.9 & 4. 3 & 7. 0 & 4.4 & 4.0 & 3.9 & 1.8 & 1.7 & 8.5 \\
\hline 13. & 10.7 & 36.5 & 33.5 & 10.6 & 4. 2 & 6.5 & 3.9 & 4. 0 & 3.6 & 1.7 & 2.7 & 6.8 \\
\hline 14. & 10.0 & 37.5 & 27.0 & 11.3 & 4. 1 & 5.7 & 3. 5 & 3.9 & 3.5 & 1.7 & 3. 6 & 5.7 \\
\hline 15. & 9.2 & 35.5 & 22.2 & 12.2 & 4. 2 & 5. 5 & 3.1 & 3.8 & 3.5 & 1.7 & 4. 1 & 5.1 \\
\hline 16. & 7. 3 & 29.0 & 19.8 & 10.8 & 4. 2 & 5. 7 & 2.9 & 3.6 & 3. 5 & 1. 6 & 3. 5 & 5.0 \\
\hline 17. & 7.8 & 21.1 & 18.1 & 9.4 & 4.5 & 5. 8 & 2.8 & 3. 5 & 35 & 1.6 & 2. 8 & 5. 2 \\
\hline 18. & 7.5 & 19.7 & 15.3 & 8.4 & 4. 7 & 6.1 & 2.7 & 3.4 & 3. 2 & 1. 5 & 2.5 & 5.3 \\
\hline 19. & 7.5 & 18.2 & 13.5 & $\therefore 2$ & 4. 7 & 6. 8 & 4. 1 & 3.0 & 2.9 & 1.5 & 2. 4 & 4. 8 \\
\hline 20 . & 7.6 & 16.5 & 12.3 & 7.9 & 4. 6 & 7. 3 & 5.0 & 3.0 & 2.7 & 1.5 & 2.5 & 4.5 \\
\hline 21. & 7.3 & 15.5 & 11.3 & 7.9 & 4. 5 & 6.8 & 4.5 & 3.4 & 2.6 & 1.5 & 2.3 & 4. 2 \\
\hline $22 \ldots$ & 8.2 & 18.8 & 10.8 & 7. 6 & 4. 3 & 6.8 & 4.0 & 4.0 & 2.5 & 1.5 & 2.4 & 4. 0 \\
\hline $23 \ldots$ & $12 . \overline{5}$ & $2+.0$ & 10.7 & 7.4 & 4. 1 & 6. 5 & 3.8 & 4. 6 & 2. 4 & 1.5 & 3.0 & 3.8 \\
\hline $24 .$. & 15.3 & 27.7 & 10.8 & 7.4 & 4. 0 & 7.1 & 3.6 & 5. 5 & 2.3 & 1.5 & 4. 6 & 3.7 \\
\hline 25. & 14. 0 & 29.0 & 10.6 & 7.5 & 3.8 & 7.4 & 3.5 & 5.6 & 2. 2 & 1. & 6.2 & 4.1 \\
\hline 26. & 13.6 & 26.7 & 10.4 & 7.5 & 3.9 & 7.6 & 3.5 & 7.7 & 2.2 & 1.5 & 6.7 & 4.9 \\
\hline 27. & 11.2 & 20.6 & J0. 5 & 7.4 & 4.0 & 6.2 & 3.5 & 8.2 & 2.1 & 1. & 6.3 & 8.1 \\
\hline & 9. 7 & 19.0 & 14.1 & 7.2 & 4.1 & 4.9 & 3.6 & 8.1 & 2. 0 & 1. & 5. 6 & 10.2 \\
\hline & 7.9 & $\ldots \ldots$ & 13.6 & 6.5 & 4.1 & 4. 3 & 3. 7 & 7.0 & 1.9 & 1.4 & 4.7 & 9.6 \\
\hline & 7.9 & $\cdots$ & 13.0 & 6,2 & 4. 7 & 4.1 & 3. 8 & 6. 4 & 1.9 & 1. 4 & 4. 0 & 8.4 \\
\hline & 8.9 & $\ldots \ldots$ & 13.1 & $\ldots \ldots$ & 5.3 & ..... & 5.7 & 6.1 & 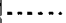 & 1.4 & $\cdots$ & 7.8 \\
\hline
\end{tabular}

Tennessee River at Chattanooga, Tennessee, for 189.

\begin{tabular}{|c|c|c|c|c|c|c|c|c|c|c|c|c|}
\hline Day. & Jan. & Feb. & Mar. & Apr. & May. & Jume. & July. & Aug. & Sept. & Oet. & Nov. & Dec. \\
\hline $1 \ldots$ & 6.6 & 6.5 & 5.7 & 9.1 & 8.7 & 5.6 & 6.6 & 4.2 & 2.1 & 2.2 & 1.1 & 4. 1 \\
\hline $2 \ldots$ & 6. 6 & 6.2 & 5.6 & 8.3 & 8.2 & 5.4 & 5.6 & 4.1 & 1.8 & 2.0 & 1.2 & 4. 0 \\
\hline $3 \ldots$ & 8.1 & 6.0 & 5.5 & 7. 4 & 7. 6 & 5.5 & 5.5 & 4.2 & 1.7 & 1.9 & 1. 2 & 3. 8 \\
\hline 4. & 8. 8 & 5.8 & 5.3 & 6.8 & 7.3 & 5.8 & 5.4 & 4.0 & 1.5 & 1.8 & 1. 6 & 3.7 \\
\hline $5 \ldots$ & 8.7 & 5.5 & 5. 1 & 6.5 & 7.4 & 8.8 & 6. 6 & 4.1 & 1.3 & 1.7 & 2.0 & 3.6 \\
\hline 6. & 8.4 & 5. 4 & 5.0 & 8.5 & 7.0 & 9.2 & 8.9 & 4.9 & 2.2 & 1.6 & 2.3 & 3.6 \\
\hline 7. & 9.0 & 5.3 & 4.9 & 21.7 & 6.6 & 9.3 & 11.2 & 4.2 & 2.1 & 1.6 & 2.4 & 3.3 \\
\hline 8. & 9.8 & 5. 8 & 5.0 & 31.6 & 6.3 & 8.7 & 11.8 & 3.8 & 2.0 & 1.5 & 2.4 & 3.2 \\
\hline ..... & 10.0 & 8.1 & 6.0 & 34.2 & 6.2 & 8.6 & 10. 1 & 3.5 & 2.0 & 1.5 & 2.9 & 3.2 \\
\hline $10 \ldots$ & 9.3 & 11,5 & 7.1 & 34.3 & 5.9 & 8.3 & 8.6 & 3.3 & 1.9 & 1.5 & 4.4 & 3.0 \\
\hline $11 \ldots$ & 8. 1 & 11.3 & 8.0 & 31.0 & 5.5 & 7.8 & 9.0 & 3.3 & 2.0 & 1.5 & 5.9 & 2.9 \\
\hline $12 \ldots$ & 8.3 & 10.5 & 7.9 & 26.6 & 5. 7 & 8.0 & 9.5 & 3.1 & 1.8 & 1. 5 & 6. 6 & 2.7 \\
\hline $13 \ldots \ldots$ & 11.2 & 8.9 & 7. 6 & 18.0 & 5.7 & 8.1 & 9.4 & 3.5 & 2. 1 & 1. 4 & 7.0 & 2.6 \\
\hline $14 \ldots .$. & 22.9 & 7.7 & 7.6 & 12.9 & 5.5 & 7.8 & 8. 9 & 3.6 & 2.1 & 1.4 & 4. 4 & 2. 6 \\
\hline $15 \ldots$ & 32.9 & 7.2 & 6.8 & 11.7 & 5.3 & 6.0 & 8.7 & 3.7 & 2. 1 & 1.4 & 4.2 & 3.3 \\
\hline $16 \ldots$. & 37.1 & 7.4 & 6.2 & 10.9 & 5. 2 & 5.3 & 8.5 & 3.5 & 3.1 & 1.4 & 4.8 & 3.4 \\
\hline $17 \ldots$ & 37.9 & 8.0 & 5. 9 & 10.0 & 5.1 & 4.8 & 8.4 & 3.1 & 4. 5 & 1.4 & 5.6 & 5. 3 \\
\hline $18 \ldots \ldots$ & 35.2 & 7.9 & 6.5 & 9.4 & 4.8 & 4.4 & 8.1 & 2.9 & 4. 1 & 1. 3 & 6. 2 & 8.1 \\
\hline $19 \ldots$ & 26.3 & 7.5 & 7.5 & 8.8 & 5.1 & 4.4 & 7.5 & 2.8 & 3.5 & 1.3 & 6.4 & 8.7 \\
\hline 20. & 18. 7 & 7. 7 & 8. 2 & 12. 3 & 5.5 & 4.3 & 7. 2 & 2.8 & 3. 0 & 1. 2 & 6.2 & 8.4 \\
\hline 21. & 19.0 & 7.9 & 8.2 & 16. 2 & 6.1 & 7.0 & 6.9 & 3.0 & 2.8 & 1.2 & 5.5 & 9.1 \\
\hline 22. & 19.0 & 8.9 & 7.8 & 16. 3 & 6.5 & 7.8 & 6. 2 & 2.9 & 3. 0 & 1.2 & 4.8 & 9. 3 \\
\hline 23.. & 17.4 & 8.9 & 7.6 & 15.5 & 6.9 & 7.4 & 5.6 & 2.9 & 2. 6 & 1. 2 & 4. 4 & 8.9 \\
\hline $24 \ldots$ & 14.9 & 8.4 & 8.4 & 14.8 & 7.1 & 7.1 & 5.4 & 3.0 & 2.3 & 1. 2 & 4.2 & 7.8 \\
\hline $25 \ldots$ & 12.2 & 7.9 & 9.6 & 13.5 & 7.6 & 6.9 & 5.2 & 2.7 & 2.3 & 1.2 & 3.9 & 6.7 \\
\hline 26. & 10.5 & 7.5 & 9.5 & 13.7 & 7.8 & 6.8 & 5.0 & 2.8 & 3.6 & 1.1 & 3. 4 & 5.8 \\
\hline 27. & 9.7 & 6.7 & 10.0 & 13.6 & 6.7 & 6.7 & 4. 6 & 3. 2 & 3.6 & 1.1 & 3.1 & 5. 2 \\
\hline 28. & 8.5 & 6.4 & 10.6 & 10.4 & 6.2 & 7.3 & 4. 5 & 3.6 & 3.1 & 1.1 & 3.0 & 4.8 \\
\hline 29. & 7.7 & 5.9 & 10.3 & 9.1 & 5. 8 & 7.5 & 4.3 & 4.4 & 2.6 & 1.1 & 3.0 & 4.3 \\
\hline 30. & 6.9 & $\mid \ldots . .$. & 9.7 & 8.8 & 5. 7 & 7.2 & 4.1 & 3.8 & 2.4 & 1.1 & 3.6 & 3.9 \\
\hline 31. & 6.8 & $\ldots \ldots$ & 9.4 & $\ldots$ & 5. & ..... & 3.8 & 3.3 & $\ldots \ldots$ & 1.1 & $\ldots$. & 3.3 \\
\hline
\end{tabular}


Tennessee River at Chattanooga, Tennessee, for 1893.

\begin{tabular}{|c|c|c|c|c|c|c|c|c|c|c|c|c|}
\hline Day. & Jan. & Feb. & Mar. & Apr. & May. & June. & Julg. & Aug. & Sept. & Oct. & Nov. & Dec. \\
\hline 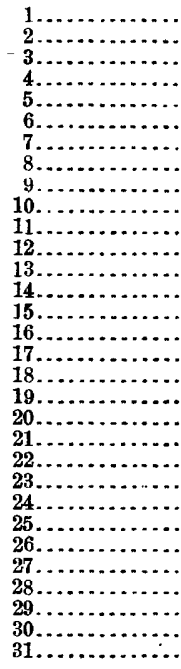 & $\begin{array}{l}3.4 \\
3.8 \\
4.7 \\
5.7 \\
5.6 \\
5.3 \\
5.2 \\
4.7 \\
4.0 \\
3.8 \\
3.4 \\
2.9 \\
2.9 \\
(a) \\
(a) \\
(a) \\
(a) \\
(a) \\
(a) \\
(a) \\
(a) \\
(a) \\
2.9 \\
3.1 \\
3.1 \\
3.4 \\
3.7 \\
3.8 \\
4.4 \\
5.3 \\
7.1\end{array}$ & \begin{tabular}{|r}
10.4 \\
12.1 \\
10.6 \\
8.6 \\
8.0 \\
7.7 \\
7.0 \\
6.5 \\
6.1 \\
6.2 \\
8.5 \\
14.7 \\
21.8 \\
23.6 \\
22.6 \\
21.3 \\
23.6 \\
29.4 \\
32.4 \\
33.4 \\
32.8 \\
28.5 \\
18.2 \\
12.3 \\
10.4 \\
9.3 \\
8.4 \\
8.2 \\
$\ldots \ldots .$. \\
$\cdots \ldots .$. \\
$\cdots \ldots .$.
\end{tabular} & $\begin{array}{r}8.4 \\
9.1 \\
8.9 \\
8.9 \\
8.7 \\
9.0 \\
9.1 \\
8.8 \\
8.8 \\
9.4 \\
11.1 \\
11.7 \\
11.5 \\
12.0 \\
10.6 \\
9.5 \\
8.4 \\
7.6 \\
7.0 \\
6.7 \\
6.3 \\
6.0 \\
5.8 \\
5.7 \\
6.4 \\
6.8 \\
6.8 \\
6.3 \\
5.9 \\
5.8 \\
5.7\end{array}$ & $\begin{array}{r}5.5 \\
5.3 \\
5.1 \\
5.1 \\
5.1 \\
5.2 \\
5.4 \\
5.4 \\
5.1 \\
5.1 \\
5.1 \\
5.0 \\
4.8 \\
10.2 \\
12.1 \\
10.4 \\
8.6 \\
7.4 \\
6.5 \\
6.4 \\
7.2 \\
7.2 \\
7.1 \\
6.8 \\
6.7 \\
6.0 \\
5.7 \\
7.0 \\
9.5 \\
10.4 \\
\ldots . . .\end{array}$ & $\begin{array}{r}10.2 \\
9.6 \\
11.0 \\
18.4 \\
24.5 \\
28.2 \\
30.0 \\
28.2 \\
18.0 \\
12.8 \\
11.7 \\
10.4 \\
9.4 \\
8.8 \\
8.1 \\
7.8 \\
9.4 \\
10.4 \\
8.9 \\
7.7 \\
7.4 \\
6.7 \\
6.1 \\
5.7 \\
5.4 \\
5.2 \\
5.0 \\
4.6 \\
5.4 \\
6.5 \\
7.4\end{array}$ & $\begin{array}{r}7.4 \\
8.0 \\
10.0 \\
8.1 \\
6.6 \\
8.3 \\
16.0 \\
20.7 \\
19.1 \\
15.2 \\
11.8 \\
8.9 \\
7.3 \\
6.8 \\
6.5 \\
6.2 \\
5.6 \\
5.4 \\
5.3 \\
5.2 \\
5.4 \\
5.6 \\
5.7 \\
5.4 \\
5.9 \\
5.6 \\
5.2 \\
5.1 \\
4.7 \\
4.1 \\
\ldots . . .\end{array}$ & $\begin{array}{l}3.9 \\
3.8 \\
4.2 \\
5.2 \\
4.8 \\
3.9 \\
3.8 \\
3.5 \\
3.4 \\
3.6 \\
3.4 \\
3.4 \\
3.3 \\
3.2 \\
3.0 \\
2.8 \\
2.7 \\
2.8 \\
3.0 \\
3.2 \\
3.5 \\
3.6 \\
4.6 \\
5.2 \\
5.5 \\
3.7 \\
3.4 \\
2.9 \\
3.7 \\
2.6 \\
2.6\end{array}$ & $\begin{array}{l}2.6 \\
2.5 \\
3.1 \\
3.5 \\
3.3 \\
3.2 \\
4.9 \\
5.0 \\
4.1 \\
3.8 \\
3.3 \\
3.0 \\
2.7 \\
2.5 \\
2.8 \\
2.9 \\
3.8 \\
5.2 \\
4.9 \\
4.0 \\
2.9 \\
2.6 \\
2.4 \\
2.3 \\
2.2 \\
1.9 \\
1.8 \\
1.6 \\
1.6 \\
1.7 \\
1.6\end{array}$ & $\begin{array}{r}8.4 \\
6.8 \\
5.0 \\
6.2 \\
6.0 \\
4.9 \\
4.3 \\
3.5 \\
3.2 \\
2.8 \\
2.8 \\
3.7 \\
5.8 \\
10.9 \\
12.7 \\
9.6 \\
8.0 \\
7.0 \\
6.1 \\
5.1 \\
4.2 \\
3.6 \\
3.4 \\
3.2 \\
3.0 \\
2.9 \\
2.7 \\
2.6 \\
2.4 \\
2.5 \\
\ldots . . .\end{array}$ & $\begin{array}{l}2.6 \\
2.5 \\
2.4 \\
2.4 \\
2.6 \\
2.5 \\
2.9 \\
3.1 \\
2.9 \\
2.9 \\
2.6 \\
2.5 \\
2.4 \\
2.5 \\
2.0 \\
1.7 \\
9.6 \\
6.4 \\
5.7 \\
5.2 \\
4.1 \\
3.4 \\
3.1 \\
2.8 \\
3.1 \\
3.3 \\
4.9 \\
4.6 \\
4.0 \\
3.5 \\
3.2\end{array}$ & $\begin{array}{r}3.1 \\
3.0 \\
2.8 \\
2.7 \\
2.6 \\
2.5 \\
2.4 \\
2.4 \\
2.3 \\
2.4 \\
2.5 \\
4.8 \\
3.8 \\
3.6 \\
3.5 \\
3.0 \\
2.8 \\
2.7 \\
2.5 \\
2.6 \\
2.6 \\
2.6 \\
2.5 \\
2.5 \\
2.4 \\
2.5 \\
2.6 \\
2.8 \\
2.7 \\
2.5 \\
\ldots \ldots .\end{array}$ & $\begin{array}{l}2.5 \\
3.9 \\
3.7 \\
4.1 \\
4.3 \\
4.7 \\
4.7 \\
4.5 \\
4.1 \\
4.0 \\
3.7 \\
3.2 \\
3.1 \\
3.0 \\
2.9 \\
3.0 \\
3.2 \\
3.5 \\
3.8 \\
3.9 \\
3.9 \\
3.5 \\
3.3 \\
3.1 \\
2.9 \\
2.8 \\
2.7 \\
2.6 \\
2.5 \\
2.7 \\
3.1\end{array}$ \\
\hline
\end{tabular}

$a$ Frozen at gage.

Tennessee River at Chattanooga, Tennessee, for 1894.

\begin{tabular}{|c|c|c|c|c|c|c|c|c|c|c|c|c|}
\hline Day. & Jan. & Feb. & Mar. & Apr. & May. & June. & July. & Aug. & Sept. & Oct. & Nov. & Dec. \\
\hline 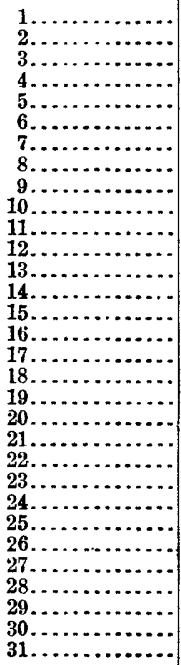 & $\begin{array}{l}2.9 \\
3.4 \\
3.8 \\
3.9 \\
3.5 \\
3.1 \\
4.9 \\
6.1 \\
9.3 \\
9.0 \\
8.5 \\
7.9 \\
8.3 \\
8.0 \\
7.8 \\
7.8 \\
7.1 \\
7.2 \\
6.3 \\
6.0 \\
5.3 \\
5.0 \\
5.0 \\
5.2 \\
5.3 \\
5.2 \\
5.4 \\
5.4 \\
5.1 \\
5.0 \\
4.9\end{array}$ & $\begin{array}{r}5.1 \\
5.0 \\
4.9 \\
5.5 \\
21.9 \\
25.5 \\
23.9 \\
19.7 \\
16.1 \\
16.0 \\
16.7 \\
15.4 \\
15.2 \\
14.1 \\
12.2 \\
10.3 \\
9.5 \\
8.6 \\
8.4 \\
8.3 \\
8.5 \\
8.7 \\
8.8 \\
8.2 \\
7.9 \\
7.6 \\
7.7 \\
7.7 \\
\cdots \cdots \cdots \\
\cdots \ldots . . \\
\cdots \cdots . .\end{array}$ & $\begin{array}{l}7.7 \\
8.2 \\
9.4 \\
9.7 \\
9.5 \\
9.3 \\
8.5 \\
8.2 \\
7.9 \\
7.2 \\
6.9 \\
6.6 \\
6.7 \\
7.2 \\
7.0 \\
6.9 \\
6.8 \\
7.3 \\
7.4 \\
7.7 \\
7.1 \\
8.8 \\
8.7 \\
8.1 \\
7.7 \\
7.3 \\
7.0 \\
6.5 \\
5.9 \\
5.7 \\
5.2\end{array}$ & $\begin{array}{r}5.0 \\
4.8 \\
5.4 \\
5.3 \\
6.8 \\
6.9 \\
7.2 \\
7.4 \\
6.6 \\
5.7 \\
5.9 \\
7.2 \\
8.5 \\
7.8 \\
7.2 \\
6.7 \\
6.3 \\
5.5 \\
5.0 \\
5.1 \\
4.9 \\
4.8 \\
4.7 \\
4.6 \\
4.5 \\
4.3 \\
4.2 \\
4.1 \\
4.0 \\
4.0 \\
\ldots . . .\end{array}$ & $\begin{array}{l}3.9 \\
3.8 \\
3.7 \\
3.7 \\
3.6 \\
3.5 \\
3.4 \\
3.3 \\
3.4 \\
3.3 \\
3.2 \\
4.7 \\
5.1 \\
4.8 \\
4.3 \\
4.0 \\
4.1 \\
5.2 \\
5.0 \\
5.4 \\
5.6 \\
6.2 \\
6.8 \\
6.9 \\
7.1 \\
6.7 \\
6.0 \\
5.6 \\
5.1 \\
4.7 \\
4.2\end{array}$ & $\begin{array}{r}3.8 \\
3.6 \\
3.5 \\
3.4 \\
3.3 \\
3.2 \\
2.9 \\
2.8 \\
2.6 \\
2.5 \\
2.5 \\
2.4 \\
2.3 \\
2.3 \\
2.2 \\
2.1 \\
2.0 \\
2.1 \\
2.4 \\
2.5 \\
2.6 \\
2.5 \\
2.3 \\
2.2 \\
2.2 \\
2.5 \\
2.6 \\
2.7 \\
2.9 \\
4.3 \\
\ldots . . .\end{array}$ & $\begin{array}{l}4.4 \\
4.0 \\
3.7 \\
4.4 \\
4.2 \\
3.7 \\
3.2 \\
3.3 \\
3.1 \\
3.3 \\
3.7 \\
3.3 \\
2.7 \\
2.4 \\
2.1 \\
1.9 \\
1.8 \\
2.8 \\
2.4 \\
2.4 \\
3.3 \\
3.7 \\
3.8 \\
3.4 \\
4.0 \\
1.4 \\
3.9 \\
3.8 \\
3.6 \\
3.3 \\
3.3\end{array}$ & $\begin{array}{l}2.9 \\
2.9 \\
2.9 \\
2.8 \\
2.9 \\
3.0 \\
2.9 \\
2.9 \\
3.0 \\
2.6 \\
2.3 \\
2.1 \\
1.9 \\
1.8 \\
2.0 \\
3.6 \\
4.6 \\
3.5 \\
3.0 \\
3.1 \\
3.6 \\
3.7 \\
4.0 \\
3.6 \\
3.0 \\
2.6 \\
2.2 \\
2.7 \\
2.4 \\
2.7 \\
4.3\end{array}$ & $\begin{array}{l}4.0 \\
3.8 \\
3.0 \\
2.6 \\
2.1 \\
2.0 \\
1.8 \\
1.7 \\
1.5 \\
1.4 \\
1.4 \\
1.4 \\
1.3 \\
1.2 \\
1.5 \\
1.8 \\
1.8 \\
2.0 \\
2.0 \\
1.6 \\
1.5 \\
1.5 \\
1.8 \\
1.8 \\
1.6 \\
1.3 \\
1.1 \\
1.0 \\
.9 \\
.8 \\
\ldots . . .\end{array}$ & $\begin{array}{r}0.9 \\
.9 \\
1.0 \\
1.5 \\
1.8 \\
1.8 \\
1.5 \\
1.3 \\
1.1 \\
1.0 \\
.8 \\
.9 \\
1.2 \\
1.9 \\
2.4 \\
2.1 \\
1.7 \\
1.4 \\
1.1 \\
1.0 \\
.9 \\
.8 \\
.8 \\
.8 \\
.8 \\
.8 \\
.7 \\
.7 \\
.7 \\
1.0 \\
1.1\end{array}$ & $\begin{array}{r}1.4 \\
1.7 \\
2.3 \\
1.6 \\
1.4 \\
1.5 \\
1.5 \\
1.3 \\
1.2 \\
1.1 \\
1.0 \\
1.0 \\
.9 \\
.8 \\
.8 \\
.8 \\
.8 \\
.7 \\
.8 \\
.9 \\
.9 \\
.9 \\
1.0 \\
1.0 \\
1.1 \\
1.2 \\
1.2 \\
1.1 \\
1.1 \\
1.1 \\
\ldots . . .\end{array}$ & $\begin{array}{r}1.0 \\
1.0 \\
.9 \\
.9 \\
.9 \\
.9 \\
.9 \\
.9 \\
1.1 \\
1.2 \\
1.6 \\
3.8 \\
8.6 \\
11.1 \\
11.2 \\
10.8 \\
8.6 \\
6.6 \\
4.7 \\
4.2 \\
3.6 \\
3.2 \\
2.8 \\
2.7 \\
2.5 \\
2.4 \\
4.2 \\
6.9 \\
8.4 \\
7.9 \\
5.8\end{array}$ \\
\hline
\end{tabular}


Tennessee River at Chattanooga, Tennessee, for 1895.

\begin{tabular}{|c|c|c|c|c|c|c|c|c|c|c|c|c|}
\hline Day. & Jan. & Feb. & Mar. & Apr. & May. & June. & July. & Aug. & Sept. & Oet. & Nov. & Dec. \\
\hline 1. & 4. 7 & 7.6 & 6.8 & 7.8 & 6.0 & 5. 6 & 3.2 & 4.4 & 3.3 & 0.9 & 1.1 & 1.4 \\
\hline & $\begin{array}{l}4.8 \\
3.9\end{array}$ & $\begin{array}{l}7.0 \\
7.2\end{array}$ & $\begin{array}{l}0.8 \\
7.8\end{array}$ & $\begin{array}{l}7.0 \\
7.4\end{array}$ & $\begin{array}{l}\text { 0. } 0 \\
\text { 5. } 8\end{array}$ & 5. 2 & $\begin{array}{l}\text {.. } \\
\text { 3. } 4\end{array}$ & $\begin{array}{l}4.4 \\
4.1\end{array}$ & $\begin{array}{l}3.0 \\
3.4\end{array}$ & $\begin{array}{r}0.8 \\
.8\end{array}$ & $\begin{array}{l}1.1 \\
1.2\end{array}$ & $\begin{array}{l}1.4 \\
1.5\end{array}$ \\
\hline 3 & $\begin{array}{l}3.8 \\
3.3\end{array}$ & $\begin{array}{l}7.2 \\
7.3\end{array}$ & 12.1 & 6.8 & 5.5 & 4.8 & $\begin{array}{l}0.4 \\
3.8\end{array}$ & $\begin{array}{l}3.1 \\
3.8\end{array}$ & $\begin{array}{l}3.4 \\
3.2\end{array}$ & .8 & $\begin{array}{l}1.2 \\
1.3\end{array}$ & $\begin{array}{l}1.4 \\
1.4\end{array}$ \\
\hline 4 & 3.2 & 7.5 & 18. 2 & 6.5 & 5.4 & 4.5 & 4.0 & 3.5 & 3.1 & .8 & 1.6 & 1.4 \\
\hline 5 & 3.1 & 7.6 & 19.9 & 6.3 & 5.7 & 4. 2 & 4.5 & 3.4 & 2.9 & .8 & 1.6 & 1.4 \\
\hline 6. & 3.1 & 7.4 & 18.2 & 6. 2 & 6.0 & 4.4 & 5.0 & 3.0 & 2.8 & .8 & 1.3 & 1.6 \\
\hline 7. & 3.3 & 6.9 & 13.4 & 6.0 & 6.5 & 4.6 & 5.0 & 3.2 & 2.8 & .8 & 1. 3 & 1.5 \\
\hline 8. & 4.0 & 6.5 & 10.5 & 9.6 & 7.0 & 5. 2 & 5.1 & 3.3 & 2.8 & .9 & 1. 2 & 1.4 \\
\hline 9. & 10.9 & 6.4 & 9.2 & 10.7 & 8.2 & 5. 1 & 5.5 & 3.3 & 2.5 & .8 & 1. 1 & 1.3 \\
\hline 10. & 20.5 & 5.0 & 8.6 & 11.4 & 8.6 & 4.6 & 5.7 & 3.0 & 2.4 & .9 & 1. 2 & 1. 3 \\
\hline 11. & 28.5 & 4. 0 & 8.1 & 13.0 & 9.0 & 4. 2 & 5.1 & 3.0 & 2.3 & 1,0 & 1. 3 & 1. 4 \\
\hline 12. & 32.1 & 3.3 & 7.5 & 12.5 & 8.8 & 3.8 & 4.4 & 2.9 & 2.4 & 1.0 & 1.7 & 1.5 \\
\hline 13. & 31.2 & 4. 2 & 7.8 & 10.4 & 8.9 & 3.6 & 3.8 & 3.2 & 2.5 & 1. 0 & 2.1 & 1.8 \\
\hline 14. & 28.3 & (a) & 8.0 & 8.8 & 9.5 & 3.5 & 3.4 & 2.9 & 2.4 & 1.0 & 2.4 & 1.9 \\
\hline 15. & 19.5 & 4.3 & 8.7 & 7. 9 & 9.0 & 3.4 & 3.2 & 2.8 & 2.3 & .9 & 2.2 & 2.0 \\
\hline 16.. & 12.3 & 4.7 & 9.4 & 7.4 & 8.2 & 3.4 & 3.2 & 2.7 & 2.2 & 1.0 & 2.0 & 1. 9 \\
\hline$\ldots \ldots$ & 10.9 & 4.7 & 9.2 & 7.0 & 7.7 & 3.8 & 3.7 & 3.1 & 2.1 & 1.0 & 1.9 & 1.8 \\
\hline $18 \ldots$ & 10.0 & 4. 2 & 9.6 & 9.0 & 7.1 & 3.7 & 3.6 & 4.3 & 2.3 & 1. 0 & 1.8 & 1.7 \\
\hline 19. & 9.7 & 4.7 & 9.4 & 11.8 & 7.0 & 3.5 & 3.3 & 4. 9 & 2.2 & .9 & 1.7 & 1. 6 \\
\hline 20. & 9.1 & 4.6 & 8.9 & 11.8 & 7. 2 & 3.2 & 3.0 & 5.7 & 2.0 & 9 & 1.5 & 1. 5 \\
\hline 21. & 9.6 & 5. 1 & 14. 3 & 9.9 & 7.1 & 3.1 & 2.7 & 5.3 & 2.1 & .8 & 1. 3 & 1.6 \\
\hline $22 \ldots$ & 10.2 & 5.6 & 20.6 & 8.6 & 6.7 & 3.2 & 2.7 & 6. 1 & 1.9 & .8 & 1. 1 & 2. 1 \\
\hline 23. & 9.9 & 6.1 & 22.7 & 7. 7 & 6.5 & 3.1 & 2.5 & 5. 3 & 1. 6 & .7 & 1. 3 & 3. 3 \\
\hline 24 & 9. 1 & 6.7 & 22.0 & 7.1 & 5.8 & 3.0 & 2.4 & 4.8 & 1.4 & .7 & 1. 4 & 4.2 \\
\hline 25. & 10.8 & 6.8 & 18.2 & 6. 7 & 5.6 & 2.9 & 2.4 & 4.6 & 1.3 & .7 & 1. 3 & 4.6 \\
\hline $26 \ldots$ & 10.8 & 6.5 & 13.0 & 6.3 & 5. 6 & 2.9 & 2.9 & 4.7 & 1. 3 & .7 & 1. 3 & 4. 3 \\
\hline 27. & 10.0 & 6.3 & 11. 3 & 6.0 & 7.0 & 2.6 & 3.8 & 4.2 & 1. 2 & .7 & 1. 3 & 4. 2 \\
\hline 28. & 9.3 & 6.3 & 10.6 & 6.0 & 7,5 & 2.5 & 10.2 & 3.7 & 1.1 & .7 & 1. 3 & 4. 5 \\
\hline 29 & 8.8 & & 9.5 & 5.9 & 7.4 & 2.5 & 10.4 & 3.5 & 1.0 & .7 & 1. 3 & 5.2 \\
\hline $30 \ldots$ & 8.6 & & 8. 9 & 5.9 & 6.7 & 2.6 & 7.4 & 3.7 & 0.9 & .7 & 1.5 & 4. 7 \\
\hline $31 \ldots$ & 8. 4 & $\ldots .$. & 8.4 & $\cdots$ & 6.0 & ... & 5. 3 & 3.4 & $\cdots$ & 1.0 & 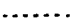 & 4. 7 \\
\hline
\end{tabular}

$a$ Frozen.

Tennessee River at Chattanooga, Tennessee, for 1896.

\begin{tabular}{|c|c|c|c|c|c|c|c|c|c|c|c|c|}
\hline Day. & Jan. & Feb. & Mar. & Apr. & May. & J une. & July. & Ang. & Sept. & Oet. & Nov. & Dec. \\
\hline 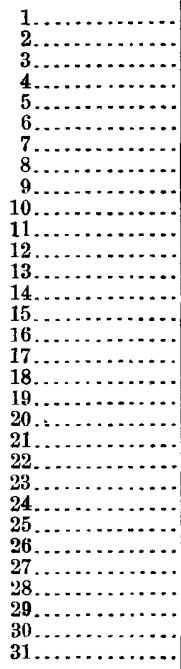 & $\begin{array}{l}4.9 \\
5.0 \\
4.9 \\
4.9 \\
4.7 \\
4.3 \\
3.6 \\
3.3 \\
3.2 \\
3.2 \\
3.1 \\
3.1 \\
2.9 \\
2.7 \\
2.6 \\
2.4 \\
2.3 \\
2.3 \\
2.3 \\
2.3 \\
2.3 \\
2.5 \\
3.1 \\
5.0 \\
6.5 \\
8.2 \\
8.0 \\
7.0 \\
6.0 \\
5.3 \\
4.8\end{array}$ & $\begin{array}{r}4.4 \\
6.2 \\
10.0 \\
11.6 \\
10.5 \\
9.3 \\
11.8 \\
14.0 \\
13.8 \\
13.2 \\
12.8 \\
11.4 \\
10.1 \\
11.1 \\
12.8 \\
13.6 \\
12.5 \\
11.0 \\
9.0 \\
7.6 \\
6.7 \\
6.0 \\
5.4 \\
4.9 \\
4.7 \\
4.6 \\
4.5 \\
4.4 \\
4.2 \\
\ldots \ldots . . \\
\ldots \ldots . .\end{array}$ & $\begin{array}{r}4.1 \\
3.9 \\
3.8 \\
3.7 \\
3.6 \\
3.5 \\
3.4 \\
3.5 \\
3.5 \\
3.4 \\
3.6 \\
3.6 \\
3.8 \\
3.8 \\
3.7 \\
3.8 \\
5.5 \\
10.1 \\
13.1 \\
15.7 \\
13.8 \\
11.2 \\
9.5 \\
8.4 \\
7.9 \\
7.5 \\
7.2 \\
6.7 \\
6.2 \\
5.8 \\
7.7\end{array}$ & $\begin{array}{r}14.8 \\
27.7 \\
34.4 \\
38.8 \\
40.5 \\
36.9 \\
23.3 \\
11.6 \\
9.0 \\
8.0 \\
7.2 \\
6.7 \\
6.2 \\
5.8 \\
5.5 \\
5.2 \\
5.0 \\
4.8 \\
4.2 \\
4.4 \\
4.2 \\
4.1 \\
4.1 \\
4.0 \\
4.0 \\
3.8 \\
3.8 \\
3.8 \\
3.6 \\
3.6 \\
\ldots . . .\end{array}$ & $\begin{array}{l}3.4 \\
3.4 \\
3.4 \\
3.5 \\
4.0 \\
4.6 \\
4.6 \\
4.3 \\
4.0 \\
3.7 \\
3.4 \\
3.1 \\
2.9 \\
2.8 \\
2.7 \\
2.6 \\
2.5 \\
2.4 \\
2.4 \\
2.2 \\
2.1 \\
2.1 \\
2.5 \\
3.2 \\
3.6 \\
3.8 \\
3.2 \\
3.1 \\
2.8 \\
2.7 \\
2.5\end{array}$ & $\begin{array}{l}2.6 \\
3.0 \\
4.4 \\
5.7 \\
5.2 \\
4.7 \\
4.1 \\
3.5 \\
3.5 \\
4.5 \\
7.0 \\
6.3 \\
5.1 \\
4.3 \\
3.6 \\
3.2 \\
3.0 \\
2.8 \\
2.9 \\
3.1 \\
3.7 \\
3.5 \\
3.5 \\
3.3 \\
3.1 \\
2.9 \\
2.6 \\
2.6 \\
2.8 \\
3.0 \\
\ldots \ldots .\end{array}$ & $\begin{array}{r}3.3 \\
3.2 \\
3.1 \\
3.2 \\
3.3 \\
3.2 \\
3.6 \\
5.0 \\
3.9 \\
14.2 \\
21.1 \\
21.6 \\
15.6 \\
11.5 \\
11.2 \\
11.4 \\
11.0 \\
13.9 \\
12.5 \\
9.6 \\
7.6 \\
6.5 \\
8.5 \\
8.8 \\
8.6 \\
7.8 \\
11.1 \\
12.2 \\
9.3 \\
7.2 \\
6.2 \\
1\end{array}$ & $\begin{array}{l}5.5 \\
5.2 \\
4.8 \\
4.6 \\
4.5 \\
4.9 \\
5.0 \\
4.2 \\
3.8 \\
3.4 \\
3.3 \\
3.4 \\
3.2 \\
3.2 \\
3.1 \\
3.0 \\
3.0 \\
2.9 \\
2.7 \\
2.6 \\
2.4 \\
2.4 \\
2.2 \\
2.2 \\
2.8 \\
2.6 \\
2.7 \\
3.2 \\
4.0 \\
3.6 \\
2.8\end{array}$ & $\begin{array}{l}2.4 \\
2.1 \\
1.9 \\
1.8 \\
1.6 \\
1.6 \\
1.5 \\
2.0 \\
2.8 \\
2.7 \\
2.4 \\
2.0 \\
1.8 \\
1.6 \\
1.6 \\
1.5 \\
1.3 \\
1.4 \\
1.4 \\
1.3 \\
1.2 \\
1.2 \\
1.3 \\
1.4 \\
1.6 \\
2.0 \\
1.7 \\
1.5 \\
1.5 \\
2.7 \\
\ldots . . .\end{array}$ & $\begin{array}{l}2.5 \\
2.3 \\
2.6 \\
3.0 \\
2.7 \\
2.6 \\
2.1 \\
1.7 \\
1.5 \\
1.4 \\
1.2 \\
1.2 \\
1.2 \\
1.2 \\
1.5 \\
1.7 \\
1.6 \\
1.6 \\
1.7 \\
1.6 \\
1.6 \\
1.4 \\
1.2 \\
1.2 \\
1.2 \\
1.2 \\
1.2 \\
1.2 \\
1.1 \\
1.1 \\
1.3\end{array}$ & $\begin{array}{r}1.2 \\
1.2 \\
1.3 \\
1.5 \\
1.5 \\
1.6 \\
2.3 \\
3.5 \\
4.2 \\
4.1 \\
3.3 \\
3.2 \\
5.8 \\
7.3 \\
6.5 \\
5.5 \\
4.9 \\
4.3 \\
3.8 \\
3.4 \\
3.0 \\
2.8 \\
2.5 \\
2.4 \\
2.3 \\
2.2 \\
2.1 \\
2.2 \\
5.3 \\
9.4 \\
\ldots . .2\end{array}$ & $\begin{array}{l}2.4 \\
2.5 \\
2.5 \\
2.6 \\
2.6 \\
2.7 \\
2.9 \\
3.0 \\
2.8 \\
2.8 \\
2.7 \\
2.6 \\
2.4 \\
2.6 \\
4.1 \\
6.5 \\
6.6 \\
6.3 \\
6.4 \\
6.8 \\
7.0 \\
7.2 \\
7.3 \\
7.0 \\
6.6 \\
5.9 \\
5.3 \\
4.8 \\
4.4 \\
3.7 \\
3.0\end{array}$ \\
\hline
\end{tabular}


Missouri River at Tounsend, Montana.

\begin{tabular}{|c|c|c|c|c|c|c|c|c|c|c|c|c|}
\hline Day. & Jan. & Feb. & Mar. & Apr. & May. & June. & July. & Ang. & Sept. & Oet. & Nor. & Dec. \\
\hline & 92. 3 & 91.6 & 88.8 & 88.9 & 89.5 & 91.8 & 92.0 & 89.1 & 88.9 & 88.9 & 89.0 & 91.5 \\
\hline 2 . & 92.2 & 91. 6 & 90.9 & 88.9 & 89.5 & 92.2 & 92.0 & 89.1 & 89.0 & 88.9 & 89.0 & 91. 8 \\
\hline $3 \ldots$ & 91.8 & 91.6 & 90.7 & 88.9 & 89.5 & 92.6 & 91.8 & 89.1 & 88.9 & 88.9 & 88.9 & 92.2 \\
\hline 4. & 91.4 & 91.6 & 90.7 & 88.9 & 89.5 & 93.0 & 91.6 & 89.1 & 88.9 & 88.9 & 88.9 & 92.8 \\
\hline $5 \ldots$ & 91.4 & 91.6 & 90.7 & 88.9 & 89.6 & 93.6 & 91.4 & 89.1 & 88.9 & 88.9 & 88.9 & 92.9 \\
\hline $6 \ldots$ & 91.6 & 91. 6 & 90.7 & 88.9 & 89.7 & 93.7 & 91.2 & 83.1 & 88.9 & 88.9 & 88.9 & 92.9 \\
\hline $7 \ldots$ & 91. 6 & 91.6 & 90.7 & 88.9 & 89.8 & 93.8 & 91.0 & 89.1 & 88.9 & 88.9 & 88.9 & 92.9 \\
\hline 8. & 91.6 & 91.6 & 90.7 & 89.0 & 89.9 & 93.7 & 91.0 & 89.1 & 88.9 & 88.9 & 88.9 & 92.9 \\
\hline 9. & 91.8 & 91.6 & 90.7 & 89.0 & 90.0 & 93.8 & 90.7 & 89.1 & 88.9 & 88.9 & 89.0 & 92.9 \\
\hline 10. & 91.8 & 91.5 & 90.7 & 89.0 & 90.0 & 93.9 & 90.6 & 89.1 & 89.0 & 88.9 & 89.0 & 92.9 \\
\hline 11. & 92.0 & 91.5 & 90.7 & 89.0 & 89.9 & 94.2 & 90.4 & 89.1 & 89.1 & 89.0 & $\cdot 89.1$ & 92.9 \\
\hline 12. & 91.9 & 91.5 & 92.2 & 89.0 & 89.8 & 94.5 & 90.4 & 89.0 & 89.1 & 88.9 & 89.1 & 92.9 \\
\hline 13. & 91.9 & 91.5 & 90.0 & 88.9 & 89.8 & 94.4 & 90.5 & 88.9 & 89. 1 & 88.9 & 89.2 & 92.8 \\
\hline 14. & 91.9 & 91.5 & 89.4 & 88.9 & 89.7 & 94.4 & 90.6 & 88.9 & 89.1 & 88.9 & 89. 2 & 92.8 \\
\hline 15. & 91.9 & 91.5 & 89.0 & 88.9 & 89.6 & 94.5 & 90.8 & 88.9 & 89.1 & 88.9 & 89.2 & 92.6 \\
\hline 16. & 91.9 & 91.5 & 88.8 & 88.9 & 89.6 & 94.6 & 90.6 & 88.9 & 89. 1 & 88.9 & 83.2 & 92.2 \\
\hline 17. & 91.9 & 91.5 & 88.6 & 88.9 & 89.6 & 94.7 & 90.5 & 88.9 & 89.1 & 88.9 & 89. 2 & 91.9 \\
\hline 18. & 91. 9 & 91.5 & 88.6 & 88.9 & 89.7 & 94.8 & 90.2 & 88.9 & 89.0 & 88.9 & 89. 1 & 91.4 \\
\hline 19. & 91.8 & 91.5 & 88.8 & 88.9 & 89.7 & 94.8 & 90.0 & 88.8 & 89.0 & 88.9 & 89.1 & 91.4 \\
\hline 20. & 91.8 & 91.5 & 89.0 & 88.9 & 89.8 & 94.9 & 89.8 & 88.8 & 89.0 & 88.9 & 89.1 & 91.4 \\
\hline 21. & 91.8 & 91.5 & 89.1 & 89.0 & 89.8 & 94.7 & 89.7 & 88.8 & 89.0 & 88.9 & 89.1 & 89.8 \\
\hline 22. & 91.8 & 90.5 & 89.1 & 89.0 & 89.8 & 94.6 & 89.7 & 88.8 & 89.1 & 88.9 & 89.1 & 89.7 \\
\hline 23. & 91.8 & 90.4 & 89.1 & 89.1 & 90.0 & 94.5 & 89.7 & 88.8 & 89. 1 & 88.8 & 89.1 & $89.5^{\circ}$ \\
\hline 24 . & 91. 8 & 90.3 & 89.1 & 89.1 & 90.0 & 94.2 & 89.6 & 88.8 & 89. 1 & 88.8 & 89. I & 89.3 \\
\hline $25 \ldots$ & 91.8 & 89.8 & 89.1 & 89.1 & 90.2 & 93.8 & 89.6 & 88.8 & 89.1 & 88.8 & 89. 1 & 89.3 \\
\hline 26. & 91.8 & 89.6 & 89.1 & 89.1 & 90.4 & 93.4 & 89.5 & 88.8 & 89.1 & 88.8 & 90.6 & 89.2 \\
\hline 27. & 91.7 & 89.2 & 89.1 & 89.2 & 90.4 & 92.8 & 89.4 & 88.7 & 89.1 & 88.8 & 90.6 & 89.2 \\
\hline 28. & 91.7 & 88.4 & 89.1 & 89.2 & 90.6 & 92.6 & 89.4 & 88.7 & 89.0 & 88.9 & 90.6 & 89.1 \\
\hline 29 . & 91.7 & 88.8 & 89.0 & 89. 4 & 91.1 & 92.4 & 89.3 & 88.7 & 88.9 & 88.9 & 90.6 & 89.0 \\
\hline 30. & 91.6 & $\ldots \ldots$ & 88.9 & 89.5 & 91.5 & 92.2 & 89.3 & 88.7 & 88.9 & 89.0 & 90.8 & 88.9 \\
\hline 31. & 91.6 & $\cdots$ & 88.9 & $\ldots .$. & 91.6 & $\ldots \ldots$ & 89.2 & 88.8 & $\ldots \ldots$ & 89.0 & $\cdots \cdots$ & 88.9 \\
\hline
\end{tabular}

Trest Gallatin River, near Salesville, Montana.

\begin{tabular}{|c|c|c|c|c|c|c|c|c|c|c|c|}
\hline Day. & Jan. & Feb. & Mar. & Apr. & May. & June. & July. & Aug. & Sept. & Oct. & Nov. \\
\hline $1 \ldots$ & 3. $G 0$ & 2. 90 & 2.70 & & 3.25 & 5.45 & 5.55 & 3. 60 & 3.40 & 3. 40 & 3.10 \\
\hline $2 \ldots$ & 3. 70 & 2.90 & 2. 60 & & 3.25 & 6.05 & 5.45 & 3. 70 & 3.40 & 3.40 & 3. 10 \\
\hline 3 & 3. 60 & 2.90 & 2.70 & & 3. 30 & 5. 95 & 5. 30 & 3.80 & 3. 40 & 3. 40 & 3.10 \\
\hline & 3. 50 & 2.90 & 2. 70 & & 3.45 & 5.85 & 5.15 & 3.75 & 3.40 & 3.35 & 3.10 \\
\hline 5. & 3.85 & 2.90 & 2. 80 & & 3.45 & 5.65 & 5.05 & 3.70 & 3. 40 & 3. 40 & 3.10 \\
\hline $6 \ldots$ & 3.90 & 2.90 & 2. 80 & & 3.45 & 5.60 & 4. 95 & 3. 70 & 3. 30 & 3.40 & 3.10 \\
\hline $7 \ldots$ & 4. 00 & 2.90 & 2.85 & & 3.40 & 5. 60 & 4. 95 & 3. 70 & 3. 30 & 3.30 & 3.05 \\
\hline 8. & 3.75 & 2.90 & $\ldots .$. & & 3.35 & $6.0,1$ & 4.85 & 3. 70 & 3. 30 & 3. 35 & 3.05 \\
\hline 9. & 3.40 & 2.90 & $\ldots$ & $\cdots$ & 3.35 & 6.95 & 4.75 & 3.65 & 3.30 & 3.45 & 3. 15 \\
\hline 10. & 3.10 & 2. 90 & ...... & 3.00 & 3.35 & 7.85 & 4. 70 & 3.55 & 3.30 & 3.50 & 3.20 \\
\hline 11. & 3.00 & 3.00 & & 3.00 & 3.35 & 7. 10 & 4. 70 & 3. 50 & 3. 40 & 3. 40 & 3.10 \\
\hline $12 \ldots$ & 2. 90 & 3.00 & & 3.00 & 3.35 & 6.65 & 4. 75 & 3.50 & 3.40 & 3. 30 & 3. 10 \\
\hline 13 & 2. 90 & 3.00 & & 2.90 & 3.30 & 6.75 & 4. 85 & 3. 50 & 3.40 & 3. 30 & 3.15 \\
\hline 14 & 2.90 & 3.00 & & 3.00 & 3.30 & 7. 15 & 4. 75 & 3.50 & 3.45 & 3. 30 & 3.20 \\
\hline 15 & 3. 0c & 3.00 & $\ldots$ & 2.90 & 3. 30 & 7.20 & 4. 60 & 3. 50 & 3. 45 & 3. 30 & 3.20 \\
\hline 10 & 3. 00 & 3.00 & . & 2.90 & 3. 30 & 7.50 & 4. 45 & 3.50 & 3.40 & 3. 30 & 3. 20 \\
\hline 17 & 3. 00 & 3.00 & & 2.90 & 3.20 & 7.50 & 4. 30 & 3.50 & 3.40 & 3. 20 & 3.20 \\
\hline 18 & 3.00 & 3.00 & & 2.90 & 3.20 & 7.90 & 4.25 & 5.50 & 3. 40 & 3. 20 & 3.10 \\
\hline 19 & 3.00 & 3.00 & & 2.90 & 3.20 & 7.8 .3 & 4. 20 & 3.50 & 3.40 & 3.20 & 3.10 \\
\hline 20 . & 2.90 & 3.00 & & 2. 90 & 3.25 & 7. 35 & 4.10 & 3. 40 & 3.40 & 3.20 & 3. 10 \\
\hline 21 & 2.90 & 3.10 & & 2.90 & 3.30 & 6.95 & 4.00 & 3. 50 & 3.30 & 3. 20 & 3. 05 \\
\hline 22 & 2.90 & 3.10 & & 3.00 & 3. 40 & 6. 50 & 4.00 & 3.45 & 3.30 & 3. 20 & 3. 20 \\
\hline 23. & 3.00 & 2.95 & & 3.00 & 3.50 & 6.20 & 4.00 & 3.40 & 3.30 & 3.20 & 3.20 \\
\hline 24 & 3.00 & 2.95 & & 3.00 & 4.10 & 6.00 & 3.90 & 3.40 & 3.30 & 3. 20 & 3. 10 \\
\hline 25 & 3. 00 & 2.95 & & 3.15 & 4. 10 & 5.90 & 3.90 & 3.40 & 3.50 & 8. 20 & 3.00 \\
\hline 26 & 3. 00 & 3.00 & & 3.20 & 4.00 & 5.85 & 3.80 & 3.35 & 3.45 & 3. 20 & 2. 80 \\
\hline & 2.90 & 2.95 & & 3.20 & 4. 10 & 5.80 & 3.70 & 3.30 & 3.40 & 3. 20 & 2.70 \\
\hline & 2. 90 & 2.90 & & 3.30 & 4.40 & 5.55 & 3.70 & 3. 30 & 3. 40 & 3. 20 & 2.70 \\
\hline & 2.90 & 2.75 & & 3. 30 & 4. 40 & 5.50 & 3.85 & 3.30 & 3.30 & 3.10 & 2. 70 \\
\hline & 2.90 & $\ldots . .$. & & 3.30 & 3.90 & 5.50 & 3.70 & $\begin{array}{r}-3.30 \\
\end{array}$ & 3.40 & 3.10 & 2.70 \\
\hline 31 & 2.90 & & & (. & 4.90 & $\ldots$ & 3.60 & 3.40 & $\ldots$. & 3. 10 & \\
\hline
\end{tabular}


Middle Creek at Bozeman, Montana.

\begin{tabular}{|c|c|c|c|c|c|c|c|}
\hline Day. & Apr. & May. & June. & July. & Aug. & Sept. & Oet. \\
\hline . . . . . & & & & & & & \\
\hline .......... & & 0.25 & $\therefore$ & & & & \\
\hline $3 \ldots \ldots \ldots$ & $\cdots$ & $\cdots$ & $\cdots$ & 0.90 & $\cdots$ & $\cdots$ & \\
\hline 5 & $\begin{array}{r}0.10 \\
\ldots . . . .\end{array}$ & $\cdots$ & 0.90 & $\cdots$ & $\cdots$ & 0.20 & \\
\hline 6 & & & & 1,05 & & & \\
\hline $8 \ldots \ldots$ & & & 1.15 & $\begin{array}{r}1.00 \\
\ldots . . .\end{array}$ & 0.35 & $\cdots$ & \\
\hline $9 \ldots \ldots \ldots \ldots \ldots \ldots \ldots \ldots \ldots$ & $\cdots$ & .33 & $\cdots$ & $\ldots \ldots$ & $\ldots$ & $\cdots$ & \\
\hline $10 \ldots \ldots$ & $\cdots$ & $\cdots$ & (n........ & $\cdots$ & $\cdots \cdots$ & & \\
\hline $11 \ldots \ldots \ldots$ & $\cdots$ & ann & 1.20 & $\cdots$ & $\ldots \ldots$ & .28 & \\
\hline 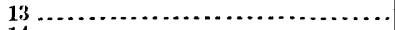 & $\ldots \ldots$ & ......... & ......... & ........ & $\ldots \ldots \ldots$ & $\cdots$ & ... \\
\hline $14 \ldots \ldots \ldots \ldots \ldots \ldots \ldots \ldots \ldots \ldots \ldots \ldots$ & $\ldots . .$. & ......... & ......... & $\ldots \ldots$ & ....... & & ... \\
\hline $15 \ldots \ldots \ldots \ldots$ & $\cdots$ & & $-\cdots$ & .88 & & & \\
\hline $16 \ldots \ldots \ldots \ldots$ & $\cdot$ & .25 & 1.30 & $\cdots$ & $\cdots \cdots$ & $\cdots$ & \\
\hline $17 \ldots \ldots \ldots \ldots \ldots \ldots \ldots \ldots \ldots \ldots$ &.- & $\cdots \ldots$ & $\cdots$ & & .28 & $\cdots \cdots$ & \\
\hline 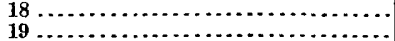 & $\ldots \ldots$ & $\cdots \ldots \ldots$ & $\because$ & .60 & $\ldots \ldots$ & .22 & $\cdots$ \\
\hline $20 \ldots \ldots$ & ... & $\ldots$ & $\theta_{1}$ & & . & & \\
\hline 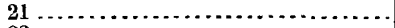 & - & $\ldots \ldots$ & $\ldots \ldots$. & & ... & ......... & .... \\
\hline $22 \ldots \ldots \ldots \ldots \ldots$ & & .48 & $\cdots \cdots$ & & 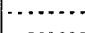 & .20 & \\
\hline 24. & .20 & .80 & & & & & \\
\hline 25 & & $\cdots$ & & & .23 & $\cdots$ & \\
\hline $26 \ldots \ldots \ldots \ldots$ & & $\cdots . . . .$. & $\ldots \ldots$ & & $\ldots$. & & $\cdots$ \\
\hline $\begin{array}{l}27 \ldots \ldots \ldots \ldots \\
28 \ldots \ldots \ldots \ldots\end{array}$ & & .93 & & & .22 & $\cdots$ & - \\
\hline 290. & & $\ldots \ldots$ & .......... & .43 & $\ldots \ldots$ & .......... & $\ldots$ \\
\hline . & $\cdots$ & 1. 80 & $\ldots \ldots . .$. & $\ldots$ & ........ & $\ldots$ & $\cdots$ \\
\hline 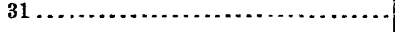 & & & & & 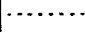 & & \\
\hline
\end{tabular}

Gallatin River at Logan, Montana.

\begin{tabular}{|c|c|c|c|c|c|c|c|c|c|c|c|}
\hline Day. & Jan. & Feb. & Apr. & May. & June. & July. & Aug. & Sept. & Oct. & Nov. & Dec. \\
\hline $1 \ldots \ldots \ldots \ldots$ & (a) & 1.00 & & 1. 30 & 3.20 & 2.75 & 1. 20 & 1.00 & 1.00 & & \\
\hline $2 \ldots \ldots \ldots \ldots$ & (a) & $: 90$ & & 1. 20 & 3.65 & 2.65 & 1. 10 & 1.00 & 1. 00 & & \\
\hline 3 & (a) & .90 & $\cdots$ & 1. 20 & 3. 80 & 2.50 & 1. 10 & 1.00 & 1.00 & & \\
\hline $4 \ldots \ldots$ & (a) & 1.00 & . & 1.20 & 3.95 & 2.50 & 1.10 & 1.00 & 1. 05 & 1.00 & \\
\hline $5 \ldots \ldots$ & (a) & .90 & $\ldots . .$. & 1.40 & 3.80 & 2.35 & 1. 10 & 1.00 & 1.10 & & \\
\hline 6. & (a) & .90 & . & 1.55 & 3.75 & 2. 20 & 1. 10 & .90 & 1. 05 & & \\
\hline $7 \ldots$ & (a) & 1. 00 & & 1.50 & 3.75 & 2.00 & 1.10 & .90 & 1.00 & 1.00 & \\
\hline 8 & (a) & 1.05 & $\ldots .$. & 1.45 & 3.70 & 1.90 & 1.10 & .90 & 1.00 & & \\
\hline $9 \ldots$. & (a) & 1.00 & 1.10 & 1. 50 & 4. 20 & 1.80 & 1.10 & .90 & 1.00 & & \\
\hline $10 \ldots \ldots$ & (a) & 1.00 & 1.10 & 1.60 & 5. 10 & 1.65 & 1.05 & 1.00 & 1.00 & & \\
\hline $11 \ldots$ & (a) & 1. 20 & 1.10 & 1.50 & 5.15 & 1.50 & 1.00 & 1.10 & 1.00 & 1.00 & 1.63 \\
\hline $12 \ldots \ldots$ & (a) & .95 & 1.05 & 1.50 & 4.50 & 1. 50 & 1. 00 & 1.20 & 1.00 & .. & 1.10 \\
\hline $13 \ldots$. & (a) & .90 & 1.00 & 1.50 & 4. 50 & 1.75 & 1. 00 & 1. 10 & 1.00 & $\ldots \ldots$ & $\ldots$ \\
\hline $14 \ldots$. & (a) & .90 & 1. 10 & 1. 40 & 4.65 & 1.60 & 1.00 & 1.05 & 1. 00 & 1.00 & \\
\hline $15 \ldots \ldots$ & (a) & .90 & 1.10 & 1. 40 & 4. 75 & 1.70 & 1. 00 & 1.00 & 1.00 & $\cdots$ & \\
\hline $16 \ldots \ldots$ & (a) & .90 & 1. 10 & 1. 30 & 5.20 & 1. 60 & 1.00 & 1.00 & .95 & & 1.10 \\
\hline 17. & (a) & 90 & 1.00 & 1.30 & 5.45 & 1.50 & 1.00 & 1.00 & 95 & & \\
\hline 18 . & (a) & .90 & 1.00 & 1. 30 & 5.60 & 1.40 & 1.00 & 1.00 & 1.00 & 1. 00 & \\
\hline 19 & (a) & .90 & 1.00 & 1. 30 & 5.10 & 1.40 & 1.00 & .95 & 1. 00 & & 1.10 \\
\hline 30. & (a) & .90 & 1.00 & 1. 30 & 4.85 & 1.40 & 1. 00 & .95 & 1.00 & $\cdots$ & \\
\hline 21. & (a) & 1.00 & 1.00 & 1. 30 & 4.50 & 1.40 & 1.00 & .95 & 1.00 & 1.00 & \\
\hline 22. & (a) & .95 & 1.00 & 1. 30 & 4.40 & 1.30 & 1.00 & .95 & 1.00 & . & \\
\hline 23. & (a) & .90 & 1.00 & 1. 30 & 4. 25 & 1.30 & 1. 00 & .95 & .95 & & \\
\hline 24. & (a) & .95 & 1.00 & 1.35 & 4. 05 & 1. 20 & 1.00 & .95 & 1. 00 & 1. 00 & 1. 00 \\
\hline 25 & (a) & 1. 00 & 1.05 & 1.70 & 3.80 & 1.10 & 1.00 & 1.10 & 1.00 & $\ldots$ & \\
\hline 26 & (a) & 1.00 & 1.10 & 1.80 & 3.55 & 1.10 & 1.00 & 1.15 & 1.00 & . & 1.00 \\
\hline 27 & (a) & 1.00 & 1.10 & 1. 80 & 3. 40 & 1.10 & 1.00 & 1.05 & 1.00 & & \\
\hline & 1. 10 & 1. 00 & 1.20 & 2. 05 & 3. 25 & 1.10 & 1.00 & 1.05 & 1. 00 & & \\
\hline 29 & 1. 10 & 1.00 & 1. 20 & 2.30 & 3.10 & 1. 20 & 1. 00 & 1.05 & 1.00 & & \\
\hline 30 & 1.10 & $\ldots$. & 1. 20 & 2.50 & 2.95 & 1. 20 & 1.00 & 1.00 & 1. 00 & & \\
\hline 31. & 1.00 & $\ldots . .$. & & 2. 70 & ....... & 1.20 & 1. 00 & ......... & 1.00 & $\ldots \ldots$ & \\
\hline
\end{tabular}

a Frozen. 
Madison River at Threeforks, Montana.

\begin{tabular}{|c|c|c|c|c|c|c|c|c|c|}
\hline Day. & Jan. & Feb. & Apr. & May. & June. & July. & Aug. & Sept. & Oet. \\
\hline 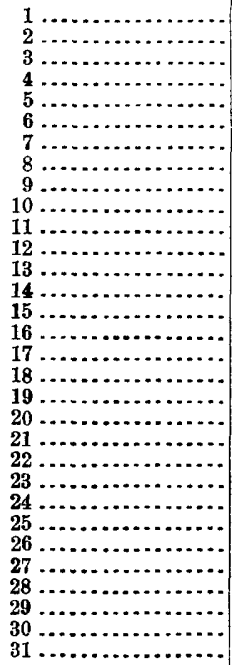 & $\begin{array}{r}1.00 \\
.90 \\
-.60 \\
-.60 \\
-.60 \\
-.60 \\
-.35 \\
.1 .95 \\
+1.20 \\
1.20 \\
1.15 \\
1.05 \\
1.00 \\
1.00 \\
1.00 \\
1.00 \\
1.10 \\
1.20 \\
1.20 \\
1.20 \\
1.20 \\
1.20 \\
1.20 \\
1.20 \\
1.20 \\
1.20 \\
1.20 \\
1.20 \\
1.10 \\
1.10\end{array}$ & $\begin{array}{r}1.10 \\
1.10 \\
1.00 \\
1.00 \\
.90 \\
.90 \\
.90 \\
.90 \\
.90 \\
.90 \\
.80 \\
.80 \\
.80 \\
.80 \\
.80 \\
.80 \\
.80 \\
.80 \\
.80 \\
.80 \\
.80 \\
.80 \\
.80 \\
.90 \\
.90 \\
.90 \\
.90 \\
.90 \\
.80 \\
\ldots . . . \\
\ldots . .\end{array}$ & 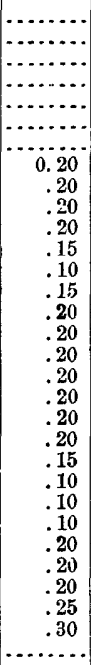 & $\begin{array}{r}0.30 \\
.30 \\
.30 \\
.30 \\
.30 \\
.30 \\
.30 \\
.35 \\
.40 \\
.40 \\
.30 \\
.30 \\
.30 \\
.30 \\
.25 \\
.20 \\
.20 \\
.20 \\
.20 \\
.25 \\
.30 \\
.35 \\
.40 \\
.45 \\
.55 \\
.70 \\
.75 \\
.85 \\
.95 \\
1.05 \\
1.20\end{array}$ & $\begin{array}{l}1.35 \\
1.45 \\
1.55 \\
1.75 \\
1.95 \\
2.05 \\
1.95 \\
1.95 \\
2.30 \\
2.55 \\
2.65 \\
2.75 \\
2.70 \\
2.75 \\
2.80 \\
2.95 \\
3.05 \\
3.15 \\
3.20 \\
3.10 \\
3.10 \\
3.00 \\
2.85 \\
2.70 \\
2.55 \\
2.45 \\
2.40 \\
2.30 \\
2.20 \\
2.15 \\
\ldots . . . .\end{array}$ & $\begin{array}{r}2.10 \\
2.00 \\
1.90 \\
1.85 \\
1.80 \\
1.70 \\
1.60 \\
1.50 \\
1.45 \\
1.40 \\
1.30 \\
1.35 \\
1.35 \\
1.30 \\
1.30 \\
1.30 \\
1.30 \\
1.20 \\
1.15 \\
1.10 \\
1.05 \\
1.00 \\
.90 \\
.90 \\
.80 \\
.80 \\
.80 \\
.70 \\
.75 \\
.80 \\
.80\end{array}$ & $\begin{array}{l}0.80 \\
.80 \\
.70 \\
.70 \\
.70 \\
.70 \\
.60 \\
.60 \\
.60 \\
.60 \\
.60 \\
.60 \\
.50 \\
.50 \\
.50 \\
.50 \\
.50 \\
.50 \\
.50 \\
.50 \\
.45 \\
.40 \\
.40 \\
.40 \\
.40 \\
.40 \\
.40 \\
.40 \\
.40 \\
.40 \\
.40\end{array}$ & $\begin{array}{r}0.50 \\
.50 \\
.50 \\
.50 \\
.50 \\
.50 \\
.50 \\
.50 \\
50 \\
.50 \\
.50 \\
.53 \\
.55 \\
.55 \\
.55 \\
.50 \\
.50 \\
.45 \\
.45 \\
.45 \\
.45 \\
.45 \\
.45 \\
.45 \\
.48 \\
.50 \\
.50 \\
.50 \\
.50 \\
.50 \\
. . . .\end{array}$ & $\begin{array}{l}0.45 \\
45 \\
.48 \\
.50 \\
.50 \\
.50 \\
.50 \\
.50 \\
.48 \\
.45 \\
.45 \\
.45 \\
.45 \\
.45 \\
.45 \\
.45 \\
.45 \\
.45 \\
.45 \\
.45 \\
.45 \\
.45 \\
.45 \\
.45 \\
.45 \\
.45 \\
.45 \\
.45 \\
.40 \\
.40 \\
.40\end{array}$ \\
\hline
\end{tabular}

Jefferson River at Šappington, Montana.

\begin{tabular}{|c|c|c|c|c|c|c|c|c|c|c|c|}
\hline Day. & Jan. & Sept. & Oct. & Nov. & Dec. & Day. & Jan. & Sept. & Oct. & Nov. & Dec. \\
\hline 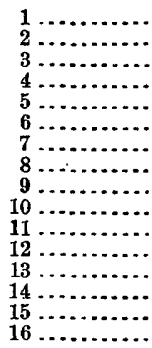 & $\begin{array}{l}(a) \\
(a) \\
(a) \\
(a) \\
(a) \\
(a) \\
(a) \\
(a) \\
(a) \\
(a) \\
(a) \\
(a) \\
(a) \\
(a) \\
(a) \\
(a)\end{array}$ & \begin{tabular}{l}
$\ldots \ldots \ldots$ \\
$\ldots \ldots \ldots$ \\
$\ldots \ldots \ldots$ \\
$\ldots \ldots \ldots$ \\
\hdashline$\ldots \ldots$ \\
$\ldots \ldots \ldots$ \\
$\ldots \ldots \ldots$ \\
0.88 \\
.90 \\
.92 \\
.97 \\
1.00 \\
1.04 \\
1.00
\end{tabular} & $\begin{array}{l}0.87 \\
.90 \\
.89 \\
.90 \\
.90 \\
.90 \\
.90 \\
.90 \\
.90 \\
.90 \\
.90 \\
.88 \\
.87 \\
.87 \\
.87 \\
.88\end{array}$ & 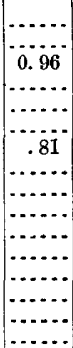 & 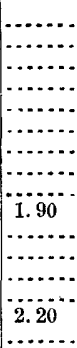 & 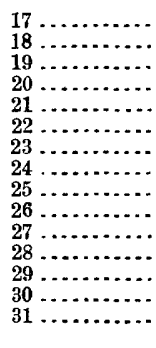 & \begin{tabular}{c}
$(a)$ \\
$(a)$ \\
0.50 \\
1.00 \\
1.50 \\
1.00 \\
$\ldots \ldots .$. \\
$(a)$ \\
$(a)$ \\
\hdashline$\ldots \ldots$ \\
$\ldots \ldots .$. \\
\hdashline$\ldots \ldots$ \\
\hdashline$\ldots \ldots$. \\
\hdashline$\ldots .$. \\
\end{tabular} & $\begin{array}{r}0.98 \\
.97 \\
.90 \\
.90 \\
.87 \\
.85 \\
.84 \\
.86 \\
.89 \\
.90 \\
.90 \\
.86 \\
.84 \\
.86 \\
\cdots . .\end{array}$ & $\begin{array}{l}0.89 \\
.89 \\
.88 \\
.87 \\
.86 \\
.87 \\
.87 \\
.87 \\
.87 \\
.86 \\
.91 \\
.93 \\
.94 \\
.94 \\
.93\end{array}$ & 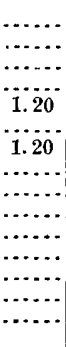 & 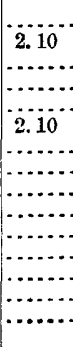 \\
\hline
\end{tabular}

a Frozen.

Little Goose Creek at Sheridan, Wyoming.

\begin{tabular}{|c|c|c|c|c|c|c|c|c|c|c|c|}
\hline Day. & May. & June. & July. & Day. & May. & June. & July. & Day. & May. & June. & July. \\
\hline $\begin{array}{r}1 . . \\
2 \ldots \\
3 \ldots \\
4 \ldots \\
5 . . \\
6 \ldots \\
7 \ldots \\
8 . . \\
9 \ldots \\
10 . . \\
11 . .\end{array}$ & $\begin{array}{l}1.70 \\
1.70 \\
1.70 \\
1.80 \\
1.80 \\
2.00 \\
2.30 \\
2.00 \\
2.20 \\
2.40 \\
2.00\end{array}$ & $\begin{array}{l}1.75 \\
2.45 \\
2.30 \\
2.50 \\
2.30 \\
2.00 \\
1.80 \\
1.75 \\
1.90 \\
1.95 \\
1.60\end{array}$ & $\begin{array}{r}0.70 \\
.70 \\
.70 \\
.75 \\
.80 \\
.75 \\
.75 \\
.75 \\
.75 \\
.80 \\
.80\end{array}$ & $\begin{array}{l}12 . . \\
13 \ldots \\
14 \ldots \\
15 . . \\
16 . . \\
17 \ldots \\
18 . . \\
19 . . \\
20 . . \\
21 \ldots \\
22 . .\end{array}$ & $\begin{array}{l}2.00 \\
1.80 \\
1.80 \\
1.80 \\
1.60 \\
1.60 \\
1.50 \\
1.40 \\
1.30 \\
1.40 \\
1.55\end{array}$ & $\begin{array}{r}1.30 \\
1.35 \\
1.30 \\
1.35 \\
1.10 \\
1.10 \\
.90 \\
.75 \\
.75 \\
.75 \\
.75\end{array}$ & $\begin{array}{r}0.80 \\
.80 \\
.85 \\
.80 \\
.85 \\
.80 \\
.95 \\
.80 \\
.80 \\
.80 \\
.80\end{array}$ & $\begin{array}{l}23 \ldots \\
24 \ldots \\
25 \ldots \\
26 . . \\
27 \ldots \\
28 \ldots \\
29 . . \\
30 . . \\
31 \ldots\end{array}$ & $\begin{array}{l}1.50 \\
1.95 \\
2.10 \\
1.70 \\
1.70 \\
1.85 \\
1.75 \\
2.50 \\
1.80\end{array}$ & $\begin{array}{r}0.75 \\
.75 \\
.75 \\
.75 \\
.75 \\
.75 \\
.75 \\
.70 \\
. . .\end{array}$ & $\begin{array}{r}0.80 \\
.85 \\
.80 \\
.80 \\
.80 \\
.90 \\
.80 \\
.80 \\
.85\end{array}$ \\
\hline
\end{tabular}

IRR $11-4$ 
Big Goose Creek at Sheridan, Wyoming.

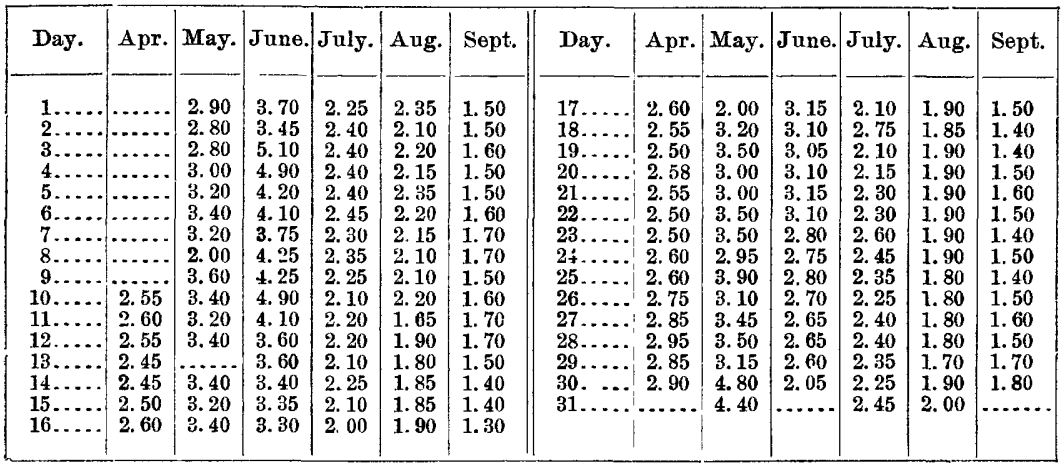

Clear Creek near Buffalo, Wyoming.

\begin{tabular}{|c|c|c|c|c|c|c|c|c|c|c|c|c|c|}
\hline Day. & May. & June. & July. & Aug. & Sept. & Oct. & Day. & May. & June. & $J u l y$. & Aug. & Sept. & Oet. \\
\hline $\begin{array}{r}1 \ldots \ldots \\
2 \ldots \ldots \\
3 \ldots \ldots \\
4 \ldots \ldots \\
5 \ldots \ldots \\
6 \ldots \ldots \\
7 \ldots \ldots \\
8 \ldots \ldots \\
9 \ldots \ldots \\
10 \ldots \ldots \\
11 \ldots \ldots \\
12 \ldots \ldots \\
13 \ldots \ldots \\
14 \ldots \ldots \\
15 \ldots \ldots \\
16 \ldots \ldots\end{array}$ & $\begin{array}{r}0.80 \\
.80 \\
.85 \\
1.00 \\
1.05 \\
1.05 \\
1.10 \\
1.00 \\
.90 \\
.80 \\
.90 \\
.90 \\
.80 \\
.70 \\
.70\end{array}$ & $\begin{array}{l}1.35 \\
1.50 \\
1.90 \\
1.60 \\
1.30 \\
1.20 \\
1.30 \\
1.30 \\
1.70 \\
1.70 \\
1.45 \\
1.45 \\
1.30 \\
1.30 \\
1.25 \\
1.25\end{array}$ & $\begin{array}{r}0.80 \\
.80 \\
.85 \\
.85 \\
.80 \\
.85 \\
.80 \\
.85 \\
.80 \\
.80 \\
.80 \\
.75 \\
.70 \\
.75 \\
1.20 \\
1.50\end{array}$ & $\begin{array}{r}1.00 \\
.90 \\
.80 \\
.95 \\
.90 \\
.80 \\
.70 \\
.90 \\
.90 \\
.75 \\
.70 \\
.68 \\
.65 \\
.65 \\
.65 \\
.65\end{array}$ & $\begin{array}{l}0.56 \\
.60 \\
.62 \\
.66 \\
.66 \\
.64 \\
.62 \\
.60 \\
.62 \\
.64 \\
.64 \\
.64 \\
.66 \\
.64 \\
.60 \\
.60\end{array}$ & $\begin{array}{c}\ldots \ldots \ldots \\
\ldots \ldots \ldots \\
\ldots \ldots \ldots \\
\ldots \ldots \ldots \\
\ldots \ldots \ldots \\
\ldots . .52 \\
0.52 \\
.54 \\
.52 \\
.50 \\
.48 \\
.48 \\
.48 \\
.48 \\
.48\end{array}$ & $\begin{array}{l}17 \ldots \ldots \\
18 \ldots \ldots \\
19 \ldots \ldots \\
20 \ldots \ldots \\
21 \ldots \ldots \\
22 \ldots \ldots \\
23 \ldots \ldots \\
24 \ldots \ldots \\
25 \ldots \ldots \\
26 \ldots \ldots \\
27 \ldots \ldots \\
29 \ldots \ldots \\
30 \ldots \ldots \\
31 \ldots \ldots\end{array}$ & $\begin{array}{r}0.70 \\
.70 \\
.70 \\
.75 \\
.70 \\
.70 \\
.85 \\
1.20 \\
1.30 \\
1.20 \\
1.10 \\
1.10 \\
1.30 \\
1.70 \\
1.70\end{array}$ & $\begin{array}{c}1.25 \\
1.30 \\
1.20 \\
\cdots \ldots . . \\
\cdots \ldots . \\
\cdots \cdots \\
\cdots \ldots . . \\
\cdots \ldots . . \\
\cdots \cdots \\
\cdots \cdots \\
.80 \\
.80 \\
.80 \\
.85 \\
\cdots \cdots\end{array}$ & $\begin{array}{r}1.40 \\
1.20 \\
1.10 \\
1.00 \\
.90 \\
.90 \\
.85 \\
.90 \\
.90 \\
.80 \\
.80 \\
.75 \\
.75 \\
.70 \\
1.15\end{array}$ & $\begin{array}{r}0.63 \\
.62 \\
.68 \\
.65 \\
.68 \\
.70 \\
.68 \\
.62 \\
.60 \\
.60 \\
.60 \\
.58 \\
.58 \\
.56 \\
.56\end{array}$ & $\begin{array}{c}0.60 \\
.62 \\
.60 \\
.60 \\
.60 \\
.58 \\
.58 \\
.58 \\
.58 \\
.58 \\
.58 \\
.58 \\
.60 \\
.62 \\
. . . .\end{array}$ & 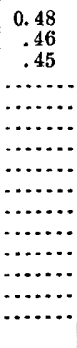 \\
\hline
\end{tabular}

Laramie River at Woods Landing, Wyoming.

\begin{tabular}{|c|c|c|c|c|c|c|c|}
\hline Day. & Apr. & May. & June. & July. & Aug. & Sept. & Oct. \\
\hline 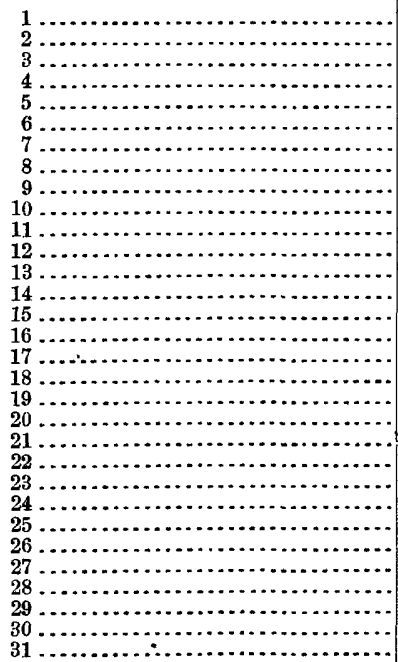 & 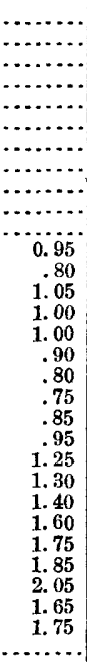 & $\begin{array}{l}1.60 \\
1.55 \\
1.55 \\
1.60 \\
1.65 \\
1.95 \\
2.15 \\
2.25 \\
2.00 \\
2.00 \\
2.05 \\
1.90 \\
1.75 \\
1.70 \\
1.65 \\
1.60 \\
1.60 \\
1.70 \\
1.85 \\
1.75 \\
1.75 \\
1.75 \\
1.83 \\
1.97 \\
2.02 \\
2.15 \\
2.00 \\
2.10 \\
2.10 \\
3.25 \\
2.85\end{array}$ & $\begin{array}{r}2.40 \\
2.28 \\
2.15 \\
2.30 \\
2.30 \\
2.05 \\
1.95 \\
1.85 \\
1.85 \\
1.90 \\
2.20 \\
1.90 \\
1.70 \\
1.90 \\
1.95 \\
1.85 \\
1.70 \\
1.65 \\
1.60 \\
1.55 \\
1.50 \\
1.45 \\
1.50 \\
1.35 \\
1.35 \\
1.35 \\
1.30 \\
1.20 \\
1.20 \\
1.20 \\
. . .2\end{array}$ & $\begin{array}{r}1.20 \\
1.10 \\
1.10 \\
1.00 \\
1.00 \\
.90 \\
.80 \\
.80 \\
.80 \\
.70 \\
.70 \\
.70 \\
.80 \\
.95 \\
1.05 \\
.85 \\
1.30 \\
1.15 \\
1.15 \\
.95 \\
.95 \\
.85 \\
1.25 \\
1.40 \\
1.30 \\
1.40 \\
1.40 \\
1.25 \\
1.00 \\
.90 \\
.80\end{array}$ & $\begin{array}{r}0.60 \\
.80 \\
.80 \\
.80 \\
.80 \\
.80 \\
.70 \\
.70 \\
.75 \\
.70 \\
.70 \\
.60 \\
.60 \\
.60 \\
.60 \\
.60 \\
.75 \\
.85 \\
1.00 \\
1.30 \\
1.30 \\
1.48 \\
1.35 \\
1.02 \\
.90 \\
.85 \\
.80 \\
.80 \\
.95 \\
1.15 \\
1.15\end{array}$ & $\begin{array}{r}1.00 \\
.90 \\
.88 \\
.80 \\
.75 \\
.70 \\
.80 \\
.80 \\
.90 \\
1.15 \\
1.25 \\
1.20 \\
1.05 \\
.95 \\
.80 \\
.85 \\
.85 \\
1.00 \\
1.00 \\
1.00 \\
1.00 \\
1.05 \\
1.10 \\
1.15 \\
1.60 \\
1.15 \\
1.30 \\
1.15 \\
1.15 \\
1.05 \\
\ldots \ldots . .\end{array}$ & 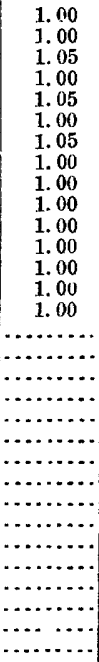 \\
\hline
\end{tabular}


Laramie River at Uva, Wyoming.

\begin{tabular}{|c|c|c|c|c|c|c|c|}
\hline Day. & Apr. & May. & June. & July. & Aug. & Sept. & Oct. \\
\hline 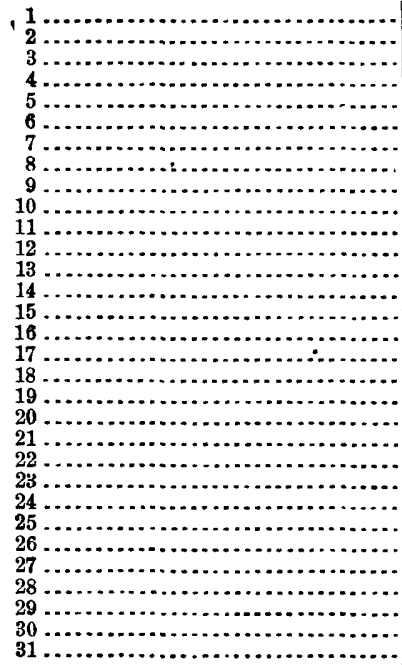 & $\begin{array}{r}\ldots \\
1.80 \\
1.80 \\
1.75 \\
1.75 \\
1.60 \\
1.60 \\
1.50 \\
1.50 \\
1.60 \\
1.70 \\
1.80 \\
1.70 \\
1.70 \\
1.40 \\
1.90 \\
1.80 \\
1.80 \\
1.70 \\
1.70 \\
1.80 \\
1.30 \\
1.70 \\
1.70 \\
2.00 \\
1.40 \\
\ldots \cdots\end{array}$ & 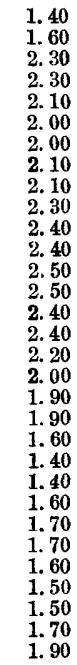 & $\begin{array}{r}2.90 \\
4.00 \\
4.60 \\
3.80 \\
3.00 \\
2.70 \\
2.70 \\
2.80 \\
2.50 \\
2.30 \\
2.00 \\
1.50 \\
1.40 \\
1.40 \\
1.30 \\
1.30 \\
1.20 \\
1.20 \\
1.10 \\
1.00 \\
1.00 \\
1.10 \\
1.00 \\
1.10 \\
1.20 \\
1.10 \\
1.00 \\
1.00 \\
1.00 \\
.90 \\
2 . .1\end{array}$ & $\begin{array}{l}0.90 \\
.80 \\
1.00 \\
.90 \\
.85 \\
.80 \\
.80 \\
.80 \\
.75 \\
.70 \\
.70 \\
.70 \\
.70 \\
.70 \\
.70 \\
.80 \\
.80 \\
2.00 \\
1.80 \\
1.70 \\
1.40 \\
1.40 \\
1.20 \\
1.10 \\
1.00 \\
1.00 \\
1.00 \\
1.00 \\
.90 \\
.90 \\
.80\end{array}$ & $\begin{array}{r}0.70 \\
.70 \\
.70 \\
.80 \\
.80 \\
.70 \\
.70 \\
.60 \\
.70 \\
.70 \\
.80 \\
.80 \\
.70 \\
.90 \\
1.00 \\
1.00 \\
1.20 \\
1.40 \\
3.20 \\
3.00 \\
1.70 \\
1.20 \\
1.00 \\
1.00 \\
.90 \\
.90 \\
.90 \\
.82 \\
.82 \\
.80 \\
.80\end{array}$ & $\begin{array}{l}0.80 \\
.80 \\
.72 \\
.72 \\
.72 \\
.72 \\
.72 \\
.72 \\
.70 \\
.70 \\
.70 \\
.70 \\
.72 \\
.72 \\
.72 \\
.80 \\
.80 \\
.82 \\
.82 \\
.82 \\
.82 \\
.90 \\
.90 \\
.90 \\
.82 \\
.82 \\
.80 \\
.80 \\
.80 \\
.90 \\
. .1 . .\end{array}$ & 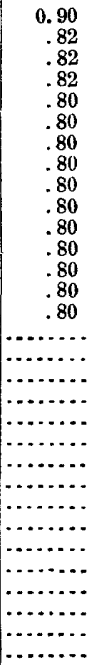 \\
\hline
\end{tabular}

North Platte River at Orin, Wyoming.

\begin{tabular}{|c|c|c|c|c|c|c|c|c|}
\hline Day. & Apr. & May. & June. & July. & Aug. & Sept. & Oet. & Dec. \\
\hline $\begin{array}{l}1 \\
1\end{array}$ & $\begin{array}{r}\ldots \ldots \\
\ldots \ldots \ldots \\
\ldots \ldots .68 \\
3.10 \\
3.47 \\
3.65 \\
3.62 \\
3.45 \\
\ldots . .65 \\
3.05 \\
3.00 \\
2.92 \\
3.10 \\
3.35 \\
3.60 \\
\ldots . .05 \\
4.05 \\
4.40 \\
4.55 \\
4.62 \\
\ldots . . . .\end{array}$ & 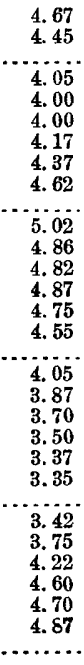 & 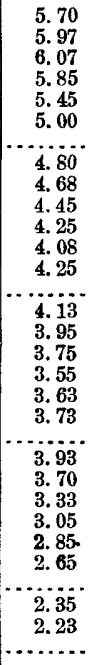 & $\begin{array}{r}2.13 \\
2.03 \\
2.00 \\
2.00 \\
\ldots 1.75 \\
1.62 \\
1.52 \\
1.48 \\
1.40 \\
1.25 \\
1.13 \\
1.10 \\
2.30 \\
2.20 \\
1.78 \\
1.65 \\
\ldots 2 . .15 \\
2.45 \\
2.35 \\
2.10 \\
2.08 \\
1.85 \\
1.73 \\
1.2 .\end{array}$ & \begin{tabular}{r}
1.68 \\
1.68 \\
1.58 \\
1.48 \\
1.38 \\
1.28 \\
1.18 \\
\hdashline 1.13 \\
.98 \\
.95 \\
.90 \\
.90 \\
.83 \\
$\ldots 1.05$ \\
.80 \\
.90 \\
1.10 \\
.78 \\
.85 \\
$\ldots . .2$ \\
.87 \\
1.95 \\
1.67 \\
1.45 \\
1.37 \\
1.42 \\
$\ldots 1.27$
\end{tabular} & $\begin{array}{r}1.20 \\
1.20 \\
1.27 \\
1.30 \\
1.40 \\
\ldots 1.17 \\
1.07 \\
1.17 \\
1.20 \\
1.07 \\
.97 \\
\ldots 1.23 \\
1.30 \\
1.35 \\
1.42 \\
1.37 \\
1.27 \\
\ldots \ldots 17 \\
1.17 \\
1.07 \\
.97 \\
.95 \\
.90 \\
.90 \\
\ldots \ldots .9 \\
1.02 \\
1.07 \\
1.10 \\
\ldots . . .\end{array}$ & $\begin{array}{r}1.17 \\
1.32 \\
1.37 \\
\ldots 1.30 \\
1.27 \\
1.22 \\
1.17 \\
1.12 \\
1.10 \\
\ldots 1.15 \\
1.10 \\
1.10 \\
1.10 \\
\ldots \ldots \ldots \\
\ldots \ldots \ldots \\
\ldots \ldots \ldots \\
\ldots \ldots \ldots \\
\ldots \ldots \ldots \\
\ldots \ldots \ldots \\
\ldots \ldots \ldots\end{array}$ & 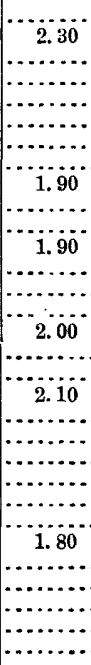 \\
\hline
\end{tabular}


North Platte River at North Platte, Nebraska.

\begin{tabular}{|c|c|c|c|c|c|c|c|c|c|c|c|}
\hline Day. & Jan. & Feb. & Apr. & May. & June. & July. & Aug. & Sept. & Oet. & Nov. & Dec. \\
\hline $1 \ldots$ & 2.50 & 2.85 & & 2. 70 & 2.75 & 2.55 & 2.20 & 1.50 & 1.90 & 2.45 & 2.40 \\
\hline & 2.50 & 2. 90 & & 2.75 & 2.90 & 2.55 & 2.15 & 1.75 & 1.80 & 2.50 & 2.40 \\
\hline $3 \ldots$ & 2.50 & 2.80 & & 2.65 & 2.75 & 2.45 & 2.25 & 1.65 & 1.75 & 2.35 & 2.40 \\
\hline$\ldots . .$. & 2.50 & 2.80 & & 2.85 & 2.55 & 2.35 & 2.30 & 1. 65 & 1. 70 & 2.20 & 2.30 \\
\hline 5 & 2.60 & 2.65 & . & 2.95 & 3.85 & 2.25 & 2.05 & 1.60 & 1.75 & 2.15 & 2. 30 \\
\hline $6 \ldots$ & 2.60 & 2.50 & & 3.15 & 4.00 & 2.20 & 2.15 & 1. 60 & 1.70 & 1.90 & 2. 30 \\
\hline $7 \ldots$ & 2.60 & 2.45 & $\cdots$ & 3.05 & 3. 85 & 2.15 & 2.25 & 1.50 & 1.85 & 1.80 & 2.40 \\
\hline $8 \ldots \ldots$ & 2.60 & 2. 05 & $\ldots .$. & 3.15 & 3. 70 & 2.00 & 2.05 & 1.50 & 1.90 & 1.70 & 2.45 \\
\hline & 2.65 & 2.00 & (n) & 3.00 & 3.55 & 2.00 & 1.80 & 1. 60 & 1.85 & 1.85 & 2.55 \\
\hline 10. & 2.75 & 2.05 & 2.30 & 2.90 & 3.45 & 1. 90 & 1. 90 & 1. 70 & 1. 90 & 1.95 & 2. 65 \\
\hline 11. & 2.80 & 1. 95 & 2.45 & 2.90 & 3. 35 & 1. 80 & 1. 70 & 1.80 & 1. 80 & 2.05 & 2.65 \\
\hline 12 & 2.70 & 2.45 & 2.65 & 2.85 & 3.35 & 1.60 & 1.60 & 1.80 & 1.85 & 2.10 & 2.75 \\
\hline 13. & 2.70 & 2.75 & 2.80 & 2.95 & 3.35 & 1. 50 & 1. 60 & 1.90 & 1.85 & 2.20 & 2. 75 \\
\hline 14. & 2. 70 & 2. 60 & 2.65 & 3.05 & 3. 20 & 1.35 & 1.50 & 2.00 & 1.85 & 2.10 & 2. 75 \\
\hline $15 \ldots$ & 2.70 & 2. 35 & 2.55 & 3.25 & 3.05 & 1.10 & 1. 45 & 2.00 & 1.85 & 2.15 & 2. 75 \\
\hline $16 \ldots$ & 2.70 & 2.35 & 2.50 & 3. 05 & 2.90 & 1.15 & 1.35 & 1.90 & 1.90 & 2.05 & 2. 65 \\
\hline $17 \ldots$ & 2.75 & 2.25 & 2.60 & 3.05 & 2.85 & 1.05 & 1. 45 & 1.80 & 1.90 & 2.25 & 2.70 \\
\hline 18. & $2.7 \mathrm{v}$ & 2.15 & 2.60 & 3. 10 & 2.85 & 1.00 & 1. 65 & 1.85 & 1.80 & 2.25 & 2. 55 \\
\hline 10 & 2.75 & 2.25 & 2.50 & 3. 00 & 3.00 & 1.15 & 1. 80 & 1.90 & 1.80 & 2.35 & 2.50 \\
\hline 20. & 2.75 & 2.45 & 2.55 & 3.05 & 2.90 & 1.25 & 1.85 & 1.85 & 1.80 & 1.90 & 2.55 \\
\hline 21. & 2.80 & 2.35 & 2.70 & 3.05 & 2.85 & 1.50 & 2.00 & 1.85 & 1.80 & 2.00 & 2.55 \\
\hline 22 . & 2.80 & 2.40 & 2.55 & 3.00 & 2.80 & 1.85 & 1. 90 & 1.90 & 1.90 & 1.85 & 2. 50 \\
\hline 23 . & 2.80 & 2.40 & 2.65 & 2.90 & 2.70 & 2.20 & 1. 80 & 1.80 & 2.00 & 1.95 & 2.60 \\
\hline 24 . & 2.80 & 2.45 & 2.55 & 2. 90 & 2.80 & 2. 45 & 1. 90 & 1.85 & 1.90 & 2.15 & 2.65 \\
\hline 25. & 2.85 & 2.35 & 2.70 & 2.85 & 2.95 & 2.05 & 1. 75 & 1.90 & 1.90 & 2.25 & 2. 75 \\
\hline 26. & 2.90 & 2.30 & 2.65 & 2.80 & 2.85 & 1.95 & 1.60 & 2.00 & 1.90 & 2.30 & 2.75 \\
\hline 27 & 2.90 & 2.35 & 2.55 & 2.70 & 2.80 & 1.85 & 1. 50 & 2.00 & 1.90 & 2.40 & 2. 75 \\
\hline 28. & 2.90 & 2.30 & 2.55 & 2. 70 & 2.80 & 1.70 & 1. 60 & 2.05 & 1.80 & 2.40 & 2. 70 \\
\hline & 2. 90 & 2. 40 & 2.65 & 2.55 & 2.90 & 2.00 & 1. 55 & 2.05 & 1. 95 & 2.40 & 2.85 \\
\hline & 2.90 & $\ldots . .$. & 2.65 & 2. 55 & 2.55 & 1.95 & 1.50 & 2.00 & 1.95 & 2.40 & 2.85 \\
\hline & 2.85 & ...... & ...... & 2.60 & ....... & 2.15 & 1.60 & ........ & 2.25 & ....... & 2.85 \\
\hline
\end{tabular}

South Platte River at Deansbury, Colorado.

\begin{tabular}{|c|c|c|c|c|c|c|c|c|c|c|c|c|}
\hline Day. & Jan. & Feb. & Mar. & Apr. & May. & $\operatorname{Tun} \theta$ & July. & Ang. & Sept. & Oct. & Nov. & Dec. \\
\hline 1. & 2.93 & 2. 96 & 3.30 & 4. 39 & 2.70 & 2. 78 & 0.98 & 1.39 & 1.80 & 1.46 & 1. 36 & 0.80 \\
\hline 2. & 2.95 & 3. 02 & 3.15 & 4. 24 & 2. 68 & 2.78 & .93 & 1.35 & 1.80 & 1.45 & 1.20 & 78 \\
\hline 3. & 2.97 & 3. 14 & 3.09 & 4. 12 & 2.64 & 2. 74 & .90 & 1.32 & 1.68 & 1. 42 & 1.20 & 1. 70 \\
\hline 4. & 2.97 & 3. 14 & 3.45 & 3.85 & 3.05 & 2. 54 & .88 & 1. 23 & 1.90 & 1. 45 & 1. 16 & $a 3.48$ \\
\hline 5. & 3.00 & 3.11 & 3. 20 & 3. 86 & 2.91 & 2. 35 & .96 & 1.18 & 1. 74 & 1. 42 & 1.07 & $\begin{array}{r}3.27 \\
\end{array}$ \\
\hline & 3.10 & 3. 12 & 3. 23 & 4.68 & 3. 30 & 2. 24 & .90 & 1.17 & 1. 27 & 1.40 & 1. 10 & 3. 06 \\
\hline & 3.35 & 3.12 & 3. 31 & 5.09 & 3. 15 & 2. 11 & .95 & 1.12 & 1. 30 & 1. 38 & .87 & 2. 96 \\
\hline & 3. 28 & 3.17 & 3. 11 & 5. 46 & 3.25 & 2. 00 & .99 & 1.02 & 1. 25 & 1. 32 & .86 & 2.90 \\
\hline 9. & 3.24 & 3.15 & 3.30 & 5.73 & 2.85 & 1.90 & 1.03 & .93 & 1. 23 & 1. 33 & 1. 11 & 3.00 \\
\hline 10. & 3.40 & 3.18 & 3. 26 & 5. 36 & 2.65 & 1.80 & .92 & .83 & 1.55 & 1. 42 & 1.20 & 2.90 \\
\hline $11 \ldots$ & 3.12 & 3.19 & 3.35 & 5. 35 & 2. 52 & 1. 68 & .82 & .77 & 1.83 & 1. 42 & 1.09 & 2. 81 \\
\hline $12 \ldots$ & 3.05 & 3. 20 & 3. 31 & 5. 53 & 2. 33 & 1.60 & .78 & .74 & 1. 74 & 1. 41 & .88 & 2. 80 \\
\hline $13 \ldots$ & 3. 03 & 3. 17 & 3. 39 & 5. 08 & 2. 30 & 1. 50 & 73 & .71 & 1. 63 & 1. 42 & 97 & 2.80 \\
\hline $14 \ldots$ & 2. 95 & 3.22 & 3. 33 & 5. 47 & 2. 44 & 1.45 & 70 & .73 & 1. 54 & 1.39 & 1.22 & 2.80 \\
\hline 15. & 2.91 & 3. 17 & 3. 16 & 6. 00 & 2. 28 & 1. 44 & 76 & .74 & 1. 43 & 1.37 & 1.19 & 2.76 \\
\hline 16. & 2.90 & 3. 26 & 3. 38 & 6.47 & 2. 30 & 1. 40 & 1. 32 & .97 & 1. 34 & 1. 31 & 1. 17 & 2.97 \\
\hline 17. & 2.96 & 3.22 & 3. 44 & 6. 21 & 2. 31 & 1.34 & 1.77 & .96 & 1. 30 & 1. 29 & 1.18 & 2. 90 \\
\hline 18. & 3.00 & 3.22 & 3. 14 & 5.72 & 2.43 & 1. 30 & 1.72 & 1. 79 & 1. 37 & 1.26 & 1.18 & 2.71 \\
\hline & 3.00 & 3. 26 & 3. 45 & 5. 36 & 2. 41 & 1. 24 & 1.92 & 1.73 & 1. 54 & 1. 33 & 1.15 & 2. 76 \\
\hline 20 & 3.05 & 3.28 & 3. 67 & 5. 41 & 2. 24 & 1. 24 & 2.09 & 1.68 & 1.71 & 1. 37 & 1.17 & 2.81 \\
\hline 21. & 3. 05 & 3.34 & 3. 80 & 5. 39 & 2.15 & 1. 27 & 2.21 & 1.34 & 1.80 & 1.27 & 1.16 & 2. 77 \\
\hline 22. & 3. 08 & 3. 33 & 3. 87 & 5.64 & 2.12 & 1. 29 & 2.11 & 1.42 & 1. 75 & 1. 28 & 1.07 & 2. 73 \\
\hline 23. & 3.02 & 3.31 & 3. 97 & 5.89 & 2. 20 & 1.25 & 1. 92 & 1. 72 & 1. 66 & 1. 40 & 1.07 & 2. 72 \\
\hline 24. & 3. 01 & 3.26 & 4. 26 & 5.97 & 2.40 & 1. 18 & 1. 62 & 1. 39 & 1.57 & 1. 37 & 1.19 & 2. 69 \\
\hline 25 & 2.95 & 3.34 & 4.75 & 6.79 & 2,43 & 1.15 & 2.14 & 1.31 & 1.56 & 1. 38 & 1.10 & 2. 67 \\
\hline 26 . & 3.02 & 3.41 & 5. 81 & a3.42. & 2. 40 & 1.10 & 2. 40 & 1.19 & 1.50 & 1.45 & .92 & 2.69 \\
\hline 27. & 2.94 & 3.42 & 6.52 & 3.59 & 2. 46 & 1. 10 & 2.35 & 1.08 & 1.50 & 1. 43 & .78 & 2.80 \\
\hline & 2.97 & 3.40 & 6.15 & 3.63 & 2.50 & 1.03 & 2. 17 & 1. 03 & 1. 51 & 1. 42 & .80 & 2. 72 \\
\hline & 3.00 & 3.23 & 5. 86 & 3.18 & 2.49 & 1.03 & 1. 81 & 1.07 & 1.55 & 1. 41 & .79 & 2.73 \\
\hline & 3.00 & $\ldots .$. & 5. 30 & 3.02 & 2.58 & 1. 03 & 1.60 & 1.17 & 1.50 & 1.31 & 81 & 2.77 \\
\hline & 2.97 & ..... & 5. 06 & $\ldots$. & 2.71 & ..... & 1.54 & 1.40 & $\ldots \ldots$ & 1. 30 & ....... & 2. 76 \\
\hline
\end{tabular}


South Platte River at Denver, Colorado.

\begin{tabular}{|c|c|c|c|c|c|c|c|c|c|c|c|c|}
\hline Day. & Jan. & Feb. & Mar. & Apr. & May. & June. & July. & Aug. & Sept. & Oct. & Nov. & Dec. \\
\hline 1. & 3.45 & 3.55 & 4. 00 & 4.45 & 5.55 & 5. 20 & 4. 65 & 4.35 & 5.10 & 4.90 & 4.65 & 4.60 \\
\hline & 3.50 . & 3.50 & 3.95 & 4. 50 & 5. 35 & 5. 30 & 4. 60 & 4.30 & 5.20 & 5. 00 & 4. 70 & 4.70 \\
\hline & 3.55 & 4. 20 & 4.15 & 4.65 & 5. 10 & 5.05 & 4. 10 & 4.60 & 5.15 & 5.00 & 4.65 & 4. 70 \\
\hline 4.. & 3.50 & 3.85 & 4.05 & 4.55 & 5. 10 & 5.00 & $(a)$ & 4.50 & 4.95 & 4. 60 & 4. 70 & 4. 65 \\
\hline 5. & 3.50 & 3.70 & 4. 00 & 4.50 & 5. 20 & 4.95 & (a) & 4. 30 & 4.85 & 4.50 & 4. 65 & 4.65 \\
\hline & 3.45 & 3.85 & 3.95 & 4. 45 & 5. 10 & 4. 90 & (a) & 4.35 & 4.85 & 4. 45 & 4. 70 & 4. 65 \\
\hline 7. & 3.40 & 3.80 & 3.95 & 4.60 & 5.00 & 4.90 & (a) & 4.30 & 4. 90 & 4.55 & 4. 65 & 4. 80 \\
\hline 8. & 3.35 & 3.75 & 4.15 & 4.55 & 5. 05 & 4. 90 & 4. 10 & 4.25 & 4. 80 & 4.50 & 4.70 & 4. 90 \\
\hline 9 & 3. 30 & 3.70 & 4.25 & 4. 70 & 4. 95 & 4.75 & 4. 20 & 4. 20 & 4. 85 & 4. 45 & 4. 73 & 4.90 \\
\hline 10. & 3. 60 & 3.85 & 4. 15 & 4.55 & 4. 70 & 4. 65 & 4. 25 & 4. 30 & 5.05 & 4.35 & 4.80 & 4. 90 \\
\hline 11.. & 3.75 & 3.35 & 4.15 & 4.45 & 4.75 & 4. 50 & 4.10 & 4. 25 & 5.05 & 4. 30 & 4.75 & 4.85 \\
\hline $12 \ldots$ & 3.80 & 3.15 & 4.15 & 4.50 & 4.45 & 4.55 & 4.00 & 4.30 & 5.10 & 4.40 & 4.85 & 4. 80 \\
\hline $13 \ldots .$. & 3. 85 & 4.00 & 4. 20 & 4.65 & 4. 35 & 4. 60 & 4.00 & 4. 20 & 505 & 4. 45 & 4.90 & 4.85 \\
\hline $14 \ldots$. & 3. 70 & 3.90 & 4.05 & 4. 90 & 4. 65 & 4.00 & 4. 30 & 4. 25 & 5.00 & 5.10 & 4.85 & 4. 90 \\
\hline $15 \ldots$ & 3.70 & 3.75 & 4.15 & 4.85 & 4. 60 & 4.40 & 4. 25 & 4. 05 & 5.05 & 5.00 & 4. 80 & 4. 80 \\
\hline $16 \ldots \ldots$ & 3.65 & 3.90 & 4.25 & 5.05 & 5.00 & 4.45 & 4.45 & 4.00 & 5.00 & 4.85 & 4. 70 & 4.80 \\
\hline $17 \ldots$ & 3.75 & 4.00 & 4. 15 & 5.10 & 4. 60 & 4.25 & 4. 40 & 4. 30 & 5.00 & 4.80 & 4. 65 & 4. 75 \\
\hline 18. & 3.85 & 4.05 & 4.00 & 4.95 & 4.85 & 4.05 & 4.70 & 4. 35 & 5.05 & 4. 90 & 4. 70 & 4. 80 \\
\hline 19. & 3.60 & 4.10 & 4.15 & 5.00 & 4.80 & 4. 10 & 4.00 & 4.50 & 5. 15 & 4.95 & 4.70 & 4. 75 \\
\hline 20 . & 3.70 & 4.05 & 4. 20 & 4.90 & 4.75 & 4. 10 & 4.30 & 4. 50 & 5.00 & 5.10 & 4. 75 & 4.85 \\
\hline 21 . & 3. 95 & 3.95 & 4.10 & 4.85 & 4. 60 & 4. 10 & 4. 45 & $(b)$ & 4. 95 & 5.10 & 4.80 & 4.80 \\
\hline 22. & 3. 80 & 3.95 & 4. 25 & 4.95 & 4. 40 & 4. 10 & 4. 50 & (b) & 5.00 & 5.15 & 4. 80 & 4.75 \\
\hline $23 \ldots$ & 3.70 & 4.00 & 4. 50 & 5.05 & 4. 40 & 4.15 & 4. 45 & $(b)$ & 4. 90 & 5.20 & 4.80 & 4. 80 \\
\hline $24 \ldots$ & 3.75 & 4.05 & 4.55 & 5.00 & 4. 40 & 4. 20 & 4.45 & (b) & 4.80 & 5.10 & 4. 90 & 4.80 \\
\hline 25. & 3.75 & 4.00 & 4.70 & 5. 10 & 4.95 & 4. 50 & 5.50 & $(b)$ & 5. 00 & 5.05 & 4. 90 & 4.75 \\
\hline 26. & 3.85 & 4. 10 & 4.85 & 5.25 & 4.85 & 4. 55 & 5.05 & 4. 80 & 5.05 & 5. 00 & 4. 90 & 4. 80 \\
\hline 27. & 3.70 & 4.05 & 4. 75 & 5. 70 & 4. 80 & 4. 50 & 4.95 & 4.85 & 5. 10 & 4. 90 & (c) & 4. 70 \\
\hline 28 . & 3.75 & 4.00 & 4. 70 & 5.65 & 4. 85 & 4. 45 & 4.80 & 4. $8 \tilde{5}$ & 5. 10 & 5. 00 & (c) & 4. 75 \\
\hline 29 . & 3. 90 & 4.05 & 4.75 & 5.75 & 4.95 & 4. 65 & 4. 90 & 4.80 & 4.95 & 4. 90 & (c) & 4. 80 \\
\hline $30 \ldots$ & 4.00 & ...... & 4. 65 & 5.60 & 4.85 & 4.65 & 4.85 & 4. 85 & 5.00 & 4. 70 & (c) & 4.65 \\
\hline $31 \ldots$ & 3. 80 & ...... & 4.55 & ...... & 5. 00 & ...... & 4. 65 & 5.00 & ...... & 4. 65 & $\ldots$. & 4. 60 \\
\hline
\end{tabular}

$a$ July 4 to 7 water below gage. $\quad b$ September 21 to 25 sand in front of gage. $\quad c$ Frozen.

South Platte River at Orchard, Colorado, for 1895-96.

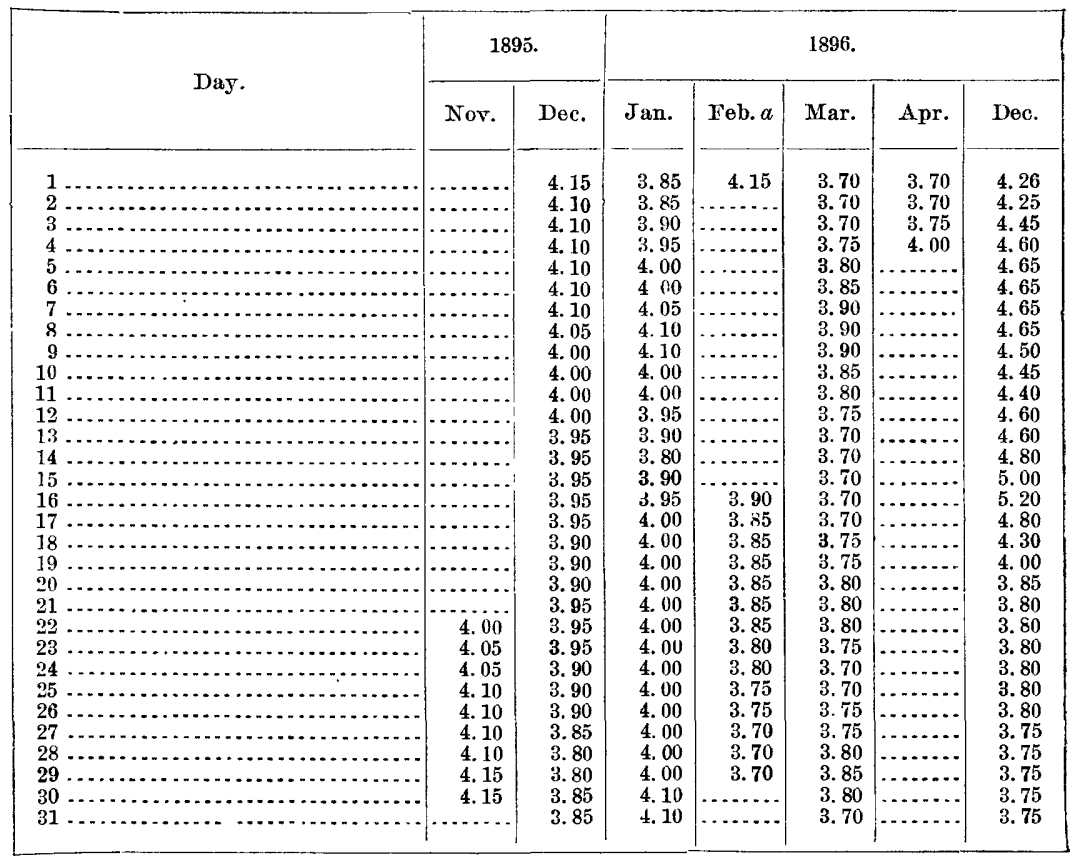

$a$ Februars 2 to 15 observer away; no record. 
Bear Creek at Morrison, Colorado.

\begin{tabular}{|c|c|c|c|c|c|c|c|}
\hline Day. & Apr. & May. & June. & July,a & Aug. $a$ & Sept. & Oet. \\
\hline 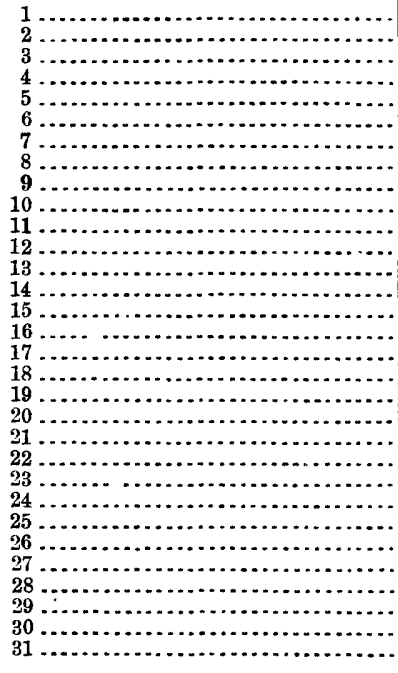 & $\begin{array}{r}\ldots \ldots \\
0.70 \\
.80 \\
.80 \\
.68 \\
.75 \\
.73 \\
.77 \\
.80 \\
.92 \\
1.00 \\
.90 \\
.95 \\
.72 \\
.75 \\
.80 \\
.97 \\
1.02 \\
1.07 \\
1.10 \\
1.15 \\
1.17 \\
1.22 \\
1.17 \\
1.11 \\
.95 \\
\ldots \ldots \ldots \\
\ldots\end{array}$ & $\begin{array}{r}0.92 \\
.87 \\
.97 \\
1.02 \\
.97 \\
1.10 \\
1.07 \\
1.02 \\
.87 \\
.90 \\
.92 \\
.87 \\
.82 \\
.80 \\
.85 \\
.82 \\
.92 \\
.92 \\
.90 \\
.87 \\
.87 \\
.92 \\
.95 \\
1.07 \\
1.05 \\
1.02 \\
.92 \\
.95 \\
.97 \\
1.02 \\
1.00\end{array}$ & $\begin{array}{r}1.00 \\
.95 \\
.90 \\
.85 \\
.83 \\
.85 \\
.85 \\
.80 \\
.78 \\
.75 \\
.75 \\
.71 \\
.72 \\
.71 \\
.73 \\
.75 \\
.74 \\
.75 \\
.75 \\
.73 \\
.73 \\
.73 \\
.70 \\
.70 \\
.78 \\
.95 \\
.80 \\
.80 \\
.75 \\
.73\end{array}$ & $\begin{array}{r}0.70 \\
.69 \\
.78 \\
.70 \\
.75 \\
.70 \\
.78 \\
.78 \\
.78 \\
.68 \\
.63 \\
.60 \\
.55 \\
.55 \\
.58 \\
.80 \\
.95 \\
1.00 \\
1.03 \\
1.03 \\
.93 \\
.90 \\
1.03 \\
.88 \\
\ldots \ldots \ldots \\
\ldots \ldots \ldots \\
\ldots \ldots \ldots \\
\ldots \ldots \ldots \\
\ldots \ldots \ldots \\
\ldots \ldots \ldots\end{array}$ & $\begin{array}{r}\ldots \ldots \\
\ldots \ldots . \\
\ldots \ldots . \\
\ldots \ldots .38 \\
3.00 \\
3.00 \\
3.00 \\
2.88 \\
2.88 \\
2.85 \\
2.83 \\
2.80 \\
2.78 \\
2.75 \\
2.75 \\
2.78 \\
2.80 \\
2.83 \\
2.85 \\
2.90 \\
2.98 \\
2.98 \\
2.88 \\
2.80 \\
2.80 \\
2.80 \\
2.78 \\
2.75 \\
2.88 \\
2.98\end{array}$ & $\begin{array}{l}2.88 \\
2.83 \\
2.90 \\
2.90 \\
2.85 \\
2.80 \\
2.83 \\
2.79 \\
2.83 \\
3.00 \\
2.95 \\
2.90 \\
2.85 \\
2.78 \\
2.75 \\
2.75 \\
2.75 \\
2.80 \\
2.98 \\
2.98 \\
2.88 \\
2.88 \\
2.83 \\
2.80 \\
2.80 \\
2.80 \\
2.90 \\
2.85 \\
2.80 \\
2.80 \\
\ldots . . . .\end{array}$ & $\begin{array}{l}2.80 \\
2.75 \\
2.75 \\
2.75 \\
2.75 \\
2.75 \\
2.75 \\
2.75 \\
2.73 \\
2.73 \\
2.70 \\
2.70 \\
2.70 \\
2.70 \\
2.70 \\
2.70 \\
2.70 \\
2.70 \\
2.70 \\
2.70 \\
2.70 \\
2.70 \\
2.70 \\
2.78 \\
2.78 \\
2.75 \\
2.75 \\
2.78 \\
2.70 \\
2.70 \\
2.65\end{array}$ \\
\hline
\end{tabular}

a From July 25 to August 5 no record; rod washed away by flood.

South Boulder Creek at Marshall, Colorado.

\begin{tabular}{|c|c|c|c|c|c|c|c|}
\hline Day. & Apr. & May. & June. & July. & Aug. & Sept. & Oct. \\
\hline 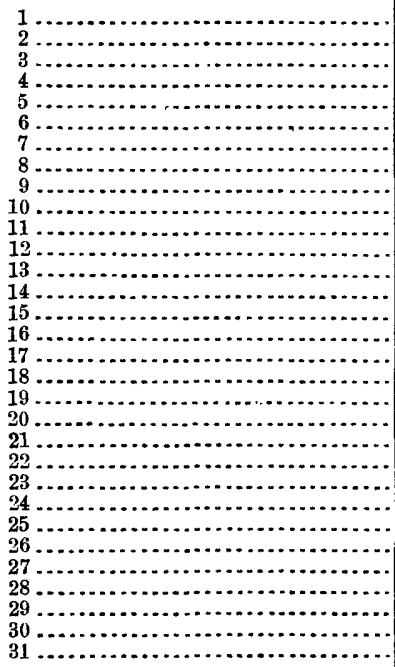 & $\begin{array}{l}0.80 \\
1.05 \\
.90 \\
.95 \\
.90 \\
.90 \\
1.05 \\
1.05 \\
1.05 \\
1.05 \\
1.15 \\
1.15 \\
1.20 \\
1.35 \\
1.35 \\
1.35 \\
1.45 \\
1.15 \\
1.20 \\
1.20 \\
1.40 \\
1.55 \\
1.60 \\
1.75 \\
1.70 \\
1.80 \\
1.90 \\
1.90 \\
1.80 \\
1.80 \\
1.2\end{array}$ & $\begin{array}{l}1.85 \\
1.55 \\
1.65 \\
1.85 \\
1.70 \\
1.90 \\
1.95 \\
2.05 \\
2.10 \\
2.10 \\
2.20 \\
1.95 \\
1.80 \\
1.75 \\
1.70 \\
1.75 \\
1.75 \\
1.70 \\
1.65 \\
1.70 \\
1.65 \\
1.70 \\
1.85 \\
2.00 \\
2.00 \\
2.30 \\
2.35 \\
2.40 \\
2.45 \\
2.55 \\
2.50\end{array}$ & $\begin{array}{l}2.30 \\
2.10 \\
2.10 \\
2.30 \\
2.15 \\
2.20 \\
2.00 \\
1.95 \\
2.20 \\
2.15 \\
2.25 \\
2.15 \\
2.10 \\
2.05 \\
2.05 \\
2.00 \\
1.95 \\
1.95 \\
1.95 \\
1.90 \\
1.90 \\
1.85 \\
1.85 \\
1.80 \\
1.75 \\
1.70 \\
1.65 \\
1.65 \\
1.65 \\
1.60 \\
\ldots \ldots . .\end{array}$ & $\begin{array}{l}1.55 \\
1.55 \\
1.55 \\
1.50 \\
1.50 \\
1.45 \\
1.45 \\
1.45 \\
1.40 \\
1.40 \\
1.40 \\
1.35 \\
1.35 \\
1.35 \\
1.30 \\
1.60 \\
1.50 \\
1.50 \\
1.50 \\
1.40 \\
1.30 \\
1.30 \\
1.30 \\
1.30 \\
1.30 \\
1.30 \\
1.30 \\
1.30 \\
1.25 \\
1.25 \\
1.20\end{array}$ & $\begin{array}{l}1.15 \\
1.05 \\
1.05 \\
1.05 \\
1.05 \\
1.05 \\
1.05 \\
1.05 \\
.95 \\
.95 \\
.95 \\
.95 \\
.95 \\
.95 \\
.95 \\
.95 \\
.95 \\
1.00 \\
1.40 \\
1.90 \\
1.45 \\
1.20 \\
1.30 \\
1.15 \\
1.10 \\
1.10 \\
1.10 \\
1.10 \\
1.10 \\
1.10 \\
1.10\end{array}$ & $\begin{array}{r}1.10 \\
1.10 \\
1.10 \\
1.10 \\
1.10 \\
1.10 \\
1.05 \\
1.05 \\
1.05 \\
1.10 \\
1.10 \\
1.10 \\
1.10 \\
1.10 \\
1.05 \\
1.05 \\
1.10 \\
1.15 \\
1.15 \\
1.05 \\
1.05 \\
1.00 \\
1.00 \\
1.00 \\
1.00 \\
1.00 \\
1.00 \\
1.00 \\
1.00 \\
1.00 \\
\cdots+. . .\end{array}$ & $\begin{array}{r}0.95 \\
.95 \\
.85 \\
.80 \\
.80 \\
.80 \\
.85 \\
1.00 \\
1.00 \\
1.00 \\
1.00 \\
1.00 \\
.95 \\
.95 \\
.90 \\
.90 \\
.90 \\
.90 \\
.90 \\
.85 \\
.80 \\
.80 \\
.90 \\
.90 \\
.90 \\
.90 \\
.90 \\
.80 \\
.80 \\
.80 \\
.80\end{array}$ \\
\hline
\end{tabular}


Boulder Creek at Boulder, Colorado.

\begin{tabular}{|c|c|c|c|c|c|c|c|}
\hline Day. & Apr. & May. & June. & July. & Aug. & Sept. & Oet. \\
\hline 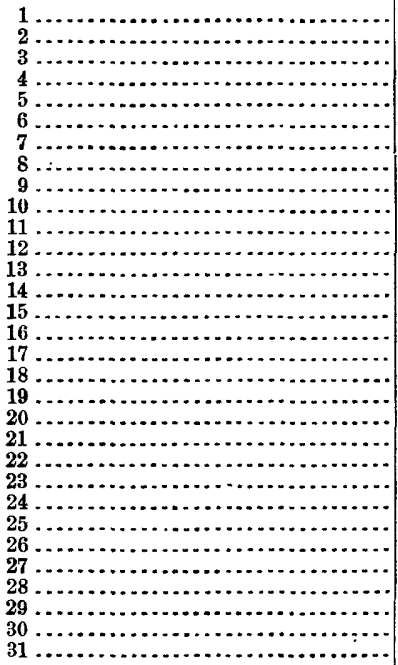 & $\begin{array}{r}0.20 \\
.35 \\
.22 \\
.27 \\
.25 \\
.27 \\
.27 \\
.30 \\
.45 \\
.47 \\
.50 \\
.50 \\
.52 \\
.57 \\
.57 \\
.77 \\
.77 \\
.77 \\
.62 \\
.75 \\
.82 \\
1.00 \\
1.07 \\
1.20 \\
1.27 \\
1.45 \\
1.80 \\
1.85 \\
1.75 \\
1.60 \\
\ldots . . .\end{array}$ & $\begin{array}{l}1.10 \\
1.10 \\
1.15 \\
1.17 \\
1.25 \\
1.35 \\
1.45 \\
1.85 \\
1.85 \\
1.75 \\
1.75 \\
1.40 \\
1.30 \\
1.17 \\
1.12 \\
1.07 \\
.95 \\
1.07 \\
1.02 \\
1.00 \\
1.12 \\
1.15 \\
1.45 \\
1.70 \\
1.90 \\
1.95 \\
2.05 \\
2.15 \\
2.20 \\
2.32 \\
2.15\end{array}$ & $\begin{array}{l}2.05 \\
1.77 \\
1.90 \\
1.92 \\
1.97 \\
1.97 \\
1.90 \\
1.88 \\
1.95 \\
1.95 \\
2.00 \\
1.88 \\
1.80 \\
1.83 \\
1.88 \\
1.80 \\
1.75 \\
1.65 \\
1.60 \\
1.55 \\
1.55 \\
1.55 \\
1.48 \\
1.45 \\
1.48 \\
1.43 \\
1.40 \\
1.38 \\
1.33 \\
1.33 \\
\ldots . .2\end{array}$ & $\begin{array}{l}1.30 \\
1.30 \\
1.30 \\
1.30 \\
1.35 \\
1.73 \\
1.57 \\
1.45 \\
1.38 \\
1.35 \\
1.28 \\
1.23 \\
1.15 \\
1.18 \\
1.32 \\
1.50 \\
1.45 \\
1.38 \\
1.35 \\
1.25 \\
1.23 \\
1.30 \\
1.35 \\
1.45 \\
1.45 \\
1.40 \\
1.38 \\
1.33 \\
1.30 \\
1.18 \\
1.05\end{array}$ & $\begin{array}{r}0.98 \\
.95 \\
.88 \\
.83 \\
.80 \\
.80 \\
.78 \\
.73 \\
.69 \\
.65 \\
.65 \\
.65 \\
.63 \\
.60 \\
.60 \\
.65 \\
.70 \\
1.23 \\
2.15 \\
1.05 \\
1.20 \\
1.35 \\
1.28 \\
1.15 \\
.98 \\
.88 \\
.88 \\
.90 \\
1.00 \\
1.10 \\
1.20\end{array}$ & $\begin{array}{r}1.08 \\
1.00 \\
1.08 \\
1.03 \\
1.00 \\
.98 \\
.93 \\
.88 \\
.85 \\
.90 \\
.90 \\
.80 \\
.73 \\
.70 \\
.65 \\
.60 \\
.63 \\
.75 \\
.90 \\
.80 \\
.80 \\
.75 \\
.78 \\
.80 \\
.75 \\
.80 \\
.80 \\
.75 \\
.70 \\
.70 \\
. . . .\end{array}$ & $\begin{array}{l}0.65 \\
.63 \\
.60 \\
.55 \\
.50 \\
.50 \\
.45 \\
.43 \\
.45 \\
.45 \\
.53 \\
.60 \\
.58 \\
.55 \\
.50 \\
.50 \\
.45 \\
.45 \\
.45 \\
.43 \\
.40 \\
.40 \\
.45 \\
.45 \\
.40 \\
.45 \\
.45 \\
.45 \\
.40 \\
.40 \\
.35\end{array}$ \\
\hline
\end{tabular}

St. Vrain Creek at Lyons, Colorado.

\begin{tabular}{|c|c|c|c|c|c|c|c|}
\hline Day. & Apr. & May. & June. & July. & Aug. & Sept. & Oet. \\
\hline 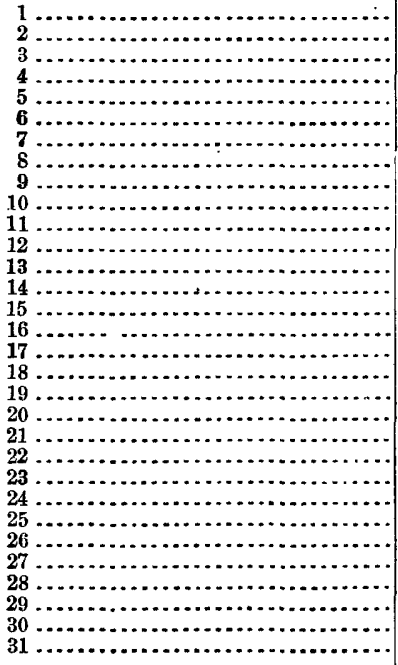 & $\begin{array}{l}1.63 \\
1.77 \\
1.85 \\
1.90 \\
1.85 \\
2.00 \\
2.05 \\
2.20 \\
2.10 \\
2.10 \\
2.15 \\
2.20 \\
2.10 \\
2.15 \\
2.30 \\
2.30 \\
2.15 \\
2.10 \\
2.00 \\
2.35 \\
2.55 \\
2.55 \\
2.60 \\
2.55 \\
2.60 \\
2.70 \\
2.95 \\
2.90 \\
2.45 \\
2.65 \\
\cdots\end{array}$ & $\begin{array}{l}2.60 \\
2.65 \\
2.45 \\
2.55 \\
2.55 \\
2.80 \\
2.95 \\
3.15 \\
3.20 \\
3.10 \\
3.10 \\
2.80 \\
2.65 \\
2.50 \\
2.50 \\
2.50 \\
2.50 \\
2.50 \\
2.60 \\
2.50 \\
2.50 \\
2.55 \\
2.85 \\
3.10 \\
3.40 \\
3.40 \\
3.35 \\
3.55 \\
3.85 \\
4.40 \\
3.85\end{array}$ & $\begin{array}{r}3.50 \\
3.45 \\
3.50 \\
3.65 \\
3.35 \\
3.60 \\
3.30 \\
3.30 \\
3.70 \\
3.70 \\
3.70 \\
3.40 \\
3.35 \\
3.35 \\
3.35 \\
3.35 \\
3.30 \\
3.30 \\
3.10 \\
3.20 \\
3.15 \\
3.15 \\
3.10 \\
2.95 \\
3.25 \\
2.85 \\
2.80 \\
2.80 \\
2.85 \\
2.75 \\
\ldots . . . .\end{array}$ & $\begin{array}{l}2.80 \\
2.85 \\
2.85 \\
2.80 \\
2.80 \\
2.80 \\
2.80 \\
2.80 \\
2.70 \\
2.70 \\
2.75 \\
2.65 \\
2.65 \\
2.65 \\
2.85 \\
3.70 \\
3.05 \\
2.95 \\
2.80 \\
2.75 \\
2.65 \\
2.70 \\
2.95 \\
3.30 \\
3.15 \\
3.05 \\
2.85 \\
2.70 \\
2.60 \\
2.60 \\
2.53\end{array}$ & $\begin{array}{l}2.50 \\
2.50 \\
2.50 \\
2.50 \\
2.48 \\
2.48 \\
2.48 \\
2.40 \\
2.38 \\
2.30 \\
2.30 \\
2.28 \\
2.23 \\
2.20 \\
2.20 \\
2.25 \\
2.38 \\
3.40 \\
3.10 \\
2.70 \\
2.80 \\
3.50 \\
3.35 \\
2.95 \\
2.75 \\
2.70 \\
2.60 \\
2.55 \\
2.50 \\
2.65 \\
2.75\end{array}$ & $\begin{array}{r}2.75 \\
2.63 \\
2.75 \\
2.55 \\
2.58 \\
2.55 \\
2.55 \\
2.30 \\
2.35 \\
2.45 \\
2.85 \\
2.80 \\
2.55 \\
2.45 \\
2.43 \\
2.35 \\
2.38 \\
2.45 \\
2.58 \\
2.50 \\
2.53 \\
2.45 \\
3.50 \\
2.58 \\
2.45 \\
2.43 \\
2.40 \\
2.43 \\
2.38 \\
2.38 \\
\cdots \ldots\end{array}$ & $\begin{array}{l}2.38 \\
2.33 \\
2.38 \\
2.38 \\
2.38 \\
2.30 \\
2.30 \\
2.28 \\
2.30 \\
2.28 \\
2.30 \\
2.25 \\
2.25 \\
2.28 \\
2.30 \\
2.28 \\
2.25 \\
2.25 \\
2.23 \\
2.25 \\
2.28 \\
1.93 \\
1.75 \\
1.85 \\
1.75 \\
1.63 \\
2.20 \\
2.23 \\
2.20 \\
2.28 \\
2.20 \\
\end{array}$ \\
\hline
\end{tabular}


Big Thompson Creek at Arkins, Colorado.

\begin{tabular}{|c|c|c|c|c|c|c|c|}
\hline Day. & Apr. & May. & June. & July. & Aug. & Sept. & Oct. \\
\hline 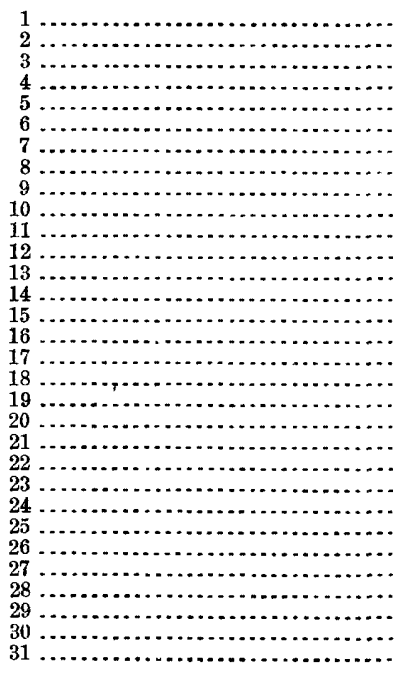 & 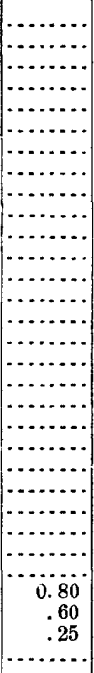 & $\begin{array}{r}0.10 \\
.50 \\
.80 \\
.85 \\
.90 \\
1.00 \\
1.10 \\
1.05 \\
1.20 \\
1.25 \\
1.35 \\
1.15 \\
1.10 \\
.95 \\
.90 \\
.85 \\
.85 \\
.85 \\
.90 \\
.95 \\
1.00 \\
1.10 \\
1.30 \\
1.70 \\
1.75 \\
1.75 \\
1.75 \\
1.65 \\
1.80 \\
2.30 \\
1.80\end{array}$ & $\begin{array}{r}1.35 \\
1.65 \\
1.80 \\
1.80 \\
1.65 \\
1.70 \\
1.50 \\
1.55 \\
1.45 \\
1.55 \\
1.65 \\
1.45 \\
1.55 \\
1.65 \\
1.55 \\
1.55 \\
1.55 \\
1.55 \\
1.30 \\
1.35 \\
1.40 \\
1.35 \\
1.40 \\
1.30 \\
1.25 \\
1.25 \\
1.20 \\
1.20 \\
1.25 \\
1.20 \\
\ldots \ldots . .\end{array}$ & $\begin{array}{l}1.20 \\
1.25 \\
1.25 \\
1.20 \\
1.20 \\
1.20 \\
1.15 \\
1.55 \\
1.20 \\
1.15 \\
1.25 \\
1.20 \\
1.10 \\
1.15 \\
1.20 \\
1.70 \\
1.55 \\
1.25 \\
1.45 \\
1.35 \\
1.30 \\
1.20 \\
1.35 \\
1.70 \\
1.45 \\
1.45 \\
1.45 \\
1.40 \\
1.40 \\
1.30 \\
1.25\end{array}$ & $\begin{array}{r}1.20 \\
1.10 \\
1.05 \\
1.00 \\
.95 \\
.90 \\
.75 \\
.75 \\
.75 \\
.70 \\
.75 \\
.75 \\
.75 \\
.75 \\
.65 \\
.80 \\
.70 \\
.75 \\
.85 \\
.95 \\
1.50 \\
1.75 \\
1.65 \\
1.55 \\
1.45 \\
1.35 \\
1.25 \\
1.15 \\
1.00 \\
1.10 \\
1.30\end{array}$ & $\begin{array}{r}1.25 \\
1.00 \\
1.00 \\
1.00 \\
1.00 \\
.85 \\
.80 \\
1.00 \\
.95 \\
.90 \\
1.00 \\
.95 \\
.85 \\
.90 \\
.90 \\
.80 \\
1.00 \\
.95 \\
.95 \\
1.00 \\
1.05 \\
1.00 \\
1.00 \\
1.05 \\
1.00 \\
1.05 \\
1.00 \\
1.05 \\
1.00 \\
1.00 \\
\ldots \ldots . .\end{array}$ & $\begin{array}{l}1.05 \\
.95 \\
.95 \\
.90 \\
.90 \\
.85 \\
.90 \\
.80 \\
.90 \\
.85 \\
.90 \\
.85 \\
.85 \\
.85 \\
.90 \\
.85 \\
.80 \\
.75 \\
.70 \\
.65 \\
.60 \\
.60 \\
.65 \\
.65 \\
.60 \\
.40 \\
.40 \\
.40 \\
.40 \\
.40 \\
.40\end{array}$ \\
\hline
\end{tabular}

Frenchman River at Palisañe, Nebraska.

[River heights on main and supplementary gages. Gage heights are all referred to original zero of main gage.]

\begin{tabular}{|c|c|c|c|c|c|c|}
\hline \multirow[b]{2}{*}{ Date. } & \multirow[b]{2}{*}{$\begin{array}{l}\text { South } \\
\text { gage. }\end{array}$} & \multirow[b]{2}{*}{$\begin{array}{l}\text { Main } \\
\text { gage. }\end{array}$} & \multirow[b]{2}{*}{$\begin{array}{l}\text { North } \\
\text { gage. }\end{array}$} & \multicolumn{3}{|c|}{ Differences in heights. } \\
\hline & & & & $\begin{array}{l}\text { South } \\
\text { gage, } \\
\text { main } \\
\text { gage. }\end{array}$ & $\begin{array}{c}\text { Main } \\
\text { gage, } \\
\text { north } \\
\text { gage. }\end{array}$ & $\begin{array}{c}\text { South } \\
\text { gage, } \\
\text { north } \\
\text { gage. }\end{array}$ \\
\hline 1896. & & & & & & \\
\hline June $18 \ldots \ldots \ldots \ldots \ldots \ldots \ldots \ldots$ & 1.85 & 1.66 & 1. 11 & 0.19 & 0.55 & 0.74 \\
\hline June $23 . \ldots \ldots$ & 2.11 & 2.05 & 1. 35 & .06 & .70 & .76 \\
\hline June $25 \ldots \ldots \ldots \ldots \ldots \ldots \ldots$ & 2.10 & 1.93 & 1.35 & .17 & .58 & .75 \\
\hline June 27 (1) & 2.06 & 1.87 & 1.34 & .19 & .53 & .72 \\
\hline July $1 \ldots \ldots \ldots \ldots \ldots \ldots . . . . . .$. & 2.14 & 1. 96 & 1. 40 & .18 & .56 & .74 \\
\hline 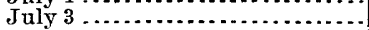 & 2.13 & 1. 94 & 1. 39 & .19 & .55 & .74 \\
\hline July $5 \ldots .$. & 2.13 & 1. 93 & 1.38 & .20 & .55 & .74 \\
\hline July $7 \ldots$ & 2.03 & 1.87 & 1. 27 & .16 & .60 & .76 \\
\hline July $9 \ldots$ & 1.97 & 1.75 & 1. 15 & .22 & .60 & .82 \\
\hline July $11 \ldots$. & 1. 94 & 1. 73 & 1.17 & .21 & .56 & .77 \\
\hline July $14 \ldots$. & 1.96 & 1.74 & 1.19 & .22 & .55 & .77 \\
\hline July $16 \ldots \ldots \ldots \ldots \ldots \ldots \ldots \ldots \ldots \ldots$ & 1. 99 & 2.05 & 1. 21 & $\rightarrow .06$ & .84 & .78 \\
\hline July $18 \ldots \ldots \ldots \ldots$ & 1.91 & 1.75 & 1.13 & +.16 & .62 & .78 \\
\hline July $21 \ldots \ldots$ & 1.89 & 1.74 & 1.11 & .15 & .63 & .78 \\
\hline July $23 \ldots$ & 1.89 & 1.74 & 1.13 & .15 & .61 & .76 \\
\hline $\begin{array}{l}\text { July } 25 \\
\text { July } 28\end{array}$ & $\begin{array}{l}1.87 \\
1.89\end{array}$ & $\begin{array}{l}1.75 \\
1.75\end{array}$ & $\begin{array}{l}1.12 \\
1.13\end{array}$ & .12 & .03 & .75 \\
\hline July $30 \ldots$ & 1.89 & 1.74 & 1.15 & .15 & .59 & .74 \\
\hline Aug. $1 \ldots . .$. & 1.89 & 1.72 & 1.14 & .17 & .58 & .75 \\
\hline Ang. $4 . . . .$. & 1. 89 & 1.70 & 1. 13 & .19 & .57 & .76 \\
\hline Aug. 6.... & 1. 89 & 1. 69 & 1.13 & .20 & .56 & .76 \\
\hline Ang. 8... & 1. 88 & 1. 69 & 1. 12 & .19 & .57 & .76 \\
\hline Ang. 25... & 1. 87 & 1.79 & 1. 08 & .08 & .71 & .79 \\
\hline Aug. $28 \ldots$ & 1. 87 & 1. 74 & 1. 06 & .13 & .68 & .81 \\
\hline Ang. $31 \ldots$ & 1.87 & 1.75 & 1. 09 & .12 & .66 & .78 \\
\hline Sept. $4 \ldots$ & 1. 83 & 1. 79 & 1. 17 & .04 & .62 & .66 \\
\hline Sept. $9 .$. & 1.89 & 1.74 & I. 13 & .15 & .61 & .76 \\
\hline Sept. $12 \ldots$ & 1.84 & 1.75 & 1.10 & .09 & .65 & .74 \\
\hline Sept. $14 \ldots$... & 1.99 & 1. 79 & 1.17 & .20 & .62 & .82 \\
\hline Sept. $20 \ldots . .$. & 1. 98 & 1. 77 & 119 & .21 & .58 & .79 \\
\hline$\ldots \ldots \ldots \ldots \ldots \ldots \ldots$ & 2.00 & 1.79 & 1. 23 & .21 & .56 & .77 \\
\hline
\end{tabular}


Repub̄lican River at Junction City, Kansas.

\begin{tabular}{|c|c|c|c|c|c|c|c|c|c|c|c|c|}
\hline Day. & Jan. & Feb. & Mar a & Apr. & May & June. & July. & Ang. & Sept. & Oct. & Nov. & Dec. \\
\hline 1. & 3. 55 & 3.65 & 3.50 & 3.50 & 4.45 & 4.90 & 3.55 & 4.00 & 3.10 & 2,80 & 4.65 & 3.70 \\
\hline & 3.40 & 3.95 & 3.50 & 3.50 & 4. 30 & 6.30 & 3.35 & 3.80 & 3.00 & 2. 80 & 4.25 & 3.85 \\
\hline 3. & 3.30 & 4.05 & 3.50 & 3.50 & 4. 25 & 7.20 & 3.30 & 3.60 & 2.95 & 2.80 & 3.90 & 3. 80 \\
\hline & 3.05 & 3.90 & 3.50 & 3.50 & 5. 25 & 6.20 & 3.40 & 3.45 & 2.85 & 2.75 & 3.55 & 3.75 \\
\hline 5. & 3.10 & 3.80 & 3.50 & 3.50 & 5.95 & 6.20 & 3.35 & 3.60 & 2.80 & 2.80 & 3.40 & 3.95 \\
\hline & 3.05 & 3.85 & 3.50 & 3.50 & 6.20 & 4.95 & 6.10 & 3. 60 & 2.80 & 2.75 & 3.30 & 3. 80 \\
\hline & 2.90 & 4.05 & 3.50 & 3.50 & 6.45 & 4. 95 & 5. 80 & 3. 45 & 2.70 & 2. 70 & 3. 20 & 3. 60 \\
\hline 8. & 3.10 & 4.00 & 3.50 & 3.50 & 6.45 & 4.55 & 5.70 & 3.35 & 2.65 & 2.70 & 3.10 & 3. 50 \\
\hline 9. & 3.05 & 3.80 & 9.50 & 3.65 & 5. 10 & 4.65 & 5. $40^{\circ}$ & 3.25 & 2.60 & 2.70 & 3.10 & 3.50 \\
\hline 10. & 3.35 & 3.75 & 3.50 & 3.45 & 4.50 & 4.55 & 5.00 & 3.15 & 2.75 & 2.70 & 3.10 & 3.50 \\
\hline $11 .$. & 3.30 & 3.85 & 3.60 & 3.40 & 4. 20 & 4. 30 & 4.85 & 3.00 & 2.75 & 2. 70 & 3.20 & 3.50 \\
\hline $12 \ldots \ldots$ & 3.30 & 3.80 & 3.50 & 3.50 & 4. 10 & 4. 15 & 4.95 & 3.55 & 2.60 & 2,70 & 3.20 & 3.50 \\
\hline $13 \ldots .$. & 3.35 & 3.75 & 3.50 & 3.45 & 4. 40 & 3.95 & 4.85 & 3.70 & 2.60 & 2.75 & 3.20 & 3.50 \\
\hline $14 \ldots$ & 3.30 & 3.70 & 3.50 & 3.50 & 5.40 & 3.85 & 4. 55 & 3.65 & 5.00 & 2.80 & 3.20 & 3.55 \\
\hline $15 \ldots$ & 3. 35 & 3. 65 & 3.50 & 4.90 & 5. 70 & 3.85 & 4. 40 & 3.20 & 3. 65 & 2. 80 & 3.20 & 3.60 \\
\hline $16 .$. & 3.50 & 3.60 & 3.50 & 6.50 & 5.90 & 3.85 & 4. 20 & 3.00 & 3.50 & 2,80 & 3.30 & 3. 60 \\
\hline 17 & 3.30 & 3. 60 & 3.50 & 6.75 & 5. 60 & 3.75 & 4. 20 & 3.00 & 3.70 & 2. 70 & 3. 30 & 3.65 \\
\hline 18. & 3.20 & 3. 60 & 8.50 & 5.65 & 5.00 & 3.60 & 10.97 & 3.00 & 3.75 & 2.70 & 3.30 & 3.50 \\
\hline 19. & 3.10 & 3.55 & 3.50 & 5.10 & 6.50 & 3.55 & 8.80 & 3. 20 & 4. 70 & 2.75 & 3. 35 & 3.30 \\
\hline 20. & 3.05 & 3.55 & 3.50 & 4.55 & 7.00 & 3.45 & 6.50 & 4. 50 & 5.40 & 2.70 & 3. 35 & 3.45 \\
\hline 21. & 3.05 & 3,55 & 3.50 & 4. 25 & 6.10 & 3.70 & 5.15 & 4. 95 & 4. 35 & 2. 70 & 3. 30 & 3.50 \\
\hline 22. & 3.20 & 3.60 & 3.50 & 4. 10 & 5.30 & 3.55 & 5.65 & 5.35 & 3. 65 & 2. 70 & 3.25 & 3. 60 \\
\hline 23. & 3.40 & 3.60 & 3.50 & 4. 10 & 4. 75 & 3.55 & 5. 10 & 4. 70 & 3.35 & 2.70 & 3.05 & 3.80 \\
\hline 24. & 3.30 & 3.55 & 3.50 & 6.20 & 4.40 & 3.85 & 4. 35 & 4. 35 & 3. 15 & 2. 70 & 3. 10 & 3. 70 \\
\hline 25. & 3.20 & 3.55 & 3.50 & 5.90 & 4. 25 & 4.05 & 4. 10 & 4. 05 & 3.05 & 2. 70 & 3.00 & 3.50 \\
\hline 26. & 3.15 & 3.50 & 3.50 & 5. 30 & 4. 05 & 3.65 & 3. 80 & 3.80 & 3. 00 & 2. 70 & 3.50 & 3.50 \\
\hline 27. & 3.05 & 3.55 & 3.50 & 5.40 & 3. 85 & 4.15 & 3.65 & 3.75 & 2. 90 & 2. 75 & 3.50 & 3. 50 \\
\hline 28. & 3. 20 & 3. 50 & 3.50 & 4.95 & 3.80 & 3.90 & 3.60 & 3.95 & 2. 80 & 2. 50 & 3.40 & 3.50 \\
\hline 29 . & 3.30 & 3.50 & 3.50 & 4.75 & 3.75 & 3.85 & 3. 55 & 3. 55 & 2.80 & 3.00 & 3. 30 & 3.70 \\
\hline & 3.40 & $\ldots . .$. & 3.50 & 4.85 & 4.05 & 3.45 & 3. 45 & 3.35 & 2. 80 & 4. 65 & 3. 50 & 3.65 \\
\hline 31 & 3. 40 & & 3.50 & ..... & 6.40 & .... & 3. 45 & 3.25 & ..... & & ....... & 3.75 \\
\hline
\end{tabular}

$a$ Figures in italies are estimated.

Solomon River at Beloit, Kansas.

\begin{tabular}{|c|c|c|c|c|c|c|c|c|c|c|c|c|}
\hline Day. & Jan. & Feb. & Mar. & Apr. & May. & June. & July. & Ang. & Sept. & Oct. & Nov. & Dec. \\
\hline 1. & 2. 70 & 2.90 & 2.00 & 2. 70 & 4.05 & 7.13 & 4. 55 & 4.95 & 3.75 & 3. 20 & 3.30 & 3. 10 \\
\hline 2. & 2.70 & 2. 82 & 3.00 & 2.35 & 4.20 & 9.50 & 4.25 & 4.75 & 3. 80 & 3.60 & $2.9 \mathrm{C}$ & 3.00 \\
\hline 3. & 2.30 & 2.92 & 2.75 & 2. 35 & 3.80 & 7.20 & 4.50 & 4.45 & 3.50 & 3. 20 & 2.70 & 3.10 \\
\hline 4. & 2.70 & 2.92 & 2.80 & 2. 40 & 3. 65 & 5.90 & 4.43 & 4.05 & 3. 40 & 2. 40 & 2.90 & 3.10 \\
\hline 5 & 1.75 & 2.92 & 2.25 & 2. 30 & 3.65 & 4.85 & 4. 80 & 3.95 & 3.40 & 3. 10 & 2.85 & 3. 10 \\
\hline 6. & 2.70 & 2.90 & 2.70 & 2.75 & 3.55 & 4. 75 & 6. 80 & 3.85 & 2.55 & 2. 60 & 2.80 & 2.30 \\
\hline 7. & 2.30 & 2. 92 & 2.70 & 2. 80 & 3. 50 & 4.90 & 6.00 & 4. 10 & 3. 25 & 3. 00 & 3.00 & 3.10 \\
\hline 8. & 2.70 & 2.95 & 2.25 & 2.75 & 3.30 & 5.55 & 4.50 & 4.60 & 3.40 & 2. 95 & 2. 20 & 3. 10 \\
\hline 9. & 2.70 & 2. 42 & 2.90 & 2. 60 & 3.20 & 4.65 & 4. 25 & 4. 35 & 2.85 & 3.15 & 3. 00 & 3.10 \\
\hline 10. & 2.70 & 2.92 & 2.70 & 2.60 & 3. 20 & 4.25 & 4.15 & 4. 20 & 3. 40 & 3.05 & 3.00 & 3.10 \\
\hline 11. & 2.30 & 3.00 & 2.80 & 2. 00 & 3. 40 & 4.15 & 5.20 & 4.00 & 3.45 & 2.15 & 3.05 & 3.10 \\
\hline 12. & 1.90 & 2. 92 & 2.75 & 4.85 & 3.25 & 4. 10 & 4.70 & 4. 15 & 3.40 & 2.80 & 3.00 & 3. 30 \\
\hline 13. & 2. 70 & 2. 55 & 2.75 & 15.50 & 3. 30 & 4.05 & 4. 25 & 4.00 & 2.85 & 2.65 & 3.00 & 2.65 \\
\hline 34. & 2. 30 & 2. 95 & 2.75 & 11.60 & 4. 60 & 3.80 & 4. 00 & 3.70 & 3.75 & 2. 60 & 3.00 & 3.30 \\
\hline 15. & 2.30 & 2. 92 & 2.25 & 11.90 & 4.80 & 3. 85 & 3.85 & 3.60 & 5. 25 & 2.60 & 2.20 & 3.25 \\
\hline $16 .$. & 2.70 & 2.37 & 2.65 & 580 & 4. 70 & 3.60 & 3.65 & 3. 65 & 4. 05 & 2.85 & 3.05 & 3. 35 \\
\hline $17 \ldots$ & 2.70 & 2. 82 & 2. 75 & 4. 50 & 8.32 & 3.80 & 3. 85 & 3.45 & 3.75 & 3. 15 & 3.00 & 3.05 \\
\hline $18 \ldots$ & 2.95 & 2.72 & 2.70 & 4. 35 & 4. 40 & 3. 80 & 4.05 & 3.70 & 3.95 & 2. 50 & 3.00 & 3.15 \\
\hline 19. & 2. 30 & 2.75 & 2.70 & 4. 20 & 4. 30 & 4. 20 & 3.95 & 3.65 & 4. 00 & 2.75 & 3.05 & 3.10 \\
\hline 20 . & 2.90 & 2.75 & 2.75 & 4.60 & 5. 40 & 4. 60 & 3. 95 & 3.90 & 4. 15 & 3.05 & 3.10 & 2.50 \\
\hline 21. & 2.95 & 2.72 & 2.75 & 4.20 & 4. 80 & 4. 15 & 3.90 & 4.55 & 3. 95 & 2. 65 & 2.70 & 3.15 \\
\hline 22. & 2. 95 & 2. 70 & 2.50 & 3. 90 & 4.25 & 4.00 & 4.60 & 4. 35 & 3.70 & 3. 05 & 2.40 & 3.15 \\
\hline 23. & 2.70 & 2.25 & 2.70 & 3.70 & 4.00 & 4.05 & 4. 05 & 4.40 & 3.60 & 3.05 & 3.10 & 3.20 \\
\hline 24 & 2.95 & 2.70 & 2. 70 & 3. 70 & 3.80 & 3. 75 & 4.05 & 4.55 & 3.85 & 2.80 & 3.10 & 3.20 \\
\hline & 2.95 & 2. 70 & 2.70 & 3. 65 & 4.35 & 3.90 & 3.95 & 4.95 & 3.55 & 2.55 & 3.10 & 2. 61) \\
\hline & 2. 30 & 2. 75 & 2.75 & 3.50 & 4. 15 & 3.95 & 4.00 & 4.40 & 3. 80 & 2.55 & 2.30 & 3. 15 \\
\hline 27. & 2. 90 & 2.70 & 3.00 & 3. 65 & 3. 65 & 4. 05 & 4.00 & 4.25 & 2. 65 & 2.55 & 3.10 & 2.50 \\
\hline $28 \ldots$ & 2. 90 & 2. 60 & 2.80 & 4.05 & 3. 70 & 4. 05 & 3.90 & 4.15 & 3.55 & 3. 10 & 2. 90 & 3.20 \\
\hline & 2.90 & 2.75 & 2. 30 & 3.75 & 3.35 & 4. 10 & 5. 30 & 3.90 & 3. 10 & 8.50 & 2.30 & 3.20 \\
\hline & 2.90 & ...... & 2.75 & 3.75 & 3. 30 & 5. 90 & 16.75 & 3. 65 & 2.75 & 5.10 & 3.05 & 3. 20 \\
\hline & 2.87 & & 2.60 & & 3.70 & & 6.25 & 3.90 & $\ldots .$. & 3.75 & ....... & 3.20 \\
\hline
\end{tabular}


Saline River at Beverly, Kansas.

\begin{tabular}{|c|c|c|c|c|c|c|c|c|c|c|c|c|}
\hline Day. & Jan. & Feb. & Mar. & Apr. & May. & June. & July. & Aug. & Sept. & Oet. & Nov. & Dec. \\
\hline & 4. 20 & 4.45 & 4.35 & 4. 15 & 5. 20 & 20.25 & 7.00 & 5. 10 & 4.40 & 4. 20 & 5. 65 & 4. 20 \\
\hline 2. & 4. 20 & 4.50 & 4.20 & 4. 30 & 5.35 & 23.00 & 6.40 & 4. 80 & 4. 50 & 4.35 & 5.25 & 4. 30 \\
\hline 3. & 4.00 & 4.45 & 4.15 & 4.35 & 5. 10 & 23.50 & 6.95 & 4. 70 & 4.45 & 4. 40 & 4.90 & 4.40 \\
\hline & 3.85 & 4.80 & 4. 10 & 4. 20 & 10.55 & 21.50 & 7.15 & 4.80 & 4. 20 & 4.20 & 4. 90 & 4. 35 \\
\hline 5. & 3. 85 & 4.70 & 4.25 & 4. 15 & 8.85 & 13.05 & 6.95 & 4. 60 & 4.30 & 4. 20 & 4. 75 & 4.30 \\
\hline & 4. 40 & 4.45 & 4.25 & 4. 20 & 5. 20 & 9.20 & 6.13 & 5. 00 & 4.15 & 4. 30 & 4.75 & 4. 30 \\
\hline & 4. 30 & 4. 35 & 4.30 & 4. 15 & 4.85 & 12.10 & 5.75 & 5.00 & 4. 35 & 4. 35 & 4.75 & 4. 15 \\
\hline & 4.40 & 4. 35 & 4. 30 & 4.60 & 4.70 & 10.55 & 7.35 & 5.05 & 4. 30 & 4. 35 & 4.65 & 4.80 \\
\hline 9. & 4. 15 & 4.25 & 4.45 & 4.90 & 4. 75 & 8.50 & 6. 00 & 4. 65 & 4. 10 & 4. 35 & 4. 45 & 4. 65 \\
\hline 10. & 4. 25 & 4. 15 & 4. 25 & 4. 65 & 4. 65 & 6.90 & 5. 75 & 4.60 & 4. 45 & 4. 35 & 4. 70 & 4.55 \\
\hline $11 \ldots$ & 4. 30 & 4. 25 & 4.50 & 4.50 & 4.65 & 6. 50 & 8. 10 & 4.85 & 4. 50 & 4. 15 & 4.65 & 4.65 \\
\hline $12 \ldots$ & 4.55 & 4.25 & 4.35 & 4. 45 & 4. 70 & 6.20 & 9.35 & 4. 55 & 4.30 & 4. 35 & 4.45 & 4.50 \\
\hline $13 \ldots$ & 4. 50 & 4. 35 & 4. 30 & 6. 40 & 7. 30 & 6.05 & 6.90 & 4.65 & 4. 40 & 4. 30 & 4. 30 & 4. 40 \\
\hline $14 \ldots$ & 4. 50 & 4. 35 & 4. 40 & 9.70 & 7.40 & 5.90 & 5. 85 & 4. 45 & 4.40 & 4. 35 & 4. 55 & 4. 05 \\
\hline $15 \ldots$ & 4. 40 & 4.30 & 4. 40 & 5.80 & 5. 60 & 5.70 & 5.55 & 4. 20 & 4. 40 & 4. 35 & 4. 30 & 4. 70 \\
\hline 16. & 4.30 & 4. 10 & 4.45 & 5. 05 & 7.25 & 5.60 & 5.45 & 4.75 & 4.50 & 4. 35 & 4.50 & 4.50 \\
\hline 17. & 4.50 & 4.15 & 4. 25 & 5.50 & 6. 50 & 5.80 & 5. 30 & 4.95 & 4.55 & 4. 30 & 4.60 & 4. 55 \\
\hline 18. & 4. 45 & 4. 20 & 4. 40 & 5. 40 & 5.80 & 5.75 & 8.50 & 4.85 & 4.45 & 4. 20 & 4.50 & 4. 25 \\
\hline 19. & 4. 50 & 4.15 & 4. 25 & 5. 05 & 5. 75 & 5.50 & 7.65 & 4. 70 & 4. 60 & 4.25 & 4. 60 & 4. 50 \\
\hline 20. & 4. 40 & 4. 40 & 4. 35 & 4.80 & 7.20 & 5.50 & 7.25 & 4.80 & 4.70 & 4. 10 & 4. 40 & 4. 25 \\
\hline 21. & 4. 55 & 4.25 & 4. 30 & 4. 90 & 6. 55 & 10.80 & 5.95 & 4.75 & 4.75 & 4. 25 & 4. 40 & 4. 35 \\
\hline & 4. 55 & 4.25 & 4. 20 & 4. 75 & 5. 75 & 7.65 & 5. 45 & 4.75 & 4.60 & 4.35 & 4.55 & 4. 30 \\
\hline 23. & 4.60 & 4. 35 & 4. 35 & 4.70 & 5. 20 & 8.85 & 5. 25 & 7. 50 & 4.50 & 4. 35 & 4.20 & 4.25 \\
\hline 24. & 4. 55 & 4.20 & 4. 20 & 4. 65 & 4.95 & 6.80 & 5.85 & 5.95 & 4.50 & 4.25 & 4. 30 & 4. 60 \\
\hline 25 . & 4. 40 & 4.15 & 4. 30 & 4. 65 & 4. 70 & 6. 20 & 5. 55 & 5.10 & 4.40 & 4. 05 & 4.55 & 4. 50 \\
\hline 26. & 4. 25 & 4.20 & 4. 40 & 6. 60 & 4. 60 & 6.75 & 5. 10 & 4.85 & 4.35 & 4. 30 & 4.55 & 4.15 \\
\hline 27. & 4. 25 & 4. 30 & 4. 30 & 9. 30 & 4.55 & 8.30 & 5.00 & 4. 65 & 4. 35 & 4. 15 & 4. 30 & ..... \\
\hline & 4. 20 & 4.40 & 4. 25 & 7. 35 & 4. 45 & 8.20 & 4. 95 & 4. 70 & 4.40 & 4. 40 & 4. 35 & \\
\hline & 4.15 & 4.25 & 4. 40 & 5. 75 & 4. 40 & 8.00 & 4. 90 & 4. 55 & 4.30 & 5.75 & 4. 30 & \\
\hline & 4. 20 & ...... & 4. 25 & 5.30 & 4. 25 & 7.90 & 6. 90 & 4.50 & 4.45 & 15. 35 & 4.25 & \\
\hline & 4. 40 & $\ldots$ & 4. 10 & ...... & 13.00 & ...... & 5.85 & 4. 45 & $\ldots$. & 7.10 & $\cdots$ & \\
\hline
\end{tabular}

Smoky Hill River at Ellsworth, Kansas.

\begin{tabular}{|c|c|c|c|c|c|c|c|c|c|c|c|c|}
\hline Day. & Jan. & Feb. & Mar. & Apr. & May. & June. & July. & Aug. & Sept. & Oet. & Nov. & Dec. \\
\hline & 1. 50 & 1. 40 & 1.30 & 1. 20 & 1. 72 & 1.85 & 2. 68 & 2. 05 & 1.68 & 1. 45 & 1.95 & 1. 33 \\
\hline 2. & 1.50 & 1.50 & 1.27 & 1. 20 & 1.67 & 1. 75 & 2.45 & 1. 90 & 1. 60 & 1. 43 & 1.85 & 1.30 \\
\hline 3. & 1. 42 & 1.50 & 1. 35 & 1. 20 & 1.65 & 1. 62 & 2.25 & 1.78 & 1.58 & 1. 40 & 1.88 & 1. 30 \\
\hline 4. & 1. 42 & 1.50 & 1. 27 & 1. 20 & 1.70 & 1.55 & 2.20 & 1. 68 & 1.53 & 1. 40 & 1.78 & 1.30 \\
\hline & 1.35 & 1.50 & 1. 30 & 1.20 & 1.62 & 1.90 & 2. 13 & 1.65 & 1. 48 & 1. 43 & 1.70 & 1. 28 \\
\hline & 1. 32 & 1. 50 & 1. 30 & 1. 20 & 1.60 & 1. 95 & 2.03 & 1.78 & 1. 45 & 1. 35 & 1.63 & 1. 28 \\
\hline & 1. 27 & 1.40 & 1. 30 & 1. 20 & 1. 52 & 4.05 & 4. 30 & 1.88 & 1. 43 & 1. 35 & 1. 53 & 1.30 \\
\hline 8. & 1. 42 & 1. 30 & 1. 30 & 2.10 & 1.50 & 7.10 & 2. 10 & 1.75 & 1. 40 & 1. 30 & 1. 50 & 1.25 \\
\hline 9. & 1. 42 & 1. 35 & 1. 30 & 1.42 & 1. 47 & 3.75 & 2.00 & 1.65 & 1. 40 & 1. 30 & 1.50 & 1. 23 \\
\hline 10. & 1.35 & 1.47 & 1.30 & 1.27 & 1.50 & 2.75 & 1.93 & 1.58 & 1.50 & 1. 40 & 1.45 & 1.25 \\
\hline 11. & 1. 35 & 1. 40 & 1.27 & 1.20 & 1.45 & 2.45 & 3.50 & 1.50 & 1.48 & 1.35 & 1.43 & 1. 25 \\
\hline 12. & 1. 32 & 1. 40 & 1.27 & 1.55 & 1. 47 & 2. 15 & 2.90 & 1. 55 & 1. 40 & 1. 33 & 1.40 & 1.25 \\
\hline 13. & 1.42 & 1. 40 & 1. 22 & 2.30 & 1. 85 & 2.05 & 2.50 & 1.58 & 1. 45 & 1. 30 & 1.40 & 1.25 \\
\hline 14. & 1. 35 & 1.40 & 1. 37 & 2.25 & 1. 72 & 1.95 & 2.23 & 1.63 & 1,43 & 1. 28 & 1.35 & 1.20 \\
\hline 15 . & 1. 32 & 1.40 & 1.37 & 1.85 & 1.67 & 1. 85 & 2.03 & 1.68 & 1. 40 & 1. 25 & 1. 35 & 1. 20 \\
\hline 16 & 1. 32 & 1.40 & 1.27 & 1. 97 & 1. 62 & 1.83 & 2. 00 & 1.60 & 1. 40 & 1. 23 & 1.35 & 1.20 \\
\hline 17. & 1. 27 & 1. 35 & 1. 32 & 2. 37 & 1.55 & 1.80 & 1. 98 & 1. 50 & 1. 40 & 1. 20 & 1. 30 & 1. 23 \\
\hline 18. & 1.27 & 1. 35 & 1. 32 & 3.00 & 1. 50 & 1.83 & 2. 90 & 1.50 & 1.45 & 1. 20 & 1. 30 & 1. 13 \\
\hline 19. & 1. 30 & 1. 35 & 1. 25 & 2.10 & 1.50 & 1.85 & 3.60 & 1.55 & 1.90 & 1. 20 & 1. 20 & 1. 35 \\
\hline 20. & 1.35 & 1. 32 & 1.30 & 1.97 & 1.50 & 1. 73 & 2.65 & 1. 50 & 1. 68 & 1.20 & 1. 20 & 1.25 \\
\hline 21 . & 1.32 & 1.32 & 1.30 & 1. 97 & 1.50 & 4.30 & 2.35 & 1.50 & 1.60 & 1. 20 & 1.28 & 1.20 \\
\hline 22. & 1.35 & 1. 35 & 1.30 & 1. 90 & 1. 47 & 2.30 & 2.08 & 3.00 & 1.63 & 1. 20 & 1. 15 & 1.20 \\
\hline 23. & 1.32 & 1.35 & 1. 30 & 1.92 & 1.60 & 2.10 & 2.15 & 2.40 & 1.68 & 1.28 & 1.23 & 1.20 \\
\hline 24. & 1. 40 & 1. 30 & 1. 30 & 1.87 & 1. 47 & 2. 65 & 2.15 & 1.85 & 1. 70 & 1. 28 & 1.25 & 1.20 \\
\hline 25. & 1. 45 & 1. 30 & 1. 27 & 4. 30 & 1. 40 & 2.50 & 2. 00 & 1.95 & 1.70 & 1.25 & 1. 25 & 1.20 \\
\hline 26. & 1. 37 & 1.30 & 1.30 & 2.80 & 1. 40 & 2.35 & 1. 93 & 2.43 & I. 68 & 1. 28 & 1. 33 & 1.25 \\
\hline 27. & 1. 52 & 1. 30 & 1.30 & 2.10 & 1. 35 & 2.45 & 1.88 & 2. 28 & 1. 58 & 1. 30 & 1. 35 & 1.25 \\
\hline 28 . & 1. 42 & 1. 30 & 1. 27 & 2.00 & 1. 30 & 3.85 & 2.00 & 2.05 & 1.53 & 1. 30 & 1. 30 & 1.20 \\
\hline 29 . & 1.40 & 1. 30 & 1. 20 & 1.85 & 1. 30 & 3. 30 & 1. 93 & 1.93 & 1.50 & 2. 63 & 1.25 & 1.20 \\
\hline 30. & I. 40 & ...... & 1. 20 & 1.80 & 1. 30 & 2. 90 & 2.40 & 1.83 & 1.48 & 2. 40 & 1. 30 & 1.20 \\
\hline 31. & 1.40 & & 1. 20 & ..... & 1.52 & $\ldots . .$. & 2. 20 & 1.73 & ...... & 2. 28 & $\cdots \cdot$ & 1.20 \\
\hline
\end{tabular}


Blue River at Manhattan, Kansas.

\begin{tabular}{|c|c|c|c|c|c|c|c|c|c|c|c|}
\hline Day. & Jan. & Feb. & Apr.a & May. & June. & July. & Aug. & Sept. & Oet. & Nov. & Dec. \\
\hline 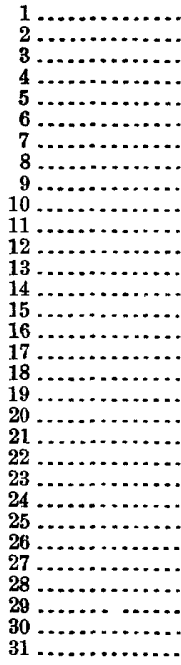 & $\begin{array}{l}\text { 3. } 70 \\
\text { 3. } 60 \\
\text { 3. } 55 \\
\text { 3. } 70 \\
\text { 3. } 70 \\
\text { 3. } 80 \\
\text { 3. } 75 \\
\text { 3.75 } \\
\text { 3. } 80 \\
\text { 3. } 85 \\
\text { 3. } 80 \\
\text { 3. } 70 \\
\text { 3. } 65 \\
\text { 3. } 50 \\
3.55 \\
3.60 \\
\text { 3. } 65 \\
\text { 3. } 60 \\
\text { 3. } 55 \\
\text { 3. } 50 \\
3.75 \\
\text { 3. } 60 \\
\text { 3. } 60 \\
\text { 3. } 30 \\
\text { 3. } 45 \\
3.65 \\
\text { 3. } 75 \\
3.80 \\
3.75 \\
\text { 3. } 70 \\
3.80\end{array}$ & 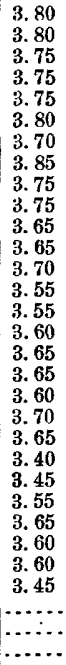 & $\begin{array}{r}\cdots 50 \\
7.20 \\
9.95 \\
11.05 \\
10.20 \\
8.35 \\
7.20 \\
6.55 \\
6.05 \\
5.70 \\
5.35 \\
5.35 \\
5.20 \\
5.20 \\
5.25 \\
\ldots \ldots\end{array}$ & $\begin{array}{r}5.60 \\
5.35 \\
5.75 \\
13.95 \\
16.75 \\
13.25 \\
9.50 \\
7.60 \\
6.20 \\
6.45 \\
6.10 \\
5.75 \\
5.65 \\
11.20 \\
13.65 \\
12.30 \\
11.50 \\
11.00 \\
11.45 \\
14.40 \\
13.25 \\
11.35 \\
10.35 \\
11.05 \\
10.05 \\
9.00 \\
8.35 \\
7.65 \\
6.90 \\
6.40 \\
14.15\end{array}$ & $\begin{array}{r}20.55 \\
20.30 \\
13.85 \\
\mathbf{1 1 . 8 5} \\
\mathbf{8 . 8 0} \\
8.75 \\
8.25 \\
\mathbf{8 . 3 5} \\
14.50 \\
14.50 \\
12.30 \\
\mathbf{9 . 5 5} \\
7.20 \\
\mathbf{7 . 2 0} \\
\mathbf{6 . 3 5} \\
\mathbf{5 . 9 5} \\
\mathbf{5 . 6 0} \\
\mathbf{5 . 2 0} \\
\mathbf{5 . 0 0} \\
\mathbf{4 . 9 0} \\
\mathbf{4 . 3 0} \\
\mathbf{4 . 0 5} \\
\mathbf{5 . 1 0} \\
\mathbf{5 . 7 5} \\
\mathbf{5 . 4 5} \\
\mathbf{9 . 2 0} \\
12.60 \\
\mathbf{1 0 . 7 0} \\
\mathbf{9 . 4 0} \\
\mathbf{8 . 2 5} \\
\ldots . . .\end{array}$ & $\begin{array}{r}11.15 \\
18.30 \\
22.25 \\
22.20 \\
19.00 \\
10.75 \\
8.15 \\
7.50 \\
7.75 \\
9.40 \\
9.25 \\
9.20 \\
9.35 \\
8.65 \\
7.75 \\
6.40 \\
6.25 \\
9.15 \\
13.90 \\
12.70 \\
12.00 \\
10.75 \\
9.35 \\
7.25 \\
5.60 \\
5.20 \\
5.40 \\
5.10 \\
4.65 \\
6.70 \\
11.60\end{array}$ & $\begin{array}{r}9.75 \\
8.80 \\
7.10 \\
6.70 \\
6.00 \\
5.70 \\
7.60 \\
8.25 \\
7.35 \\
7.35 \\
5.85 \\
5.45 \\
5.60 \\
8.10 \\
10.75 \\
9.70 \\
7.80 \\
6.95 \\
10.15 \\
15.85 \\
10.25 \\
7.75 \\
5.95 \\
6.75 \\
8.40 \\
6.90 \\
5.75 \\
5.45 \\
5.45 \\
5.10 \\
4.95\end{array}$ & $\begin{array}{r}4.80 \\
4.60 \\
4.85 \\
5.50 \\
5.20 \\
5.15 \\
4.95 \\
4.85 \\
5.05 \\
5.15 \\
5.00 \\
5.20 \\
5.15 \\
7.50 \\
9.75 \\
8.15 \\
6.65 \\
6.15 \\
10.50 \\
10.25 \\
7.60 \\
6.15 \\
5.85 \\
6.10 \\
5.40 \\
5.05 \\
5.00 \\
4.95 \\
4.80 \\
4.70 \\
\ldots . .2\end{array}$ & $\begin{array}{r}4.70 \\
4.60 \\
4.55 \\
4.70 \\
4.85 \\
5.05 \\
4.90 \\
4.50 \\
4.25 \\
4.00 \\
4.30 \\
4.00 \\
3.90 \\
3.70 \\
3.60 \\
3.70 \\
3.30 \\
3.20 \\
3.15 \\
3.00 \\
3.00 \\
3.00 \\
3.00 \\
3.05 \\
3.00 \\
3.10 \\
3.00 \\
2.80 \\
10.00 \\
12.75 \\
12.25\end{array}$ & 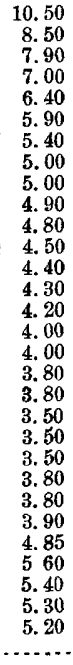 & $\begin{array}{l}5.00 \\
5.00 \\
4.80 \\
4.65 \\
4.00 \\
4.00 \\
4.25 \\
4.55 \\
4.60 \\
4.80 \\
4.90 \\
5.00 \\
5.00 \\
5.00 \\
4.80 \\
4.80 \\
4.70 \\
4.70 \\
4.70 \\
4.50 \\
4.30 \\
4.00 \\
4.00 \\
3.80 \\
3.70 \\
3.50 \\
3.00 \\
3.00 \\
3.00 \\
3.65 \\
4.00\end{array}$ \\
\hline
\end{tabular}

$a$ Observations discontinued from March 1 to April 15; observer moved away.

Kansas River at Lecompton, Kansas.

\begin{tabular}{|c|c|c|c|c|c|c|c|c|c|c|c|}
\hline Day. & Jan. & Feb. & Mar. & Apr. & May. & June. & July. & Aug. & Sept. & Oet. & Nov. \\
\hline 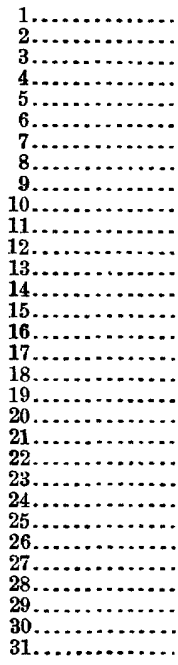 & $\begin{array}{l}0.82 \\
.76 \\
.85 \\
.65 \\
.42 \\
.49 \\
.57 \\
.56 \\
.63 \\
.65 \\
.77 \\
.88 \\
.87 \\
.94 \\
.85 \\
.66 \\
.60 \\
.54 \\
.53 \\
.58 \\
.53 \\
.51 \\
.54 \\
.55 \\
.18 \\
.34 \\
.87 \\
.60 \\
.53 \\
.98 \\
.87\end{array}$ & $\begin{array}{r}0.72 \\
.70 \\
.72 \\
.77 \\
.85 \\
.93 \\
.93 \\
.88 \\
.95 \\
.92 \\
.88 \\
.79 \\
.75 \\
.74 \\
.82 \\
.75 \\
.72 \\
.73 \\
.69 \\
.70 \\
.67 \\
.66 \\
.64 \\
.63 \\
.58 \\
.58 \\
.62 \\
.60 \\
.58 \\
\ldots . . \\
\ldots . . \\
.52\end{array}$ & \begin{tabular}{|l}
0.58 \\
.73 \\
.75 \\
.60 \\
.83 \\
.79 \\
.67 \\
.38 \\
.42 \\
.42 \\
.46 \\
.48 \\
.48 \\
.50 \\
.58 \\
.50 \\
.42 \\
.65 \\
.75 \\
.50 \\
.50 \\
.52 \\
.52 \\
.54 \\
.58 \\
.58 \\
.65 \\
.73 \\
.88 \\
.83 \\
.75
\end{tabular} & $\begin{array}{r}0.73 \\
.71 \\
.67 \\
.67 \\
.65 \\
.67 \\
.79 \\
.77 \\
.83 \\
.75 \\
.75 \\
1.08 \\
1.25 \\
1.02 \\
.94 \\
.92 \\
.92 \\
1.50 \\
4.71 \\
5.00 \\
4.67 \\
3.83 \\
3.29 \\
2.83 \\
3.33 \\
4.04 \\
4.79 \\
3.92 \\
2.96 \\
2.60 \\
\ldots \ldots . .\end{array}$ & $\begin{array}{l}2.37 \\
2.37 \\
2.35 \\
2.42 \\
4.92 \\
6.54 \\
5.42 \\
4.33 \\
3.83 \\
3.60 \\
3.04 \\
2.58 \\
2.42 \\
3.08 \\
3.88 \\
8.46 \\
8.13 \\
6.58 \\
5.63 \\
8.67 \\
8.92 \\
8.13 \\
6.13 \\
5.67 \\
5.29 \\
5.13 \\
4.42 \\
3.83 \\
3.38 \\
2.98 \\
3.71\end{array}$ & $\begin{array}{r}8.50 \\
9.33 \\
8.67 \\
7.50 \\
6.17 \\
7.13 \\
6.92 \\
6.42 \\
5.92 \\
6.67 \\
6.79 \\
5.58 \\
4.92 \\
4.21 \\
3.54 \\
3.08 \\
2.79 \\
2.58 \\
2.38 \\
2.21 \\
2.17 \\
2.13 \\
2.25 \\
2.29 \\
2.67 \\
3.21 \\
3.54 \\
4.48 \\
4.46 \\
3.88 \\
\ldots . .2\end{array}$ & $\begin{array}{r}3.25 \\
3.50 \\
5.96 \\
8.00 \\
8.25 \\
7.58 \\
5.13 \\
3.88 \\
3.58 \\
3.42 \\
3.54 \\
3.67 \\
3.75 \\
3.38 \\
2.96 \\
2.63 \\
2.40 \\
3.46 \\
10.25 \\
11.63 \\
9.96 \\
7.42 \\
5.58 \\
4.88 \\
4.00 \\
3.46 \\
3.08 \\
2.75 \\
2.50 \\
2.42 \\
2.29\end{array}$ & $\begin{array}{l}3.08 \\
3.65 \\
3.21 \\
2.85 \\
2.92 \\
2.92 \\
2.50 \\
2.85 \\
2.94 \\
2.71 \\
2.25 \\
2.17 \\
2.08 \\
1.96 \\
1.92 \\
3.04 \\
3.21 \\
4.46 \\
4.25 \\
7.13 \\
6.63 \\
4.50 \\
3.79 \\
4.44 \\
3.75 \\
3.33 \\
3.04 \\
2.58 \\
2.42 \\
2.33 \\
2.29\end{array}$ & \begin{tabular}{|}
2.21 \\
2.04 \\
1.88 \\
1.58 \\
1.46 \\
1.25 \\
1.17 \\
1.17 \\
1.17 \\
1.13 \\
1.08 \\
1.19 \\
1.48 \\
1.38 \\
2.92 \\
4.71 \\
3.96 \\
2.67 \\
2.25 \\
2.08 \\
3.83 \\
3.08 \\
2.46 \\
2.33 \\
2.08 \\
1.81 \\
1.63 \\
1.52 \\
1.47 \\
1.38 \\
$\ldots \ldots .$.
\end{tabular} & $\begin{array}{l}1.25 \\
1.21 \\
1.17 \\
1.21 \\
1.08 \\
1.15 \\
1.04 \\
.98 \\
.92 \\
.95 \\
.97 \\
1.00 \\
1.03 \\
1.00 \\
.95 \\
.90 \\
.92 \\
.86 \\
.85 \\
.83 \\
.83 \\
.81 \\
.77 \\
.75 \\
.75 \\
.74 \\
.75 \\
.75 \\
1.00 \\
1.29 \\
3.81\end{array}$ & 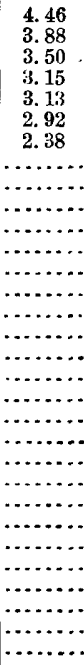 \\
\hline
\end{tabular}


Kansas River at Lawrence, Kansas.

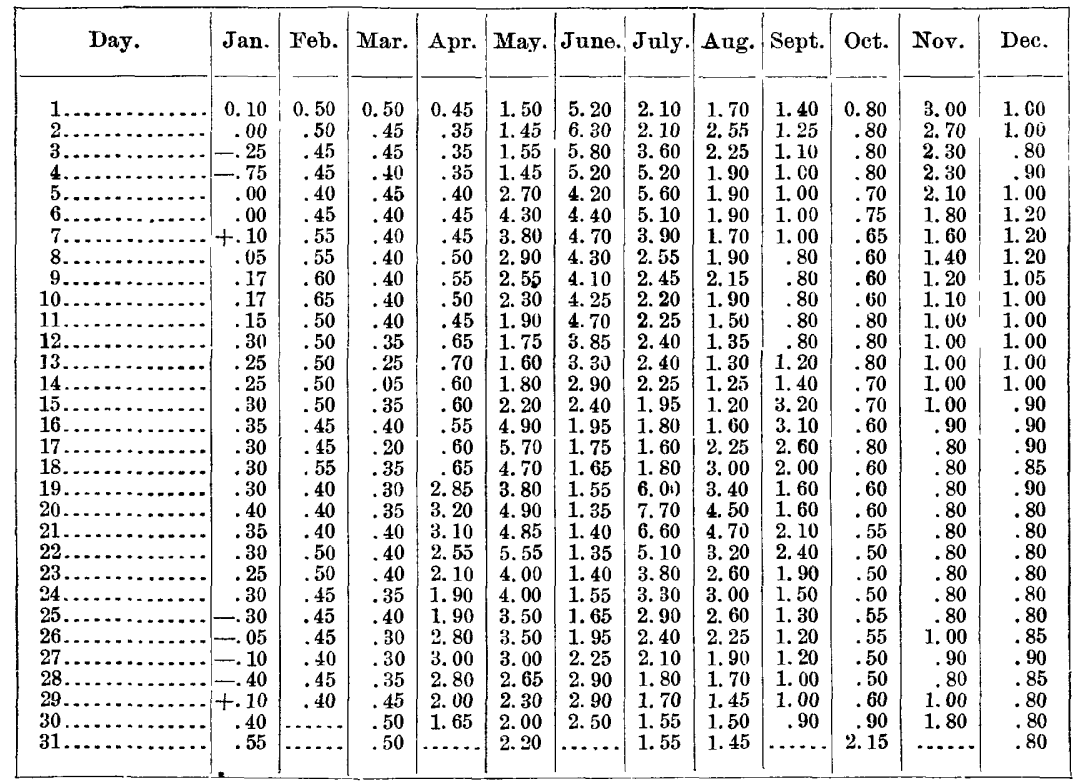

Arkansas River at Canyon, Colorado.

\begin{tabular}{|c|c|c|c|c|c|c|c|c|c|c|c|c|}
\hline Day. & Jan. & Feb. & Mar. & Apr. & May. & June. & July. & Aug. & Sept. & Oet. & Nov. & Dec. \\
\hline 1. & 2.45 & 2.40 & 2.40 & 2.50 & 3.05 & 4. 45 & 2. 30 & 2.40 & 2.60 & 2.15 & 2.30 & 3. 55 \\
\hline $2 \ldots \ldots \ldots \ldots$ & 2.50 & 2.45 & 2.30 & 2.60 & 3.05 & 4.00 & 2.30 & 2.30 & 2.50 & 2.10 & 2.30 & 5.50 \\
\hline$\ldots . .$. & 2.50 & 2.40 & 2.30 & 2.60 & 3.30 & 3.95 & 2.25 & 2.20 & 2.25 & 2.10 & 2.30 & 3.00 \\
\hline $4 \ldots$ & 2.50 & 2.50 & 2.30 & 2.50 & 3.35 & 3.70 & 2. 30 & 2.00 & 2.20 & 2.10 & 2.30 & \\
\hline $5 \ldots$ & 2. 55 & 2.50 & 2.40 & 2.50 & 3.40 & 3. 65 & 2.30 & 1.95 & 2.20 & 2.10 & 2.30 & 2.80 \\
\hline $6 \ldots$ & 245 & 2.50 & 2.40 & 2.50 & 3.60 & 3. 70 & 2.20 & 2.50 & 2.10 & 2.10 & 2.35 & 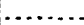 \\
\hline & 2.50 & 2.60 & 2.40 & 2.50 & 3.80 & 3.50 & 2.20 & 2.35 & 2.10 & 2.05 & 2.40 & \\
\hline & 2.50 & 2.60 & 2.40 & 2. 50 & 3.90 & 3.40 & 2.20 & 2.00 & 2.05 & 2.15 & 2.30 & \\
\hline 9. & 2.50 & 2.50 & 2.50 & 2.40 & 3. 75 & 3. 30 & 2.20 & 1. 90 & 1.95 & 2. 20 & 2. 35 & \\
\hline 10. & 2. 50 & 2.50 & 2.50 & 2.50 & 3. 60 & 3.35 & 2. 15 & 1. 90 & 2. 25 & 2. 20 & 2.40 & \\
\hline 11. & 2. 50 & 2. 50 & 2.50 & 2. 40 & 3.55 & 3.40 & 2. 20 & 1. 90 & 2.30 & 2.20 & 2.50 & \\
\hline 12. & 2.45 & 2.35 & 2. 40 & 2. 40 & 3.50 & 3.30 & 2. 20 & 1.80 & 2.25 & 2.20 & 2.60 & 2.50 \\
\hline 13. & 2.50 & 2.35 & 2. 40 & 2.40 & 3.40 & 3. 20 & 2.15 & 1.80 & 2.20 & 2.20 & 2.50 & \\
\hline 14. & 2.50 & 2.45 & 2. 40 & 2. 60 & 3.20 & 3,10 & 2. 10 & 1.80 & 2.15 & 2.20 & 2.50 & \\
\hline $15 \ldots$ & 2. 50 & 2.45 & 2. 50 & 2.70 & 3. 10 & 3,10 & 2.05 & 1. 80 & 2.10 & 2.20 & 2.50 & \\
\hline $16 .$. & 2. 50 & 2.45 & 2.50 & 2.80 & 3.00 & 3. 00 & 2. 96 & 1.80 & 2.10 & 2.20 & 2.50 & \\
\hline 17. & 2. 50 & 2.45 & 2. 60 & 2. 90 & 2,90 & 3. 05 & 3. 57 & 2.75 & 2.10 & 2.20 & 2.50 & \\
\hline 18. & 2. 50 & 2.45 & 2.60 & 2. $8 \tilde{7}$ & 2.80 & 3. 00 & 3. 20 & 2.45 & 2. 20 & 2.10 & 2.40 & \\
\hline 19. & 2.50 & 2.45 & 2. 50 & 2. 75 & 2.80 & 3.00 & 3. 20 & 2. 50 & 2.35 & 2. 10 & 2.45 & 2.40 \\
\hline 20. & 2.50 & 2.45 & 2.50 & 2. 70 & 2.85 & 2.95 & 2.80 & 2. 40 & 2.45 & 2. 10 & 2.50 & \\
\hline 21. & 2.50 & 2. 45 & 2. 40 & 2. 60 & 2. 95 & 2. 90 & 2. 70 & 2.60 & 2. 30 & 2.10 & 2.60 & \\
\hline 22. & 2.55 & 2. 45 & 2.50 & 2. 60 & 3. 05 & 2.80 & 2. 70 & 2. 50 & 2.30 & 2. 15 & 2. 50 & \\
\hline 23. & 2. 55 & 2.55 & 2. 60 & 2.50 & 3. 15 & 2.80 & 2. 60 & 2.50 & 2.30 & 2.20 & 2.50 & \\
\hline $24 .$. & 2,50 & 2.55 & $3: 60$ & 2. 60 & 3.70 & 2.70 & 4. 13 & 2.40 & 2. 20 & 2.20 & 2.40 & $\ldots$ \\
\hline 25. & 2. 5i) & 2.50 & 2. 80 & 2.75 & 3.80 & 2. 70 & 2.90 & 2. 35 & 2.20 & 2.20 & 2.40 & \\
\hline 26. & 2.45 & 2.45 & 2.80 & 2.85 & 4. 00 & 2. 60 & 2.80 & 2.15 & 2. 20 & 2. 20 & 2. 20 & 2. 70 \\
\hline 27 . & 2. 45 & 2. 45 & 2. 90 & 2.95 & 4.00 & 2. 50 & 2. 70 & 2.00 & 2.20 & 2.20 & 2.10 & \\
\hline 28. & 2.45 & 2.40 & 3.20 & 3. 00 & 4. 20 & 2.50 & 2.60 & 1. 95 & 2.20 & 2. 20 & 2.00 & \\
\hline 29. & 2.50 & 2.40 & 2.50 & 3.05 & 4.25 & 2. 10 & 2. 60 & 2.00 & 2. 20 & 2.20 & 2.00 & \\
\hline 30. & 2.50 & $\ldots \ldots$ & 2. 50 & 3. 10 & 4. 50 & 2.40 & 2. 45 & 4. 60 & 2.20 & 2. 30 & 2.00 & \\
\hline 31. & 2. 45 & & 2.40 & $\ldots \ldots$ & 4. 60 & $\ldots . .$. & 2.40 & 2.80 & ...... & 2. 30 & .... & \\
\hline
\end{tabular}


Arkansas River at Pueblo, Colorado.

\begin{tabular}{|c|c|c|c|c|c|c|c|c|c|c|c|c|}
\hline Day. & Jan. & Feb. & Mar. & Apr. & May. & June. & July. & Aug. & Sept. & Oct. & Nov. & Dec. \\
\hline 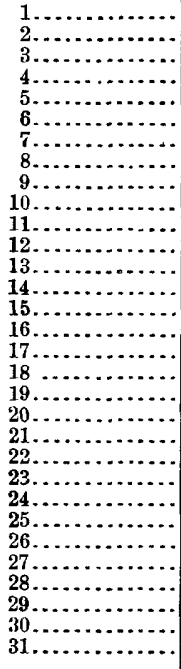 & $\begin{array}{l}0.35 \\
.40 \\
.35 \\
.35 \\
.70 \\
.60 \\
.60 \\
.60 \\
.60 \\
.60 \\
.60 \\
.60 \\
.60 \\
.60 \\
.60 \\
.60 \\
.60 \\
.60 \\
.60 \\
.60 \\
.60 \\
.60 \\
.60 \\
.60 \\
.60 \\
.60 \\
.60 \\
.60 \\
.60 \\
.60 \\
.60\end{array}$ & $\begin{array}{r}0.60 \\
.60 \\
.60 \\
.60 \\
.60 \\
.60 \\
.60 \\
.60 \\
.60 \\
.60 \\
.60 \\
.60 \\
.50 \\
.50 \\
.60 \\
.60 \\
.50 \\
.40 \\
.30 \\
.30 \\
.30 \\
.30 \\
.30 \\
.30 \\
.30 \\
.30 \\
.30 \\
.30 \\
.30 \\
\ldots \ldots \\
\ldots \ldots\end{array}$ & $\begin{array}{l}0.40 \\
.40 \\
.40 \\
.40 \\
.40 \\
.40 \\
.40 \\
.35 \\
.30 \\
.30 \\
.30 \\
.20 \\
.20 \\
.40 \\
.30 \\
.40 \\
.35 \\
.40 \\
.40 \\
.40 \\
.35 \\
.40 \\
.40 \\
.40 \\
.40 \\
.40 \\
.50 \\
.50 \\
.40 \\
.35 \\
.30\end{array}$ & $\begin{array}{r}0.30 \\
.30 \\
.30 \\
.30 \\
.30 \\
.30 \\
.30 \\
.40 \\
.30 \\
.30 \\
.30 \\
.40 \\
.40 \\
.40 \\
.40 \\
.40 \\
.40 \\
.40 \\
.35 \\
.35 \\
.25 \\
.15 \\
.20 \\
.30 \\
.60 \\
.80 \\
1.45 \\
1.40 \\
1.25 \\
.90 \\
. . .2\end{array}$ & $\begin{array}{r}0.75 \\
.65 \\
.50 \\
.85 \\
1.25 \\
1.40 \\
1.65 \\
1.85 \\
1.80 \\
1.50 \\
1.40 \\
1.45 \\
1.45 \\
1.30 \\
1.00 \\
.90 \\
.90 \\
.85 \\
.80 \\
.75 \\
.75 \\
.85 \\
1.05 \\
1.30 \\
1.70 \\
1.80 \\
1.80 \\
1.85 \\
2.05 \\
2.05 \\
2.50\end{array}$ & $\begin{array}{l}2.30 \\
1.95 \\
1.75 \\
1.70 \\
1.65 \\
1.60 \\
1.60 \\
1.45 \\
1.25 \\
1.35 \\
1.25 \\
1.05 \\
.95 \\
.85 \\
.90 \\
.95 \\
.95 \\
.90 \\
.80 \\
.80 \\
.50 \\
.80 \\
.70 \\
.60 \\
.60 \\
.60 \\
.60 \\
.45 \\
.50 \\
.40 \\
. .2\end{array}$ & $\begin{array}{r}0.40 \\
.40 \\
.30 \\
.40 \\
.30 \\
.30 \\
.25 \\
.65 \\
.45 \\
.30 \\
.35 \\
.30 \\
.25 \\
.20 \\
.20 \\
.20 \\
1.30 \\
1.35 \\
1.10 \\
1.10 \\
.75 \\
.70 \\
.60 \\
2.83 \\
2.05 \\
.80 \\
.70 \\
.80 \\
.60 \\
.50 \\
.40\end{array}$ & $\begin{array}{r}0.30 \\
.20 \\
.10 \\
.10 \\
.10 \\
.10 \\
.10 \\
.10 \\
.05 \\
.00 \\
.00 \\
.00 \\
.00 \\
.00 \\
.00 \\
.00 \\
1.40 \\
5.05 \\
1.00 \\
.80 \\
.40 \\
2.40 \\
1.05 \\
.75 \\
.55 \\
.40 \\
.40 \\
.30 \\
.25 \\
.25 \\
1.65\end{array}$ & $\begin{array}{r}0.60 \\
.40 \\
.35 \\
.40 \\
.20 \\
.15 \\
.05 \\
.15 \\
.10 \\
.10 \\
.25 \\
.35 \\
.35 \\
.30 \\
.20 \\
.20 \\
.15 \\
.15 \\
.45 \\
.50 \\
.40 \\
.65 \\
.40 \\
.25 \\
.25 \\
.30 \\
.50 \\
.40 \\
.40 \\
.40 \\
. .2\end{array}$ & $\begin{array}{r}0.35 \\
.30 \\
.30 \\
.20 \\
.20 \\
.20 \\
.20 \\
.20 \\
.20 \\
.30 \\
.30 \\
.30 \\
.30 \\
.30 \\
.35 \\
.30 \\
.30 \\
.30 \\
.30 \\
.20 \\
.20 \\
.20 \\
.25 \\
.30 \\
.30 \\
.30 \\
.30 \\
.20 \\
.40 \\
.30 \\
.30\end{array}$ & $\begin{array}{r}0.30 \\
.30 \\
.30 \\
.30 \\
.30 \\
.30 \\
.30 \\
.25 \\
.25 \\
.30 \\
.40 \\
.40 \\
.30 \\
.30 \\
.30 \\
.35 \\
.35 \\
.30 \\
.25 \\
.30 \\
.30 \\
.30 \\
.50 \\
.45 \\
.40 \\
.40 \\
.75 \\
.35 \\
.10 \\
.10 \\
. . . .\end{array}$ & $\begin{array}{r}0.20 \\
.30 \\
.55 \\
.60 \\
.55 \\
.50 \\
.50 \\
.60 \\
.55 \\
.50 \\
.40 \\
.35 \\
.30 \\
.45 \\
.40 \\
.40 \\
.35 \\
.30 \\
.30 \\
.30 \\
.30 \\
.40 \\
.30 \\
.20 \\
.20 \\
.35 \\
.40 \\
.30 \\
.30 \\
.25 \\
.30\end{array}$ \\
\hline
\end{tabular}

Purgatoire River at Trinidad, Colorado.

\begin{tabular}{|c|c|c|c|c|c|c|c|}
\hline Day. & May. & June $a$ & July. & Aug. & Sept. & Oet. & Nov. \\
\hline 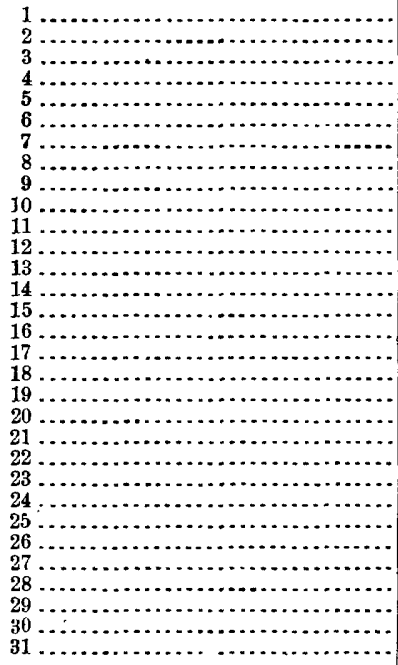 & $\begin{array}{l}\text { 3. } 15 \\
\text { 3. } 15 \\
\text { 3. } 10 \\
\text { 3. } 15 \\
\text { 3. } 10 \\
\text { 3. } 20 \\
\text { 3. } 10 \\
\text { 3. } 15 \\
\text { 3. } 15 \\
\text { 3. } 15 \\
\text { 3. } 10 \\
\text { 3. } 15 \\
\text { 3. } 20 \\
\text { 3. } 30 \\
\text { 3. } 15 \\
\text { 3. } 20 \\
\text { 3. } 20 \\
\text { 3. } 15 \\
\text { 3. } 15 \\
\text { 3. } 15 \\
\text { 3. } 05 \\
\text { 3. } 00 \\
\text { 3. } 00 \\
\text { 3.10 } \\
\text { 3. } 10 \\
\text { 3. } 05 \\
\text { 3. } 15 \\
\text { 3. } 10 \\
\text { 3. } 15 \\
\text { 3. } 20 \\
\text { 3. } 35\end{array}$ & 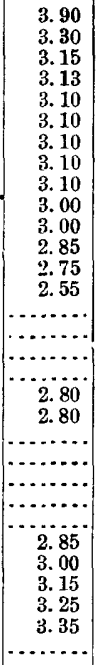 & $\begin{array}{l}3.45 \\
3.30 \\
3.20 \\
3.25 \\
3.25 \\
3.20 \\
3.05 \\
2.75 \\
2.80 \\
. . . . \\
3.35 \\
3.10 \\
2.95 \\
2.80 \\
2.80 \\
3.80 \\
3.40 \\
3.45 \\
3.50 \\
3.40 \\
3.60 \\
3.30 \\
3.30 \\
4.80 \\
5.45 \\
4.15 \\
3.90 \\
3.80 \\
3.80 \\
3.70 \\
3.70\end{array}$ & $\begin{array}{l}3.65 \\
3.80 \\
3.70 \\
3.55 \\
3.60 \\
3.70 \\
3.55 \\
3.50 \\
3.40 \\
3.40 \\
3.30 \\
3.30 \\
3.60 \\
3.25 \\
3.30 \\
3.30 \\
3.25 \\
4.65 \\
3.40 \\
3.50 \\
3.70 \\
3.60 \\
3.75 \\
3.65 \\
3.50 \\
3.50 \\
3.45 \\
3.40 \\
3.40 \\
\text { 3. 40 } \\
5.05\end{array}$ & $\begin{array}{r}3.60 \\
3.60 \\
3.80 \\
3.60 \\
3.65 \\
3.55 \\
3.40 \\
3.40 \\
3.45 \\
3.45 \\
3.40 \\
3.35 \\
3.30 \\
3.30 \\
3.30 \\
3.30 \\
3.70 \\
3.80 \\
3.85 \\
3.80 \\
3.75 \\
4.20 \\
4.00 \\
3.90 \\
3.70 \\
3.65 \\
3.70 \\
3.70 \\
3.70 \\
3.65 \\
\ldots \ldots . .\end{array}$ & 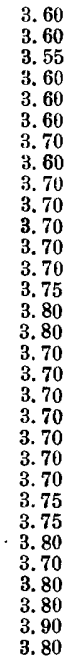 & $\begin{array}{r}\text { 3. } 70 \\
3.70 \\
3.70 \\
3.60 \\
3.60 \\
3.60 \\
3.70 \\
3.70 \\
3.70 \\
3.70 \\
3.70 \\
3.65 \\
3.60 \\
3.60 \\
3.60 \\
3.60 \\
3.60 \\
3.60 \\
3.60 \\
3.60 \\
3.60 \\
3.60 \\
3.60 \\
3.60 \\
3.60 \\
\text { 3. } 60 \\
3.60 \\
3.60 \\
3.60 \\
3.60 \\
\ldots . .6\end{array}$ \\
\hline
\end{tabular}

a June 15 to 18,20 to 26 , river dry. 
Arkansas River at Hutchinson, Kansas.

\begin{tabular}{|c|c|c|c|c|c|c|c|c|c|c|c|c|}
\hline Day. & Jan. & Feb. & Mar. & Apr. & Mas. & June. & July. & Aug. & Sept. & Oct. & Nov. & Dec. \\
\hline $1 .$. & 1.95 & 2. 50 & 1.90 & 1.50 & 1.60 & 1.30 & 1. 55 & 1. 80 & 1. 20 & 1.30 & 1.75 & (a) \\
\hline $2 \ldots$ & 1. 90 & 2.50 & 1.85 & 1.50 & 1. 55 & 1. 30 & 1.50 & 1.65 & 1. 20 & 1. 30 & 1. 60 & (a) \\
\hline 3. & 1. 85 & 2.45 & 1.70 & 1.50 & 1. 50 & 1. 30 & 1. 55 & 1.80 & 1. 20 & 1. 30 & 1. 60 & 1.70 \\
\hline 4. & 1. 80 & 2.40 & 1. 70 & 1.50 & 1. 50 & 1. 30 & 1. 60 & 1.90 & 1. 20 & 1.30 & 1.60 & 1. 60 \\
\hline $5 \ldots$ & 2. 15 & 2.40 & 1.85 & 1.50 & 1.45 & 1.30 & 1.50 & 1.85 & 1. 20 & 1. 30 & 1. 60 & 1. 60 \\
\hline 6. & 2. 20 & 2.40 & 1.90 & 1.50 & 1.40 & 1. 30 & 1.50 & 1.80 & 1.20 & 1.30 & 1. 60 & 1. 60 \\
\hline & 1.95 & 2.40 & 1.80 & 1. 45 & 1.40 & 2.35 & 1. 50 & 1.80 & 1. 20 & 1. 30 & 1. 60 & 1. 60 \\
\hline & 1. 90 & 2.40 & 1.80 & 1.50 & 1.40 & 2.65 & 1. 50 & 3.80 & 1. 20 & 1. 30 & 1. 50 & 1.00 \\
\hline & 1.85 & 2. 30 & 1.90 & 1.50 & 1. 40 & 2.00 & 1.50 & 1.65 & 1. 20 & 1. 30 & 1. 50 & 1.60 \\
\hline 10. & 1.85 & 2. 20 & 1.95 & 1. 45 & 1.50 & 1.80 & 1.55 & 1. 60 & 1. 20 & 1. 40 & 1. 50 & 1. 60 \\
\hline 11. & 1. 80 & 2. 20 & 1. 90 & 1.45 & 1.45 & 1.70 & 1.50 & 1. 50 & 1.20 & 1. 40 & 1. 50 & 1. 60 \\
\hline 12. & 1.90 & 2.15 & 1. 90 & 1.55 & 1. 40 & 1.70 & 1.45 & 1.50 & ․ 20 & 1. 30 & 1. 50 & 1. 60 \\
\hline 13. & 2.10 & 2.10 & 1. 85 & 1. 70 & 1. 40 & 1. 65 & 1. 40 & 1.50 & 1. 20 & 1. s0 & 1. 50 & 1.60 \\
\hline 14. & 2. 25 & 2.10 & 1. 90 & 1.65 & 1.50 & 1. 55 & 1. 40 & 1. 40 & 1. 50 & 1. 30 & 1. 50 & 1. 60 \\
\hline 15. & 2. 40 & 2. 10 & 1. 90 & 1. 60 & 1. 60 & 1.50 & 1.40 & 1. 40 & 1.45 & 1.30 & 1. 50 & 1. 60 \\
\hline 16. & 2.40 & 2.00 & 2.25 & 1. 65 & t. 70 & 1. 50 & 1. 50 & 1. 40 & 1. 35 & 1. 30 & 1. 50 & 1.60 \\
\hline 17. & 2. 40 & 2.00 & 1.85 & 1. 90 & 1. 70 & 1. 60 & 1.60 & 1. 40 & 1. 30 & 1. 30 & 1. 50 & 1. 60 \\
\hline 18. & 2.30 & 2.00 & 1. 90 & 2. 20 & 1. 65 & 1. 55 & 2.30 & 1. 40 & 1. 40 & 1.30 & 1. 50 & 1. 80 \\
\hline 19. & 2. 35 & 2.05 & 1.95 & 2.15 & 1. 55 & 1. 50 & 3.15 & 1. 40 & 1. 55 & 1. 30 & 1. 50 & 1. 70 \\
\hline 20. & 2. $4 u$ & 2. 10 & 1.90 & 2. 10 & 1. 55 & 1. 40 & 2.65 & 1.40 & 1.40 & 1. 30 & 1. 50 & 1. 60 \\
\hline 21. & 2.40 & 2. 10 & 1. 80 & 2.00 & 1. 50 & 1. 60 & 2. 25 & 1.40 & 1. 35 & 1. 30 & 1. 50 & 1. 60 \\
\hline 22. & 2.40 & 2.15 & 1.80 & 2.00 & 1. 50 & 1.80 & 2. 22 & 1. 40 & 1. 30 & 1. 50 & 1. 50 & 1. 50 \\
\hline 23. & 2.50 & 2.10 & 1.80 & 2.05 & 1. 50 & 2. 35 & 2.20 & 1. 30 & 1. 30 & 1. 50 & 1. 50 & 1. 50 \\
\hline 24. & 2. 45 & 2.10 & 1. 75 & 1. 95 & 1. 50 & 2.65 & 2.85 & 1. 30 & 1.30 & 1.50 & 1.40 & 1. 50 \\
\hline 25 . & 2.40 & 2. 10 & 1.70 & 1.90 & 1. 40 & 2. 30 & 3.00 & 1. 30 & 1. 30 & 1. 50 & 1. 40 & 1. 50 \\
\hline 26 . & 2.85 & 2.10 & 1.70 & 1. 80 & 1. 50 & 1. 95 & 2. 40 & 1. 30 & 1.30 & 1. 50 & 2. 00 & 1.50 \\
\hline & 2. 90 & 2.15 & 1. 60 & 1.80 & 1. 60 & 1.75 & 2.15 & 1. 30 & 1.30 & 1.50 & 2.00 & 1. 55 \\
\hline & 2.80 & 1.95 & 1. 60 & 1.85 & 1.45 & 1.70 & 2. 00 & 1. 30 & 1. 30 & 1. 50 & $(a)$ & 1. 60 \\
\hline & 2.75 & 1. 90 & 1. 69 & 1.75 & 1. 40 & 1. 70 & 1.95 & 1. 30 & 1.30 & 2. 00 & (a) & 1. 60 \\
\hline & 2.60 & ...... & 1. 55 & 1.65 & 1. 30 & 1.60 & 1.85 & 1. 30 & 1.30 & 2. 05 & (a) & 1. 50 \\
\hline 31. & 2.50 & $\ldots .$. & 1.50 & $\ldots . .$. & 1. 30 & $\cdots$ & 1.80 & 1. 30 & ....... & 1. 85 & & 1.60 \\
\hline
\end{tabular}

$a$ Ico.

Verdigris River at Liberty, Kansas.

\begin{tabular}{|c|c|c|c|c|c|c|c|c|c|c|c|c|}
\hline Day. & Jan. & Feb. & Mar. & Apr. & May. & June. & July. & Aug. & Sept. & Uct. & Nov. & Dec. \\
\hline 1. & 4. 60 & 3.50 & 3.00 & 3.00 & 3.50 & 4. 55 & 4.00 & 2.90 & 1.90 & 2.35 & 5.40 & 4. 40 \\
\hline 2. & 4.45 & 3. 80 & 2.90 & 2.90 & 3.40 & 4. 35 & 3.65 & 2.80 & 1. 90 & 2.25 & 4.45 & 4. 10 \\
\hline 3. & 4.05 & 4.00 & 2.80 & 2.80 & 3. 40 & 4.20 & 3.45 & 2.70 & 1.90 & 2.15 & 3.90 & 3.90 \\
\hline 4. & 3.85 & 3.90 & 2.80 & 2.80 & $\begin{array}{l}3.80 \\
3.80\end{array}$ & 4.05 & $\begin{array}{l}3.40 \\
0\end{array}$ & 2.60 & 1.90 & 2.10 & 3. 65 & 3.90 \\
\hline 5. & 4.00 & 3.80 & 2. 80 & 2.80 & 6.05 & 3.85 & 6.70 & 2.55 & 1.90 & 2.10 & 3.45 & 3.80 \\
\hline & 3.90 & 3. 60 & 2.80 & 2.80 & 4. 20 & 3. 80 & 5.40 & 2.45 & 1. 90 & 2.00 & $\begin{array}{l}3.40 \\
3.40\end{array}$ & \\
\hline & 3.90 & 3.60 & 2. 80 & 2. 80 & 3.55 & 3.65 & 4.35 & 2.35 & 1.95 & 2.00 & 3.30 & 3.80 \\
\hline & 3.90 & 3.50 & 2.80 & 2.80 & 3.25 & 14.75 & 3.80 & 2.30 & 1.95 & 2.00 & 3.20 & $\ldots . .$. \\
\hline & 3. 90 & 3. 40 & 2. 80 & 2. 80 & 3. 05 & 21.66 & 3.50 & 2. 10 & 1.90 & 1. 95 & 3. 20 & \\
\hline 10. & 3.90 & 3.30 & 2.80 & 3.00 & 2.90 & 12.75 & 3.40 & 2.10 & 1. 80 & 1. 90 & 3. 10 & 3.80 \\
\hline ...... & 3.90 & 3.40 & 2.80 & 5.40 & 2.80 & 5. 25 & 3.30 & 2.10 & 1.80 & 1.90 & 3. 10 & \\
\hline 12. & 3. 90 & 3. 40 & 2. 80 & 4.40 & 2. 70 & 4.65 & 3. 20 & 2.10 & 1.70 & 1.90 & 3.00 & 3.60 \\
\hline 13. & 3.90 & 3.40 & 2.80 & 3. 80 & 2. 70 & 4.15 & 3.10 & 2.10 & 1.70 & 1.90 & 2. 95 & $\ldots$ \\
\hline 14.. & 3.90 & 3. 40 & 2.80 & 3.50 & 2.70 & 3.95 & 3.00 & 2. 10 & 1. 70 & 1. 90 & 2.90 & 3.40 \\
\hline 15. & 3.90 & 3.40 & 2.80 & 3. 40 & 2.70 & 3.75 & 2.90 & 2.10 & 1. 70 & 1.85 & 2.80 & \\
\hline 16. & 3. 90 & 3. 30 & 2.80 & 3. 30 & 8.45 & 3.55 & 2.80 & 2. 10 & 1. 70 & 1. 80 & 2.80 & 3.20 \\
\hline 17. & 3. 80 & 3.30 & 3.00 & 3.20 & 11.25 & 3. 40 & 2.70 & 2.00 & 1. 70 & 1. 80 & 2. 70 & \\
\hline 18. & 3.70 & 3.30 & 3.10 & 3.10 & 10.65 & 3.30 & 2.70 & 2.00 & 1. 70 & 1.80 & 2.70 & 3.10 \\
\hline 19. & 3. 70 & 3. 20 & 3. 20 & 3. 00 & 6. 80 & 3.20 & 7.00 & 2.00 & 2. 60 & 1.80 & 2.65 & $\ldots \ldots$ \\
\hline 20 . & 3. 70 & 3.20 & 3.20 & 3.00 & 12.25 & 3. 10 & 16. 25 & 2.00 & 3. 35 & 1. 80 & 2.60 & 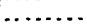 \\
\hline 21. & 3. 70 & 3.10 & 3.20 & 3.10 & 9.95 & 3. 10 & 16.50 & 2.00 & 3.20 & 1.80 & 2. 60 & 3. 00 \\
\hline 22 . & 3.70 & 3.00 & 3. 20 & 3.15 & 6.45 & 3.15 & 15. 25 & 2.00 & 3.00 & 1.90 & 2.60 & \\
\hline 23. & 3.70 & 3.00 & 3.20 & 3.50 & 12.65 & 3.20 & 7.00 & 2.00 & 2. 85 & 2. 40 & 2.60 & 3. 00 \\
\hline 24. & 3. 70 & 3.00 & 3.20 & 3.15 & 23.75 & 3.20 & 4.70 & 2.00 & 2.65 & 3.70 & 2.60 & \\
\hline 25. & 3. 60 & 3.00 & 3. 10 & 5.55 & 22.75 & 3. 25 & 4. 10 & 2. 00 & 2. 50 & 4. 50 & 2.60 & 3.00 \\
\hline 26. & 3. 50 & 3.00 & 3. 10 & 8.10 & 20.50 & 3.45 & 3. 90 & 2.00 & 2. 50 & 3.85 & 2. 80 & ...... \\
\hline & 3. 40 & 3.00 & 3.10 & 6.20 & 19.25 & 8.40 & 3.80 & 2.00 & 2.50 & 3. 30 & 14.50 & \\
\hline 28. & 3. 40 & 3.00 & 3.10 & 4.85 & 13.00 & 12.50 & 3.65 & 2. 00 & 2. 50 & 3.05 & 14.00 & \\
\hline & 3. 40 & 3. 00 & 3.00 & 4. 45 & 6.55 & 8. 10 & 3.45 & 2.00 & 2.45 & 5.25 & 6.85 & \\
\hline 30 & 3. 40 & ...... & 3. 00 & 3.90 & 5. 25 & 4. 65 & 3.15 & 1. 90 & 2.40 & 14.00 & 4.80 & \\
\hline 31 & 3. 40 & $\cdots$ & 3.00 & $\ldots$. & 4. 80 & $\ldots$. & 3.00 & 1.90 & $\ldots \ldots$ & 8.50 & $\ldots$ & \\
\hline
\end{tabular}


Neosho River at Iola, Kansas.

\begin{tabular}{|c|c|c|c|c|c|c|c|c|c|c|c|c|}
\hline Day. & Jan. & Feb. & Mar. & Apr. & May. & June. & July. & Aug: & Sept. & Oet. & Nov. & Dec. \\
\hline & 2.95 & 2. 95 & 2.40 & 2. 30 & 3. 60 & 4.40 & 3.30 & 3. 00 & 2.10 & 2.10 & 3. 70 & 3.25 \\
\hline & 2.80 & 2.95 & 2. 40 & 2.30 & 3. 40 & 4.00 & 3.30 & 2.85 & 2.10 & 2. 10 & 3. 35 & 3.15 \\
\hline & 2.60 & 2.90 & 2.40 & 2. 30 & 3.15 & 3.90 & 3. 30 & 2.80 & 2.10 & 2.10 & 2.60 & 2.85 \\
\hline 4 & 2.50 & 2.70 & 2.40 & 2.30 & 7.58 & 3. 80 & 4.35 & 2.70 & 2.10 & 2.10 & 2.40 & 2.65 \\
\hline 5 & 2.70 & 2.70 & 2.45 & 2.30 & 5.00 & 3.70 & 4. 95 & 2.60 & 2.10 & 2.00 & 2.35 & 2.55 \\
\hline 6. & 2.65 & 2.70 & 2.45 & 2. 30 & 3. 85 & 3. 70 & 3. 90 & 2.55 & 2.10 & 2.00 & 2.35 & 2. 50 \\
\hline 7 & 2.55 & 2.65 & 2.40 & 2.30 & 3. 75 & 3. 80 & 9. 80 & 2.50 & 2.10 & 2.00 & 2.45 & 2.50 \\
\hline & 2.50 & 2.55 & 2.40 & 3. 95 & 3. 40 & 6.45 & 3.50 & 2.40 & 2. 10 & 2. 00 & 2.40 & 2.50 \\
\hline & 2.55 & 2.50 & 2.40 & 10.25 & 3.00 & 4. 85 & 3. 35 & 2.30 & 2. 10 & 2.00 & 2.40 & 2.50 \\
\hline 10. & 2.50 & 2.50 & 2.40 & 8.10 & 2.90 & 4.30 & 3.20 & 2.30 & 2.10 & 2. 00 & 2.30 & 2.45 \\
\hline 11. & 2. 50 & 2.50 & 2.30 & 6.85 & 2.85 & 4. 00 & 2.95 & 2.30 & 2.10 & 2.00 & 2.30 & 2. 40 \\
\hline 12 & 2.55 & 2.50 & 2.30 & 4.50 & 2.80 & 3.95 & 2.80 & 2.30 & 2.10 & 2. 00 & 2.30 & 2.40 \\
\hline 13 & 2.50 & 2.50 & 2.30 & 3.75 & 2.70 & 3.70 & 2.75 & 2.30 & 2.00 & 2.00 & 2.30 & 2.40 \\
\hline 14 & 2.55 & 2,50 & 2.20 & 3. 50 & 2.70 & 3.45 & 2.70 & 2.20 & 2.00 & 2.00 & 2.25 & 2.40 \\
\hline & 2.50 & 2.50 & 2.20 & 3.35 & 3.75 & 3. 35 & 2.60 & 2.20 & 2.00 & 2.00 & 2. 20 & 2.40 \\
\hline 16 & 2.50 & 2.50 & 2.30 & 3.35 & 11.55 & 3.25 & 2.60 & 2. 20 & 2. 00 & 2.10 & 2.20 & 2.40 \\
\hline & 2.55 & 2.40 & 2.30 & 3. 45 & 9.45 & 3.15 & 2.60 & 2.20 & 2. 00 & 2.10 & 2.20 & 2.35 \\
\hline 18 & 2.60 & 2.40 & 2.30 & 3. 20 & 7. 40 & 3.05 & 2.65 & 2.20 & 2.00 & 2. 00 & 2. 20 & 2. 30 \\
\hline 19 & 2.60 & 2. 40 & 2.30 & 3. 05 & 6.40 & 3.00 & 4.45 & 2.15 & 2.00 & 2. 10 & 2.20 & 2.30 \\
\hline 20 & 2.60 & 2.40 & 2.20 & 3.00 & 5.55 & .3 .00 & 5,45 & 2.10 & 2.00 & 2. 10 & 2.20 & 2.30 \\
\hline 21 & 2.60 & 2.40 & 2.20 & 2.80 & 4.85 & 2.05 & 7.20 & 2.10 & 2.00 & 2. 10 & 2.20 & 2.30 \\
\hline 22 & 2.60 & 2.40 & 2.20 & 2. 90 & 16. 25 & 2.90 & 7.75 & 2.10 & 2.00 & 2.05 & 2. 10 & 2. 30 \\
\hline 23 & 2.60 & 2.40 & 2.30 & 4.25 & 18.25 & 2. 90 & 5.65 & 2.10 & 2.00 & 2. 05 & 2. 16 & 2. 20 \\
\hline & 2.60 & 2.40 & 2.40 & 3.00 & 20.10 & 2.90 & 4. 30 & 2.10 & 2.10 & 2.00 & 2.10 & 2. 20 \\
\hline & 2.60 & 2.40 & 2.60 & 9.50 & 19.05 & 3.40 & 4.00 & 2.10 & 2.10 & 2.00 & 2.15 & 2.20 \\
\hline & 2.60 & 2.40 & 2.50 & 7. 70 & 16.50 & 3. 60 & 3.65 & 2.10 & 2.10 & 2.00 & 2.80 & 2. 20 \\
\hline & 2.50 & 2.40 & 2.40 & 9.40 & 15. 25 & 3. 45 & 3.50 & 2.10 & 2.10 & 2. 00 & 4.85 & 2.20 \\
\hline & 2.50 & 2.40 & 2.40 & 6.95 & 10.75 & 3.65 & 3. 30 & 2.10 & 2.10 & 2.00 & 4. 00 & 2. 20 \\
\hline & 2.50 & 2.40 & 2.35 & 4.50 & 5.70 & 3.45 & 3. 30 & 2.10 & 2. 10 & 2.00 & 3.55 & 2.20 \\
\hline & 2.55 & $\ldots \ldots$ & 2.30 & 3.85 & 5.05 & 3. 40 & 3.20 & 2.10 & 2. 10 & 2.05 & 3.45 & 2.20 \\
\hline & 2. 75 & $\ldots . .$. & 2.30 & $\ldots \ldots$ & 4.65 & $\ldots$ & 3.10 & & & 2.85 & 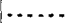 & 2.20 \\
\hline
\end{tabular}

Medicine River at Kiowa, Kansas.

\begin{tabular}{|c|c|c|c|c|c|c|c|c|c|c|}
\hline Day. & Jan. & Feb. & Mar. & Apr. & May. & June. $a$ & July. & Aug. & Sept. $b$ & Oet. \\
\hline & 2.50 & 2.50 & 2. 40 & 2.40 & 2.80 & 2.30 & 3. 65 & 2.40 & 1.40 & 2. 10 \\
\hline 2. & 2.60 & 2.50 & 2.40 & 2.40 & 2.50 & 2.30 & 3.50 & 2. 40 & 1.40 & 2.20 \\
\hline 3. & 2. 60 & 2.50 & 2.40 & 2.40 & 2.50 & 2.20 & 3. 10 & 2. 40 & 1. 40 & 2. 20 \\
\hline 4. & 2. 70 & 2.60 & 2.40 & 2.40 & 2.50 & 2.20 & 2. 80 & 2. 40 & 1.40 & 2.20 \\
\hline 5. & 2.70 & 2.60 & 2.30 & 2. 20 & 2.50 & 2,10 & 2.80 & 2.40 & 1.40 & 2.20 \\
\hline 6. & 2. 60 & 2.60 & 2.30 & 2.20 & 2.40 & 1.90 & 2. 70 & 2.40 & 1.40 & 2. 10 \\
\hline & 2. 60 & 2.70 & 2.30 & 2.00 & 2.40 & 1.65 & 2. 60 & 2. 30 & 1.40 & 2. 20 \\
\hline 8. & 2. 60 & 2.70 & 2.40 & 2.00 & 2.40 & 1.30 & 2. 60 & 2. 30 & 1. 40 & 2.20 \\
\hline 9. & 2. 60 & 2. 70 & 2.40 & 2.00 & 2.30 & 1.40 & 2. 60 & 2. 00 & $\ldots . .$. & 2. 20 \\
\hline $10 \ldots$ & 2. 70 & 2.70 & 2. 40 & 1. 90 & 2.20 & 1. 20 & 2.90 & 2.00 & $\ldots \ldots$ & 2.30 \\
\hline $11 .$. & 2. 80 & 2.60 & 2.40 & 1. 90 & 2.20 & 1.20 & 2.95 & 2.00 & ........ & 2.35 \\
\hline 12. & 2. 70 & 2.60 & 2.50 & 2.20 & 2.20 & 1. $\dot{2} 0$ & 2. 90 & 1.90 & 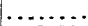 & 2.40 \\
\hline $13 \ldots \ldots$ & 2. 60 & 2. 60 & 2.60 & 2.50 & 2. 30 & 1.20 & 2.85 & 1. 90 & 2. 00 & 2. 40 \\
\hline $14 \ldots$ & 2. 60 & 2.60 & 2.60 & 2.55 & 2.30 & $\ldots \ldots \ldots$ & 3. 25 & 1. 80 & 2. 00 & 2.40 \\
\hline 15. & 2.60 & 2.60 & 2.70 & 2.60 & 2.30 & ...... & 3.80 & 1.80 & 2.00 & 2.40 \\
\hline 16. & 2.70 & 2.50 & 2.65 & 2.60 & 2. 20 & ..... & 5.90 & 1.80 & 2. 00 & 2. 40 \\
\hline 17. & 2. 80 & 2.50 & 2.60 & 2. 60 & 2.10 & & 5. 00 & 1. 80 & 2.00 & 2.30 \\
\hline 18. & 2. 75 & 2. 60 & 2.50 & 2. 75 & 2.10 & . & 4. 70 & 1.80 & 2. 00 & 2. 30 \\
\hline 19. & 2.65 & 2.70 & 2.50 & 3.75 & 2.15 & , & 5. 60 & 1. 80 & 2. 10 & 2.30 \\
\hline 20. & 2.60 & 2. 70 & 2.50 & 3.70 & 2. 20 & $\ldots . . .$. & 4.90 & 1.70 & 2.35 & 2. 40 \\
\hline 21. & 2.50 & 2.70 & 2.50 & 3. 15 & 2.35 & 2.00 & 3. 70 & 1. 70 & 2.40 & 2.40 \\
\hline 22. & 2.50 & 2.60 & 2.50 & 2.75 & 2.40 & 2.20 & 3. 60 & 1. 70 & 2. 20 & 2.55 \\
\hline 23. & 2.50 & 2.50 & 2.60 & 2.70 & 3.20 & 4.00 & 3. 40 & 1. 70 & 2.20 & 2. 80 \\
\hline 24. & 2. 50 & 2.50 & 2.60 & 2.65 & 3.00 & 2. 95 & 3.00 & 1. 60 & 2. 20 & 2.90 \\
\hline 25. & 2.60 & 2.40 & 2.60 & 2.50 & 2.80 & 2.93 & 2. 75 & 1. 60 & 2.20 & 2.85 \\
\hline 26. & 2.60 & 2.40 & 2.60 & 2.50 & 2. 70 & 5. 50 & 2.75 & 1. 50 & 2.20 & 2.80 \\
\hline $27 \ldots$ & 2.60 & 2.40 & 2. 60 & 2.60 & 2. 60 & 5. 35 & 2.60 & 1. 50 & 2.10 & 2.60 \\
\hline 28. & 2.60 & 2.50 & 2.50 & 2. 80 & 2. 55 & 4.75 & 2. 50 & 1.40 & 2. 10 & 2. 60 \\
\hline 29. & 2.60 & 2.50 & 2.45 & 2.85 & 2.45 & 4. 25 & 2.50 & 1. 40 & 2.10 & 3. 00 \\
\hline 30. & 2.50 & ........ & 2.40 & 2.80 & 2.40 & 3.95 & 2.55 & 1. 40 & 2.10 & 2.95 \\
\hline 31. & 2.50 & $\ldots \ldots$ & 2.40 & $\ldots \ldots \ldots$ & 2.30 & ...... & 2.50 & 1. 40 & $\ldots .$. & 2.75 \\
\hline
\end{tabular}

$a$ Water standing in pools from June 14 to 21 .

$b$ Water standing in pools from September 9 to 13. 
Cimarron River at Arkalon, Kansas.

\begin{tabular}{|c|c|c|c|c|c|c|c|c|c|c|}
\hline Day. & Jan. & Feb. & Mar. & Apr. & May. & June. & July. & Aug. & Sept. & Oet. \\
\hline & 2.50 & 2.50 & 2.50 & 2.50 & 2. 50 & 2.50 & 2.40 & 2. 40 & 2. 30 & 2.30 \\
\hline & 2.50 & 2.50 & 2.50 & 2.50 & 2. 50 & 2.40 & 2. 40 & 2.40 & 2. 30 & 2. 30 \\
\hline & 2. 50 & 2.50 & 2.50 & 2.50 & 2. 50 & 2.40 & 2.40 & 2.40 & 2.30 & 2. 30 \\
\hline & 2.50 & 2.50 & 2.50 & 2.50 & 2.50 & 2.40 & 2.40 & 2. 40 & 2. 30 & 2. 30 \\
\hline & 2. 50 & 2.50 & 2.50 & 2.50 & 2. 50 & 2.40 & 2. 40 & 2.40 & 2. 30 & 2.30 \\
\hline & 2.50 & 2.50 & 2.50 . & 2.50 & 2. 50 & 2. 40 & 2. 40 & 2.40 & 2. 30 & 2. 30 \\
\hline & 2.50 & 2.50 & $2.50^{\circ}$ & 2.50 & 2. 50 & 2. 40 & 2. 40 & 2. 40 & 2. 30 & 2.30 \\
\hline & 2.50 & 2.50 & 2.50 & 2.50 & 2.50 & 2. 40 & 2.30 & 2. 40 & 2. 30 & 2. 30 \\
\hline 9. & 2.50 & 2.50 & 2.50 & 2. 50 & 2.50 & 2.40 & 2.30 & 2. 40 & 2. 30 & 2. 30 \\
\hline 10. & 2.50 & 2.50 & 2.50 & 2.50 & 2. 40 & 2. 40 & 2. 30 & 2. 40 & 2. 30 & 2.30 \\
\hline $11 \ldots \ldots$ & 2. 50 & 2.50 & 2.50 & 3.00 & 2.40 & 2.40 & 2.30 & 2.40 & 2.30 & 2.30 \\
\hline $12 \ldots \ldots$ & 2.50 & 2.50 & 2.50 & 4. 15 & 2.50 & 2.40 & 2.30 & 2. 40 & 2.30 & 2.30 \\
\hline $13 \ldots . .$. & 2.50 & 2.50 & 2.50 & 3.00 & 2. 50 & 2.40 & 2.30 & 2.40 & 2. 30 & 2.30 \\
\hline $14 \ldots \ldots$ & 2.50 & 2.50 & 2.50 & 2.80 & 2.50 & 2. 40 & 2.30 & 2.40 & 2. 30 & 2. 30 \\
\hline 15. & 2.40 & 2.50 & 2.50 & 2.70 & 2.50 & 2.40 & 2.85 & 2. 40 & 2. 30 & 2.50 \\
\hline 16. & 2. 50 & 2.50 & 2.50 & 2. 6i) & 2. 50 & 2.40 & 2.65 & 2.35 & 2. 30 & 2. 50 \\
\hline 17. & 2. 50 & 2.50 & 2. 50 & 2.60 & 2.50 & 2.40 & 2.50 & 2. 30 & 2. 30 & 2.50 \\
\hline 18. & 2.50 & 2.50 & 2.50 & 2.60 & 2.50 & 2.40 & 2.50 & 2. 30 & 2. 30 & 2.50 \\
\hline & 2. 50 & 2.50 & 2.50 & 2.50 & 2.50 & 2.30 & 2.50 & 2. 30 & 2.30 & 2. 50 \\
\hline 20 . & 2. 50 & 2. 50 & 2. 50 & 2.50 & 2. 50 & 2.30 & 2.50 & 2. 30 & 2. 30 & 2.50 \\
\hline 21. & 2. 50 & 2.50 & 2.50 & 2.50 & 2. 50 & 2.40 & 2.50 & 2. 30 & 2. 30 & 2.50 \\
\hline 22. & 2. 50 & 2.50 & 2.50 & 2.40 & 2.50 & 2. 40 & 2.50 & 2.30 & 2.30 & 2.50 \\
\hline 23. & 2. 50 & 2.50 & 2.50 & 2.40 & 2. 50 & 2. 4.0 & 2.50 & 2. 30 & 2.30 & 2. 50 \\
\hline $24 \ldots$ & 2.50 & 2.50 & 2.50 & 2.40 & 2.50 & 2.40 & 2.50 & 2. 30 & 2. 30 & 2. 50 \\
\hline $25 \ldots \ldots \ldots$ & 2.50 & 2.50 & 2.50 & 2.40 & 2. 50 & 2.40 & 2. 50 & 2.30 & 2. 30 & 2.50 \\
\hline 26. & 2.50 & 2.50 & 2.50 & 2.50 & 2.50 & 2.40 & 2. 50 & 2.30 & 2.30 & 2. 50 \\
\hline 27. & 2.50 & 2.50 & 2.50 & 2.50 & 2.50 & 2.40 & 2.50 & 2. 30 & 2.30 & 2. 50 \\
\hline 28. & 2.50 & 2.50 & 2.50 & 2.50 & 2.50 & 2.40 & 2.45 & 2. 30 & 2. 30 & 2.50 \\
\hline & 2.50 & 2.50 & 2.50 & 2.50 & 2. 50 & 2.40 & 2. 40 & 2. 30 & 2.30 & 2.50 \\
\hline & 2.40 & $\ldots . .$. & 2.50 & 2. 50 & 2. 50 & 2.40 & 2.40 & 2.30 & 2. 30 & 2.50 \\
\hline & 2.40 & (2) & 2.50 & & 2.50 & & 2.40 & 2. 30 & ....... & 2. 50 \\
\hline
\end{tabular}

Mora River at Watrous, New Mexico.

\begin{tabular}{|c|c|c|c|c|c|c|c|c|c|c|c|}
\hline Day. & Jan. & Feb. & Day. & Jan. & Feb. & Day. & Jan. & Feb. & Day. & Jan. & Feb. \\
\hline $\begin{array}{l}1 \ldots \\
2 \ldots \\
3 \ldots \ldots \\
4 \ldots \\
5 \ldots \\
6 \ldots \\
7 \ldots \\
8 \ldots\end{array}$ & $\begin{array}{l}1.90 \\
1.90 \\
1.90 \\
1.90 \\
2.02 \\
2.00 \\
1.90 \\
1.90\end{array}$ & $\begin{array}{l}1.95 \\
1.95 \\
1.95 \\
1.92 \\
1.92 \\
1.92 \\
1.92 \\
1.92\end{array}$ & $\begin{array}{r}9 \ldots \\
10 \ldots \\
11 \ldots \\
12 \ldots \\
13 \ldots \\
14 \ldots \\
15 \ldots \\
16 \ldots\end{array}$ & $\begin{array}{l}1.90 \\
1.90 \\
1.90 \\
1.90 \\
1.90 \\
1.90 \\
1.90 \\
1.90\end{array}$ & $\begin{array}{l}1.90 \\
1.90 \\
1.90 \\
1.90 \\
1.90 \\
1.90 \\
1.90 \\
1.90\end{array}$ & $\begin{array}{l}17 \ldots \\
18 \ldots \\
19 \ldots \\
20 \ldots \\
21 \ldots \\
22 \ldots \\
23 \ldots \\
24 \ldots\end{array}$ & $\begin{array}{l}1.90 \\
1.95 \\
1.95 \\
1.95 \\
1.95 \\
1.90 \\
1.90 \\
1.90\end{array}$ & $\begin{array}{l}1.90 \\
1.90 \\
1.90 \\
1.90 \\
1.90 \\
1.90 \\
1.90 \\
1.90\end{array}$ & $\begin{array}{l}25 \ldots \\
26 \ldots \\
27 \ldots \\
28 \ldots \\
29 \ldots \\
30 \ldots \\
31 \ldots\end{array}$ & $\begin{array}{l}1.90 \\
1.90 \\
1.95 \\
1.95 \\
1.95 \\
1.95 \\
1.95\end{array}$ & $\begin{array}{c}1.90 \\
1.90 \\
1.90 \\
1.90 \\
1.90 \\
\ldots . . .\end{array}$ \\
\hline
\end{tabular}

Rio Grande at Del Norte, Colorado.

\begin{tabular}{|c|c|c|c|c|c|c|c|c|c|c|c|c|}
\hline Day. & Jan. & Feb. & Mar. & Apr. & May. & June. & July. & Aug. & Sept. & Oet. & Nov. & Dec. \\
\hline & 3. 12 & 2. 96 & 3. 12 & 2.08 & 4. 18 & 3.62 & 1.74 & 1.56 & 1. 60 & 2.06 & 1. 72 & \\
\hline 3. & 3.24 & $\begin{array}{l}2.00 \\
3.02\end{array}$ & 3.18 & 2.14 & 4. 56 & $\begin{array}{l}3.02 \\
3.38\end{array}$ & 1.70 & 1.52 & 1.50 & 2.00 & 1. 64 & \\
\hline 5. & 3.28 & 2.98 & 3. 10 & 2.46 & 4. 52 & 3.06 & 1. 64 & 1.54 & 1. 54 & 1.98 & 1. 60 & 1. 62 \\
\hline 7. & 3. 16 & 2.86 & 3.14 & 3. 08 & 4. 30 & 2.88 & 1. 62 & 1. 52 & 1. 48 & 1.94 & 1. 58 & $\cdots$ \\
\hline 9. & 3.20 & 2. 80 & 3.10 & 3. 20 & 3. 96 & 2. 72 & 1. 68 & 1. 48 & 1. 50 & 1.88 & 1. 60 & \\
\hline 11. & 3.22 & 2.90 & 3.04 & 3.02 & 2. 66 & 2.52 & 1. 64 & 1. 46 & 1. 74 & 1.86 & 1.72 & \\
\hline 12. & $3 . .$. & $\dddot{2} 78$ & 316 & $\because 98$ & 332 & 234 & 179 & 144 & $16 ?$ & 182 & 158 & \\
\hline $13 .$. & $\begin{array}{l}5.08 \\
3.12\end{array}$ & $\begin{array}{l}2.78 \\
2.72\end{array}$ & $\begin{array}{l}5.10 \\
3.02\end{array}$ & $\begin{array}{l}2.98 \\
3.04\end{array}$ & $\begin{array}{l}3.32 \\
3.24\end{array}$ & $\begin{array}{l}2.34 \\
2.20\end{array}$ & 1. 72 & $\begin{array}{l}1.44 \\
1.42\end{array}$ & $\begin{array}{l}1.62 \\
1.58\end{array}$ & $\begin{array}{l}1.82 \\
1.94\end{array}$ & $\begin{array}{l}1.58 \\
1.56\end{array}$ & . \\
\hline $17 \ldots$ & 3.18 & 2.78 & 3.84 & 2.94 & 3.06 & 2.12 & 1. 92 & 1. 42 & 1.50 & 1.84 & 1. 54 & \\
\hline $19 .$. & 3.26 & 3.20 & 2.76 & 2.76 & 3.22 & 2.10 & 2.22 & 1. 44 & 1.52 & 1.82 & 1. 52 & 1.72 \\
\hline 21. & 3.22 & 3.24 & 2.54 & 2.78 & 3.54 & 1.98 & 1.92 & 1.42 & 2.00 & 1.78 & 1.50 & \\
\hline 23. & 3. 16 & 3.18 & 2.50 & 3. 04 & 3.80 & 1. 92 & 1. 80 & 1. 46 & 2.28 & 1.78 & 1. 54 & \\
\hline 25 . & 3. 12 & 3. 24 & 2.68 & 3.90 & 4.00 & 1.86 & 1. 78 & 1. 44 & 3.16 & 1.80 & 1. 52 & \\
\hline & $\ddot{3} \cdots$ & $\cdots$ & 2 & 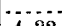 & $300^{\circ}$ & $\because \ldots$ & 130 & 30 & 0 & & & \\
\hline 27 & 3.06 & 3.20 & 2.54 & 4.22 & 4.08 & 1.82 & 1. 70 & 1.40 & 2.68 & 1.84 & 1.48 & - \\
\hline 30. & 3. 00 & $\begin{array}{l}3.11 \\
\ldots . \ldots\end{array}$ & $2.5=$ & 4.04 & 4.02 & 1.78 & 1.64 & 1. 74 & 2.18 & 1.82 & $\begin{array}{l}1.52 \\
1.64\end{array}$ & \\
\hline $31 .$. & 2.98 & .... & 2.50 & 4.12 & 3.94 & & 1 & 1.62 & $\cdots$ & 1.76 & 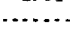 & \\
\hline
\end{tabular}


Rio Grande at Embudo, New Mexico.

\begin{tabular}{|c|c|c|c|c|c|c|c|c|c|c|c|c|}
\hline Day. & Jan. & Feb. & Mar. & Apr. & May. & June. & July. & Aug. & Sept. & Oct. & Nov. & Dec. \\
\hline 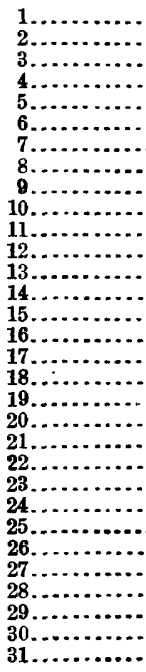 & $\begin{array}{l}8.35 \\
8.25 \\
8.20 \\
8.35 \\
8.30 \\
8.20 \\
8.30 \\
8.35 \\
8.30 \\
8.30 \\
8.50 \\
8.55 \\
8.10 \\
8.00 \\
8.00 \\
8.10 \\
8.05 \\
8.10 \\
8.00 \\
8.00 \\
8.00 \\
8.10 \\
8.10 \\
8.10 \\
8.00 \\
8.00 \\
8.10 \\
8.20 \\
8.25 \\
8.25 \\
8.30\end{array}$ & $\begin{array}{l}8.30 \\
8.30 \\
8.10 \\
8.00 \\
8.05 \\
8.20 \\
8.20 \\
8.30 \\
8.30 \\
8.20 \\
8.15 \\
8.10 \\
8.20 \\
8.25 \\
8.10 \\
8.05 \\
8.20 \\
8.25 \\
8.25 \\
8.30 \\
8.20 \\
8.25 \\
8.20 \\
8.25 \\
8.30 \\
\mathbf{8 . 3 0} \\
\mathbf{8 . 4 0} \\
\mathbf{8 . 4 5} \\
\mathbf{8 . 4 5}\end{array}$ & \begin{tabular}{|r|}
8.50 \\
8.50 \\
8.55 \\
8.40 \\
8.35 \\
8.35 \\
8.40 \\
8.35 \\
8.30 \\
8.40 \\
8.50 \\
8.60 \\
8.55 \\
8.65 \\
8.70 \\
8.75 \\
8.75 \\
8.60 \\
9.10 \\
9.10 \\
9.05 \\
8.85 \\
9.15 \\
9.35 \\
9.55 \\
9.60 \\
9.70 \\
10.10 \\
10.30 \\
10.20 \\
10.00 \\
.00
\end{tabular} & \begin{tabular}{|r}
9.50 \\
9.50 \\
9.50 \\
9.45 \\
9.55 \\
9.70 \\
10.05 \\
10.10 \\
10.10 \\
10.20 \\
10.30 \\
10.30 \\
10.30 \\
10.30 \\
10.30 \\
10.30 \\
10.30 \\
10.30 \\
10.05 \\
9.90 \\
9.70 \\
9.70 \\
9.70 \\
9.70 \\
9.80 \\
9.80 \\
9.95 \\
10.65 \\
10.80 \\
10.80 \\
\end{tabular} & $\begin{array}{l}10.70 \\
10.65 \\
10.45 \\
10.55 \\
10.85 \\
11.00 \\
10.90 \\
10.65 \\
10.40 \\
10.25 \\
10.10 \\
9.85 \\
9.65 \\
9.60 \\
9.50 \\
9.40 \\
9.40 \\
9.40 \\
9.25 \\
9.10 \\
9.00 \\
8.90 \\
8.90 \\
8.90 \\
8.90 \\
9.00 \\
9.50 \\
9.50 \\
9.50 \\
9.50 \\
9.50\end{array}$ & $\begin{array}{l}9.15 \\
8.95 \\
8.80 \\
8.45 \\
8.40 \\
8.25 \\
8.20 \\
8.00 \\
7.90 \\
7.75 \\
7.60 \\
7.60 \\
7.50 \\
7.50 \\
7.50 \\
7.50 \\
7.40 \\
7.40 \\
7.35 \\
7.30 \\
7.30 \\
7.30 \\
7.30 \\
7.30 \\
7.30 \\
7.30 \\
7.30 \\
7.30 \\
7.30 \\
7.30 \\
7.30\end{array}$ & $\begin{array}{l}7.30 \\
7.30 \\
7.30 \\
7.30 \\
7.30 \\
7.30 \\
7.30 \\
7.30 \\
7.30 \\
7.30 \\
7.30 \\
7.30 \\
9.65 \\
7.30 \\
7.30 \\
7.30 \\
7.45 \\
7.50 \\
7.50 \\
7.60 \\
8.70 \\
7.30 \\
8.05 \\
8.00 \\
7.40 \\
7.40 \\
7.50 \\
7.50 \\
7.40 \\
7.40 \\
7.30\end{array}$ & $\begin{array}{l}7.30 \\
7.30 \\
7.30 \\
7.30 \\
7.30 \\
7.30 \\
7.30 \\
7.30 \\
7.30 \\
7.30 \\
7.30 \\
7.30 \\
7.30 \\
7.30 \\
7.30 \\
7.30 \\
7.30 \\
7.30 \\
7.30 \\
7.30 \\
7.60 \\
7.40 \\
7.40 \\
7.35 \\
7.30 \\
7.30 \\
7.30 \\
7.30 \\
7.30 \\
7.30\end{array}$ & $\begin{array}{l}7.30 \\
7.30 \\
7.30 \\
7.30 \\
7.30 \\
7.30 \\
7.30 \\
7.30 \\
7.30 \\
7.30 \\
7.30 \\
7.30 \\
7.30 \\
7.30 \\
7.30 \\
7.30 \\
7.30 \\
7.30 \\
7.30 \\
7.30 \\
7.30 \\
7.30 \\
7.30 \\
7.30 \\
7.30 \\
7.30 \\
7.30 \\
7.45 \\
7.70 \\
8.30\end{array}$ & $\begin{array}{l}9.30 \\
8.65 \\
7.45 \\
7.50 \\
7.50 \\
7.50 \\
7.50 \\
7.50 \\
7.50 \\
7.50 \\
7.50 \\
7.50 \\
7.50 \\
7.50 \\
7.50 \\
7.50 \\
7.50 \\
7.50 \\
7.50 \\
8.00 \\
7.85 \\
7.70 \\
7.70 \\
7.70 \\
7.70 \\
7.70 \\
7.70 \\
7.70 \\
7.70 \\
7.70 \\
7.70\end{array}$ & $\begin{array}{l}7.50 \\
7.40 \\
7.40 \\
7.30 \\
7.30 \\
7.30 \\
7.30 \\
7.30 \\
7.40 \\
7.45 \\
7.50 \\
7.50 \\
7.60 \\
7.40 \\
7.55 \\
7.95 \\
8.30 \\
8.40 \\
8.40 \\
8.35 \\
8.50 \\
8.50 \\
8.35 \\
8.25 \\
8.20 \\
8.20 \\
8.10 \\
8.10 \\
8.10\end{array}$ & $\begin{array}{l}8.10 \\
8.10 \\
8.00 \\
8.00 \\
8.00 \\
8.00 \\
8.00 \\
8.00 \\
8.00 \\
7.90 \\
7.90 \\
7.90 \\
7.90 \\
7.99 \\
7.90 \\
7.90 \\
7.85 \\
7.80 \\
7.80 \\
7.80 \\
7.80 \\
7.80 \\
7.80 \\
7.80 \\
7.80 \\
.80\end{array}$ \\
\hline
\end{tabular}

Chama River at Abiquiu, New Mexico.

\begin{tabular}{|c|c|c|c|c|c|c|c|c|c|c|c|c|}
\hline Day. & Jan. & Feb. & Mar. & Apr. & May. & June. & July. & Ang. & Sept. & Oet. & Nov. & Dec. \\
\hline 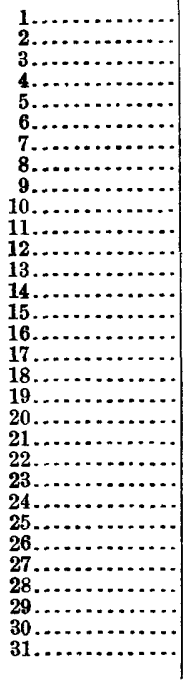 & $\begin{array}{l}1.90 \\
1.80 \\
1.80 \\
1.85 \\
1.75 \\
1.70 \\
1.75 \\
1.70 \\
1.65 \\
1.55 \\
1.70 \\
1.75 \\
1.80 \\
1.75 \\
1.70 \\
1.80 \\
1.75 \\
1.75 \\
1.65 \\
1.45 \\
1.70 \\
1.35 \\
1.65 \\
1.70 \\
1.55 \\
1.55 \\
1.55 \\
1.65 \\
1.35 \\
1.90 \\
1.10\end{array}$ & $\begin{array}{l}1.05 \\
1.95 \\
1.15 \\
1.20 \\
1.15 \\
1.00 \\
1.10 \\
1.55 \\
1.55 \\
1.20 \\
1.10 \\
1.30 \\
1.45 \\
1.55 \\
1.10 \\
.95 \\
.95 \\
1.00 \\
1.45 \\
1.40 \\
1.20 \\
1.10 \\
1.50 \\
1.75 \\
1.55 \\
1.35 \\
1.60 \\
2.05 \\
2.15 \\
\cdots \ldots . . \\
\cdots \cdots\end{array}$ & \begin{tabular}{|l|}
$\ldots \ldots$ \\
$\cdots \ldots \ldots$ \\
$\cdots \ldots$. \\
$\cdots \ldots \ldots$ \\
$\cdots \ldots \ldots$ \\
$\cdots \ldots \ldots$ \\
$\cdots \ldots \ldots$ \\
\hdashline 1.60 \\
1.75 \\
1.90 \\
1.70 \\
1.95 \\
1.50 \\
1.50 \\
1.55 \\
1.30 \\
1.30 \\
1.35 \\
1.35 \\
1.45 \\
1.55 \\
1.50 \\
1.70 \\
2.55 \\
2.25 \\
1.85 \\
1.95 \\
1.70
\end{tabular} & $\begin{array}{l}1.90 \\
2.45 \\
1.80 \\
1.95 \\
2.95 \\
2.85 \\
3.25 \\
4.13 \\
3.60 \\
2.85 \\
2.70 \\
2.80 \\
2.35 \\
3.65 \\
3.05 \\
3.35 \\
3.30 \\
3.40 \\
2.55 \\
3.40 \\
3.85 \\
4.10 \\
3.85 \\
2.95 \\
3.70 \\
3.85 \\
3.85 \\
3.55 \\
3.35 \\
3.25 \\
\ldots . . \\
.9\end{array}$ & $\begin{array}{l}3.05 \\
3.35 \\
2.85 \\
2.65 \\
2.65 \\
2.50 \\
2.90 \\
2.85 \\
2.75 \\
2.75 \\
2.65 \\
2.50 \\
2.45 \\
2.55 \\
2.35 \\
2.15 \\
2.55 \\
2.25 \\
2.35 \\
2.15 \\
1.95 \\
1.85 \\
2.05 \\
2.15 \\
2.10 \\
2.15 \\
1.55 \\
1.85 \\
2.10 \\
1.85 \\
1.55\end{array}$ & $\begin{array}{l}1.90 \\
1.90 \\
1.85 \\
1.05 \\
1.10 \\
1.05 \\
1.45 \\
1.35 \\
1.45 \\
1.75 \\
1.45 \\
1.55 \\
1.35 \\
1.30 \\
1.60 \\
1.45 \\
1.35 \\
1.30 \\
1.40 \\
1.10 \\
1.10 \\
1.10 \\
1.25 \\
1.30 \\
1.40 \\
1.25 \\
1.50 \\
1.70 \\
1.95 \\
1.70 \\
\cdots . . .\end{array}$ & $\begin{array}{l}1.70 \\
1.50 \\
1.50 \\
1.50 \\
1.35 \\
1.30 \\
1.30 \\
1.35 \\
1.80 \\
1.50 \\
1.55 \\
1.45 \\
1.45 \\
3.75 \\
2.75 \\
3.15 \\
3.55 \\
3.00 \\
3.25 \\
3.35 \\
2.85 \\
3.60 \\
2.70 \\
3.50 \\
2.95 \\
2.85 \\
2.75 \\
2.75 \\
2.95 \\
2.85 \\
2.75\end{array}$ & $\begin{array}{l}2.25 \\
2.10 \\
2.05 \\
1.95 \\
1.80 \\
1.70 \\
1.65 \\
1.50 \\
1.60 \\
1.55 \\
1.55 \\
1.60 \\
1.50 \\
1.50 \\
1.45 \\
1.50 \\
1.55 \\
1.45 \\
1.50 \\
1.35 \\
1.45 \\
1.50 \\
1.85 \\
1.85 \\
1.80 \\
1.90 \\
1.35 \\
1.35 \\
1.45 \\
1.45 \\
1.15\end{array}$ & $\begin{array}{l}2.45 \\
2.85 \\
2.75 \\
2.85 \\
2.75 \\
2.20 \\
2.65 \\
3.35 \\
3.40 \\
4.40 \\
4.40 \\
3.65 \\
3.45 \\
2.90 \\
2.75 \\
2.55 \\
3.15 \\
3.15 \\
3.45 \\
3.05 \\
2.80 \\
2.60 \\
2.55 \\
2.55 \\
2.50 \\
2.30 \\
2.25 \\
2.35 \\
2.30 \\
2.20 \\
\ldots . . . \\
\end{array}$ & $\begin{array}{l}2.25 \\
2.35 \\
2.25 \\
2.85 \\
2.70 \\
2.85 \\
3.30 \\
3.40 \\
3.15 \\
2.90 \\
2.80 \\
2.75 \\
2.65 \\
2.70 \\
2.75 \\
2.70 \\
2.65 \\
2.60 \\
2.75 \\
2.70 \\
2.75 \\
2.65 \\
2.75 \\
2.85 \\
2.85 \\
2.85 \\
2.85 \\
2.55 \\
2.65 \\
2.75 \\
2.60\end{array}$ & $\begin{array}{r}2.25 \\
2.45 \\
2.25 \\
2.25 \\
2.40 \\
2.30 \\
2.25 \\
2.30 \\
2.25 \\
2.05 \\
2.10 \\
2.25 \\
2.15 \\
2.30 \\
2.25 \\
2.15 \\
2.25 \\
2.25 \\
2.10 \\
2.15 \\
2.25 \\
2.10 \\
2.10 \\
2.00 \\
2.35 \\
2.35 \\
2.30 \\
2.35 \\
2.15 \\
2.20 \\
=2 . .\end{array}$ & $\begin{array}{r}2.15 \\
1.90 \\
2.05 \\
2.20 \\
2.10 \\
2.00 \\
1.90 \\
2.05 \\
2.00 \\
2.00 \\
1.95 \\
2.10 \\
1.90 \\
2.05 \\
2.05 \\
2.10 \\
1.95 \\
2.00 \\
1.95 \\
2.05 \\
2.15 \\
2.15 \\
2.05 \\
1.95 \\
1.85 \\
2.00 \\
\ldots \ldots . \\
\ldots \ldots \\
\ldots \ldots .\end{array}$ \\
\hline
\end{tabular}

IRR $11-5$ 
Rio Grande at Rio Grande, New Mexico.

\begin{tabular}{|c|c|c|c|c|c|c|c|c|c|c|c|c|}
\hline Day. & Jan. & Feb. & Mar. & Apr. & May. & June. & July. & Aug. & Sept. & Oet. & Nov. & Dec. \\
\hline 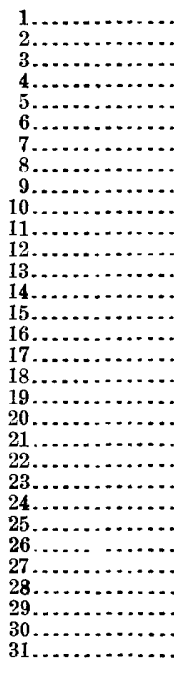 & $\begin{array}{c}5.00 \\
4.90 \\
5.05 \\
5.15 \\
\ldots \ldots\end{array}$ & \begin{tabular}{|c}
$\ldots \ldots$ \\
$\ldots \ldots \ldots$ \\
$\ldots \ldots$ \\
$\ldots \ldots$ \\
$\ldots \ldots$ \\
$\ldots \ldots \ldots$ \\
$\ldots \ldots$ \\
$\ldots \ldots$ \\
$\ldots \ldots$ \\
$\ldots \ldots$ \\
$\ldots \ldots$ \\
$\ldots \ldots$ \\
5.20 \\
5.20 \\
5.20 \\
5.20 \\
5.20 \\
5.20 \\
$\ldots \ldots \ldots$ \\
$\ldots \ldots$ \\
$\ldots \ldots$
\end{tabular} & 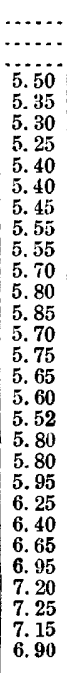 & $\begin{array}{l}6.65 \\
6.50 \\
6.45 \\
6.45 \\
6.40 \\
6.55 \\
6.85 \\
7.02 \\
7.50 \\
7.60 \\
7.80 \\
7.87 \\
7.80 \\
7.65 \\
7.80 \\
7.80 \\
7.95 \\
7.90 \\
7.75 \\
7.50 \\
7.45 \\
7.60 \\
7.70 \\
7.60 \\
7.85 \\
7.95 \\
8.15 \\
8.35 \\
8.40 \\
8.45 \\
\ldots . . .\end{array}$ & $\begin{array}{l}8.25 \\
7.95 \\
7.85 \\
7.90 \\
8.10 \\
8.15 \\
8.35 \\
8.50 \\
8.35 \\
7.75 \\
7.45 \\
7.25 \\
7.10 \\
7.00 \\
6.90 \\
6.55 \\
6.45 \\
6.30 \\
6.00 \\
5.95 \\
5.85 \\
5.80 \\
5.80 \\
5.85 \\
6.00 \\
6.25 \\
6.35 \\
6.40 \\
6.35 \\
6.45 \\
6.30\end{array}$ & 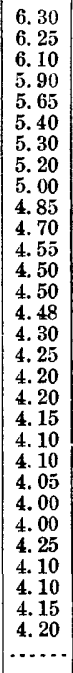 & $\begin{array}{l}4.20 \\
4.30 \\
4.25 \\
4.25 \\
4.20 \\
4.25 \\
4.10 \\
4.10 \\
4.08 \\
4.03 \\
4.00 \\
4.00 \\
4.60 \\
5.10 \\
4.30 \\
4.65 \\
4.85 \\
4.70 \\
5.40 \\
4.75 \\
5.00 \\
5.40 \\
5.15 \\
4.95 \\
4.65 \\
4.65 \\
4.50 \\
4.25 \\
4.20 \\
4.30 \\
4.25\end{array}$ & $\begin{array}{l}4.10 \\
4.10 \\
4.00 \\
4.00 \\
3.98 \\
3.90 \\
3.90 \\
3.90 \\
3.90 \\
3.88 \\
3.83 \\
3.85 \\
3.80 \\
3.80 \\
3.80 \\
3.80 \\
3.80 \\
3.80 \\
3.80 \\
3.80 \\
3.73 \\
3.70 \\
4.33 \\
3.95 \\
3.90 \\
4.00 \\
4.00 \\
4.00 \\
4.00 \\
4.13\end{array}$ & $\begin{array}{l}4.25 \\
4.20 \\
4.15 \\
4.10 \\
4.10 \\
4.18 \\
4.20 \\
4.05 \\
4.00 \\
4.35 \\
4.55 \\
4.95 \\
4.40 \\
4.40 \\
4.30 \\
4.20 \\
4.20 \\
4.80 \\
4.30 \\
4.45 \\
4.50 \\
4.40 \\
4.40 \\
4.45 \\
4.35 \\
4.25 \\
4.20 \\
4.30 \\
\mathbf{5 . 3 5} \\
5.10 \\
\ldots . . . .\end{array}$ & $\begin{array}{l}4.85 \\
4.80 \\
4.75 \\
4.70 \\
4.65 \\
4.60 \\
4.53 \\
4.95 \\
4.85 \\
5.00 \\
4.80 \\
4.70 \\
4.80 \\
4.90 \\
5.00 \\
4.98 \\
4.75 \\
4.65 \\
4.75 \\
4.70 \\
4.70 \\
4.75 \\
5.15 \\
\text { 5. } 00 \\
5.00 \\
4.90 \\
4.90 \\
5.05 \\
4.90 \\
4.80 \\
4.80\end{array}$ & 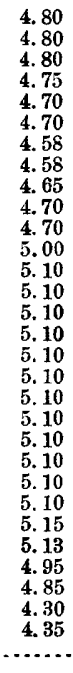 & $\begin{array}{l}4.40 \\
4.70 \\
4.90 \\
4.90 \\
4.80 \\
4.90 \\
4.95 \\
5.00 \\
5.05 \\
5.05 \\
5.05 \\
5.00 \\
5.05 \\
5.10 \\
4.95 \\
5.00 \\
5.10 \\
5.00 \\
4.85 \\
4.75 \\
4.80 \\
4.80 \\
4.80 \\
4.75 \\
4.70 \\
4.55 \\
\ldots \ldots . .\end{array}$ \\
\hline
\end{tabular}

Rio Grande at San Marcial, New Mexico.

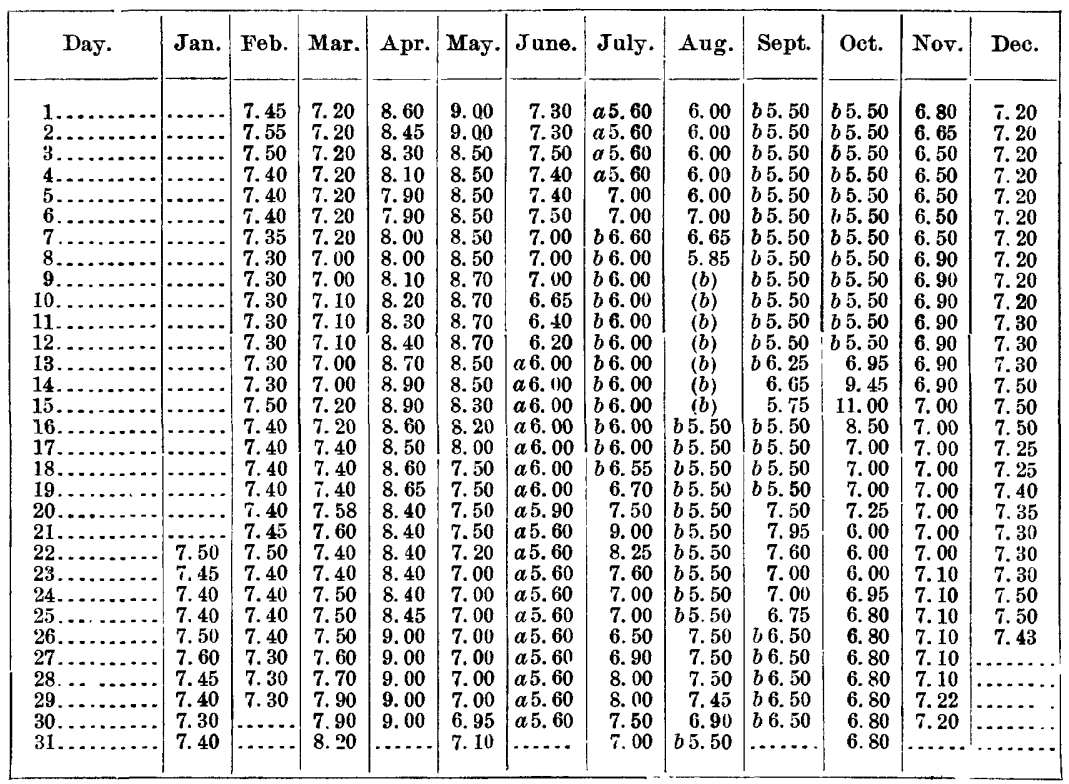


Rio Grande at El Paso, Texas.

\begin{tabular}{|c|c|c|c|c|c|c|c|c|c|c|c|c|}
\hline Day. & Jan. & Feb. & Mar. & Apr. & May. & June. & July. & Aug. & Sept. & Oct. & Nov. & Dec. \\
\hline 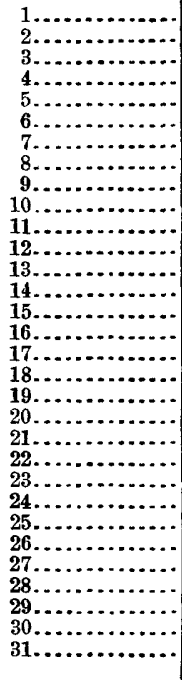 & 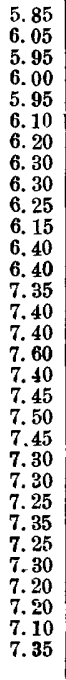 & $\begin{array}{l}7.30 \\
7.30 \\
7.30 \\
7.30 \\
7.30 \\
7.30 \\
7.35 \\
7.50 \\
7.45 \\
7.35 \\
7.25 \\
7.20 \\
7.10 \\
7.10 \\
7.05 \\
7.20 \\
7.10 \\
7.00 \\
6.90 \\
6.95 \\
7.15 \\
7.25 \\
7.20 \\
7.05 \\
7.00 \\
7.00 \\
7.00 \\
6.80 \\
6.70 \\
\cdots \ldots . . \\
\cdots \ldots\end{array}$ & $\begin{array}{l}6.60 \\
6.70 \\
6.85 \\
6.90 \\
6.80 \\
6.70 \\
6.70 \\
6.70 \\
6.75 \\
7.70 \\
7.35 \\
7.30 \\
7.10 \\
7.00 \\
6.80 \\
6.50 \\
6.40 \\
6.30 \\
6.20 \\
6.10 \\
6.10 \\
6.10 \\
6.05 \\
6.35 \\
6.75 \\
6.65 \\
6.35 \\
6.20 \\
6.10 \\
6.00 \\
5.90\end{array}$ & $\begin{array}{l}6.00 \\
7.20 \\
8.55 \\
8.60 \\
8.45 \\
8.35 \\
8.15 \\
7.90 \\
7.70 \\
7.55 \\
7.45 \\
7.30 \\
7.50 \\
8.05 \\
8.95 \\
8.90 \\
8.65 \\
8.50 \\
8.45 \\
8.45 \\
8.65 \\
8.90 \\
8.80 \\
8.60 \\
8.40 \\
8.50 \\
8.45 \\
8.50 \\
8.55 \\
8.55 \\
\ldots . . .\end{array}$ & $\begin{array}{l}8.65 \\
8.80 \\
8.80 \\
8.80 \\
8.80 \\
8.80 \\
8.80 \\
8.70 \\
8.50 \\
8.50 \\
8.65 \\
8.75 \\
8.85 \\
8.75 \\
8.65 \\
8.55 \\
8.35 \\
8.10 \\
8.00 \\
7.85 \\
7.65 \\
7.30 \\
7.15 \\
7.00 \\
6.85 \\
6.65 \\
6.45 \\
6.15 \\
5.95 \\
5.70 \\
5.45\end{array}$ & 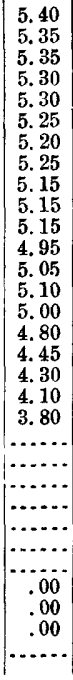 & $\begin{array}{l}5.10 \\
5.80 \\
5.25 \\
5.15 \\
5.10 \\
5.10 \\
4.90 \\
.00 \\
.00 \\
.00 \\
.00 \\
.00 \\
.00 \\
.00 \\
.00 \\
.00 \\
5.15 \\
8.38 \\
7.35 \\
7.25 \\
7.10 \\
7.90 \\
7.70 \\
9.60 \\
8.70 \\
8.20 \\
7.55 \\
7.10 \\
7.20 \\
7.10 \\
7.00\end{array}$ & $\begin{array}{l}6.70 \\
6.55 \\
6.40 \\
6.30 \\
6.05 \\
5.35 \\
5.55 \\
5.45 \\
5.35 \\
5.35 \\
5.25 \\
5.25 \\
5.20 \\
5.20 \\
5.00 \\
4.90 \\
4.75 \\
4.60 \\
4.60 \\
4.40 \\
4.30 \\
4.60 \\
4.40 \\
.00 \\
.00 \\
.00 \\
.00 \\
.00 \\
.00 \\
.00 \\
.00\end{array}$ & \begin{tabular}{|r}
0.00 \\
5.70 \\
5.25 \\
5.25 \\
5.05 \\
4.80 \\
.00 \\
.00 \\
.00 \\
.00 \\
.00 \\
.00 \\
.00 \\
.00 \\
.00 \\
.00 \\
.00 \\
.00 \\
.00 \\
.00 \\
.00 \\
.00 \\
5.50 \\
5.70 \\
7.35 \\
6.80 \\
6.20 \\
6.05 \\
6.00 \\
5.75 \\
$\cdots .$. \\
\end{tabular} & $\begin{array}{r}5.55 \\
7.05 \\
7.60 \\
7.50 \\
8.00 \\
7.45 \\
7.50 \\
7.30 \\
7.05 \\
7.40 \\
7.20 \\
7.00 \\
6.90 \\
7.00 \\
8.65 \\
10.15 \\
10.60 \\
10.55 \\
8.75 \\
8.15 \\
7.75 \\
7.65 \\
7.55 \\
7.30 \\
7.75 \\
8.10 \\
8.00 \\
8.20 \\
7.70 \\
7.45 \\
7.25\end{array}$ & $\begin{array}{r}7.25 \\
7.15 \\
6.90 \\
6.80 \\
6.70 \\
6.65 \\
6.60 \\
6.60 \\
6.50 \\
6.40 \\
6.35 \\
6.30 \\
6.20 \\
6.10 \\
6.10 \\
6.10 \\
6.10 \\
6.10 \\
6.10 \\
6.10 \\
6.10 \\
6.10 \\
6.10 \\
6.10 \\
6.30 \\
6.30 \\
6.40 \\
6.60 \\
6.55 \\
6.50 \\
\ldots . . .\end{array}$ & $\begin{array}{l}6.55 \\
6.60 \\
6.60 \\
6.60 \\
6.50 \\
6.50 \\
6.50 \\
6.60 \\
6.55 \\
6.55 \\
6.50 \\
6.40 \\
6.35 \\
6.40 \\
6.50 \\
6.40 \\
6.40 \\
6.35 \\
6.50 \\
6.70 \\
6.70 \\
6.70 \\
6.70 \\
6.70 \\
6.65 \\
6.70 \\
\ldots . .2 \\
\ldots \ldots . . \\
\ldots . .2 \\
\ldots . .2 \\
\ldots . .2 \\
6\end{array}$ \\
\hline
\end{tabular}

Grand River at Grand Junction, Colorado.

\begin{tabular}{|c|c|c|c|c|c|c|c|c|c|}
\hline Day. & Apr. & May. & June. & Juls. & Ang. & Sept. & Oet. & Nor. & Dec. \\
\hline 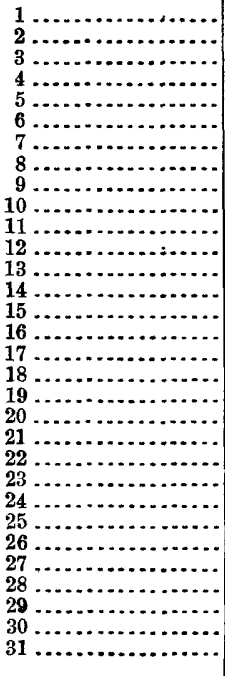 & 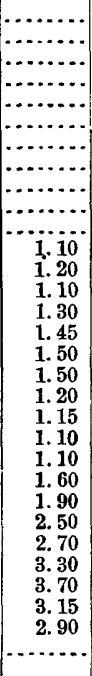 & $\begin{array}{l}2.70 \\
2.65 \\
2.65 \\
3.20 \\
3.30 \\
3.90 \\
4.00 \\
4.30 \\
3.90 \\
3.80 \\
3.70 \\
3.55 \\
3.20 \\
2.90 \\
2.50 \\
2.30 \\
1.90 \\
1.90 \\
1.85 \\
1.85 \\
1.90 \\
1.90 \\
2.35 \\
3.25 \\
\text { 3. } 0 \\
3.90 \\
4.05 \\
4.45 \\
4.30 \\
4.55 \\
4.40\end{array}$ & $\begin{array}{r}4.65 \\
4.10 \\
3.65 \\
3.45 \\
3.30 \\
3.30 \\
3.25 \\
3.15 \\
2.90 \\
2.75 \\
3.05 \\
2.95 \\
2.80 \\
2.45 \\
2.35 \\
2.20 \\
2.15 \\
2.05 \\
2.00 \\
2.00 \\
1.85 \\
1.80 \\
1.75 \\
1.65 \\
1.55 \\
1.50 \\
1.50 \\
1.40 \\
1.30 \\
1.30 \\
\ldots \ldots . . .\end{array}$ & $\begin{array}{r}1.20 \\
1.20 \\
1.10 \\
1.10 \\
1.05 \\
1.00 \\
1.05 \\
1.00 \\
.90 \\
.85 \\
.80 \\
.80 \\
.80 \\
.85 \\
.90 \\
.85 \\
.85 \\
1.00 \\
1.25 \\
1.25 \\
1.10 \\
1.00 \\
.85 \\
.80 \\
1.00 \\
1.40 \\
1.45 \\
1.55 \\
1.55 \\
1.35 \\
1.15\end{array}$ & $\begin{array}{r}\mathbf{1 . 0 5} \\
.95 \\
.85 \\
.85 \\
.70 \\
.70 \\
.65 \\
.55 \\
.45 \\
.40 \\
.25 \\
.15 \\
.05 \\
.00 \\
.00 \\
.00 \\
.00 \\
.00 \\
.00 \\
.00 \\
\mathbf{1 . 6 3} \\
.40 \\
a \mathbf{3 . 4 5} \\
\mathbf{3 . 5 5} \\
\mathbf{3 . 9 0} \\
\mathbf{3 . 7 5} \\
\mathbf{3 . 5 5} \\
\mathbf{3 . 3 5} \\
\mathbf{3 . 3 0} \\
\mathbf{3 . 3 0} \\
\mathbf{3 . 3 0}\end{array}$ & 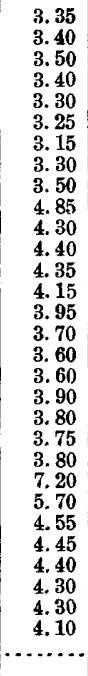 & $\begin{array}{l}4.10 \\
4.00 \\
3.95 \\
3.85 \\
3.80 \\
3.80 \\
3.80 \\
3.70 \\
3.70 \\
3.70 \\
3.70 \\
3.70 \\
3.70 \\
3.70 \\
3.65 \\
3.60 \\
3.60 \\
3.55 \\
3.50 \\
3.50 \\
3.50 \\
3.50 \\
3.50 \\
3.50 \\
3.50 \\
3.50 \\
3.40 \\
3.50 \\
3.50 \\
3.50 \\
3.50\end{array}$ & $\begin{array}{r}3.50 \\
3.50 \\
3.50 \\
3.40 \\
3.30 \\
3.20 \\
3.20 \\
3.10 \\
3.00 \\
3.20 \\
3.30 \\
3.30 \\
3.30 \\
3.20 \\
3.30 \\
3.40 \\
3.40 \\
3.25 \\
3.10 \\
3.25 \\
3.40 \\
3.40 \\
3.40 \\
3.40 \\
3.40 \\
3.40 \\
3.30 \\
3.10 \\
\mathbf{2 . 7 0} \\
\mathbf{2 . 5 0} \\
. . . . .\end{array}$ & 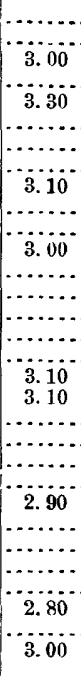 \\
\hline
\end{tabular}

a New rod placed in position reading 3 feet higher. 
Dolores River at Dolores, Colorado.

\begin{tabular}{|c|c|c|c|c|c|c|c|c|c|}
\hline Day. & Mar. & Apr. & May. & June. & July. & Aug. & Sept. & Oct. & Nov. \\
\hline 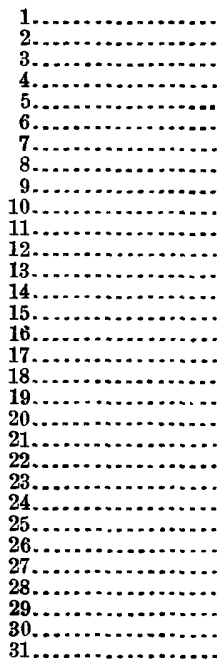 & $\begin{array}{l}3.20 \\
3.10 \\
3.00 \\
2.90 \\
2.80 \\
2.75 \\
2.60 \\
2.50 \\
2.55 \\
2.60 \\
2.65 \\
2.65 \\
2.70 \\
2.70 \\
2.75 \\
2.70 \\
2.75 \\
2.60 \\
2.60 \\
2.70 \\
2.70 \\
2.85 \\
2.90 \\
3.10 \\
3.40 \\
3.45 \\
3.25 \\
3.20 \\
3.10 \\
3.05 \\
3.05\end{array}$ & \begin{tabular}{|l|} 
2. 65 \\
2.35 \\
3.20 \\
$\mathbf{3 . 2 5}$ \\
$\mathbf{3 . 4 0}$ \\
$\mathbf{3 . 4 5}$ \\
$\mathbf{3 . 7 0}$ \\
$\mathbf{3 . 7 5}$ \\
$\mathbf{3 . 8 0}$ \\
$\mathbf{3 . 5 0}$ \\
$\mathbf{3 . 5 0}$ \\
$\mathbf{3 . 4 5}$ \\
$\mathbf{3 . 4 5}$ \\
$\mathbf{3 . 3 5}$ \\
$\mathbf{3 .} 60$ \\
$\mathbf{3 . 5 5}$ \\
$\mathbf{3 . 6 5}$ \\
$\mathbf{3 . 3 0}$ \\
$\mathbf{3 . 4 5}$ \\
$\mathbf{3 . 4 5}$ \\
$\mathbf{3 . 4 0}$ \\
$\mathbf{3 . 9 5}$ \\
$\mathbf{4 . 0 5}$ \\
$\mathbf{4 . 2 5}$ \\
$\mathbf{4 . 3 5}$ \\
$\mathbf{4 . 5 0}$ \\
$\mathbf{4 . 4 0}$ \\
$\mathbf{4 . 3 0}$ \\
$\mathbf{3 . 9 5}$ \\
$\mathbf{3 . 9 0}$ \\
$\ldots \ldots .$.
\end{tabular} & $\begin{array}{l}4.05 \\
4.30 \\
4.40 \\
4.35 \\
4.05 \\
4.30 \\
4.00 \\
4.05 \\
4.10 \\
4.10 \\
3.60 \\
3.80 \\
3.65 \\
3.40 \\
3.20 \\
3.15 \\
3.25 \\
3.30 \\
3.25 \\
3.75 \\
3.80 \\
3.90 \\
4.00 \\
4.10 \\
4.20 \\
4.25 \\
4.15 \\
4.30 \\
4.25 \\
4.10 \\
3.90\end{array}$ & $\begin{array}{l}3.75 \\
3.60 \\
3.45 \\
3.40 \\
3.30 \\
3.20 \\
3.20 \\
3.20 \\
3.10 \\
3.10 \\
3.10 \\
3.00 \\
3.00 \\
2.95 \\
2.90 \\
2.90 \\
2.90 \\
3.00 \\
2.90 \\
2.90 \\
2.80 \\
2.80 \\
2.80 \\
2.70 \\
2.70 \\
2.60 \\
2.60 \\
2.65 \\
2.70 \\
2.70 \\
2 . .2 .\end{array}$ & $\begin{array}{l}2.70 \\
2.65 \\
2.60 \\
2.60 \\
2.70 \\
2.75 \\
2.85 \\
2.90 \\
3.00 \\
3.00 \\
3.15 \\
3.00 \\
2.95 \\
3.00 \\
3.10 \\
3.50 \\
3.05 \\
3.00 \\
3.05 \\
3.00 \\
2.85 \\
2.80 \\
2.85 \\
2.80 \\
2.75 \\
2.70 \\
2.65 \\
2.60 \\
2.60 \\
2.60 \\
2.60\end{array}$ & $\begin{array}{l}2.50 \\
2.40 \\
2.40 \\
2.50 \\
2.60 \\
2.50 \\
2.50 \\
2.40 \\
2.40 \\
2.35 \\
2.30 \\
2.30 \\
2.30 \\
2.30 \\
2.25 \\
2.20 \\
2.35 \\
2.45 \\
2.55 \\
2.55 \\
2.60 \\
2.60 \\
2.60 \\
2.60 \\
2.53 \\
2.50 \\
2.50 \\
2.85 \\
3.00 \\
2.85 \\
2.80\end{array}$ & $\begin{array}{l}2.70 \\
2.70 \\
2.60 \\
2.60 \\
2.60 \\
2.60 \\
2.50 \\
2.70 \\
2.70 \\
2.80 \\
3.10 \\
2.90 \\
2.75 \\
2.75 \\
2.75 \\
2.70 \\
2.70 \\
2.70 \\
2.70 \\
2.80 \\
2.80 \\
2.65 \\
4.17 \\
4.05 \\
4.40 \\
3.20 \\
3.10 \\
3.10 \\
3.10 \\
3.00 \\
\cdots . .1\end{array}$ & $\begin{array}{l}3.00 \\
3.00 \\
3.00 \\
2.90 \\
2.90 \\
2.90 \\
2.85 \\
2.80 \\
2.80 \\
2.80 \\
2.80 \\
2.80 \\
2.85 \\
2.90 \\
2.90 \\
2.80 \\
2.80 \\
2.80 \\
2.80 \\
2.80 \\
2.80 \\
2.85 \\
2.80 \\
2.80 \\
2.80 \\
2.80 \\
2.80 \\
2.90 \\
2.80 \\
2.80 \\
2.80\end{array}$ & $\begin{array}{l}2.80 \\
2.80 \\
2.95 \\
2.95 \\
3.20 \\
3.40 \\
3.70 \\
3.35 \\
2.90 \\
2.90 \\
2.90 \\
3.00 \\
3.00 \\
2.90 \\
2.75 \\
2.75 \\
2.55 \\
2.65 \\
2.80 \\
2.75 \\
2.75 \\
2.70 \\
2.70 \\
2.70 \\
2.80 \\
3.20 \\
3.20 \\
3.20 \\
3.20 \\
3.20 \\
\ldots . .1 .\end{array}$ \\
\hline
\end{tabular}

Fall Creek at Seymour, Colorado.

\begin{tabular}{|c|c|c|c|c|c|c|c|c|}
\hline Day. & Apr. & May. & June. & July. & Aug. & Sept. & Oct. & Nov. \\
\hline $\begin{array}{l}1 \\
1\end{array}$ & 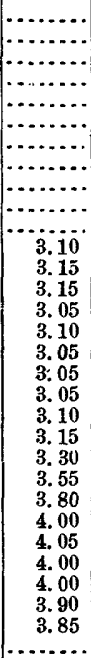 & $\begin{array}{l}4.00 \\
4.00 \\
3.95 \\
3.90 \\
3.95 \\
4.05 \\
4.05 \\
4.15 \\
4.20 \\
4.05 \\
4.00 \\
3.80 \\
3.80 \\
3.70 \\
3.70 \\
3.75 \\
3.75 \\
3.60 \\
3.55 \\
3.55 \\
3.80 \\
3.95 \\
4.35 \\
4.35 \\
4.60 \\
4.90 \\
4.95 \\
4.85 \\
4.75 \\
4.70 \\
4.40\end{array}$ & $\begin{array}{l}4.20 \\
4.15 \\
4.15 \\
4.15 \\
4.15 \\
4.05 \\
4.00 \\
4.10 \\
4.10 \\
3.95 \\
3.85 \\
3.80 \\
3.85 \\
3.90 \\
3.40 \\
3.40 \\
3.45 \\
3.40 \\
3.35 \\
3.30 \\
3.10 \\
3.10 \\
3.05 \\
3.00 \\
3.00 \\
3.00 \\
3.00 \\
3.00 \\
3.00 \\
3.00 \\
\ldots . . .\end{array}$ & $\begin{array}{l}3.00 \\
3.00 \\
2.95 \\
2.90 \\
2.90 \\
2.90 \\
2.90 \\
2.90 \\
3.20 \\
3.60 \\
3.55 \\
3.50 \\
3.45 \\
3.20 \\
3.00 \\
3.00 \\
3.20 \\
3.30 \\
3.30 \\
3.15 \\
3.00 \\
2.90 \\
2.90 \\
2.90 \\
2.85 \\
2.80 \\
2.80 \\
2.80 \\
2.80 \\
2.80 \\
2.80\end{array}$ & $\begin{array}{l}2.70 \\
2.60 \\
2.60 \\
2.60 \\
2.60 \\
2.60 \\
2.60 \\
2.60 \\
2.60 \\
2.60 \\
2.60 \\
2.60 \\
2.60 \\
2.60 \\
2.60 \\
2.60 \\
2.50 \\
2.60 \\
2.60 \\
2.60 \\
2.60 \\
2.60 \\
2.55 \\
2.55 \\
2.55 \\
2.60 \\
2.55 \\
2.90 \\
2.85 \\
2.75 \\
2.70\end{array}$ & $\begin{array}{r}2.65 \\
2.60 \\
2.60 \\
2.65 \\
2.60 \\
2.60 \\
2.60 \\
2.60 \\
2.60 \\
2.95 \\
3.00 \\
2.90 \\
2.80 \\
2.80 \\
2.70 \\
2.70 \\
2.70 \\
2.70 \\
2.75 \\
2.85 \\
2.80 \\
2.80 \\
4.45 \\
4.05 \\
4.75 \\
3.50 \\
3.35 \\
3.25 \\
3.10 \\
3.10 \\
\ldots . .7\end{array}$ & $\begin{array}{l}3.00 \\
3.00 \\
2.95 \\
2.90 \\
2.85 \\
2.80 \\
2.80 \\
2.80 \\
2.80 \\
2.75 \\
2.70 \\
2.70 \\
2.70 \\
2.70 \\
2.70 \\
2.70 \\
2.70 \\
2.70 \\
2.60 \\
2.60 \\
2.60 \\
2.65 \\
2.65 \\
2.60 \\
2.60 \\
2.60 \\
2.60 \\
2.60 \\
2.60 \\
2.85 \\
2.50\end{array}$ & $\begin{array}{l}2.60 \\
2.50 \\
2.55 \\
2.55 \\
2.55 \\
2.55 \\
2.25 \\
2.40 \\
2.60 \\
2.50 \\
2.50 \\
2.50 \\
2.55 \\
2.45 \\
2.65 \\
2.55 \\
2.45 \\
2.50 \\
2.50 \\
2.45 \\
2.50 \\
2.60 \\
2.60 \\
2.60 \\
2.60 \\
2.65 \\
2.30 \\
2.60 \\
2.90 \\
3.05\end{array}$ \\
\hline
\end{tabular}


Uncompahgre River at Uncompahgre, Colorado.

\begin{tabular}{|c|c|c|c|c|c|c|c|}
\hline Day. & May. & June. & July. & Aug. & Sept. & Oet. & Nov. \\
\hline $\begin{array}{l}1 \\
1\end{array}$ & 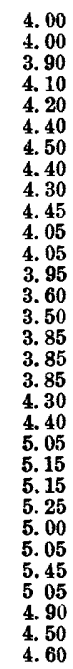 & $\begin{array}{r}\text { 5. } 00 \\
\mathbf{4 .} 80 \\
\mathbf{4 . 2 0} \\
\mathbf{4 . 4 0} \\
\mathbf{4 . 3 0} \\
\mathbf{4 . 2 5} \\
\mathbf{4 . 2 5} \\
\mathbf{4 . 4 5} \\
\mathbf{4 . 4 0} \\
\mathbf{4 . 3 0} \\
\mathbf{4 . 1 0} \\
\mathbf{4 . 1 5} \\
\mathbf{4 . 1 0} \\
\mathbf{4 . 1 5} \\
\mathbf{4 . 0 0} \\
\mathbf{3 . 8 5} \\
\mathbf{3 . 8 5} \\
\mathbf{3 . 9 0} \\
\mathbf{3 . 7 5} \\
\mathbf{3 . 8 0} \\
\mathbf{3 . 7 5} \\
\mathbf{3 . 7 0} \\
\mathbf{3 . 5 0} \\
\mathbf{3 . 7 5} \\
\mathbf{3 . 7 5} \\
\mathbf{3 . 6 0} \\
\mathbf{3 . 5 0} \\
\mathbf{3 . 6 0} \\
\mathbf{3 . 5 0} \\
\mathbf{3 . 4 5} \\
\text {... } . . .\end{array}$ & 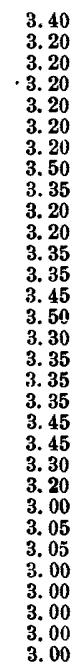 & $\begin{array}{l}3.00 \\
2.85 \\
2.80 \\
2.80 \\
2.90 \\
2.90 \\
2.90 \\
2.90 \\
2.85 \\
2.75 \\
2.75 \\
2.70 \\
2.70 \\
2.70 \\
2.70 \\
2.70 \\
2.70 \\
2.70 \\
2.70 \\
2.80 \\
2.75 \\
2.85 \\
3.05 \\
3.05 \\
3.03 \\
3.08 \\
3.00 \\
3.08 \\
3.15 \\
3.10 \\
3.18\end{array}$ & $\begin{array}{r}3.08 \\
3.10 \\
3.18 \\
3.10 \\
3.10 \\
3.10 \\
3.05 \\
3.00 \\
3.13 \\
3.13 \\
3.48 \\
3.35 \\
3.23 \\
3.20 \\
3.23 \\
3.18 \\
3.10 \\
3.15 \\
3.18 \\
3.23 \\
3.25 \\
\mathbf{3 . 4 0} \\
\mathbf{3 . 9 0} \\
\mathbf{3 . 9 8} \\
\mathbf{3 . 6 5} \\
\mathbf{3 . 5 8} \\
\mathbf{3 . 4 8} \\
\mathbf{3 . 4 5} \\
\mathbf{3 . 4 3} \\
\mathbf{3 . 4 0} \\
\ldots . . . .\end{array}$ & $\begin{array}{l}3.35 \\
3.43 \\
3.35 \\
3.30 \\
3.25 \\
3.20 \\
3.23 \\
3.25 \\
3.23 \\
3.23 \\
3.23 \\
3.23 \\
3.18 \\
3.18 \\
3.18 \\
3.13 \\
3.10 \\
3.10 \\
3.13 \\
3.08 \\
3.10 \\
3.08 \\
3.08 \\
3.05 \\
3.03 \\
3.03 \\
3.08 \\
3.10 \\
3.10 \\
3.13 \\
3.18\end{array}$ & 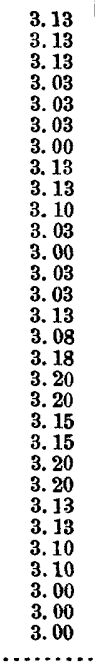 \\
\hline
\end{tabular}

Black's Fork River at Granger, Wyoming.

\begin{tabular}{|c|c|c|c|c|c|c|c|c|c|}
\hline Day. & Apr. & May. & June. & July. & Aug. & Sept. & Oet. & Nov. & Dac. \\
\hline 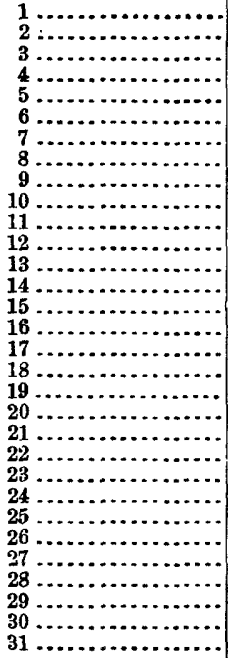 & $\begin{array}{r}3.00 \\
2.60 \\
2.90 \\
3.30 \\
3.30 \\
3.40 \\
3.20 \\
3.20 \\
\cdots 3.40 \\
3.50 \\
\text { 3.50 } \\
\text { 3. } 20\end{array}$ & $\begin{array}{r}\mathbf{3 . 2 0} \\
\mathbf{3 . 5 0} \\
\ldots . .2 \\
\mathbf{3 . 4 0} \\
\mathbf{3 . 4 0} \\
\mathbf{4 . 0 0} \\
\mathbf{5 . 1 0} \\
\mathbf{5 . 0 0} \\
\mathbf{5 . 3 0} \\
\ldots \ldots .30 \\
\mathbf{4 . 9} \\
\mathbf{4 . 7 0} \\
\mathbf{4 . 3 0} \\
\mathbf{3 . 8 0} \\
\mathbf{3 . 6 0} \\
\mathbf{3 . 5 0} \\
\ldots .30 \\
\mathbf{3 .} 30 \\
\mathbf{3 . 3 0} \\
\mathbf{3 . 3 0} \\
\mathbf{3 . 3 0} \\
\mathbf{3 . 6 0} \\
\mathbf{4 . 0 0} \\
\mathbf{4 . 6 0} \\
\mathbf{6 . 0 0} \\
\mathbf{6 . 6 0} \\
\mathbf{7 . 0 0} \\
\mathbf{7 . 7 0} \\
\mathbf{8 . 0 0} \\
\mathbf{8 . 4 0}\end{array}$ & 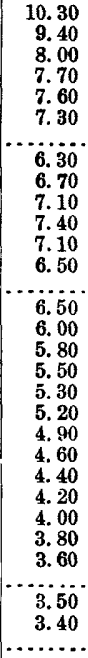 & $\begin{array}{r}3.20 \\
3.10 \\
3.00 \\
2.90 \\
2.80 \\
2.70 \\
2.60 \\
2.60 \\
2.60 \\
2.50 \\
2.40 \\
\ldots .70 \\
2.40 \\
2.70 \\
3.30 \\
3.10 \\
2.90 \\
3.30 \\
\cdots 3.0 \\
3.00 \\
2.80 \\
2.60 \\
3.70 \\
2.90 \\
2.90 \\
\cdots 2.90 \\
2.80 \\
2.80 \\
2.70 \\
2.60 \\
2.40\end{array}$ & \begin{tabular}{|l}
2.40 \\
2.40 \\
2.40 \\
2.40 \\
2.30 \\
2.30 \\
2.20 \\
2.00 \\
$\cdots \ldots .7$ \\
1.80 \\
1.70 \\
1.70 \\
1.60 \\
1.50 \\
1.50 \\
$\cdots 3.30$ \\
3.30 \\
4.90 \\
520 \\
4.10 \\
5.25 \\
4.40 \\
$\cdots 3.90$ \\
3.90 \\
2.50 \\
2.20 \\
2.10 \\
2.00 \\
1.90 \\
2.40 \\
2.10
\end{tabular} & 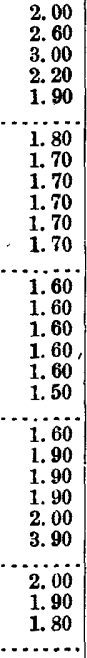 & 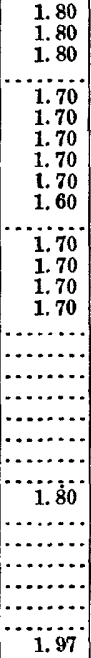 & $\begin{array}{r}1.90 \\
2.40 \\
2.60 \\
2.80 \\
2.90 \\
2.90 \\
2.90\end{array}$ & 2.90 \\
\hline
\end{tabular}


Green River at Green River, Wyoming.

\begin{tabular}{|c|c|c|c|c|c|c|c|c|}
\hline Day. & Apr. & May. & June. & July. & Aug. & Sept. & Oet. & Dec. \\
\hline 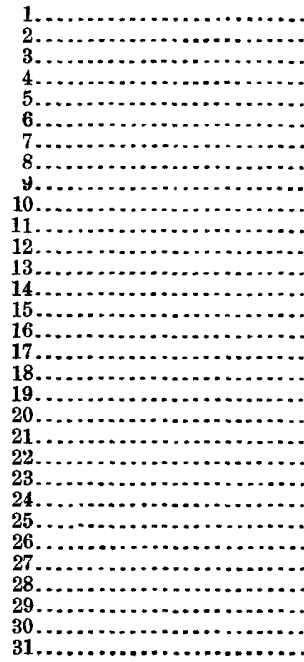 & $\begin{array}{r}\ldots . .30 \\
1.55 \\
1.60 \\
\mathbf{1 . 5 5} \\
1.50 \\
\mathbf{1 . 5 7} \\
\mathbf{1 . 6 7} \\
\mathbf{1 .} .70 \\
\mathbf{1 . 7 0} \\
\mathbf{1 . 6 2} \\
\mathbf{1 . 5 5} \\
\mathbf{1 . 5 2} \\
\mathbf{1 . 5 7} \\
\mathbf{1 . 5 7} \\
\mathbf{1 . 5 5} \\
\mathbf{1 . 5 5} \\
\mathbf{1 . 5 0} \\
\mathbf{1 . 5 7} \\
\mathbf{1 . 6 7} \\
\mathbf{1 . 8 5} \\
\mathbf{1 . 9 2} \\
\mathbf{1 . 9 0} \\
\ldots . . . .\end{array}$ & $\begin{array}{l}1.90 \\
1.85 \\
1.87 \\
1.90 \\
1.95 \\
1.90 \\
2.00 \\
2.15 \\
2.22 \\
2.25 \\
2.20 \\
2.15 \\
2.15 \\
2.10 \\
2.05 \\
2.05 \\
2.05 \\
2.00 \\
2.00 \\
1.95 \\
1.90 \\
1.95 \\
2.02 \\
2.30 \\
2.65 \\
2.85 \\
2.90 \\
2.97 \\
3.37 \\
3.87 \\
4.27\end{array}$ & $\begin{array}{r}4.77 \\
4.97 \\
4.85 \\
5.15 \\
5.45 \\
5.35 \\
5.25 \\
5.02 \\
4.80 \\
4.90 \\
5.35 \\
5.75 \\
5.92 \\
5.82 \\
5.87 \\
5.97 \\
5.97 \\
6.05 \\
6.05 \\
6.18 \\
6.20 \\
6.00 \\
5.70 \\
5.45 \\
5.10 \\
4.93 \\
4.73 \\
4.55 \\
4.43 \\
4.23 \\
\ldots . . . .\end{array}$ & $\begin{array}{l}4.0 \varepsilon \\
3.98 \\
3.88 \\
3.80 \\
3.78 \\
3.70 \\
3.63 \\
3.60 \\
3.55 \\
3.50 \\
3.45 \\
3.40 \\
3.35 \\
3.35 \\
3.45 \\
3.70 \\
3.70 \\
3.55 \\
3.50 \\
3.43 \\
3.33 \\
3.25 \\
3.08 \\
2.98 \\
2.90 \\
2.88 \\
2.80 \\
2.73 \\
2.65 \\
2.60 \\
2.53\end{array}$ & $\begin{array}{l}2.58 \\
2.53 \\
2.43 \\
2.40 \\
2.35 \\
2.28 \\
2.20 \\
2.13 \\
2.08 \\
2.03 \\
1.93 \\
1.90 \\
1.85 \\
1.78 \\
1.73 \\
1.70 \\
1.70 \\
2.00 \\
1.83 \\
1.90 \\
2.08 \\
2.10 \\
2.15 \\
2.08 \\
1.93 \\
1.88 \\
1.78 \\
1.68 \\
1.58 \\
1.57 \\
1.60\end{array}$ & $\begin{array}{l}1.62 \\
1.65 \\
1.60 \\
1.60 \\
1.55 \\
1.55 \\
1.50 \\
1.50 \\
1.50 \\
1.50 \\
1.50 \\
1.50 \\
1.50 \\
1.45 \\
1.45 \\
1.40 \\
1.35 \\
1.35 \\
1.27 \\
1.25 \\
1.25 \\
1.30 \\
1.25 \\
1.20 \\
1.20 \\
1.20 \\
1.20 \\
1.25 \\
1.30 \\
1.30 \\
\cdots 1 . . . .\end{array}$ & 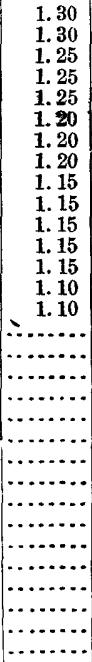 & $\begin{array}{l}1.60 \\
1.60 \\
1.60 \\
1.65 \\
1.65 \\
1.65 \\
1.65 \\
1.70 \\
1.70 \\
1.70 \\
1.70 \\
1.70 \\
1.70 \\
1.70 \\
1.70 \\
1.70 \\
1.70 \\
1.70 \\
1.70 \\
1.70 \\
1.80 \\
1.80 \\
1.80 \\
1.80 \\
1.80 \\
1.80 \\
1.80 \\
1.2 .\end{array}$ \\
\hline
\end{tabular}

Green River at Blake, Utah.

\begin{tabular}{|c|c|c|c|c|c|c|c|c|c|c|c|c|}
\hline Day. & Jan. & Feb. & Mar. & Apr. & May. & June. & July. & Aug. & Sept. & Oet. & Nov. & Dec. \\
\hline & 55 & 80 & 180 & 60 & 35 & 885 & 505 & 10 & 205 & 3.10 & 255 & \\
\hline $2 \ldots$ & $\begin{array}{l}1.05 \\
1.65\end{array}$ & $\begin{array}{l}1.80 \\
1.70\end{array}$ & $\begin{array}{l}1.80 \\
1.80\end{array}$ & 3.60 & $\begin{array}{l}0.35 \\
6.35\end{array}$ & $\begin{array}{l}0.80 \\
9.30\end{array}$ & 5.75 & 4. 30 & 2.85 & $\begin{array}{l}0.10 \\
3.20\end{array}$ & 2.50 & \\
\hline 3.. & 1. 75 & 1.70 & 1.90 & 3.50 & 6.00 & 9. 65 & 5.55 & 3.95 & 2.75 & 3. 05 & 2.50 & $\cdots$ \\
\hline 4. & 1.85 & 1.70 & 2.00 & 3. 45 & 5. 75 & 9.65 & 5.35 & 3. 75 & 2. 65 & 3. 00 & 2.50 & $\cdots$ \\
\hline 5. & 1. 70 & 1.60 & 1.95 & 3.40 & 5. 55 & 9.45 & 5. 15 & 3.65 & 2.50 & 3.00 & 2. 40 & \\
\hline 6. & 1.70 & 1.70 & 2.00 & 3.20 & 5. 55 & 9.25 & 5.05 & 3.60 & 2.50 & 2. 90 & 2. 40 & \\
\hline 7. & 1.60 & 1.75 & 2.00 & 3. 00 & 5.80 & 9.05 & 5.00 & 3.50 & 2. 50 & 2.85 & 2.40 & \\
\hline & 1. 65 & 1.70 & 2.00 & 2.90 & 6. 20 & 8.95 & 4.80 & 3. 45 & 2.45 & 2.70 & 2.40 & \\
\hline & 1. 70 & 1.70 & 2. 05 & 2.90 & 6. 55 & 8.75 & 4.70 & 3. 35 & 2.40 & 2. 70 & 2.35 & $\cdots$ \\
\hline 10. & 1. 60 & 1. 80 & 2. 10 & 2. 90 & 6. 75 & 8.70 & 4. 60 & 3. 30 & 2.70 & 2.70 & 2.30 & \\
\hline 11. & 1.65 & 1.70 & 2.10 & 3.00 & 6. 85 & 8.70 & 4. 95 & 3. 40 & 2.65 & 2. 70 & 2.25 & \\
\hline 12. & 1. 70 & 1.60 & 2.10 & 3. 10 & 6. 90 & 8.45 & 4.35 & 3. 35 & 2.85 & 2.60 & 2.20 & \\
\hline 13. & 1. 60 & 1.60 & 2.10 & 3. 20 & 6.75 & 8.25 & 4. 20 & 3.15 & 2. 90 & 2.60 & 2. 20 & \\
\hline 14. & 1. 50 & 1.50 & 2.20 & 3. 45 & 6.55 & 8. 20 & 4. 25 & 3. 00 & 2. 70 & 2. 60 & 2. 20 & \\
\hline 15. & 1. 45 & 1.50 & 2.30 & 3. 50 & 6.40 & 8.30 & 4. 40 & 2. 90 & 2. 70 & 2. 60 & 2.15 & . \\
\hline 16. & 1.55 & 1.60 & 2. 35 & 3.70 & 6.10 & 8.25 & 4.30 & 2.80 & 2. 70 & 2. 60 & 2.10 & \\
\hline 17 & 1. 65 & 1.70 & 2. 40 & 3.75 & 5.70 & 8.15 & 4.70 & 2.65 & 2.80 & 2.60 & 2.20 & \\
\hline 18. & 1. 75 & 1. 70 & 2.55 & 3. 90 & 5. 45 & 8.00 & 4. 30 & 2. 60 & 2.65 & 255 & 2. 30 & \\
\hline 19. & 1.60 & 1.80 & 2.85 & 3. 85 & 5. 20 & 7.95 & 4.45 & 2. 50 & 2. 65 & 2. 50 & 2. 40 & \\
\hline 20. & 1. 65 & 1.80 & 3. 15 & 3.95 & 5. 00 & 7.75 & 4.80 & 2. 50 & 3. 45 & 2. 50 & 2. 50 & \\
\hline 21 . & 1. 70 & 1.90 & 3. 10 & 4. 10 & 4. 85 & 7.70 & 4.95 & 2.65 & 4. 45 & 2.50 & 2. 40 & $\cdots$ \\
\hline & 1.75 & 1.90 & 2. 75 & 4. 45 & 4.70 & 7.55 & 4. 70 & 2. 85 & 3. 00 & 2.50 & 2. 30 & \\
\hline & 1.75 & 1. 91$)$ & 2. 70 & 4. 10 & 4. 70 & 7.50 & 4.55 & 3. 05 & 5. 55 & 2.50 & 2.40 & $\cdots$ \\
\hline 24 & 1. 70 & 1.80 & 2.80 & 3. 80 & 4. 70 & 7. 40 & 4.45 & 3. 20 & 4. 75 & 2.45 & 2.50 & \\
\hline & 1. 60 & 1.80 & 2.85 & 3. 60 & 4. 85 & 7. 35 & 4. 40 & 3.15 & 4.00 & 2.40 & 2. 60 & \\
\hline 26 & 1.60 & 1. 70 & 3. 05 & 3.75 & 5. 35 & 7.15 & 4.60 & 3. 30 & 4. 25 & 2.40 & 2. 70 & \\
\hline & 1.60 & 1.75 & 3. 25 & 4. 10 & 5. 80 & 6.90 & 4.60 & 3. 40 & 3.70 & 2.40 & 2.65 & \\
\hline & 1.70 & 1.80 & 3.40 & 5. 00 & 6. 65 & 6. 55 & 4. 65 & 3. 65 & 3. 45 & 2.55 & 2.45 & \\
\hline & 1. 70 & 1.80 & 3.55 & 5. 70 & 7.45 & 6.35 & 4. 60 & 4. 15 & 3. 35 & 2. 40 & $\ldots$. & \\
\hline & 1. 80 & & 3. 70 & 6. 10 & 8.00 & 6. 15 & 4.50 & 3. 45 & 3. 20 & 2. 55 & $\cdots$ & 1.80 \\
\hline & 1.80 & $\cdots$ & 3. 60 & $\ldots$ & 8.40 & ..... & 4. 50 & 3. 15 & ...... & 2. 65 & $\cdots$ & $\cdots$ \\
\hline
\end{tabular}


San Juan River at Arboles, Colorado.

\begin{tabular}{|c|c|c|c|c|c|c|c|c|}
\hline Day. & Apr. & May. & June. & July. & Aug. & Sept. & Oct. & Nov. \\
\hline $\begin{array}{l}1 \\
1\end{array}$ & 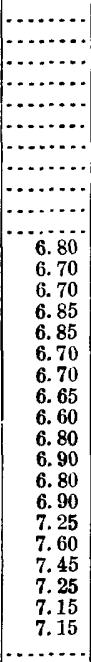 & $\begin{array}{l}7.15 \\
7.20 \\
7.50 \\
7.60 \\
7.65 \\
7.75 \\
7.65 \\
7.40 \\
7.25 \\
7.35 \\
7.25 \\
7.10 \\
7.05 \\
6.85 \\
6.75 \\
6.65 \\
6.60 \\
6.60 \\
6.80 \\
6.95 \\
7.25 \\
7.25 \\
7.35 \\
7.50 \\
7.60 \\
7.70 \\
7.60 \\
7.55 \\
7.35 \\
7.40 \\
7.40\end{array}$ & $\begin{array}{r}7.10 \\
\mathbf{7 . 0 0} \\
\mathbf{6 . 9 0} \\
\mathbf{6 . 8 0} \\
6.65 \\
6.60 \\
6.50 \\
6.50 \\
6.50 \\
\mathbf{6 . 4 0} \\
\mathbf{6 . 3 0} \\
\mathbf{6 . 3 0} \\
\mathbf{6 . 2 0} \\
\mathbf{6 . 2 0} \\
6.10 \\
\mathbf{6 . 0 0} \\
\mathbf{6 . 0 0} \\
\mathbf{6 . 0 0} \\
5.95 \\
\mathbf{5 . 9 5} \\
\mathbf{5 . 9 0} \\
\mathbf{5 . 9 0} \\
\mathbf{5 . 8 0} \\
\mathbf{5 . 8 0} \\
\mathbf{5 . 8 0} \\
\mathbf{5 . 7 0} \\
\mathbf{5 . 6 5} \\
\mathbf{5} \mathbf{7 0} \\
\mathbf{5 . 7 0} \\
\mathbf{5 . 7 0} \\
\mathbf{2 . 2}\end{array}$ & 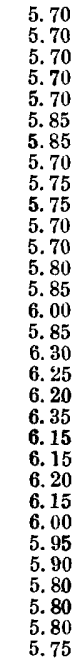 & $\begin{array}{l}5.70 \\
5.65 \\
5.60 \\
5.60 \\
5.75 \\
5.75 \\
5.65 \\
5.60 \\
5.50 \\
5.50 \\
5.50 \\
5.50 \\
5.50 \\
5.50 \\
5.50 \\
5.50 \\
5.50 \\
5.50 \\
5.50 \\
5.40 \\
5.40 \\
5.40 \\
5.40 \\
5.40 \\
5.50 \\
5.50 \\
5.50 \\
6.50 \\
6.20 \\
6.05 \\
5.90\end{array}$ & 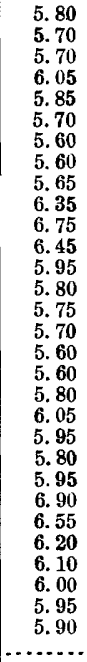 & $\begin{array}{l}\text { 5. } 85 \\
5.85 \\
5.90 \\
5.90 \\
5.85 \\
5.80 \\
6.00 \\
5.90 \\
5.80 \\
5.95 \\
\text { 5. 90 } \\
5.80 \\
5.90 \\
5.80 \\
5.80 \\
5.80 \\
\text { 5. } 80 \\
5.80 \\
5.80 \\
5.80 \\
5.80 \\
\text { 5. 75 } \\
6.05 \\
6.15 \\
\text { 5. 95 } \\
\text { 5. 85 } \\
5.85 \\
6.40 \\
6.10 \\
6.05 \\
5.95\end{array}$ & $\begin{array}{r}5.90 \\
5.90 \\
5.85 \\
5.80 \\
5.70 \\
5.60 \\
5.55 \\
5.50 \\
5.50 \\
5.65 \\
5.75 \\
5.70 \\
5.70 \\
5.70 \\
5.70 \\
5.75 \\
5.90 \\
5.85 \\
5.80 \\
5.80 \\
5.80 \\
5.80 \\
5.80 \\
5.80 \\
5.80 \\
5.80 \\
5.80 \\
5.80 \\
\mathbf{5 . 8 0} \\
\mathbf{5 . 8 0}\end{array}$ \\
\hline
\end{tabular}

Piedras River at Arboles, Colorado.

\begin{tabular}{|c|c|c|c|c|c|c|c|c|}
\hline Day. & Apr. & May. & June. & July. & Aug. & Sept. & Oct. & Nov. \\
\hline $\begin{array}{l}1 \\
1\end{array}$ & \begin{tabular}{|l}
$\ldots \ldots \ldots$ \\
$\ldots \ldots \ldots$ \\
$\cdots \ldots \ldots$ \\
$\cdots \ldots \ldots$ \\
$\cdots \ldots \ldots$ \\
$\cdots \ldots \ldots$ \\
$\cdots \ldots \ldots$ \\
$\cdots \ldots \ldots$ \\
4.00 \\
3.90 \\
3.90 \\
4.05 \\
4.05 \\
3.90 \\
3.85 \\
3.70 \\
3.55 \\
3.65 \\
3.85 \\
3.75 \\
4.00 \\
4.50 \\
4.75 \\
4.60 \\
4.45 \\
4.65 \\
4.65 \\
$\ldots \ldots \ldots$
\end{tabular} & 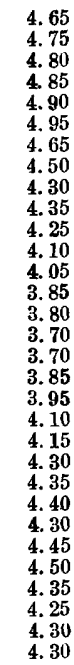 & \begin{tabular}{|l}
4.05 \\
3.85 \\
3.80 \\
3.70 \\
3.60 \\
3.50 \\
3.45 \\
3.40 \\
3.30 \\
3.30 \\
3.20 \\
3.20 \\
3.20 \\
3.10 \\
3.00 \\
3.00 \\
3.00 \\
3.00 \\
2.90 \\
2.90 \\
2.90 \\
2.80 \\
2.80 \\
2.80 \\
2.75 \\
2.70 \\
2.65 \\
2.70 \\
2.70 \\
2.70 \\
$\ldots . .2$
\end{tabular} & $\begin{array}{l}2.70 \\
2.70 \\
2.70 \\
2.60 \\
2.60 \\
2.60 \\
2.60 \\
2.70 \\
2.70 \\
2.70 \\
2.70 \\
2.70 \\
2.75 \\
2.85 \\
2.80 \\
2.70 \\
2.75 \\
3.00 \\
3.00 \\
2.95 \\
2.80 \\
2.85 \\
2.95 \\
3.05 \\
3.00 \\
2.85 \\
2.80 \\
2.70 \\
2.70 \\
2.70 \\
2.65\end{array}$ & $\begin{array}{l}2.60 \\
2.60 \\
2.50 \\
2.50 \\
2.60 \\
2.60 \\
2.55 \\
2.50 \\
2.50 \\
2.50 \\
2.40 \\
2.40 \\
2.40 \\
2.40 \\
2.40 \\
2.40 \\
2.45 \\
2.50 \\
2.50 \\
2.40 \\
2.40 \\
2.40 \\
2.45 \\
2.50 \\
2.50 \\
2.50 \\
2.50 \\
3.15 \\
3.30 \\
2.80 \\
2.90\end{array}$ & $\begin{array}{l}2.80 \\
2.70 \\
2.70 \\
2.75 \\
2.80 \\
2.70 \\
2.60 \\
2.60 \\
2.60 \\
3.05 \\
3.25 \\
3.00 \\
2.90 \\
2.80 \\
2.75 \\
2.70 \\
2.65 \\
2.60 \\
2.75 \\
3.65 \\
3.15 \\
3.00 \\
4.60 \\
\mathbf{5 . 3 5} \\
4.45 \\
3.95 \\
3.70 \\
3.55 \\
3.40 \\
3.30 \\
. . .2 . .\end{array}$ & $\begin{array}{l}3.20 \\
3.15 \\
3.05 \\
3.10 \\
3.05 \\
3.00 \\
3.00 \\
3.00 \\
3.00 \\
3.00 \\
3.00 \\
2.90 \\
3.00 \\
3.00 \\
3.00 \\
3.00 \\
3.00 \\
2.90 \\
2.90 \\
2.90 \\
2.80 \\
2.80 \\
2.90 \\
3.05 \\
\text { 3. } 05 \\
3.00 \\
3.00 \\
3.10 \\
3.00 \\
3.00 \\
3.00\end{array}$ & $\begin{array}{l}3.00 \\
3.00 \\
3.00 \\
2.95 \\
2.85 \\
2.80 \\
2.80 \\
2.75 \\
2.70 \\
2.85 \\
2.80 \\
2.75 \\
2.70 \\
2.70 \\
2.70 \\
2.75 \\
2.80 \\
2.80 \\
2.80 \\
2.80 \\
2.80 \\
2.80 \\
2.70 \\
2.80 \\
2.80 \\
2.80 \\
2.80 \\
2.80 \\
2.80 \\
2.80 \\
\ldots . .2\end{array}$ \\
\hline
\end{tabular}


Animas River at Durango, Colorado.

\begin{tabular}{|c|c|c|c|c|c|c|c|c|c|}
\hline Day. & Apr. & . May. & June. & July. & Aug. & Sept. & Oet. & Nov. & Dec. \\
\hline 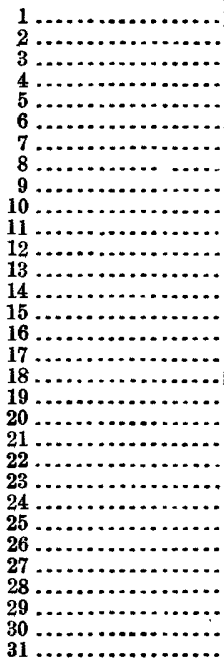 & 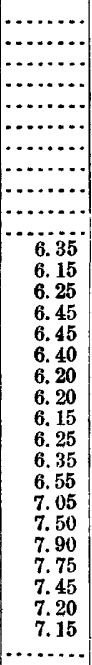 & $\begin{array}{l}7.20 \\
7.50 \\
7.65 \\
7.70 \\
7.95 \\
8.00 \\
7.55 \\
7.30 \\
7.05 \\
7.10 \\
6.90 \\
6.65 \\
6.40 \\
6.40 \\
6.35 \\
6.25 \\
6.25 \\
6.45 \\
6.65 \\
6.95 \\
6.95 \\
7.15 \\
7.30 \\
7.70 \\
7.35 \\
7.80 \\
7.75 \\
7.85 \\
7.65 \\
7.55 \\
7.35\end{array}$ & $\begin{array}{r}\mathbf{7 . 0 5} \\
\mathbf{6 . 8 5} \\
\mathbf{6 . 8 5} \\
\mathbf{6 . 8 5} \\
\mathbf{6 . 7 5} \\
\mathbf{6 . 6 5} \\
\mathbf{6 . 6 5} \\
\mathbf{6 . 5 5} \\
\mathbf{6 . 4 5} \\
\mathbf{6 . 4 0} \\
\mathbf{6 . 2 5} \\
\mathbf{6 . 1 5} \\
\mathbf{6 . 1 5} \\
\mathbf{6 . 1 0} \\
\mathbf{6 . 0 5} \\
\mathbf{6 . 0 0} \\
\mathbf{5 . 9 5} \\
\mathbf{5 . 9 0} \\
\mathbf{5 . 8 0} \\
\mathbf{5 . 8 0} \\
\mathbf{5 . 7 0} \\
\mathbf{5 . 7 0} \\
\mathbf{5 . 6 5} \\
\mathbf{5 . 6 0} \\
\mathbf{5 . 6 0} \\
\mathbf{5 . 5 5} \\
\mathbf{5 . 5 0} \\
\mathbf{5 . 4 5} \\
\mathbf{5 . 5 0} \\
\mathbf{5 . 4 0} \\
\mathbf{6 . . 2 .}\end{array}$ & $\begin{array}{l}\text { 5.40 } \\
\mathbf{5 . 4 0} \\
\mathbf{5 . 3 0} \\
\mathbf{5 . 3 0} \\
\mathbf{5 . 3 0} \\
\mathbf{5 . 3 0} \\
\mathbf{5 . 2 5} \\
\mathbf{5 . 2 5} \\
\mathbf{5 . 3 0} \\
\mathbf{5 . 3 0} \\
\mathbf{5 . 4 5} \\
\mathbf{5 . 4 5} \\
\mathbf{5 . 4 5} \\
\mathbf{5 . 5 0} \\
\mathbf{5 . 5 0} \\
\mathbf{5 . 5 0} \\
\mathbf{5 . 5 5} \\
\mathbf{5 . 6 5} \\
\mathbf{5 . 7 0} \\
\mathbf{5 . 5 5} \\
\mathbf{5 . 5 0} \\
\mathbf{5 . 4 5} \\
\mathbf{5 . 5 0} \\
\mathbf{5 . 5 5} \\
\mathbf{5 . 5 0} \\
\mathbf{5 . 4 0} \\
\mathbf{5 . 4 0} \\
\mathbf{5 . 4 0} \\
\mathbf{5 . 3 5} \\
\mathbf{5 . 3 0} \\
\mathbf{5 . 2 5}\end{array}$ & $\begin{array}{l}\text { 5. } 20 \\
5.20 \\
5.20 \\
5.15 \\
5.10 \\
5.10 \\
5.10 \\
5.10 \\
5.10 \\
5.10 \\
5.05 \\
5.00 \\
5.00 \\
5.00 \\
4.95 \\
4.90 \\
4.90 \\
4.90 \\
4.90 \\
4.90 \\
4.90 \\
4.90 \\
4.90 \\
4.90 \\
4.90 \\
4.80 \\
4.80 \\
4.95 \\
5.25 \\
5.30 \\
5.20\end{array}$ & $\begin{array}{r}\mathbf{5 . 2 0} \\
\mathbf{5 . 1 5} \\
\mathbf{5 . 5 0} \\
\mathbf{5 . 1 0} \\
\mathbf{5 . 1 0} \\
\mathbf{5 . 1 0} \\
\mathbf{5 . 1 0} \\
\mathbf{5 . 1 0} \\
\mathbf{5 . 1 5} \\
\mathbf{5 . 4 0} \\
\mathbf{5 . 9 0} \\
\mathbf{5 . 6 5} \\
\mathbf{5 . 6 0} \\
\mathbf{5 . 5 5} \\
\mathbf{5 . 5 0} \\
\mathbf{5 . 4 0} \\
\mathbf{5 . 4 0} \\
\mathbf{5 . 3 5} \\
\mathbf{5 . 4 0} \\
\mathbf{6 . 3 5} \\
\mathbf{6 . 0 5} \\
\mathbf{5 . 9 0} \\
\mathbf{8 . 4 0} \\
\mathbf{9 . 3 0} \\
\mathbf{7 . 4 0} \\
\mathbf{6 . 9 0} \\
\mathbf{6 . 6 5} \\
\mathbf{6 . 4 5} \\
\mathbf{6 . 2 0} \\
\mathbf{6 . 1 0} \\
\mathbf{7 . . . .}\end{array}$ & $\begin{array}{l}\mathbf{6 . 1 0} \\
\mathbf{6 . 0 0} \\
\mathbf{5} .95 \\
5.90 \\
\mathbf{5 . 8 5} \\
\mathbf{5 . 8 0} \\
\mathbf{5 . 8 5} \\
\mathbf{5 . 7 0} \\
\mathbf{5 . 7 0} \\
\mathbf{5 . 6 5} \\
\mathbf{5 . 6 0} \\
\mathbf{5 . 6 0} \\
\mathbf{5 . 6 5} \\
\mathbf{5 . 7 0} \\
\mathbf{5 . 6 0} \\
\mathbf{5 . 6 0} \\
\mathbf{5 . 6 0} \\
\mathbf{5 . 5 5} \\
\mathbf{5 . 5 0} \\
\mathbf{5 . 5 0} \\
5.45 \\
\mathbf{5 . 6 0} \\
\mathbf{5 .} 50 \\
\mathbf{5 . 5 0} \\
\mathbf{5 . 5 0} \\
\mathbf{5 . 4 0} \\
\mathbf{5 . 4 5} \\
\mathbf{5 . 5 0} \\
\mathbf{5 . 5 0} \\
\mathbf{5 . 4 5} \\
\mathbf{5 . 4 0}\end{array}$ & $\begin{array}{l}\text { 5. 40 } \\
\mathbf{5 . 4 0} \\
\mathbf{5 . 4 0} \\
\mathbf{5 . 3 0} \\
\mathbf{5 . 3 0} \\
\mathbf{5 . 2 5} \\
\mathbf{5 . 2 5} \\
\mathbf{5 . 2 0} \\
\mathbf{5 . 2 5} \\
\mathbf{5 . 2 5} \\
\mathbf{5 . 3 0} \\
\mathbf{5 . 3 0} \\
\mathbf{5 . 2 5} \\
\mathbf{5 . 2 5} \\
\mathbf{5 . 3 0} \\
\mathbf{5 . 2 5} \\
\mathbf{5 . 3 0} \\
\mathbf{5 . 2 5} \\
\mathbf{5 . 3 0} \\
\mathbf{5 . 3 0} \\
\mathbf{5 . 3 0} \\
\mathbf{5 . 2 5} \\
\mathbf{5 . 2 0} \\
\mathbf{5 . 2 5} \\
\mathbf{5 . 2 0} \\
\mathbf{5 . 2 0} \\
\mathbf{5 . 1 5} \\
\mathbf{5 . 0 5} \\
\mathbf{5 . 1 0} \\
\mathbf{5 . 1 0} \\
\ldots . . .\end{array}$ & 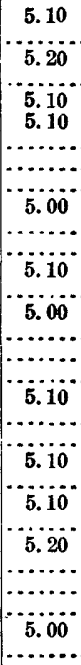 \\
\hline
\end{tabular}

Gila River at Buttes, Arizona.

\begin{tabular}{|c|c|c|c|c|c|c|c|c|c|c|c|c|}
\hline Day. & Jan. & Feb. & Mar. & Apr. & May. & June. & July. & Ang. & Sept. & Oct. & Nov. & Dec. \\
\hline & 2. 20 & 1.90 & 1.71 & 1. 76 & 1. 48 & 1.11 & 0.93 & 2.75 & 2.50 & 2. 80 & 3. 80 & 2.40 \\
\hline & 2. 20 & 1.85 & 1.71 & 1.86 & 1.48 & 1.10 & .90 & 3.70 & 2. 38 & 4. 70 & 3. 65 & 2. 38 \\
\hline & 2. 20 & 1. 80 & 1.72 & 1.87 & 1.47 & 1.09 & .91 & 2. 90 & 2. 25 & 3. 80 & 3. 50 & 2. 38 \\
\hline & 2.15 & 1.85 & 1. 80 & 1. 88 & 1. 46 & 1. 08 & 91 & 4.40 & 2.38 & 5. 20 & 3. 30 & 2. 35 \\
\hline & 2.10 & 1.85 & 1.79 & 1.90 & 1. 44 & 1.07 & 88 & 3.20 & 2.40 & 3. 70 & 3. 15 & 2. 31 \\
\hline & 2. 10 & 1.80 & 1.79 & 1.90 & 1.41 & 1.06 & 86 & 3. 60 & 2.25 & 3. 80 & 3.05 & 2. 30 \\
\hline & 2.00 & 1.80 & 1.73 & 1.89 & 1.40 & 1. 04 & 86 & 2.50 & 2.13 & 3.75 & 2. 95 & 2. 30 \\
\hline 8. & 2.05 & 1. 75 & 1.79 & 1.85 & 1. 40 & 1. $0 \mathrm{~s}$ & 1. 36 & 2.25 & 2. 70 & 4.80 & 2.90 & 2. 30 \\
\hline 9 & 2. 05 & 1. 70 & 1.80 & 1. 81 & 1. 38 & $1.0 \%$ & 1.20 & 2.13 & 3.95 & 3.90 & 2.80 & 2. 30 \\
\hline $10 \ldots$ & 2.05 & 1.70 & 1. 80 & 1. 80 & 1. 37 & 1.00 & 1. 10 & 1. 98 & 4. 20 & 3. 30 & 2. 73 & 2. 29 \\
\hline $11 .$. & 2.05 & 1. 75 & 1.79 & 1. 74 & 1. 38 & 1.00 & 1.04 & 1.90 & 3.15 & 3.00 & 2.70 & 2.28 \\
\hline 12. & 2.05 & 1.75 & 1.87 & 1.70 & 1. 36 & .99 & 1.29 & 2.00 & 2. 85 & 3.25 & 2. 65 & 2.28 \\
\hline 13. & 2.00 & 1. 70 & 1.88 & 1. 70 & 1. 34 & .98 & 1. 24 & 1. 90 & 2. 95 & 5. 00 & 2.60 & 2.28 \\
\hline 14. & 2.00 & 1. 70 & 1. 90 & 1.67 & 1. 32 & 97 & 1.20 & 1. 74 & 2.95 & 9.35 & 2.60 & 2. 27 \\
\hline 15. & 2.00 & 1. 70 & 1.91 & 1. 64 & 1.31 & .95 & 1.45 & 1.70 & 2.70 & 7. 90 & 2.58 & 2. 27 \\
\hline 16. & 2.00 & 1.70 & 1. 92 & . 1.62 & 1. 28 & .93 & 4. 50 & 1. 86 & 2.56 & 6.90 & 2. 55 & 2.29 \\
\hline 17. & 1. 95 & 1. 70 & 1. 91 & 1. 62 & 1. 27 & .92 & 2. 30 & 1. 72 & 2.20 & 5.60 & 2.50 & 2.30 \\
\hline 18 & 1.95 & 1.70 & 1.87 & 1. 62 & 1.25 & .91 & 2.10 & 1.85 & 2. 23 & 4. 80 & 2. 50 & 2. 30 \\
\hline 19 & 1.95 & 1.70 & 1.86 & 1. 61 & 1.24 & .91 & 2.10 & 2.06 & 2.50 & 4. 10 & 2. 48 & 2. 30 \\
\hline 20. & 1. 95 & 1. 70 & 1. 84 & 1. 61 & 1.24 & .91 & 3.00 & 2. 22 & 3. 43 & 3.70 & 2.46 & 2.28 \\
\hline 21. & 1.95 & 1.70 & 1.81 & 1. 60 & 1. 23 & .91 & 5. 40 & 2.00 & 3.10 & 3.35 & 2.44 & 2.26 \\
\hline 22. & 1. 90 & 1.70 & 1.79 & 1.60 & 1. 20 & .91 & 8. 10 & 2.50 & 2.80 & 3. 30 & 2. 42 & 2.24 \\
\hline 23. & 1.85 & 1.74 & 1. 75 & 1. 58 & 1.21 & .90 & 5. 10 & 2. 24 & 3.00 & 6.75 & 2.40 & 2.23 \\
\hline 24. & 1.80 & 1.72 & 1. 71 & 1.55 & 1.21 & 89 & 9.30 & 2. 41 & 2.35 & 5.50 & 2. 38 & 2.22 \\
\hline & 1.80 & 1.72 & 1. 71 & 1.53 & 1. 20 & 86 & 4.60 & 2.41 & 2.40 & 6.30 & 2.40 & 2. 22 \\
\hline & 1.80 & 1. 72 & 1. 71 & 1.50 & 1. 18 & 90 & 3.00 & 2. 20 & 2.25 & 6.50 & 2.46 & 2. 23 \\
\hline & 1.80 & 1. 72 & 1.70 & 1.50 & 1. 16 & 1. 32 & 2.80 & 2. 18 & 2. 38 & 5.50 & 2. 53 & 2.23 \\
\hline & 1.80 & 1. 72 & 1. 70 & 1. 51 & 1.15 & 1.10 & 2.50 & 2.10 & 2.32 & 6. 60 & 2.50 & 2.23 \\
\hline & 1.90 & 1. 72 & 1. 68 & 1.50 & 1. 14 & .98 & 2. 15 & 2.05 & 2.15 & 5.00 & 2. 48 & 2.30 \\
\hline & 1. 85 & & 1. 66 & 1.48 & 1. 12 & .96 & 2. 10 & 3.70 & 2.05 & 4. 60 & 2.44 & 2.30 \\
\hline & 1.90 & 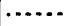 & 1.70 & $\cdots$ & 1.12 & .94 & 2.00 & 2.90 & $\ldots \ldots$ & 4. 10 & $\ldots$. & 2.34 \\
\hline
\end{tabular}


Colorado River at Fuma, Arizona.

\begin{tabular}{|c|c|c|c|c|c|c|c|c|c|c|c|c|}
\hline Day. & Jan. & Feb. & Mar. & Apr. & May & June. & July. & Ang. & Sept. & Oet. & Nov. & Dec. \\
\hline 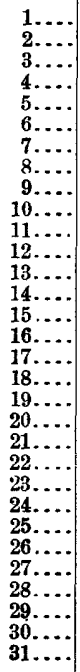 & $\begin{array}{l}18.25 \\
18.25 \\
18.17 \\
18.08 \\
18.00 \\
18.00 \\
18.00 \\
18.00 \\
18.00 \\
18.00 \\
18.00 \\
17.92 \\
17.92 \\
17.92 \\
18.00 \\
18.17 \\
18.17 \\
18.17 \\
18.17 \\
18.17 \\
18.00 \\
18.25 \\
18.42 \\
18.50 \\
18.50 \\
18.50 \\
18.50 \\
18.67 \\
18.75 \\
18.83 \\
18.92\end{array}$ & $\begin{array}{l}19.00 \\
19.08 \\
19.17 \\
19.42 \\
19.25 \\
19.25 \\
19.17 \\
19.08 \\
18.92 \\
18.92 \\
18.83 \\
18.83 \\
18.83 \\
18.83 \\
18.83 \\
18.75 \\
18.67 \\
18.67 \\
18.60 \\
18.50 \\
18.50 \\
18.50 \\
18.67 \\
18.75 \\
18.75 \\
18.83 \\
18.92 \\
18.92 \\
18.92 \\
\ldots \ldots . . . \\
\ldots \ldots . . .\end{array}$ & $\begin{array}{l}19.08 \\
19.17 \\
19.25 \\
19.33 \\
19.42 \\
19.42 \\
19.42 \\
19.42 \\
19.42 \\
19.42 \\
19.42 \\
19.25 \\
19.17 \\
19.17 \\
19.17 \\
19.17 \\
19.17 \\
19.17 \\
19.08 \\
19.08 \\
19.08 \\
19.08 \\
19.00 \\
19.00 \\
19.00 \\
19.08 \\
19.17 \\
19.25 \\
19.33 \\
19.50 \\
19.50\end{array}$ & $\begin{array}{l}19.42 \\
19.42 \\
19.83 \\
19.42 \\
19.42 \\
20.00 \\
20.00 \\
19.92 \\
19.83 \\
19.83 \\
19.75 \\
19.67 \\
19.75 \\
19.83 \\
19.67 \\
19.75 \\
19.75 \\
19.83 \\
20.00 \\
20.00 \\
19.92 \\
19.92 \\
19.83 \\
19.83 \\
19.83 \\
19.92 \\
20.00 \\
20.00 \\
20.00 \\
19.00 \\
\ldots \ldots . .\end{array}$ & $\begin{array}{l}20.00 \\
20.00 \\
21.50 \\
22.17 \\
22.42 \\
22.60 \\
22.33 \\
22.33 \\
22.25 \\
22.00 \\
22.25 \\
22.33 \\
22.42 \\
22.50 \\
22.60 \\
22.75 \\
22.75 \\
22.60 \\
22.33 \\
22.17 \\
21.92 \\
21.60 \\
21.33 \\
21.08 \\
20.83 \\
20.67 \\
20.50 \\
20.50 \\
20.75 \\
20.67 \\
21.08\end{array}$ & $\begin{array}{l}21.83 \\
22.00 \\
22.50 \\
23.25 \\
23.50 \\
23.83 \\
23.92 \\
24.00 \\
24.08 \\
24.00 \\
23.83 \\
23.75 \\
23.42 \\
23.17 \\
22.92 \\
22.75 \\
22.75 \\
22.67 \\
22.42 \\
22.42 \\
22.17 \\
21.92 \\
21.92 \\
21.83 \\
21.67 \\
21.60 \\
21.50 \\
21.50 \\
21.33 \\
21.25 \\
\ldots . . .\end{array}$ & $\begin{array}{l}21.25 \\
21.17 \\
21.00 \\
20.83 \\
20.67 \\
20.42 \\
20.42 \\
20.25 \\
20.25 \\
20.25 \\
20.25 \\
20.00 \\
20.00 \\
20.00 \\
20.00 \\
20.00 \\
20.00 \\
20.25 \\
20.33 \\
21.00 \\
20.33 \\
20.08 \\
20.67 \\
20.75 \\
20.67 \\
20.50 \\
20.60 \\
20.50 \\
20.50 \\
20.83 \\
20.75\end{array}$ & $\begin{array}{l}19.83 \\
19.42 \\
19.50 \\
19.60 \\
19.60 \\
19.60 \\
19.75 \\
20.00 \\
19.92 \\
19.75 \\
19.75 \\
19.75 \\
19.50 \\
19.33 \\
19.25 \\
19.17 \\
19.00 \\
19.08 \\
19.42 \\
19.75 \\
19.00 \\
19.00 \\
19.00 \\
19.00 \\
18.75 \\
18.75 \\
19.00 \\
19.00 \\
18.75 \\
18.42 \\
18.42\end{array}$ & \begin{tabular}{|l|}
19.83 \\
19.67 \\
19.67 \\
20.33 \\
20.67 \\
19.75 \\
19.60 \\
19.67 \\
19.42 \\
19.00 \\
18.83 \\
18.92 \\
19.33 \\
18.83 \\
18.60 \\
18.42 \\
18.42 \\
18.60 \\
18.83 \\
18.92 \\
18.60 \\
18.92 \\
19.33 \\
19.25 \\
19.50 \\
18.83 \\
18.42 \\
19.50 \\
23.17 \\
24.50 \\
$\ldots \ldots .$. \\
\end{tabular} & $\begin{array}{l}22.08 \\
20.83 \\
20.00 \\
19.67 \\
19.33 \\
19.00 \\
19.50 \\
19.00 \\
18.67 \\
18.67 \\
18.67 \\
18.75 \\
18.67 \\
18.67 \\
18.60 \\
18.50 \\
19.00 \\
19.42 \\
18.83 \\
18.83 \\
18.75 \\
19.25 \\
20.00 \\
19.17 \\
19.67 \\
19.19 \\
19.33 \\
19.17 \\
19.33 \\
19.17 \\
19.33\end{array}$ & $\begin{array}{l}19.42 \\
19.83 \\
19.17 \\
19.00 \\
18.83 \\
18.83 \\
18.75 \\
19.08 \\
18.92 \\
18.75 \\
18.75 \\
18.83 \\
18.75 \\
18.67 \\
18.50 \\
18.50 \\
18.50 \\
17.92 \\
18.42 \\
18.42 \\
18.42 \\
18.42 \\
18.33 \\
18.25 \\
18.33 \\
18.42 \\
18.50 \\
18.50 \\
18.83 \\
18.83 \\
\ldots . . .\end{array}$ & $\begin{array}{l}18.92 \\
18.92 \\
19.00 \\
18.83 \\
18.83 \\
18.83 \\
18.83 \\
18.83 \\
18.92 \\
18.83 \\
18.67 \\
18.42 \\
18.08 \\
17.83 \\
17.75 \\
17.50 \\
17.42 \\
17.42 \\
17.50 \\
17.83 \\
18.00 \\
18.17 \\
18.25 \\
18.25 \\
18.33 \\
18.33 \\
18.42 \\
18.42 \\
18.50 \\
18.50 \\
18.50\end{array}$ \\
\hline
\end{tabular}

Humboldt River at Elko, Nevada.

\begin{tabular}{|c|c|c|c|c|c|c|c|c|c|c|c|c|}
\hline Dảy. & Jan. & Feb. & Mar.a & Apr.a & May. & June. & July. & Ang. & Sept. & Oot. & Nov. & Dec. \\
\hline 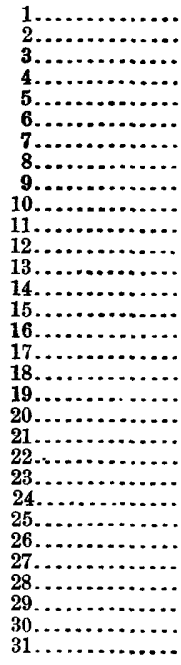 & $\begin{array}{l}2.17 \\
2.15 \\
2.14 \\
2.15 \\
2.15 \\
2.17 \\
2.00 \\
2.00 \\
2.00 \\
2.00 \\
2.00 \\
2.00 \\
2.00 \\
2.00 \\
2.00 \\
2.17 \\
2.15 \\
2.10 \\
2.14 \\
2.27 \\
2.27 \\
2.25 \\
2.20 \\
2.20 \\
2.25 \\
2.25 \\
2.25 \\
2.24 \\
2.35 \\
2.30 \\
2.35\end{array}$ & \begin{tabular}{|l}
2.40 \\
2.45 \\
2.45 \\
2.30 \\
2.47 \\
2.47 \\
2.30 \\
2.34 \\
2.30 \\
2.47 \\
2.34 \\
2.30 \\
2.35 \\
2.30 \\
2.30 \\
2.34 \\
2.20 \\
2.20 \\
2.20 \\
2.20 \\
2.20 \\
2.20 \\
2.25 \\
2.25 \\
2.25 \\
2.30 \\
2.30 \\
2.30 \\
2.30 \\
$\cdots \ldots$ \\
$\cdots \ldots$. \\
2.7
\end{tabular} & 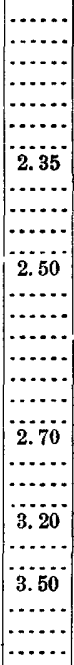 & 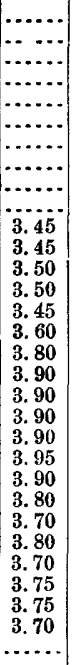 & $\begin{array}{l}\text { 3. } 60 \\
3.65 \\
3.50 \\
3.50 \\
3.50 \\
3.55 \\
3.60 \\
3.70 \\
3.75 \\
3.80 \\
3.90 \\
4.15 \\
4.20 \\
4.20 \\
4.20 \\
4.10 \\
4.00 \\
3.90 \\
3.90 \\
3.80 \\
3.70 \\
3.60 \\
3.50 \\
3.55 \\
3.55 \\
3.55 \\
3.60 \\
3.70 \\
3.95 \\
4.30 \\
4.55 \\
3.5\end{array}$ & \begin{tabular}{|c|}
$\mathbf{5 . 3 0}$ \\
$\mathbf{5 . 9 0}$ \\
$\mathbf{6 . 5 0}$ \\
$\mathbf{7 . 3 5}$ \\
$\mathbf{7 . 3 0}$ \\
7.50 \\
7.70 \\
$\mathbf{7 . 6 5}$ \\
7.55 \\
7.55 \\
7.10 \\
7.20 \\
7.40 \\
7.35 \\
7.30 \\
7.35 \\
7.45 \\
7.45 \\
7.45 \\
7.50 \\
7.55 \\
7.40 \\
7.20 \\
7.10 \\
6.90 \\
6.70 \\
6.40 \\
610 \\
5.90 \\
5.70 \\
$\cdots . .2$ \\
7
\end{tabular} & $\begin{array}{r}5.50 \\
5.40 \\
5.20 \\
5.00 \\
4.55 \\
4.50 \\
4.45 \\
4.35 \\
4.20 \\
4.15 \\
3.95 \\
3.90 \\
3.90 \\
3.80 \\
3.75 \\
3.65 \\
3.60 \\
3.50 \\
.3 .40 \\
3.20 \\
3.20 \\
3.10 \\
2.95 \\
2.90 \\
2.80 \\
2.75 \\
2.75 \\
2.70 \\
2.70 \\
2.65 \\
2.60\end{array}$ & $\begin{array}{l}2.60 \\
2.60 \\
2.60 \\
2.55 \\
2.50 \\
2.45 \\
2.40 \\
2.40 \\
2.30 \\
2.27 \\
2.25 \\
2.25 \\
2.25 \\
2.25 \\
2.20 \\
2.20 \\
2.20 \\
2.15 \\
2.10 \\
2.10 \\
2.10 \\
2.10 \\
2.10 \\
2.10 \\
2.05 \\
2.05 \\
2.05 \\
2.05 \\
2.05 \\
2.00 \\
2.00\end{array}$ & $\begin{array}{l}2.00 \\
2.00 \\
2.00 \\
2.00 \\
2.00 \\
2.00 \\
1.97 \\
1.95 \\
1.95 \\
1.93 \\
1.93 \\
1.93 \\
1.93 \\
1.93 \\
1.93 \\
1.90 \\
1.90 \\
1.90 \\
1.90 \\
1.90 \\
1.90 \\
1.90 \\
1.93 \\
1.90 \\
1.90 \\
1.90 \\
1.90 \\
1.90 \\
1.90 \\
1.90 \\
\ldots . .2\end{array}$ & $\begin{array}{l}1.95 \\
1.97 \\
1.97 \\
1.98 \\
1.98 \\
2.00 \\
2.00 \\
2.00 \\
2.00 \\
2.00 \\
2.00 \\
2.00 \\
2.05 \\
2.05 \\
2.05 \\
2.10 \\
2.10 \\
2.10 \\
2.10 \\
2.10 \\
2.10 \\
2.10 \\
2.10 \\
2.15 \\
2.15 \\
2.15 \\
2.15 \\
2.15 \\
2.15 \\
2.15 \\
2.15\end{array}$ & $\begin{array}{r}2.00 \\
2.00 \\
2.00 \\
2.00 \\
2.00 \\
2.00 \\
2.00 \\
2.10 \\
2.20 \\
2.20 \\
2.25 \\
2.25 \\
2.30 \\
2.30 \\
2.35 \\
2.35 \\
2.35 \\
2.40 \\
2.40 \\
2.40 \\
2.40 \\
2.40 \\
2.40 \\
2.00 \\
2.40 \\
2.40 \\
2.40 \\
2.40 \\
2.40 \\
2.35 \\
\ldots \ldots . \\
\ldots\end{array}$ & $\begin{array}{l}2.35 \\
2.35 \\
2.35 \\
2.40 \\
2.40 \\
2.40 \\
2.40 \\
2.35 \\
2.30 \\
2.35 \\
2.40 \\
2.45 \\
2.45 \\
2.45 \\
2.45 \\
2.45 \\
2.50 \\
2.50 \\
2.52 \\
2.50 \\
2.50 \\
2.45 \\
2.45 \\
2.45 \\
2.50 \\
2.50 \\
2.50 \\
2.45 \\
2.45 \\
2.45 \\
2.50\end{array}$ \\
\hline
\end{tabular}

a Observations discontinued from March 1 to April 11; gage taken by flood. 
Humboldt River at Battle Mountain, Nevada.

\begin{tabular}{|c|c|c|c|c|c|c|c|}
\hline Day. & June. & July. & Aug. & Sept. & Oct. & Nov. & Dec. \\
\hline 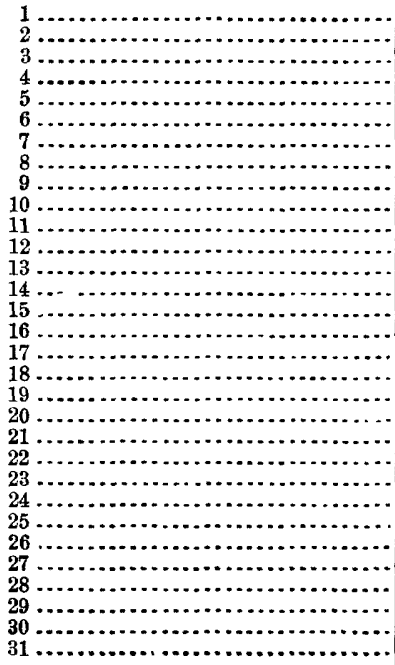 & 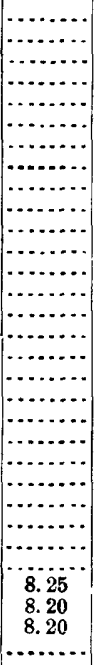 & $\begin{array}{l}8.15 \\
8.10 \\
8.10 \\
8.05 \\
7.50 \\
7.30 \\
7.10 \\
6.80 \\
6.40 \\
6.00 \\
5.60 \\
5.30 \\
5.00 \\
4.80 \\
4.60 \\
4.40 \\
4.20 \\
4.10 \\
4.00 \\
3.80 \\
3.70 \\
3.60 \\
3.50 \\
3.30 \\
3.10 \\
3.00 \\
2.80 \\
2.70 \\
2.60 \\
2.40 \\
2.30\end{array}$ & $\begin{array}{l}2.10 \\
2.00 \\
1.90 \\
1.80 \\
1.75 \\
1.70 \\
1.60 \\
1.50 \\
1.45 \\
1.40 \\
1.40 \\
1.35 \\
1.30 \\
1.25 \\
1.20 \\
1.15 \\
1.15 \\
1.10 \\
1.05 \\
1.00 \\
1.00 \\
1.00 \\
.95 \\
.95 \\
.90 \\
.90 \\
.90 \\
.85 \\
.85 \\
.80 \\
.80\end{array}$ & $\begin{array}{r}0.80 \\
.85 \\
.90 \\
.90 \\
.90 \\
.85 \\
.85 \\
.85 \\
.80 \\
.80 \\
.80 \\
.80 \\
.80 \\
.80 \\
.80 \\
.80 \\
.75 \\
.75 \\
.75 \\
.75 \\
.75 \\
1.10 \\
1.00 \\
.90 \\
.90 \\
.85 \\
.85 \\
.85 \\
.85 \\
.80 \\
. .2 \\
.9\end{array}$ & $\begin{array}{l}0.80 \\
.80 \\
.80 \\
.80 \\
.80 \\
.80 \\
.80 \\
.80 \\
.85 \\
.85 \\
.85 \\
.85 \\
.85 \\
.85 \\
.90 \\
.90 \\
.95 \\
.95 \\
.95 \\
1.00 \\
1.10 \\
1.10 \\
1.15 \\
1.15 \\
1.20 \\
1.20 \\
1.30 \\
1.35 \\
1.35 \\
1.40 \\
1.40\end{array}$ & $\begin{array}{l}1.40 \\
1.40 \\
1.45 \\
1.45 \\
1.50 \\
1.50 \\
1.50 \\
1.50 \\
1.55 \\
1.60 \\
1.70 \\
1.70 \\
1.70 \\
1.65 \\
1.65 \\
1.70 \\
1.70 \\
1.70 \\
1.70 \\
1.70 \\
1.75 \\
1.75 \\
1.80 \\
1.80 \\
1.80 \\
1.80 \\
1.80 \\
1.80 \\
1.85 \\
1.85 \\
\therefore 1.2 \\
1.1\end{array}$ & $\begin{array}{l}1.90 \\
1.90 \\
1.90 \\
1.90 \\
1.85 \\
1.80 \\
1.80 \\
1.80 \\
1.85 \\
1.85 \\
1.90 \\
1.90 \\
1.90 \\
1.90 \\
2.00 \\
2.00 \\
2.00 \\
2.00 \\
2.00 \\
2.05 \\
2.05 \\
2.05 \\
2.10 \\
2.10 \\
2.10 \\
2.10 \\
2.10 \\
2.15 \\
2.15 \\
2.15 \\
2.20\end{array}$ \\
\hline
\end{tabular}

Humboldt River at Golconda, Nevada.

\begin{tabular}{|c|c|c|c|c|c|c|c|c|c|c|c|c|}
\hline Day. & Jan. & Feb. & Mar. & Apr. & May. & Jane. & July. & Aug. & Sept. & Oet. & Nov. & Dec. \\
\hline 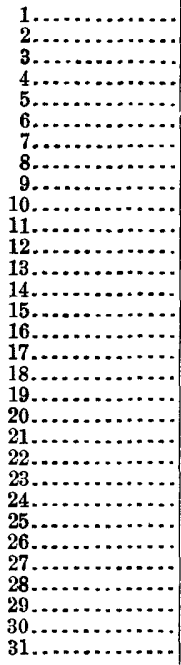 & $\begin{array}{l}0.65 \\
.63 \\
.60 \\
.58 \\
.50 \\
.50 \\
.55 \\
.53 \\
.53 \\
.58 \\
.58 \\
.55 \\
.58 \\
.53 \\
.60 \\
.60 \\
.60 \\
.65 \\
.70 \\
.78 \\
.85 \\
.85 \\
1.05 \\
1.70 \\
1.10 \\
1.00 \\
1.30 \\
1.35 \\
1.40 \\
1.50 \\
1.50\end{array}$ & $\begin{array}{l}1.50 \\
1.50 \\
1.60 \\
1.75 \\
1.70 \\
1.70 \\
1.65 \\
1.70 \\
1.40 \\
1.60 \\
1.70 \\
1.70 \\
1.60 \\
1.50 \\
1.40 \\
1.40 \\
1.35 \\
1.37 \\
1.37 \\
1.25 \\
1.20 \\
1.15 \\
1.00 \\
1.00 \\
.90 \\
.80 \\
.85 \\
.75 \\
.60 \\
\ldots \ldots . . \\
\ldots \ldots\end{array}$ & $\begin{array}{l}0.50 \\
.50 \\
.50 \\
.50 \\
.50 \\
.50 \\
.50 \\
.50 \\
.50 \\
.55 \\
.60 \\
.55 \\
.50 \\
.50 \\
.50 \\
.50 \\
.40 \\
.30 \\
.20 \\
.20 \\
.20 \\
.20 \\
.20 \\
.20 \\
.40 \\
.75 \\
.75 \\
.50 \\
.35 \\
.35 \\
.30 \\
.50 \\
.50 \\
.50 \\
.50 \\
.50 \\
.50 \\
.50 \\
.50 \\
.50 \\
.50 \\
.50\end{array}$ & $\begin{array}{r}0.30 \\
.35 \\
.40 \\
.50 \\
.70 \\
.80 \\
1.60 \\
2.00 \\
2.40 \\
2.10 \\
2.00 \\
1.90 \\
1.70 \\
1.60 \\
1.50 \\
1.55 \\
1.60 \\
1.90 \\
1.70 \\
1.70 \\
2.10 \\
2.00 \\
2.00 \\
2.20 \\
2.30 \\
2.30 \\
2.40 \\
2.50 \\
2.60 \\
2.60 \\
\ldots . . .\end{array}$ & $\begin{array}{l}2.65 \\
2.70 \\
2.70 \\
2.70 \\
2.70 \\
2.80 \\
2.90 \\
3.20 \\
3.20 \\
3.20 \\
3.30 \\
3.30 \\
3.30 \\
3.30 \\
3.30 \\
3.30 \\
3.30 \\
3.35 \\
3.40 \\
3.45 \\
3.40 \\
3.45 \\
3.50 \\
3.50 \\
3.50 \\
3.50 \\
3.50 \\
3.50 \\
3.70 \\
3.80 \\
3.90\end{array}$ & $\begin{array}{l}4.00 \\
4.00 \\
4.30 \\
4.50 \\
4.60 \\
4.80 \\
4.90 \\
4.90 \\
4.90 \\
5.00 \\
5.30 \\
5.75 \\
5.70 \\
5.90 \\
6.00 \\
6.10 \\
6.40 \\
6.70 \\
6.70 \\
6.80 \\
6.90 \\
6.90 \\
7.00 \\
7.00 \\
7.10 \\
7.10 \\
7.20 \\
7.20 \\
7.30 \\
7.40 \\
. . . . .\end{array}$ & $\begin{array}{l}7.40 \\
7.40 \\
7.30 \\
7.20 \\
7.00 \\
6.90 \\
6.60 \\
6.50 \\
6.10 \\
5.80 \\
5.80 \\
5.50 \\
5.20 \\
5.00 \\
4.95 \\
4.85 \\
4.80 \\
4.70 \\
4.50 \\
4.30 \\
4.00 \\
3.90 \\
3.90 \\
3.60 \\
\mathbf{3 . 5 0} \\
\mathbf{3 . 5 0} \\
\mathbf{3 . 4 0} \\
\mathbf{3 . 4 0} \\
3.40 \\
\mathbf{3 . 1 0} \\
\mathbf{3 .} 00\end{array}$ & $\begin{array}{r}3.00 \\
2.90 \\
2.80 \\
2.60 \\
2.50 \\
2.40 \\
2.20 \\
2.20 \\
2.10 \\
2.10 \\
2.00 \\
1.90 \\
1.80 \\
1.80 \\
1.60 \\
1.50 \\
1.50 \\
1.40 \\
1.35 \\
1.30 \\
1.20 \\
1.05 \\
1.00 \\
1.00 \\
1.00 \\
.90 \\
.80 \\
.80 \\
.75 \\
.70 \\
.70\end{array}$ & $\begin{array}{l}0.70 \\
.70 \\
.60 \\
.60 \\
.50 \\
.50 \\
.45 \\
.40 \\
.40 \\
.35 \\
.35 \\
.30 \\
.30 \\
.25 \\
.25 \\
.25 \\
.25 \\
.20 \\
.20 \\
.20 \\
.20 \\
.20 \\
.20 \\
.30 \\
.30 \\
.30 \\
.30 \\
.30 \\
.30 \\
.30 \\
. . . .\end{array}$ & $\begin{array}{l}0.30 \\
.30 \\
.35 \\
.40 \\
.40 \\
.50 \\
.60 \\
.60 \\
.60 \\
.60 \\
.60 \\
.60 \\
.60 \\
.60 \\
.60 \\
.60 \\
.60 \\
.60 \\
.70 \\
.70 \\
.70 \\
.70 \\
.70 \\
.70 \\
.75 \\
.80 \\
.80 \\
.80 \\
.90 \\
.90 \\
.90\end{array}$ & \begin{tabular}{|}
0.95 \\
1.00 \\
1.00 \\
1.00 \\
1.10 \\
1.10 \\
1.20 \\
1.20 \\
1.25 \\
1.20 \\
1.20 \\
1.30 \\
1.40 \\
1.50 \\
1.50 \\
1.60 \\
1.60 \\
1.60 \\
1.60 \\
1.60 \\
1.60 \\
1.60 \\
1.70 \\
1.70 \\
1.70 \\
1.70 \\
1.60 \\
1.50 \\
1.50 \\
1.55 \\
$\ldots \ldots .$.
\end{tabular} & $\begin{array}{l}1.60 \\
1.60 \\
1.60 \\
1.70 \\
1.70 \\
1.70 \\
1.80 \\
1.80 \\
1.80 \\
1.85 \\
1.85 \\
1.80 \\
1.90 \\
1.90 \\
2.00 \\
2.00 \\
2.00 \\
2.10 \\
2.10 \\
2.15 \\
2.20 \\
2.20 \\
2.20 \\
2.20 \\
2.20 \\
2.20 \\
2.00 \\
2.10 \\
2.15 \\
2.20 \\
2.20\end{array}$ \\
\hline
\end{tabular}


Humboldt River at Oreana, Nevada.

\begin{tabular}{|c|c|c|c|c|c|c|c|c|c|c|c|c|}
\hline Day. & Jan. & Feb. & Mar. & Apr. & May & June. & July. & Ang. & Sept. & Oct. & Nov. & Dec. \\
\hline 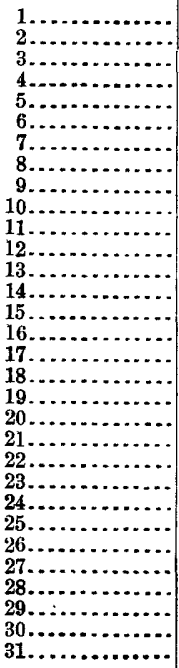 & \begin{tabular}{l}
$\cdots$ \\
\hdashline 2.70 \\
2.70 \\
2.70 \\
2.71 \\
2.72
\end{tabular} & $\begin{array}{l}2.72 \\
2.72 \\
2.72 \\
2.73 \\
2.73 \\
2.78 \\
2.80 \\
2.80 \\
2.80 \\
2.80 \\
2.80 \\
2.80 \\
2.80 \\
2.81 \\
2.85 \\
2.81 \\
2.81 \\
2.82 \\
2.83 \\
2.84 \\
2.84 \\
2.85 \\
2.83 \\
2.82 \\
2.81 \\
2.81 \\
2.81 \\
2.81 \\
2.80\end{array}$ & $\begin{array}{l}2.80 \\
2.80 \\
2.70 \\
2.70 \\
2.50 \\
2.70 \\
2.70 \\
2.60 \\
2.60 \\
2.60 \\
2.60 \\
2.60 \\
2.60 \\
2.60 \\
2.50 \\
2.50 \\
2.50 \\
2.50 \\
2.50 \\
2.50 \\
2.50 \\
2.50 \\
2.50 \\
2.60 \\
2.60 \\
2.60 \\
2.65 \\
2.65 \\
2.55 \\
2.40 \\
2.40\end{array}$ & $\begin{array}{l}2.40 \\
2.40 \\
2.40 \\
2.30 \\
2.30 \\
2.30 \\
2.30 \\
2.20 \\
2.05 \\
2.05 \\
2.05 \\
2.04 \\
2.04 \\
2.04 \\
2.04 \\
2.04 \\
2.03 \\
2.03 \\
2.03 \\
2.03 \\
2.03 \\
2.03 \\
2.03 \\
2.03 \\
2.03 \\
2.03 \\
2.04 \\
2.04 \\
2.04 \\
2.04\end{array}$ & $\begin{array}{l}2.04 \\
2.04 \\
2.04 \\
2.04 \\
2.04 \\
2.04 \\
2.04 \\
2.04 \\
2.04 \\
2.05 \\
2.05 \\
2.06 \\
2.06 \\
2.07 \\
2.08 \\
2.09 \\
2.10 \\
2.10 \\
2.10 \\
2.80 \\
3.20 \\
3.40 \\
3.70 \\
4.90 \\
4.80 \\
4.60 \\
4.50 \\
4.25 \\
4.05 \\
3.95 \\
3.95\end{array}$ & \begin{tabular}{|l|}
3.80 \\
3.70 \\
3.60 \\
3.50 \\
3.40 \\
3.30 \\
3.35 \\
3.50 \\
3.50 \\
4.20 \\
4.50 \\
4.60 \\
4.70 \\
4.80 \\
4.80 \\
4.90 \\
5.10 \\
5.20 \\
5.40 \\
5.70 \\
5.70 \\
5.90 \\
6.10 \\
6.20 \\
6.30 \\
6.50 \\
6.50 \\
6.60 \\
6.80 \\
6.90
\end{tabular} & $\begin{array}{l}7.10 \\
7.10 \\
7.20 \\
7.30 \\
7.40 \\
7.50 \\
7.50 \\
7.50 \\
7.60 \\
7.65 \\
7.60 \\
7.50 \\
7.30 \\
7.10 \\
6.90 \\
6.70 \\
6.60 \\
6.40 \\
6.30 \\
6.10 \\
6.00 \\
5.80 \\
5.70 \\
5.60 \\
5.40 \\
5.30 \\
5.20 \\
5.10 \\
5.10 \\
5.00 \\
4.90\end{array}$ & \begin{tabular}{|l|}
4.70 \\
4.70 \\
4.60 \\
4.45 \\
4.40 \\
4.30 \\
4.20 \\
4.15 \\
4.10 \\
4.10 \\
4.00 \\
4.00 \\
3.90 \\
3.80 \\
3.80 \\
3.70 \\
3.60 \\
3.60 \\
3.50 \\
3.40 \\
3.50 \\
3.40 \\
3.30 \\
3.30 \\
3.20 \\
3.10 \\
3.00 \\
3.10 \\
3.20 \\
3.20 \\
3.20 \\
\end{tabular} & $\begin{array}{l}3.20 \\
3.20 \\
3.20 \\
3.10 \\
3.10 \\
3.10 \\
3.00 \\
2.90 \\
2.80 \\
2.70 \\
2.70 \\
2.70 \\
2.70 \\
2.65 \\
2.65 \\
2.65 \\
2.60 \\
2.60 \\
2.60 \\
2.55 \\
2.55 \\
3.10 \\
3.00 \\
2.90 \\
2.60 \\
2.60 \\
2.55 \\
2.55 \\
2.50 \\
2.50\end{array}$ & $\begin{array}{l}2.45 \\
2.40 \\
2.40 \\
2.30 \\
2.20 \\
2.15 \\
2.10 \\
2.10 \\
2.05 \\
2.05 \\
2.05 \\
2.05 \\
2.05 \\
2.00 \\
2.00 \\
2.00 \\
1.95 \\
1.95 \\
1.90 \\
1.90 \\
2.20 \\
2.25 \\
2.25 \\
2.20 \\
2.20 \\
2.60 \\
2.50 \\
2.40 \\
2.40 \\
2.40 \\
2.40\end{array}$ & 2.40 & 2.80 \\
\hline
\end{tabular}

Rock Creek at Battle Mountain, Nevada.

\begin{tabular}{|c|c|c|c|c|c|c|c|}
\hline Day. & Feb. & Mar. & Apr. & May. & June. & July. & Aug. \\
\hline & 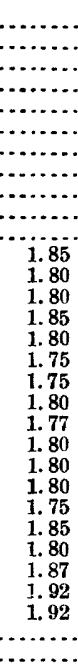 & $\begin{array}{l}1.90 \\
1.70 \\
1.77 \\
1.80 \\
1.75 \\
2.05 \\
1.92 \\
1.90 \\
2.05 \\
2.02 \\
2.05 \\
2.00 \\
2.02 \\
2.14 \\
2.15 \\
1.97 \\
2.10 \\
2.35 \\
2.55 \\
2.80 \\
3.65 \\
3.32 \\
3.37 \\
3.45 \\
3.65 \\
3.85 \\
4.40 \\
5.20 \\
4.60 \\
4.25 \\
4.00\end{array}$ & $\begin{array}{l}3.97 \\
3.50 \\
3.35 \\
3.27 \\
3.22 \\
2.80 \\
2.60 \\
2.55 \\
2.55 \\
2.50 \\
2.40 \\
2.30 \\
2.35 \\
2.57 \\
2.82 \\
2.55 \\
2.50 \\
2.47 \\
2.55 \\
2.50 \\
2.50 \\
2.50 \\
2.57 \\
2.50 \\
2.50 \\
2.70 \\
2.65 \\
2.60 \\
2.60 \\
2.68\end{array}$ & $\begin{array}{l}2.70 \\
2.75 \\
2.85 \\
2.85 \\
2.95 \\
3.05 \\
3.10 \\
3.10 \\
3.15 \\
3.20 \\
3.35 \\
3.50 \\
3.65 \\
3.85 \\
4.10 \\
4.20 \\
4.35 \\
4.60 \\
4.95 \\
5.10 \\
5.15 \\
5.20 \\
5.25 \\
5.55 \\
5.80 \\
5.88 \\
5.95 \\
6.00 \\
6.00 \\
6.10 \\
6.15\end{array}$ & $\begin{array}{l}5.90 \\
5.70 \\
5.50 \\
5.15 \\
4.95 \\
4.65 \\
4.40 \\
4.10 \\
3.85 \\
3.75 \\
3.50 \\
3.25 \\
3.10 \\
3.08 \\
3.05 \\
3.00 \\
3.00 \\
2.95 \\
2.93 \\
2.86 \\
2.88 \\
2.88 \\
2.85 \\
2.83 \\
2.83 \\
2.80 \\
2.78 \\
2.75 \\
2.75 \\
2.75\end{array}$ & $\begin{array}{l}2.73 \\
2.70 \\
2.68 \\
2.65 \\
2.63 \\
2.60 \\
2.58 \\
2.55 \\
2.55 \\
2.50 \\
2.50 \\
2.45 \\
2.40 \\
2.40 \\
2.35 \\
2.35 \\
2.30 \\
2.25 \\
2.20 \\
2.15 \\
2.10 \\
2.00 \\
2.00 \\
1.95 \\
1.90 \\
1.90 \\
1.85 \\
1.85 \\
1.80 \\
1.80 \\
1.80\end{array}$ & 1.80 \\
\hline
\end{tabular}


South Fork of Humboldt River at Masons Ranch, Nevada.

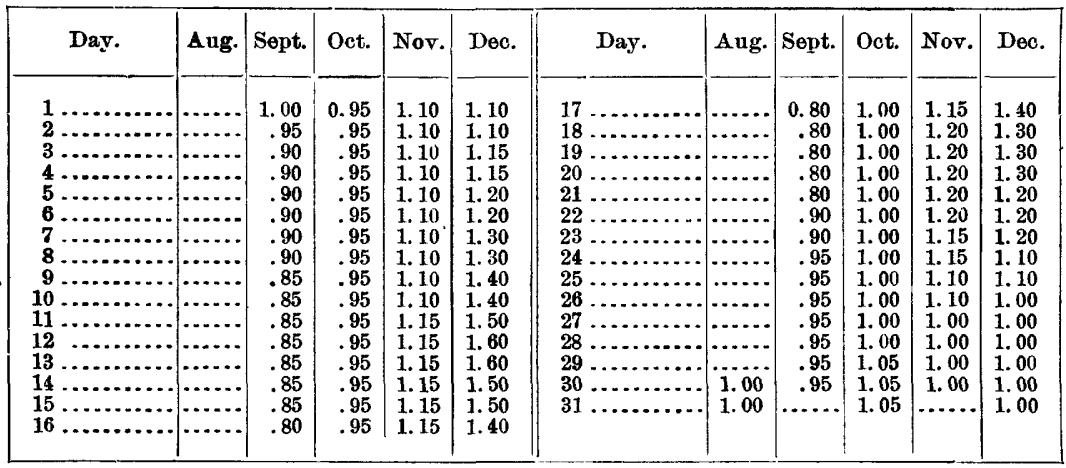

Bear River at Soda Springs, Idaho.

\begin{tabular}{|c|c|c|c|c|c|c|c|c|c|c|c|c|c|}
\hline Day. & Cay. & June & July & Aug & Sept. & Oct. & Day. & May. & June & July. & Ang & Sept. & Oct. \\
\hline \multirow{3}{*}{ 3.... } & & & & \begin{tabular}{l|l|} 
& 5.90
\end{tabular} & \multirow[b]{2}{*}{ 5. 40} & \multirow[b]{2}{*}{\begin{tabular}{|l}
5.35 \\
$\ldots$
\end{tabular}} & \multirow{4}{*}{$\begin{array}{l}17 \ldots . \\
18 \ldots \\
19 \ldots \ldots \\
20 \ldots \\
22 \ldots\end{array}$} & & $\begin{array}{c}8.20 \\
\ldots \ldots\end{array}$ & \multirow[t]{2}{*}{5.90} & \multirow[t]{2}{*}{5.30} & \multirow[b]{2}{*}{5.30} & \multirow[b]{2}{*}{$\cdots .}$. \\
\hline & & \begin{tabular}{l|l} 
& 7.53 \\
\end{tabular} & & 5.85 & & & & & 8.00 & & & & \\
\hline & & & & 5.80 & \multirow{2}{*}{$\begin{array}{l}5.35 \\
5.30\end{array}$} & & & & 7.90 & 5.85 & 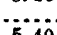 & & \\
\hline & $\cdots$ & 7.70 & $\begin{array}{l}7.40 \\
7.30\end{array}$ & 5.70 & & & & & 780 & 5.80 & $\begin{array}{l}5.40 \\
5.40\end{array}$ & \multirow{2}{*}{$\dddot{5} .35$} & \\
\hline \multirow{2}{*}{$\begin{array}{l}7 \ldots \\
8 \ldots\end{array}$} & $\ldots$ & 7.93 & 7.00 & 5.65 & \multicolumn{2}{|l|}{5.30} & & & 100 & 00 & \multirow[b]{2}{*}{5.45} & & \\
\hline & 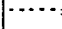 & 8.40 & 6.90 & & \multicolumn{2}{|l|}{5.30} & & \multirow{3}{*}{\begin{tabular}{|l|}
6.50 \\
6.52
\end{tabular}} & 7.70 & 5.80 & & \multirow{2}{*}{5.40} & \\
\hline \multirow{2}{*}{$\begin{array}{l}10 . \\
11 .\end{array}$} & $\ldots \ldots$ & $\begin{array}{l}8.30 \\
8.32\end{array}$ & 6. 30 & 5.60 & \multirow{2}{*}{5.30} & & & & 7.60 & & 5.40 & & \\
\hline & & & 6.00 & & & & & & & \multirow{3}{*}{5.80} & \multirow[b]{2}{*}{$\begin{array}{l}5.40 \\
5.40\end{array}$} & \multirow{2}{*}{$\begin{array}{l}5.35 \\
\ldots\end{array}$} & \\
\hline 13 & $\ldots$ & 8.30 & & b.50 & $\cdots$ & & & & & & & & \\
\hline & & & 6.00 & & 5.30 & & 30 . & 7.10 & & & & \multirow{2}{*}{$\overline{5} .35$} & \\
\hline & & 8.40 & 5.90 & & & & & & $\cdots$ & 5.95 & 5.40 & & \\
\hline & & & & & 5. 30 & & & & & & & & \\
\hline
\end{tabular}

Bear River at Battle Creek, Idaho.

\begin{tabular}{|c|c|c|c|c|c|c|c|c|c|c|c|c|}
\hline Day & an. & Fob. & Mar. & Apr & May. & June. & July. & Aug. & Sept. & Oct. & Nov. & Dec. \\
\hline $\begin{array}{r}8 . \\
9 \\
10\end{array}$ & \begin{tabular}{|}
1.40 \\
1.40 \\
1.40 \\
1.40 \\
1.40 \\
1.40 \\
1.40 \\
1.40 \\
1.40 \\
1.40 \\
1.40 \\
1.40 \\
1.40 \\
1.40 \\
1.40 \\
1.40 \\
1.40 \\
1.40 \\
1.40 \\
1.40 \\
1.40 \\
1.40 \\
1.40 \\
1.40 \\
1.40 \\
1.40 \\
1.40 \\
1.30 \\
1.30
\end{tabular} & $\begin{array}{l}1.30 \\
1.30 \\
1.30 \\
1.30 \\
1.30 \\
1.30 \\
1.30 \\
1.30 \\
1.30 \\
1.30 \\
1.30 \\
1.30 \\
1.40 \\
1.40 \\
1.40 \\
1.40 \\
1.40 \\
1.40 \\
1.40 \\
1.40 \\
1.40 \\
1.40 \\
1.40 \\
1.40 \\
1.40 \\
1.40 \\
1.40 \\
1.40 \\
1.40\end{array}$ & $\begin{array}{l}1.40 \\
1.40 \\
1.40 \\
1.40 \\
1.40 \\
1.40 \\
1.40 \\
1.40 \\
1.40 \\
1.40 \\
1.40 \\
1.40 \\
1.40 \\
1.40 \\
1.40 \\
1.40 \\
1.40 \\
1.40 \\
1.50 \\
1.50 \\
1.50 \\
1.60 \\
1.70 \\
1.70 \\
1.80 \\
1.90 \\
2.00 \\
2.10 \\
2.10 \\
2.20 \\
2.30\end{array}$ & $\begin{array}{l}2.40 \\
2.60 \\
2.60 \\
2.70 \\
2.80 \\
2.80 \\
2.80 \\
2.70 \\
2.70 \\
2.70 \\
2.70 \\
2.70 \\
2.70 \\
2.70 \\
2.70 \\
2.70 \\
2.70 \\
2.70 \\
2.70 \\
2.60 \\
2.60 \\
2.60 \\
2.60 \\
2.60 \\
2.60 \\
2.70 \\
2.70 \\
2.70\end{array}$ & $\begin{array}{l}2.70 \\
2.80 \\
2.80 \\
2.80 \\
2.80 \\
2.90 \\
2.90 \\
3.00 \\
3.00 \\
3.00 \\
3.00 \\
3.00 \\
3.00 \\
3.00 \\
3.00 \\
3.00 \\
3.00 \\
2.90 \\
2.90 \\
2.80 \\
2.80 \\
2.70\end{array}$ & $\begin{array}{l}4.45 \\
4.65 \\
4.70 \\
4.70 \\
4.80 \\
4.90 \\
4.80 \\
5.05 \\
5.15 \\
5.45 \\
5.60 \\
5.50\end{array}$ & $\begin{array}{l}3.40 \\
3.25 \\
3.10 \\
2.95 \\
2.80 \\
2.70 \\
9\end{array}$ & $\begin{array}{l}2.10 \\
2.10 \\
2.00 \\
2.00 \\
2.00 \\
1.90 \\
1.90 \\
1.80 \\
1.80 \\
1.80 \\
1.80 \\
1.80 \\
1.70 \\
1.70 \\
1.70 \\
1.70 \\
1.70 \\
1.70\end{array}$ & $\begin{array}{l}1.60 \\
1.60 \\
1.60 \\
1.60 \\
1.60 \\
1.60 \\
1.60 \\
1.60 \\
1.60 \\
1.60 \\
1.60 \\
1.60 \\
1.60 \\
1.60 \\
1.60 \\
1.60 \\
1.60 \\
1.60 \\
1.60 \\
1.60 \\
1.60 \\
1.60 \\
1.60 \\
1.60 \\
1.60 \\
1.60 \\
1.60 \\
1.60 \\
1.60\end{array}$ & \begin{tabular}{|}
1.60 \\
1.60 \\
1.60 \\
1.60 \\
1.60 \\
1.60 \\
1.60 \\
1.60 \\
1.60 \\
1.60 \\
1.60 \\
1.70 \\
1.70 \\
1.70 \\
1.70 \\
1.70
\end{tabular} & $\begin{array}{l}1.90 \\
1.90 \\
1.90 \\
1.90 \\
1.90 \\
1.90\end{array}$ & $\begin{array}{l}2.60 \\
1.90 \\
1.90 \\
1.90 \\
1.90\end{array}$ \\
\hline
\end{tabular}


Bear River at Collinston, Utah.

\begin{tabular}{|c|c|c|c|c|c|c|c|c|c|c|c|c|}
\hline Day. & Jan. & Feb. & Mar. & Apr. & May. & June. & July. & Ang. & Sept. & Oet. & Nov. & Dec. \\
\hline & & & & & & 5.60 & & & & 2.00 & & \\
\hline & 1. 90 & 1.80 & 1. 70 & 3. 30 & 3. 60 & & 3.95 & 2.30 & 1. 80 & 1. 95 & 00 & 2.30 \\
\hline & 1. 90 & 1.80 & 1.70 & 3.10 & 3. 75 & 5.90 & 3.75 & 30 & 1.80 & 1. 95 & 00 & \\
\hline & 1.95 & 1.70 & 1.70 & 3. 00 & 3.75 & 6.05 & 3.55 & 2.30 & 1.80 & 1.95 & 2. 00 & 2. 20 \\
\hline & 1. 90 & 1.70 & 1.70 & 3.00 & 3. 85 & 6.15 & 3.40 & 2.30 & 1. 80 & 1. 95 & 2.05 & \\
\hline & 1. 90 & 1.70 & 1.70 & 3.10 & 3. 95 & 6.30 & 3.20 & 2. 20 & 1. 80 & 1. 90 & 2.05 & \\
\hline & 1.90 & 1.70 & 1.70 & 3. 10 & 4.00 & 6.30 & 3.10 & 2.20 & 1.75 & 1.90 & 2.05 & \\
\hline & 1.90 & 1.70 & 1.70 & 3. 20 & 4.00 & $\begin{array}{l}6.20 \\
6\end{array}$ & 3. 00 & 2.20 & 1.75 & 1. 90 & 2.05 & \\
\hline & 1.90 & 1.70 & 1.70 & $\begin{array}{l}3.30 \\
3.30\end{array}$ & 4. 10 & 6.30 & 2.90 & 2. 10 & 1.80 & 1.90 & 2. 05 & \\
\hline & $\begin{array}{l}1.90 \\
1.90\end{array}$ & $\begin{array}{l}1.70 \\
1.70\end{array}$ & $\begin{array}{l}.70 \\
1.70\end{array}$ & $\begin{array}{l}\text { 3. 30 } \\
\text { 3. } 30\end{array}$ & $\begin{array}{l}4.10 \\
4.00\end{array}$ & $\begin{array}{l}6.40 \\
6.40\end{array}$ & \begin{tabular}{|l|}
2.80 \\
2.70
\end{tabular} & $\begin{array}{l}2.00 \\
\text { 2.00 }\end{array}$ & $\begin{array}{l}1.80 \\
1.80\end{array}$ & $\begin{array}{l}\text { 1. } 90 \\
\text { 1. } 90\end{array}$ & $\begin{array}{l}2.10 \\
2.15\end{array}$ & \\
\hline 12 & 1.90 & 1.70 & 1. 70 & 3. 40 & $\begin{array}{l}\text { 4. } \\
\text { 3. } 95\end{array}$ & $\begin{array}{l}0.40 \\
6.40\end{array}$ & 2.70 & $\begin{array}{l}2.00 \\
1.95\end{array}$ & $\begin{array}{l}1.00 \\
1.80\end{array}$ & $\begin{array}{l}1.90 \\
1.90\end{array}$ & & \\
\hline & & 1. 70 & & 3. 40 & 3. 80 & & & 1.90 & & & 10 & \\
\hline & 2.00 & 1.70 & 1. 70 & 3.50 & 3.75 & 6.55 & 2.55 & 1.85 & 1. 90 & 1. 90 & 2.00 & \\
\hline & 2.00 & 1.70 & 1. 70 & 3.50 & 3. 60 & 6.55 & 2.50 & 1.80 & 1.90 & 1. 90 & 2.00 & 2. 20 \\
\hline & 2.00 & 1.70 & 1.70 & 3. 50 & 3.60 & 6.30 & 2.50 & 1.80 & 1.85 & 1.90 & 2.00 & \\
\hline 17 & 2.10 & 1.70 & 1.70 & 3. 50 & 3. 60 & 6.00 & 2.50 & 1.75 & 1.85 & 1.90 & 2.05 & \\
\hline & 2.10 & 1.70 & 1. 70 & 3. 60 & 3.50 & 5.90 & 2.50 & 1.70 & 1.85 & 1.90 & 2.10 & 2.00 \\
\hline & 2.20 & 1.70 & 1. 70 & $\begin{array}{l}3.35 \\
3.35\end{array}$ & 3. 50 & 5.80 & 2.50 & 1.70 & 1. 80 & 1.90 & 2.10 & \\
\hline & $\begin{array}{l}2.30 \\
2.30\end{array}$ & $\begin{array}{l}1.70 \\
1.70\end{array}$ & $\begin{array}{l}1.70 \\
1.70\end{array}$ & $\begin{array}{l}\text { 3. } 25 \\
3.15\end{array}$ & $\begin{array}{l}3.40 \\
3.30\end{array}$ & \begin{tabular}{|l}
5.70 \\
5.70
\end{tabular} & 2.40 & 1.75 & 1. 80 & $\begin{array}{l}1.90 \\
1.90\end{array}$ & 2.10 & \\
\hline & $\begin{array}{l}2.30 \\
2.20\end{array}$ & $\begin{array}{l}\text { 1. } 70 \\
\text { 1. }\end{array}$ & $\begin{array}{l}1.70 \\
1.70\end{array}$ & $\begin{array}{l}\text { 3.15 } \\
\text { 3.10 }\end{array}$ & $\begin{array}{l}\text { 3. } 40 \\
\text { 3. } 40\end{array}$ & $\begin{array}{l}5.60 \\
5.50\end{array}$ & $\begin{array}{l}2.30 \\
2.20\end{array}$ & $\begin{array}{l}1.80 \\
1.90\end{array}$ & \begin{tabular}{|l}
1.80 \\
1.85
\end{tabular} & $\begin{array}{l}1.90 \\
1.90\end{array}$ & $\begin{array}{l}2.10 \\
\text { 2.15 }\end{array}$ & \\
\hline & 2.10 & 1.70 & 1.90 & 3.10 & 3. 40 & 5.40 & 2. 20 & 1. 90 & 1.90 & 1. 90 & 2.20 & \\
\hline & 2.00 & 1.70 & 2.00 & 3.20 & 3. 65 & 5.35 & 2. 10 & 1.85 & 1.90 & 1.90 & 2. 25 & \\
\hline & 2.00 & 1.70 & 2. 10 & 3. 20 & 3. 90 & 5.20 & 2. 20 & 1.80 & 1.90 & 1.90 & 2. & \\
\hline & 1.70 & 1.70 & 2.20 & 3.10 & 4. 10 & 5.10 & 2.25 & 1.85 & 1.95 & 1.90 & 2.30 & \\
\hline & 1.70 & 1.70 & 2.30 & 3.30 & 445 & 4.80 & 2.30 & 1.85 & 1.95 & 1.90 & 2. 30 & \\
\hline & 1.70 & 1.70 & 2.40 & 3. 40 & 4.65 & 4.80 & 2.25 & 1.90 & 1.95 & 2.00 & 2.30 & 2. 20 \\
\hline & $\begin{array}{l}1.70 \\
1.70\end{array}$ & & $\begin{array}{l}2.70 \\
2.90\end{array}$ & $\begin{array}{l}\begin{array}{l}3.40 \\
3.50\end{array} \\
3.50\end{array}$ & $\begin{array}{l}\text { 4. } 45 \\
\text { 4. } 95\end{array}$ & $\begin{array}{l}\text { 4. } 60 \\
\text { 4. } 40\end{array}$ & $\begin{array}{l}2.20 \\
\text { 2. } 20\end{array}$ & $\begin{array}{l}1.90 \\
1.85\end{array}$ & $\begin{array}{l}2.00 \\
2.00\end{array}$ & $\begin{array}{l}\text { 2. } 00 \\
\text { 2. } 00\end{array}$ & 2.30 & 220 \\
\hline & & & & & 5.20 & & 2.20 & 1. 80 & & 2. 00 & & \\
\hline
\end{tabular}

Logan River at Logan, Utah.

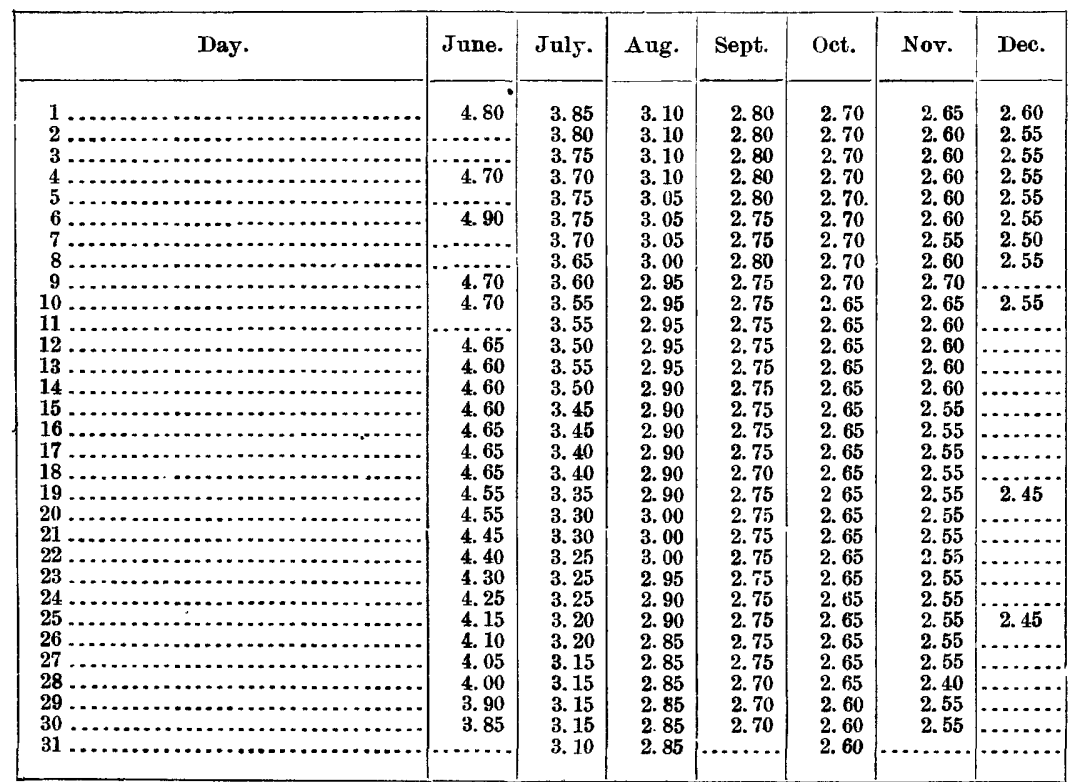


Ogden River at Eden, Utah.

\begin{tabular}{|c|c|c|c|c|c|c|c|c|c|c|c|c|}
\hline Day. & Jan. & Feb. & Mar. & Apr. & May. & June. & July. & Aug. & Sept. & Oct. & Nov. & Dec. \\
\hline & & & & 3.11 & $5.50^{\circ}$ & 10.30 & & & & & 1.62 & \\
\hline & 1.49 & 1.67 & 1.70 & & 5.90 & 10.20 & 2. 25 & 1. 80 & 1. 60 & 1.60 & 1.63 & \\
\hline & 1.54 & 1. 64 & & 3.15 & 5.90 & 10.20 & 2.20 & 1. 80 & 1.60 & 1. 60 & 1. 63 & 1.60 \\
\hline 4 & $\begin{array}{l}1.54 \\
1.52\end{array}$ & $\begin{array}{l}1.62 \\
1.62\end{array}$ & 1.65 & & 6. 20 & $\begin{array}{l}9.70 \\
88.90\end{array}$ & $\begin{array}{l}2.20 \\
2.19\end{array}$ & $\begin{array}{l}1.80 \\
1.79\end{array}$ & $\begin{array}{l}1.60 \\
1\end{array}$ & 1.60 & $\begin{array}{l}1.63 \\
1.63\end{array}$ & \\
\hline & $\begin{array}{l}1.02 \\
1.50\end{array}$ & 162 & 1.00 & 3.60 & 7.50 & $\begin{array}{l}8.90 \\
8.60\end{array}$ & 2.19 & 1. 75 & $\begin{array}{l}1.00 \\
1.61\end{array}$ & $\begin{array}{l}1.00 \\
1.60\end{array}$ & $\begin{array}{l}\text { 1. } 63 \\
\text { 1. }\end{array}$ & \\
\hline & 1.40 & & & & 6. 80 & 8.00 & 2.15 & 1.73 & 1.61 & 1.60 & 1. 63 & \\
\hline & $\begin{array}{l}1.43 \\
1.45\end{array}$ & \begin{tabular}{|l|}
1.67 \\
1.70
\end{tabular} & 1.65 & 4.00 & 6.40 & $\begin{array}{l}\text { 7. } 50 \\
6.90\end{array}$ & 2.00 & 1.70 & 1.62 & 1.60 & 1.63 & \\
\hline & $\begin{array}{l}1.49 \\
1.44\end{array}$ & $\begin{array}{l}1.70 \\
1.70\end{array}$ & & & $\begin{array}{l}6.70 \\
6.30\end{array}$ & 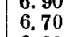 & 1.90 & $\begin{array}{l}1.69 \\
1.65\end{array}$ & $\begin{array}{l}1.64 \\
1.64\end{array}$ & $\begin{array}{l}1.60 \\
1.60\end{array}$ & 2.00 & 1.60 \\
\hline & 1.45 & 1.70 & 1.66 & & 5.80 & 6. 00 & 1.90 & 1.63 & 1.65 & 1.60 & 1. 83 & \\
\hline & 1. 45 & 1.69 & & 3.80 & 6. 20 & 5.50 & 1. 89 & 1.63 & 1.65 & 1.60 & 1. 80 & \\
\hline & 1.45 & & & & & & & & & 1.60 & 1. 78 & \\
\hline & 1. 45 & 1.67 & 1.65 & 8.50 & 5.45 & 4. 90 & 2.00 & 1.60 & 1. 65 & 1. 60 & 1.77 & \\
\hline & 1.46 & 1.64 & & & & 4.70 & 2.10 & 1.60 & 1.64 & 1. 60 & 1. 70 & \\
\hline & 1.48 & & & & 4.80 & 4. 40 & 2. 00 & 1.60 & 1.64 & 1.60 & 1. 71 & 1.65 \\
\hline & $\begin{array}{l}1.54 \\
1.60\end{array}$ & 1.61 & 1.67 & & & $\begin{array}{l}4.10 \\
4.00\end{array}$ & $\begin{array}{l}2.00 \\
1.95\end{array}$ & 60 & $\begin{array}{l}1.63 \\
1.60\end{array}$ & $\begin{array}{l}1.60 \\
1.60\end{array}$ & $\begin{array}{l}1.73 \\
1.77\end{array}$ & \\
\hline & 1.71 & 1.61 & {$[\cdots$} & $\begin{array}{l}0.20 \\
4.80\end{array}$ & 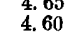 & $\begin{array}{l}\text { 4. } \\
\text { 3. } 80\end{array}$ & $\begin{array}{l}1.90 \\
1.90\end{array}$ & $\begin{array}{l}1.00 \\
1.57\end{array}$ & $\begin{array}{l}1.00 \\
1.60\end{array}$ & $\begin{array}{l}1.00 \\
1.60\end{array}$ & 1.76 & \\
\hline & 1.69 & 1. 62 & & 4.50 & 4.50 & 3. 50 & 1.85 & & 1.60 & 1. 60 & & \\
\hline & 1.80 & 1.62 & 1.85 & 4. 35 & 4.70 & 3. 25 & 1.85 & & 1.60 & & 1.75 & \\
\hline & & 1.6 & & 4. & & 3.15 & 1.85 & & 1.61 & 1.60 & & \\
\hline & & 1.6 & & 4.40 & 30 & 2.90 & 1.85 & 1.63 & 1.63 & 1.60 & & \\
\hline & 1. 80 & 1.63 & 2. 80 & & & 2.70 & 1.85 & 1.63 & 1.62 & 1.60 & & 1.60 \\
\hline & 1.73 & 1.6 & & & & & & & & & & \\
\hline & 1.73 & 1. 64 & 4. 50 & & 9.20 & 2.55 & 1.85 & 1.62 & 1.61 & 30 & & \\
\hline & & 65 & & & 10.00 & & 1.80 & 62 & 1.60 & 11 & & \\
\hline & 1.70 & 1.70 & & & 10.50 & 2.48 & 1.78 & 1.61 & 1.60 & & & \\
\hline & $\begin{array}{l}1.08 \\
1.68\end{array}$ & & $\because 315$ & $\begin{array}{l}5.40 \\
6.10\end{array}$ & $\begin{array}{l}10.50 \\
1120\end{array}$ & $\begin{array}{l}2.38 \\
2.30\end{array}$ & $\begin{array}{l}\text { J. } \\
1.80\end{array}$ & $\begin{array}{l}1.62 \\
1.62\end{array}$ & $\begin{array}{l}1.61 \\
1.61\end{array}$ & $\begin{array}{l}\text { 1. } 62 \\
1.62\end{array}$ & $\begin{array}{l}1.50 \\
1.50\end{array}$ & \\
\hline & 1.67 & & & & 11.00 & & 1. 90 & 1.61 & & 69 & & 2. 60 \\
\hline
\end{tabular}

Weber River at Uinta, Utah.

\begin{tabular}{|c|c|c|c|c|c|c|c|c|c|c|c|c|}
\hline Day. & Jan. & Feb. & Mar. & Apr. & May. & June. & July. & Aug. & Sept. & Oct. & Nov. & Dec. \\
\hline & 1.60 & 1. 70 & 1. 70 & 2. 10 & 3.40 & 8.75 & 2.00 & 1.86 & 1.30 & 1. 40 & 1.60 & \\
\hline 2. & 1.70 & 1. 70 & 1. 70 & 2. 25 & 3. 30 & 8.50 & 1.93 & 1. 90 & 1. 30 & 1. 40 & 1. 70 & \\
\hline & 1. 70 & 1. 70 & 1.70 & 2.35 & 3.35 & 7. 80 & 1.85 & 1. 90 & 1. 30 & 1.40 & 1.80 & \\
\hline & 1.70 & 1.70 & 1. 70 & 2.45 & 3.55 & 7. 30 & 1.78 & 1. 80 & 1. 30 & 1.40 & 1. 80 & \\
\hline & 1. 70 & 1.70 & 1.70 & 2.55 & 3.90 & 7.05 & 1. 71 & 1. 70 & 1.30 & 1.40 & 1. 80 & 1. 70 \\
\hline 6. & 1. 70 & 1.70 & 1. 70 & 2.65 & 4. 55 & 6. 70 & 1.71 & 1. 60 & 1. 30 & 1.40 & 1. 80 & \\
\hline & 1. 70 & 1. 70 & 1. 70 & 2.85 & 4. 45 & 5.75 & 1. 71 & 1.60 & 1. 30 & 1. 40 & 1. 80 & \\
\hline & 1. 70 & 1. 70 & 1. 70 & 2.95 & 4.40 & 5.95 & 1.71 & 1. 50 & 1. 30 & 1. 40 & 1. 90 & 1. 70 \\
\hline 9. & 1. 70 & 1. 70 & 1.70 & 3.00 & 4. 30 & 6. 25 & 1. 64 & 1. 40 & 1. 30 & 1. 40 & 2.00 & \\
\hline 10. & 1. 70 & 1. 70 & 1. 70 & 2. 85 & 2.95 & 5.90 & 1.57 & 1. 30 & 1. 30 & 1. 40 & 2.00 & \\
\hline & 1.70 & 1.70 & 1.70 & 2.65 & 2.85 & 5.50 & 1. 49 & 1. 20 & 1. 30 & 1. 40 & 2.00 & \\
\hline 12 & 1. 70 & 1.70 & 1.70 & 2.50 & 3.55 & 5.15 & 1. 49 & 1. 10 & 1. 30 & 1.40 & 2.00 & 1. 70 \\
\hline 13. & 1.70 & 1.70 & 1.80 & 4. 10 & 3.60 & 4. 75 & 1. 49 & 1. 10 & 1. 30 & 1.40 & 2.00 & \\
\hline 14. & 1. 70 & 1. 70 & 1. 90 & 4. 40 & 3.35 & 4.65 & 1.71 & 1.00 & 1,30 & 1. 40 & 2.00 & 1. 60 \\
\hline 15. & 1. 70 & 1. 70 & 1. 90 & 4.40 & 2. 95 & 4. 40 & 1. 64 & 1. 00 & 1. 30 & 1. 40 & 1. 90 & \\
\hline 16. & 1. 70 & 1.70 & 2.00 & 3.55 & 3.00 & 3. 75 & 1. 49 & 1.00 & 1. 30 & 1. 40 & 1.80 & \\
\hline 17. & 1. 70 & 1.70 & 2.15 & 3. 10 & 2.80 & 3. 19 & 1.49 & 1. 00 & 1. 30 & 1.40 & 1. 70 & \\
\hline 18. & 1. 70 & 1. 70 & 2. 30 & 2.55 & 2.80 & 3.10 & 1. 49 & 1. 00 & 1. 30 & 1.60 & 1. 70 & 1. 60 \\
\hline 19. & 1. 70 & 1.70 & 2. 55 & 2.65 & 2.70 & 3.10 & 1. 93 & 1.00 & 1. 30 & 1.60 & 1. 70 & \\
\hline 20 . & 1. 70 & 1.70 & 2.75 & 2. 70 & 2.70 & 3.01 & 2.00 & 1. 00 & 1. 30 & 1. 60 & 1.70 & \\
\hline & 1. 70 & 1.70 & 2. 90 & 2.80 & 2.85 & 2.84 & 2.00 & 1.00 & 1. 30 & 1.60 & 1. 70 & \\
\hline 22 & 1. 70 & 1. 70 & 3.10 & 2.85 & 2.90 & 2.66 & 2.00 & 1.00 & 1.30 & 1.60 & 1. 70 & \\
\hline & 1.70 & 1. 70 & 3.25 & 2.60 & 2.90 & 2.57 & 2.00 & a1.30 & 1. 30 & 1. 60 & 1. 70 & \\
\hline & 1.70 & 1.70 & 2.95 & 2.60 & 4.85 & 2.48 & 2.00 & 1. 30 & 1. 30 & 1.60 & 1. 70 & 1. 50 \\
\hline & 1.70 & 1. 70 & 2.90 & 2. 60 & 5.70 & 2. 48 & 1.93 & 1. 30 & 1. 30 & 1. 60 & 1.80 & $\ldots . .$. \\
\hline 26. & 1. 70 & 1.70 & 2.75 & 2.75 & 6.50 & 2.66 & 1.93 & 1. 30 & 1. 30 & 1.60 & 1.80 & \\
\hline & 1. 70 & 1.70 & 2. 65 & 2. 95 & 6.95 & 2. 22 & 1. 93 & 1. 30 & 1. 30 & 1.60 & 1. 70 & \\
\hline & 1. 70 & 1.70 & 2.55 & 3. 15 & 7. 95 & 2. 22 & 1. 86 & 1. 30 & 1. 40 & 1.60 & 1. 70 & \\
\hline & 1.70 & 1. 70 & 2.45 & 3.35 & 8.10 & 2.14 & 1.86 & 1. 30 & 1. 40 & 1.60 & 1. 70 & 1. 50 \\
\hline & 1. 70 & ..... & 2.45 & 3.50 & 8.20 & 2.07 & 1.86 & 1. 30 & 1.40 & 1. 60 & 1. 70 & \\
\hline & 1.70 & 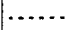 & 2.40 & ..... & 8.80 & +..... & 1.86 & 1. 30 & $\ldots$ & 1.60 & ...... & \\
\hline
\end{tabular}

a New gage put in August 23. 
Provo River at Provo Canyon, Utah.

\begin{tabular}{|c|c|c|c|c|c|c|c|c|c|c|c|c|}
\hline Day. & Jan. & Feb. & Mar. & Apr. & May. & June. & July. & Aug & Sept. & Oct. & Nov. & Dec. \\
\hline 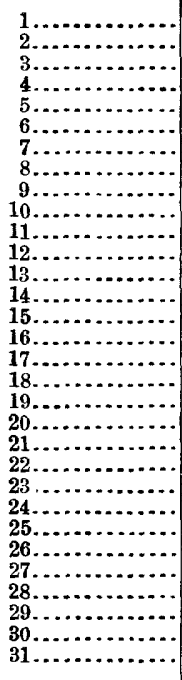 & 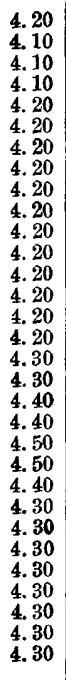 & \begin{tabular}{|l|}
4.30 \\
4.20 \\
4.20 \\
4.20 \\
4.20 \\
4.20 \\
4.20 \\
4.20 \\
4.20 \\
4.20 \\
4.20 \\
4.20 \\
4.30 \\
4.30 \\
4.30 \\
4.20 \\
4.20 \\
4.20 \\
4.20 \\
4.20 \\
4.20 \\
4.20 \\
4.20 \\
4.20 \\
4.20 \\
4.20 \\
4.20 \\
4.20 \\
4.20 \\
$\cdots .$. \\
.2
\end{tabular} & $\begin{array}{l}4.20 \\
4.20 \\
4.20 \\
4.20 \\
4.20 \\
4.20 \\
4.20 \\
4.20 \\
4.20 \\
4.20 \\
4.20 \\
4.20 \\
4.20 \\
4.20 \\
4.30 \\
4.30 \\
4.30 \\
4.40 \\
4.40 \\
4.40 \\
4.50 \\
4.60 \\
4.60 \\
4.60 \\
4.70 \\
4.70 \\
4.80 \\
4.90 \\
4.60 \\
4.60 \\
4.60\end{array}$ & $\begin{array}{l}4.50 \\
4.50 \\
4.50 \\
4.50 \\
4.50 \\
4.50 \\
4.50 \\
4.50 \\
4.40 \\
4.40 \\
4.30 \\
5.30 \\
5.80 \\
5.90 \\
5.80 \\
5.30 \\
4.70 \\
4.70 \\
4.60 \\
4.60 \\
4.60 \\
4.60 \\
4.60 \\
4.60 \\
4.60 \\
4.70 \\
4.70 \\
4.70 \\
4.70 \\
4.80 \\
\cdots \\
\cdots .7\end{array}$ & $\begin{array}{l}4.80 \\
4.80 \\
4.80 \\
4.90 \\
5.00 \\
5.20 \\
5.40 \\
5.40 \\
5.50 \\
5.30 \\
5.30 \\
5.20 \\
5.20 \\
4.90 \\
4.70 \\
4.70 \\
4.70 \\
4.70 \\
4.70 \\
4.70 \\
4.70 \\
4.70 \\
4.70 \\
5.90 \\
5.60 \\
6.00 \\
6.20 \\
6.50 \\
7.00 \\
7.80 \\
8.50\end{array}$ & $\begin{array}{l}7.30 \\
7.30 \\
7.80 \\
7.60 \\
7.30 \\
6.90 \\
6.90 \\
6.90 \\
6.80 \\
6.80 \\
6.60 \\
6.50 \\
6.50 \\
6.20 \\
6.00 \\
5.80 \\
5.60 \\
5.00 \\
4.80 \\
4.60 \\
4.60 \\
4.90 \\
4.80 \\
4.80 \\
4.70 \\
4.60 \\
4.60 \\
4.60 \\
4.60 \\
4.50 \\
\ldots . . . \\
4\end{array}$ & $\begin{array}{l}4.50 \\
4.40 \\
4.30 \\
4.30 \\
4.30 \\
4.30 \\
4.30 \\
4.20 \\
4.20 \\
4.20 \\
4.20 \\
4.20 \\
4.20 \\
4.20 \\
4.30 \\
4.30 \\
4.30 \\
4.30 \\
4.20 \\
4.20 \\
4.30 \\
4.90 \\
4.80 \\
4.70 \\
4.50 \\
4.50 \\
4.50 \\
4.50 \\
4.50 \\
4.50 \\
4.50\end{array}$ & $\begin{array}{l}4.40 \\
4.40 \\
4.40 \\
4.30 \\
4.30 \\
4.30 \\
4.20 \\
4.20 \\
4.20 \\
4.20 \\
4.20 \\
4.20 \\
4.20 \\
4.20 \\
4.20 \\
4.20 \\
4.20 \\
4.20 \\
4.20 \\
4.20 \\
4.20 \\
4.20 \\
4.20 \\
4.20 \\
4.20 \\
4.20 \\
4.20 \\
4.20 \\
4.20 \\
4.20 \\
4.20\end{array}$ & $\begin{array}{l}4.20 \\
4.20 \\
4.20 \\
4.20 \\
4.20 \\
4.20 \\
4.20 \\
4.20 \\
4.20 \\
4.20 \\
4.20 \\
4.20 \\
4.20 \\
4.20 \\
4.20 \\
4.20 \\
4.20 \\
4.20 \\
4.20 \\
4.30 \\
4.30 \\
4.30 \\
4.30 \\
4.30 \\
4.30 \\
4.30 \\
4.30 \\
4.30 \\
\text { 4. } 30 \\
4.30 \\
\text {-. .. }\end{array}$ & $\begin{array}{r}4.30 \\
4.30 \\
4.30 \\
4.30 \\
4.30 \\
4.30 \\
4.30 \\
4.30 \\
4.30 \\
4.30 \\
4.30 \\
4.30 \\
4.30 \\
4.30 \\
4.30 \\
4.30 \\
4.30 \\
4.30 \\
4.30 \\
4.30 \\
4.30 \\
4.30 \\
4.30 \\
4.30 \\
4.30 \\
4.30 \\
4.30 \\
4.30 \\
4.30 \\
4.30 \\
4.30\end{array}$ & $\begin{array}{r}4.30 \\
4.30 \\
4.30 \\
4.30 \\
4.30 \\
4.30 \\
4.30 \\
4.60 \\
4.60 \\
4.60 \\
4.60 \\
4.50 \\
4.50 \\
4.50 \\
4.40 \\
4.40 \\
4.40 \\
4.40 \\
4.40 \\
4.40 \\
4.40 \\
4.50 \\
4.50 \\
4.50 \\
4.50 \\
4.50 \\
4.40 \\
4.40 \\
\ldots \ldots . . \\
\ldots \ldots . .\end{array}$ & 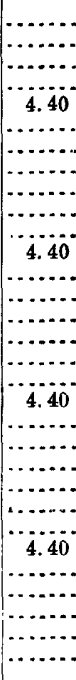 \\
\hline
\end{tabular}

Portneuf River at MoCammon, Idaho.

\begin{tabular}{|c|c|c|c|c|c|c|c|c|c|c|c|c|c|c|}
\hline Day. & Apr. & May. & June. & July. & Day. & Apr. & May. & Tune. & July. & Day. & Apr. & May. & June. & July. \\
\hline $\begin{array}{r}1 . . \\
2 . . \\
3 . . \\
4 \ldots \\
5 . . \\
6 \ldots \\
7 \ldots \\
8 \ldots \\
9 \ldots \\
10 . . \\
11 . .\end{array}$ & $\begin{array}{c}\ldots \\
\ldots \\
\cdots \\
\cdots \\
\cdots \\
\cdots \\
\cdots \\
\\
\end{array}$ & $\begin{array}{l}3.50 \\
3.50 \\
3.50 \\
3.58 \\
3.63 \\
3.83 \\
3.92 \\
3.83 \\
3.92 \\
3.92 \\
\mathbf{3 . 9 2}\end{array}$ & $\begin{array}{l}4.50 \\
4.50 \\
4.46 \\
4.42 \\
4.50 \\
4.42 \\
4.42 \\
4.25 \\
4.25 \\
4.25 \\
4.25\end{array}$ & $\begin{array}{l}3.25 \\
3.08 \\
3.08 \\
3.00 \\
3.00 \\
3.00 \\
2.92 \\
2.92 \\
2.88 \\
2.88 \\
2.88\end{array}$ & $\begin{array}{l}12 . . \\
13 . . \\
14 . . \\
15 . . \\
16 . . \\
17 \ldots \\
18 . . \\
19 . . \\
20 \ldots \\
21 \ldots \\
22 \ldots\end{array}$ & $\begin{array}{l}\ldots \ldots \\
\cdots 3.50 \\
3.67 \\
3.67 \\
3.67 \\
3.50 \\
3.50 \\
3.50 \\
3.50 \\
3.46\end{array}$ & $\begin{array}{l}3.83 \\
\text { 3. } 75 \\
\text { 3. } 75 \\
3.58 \\
\text { 3. } 58 \\
\text { 3.50 } \\
3.50 \\
\text { 3. 50 } \\
3.54 \\
\text { 3.54 } \\
\text { 3.50 }\end{array}$ & $\begin{array}{l}4.17 \\
4.08 \\
4.08 \\
4.00 \\
4.00 \\
4.04 \\
4.00 \\
4.00 \\
3.92 \\
3.83 \\
3.83\end{array}$ & 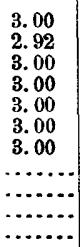 & $\begin{array}{l}23 . . \\
24 \ldots \\
25 \ldots \\
26 . . \\
27 \ldots \\
28 . \\
29 . . \\
30 . \\
31 \ldots\end{array}$ & $\begin{array}{c}3.46 \\
3.50 \\
3.50 \\
3.50 \\
3.46 \\
3.58 \\
3.58 \\
3.50 \\
\ldots \ldots . .\end{array}$ & $\begin{array}{l}3.50 \\
3.83 \\
4.00 \\
4.08 \\
4.17 \\
4.25 \\
4.25 \\
4.29 \\
4.50\end{array}$ & $\begin{array}{c}3.58 \\
3.58 \\
3.54 \\
3.50 \\
3.46 \\
3.42 \\
3.33 \\
3.25 \\
\ldots . \ldots\end{array}$ & \\
\hline
\end{tabular}

Malad River at Taponis, Idaho.

\begin{tabular}{|c|c|c|c|c|c|c|c|c|c|c|c|c|c|c|}
\hline Day. & June. & July. & Ang & Sept. & Day. & June. & July. & Aug. & Sept. & Day & June. & July. & A'ig. & Sept. \\
\hline & \multirow{3}{*}{$\begin{array}{c}9.40 \\
\ldots . .\end{array}$} & & & 1.80 & 12. & 8.00 & & & & & \multirow{2}{*}{\begin{tabular}{|c}
8.60 \\
$\ldots$
\end{tabular}} & & & \\
\hline & & \multirow{2}{*}{$\begin{array}{l}7.20 \\
\ldots \ldots . .\end{array}$} & \multirow{2}{*}{2.30} & 10 & 14. & 8.60 & \multirow[t]{2}{*}{5.60} & \multirow[t]{2}{*}{1.80} & \multirow{2}{*}{1.80} & \multirow{4}{*}{$\begin{array}{l}24 . \\
25 . \\
26 . \\
28 . \\
29 .\end{array}$} & & \multirow{2}{*}{7.67} & & \multirow{2}{*}{1.80} \\
\hline & & & & 1.60 & 15. & 8.80 & & & & & 760 & & & \\
\hline & 9.60 & $\dddot{6} .40$ & $\because 2.2 \dot{0}$ & & 17. & 0.00 & \multirow[t]{2}{*}{6.25} & \multirow{3}{*}{2.00} & & & \multirow[t]{2}{*}{\begin{tabular}{|l|}
7.40 \\
\end{tabular}} & \multirow[t]{2}{*}{$\dddot{8.50}$} & & \multirow{3}{*}{1.90} \\
\hline & & & & 1.70 & 18. & & & & \multirow{2}{*}{1.80} & & & & [....... & \\
\hline 19. & $\begin{array}{r}7.80 \\
\end{array}$ & 6.20 & & & 19. & $\begin{array}{l}9.60 \\
8.80\end{array}$ & 717 & & & ${ }_{31}^{30 .}$ & 7.40 & 9 & & \\
\hline 11 . & & & 2.00 & 1.70 & 22 . & & & & 1.80 & & & & & \\
\hline
\end{tabular}


Snake River at Montgomerys Ferry, Idaho.

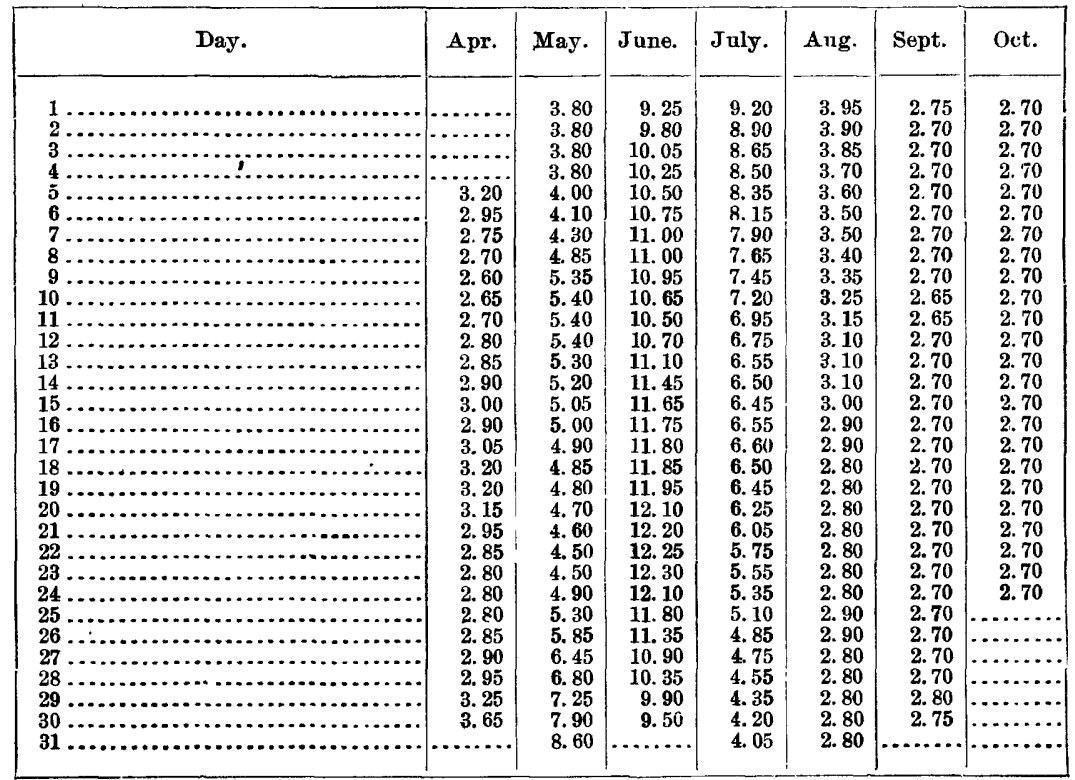

Little Camas Creek at Little Camas Store, Idaho.

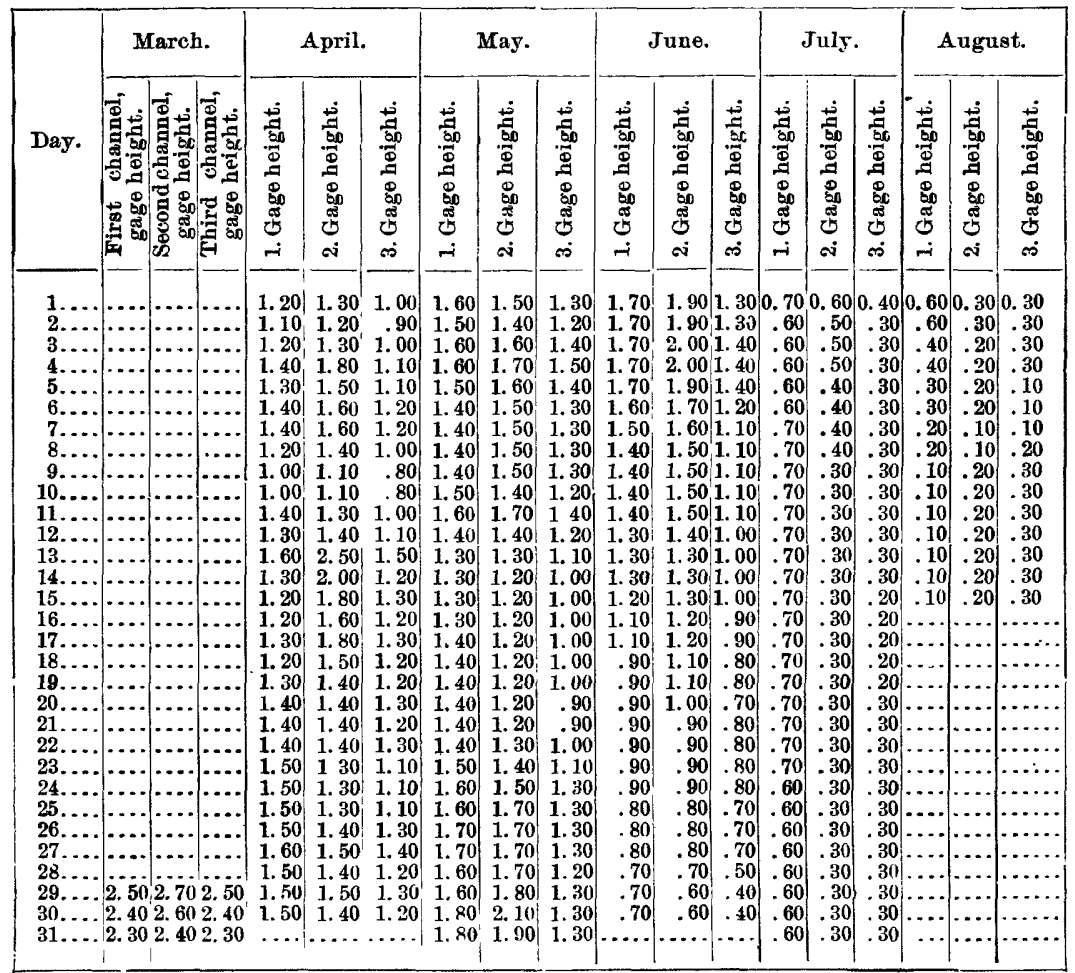


Little Wood River at Taponis, Idaho.

\begin{tabular}{|c|c|c|c|c|c|c|c|c|c|c|c|c|c|c|}
\hline Day. & June. & July. & Aug. & Sept. & Day. & June. & July. & Aug. & Sept. & Day. & June. & July. & Ang. & Sept. \\
\hline 1. & & & & 1.50 & $11 .$. & & & 1.20 & 1. 33 & $22 .$. & & & & 2.58 \\
\hline & 6.90 & & & ........ & 12. & 6.40 & & & 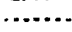 & 23. & 6.40 & & & 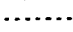 \\
\hline 3 & $\cdots$ & 4.40 & 120 & $\cdots$ & 14.. & 6.40 & 4.30 & 1.20 & 9 17 & $24 .$. & $\ldots$ & 5.67 & .... & 067 \\
\hline 5 & 7.10 & & & 2.08 & $\begin{array}{l}15 . . \\
16 . .\end{array}$ & 6.70 & & 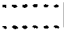 & 2.17 & $26 \ldots$ & 5.20 & 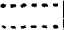 & & 2.67 \\
\hline 7. & ....... & 3.90 & 1.00 & $\cdots$ & 17. & ..... & 4.50 & & & 28 .. & 5.30 & 6.33 & & \\
\hline & & & ...... & 1.33 & 18. & & & 1.20 & 2.33 & 29 . & & & $\cdots$ & 2.75 \\
\hline 9. & 6.40 & $\because 3$ & $\ldots$. & ......... & 19. & 6.90 & $=\cdots$ & $\cdots$ & $\cdots$ & $30 .$. & 4.10 & 17 & $\cdots$ & ....... \\
\hline 10. & & 4.30 & $\cdots$ & & 21 & 6.70 & 5.00 & & & 31. & $\cdots$ & 7.17 & & \\
\hline
\end{tabular}

Bruneau River at Owyhee, Idaho.

\begin{tabular}{|c|c|c|c|c|c|c|c|c|c|c|c|c|c|}
\hline Day. & Jan. & Feb. & Mar. & Apr. & May. & June. & Day. & Jan. & Feb. & Mar. & Apr. & May. & June. \\
\hline $\begin{array}{r}1 \ldots . . \\
2 \ldots \ldots \\
3 \ldots \ldots \\
4 \ldots \ldots \\
5 \ldots \ldots \\
6 \ldots \ldots \\
7 \ldots \ldots \\
8 \ldots \ldots \\
9 \ldots \ldots \\
10 \ldots \ldots \\
11 \ldots \ldots \\
12 \ldots \ldots \\
13 \ldots \ldots \\
14 \ldots \ldots \\
15 \ldots \ldots \\
16 \ldots . .\end{array}$ & $\begin{array}{l}1.65 \\
1.65 \\
1.65 \\
1.65 \\
1.60 \\
1.60 \\
1.60 \\
1.65 \\
1.65 \\
1.65 \\
1.65 \\
1.65 \\
1.65 \\
1.65 \\
1.65 \\
1.65\end{array}$ & $\begin{array}{l}1.75 \\
1.75 \\
1.75 \\
1.75 \\
1.75 \\
1.75 \\
1.75 \\
1.75 \\
1.70 \\
1.70 \\
1.70 \\
1.70 \\
1.70 \\
1.70 \\
1.70 \\
1.70\end{array}$ & $\begin{array}{l}1.75 \\
1.75 \\
1.75 \\
1.75 \\
1.75 \\
1.80 \\
1.80 \\
1.80 \\
1.80 \\
1.80 \\
1.75 \\
1.75 \\
1.75 \\
1.75 \\
1.75 \\
1.75\end{array}$ & $\begin{array}{l}2.45 \\
2.40 \\
2.40 \\
2.35 \\
2.35 \\
2.35 \\
2.40 \\
2.55 \\
2.60 \\
2.65 \\
2.65 \\
2.55 \\
2.40 \\
2.80 \\
3.85 \\
3.60\end{array}$ & $\begin{array}{l}2.90 \\
2.90 \\
2.95 \\
2.90 \\
2.90 \\
3.30 \\
3.25 \\
3.20 \\
3.15 \\
3.00 \\
3.00 \\
3.00 \\
3.15 \\
3.00 \\
2.95 \\
2.95\end{array}$ & $\begin{array}{l}6.40 \\
6.70 \\
6.00 \\
5.90 \\
5.35 \\
5.00 \\
4.95 \\
4.80 \\
4.70 \\
5.00 \\
5.20 \\
4.90 \\
4.65 \\
4.60 \\
4.65 \\
4.60\end{array}$ & $\begin{array}{l}17 \ldots \ldots \\
18 \ldots \ldots \\
19 \ldots \ldots \\
20 \ldots \ldots \\
21 \ldots \ldots \\
22 \ldots \ldots \\
23 \ldots \ldots \\
24 \ldots \ldots \\
25 \ldots \ldots \\
26 \ldots \ldots \\
27 \ldots \ldots \\
28 \ldots \ldots \\
29 \ldots \ldots \\
30 \ldots \ldots \\
31 \ldots \ldots\end{array}$ & $\begin{array}{l}1.65 \\
1.70 \\
1.70 \\
1.75 \\
1.75 \\
1.75 \\
1.75 \\
1.75 \\
1.75 \\
1.75 \\
1.75 \\
1.75 \\
1.75 \\
1.75 \\
1.75\end{array}$ & $\begin{array}{l}1.60 \\
1.60 \\
1.65 \\
1.65 \\
1.70 \\
1.70 \\
1.70 \\
1.70 \\
1.70 \\
1.70 \\
1.70 \\
1.70 \\
1.70 \\
\ldots \ldots . . \\
\ldots \ldots .\end{array}$ & $\begin{array}{l}1.75 \\
1.75 \\
1.75 \\
1.80 \\
1.80 \\
1.95 \\
2.40 \\
2.45 \\
2.75 \\
3.20 \\
3.35 \\
3.55 \\
3.35 \\
3.00 \\
2.85\end{array}$ & $\begin{array}{l}3.20 \\
3.00 \\
2.90 \\
2.85 \\
2.75 \\
2.75 \\
2.90 \\
2.90 \\
2.90 \\
2.90 \\
3.00 \\
3.15 \\
3.00 \\
2.90 \\
\ldots \ldots . .\end{array}$ & $\begin{array}{l}2.80 \\
2.70 \\
2.65 \\
2.60 \\
2.60 \\
2.60 \\
2.60 \\
2.90 \\
2.95 \\
3.20 \\
3.80 \\
4.00 \\
4.50 \\
5.30 \\
6.00\end{array}$ & $\begin{array}{l}4.70 \\
4.60 \\
4.60 \\
4.30 \\
4.20 \\
4.00 \\
3.80 \\
3.70 \\
3.60 \\
3.45 \\
3.35 \\
3.30 \\
3.20 \\
\text { 3. } 15 \\
\ldots . . .\end{array}$ \\
\hline
\end{tabular}

Boise River at Boise, Idaho.

\begin{tabular}{|c|c|c|c|c|c|c|c|c|c|c|c|c|}
\hline Day. & Jau. & Feb. & Mar. & Apr. & May. & June. & July. & Aug. & Sept. & Oet. & Nov. & Dec. \\
\hline 1. & 1.80 & 2. 70 & 2. 60 & 4.40 & 5.30 & 9. 00 & 6. 30 & 3.30 & 2.20 & 1. 70 & 1.90 & 1.20 \\
\hline 2. & 2. 00 & 2. 40 & 2.40 & 3. 90 & 5.00 & 9.00 & 6. 80 & 3.00 & 2.20 & 1. 80 & 2.00 & 1.10 \\
\hline 3. & 1.40 & 2.40 & 2.40 & 3. 80 & 5.30 & 9.60 & 700 & 3.30 & 2.00 & 1.60 & 1. 90 & 1.10 \\
\hline $4 .$. & 1.40 & 2.00 & 2.30 & 3. 80 & 6.00 & 9.30 & 7.00 & 2.90 & 1.90 & 1. 60 & 1.90 & 1.40 \\
\hline 5. & 1. 40 & 2.00 & 2.40 & 3.90 & 6.00 & 9.00 & 6. 80 & 2. 80 & 1. 80 & 1.70 & 1. 70 & 2.00 \\
\hline 6. & 1. 40 & 1.80 & 2.00 & 3. 90 & 5.60 & 8. 70 & 6. 70 & 2.80 & 1.80 & 1. 60 & 1. 70 & 2.40 \\
\hline & 1. 40 & 1. 80 & 2.00 & 4. 60 & 5.70 & 8.70 & 6.60 & 2.70 & 1. 80 & 1. 80 & 1. 50 & 2. 40 \\
\hline 8 & 1.80 & 2.00 & 2.20 & 4. 80 & 5.80 & 8.80 & 6.50 & 2.70 & 1.70 & 1. 70 & 1.90 & 2.60 \\
\hline 9. & 1. 80 & 2.00 & 2. 40 & 4.40 & 5.60 & 8.60 & 6.30 & 2.70 & 1. 70 & 1.60 & 2.60 & 2.60 \\
\hline 10. & 1.80 & 2.10 & 2.20 & 5. 00 & 5. 40 & 8.80 & 6.20 & 2.60 & 1. 70 & 1. 60 & 2.30 & 2.40 \\
\hline $11 .$. & 1.80 & 1.90 & 2. 30 & 4.50 & 5.50 & 8.50 & 6.10 & 2.60 & 1.80 & 1.70 & 2. 00 & 2.00 \\
\hline -12... & 2. 00 & 2.00 & 2. 30 & 4.50 & 5. 40 & 8.50 & 6.10 & 2.40 & 1.80 & 1.60 & 1.90 & 2.60 \\
\hline 13. & 1. 80 & 2.00 & 2.40 & 5.70 & 5.20 & 9.30 & 5. 90 & 2.30 & 1. 70 & 1. 60 & 1. 90 & 2. 70 \\
\hline 14 & 1.40 & 1.90 & 2.40 & 6. 70 & 5. 00 & 10.00 & 5.80 & 2.20 & 1.80 & 1. 60 & 1. 90 & 2. 80 \\
\hline 15. & 1. 40 & 2.00 & 2.40 & 5.80 & 5.00 & 9.60 & 5. 60 & 2. 20 & 1.80 & 1.70 & 2.00 & 2.50 \\
\hline 16. & 1. 20 & 2.00 & 2.40 & 5.50 & 5.00 & 9.60 & 5.40 & 2.20 & 1.80 & 1. 60 & 1.90 & 2. 50 \\
\hline 17. & 1.90 & 1.90 & 2.30 & 5.40 & 4.90 & 9.60 & 5.00 & 2.20 & 1.70 & 1. 60 & 1. 90 & 2.40 \\
\hline 18. & 2. 30 & 1.90 & 2.40 & 5. 00 & 4.80 & 9.50 & 4.80 & 2.00 & 1. 70 & 1. 70 & 2.00 & 2.40 \\
\hline 19. & 2.40 & 2.00 & 2.40 & 4. 80 & 4. 70 & 9.40 & 4. 60 & 2.00 & 1.60 & 1. 70 & 2. 60 & 2.20 \\
\hline & 2.50 & 2.00 & 2. 70 & 4.50 & 4.70 & 9.00 & 4.00 & 1. 90 & 1.70 & 1. 60 & 2.80 & 2.20 \\
\hline 21. & 3.00 & 2. 00 & 3.50 & 4.40 & 4. 60 & 9.00 & 3. 60 & 1. 90 & 1.70 & 1.60 & 2. 40 & 2. 00 \\
\hline 22. & 3.00 & 2.10 & 3. 90 & 5.00 & 5.00 & 8.50 & 3.00 & 1.80 & 1.60 & 1. 70 & 2.70 & 2.00 \\
\hline 23. & 3.00 & 2. 10 & 4. 00 & 5. 10 & 5.80 & 8.00 & 3. 60 & 1.90 & 1.70 & 1.70 & 2.70 & 1. 90 \\
\hline & 2.40 & 2. 10 & 4.60 & 5. 10 & 6.70 & 7.50 & 4. 00 & 1. 90 & 1.90 & 1. 70 & 2.90 & 1.90 \\
\hline & 2.30 & 2.20 & 4.60 & 5.40 & 7. 00 & 7. 30 & 4. 00 & 1.80 & 2.00 & 1. 60 & 2.60 & 1.80 \\
\hline 26 & 2.60 & 2.20 & 5.60 & 5.80 & 7. 40 & 7. 30 & 3.40 & 1.90 & $\ldots .$. & 1. 60 & 2.40 & 1.80 \\
\hline & 2.80 & 2. 30 & 6.10 & 5. 30 & 7.40 & 9.00 & 3. 20 & 1.80 & 1.90 & 1.80 & 2.00 & 2. 00 \\
\hline & 2. 90 & 2. 90 & 5.70 & 5. 20 & 8.00 & 6.80 & 3.00 & 1.90 & 1.90 & 1.80 & 1. 60 & 2.00 \\
\hline & 2.80 & 3.00 & 5. 50 & 5.30 & 810 & 6.40 & 3.40 & 1.90 & 1.80 & 1.80 & 1. 40 & 2.10 \\
\hline & 2.80 & $\ldots \ldots$ & 4.60 & 5.10 & 8.60 & 6.40 & 3.40 & 2.00 & 1. 70 & 1.60 & 1. 40 & 2. 10 \\
\hline & 2.70 & $\ldots . .$. & 4. 50 & $\ldots . .$. & 9.50 & ...... & 3. 30 & 2.20 & $\ldots . .$. & 1.90 & $\ldots . .$. & 2.10 \\
\hline
\end{tabular}

IRR $11-6$ 
Boise River at Caldwell, Idaho.

\begin{tabular}{|c|c|c|c|c|c|c|c|}
\hline Day. & Mar. & Apr. & May. & June. & July. & Aug. & Sept. \\
\hline 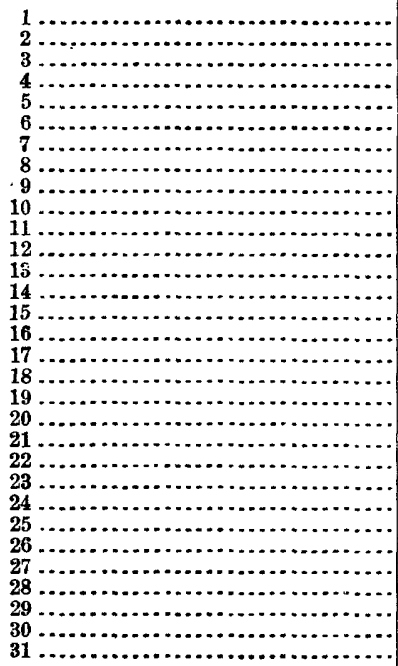 & $\begin{array}{l}\cdots \ldots \ldots \\
4.40 \\
4.40 \\
4.30 \\
4.30 \\
4.30 \\
4.30 \\
4.35 \\
4.40 \\
4.40 \\
4.40 \\
4.50 \\
4.45 \\
4.45 \\
4.50 \\
4.55 \\
4.60 \\
4.85 \\
5.40 \\
5.70 \\
5.75 \\
6.00 \\
7.10 \\
7.10 \\
7.70 \\
7.30 \\
6.70 \\
6.35 \\
5.90\end{array}$ & $\begin{array}{l}5.75 \\
5.60 \\
5.55 \\
5.50 \\
5.60 \\
5.80 \\
5.90 \\
6.00 \\
6.00 \\
5.90 \\
5.80 \\
5.90 \\
6.30 \\
8.00 \\
7.40 \\
6.90 \\
6.60 \\
6.50 \\
6.20 \\
6.20 \\
6.15 \\
6.10 \\
6.15 \\
6.30 \\
6.60 \\
6.80 \\
6.70 \\
6.60 \\
6.50 \\
6.55 \\
\ldots . .7\end{array}$ & $\begin{array}{r}6.40 \\
6.50 \\
6.40 \\
7.00 \\
7.60 \\
7.40 \\
7.10 \\
7.00 \\
7.00 \\
6.90 \\
7.00 \\
6.80 \\
6.60 \\
6.50 \\
6.40 \\
6.30 \\
6.40 \\
6.20 \\
6.10 \\
6.00 \\
6.00 \\
6.10 \\
6.80 \\
7.60 \\
8.00 \\
8.50 \\
8.60 \\
8.90 \\
9.10 \\
10.00 \\
11.00\end{array}$ & $\begin{array}{r}11.00 \\
11.00 \\
11.30 \\
11.00 \\
10.00 \\
9.90 \\
9.50 \\
9.20 \\
9.90 \\
10.20 \\
9.80 \\
9.60 \\
9.80 \\
10.00 \\
9.90 \\
10.00 \\
10.00 \\
10.30 \\
10.00 \\
9.80 \\
9.40 \\
9.00 \\
8.60 \\
8.40 \\
8.30 \\
8.10 \\
8.00 \\
8.20 \\
8.10 \\
8.00 \\
\ldots \ldots . .\end{array}$ & $\begin{array}{l}8.20 \\
7.80 \\
7.70 \\
7.60 \\
7.40 \\
7.40 \\
7.30 \\
7.20 \\
7.00 \\
6.95 \\
6.80 \\
6.70 \\
6.75 \\
6.60 \\
6.50 \\
6.30 \\
6.10 \\
5.90 \\
5.70 \\
5.40 \\
5.30 \\
5.20 \\
5.10 \\
5.00 \\
4.90 \\
4.80 \\
4.70 \\
4.70 \\
4.60 \\
4.50 \\
4.50\end{array}$ & $\begin{array}{l}4.40 \\
4.40 \\
4.30 \\
4.25 \\
4.20 \\
4.20 \\
4.15 \\
4.10 \\
4.10 \\
4.00 \\
3.90 \\
3.90 \\
3.85 \\
3.80 \\
3.70 \\
3.70 \\
3.65 \\
3.60 \\
3.60 \\
3.55 \\
3.50 \\
3.50 \\
3.50 \\
3.45 \\
3.40 \\
3.35 \\
3.30 \\
3.25 \\
3.20 \\
3.20 \\
3.30\end{array}$ & $\begin{array}{r}3.65 \\
3.60 \\
3.60 \\
3.55 \\
3.50 \\
3.50 \\
3.45 \\
3.45 \\
3.45 \\
3.45 \\
3.45 \\
3.45 \\
3.50 \\
3.50 \\
3.45 \\
3.40 \\
3.40 \\
3.40 \\
3.45 \\
3.40 \\
3.45 \\
3.45 \\
3.50 \\
3.65 \\
3.70 \\
3.65 \\
3.60 \\
3.60 \\
3.55 \\
3.50 \\
\ldots . . .5\end{array}$ \\
\hline
\end{tabular}

Owyhee River at Nyssa, Oregon.

\begin{tabular}{|c|c|c|c|c|c|c|c|c|c|c|}
\hline Day. & Jan. & Feb. & Apr. & May. & June. & July. & Aug. & Sept. & Oet. & Nov. \\
\hline 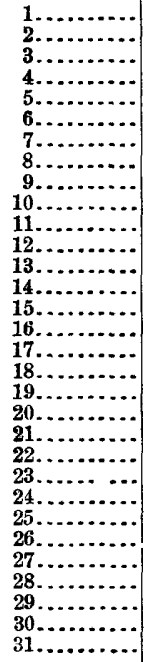 & $\begin{array}{l}1.30 \\
1.30 \\
1.30 \\
1.30 \\
1.40 \\
1.40 \\
1.40 \\
1.40 \\
1.40 \\
1.40 \\
1.40 \\
1.40 \\
1.40 \\
1.55 \\
1.75 \\
2.50 \\
5.65 \\
3.80 \\
3.35 \\
3.05 \\
2.75 \\
2.55 \\
2.45 \\
2.40 \\
2.35 \\
2.05 \\
1.85 \\
1.80 \\
1.75 \\
1.70 \\
1.60\end{array}$ & $\begin{array}{r}1.60 \\
1.50 \\
1.50 \\
1.40 \\
1.40 \\
1.50 \\
1.50 \\
1.50 \\
1.40 \\
1.40 \\
1.40 \\
1.40 \\
1.35 \\
1.30 \\
1.30 \\
1.30 \\
1.30 \\
1.30 \\
1.30 \\
1.40 \\
1.40 \\
1.40 \\
1.50 \\
1.50 \\
1.55 \\
1.60 \\
1.60 \\
1.60 \\
1.60 \\
\ldots \ldots . .2 \\
\cdots \ldots . .\end{array}$ & 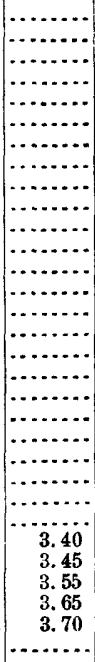 & $\begin{array}{l}\text { 3. } 65 \\
3.60 \\
\text { 3. } 85 \\
4.05 \\
4.35 \\
4.45 \\
4.65 \\
4.80 \\
4.90 \\
4.80 \\
4.80 \\
4.70 \\
4.65 \\
4.60 \\
4.55 \\
4.50 \\
4.40 \\
4.40 \\
4.55 \\
4.60 \\
4.65 \\
4.75 \\
4.85 \\
5.05 \\
5.25 \\
5.45 \\
5.65 \\
5.85 \\
5.95 \\
6.05 \\
6.00\end{array}$ & $\begin{array}{l}5.75 \\
5.45 \\
5.00 \\
4.70 \\
4.55 \\
4.45 \\
4.35 \\
4.30 \\
4.25 \\
4.15 \\
4.15 \\
4.05 \\
3.95 \\
3.55 \\
3.45 \\
3.35 \\
3.30 \\
3.25 \\
3.15 \\
3.05 \\
2.95 \\
2.90 \\
2.85 \\
2.80 \\
2.75 \\
2.70 \\
2.65 \\
2.45 \\
2.40 \\
2.35 \\
\ldots \ldots . . .\end{array}$ & $\begin{array}{l}2.25 \\
2.15 \\
2.05 \\
1.95 \\
1.90 \\
1.85 \\
1.80 \\
1.75 \\
1.70 \\
1.65 \\
1.60 \\
1.50 \\
1.45 \\
1.40 \\
1.40 \\
1.35 \\
1.30 \\
1.25 \\
1.20 \\
1.20 \\
1.20 \\
1.10 \\
1.10 \\
1.10 \\
1.00 \\
1.20 \\
1.20 \\
1.15 \\
1.10 \\
1.10 \\
1.10\end{array}$ & $\begin{array}{l}1.05 \\
1.00 \\
1.00 \\
1.00 \\
1.00 \\
1.00 \\
1.00 \\
1.00 \\
.95 \\
.95 \\
.95 \\
.95 \\
.95 \\
.95 \\
.95 \\
.90 \\
.90 \\
.90 \\
.90 \\
.90 \\
.90 \\
.90 \\
1.00 \\
1.00 \\
1.10 \\
1.10 \\
1.20 \\
1.20 \\
1.30 \\
1.40 \\
1.40\end{array}$ & $\begin{array}{r}1.30 \\
1.30 \\
1.30 \\
1.20 \\
1.20 \\
1.10 \\
1.10 \\
1.00 \\
1.00 \\
.90 \\
.90 \\
.90 \\
.95 \\
.95 \\
1.00 \\
1.00 \\
1.00 \\
1.00 \\
1.00 \\
1.00 \\
1.00 \\
1.00 \\
.95 \\
1.95 \\
1.00 \\
1.00 \\
1.00 \\
1.00 \\
1.00 \\
1.00 \\
\ldots \ldots . . .\end{array}$ & 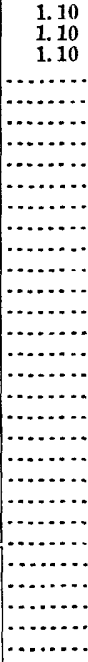 & 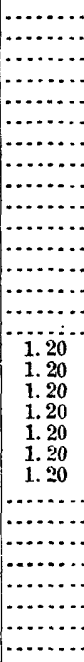 \\
\hline
\end{tabular}


Malheur River at Vale, Oregon.

\begin{tabular}{|c|c|c|c|c|c|c|c|c|}
\hline Day. & $\operatorname{Jan} \cdot \boldsymbol{a}$ & Feb. & Apr. & May. & June. & July. & Aug. & Sept. \\
\hline 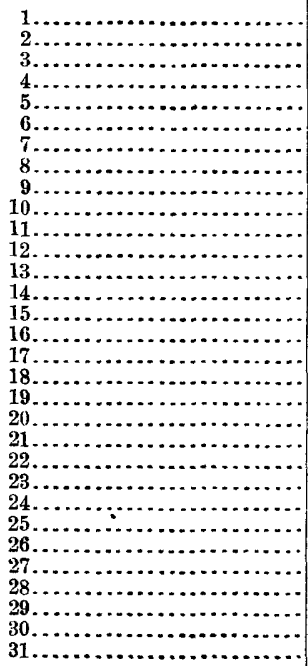 & $\begin{array}{r}1.70 \\
1.75 \\
1.80 \\
1.90 \\
1.90 \\
1.90 \\
1.85 \\
1.90 \\
1.85 \\
1.85 \\
1.70 \\
1.70 \\
1.70 \\
1.70 \\
1.70 \\
1.75 \\
5.00 \\
\ldots \ldots \ldots \\
\ldots \ldots \ldots \\
\ldots \ldots \ldots \\
\ldots \ldots \ldots \\
\ldots \ldots \ldots \\
\ldots 3.20 \\
3.10 \\
2.90 \\
2.75 \\
2.60 \\
2.55 \\
2.65\end{array}$ & $\begin{array}{l}2.55 \\
2.65 \\
2.70 \\
2.60 \\
2.60 \\
2.50 \\
2.50 \\
2.40 \\
2.35 \\
2.25 \\
2.20 \\
2.10 \\
2.00 \\
2.00 \\
2.00 \\
2.00 \\
2.00 \\
2.05 \\
2.10 \\
2.10 \\
2.00 \\
1.95 \\
2.05 \\
2.20 \\
2.30 \\
2.15 \\
2.20 \\
2.30 \\
2.95 \\
\ldots \ldots . . \\
\ldots \ldots .\end{array}$ & 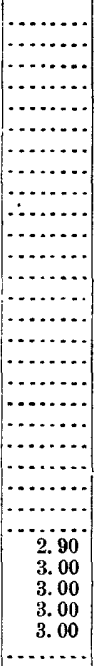 & $\begin{array}{l}3.00 \\
3.00 \\
3.05 \\
3.10 \\
3.85 \\
4.90 \\
4.65 \\
4.05 \\
3.85 \\
3.80 \\
3.70 \\
3.70 \\
3.75 \\
3.65 \\
3.45 \\
3.30 \\
3.30 \\
3.30 \\
3.30 \\
3.20 \\
3.15 \\
3.10 \\
3.10 \\
3.25 \\
3.55 \\
3.75 \\
3.90 \\
4.00 \\
4.10 \\
4.20 \\
4.20\end{array}$ & $\begin{array}{l}4.30 \\
4.35 \\
4.50 \\
4.50 \\
4.45 \\
4.30 \\
4.25 \\
4.25 \\
4.10 \\
4.05 \\
3.90 \\
3.75 \\
3.70 \\
3.60 \\
3.50 \\
3.50 \\
3.50 \\
3.40 \\
3.35 \\
3.40 \\
3.35 \\
3.30 \\
3.25 \\
3.15 \\
3.05 \\
3.40 \\
3.35 \\
2.75 \\
2.65 \\
2.55 \\
\ldots . . . \\
4\end{array}$ & $\begin{array}{l}2.45 \\
2.35 \\
2.30 \\
2.25 \\
2.15 \\
2.10 \\
2.10 \\
2.05 \\
1.95 \\
1.90 \\
1.85 \\
1.80 \\
1.85 \\
1.90 \\
1.95 \\
1.90 \\
1.90 \\
1.65 \\
1.60 \\
1.50 \\
1.50 \\
1.45 \\
1.40 \\
1.40 \\
1.30 \\
1.30 \\
1.25 \\
1.20 \\
1.15 \\
1.10 \\
1.00\end{array}$ & $\begin{array}{r}1.00 \\
.95 \\
.90 \\
.90 \\
.90 \\
.85 \\
.75 \\
.70 \\
.70 \\
.71 \\
.72 \\
.72 \\
.71 \\
.71 \\
.71 \\
.70 \\
.70 \\
.70 \\
.70 \\
.70 \\
.70 \\
.70 \\
.70 \\
.70 \\
.69 \\
.68 \\
.68 \\
.70 \\
.70 \\
.70 \\
2.20\end{array}$ & $\begin{array}{l}1.60 \\
1.45 \\
1.20 \\
1.10 \\
1.10 \\
1.10 \\
1.10 \\
1.10 \\
1.10 \\
1.10 \\
1.10 \\
1.10 \\
1.10 \\
1.10 \\
1.10 \\
1.10 \\
1.10 \\
1.10 \\
1.10 \\
1.15 \\
1.20 \\
1.20 \\
1.20 \\
1.20 \\
1.20 \\
1.20 \\
1.25 \\
1.25 \\
1.25 \\
1.25 \\
\cdots . .2 . .\end{array}$ \\
\hline
\end{tabular}

a January 16 to 25 gage broken; no readings.

Payette River at Payette, Idaho.

\begin{tabular}{|c|c|c|c|c|c|c|c|c|c|c|c|}
\hline Day. & May. & June. & July. & Aug. & Sept. & Day. & May. & June. & July. & Ang. & Sept. \\
\hline 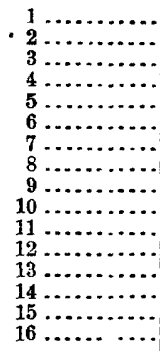 & 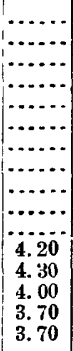 & $\begin{array}{l}8.20 \\
8.30 \\
9.10 \\
\mathbf{9 . 3 0} \\
\mathbf{9 . 1 0} \\
\mathbf{9 . 2 0} \\
\mathbf{8 . 6 0} \\
8.40 \\
8.70 \\
5.90 \\
\mathbf{8 . 5 0} \\
8.40 \\
\mathbf{8 . 3 0} \\
8.70 \\
\mathbf{8 . 8 0} \\
\mathbf{8 . 8 0}\end{array}$ & $\begin{array}{l}7.50 \\
7.40 \\
7.20 \\
6.80 \\
6.80 \\
6.50 \\
6.40 \\
6.20 \\
6.00 \\
5.70 \\
5.70 \\
5.50 \\
6.20 \\
5.90 \\
5.50 \\
5.30\end{array}$ & $\begin{array}{l}2.20 \\
2.20 \\
2.10 \\
2.10 \\
2.10 \\
2.00 \\
2.00 \\
1.90 \\
1.90 \\
1.80 \\
1.80 \\
1.80 \\
1.80 \\
1.70 \\
1.70 \\
1.70\end{array}$ & $\begin{array}{l}1.90 \\
1.80 \\
1.70 \\
1.40 \\
1.30 \\
1.30 \\
1.20 \\
1.20 \\
1.10 \\
1.10 \\
1.10 \\
1.10 \\
1.10 \\
1.10 \\
1.10 \\
1.10\end{array}$ & 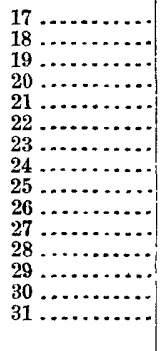 & $\begin{array}{l}3.70 \\
3.60 \\
3.60 \\
3.30 \\
3.40 \\
4.20 \\
5.40 \\
5.40 \\
5.60 \\
5.90 \\
6.10 \\
6.50 \\
6.90 \\
7.20 \\
7.70\end{array}$ & $\begin{array}{l}8.90 \\
9.20 \\
9.20 \\
9.10 \\
9.00 \\
8.80 \\
7.60 \\
7.90 \\
7.50 \\
7.40 \\
7.40 \\
7.20 \\
7.40 \\
7.50 \\
\cdots . . .\end{array}$ & $\begin{array}{l}4.90 \\
4.30 \\
3.90 \\
3.70 \\
3.50 \\
3.30 \\
3.10 \\
2.90 \\
2.80 \\
2.70 \\
2.60 \\
2.50 \\
2.50 \\
2.40 \\
2.30\end{array}$ & $\begin{array}{l}1.60 \\
1.60 \\
1.50 \\
1.50 \\
1.40 \\
1.40 \\
1.40 \\
1.30 \\
1.30 \\
1.20 \\
1.20 \\
1.10 \\
1.10 \\
1.20 \\
1.30\end{array}$ & $\begin{array}{r}1.10 \\
1.10 \\
1.00 \\
1.00 \\
1.00 \\
1.00 \\
1.10 \\
1.10 \\
1.10 \\
1.10 \\
1.00 \\
.90 \\
.90 \\
.90 \\
\ldots . .2\end{array}$ \\
\hline
\end{tabular}

Yakima River at Kiona, Washington.

\begin{tabular}{|c|c|c|c|c|c|c|c|c|c|c|c|c|c|}
\hline Day. & $\mathbf{J} \mathbf{a n}$. & Aug. & Sept. & Oct. & Nov. & Dec. & Day. & Jan. & Aug. & Sept. & Oet. & Nov. & Dec. \\
\hline $\begin{array}{r}1 \ldots . \\
2 \ldots \ldots \\
3 \ldots \ldots \\
4 \ldots \\
5 \ldots \\
6 \ldots . \\
7 \ldots \ldots \\
8 \ldots \ldots \\
9 \ldots \ldots \\
10 \ldots \ldots \\
11 \ldots \ldots \\
12 \ldots \ldots \\
13 \ldots \ldots \\
14 \ldots \ldots \\
15 \ldots \ldots \\
16 \ldots \ldots\end{array}$ & $\begin{array}{l}5.30 \\
5.35 \\
5.20 \\
5.10 \\
5.35 \\
5.40 \\
5.55 \\
5.90 \\
6.70 \\
8.20 \\
8.80 \\
8.80 \\
8.00 \\
7.60 \\
7.15 \\
\ldots . \ldots\end{array}$ & $\begin{array}{l}7.10 \\
6.15 \\
6.10 \\
6.05 \\
6.00 \\
5.90 \\
5.80 \\
5.70 \\
5.60 \\
5.60 \\
5.50 \\
5.35 \\
5.30 \\
5.20 \\
5.15\end{array}$ & $\begin{array}{l}4.73 \\
4.73 \\
4.70 \\
4.70 \\
4.70 \\
4.68 \\
4.65 \\
4.65 \\
4.63 \\
4.55 \\
4.55 \\
4.50 \\
4.50 \\
4.48 \\
4.50 \\
4.50\end{array}$ & $\begin{array}{l}4.08 \\
4.05 \\
4.05 \\
4.10 \\
4.10 \\
4.05 \\
4.08 \\
4.10 \\
4.08 \\
4.05 \\
4.08 \\
4.04 \\
4.00 \\
4.00 \\
4.01 \\
3.95\end{array}$ & $\begin{array}{r}4.24 \\
4.35 \\
4.40 \\
4.46 \\
4.47 \\
4.51 \\
4.52 \\
4.52 \\
4.56 \\
5.80 \\
5.80 \\
5.72 \\
5.60 \\
5.60 \\
8.68 \\
11.91\end{array}$ & \begin{tabular}{r}
5.95 \\
6.10 \\
6.50 \\
7.10 \\
8.90 \\
9.80 \\
11.45 \\
11.55 \\
\hdashline 11.30 \\
10.80 \\
10.60 \\
10.65 \\
10.70 \\
10.07 \\
9.50
\end{tabular} & $\begin{array}{l}17 . \\
18 . \\
19 . \\
20 . \\
21 . \\
22 . \\
23 . \\
24 . \\
25 . \\
26 . \\
27 . \\
28 . \\
29 . \\
30 . \\
31 .\end{array}$ & \begin{tabular}{c}
$\ldots \ldots$ \\
$\cdots \ldots$ \\
\hdashline$\ldots .35$ \\
\hdashline 6.15 \\
6.05 \\
\hdashline$\ldots \ldots$ \\
\hdashline$\ldots \ldots$ \\
$\cdots \ldots \ldots$
\end{tabular} & $\begin{array}{l}5.10 \\
5.05 \\
5.00 \\
4.95 \\
4.90 \\
4.85 \\
4.80 \\
4.75 \\
4.75 \\
4.70 \\
4.70 \\
4.70 \\
4.70 \\
4.70 \\
4.70\end{array}$ & \begin{tabular}{|c|}
4.48 \\
4.50 \\
4.55 \\
4.45 \\
4.35 \\
4.30 \\
4.30 \\
4.25 \\
4.20 \\
4.20 \\
4.20 \\
4.18 \\
4.15 \\
4.13 \\
$\cdots \ldots .$.
\end{tabular} & $\begin{array}{l}4.00 \\
3.96 \\
\text { 3. } 94 \\
3.93 \\
3.92 \\
3.89 \\
3.87 \\
\text { 3. } 87 \\
\text { 3. 94 } \\
3.91 \\
\text { 3. } 93 \\
\text { 3. } 88 \\
\text { 3. } 94 \\
\text { a } \\
\text { 3. } 94\end{array}$ & $\begin{array}{r}15.18 \\
16.07 \\
13.35 \\
10.91 \\
9.50 \\
9.20 \\
8.30 \\
7.93 \\
7.62 \\
7.283 \\
6.65 \\
5.93 \\
5.95 \\
5.95 \\
\ldots \ldots\end{array}$ & $\begin{array}{l}9.20 \\
8.70 \\
8.52 \\
8.43 \\
8.31 \\
8.30 \\
8.21 \\
8.03 \\
7.90 \\
7.70 \\
7.55 \\
7.45 \\
7.35 \\
7.15 \\
7.05\end{array}$ \\
\hline
\end{tabular}


Weiser River at Weiser, Idaho.

\begin{tabular}{|c|c|c|c|c|c|c|c|c|c|c|}
\hline Day. & Jan. & Feb. & Mar. & Apr. & May. & June. & July. & Aug. & Sept. & Oet. \\
\hline & 2.30 & 4.00 & 3. 80 & 4. 30 & 4. 10 & 7,10 & 4. 90 & 2.00 & 1,80 & 1.80 \\
\hline 2. & 2.30 & 3. 70 & $\begin{array}{l}0.00 \\
3.50\end{array}$ & 4.00 & 5.20 & 7.10 & 4. 70 & 2.00 & $\begin{array}{l}1.80 \\
1.80\end{array}$ & 1.80 \\
\hline $3 \ldots$ & 2. 30 & 3. 00 & 3. 20 & 3.80 & 5. 10 & 7.10 & 4. 60 & 2.00 & 1.80 & 1. 70 \\
\hline 4.. & 2.30 & 3.20 & 3.00 & 3. 70 & 8. 30 & 7.40 & 4. 40 & 2.00 & 1. 90 & 1.70 \\
\hline 5 & 2.30 & 3.10 & 3.00 & 3.70 & 9.20 & 7.00 & 4. 20 & 2.00 & 1.90 & 1. 70 \\
\hline 6. & 2. 30 & 3. 00 & 2.90 & 3. 80 & 9. 10 & 7.90 & 4.00 & 2.00 & 1. 90 & 1.70 \\
\hline 7. & 2.40 & 2.90 & 2. 90 & 4. 10 & 8.50 & 8.90 & 3.80 & 2.00 & 1. 90 & 1. 70 \\
\hline 8. & 2,40 & 2.80 & 3.00 & 4.50 & 7.40 & 7.60 & 3. 60 & 2.00 & 1.90 & 1. 70 \\
\hline $9 \ldots$ & 2.40 & 2.70 & 3.30 & 4.70 & 6.70 & 6.70 & 3. 50 & 2.00 & 1.90 & 1. 70 \\
\hline $10 \ldots$ & 2.30 & 2.60 & 3.70 & 4.50 & 5. 90 & 6. 70 & 3.40 & 1.90 & 1.90 & 1.70 \\
\hline $11 \ldots$ & 2.30 & 2.60 & 4.00 & 4. 30 & 6.00 & 6.60 & 3. 30 & 1. 90 & 1. 90 & 1.70 \\
\hline 12. & 2. 30 & 2.50 & 3.80 & 410 & 6.40 & 6.60 & 3. 20 & 1.90 & 1.90 & 1.70 \\
\hline 13. & 2.30 & 2.50 & 3.80 & 4.60 & 5.50 & 6.50 & 3.10 & 1.90 & 2.00 & 1. 70 \\
\hline 14 & 2.30 & 2.50 & 3. 70 & 4.50 & 5. 00 & 6.40 & 3.00 & 1.90 & 2.00 & 1. 70 \\
\hline 15. & 2. 30 & 2.50 & 3.50 & 5.20 & 4. 70 & 6.40 & 2.90 & 1.80 & 2.00 & 1. 70 \\
\hline 16. & 2. 50 & 2.60 & 3.40 & 4.80 & 4.60 & 6.40 & 2.80 & 1. 80 & 2.00 & 1. 70 \\
\hline 17. & 5. 10 & 2.60 & $3 .: 30$ & 4.50 & 4. 70 & 6.40 & 2.70 & 1. 80 & 2.00 & 1.70 \\
\hline 18. & 5.50 & 2.70 & 3.30 & 4. 10 & 5. 20 & 6.50 & 2.60 & 1.80 & 2.00 & \\
\hline 19. & 6.50 & 2.70 & 3. 40 & 4.00 & 4.70 & 6.60 & 2.50 & 1.80 & 2.00 & \\
\hline 20 . & 7. 60 & 2.70 & 3.80 & 3.90 & 4. 60 & 6.40 & 2. 40 & 1.80 & 1.90 & \\
\hline 21. & 8.30 & 2.70 & 4. 60 & 3.80 & 4. 60 & 6.20 & 2.40 & 190 & 1. 90 & \\
\hline 22. & 7.40 & 2. 80 & 4. 70 & $\begin{array}{l}0.70 \\
3.70\end{array}$ & 4.70 & 5.90 & 2.30 & 1. 80 & 1.90 & \\
\hline 23. & 5. 80 & 2.90 & 4. 70 & 3.80 & 5. 10 & 5.80 & 2. 30 & 1.80 & 1. 90 & \\
\hline 24. & 4.50 & 2.90 & 5.20 & 3.90 & 5.60 & 5.60 & 2.20 & 1.80 & 1. 80 & \\
\hline 25. & 3. 80 & 3.00 & 6.00 & 4. 10 & 6,10 & 5.40 & 2. 20 & 1.80 & 1.80 & \\
\hline 26 . & 3.80 & 3.20 & 6.00 & 4.40 & 6.10 & 5.20 & 2. 20 & 1.80 & 1.80 & \\
\hline 27. & 4.00 & 4.00 & 6.50 & 4.70 & 6.10 & 4. 90 & 2.10 & 1.80 & 1.80 & \\
\hline 28. & 4. 40 & 4.45 & 6.20 & 4.90 & 6.20 & 5. 20 & 2.10 & 1.80 & 1.80 & \\
\hline 29. & 6.40 & 4. 10 & 5.60 & 4. 50 & 6.20 & 5.30 & 2.10 & 1.80 & 1.80 & $\cdots$ \\
\hline & 5. 70 & & 5.00 & 4.20 & 6.60 & 5. 20 & 2. 10 & 1.80 & 1.80 & \\
\hline 31. & 4.50 & $\ldots$ & 4.60 & (2) & 6.90 & 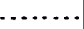 & 2.00 & 1.80 & ........ & $\ldots$ \\
\hline
\end{tabular}

Natches River at North Yakima, Washington.

\begin{tabular}{|c|c|c|c|c|c|c|c|c|}
\hline Day. & Jan. & Feb. & July. & Aug. & Sept. & Oet. & Nov. & Dec. \\
\hline 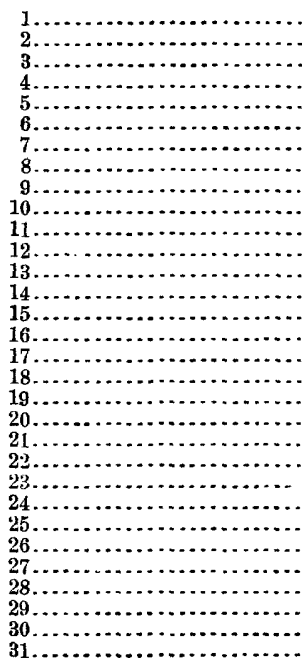 & 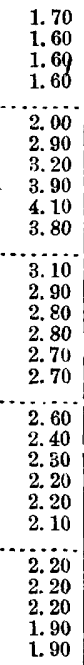 & $\begin{array}{r}1.90 \\
1.90 \\
1.90 \\
1.90 \\
2.00 \\
2.00 \\
1.90 \\
\ldots \\
1.90 \\
1.90 \\
1.90 \\
1.90 \\
2.00 \\
1.90 \\
\ldots 2.10 \\
2.10 \\
2.70 \\
2.80 \\
2.90 \\
3.20 \\
\ldots 3.20 \\
3.90 \\
5.60 \\
6.20 \\
5.80 \\
5.80 \\
\ldots \ldots . . \\
\ldots \ldots\end{array}$ & 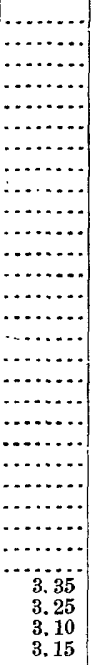 & $\begin{array}{l}3.25 \\
3.10 \\
3.00 \\
2.90 \\
3.00 \\
2.85 \\
2.80 \\
2.70 \\
2.65 \\
2.45 \\
2.35 \\
2.20 \\
2.10 \\
2.05 \\
2.00 \\
1.90 \\
1.80 \\
1.80 \\
1.70 \\
1.70 \\
1.60 \\
1.60 \\
1.50 \\
1.50 \\
1.50 \\
1.50 \\
1.50 \\
1.50 \\
1.50 \\
1.50 \\
1.50\end{array}$ & $\begin{array}{r}1.50 \\
1.40 \\
1.40 \\
1.40 \\
1.40 \\
1.30 \\
1.30 \\
1.30 \\
1.30 \\
1.25 \\
1.20 \\
1.30 \\
1.30 \\
1.35 \\
1.50 \\
1.40 \\
1.30 \\
1.30 \\
1.20 \\
1.20 \\
1.20 \\
1.25 \\
1.30 \\
1.20 \\
1.10 \\
.90 \\
.90 \\
.90 \\
1.90 \\
1.10 \\
1 . .2\end{array}$ & $\begin{array}{r}1.10 \\
1.20 \\
1.20 \\
1.20 \\
1.10 \\
.90 \\
.89 \\
.88 \\
.87 \\
.86 \\
.85 \\
.84 \\
.83 \\
.82 \\
.81 \\
.80 \\
.79 \\
.72 \\
.74 \\
.76 \\
.76 \\
.60 \\
.60 \\
.60 \\
.50 \\
.50 \\
.60 \\
.75 \\
.95 \\
1.15 \\
1.40\end{array}$ & \begin{tabular}{|l|}
1.50 \\
1.40 \\
1.30 \\
1.20 \\
1.10 \\
1.00 \\
1.20 \\
2.20 \\
2.50 \\
2.40 \\
2.30 \\
2.15 \\
2.00 \\
4.25 \\
8.00 \\
7.75 \\
6.25 \\
5.25 \\
4.70 \\
4.30 \\
4.00 \\
3.80 \\
3.70 \\
3.60 \\
3.50 \\
3.35 \\
3.15 \\
3.00 \\
3.20 \\
3.40 \\
$\ldots \ldots .$.
\end{tabular} & 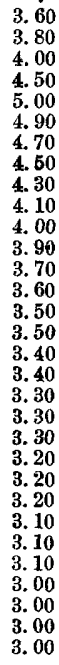 \\
\hline
\end{tabular}


Fakima River at Yakima, Washington.

\begin{tabular}{|c|c|c|c|c|c|c|c|}
\hline Day. & Jan. & Feb. & Ang. & Sept. & Oet. & Nov. & Dec. \\
\hline 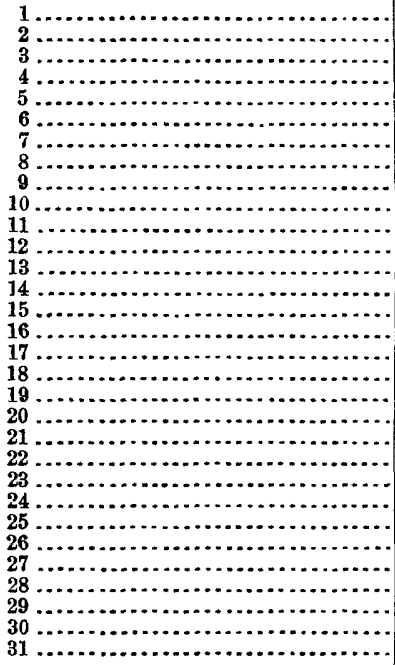 & 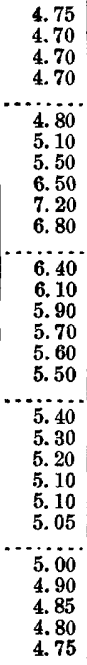 & 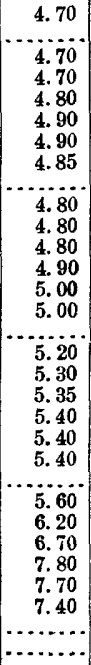 & $\begin{array}{r}\mathbf{5 .} 80 \\
\mathbf{5 . 8 0} \\
\mathbf{5 . 7 0} \\
\mathbf{5 . 7 0} \\
\mathbf{5 . 6 0} \\
\mathbf{5 . 6 0} \\
\mathbf{5 . 5 0} \\
\mathbf{5 . 5 0} \\
\mathbf{5 . 5 0} \\
\mathbf{5 . 4 0} \\
\mathbf{5 . 4 0} \\
\mathbf{5 . 3 0} \\
\mathbf{5 . 3 0} \\
\mathbf{5 . 2 0} \\
\mathbf{5 . 2 0} \\
\mathbf{5 . 1 0} \\
5.10 \\
5.10 \\
\mathbf{5 . 0 0} \\
\mathbf{5 . 0 0} \\
\mathbf{5 . 0 0} \\
\mathbf{4 . 9 0} \\
\mathbf{4 . 9 0} \\
\mathbf{4 . 9 0} \\
\mathbf{4 . 9 0} \\
\mathbf{4 . 8 0} \\
\mathbf{4 . 8 0} \\
\mathbf{4 . 8 0} \\
\mathbf{2 . 1 .}\end{array}$ & 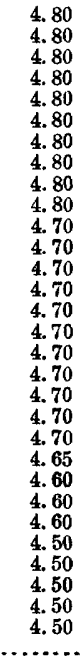 & $\begin{array}{l}4.50 \\
4.50 \\
4.50 \\
4.50 \\
4.50 \\
4.50 \\
4.50 \\
4.50 \\
4.40 \\
4.40 \\
4.40 \\
4.40 \\
4.40 \\
4.40 \\
4.40 \\
4.40 \\
4.40 \\
4.40 \\
4.40 \\
4.40 \\
4.40 \\
4.40 \\
4.40 \\
4.40 \\
4.40 \\
4.40 \\
4.40 \\
4.40 \\
4.40 \\
4.40 \\
4.50\end{array}$ & 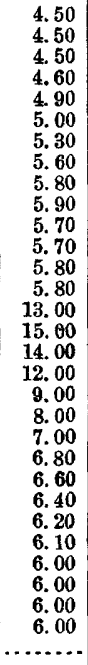 & $\begin{array}{l}6.60 \\
7.60 \\
8.20 \\
7.70 \\
6.60 \\
6.70 \\
6.80 \\
6.90 \\
8.00 \\
8.20 \\
8.60 \\
8.40 \\
8.20 \\
8.00 \\
7.60 \\
7.40 \\
7.10 \\
6.80 \\
6.20 \\
6.10 \\
6.10 \\
6.00 \\
6.00 \\
5.90 \\
\text { 5.90 } \\
\mathbf{5 . 8 0} \\
\mathbf{5 . 8 0} \\
\text { 5.70 } \\
\mathbf{5 . 7 0} \\
5.60 \\
\mathbf{5 . 6 0}\end{array}$ \\
\hline
\end{tabular}

Spokane River at dam of the Washington Water Pover Company, Spokane, Washington, for 1891 .

\begin{tabular}{|c|c|c|c|c|c|c|c|c|c|c|}
\hline Day. & Mar. & Apr. & May. & Jnne. & $\mathbf{J u l y}$ & Aug. & Sept. & Oet. & Nov. & Dec. \\
\hline 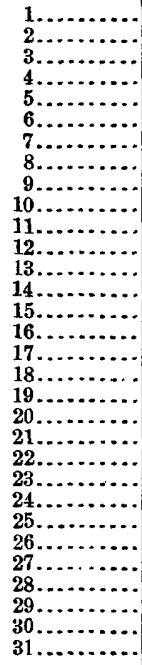 & 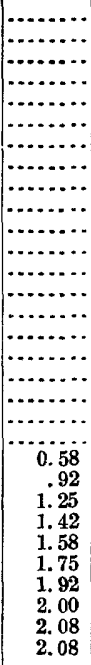 & $\begin{array}{l}2.08 \\
2.08 \\
2.17 \\
2.17 \\
2.17 \\
2.17 \\
2.17 \\
2.50 \\
2.75 \\
2.92 \\
2.92 \\
2.92 \\
3.17 \\
3.33 \\
3.50 \\
3.75 \\
3.92 \\
4.08 \\
4.50 \\
4.92 \\
4.50 \\
4.58 \\
4.67 \\
4.83 \\
4.83 \\
4.83 \\
4.83 \\
4.83 \\
4.83 \\
5.00 \\
\ldots \ldots .7\end{array}$ & $\begin{array}{l}\text { 5. } 00 \\
\mathbf{5 . 0 0} \\
\mathbf{4 . 9 2} \\
\mathbf{4 . 6 7} \\
\mathbf{4 . 5 0} \\
\mathbf{4 . 5 0} \\
\mathbf{4 . 6 7} \\
\mathbf{4 . 8 3} \\
\mathbf{5 . 0 0} \\
\mathbf{5 . 0 0} \\
\mathbf{5 . 2 5} \\
\mathbf{5 . 0 0} \\
\mathbf{5 . 0 0} \\
\mathbf{5 . 0 0} \\
\mathbf{5 . 0 0} \\
\mathbf{5 . 1 7} \\
\mathbf{5 . 1 7} \\
\mathbf{5 . 2 5} \\
\mathbf{5 . 2 5} \\
\mathbf{5 . 1 7} \\
\mathbf{5 . 0 8} \\
\mathbf{5 . 0 0} \\
\mathbf{4 . 8 3} \\
\mathbf{4 . 8 3} \\
\mathbf{4 . 5 0} \\
\mathbf{4 . 4 2} \\
\mathbf{4 . 2 5} \\
\mathbf{4 . 0 0} \\
\mathbf{4 . 0 0} \\
\mathbf{4 . 0 0} \\
\mathbf{3 .} 83\end{array}$ & \begin{tabular}{|l} 
3. 83 \\
3.67 \\
3.50 \\
3.33 \\
3.17 \\
3.17 \\
3.00 \\
3.00 \\
2.92 \\
2.83 \\
2.75 \\
2.67 \\
2.50 \\
2.50 \\
2.50 \\
2.42 \\
2.33 \\
2.25 \\
2.17 \\
2.08 \\
2.00 \\
2.00 \\
1.92 \\
1.92 \\
1.83 \\
1.75 \\
1.67 \\
1.67 \\
1.67 \\
1.67 \\
$\cdots \cdots .$.
\end{tabular} & $\begin{array}{r}1.50 \\
1.58 \\
1.58 \\
1.50 \\
1.50 \\
1.50 \\
1.33 \\
1.33 \\
1.25 \\
1.25 \\
1.25 \\
1.25 \\
1.17 \\
1.17 \\
1.17 \\
1.17 \\
1.08 \\
1.00 \\
1.00 \\
1.00 \\
.92 \\
.83 \\
.83 \\
.75 \\
.67 \\
.67 \\
.67 \\
.50 \\
.42 \\
.42 \\
.33\end{array}$ & $\begin{array}{l}0.33 \\
.25 \\
.25 \\
.25 \\
.17 \\
.17 \\
.17 \\
.08 \\
.08 \\
.08 \\
.08 \\
.00 \\
.00 \\
1.33 \\
1.33 \\
1.33 \\
1.33 \\
1.33 \\
1.33 \\
1.25 \\
1.25 \\
1.25 \\
1.25 \\
1.17 \\
1.17 \\
1.17 \\
1.17 \\
1.08 \\
1.08 \\
1.08 \\
1.08\end{array}$ & $\begin{array}{r}1.00 \\
1.00 \\
\mathbf{1 . 0 0} \\
\mathbf{1 . 0 0} \\
.92 \\
.92 \\
.92 \\
.92 \\
.83 \\
.83 \\
.83 \\
.83 \\
.83 \\
.75 \\
.75 \\
.75 \\
.75 \\
.75 \\
.67 \\
.67 \\
.67 \\
.58 \\
.58 \\
.58 \\
.58 \\
.50 \\
.50 \\
.50 \\
.50 \\
.50 \\
. . . .\end{array}$ & $\begin{array}{r}0.50 \\
.50 \\
.50 \\
.50 \\
.50 \\
.50 \\
.50 \\
.50 \\
.50 \\
.50 \\
.50 \\
.50 \\
.50 \\
.50 \\
.50 \\
.50 \\
.50 \\
.50 \\
1.08 \\
2.67 \\
2.67 \\
2.67 \\
2.67 \\
2.67 \\
2.67 \\
2.67 \\
2.67 \\
2.67 \\
2.67 \\
2.67 \\
2.75\end{array}$ & 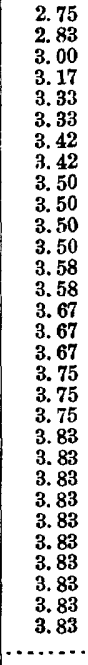 & 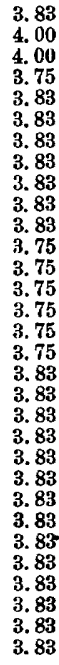 \\
\hline
\end{tabular}

March 22, waste gates almost entirely closed. April 21, waste gates opened, which lowered the water about 1 foot. A ugust 14, waste gates partly closed, oue open about 2 feet 6 inches, the other, 3 foet 6 inches. October 19 , waste gates further closed, one open about 8 inches, the other 1 foot. Octo. ber 20, flashboards pnt on dam about three-fourths of the way across. December 4, about one-third of the tlashboards were carried out. 
Spokane River at dam of the Washington Water Power Company, Spokane, Washington, for 1892.

\begin{tabular}{|c|c|c|c|c|c|c|c|c|c|c|c|c|}
\hline Day. & Jan. & Fob. & Mar. & Apr. & May. & June & July. & Ang. & Sept. & Oct. & Nov. & Dec. \\
\hline & 3.83 & 3.33 & 3.08 & 4. 58 & 4.42 & 8.00 & 4.33 & 1.58 & 0.92 & 3.00 & 3.00 & 4.00 \\
\hline & 3.67 & 3.17 & $\begin{array}{l}0.00 \\
3.17\end{array}$ & 4. 42 & 4.42 & 8.00 & 4. 25 & 2. 33 & $\begin{array}{r}.92 \\
\end{array}$ & $\begin{array}{l}\text { 3. } 00 \\
\text { 3. }\end{array}$ & $\begin{array}{l}3.00 \\
3.00\end{array}$ & 4.00 \\
\hline & 3.67 & 3,17 & 3.25 & 4. 33 & $\begin{array}{l}4.42 \\
\text {. }\end{array}$ & 8.00 & 4.17 & 2.33 & .92 & 3.00 & 3.00 & 4.08 \\
\hline 4. & 3.67 & 3. 17 & 3. 33 & 4.08 & 4.42 & 7.92 & 4.08 & 2.17 & .83 & 3.00 & 3.00 & 4. 08 \\
\hline 5. & 3. 67 & 3.17 & 3. 42 & 3.92 & 4.50 & 7.83 & 4.00 & 2.08 & .83 & 3.00 & 3.00 & 4.17 \\
\hline & 3.67 & 3.17 & 3.50 & 3.83 & 4. 58 & 7. 67 & 3. 92 & 2. 00 & .75 & 3. 00 & 3.00 & 4.17 \\
\hline & 3.67 & 3.17 & 3.50 & 3.75 & 4.67 & 7. 58 & 3. 83 & 1. 92 & .75 & 3. 00 & 3.00 & 4. 25 \\
\hline 8. & 3.67 & 3.17 & 3.50 & 3.75 & 4.75 & 7.42 & 3.75 & 1.83 & .75 & 3.00 & 3.00 & 4. 25 \\
\hline 9. & 3.67 & 3.17 & 3.58 & 3.75 & 4.83 & 7.25 & 3.67 & 1. 75 & .67 & 3. 00 & 3.00 & 4.25 \\
\hline 10. & 3.67 & 3.17 & 3.67 & 3.75 & 4.92 & 7.00 & 3.58 & 1.67 & .67 & 3.00 & 3.00 & 4.25 \\
\hline 11. & 3.50 & 3.17 & 3.83 & 3.75 & 5.08 & 6.75 & 3.50 & 1.67 & 67 & 3.00 & 3.00 & 4. 25 \\
\hline & 3.50 & 3.25 & 4.00 & 3.75 & 5. 33 & 6.67 & 3.33 & 1.58 & .58 & 3. 00 & 3.00 & 4. 25 \\
\hline 13. & 3.50 & 3. 25 & 4.33 & 3.75 & 5. 58 & 6.58 & 3.25 & 1.58 & .50 & 3.00 & 3.00 & 4. 25 \\
\hline 14. & 3.50 & 3.25 & 4.67 & 3.83 & 5. 75 & 6.50 & 3.17 & 1.50 & 42 & 3. 00 & 3.00 & 4.25 \\
\hline & 3.50 & 3. 25 & 4.92 & 3.92 & 5.92 & 6. 33 & 3. 08 & 1. 50 & .33 & 3. 00 & 3.00 & 4. 17 \\
\hline 16. & 3. 50 & 3.25 & 5.17 & 4.00 & 6.00 & 6.25 & 3. 00 & 1.50 & .25 & 3.00 & 3.08 & 4.17 \\
\hline 17. & 3.50 & 3.25 & 5.33 & 4.08 & 6. 08 & 6.08 & 2.92 & 1.42 & .00 & 3. 00 & 3.17 & 4. 17 \\
\hline & 3.50 & 3.25 & 5.50 & 4.17 & 6. 17 & 6.00 & 2.83 & 1.42 & .00 & 3. 00 & 3. 17 & 4. 08 \\
\hline 19. & 3.50 & 3.25 & 5. 67 & 4. 25 & 6.25 & 5.83 & 2.75 & 1.42 & 3.00 & 3.00 & 3.25 & 4.08 \\
\hline & 3.50 & 3.25 & 5.75 & 4.25 & 6. 33 & 5.67 & 2.67 & 1.33 & 3.00 & 3.00 & 3. 25 & 4. 0 \\
\hline 21 . & 3.50 & 3.25 & 5.75 & 4. 25 & 6.42 & 5.50 & 2.58 & 1.33 & 3.00 & 3. 00 & 3. 33 & 3. 92 \\
\hline 22. & 3.50 & 3. 25 & 5. 75 & 4. 25 & 6. 58 & 5. 33 & 2.50 & 1. 23 & 3.00 & 3. 00 & 3. 33 & 3. 92 \\
\hline & 3.50 & 3. 33 & 5.67 & 4. 25 & 6.83 & 5. 25 & 2.33 & 1. 25 & 3.00 & 3.00 & 3.42 & 3.83 \\
\hline & 3. 33 & 3.50 & 4.75 & 4. 25 & 7. 00 & 5. 08 & 2.17 & 1.25 & 3.00 & 3.00 & 3.50 & 3.83 \\
\hline & 3.33 & 3.75 & 4.83 & 4. 25 & 7.33 & 5.00 & 2.00 & 1. 17 & 3.00 & 3,00 & 3.58 & 3. 83 \\
\hline & 3.33 & 3.92 & 4.83 & 4. 33 & 7.67 & 4. 83 & 1. 83 & 1. 17 & 3. 00 & 3. 00 & 3.67 & 3. 92 \\
\hline 27 & 3.33 & 4. 00 & 4.83 & 4.33 & 7.75 & 4.75 & 1.75 & 1.08 & 3.00 & 3.00 & 3.75 & 3.92 \\
\hline & 3. 33 & 3.75 & 4.83 & 4. 33 & 7.92 & 4.67 & 1.67 & 1.08 & 3.00 & 3.00 & 3. 83 & 3.92 \\
\hline & 3. 33 & 3. 00 & 4.83 & 4. 33 & 8.00 & 4.58 & 1.58 & 1.00 & 3. 00 & 3. 00 & 3.92 & $4: 00$ \\
\hline & 3.33 & & 4.83 & 4.42 & 8.00 & 4.50 & 1.58 & 1.00 & 3.00 & 3.00 & 3.92 & 4.00 \\
\hline & 3.33 & & 4.75 & & 8.00 & & 1.58 & 1.00 & 3.00 & 3.00 & & 4.08 \\
\hline
\end{tabular}

February 28, part of the flashboards taken out. February 29, balance taken out. March 24, waste gates opened full. Angust 2, one waste gate partly closed, leaving opening 3 foet 6 inches: openings partly closed with drift logs. September 17, logs removed from waste gates. September 19 , waste gates closed and flashboards put on dam.

Spokane River at dam of the Washington Water Power Company, Spokane, Washington, for 1893 .

\begin{tabular}{|c|c|c|c|c|c|c|c|c|c|c|c|c|}
\hline Day. & Jan. & Feb. & Mar. & Apr. & May. & June. & July. & Ang. & Sept. & Oct. & Nov.! & Dec. \\
\hline & 4. 08 & 3.92 & 3.58 & 3.50 & 6.17 & 8.25 & 5.25 & 1.83 & 0.33 & 3.25 & 5.00 & 4.92 \\
\hline & 4.17 & 3.83 & 3.58 & 3.75 & 6. 33 & 8.25 & 5.17 & 1.75 & .33 & 3.33 & 5. 00 & 4.92 \\
\hline & 4.17 & 3.83 & 3.58 & 4. 08 & 6.50 & 8. 25 & 5.00 & 1.75 & .25 & 3.42 & 4.92 & 4.83 \\
\hline & 4.25 & 3.83 & 3.58 & 4. 33 & 6.67 & 8.25 & 5.00 & 1.67 & .25 & 3.50 & 4.83 & 4.83 \\
\hline & 4. 33 & 3.75 & 3.58 & 4. 67 & 6. 75 & 8.25 & 4.92 & 1.67 & .25 & 3.58 & 4.83 & 4.83 \\
\hline & 4.42 & 3. 75 & 3. 58 & 4.00 & 6. 83 & 8.00 & 4.83 & 1.67 & .17 & 3.67 & 4. 92 & 4.83 \\
\hline & 4.50 & 3.75 & 3.58 & 4.42 & 6. 92 & 8.00 & 4.75 & 1.58 & .08 & 3.83 & 5.17 & 4.83 \\
\hline 8. & 4. 50 & 3.75 & 3.67 & 4.75 & 7.00 & 8.00 & 4.67 & 1.58 & .08 & 4.00 & 5.67 & 4.83 \\
\hline & 4.58 & 3.67 & .67 & 4.83 & 7.17 & 8.00 & 4.58 & 1.50 & .00 & 4. 25 & 6.17 & 5.17 \\
\hline 10. & 4.58 & 3.67 & .67 & 5.08 & 7. 33 & 8.00 & 4.42 & 1. 50 & .00 & 4. 50 & 6.50 & 5.67 \\
\hline 11. & 4.58 & 3.58 & .67 & 5.08 & 7.50 & 7.92 & 4.25 & 1.50 & .00 & 4. 75 & 6.67 & 6.00 \\
\hline 12. & 4.58 & 3.58 & .67 & 5.08 & 7. 75 & 7.83 & 4.00 & 1.42 & 3.25 & 5.00 & 6. 75 & 6.83 \\
\hline 13. & 4.58 & 3.58 & 1.33 & 4.92 & 7. 75 & 7. 75 & 3.92 & 1.42 & 3.25 & 5.00 & 6.83 & 7.00 \\
\hline 14. & 4. 50 & 3.58 & 1.50 & 4.92 & 8.00 & 7.58 & 3.75 & 1.33 & 3.17 & 5. 00 & 6. 83 & 7.00 \\
\hline 15. & 4.50 & 3.58 & 1.67 & 4.75 & 8.25 & 7.42 & 3. 58 & 1.25 & 3.17 & 5.00 & 6.83 & 6. 02 \\
\hline 16. & 4.50 & 3.58 & 1.67 & 4. 67 & 8.75 & 7.25 & 3.42 & 1. 17 & 3.17 & 5.08 & 6. 75 & 6.83 \\
\hline & 4.42 & 3. 58 & 1. 67 & 4.50 & 9. 00 & 7.00 & 3.25 & 1.08 & 3.17 & 5.08 & 6.67 & 6.67 \\
\hline 18. & 4.42 & 3. 58 & 1. 67 & 4. 42 & 9. 25 & 6.92 & 3.08 & 1.00 & 3.17 & 5. 08 & 6. 50 & 6. 50 \\
\hline 19. & 4. 33 & 3.58 & 1.67 & 4.33 & 10.00 & 6.75 & 3.00 & .92 & 3.17 & 5. 08 & 6. 33 & 6. 33 \\
\hline 20 & 4. 25 & 3.58 & 1.67 & 4. 25 & 10.25 & 6. 58 & 2.92 & .83 & 3.17 & 5.17 & 6.08 & 6.17 \\
\hline $2 r$ & 4. 25 & 3.58 & 1.67 & 4. 25 & 10.25 & 6.42 & 2.83 & .83 & 3.08 & 5. 17 & 5.83 & 6. 17 \\
\hline & 4.17 & 3.58 & 1.75 & 4. 33 & 10.17 & 6.33 & 2. & .75 & 3.08 & 5.17 & 5. 58 & 6.17 \\
\hline & 4.17 & 3. 58 & 1. 75 & 4. 42 & 9.75 & 6.17 & 2.50 & .75 & 3.08 & 5.17 & 5.42 & 6. 08 \\
\hline 24. & 4. 17 & 3.58 & 1.83 & 4. 67 & 9.50 & 6.00 & 2.42 & .67 & 3.08 & 5. 17 & 5. 25 & 6. 00 \\
\hline & 4.08 & 3.58 & 1.83 & 5.0 & 9.50 & 6.00 & 2.33 & .58 & 3.08 & 5.17 & 5.17 & 6.00 \\
\hline 26 . & 4. 08 & 3.58 & 2.00 & 5.42 & 9.17 & 5.83 & 2.17 & .50 & 3.08 & 5.17 & 5. 00 & 5.92 \\
\hline & 4.00 & 3.58 & 2.17 & 5.58 & 9.00 & 5.83 & 2.00 & .42 & 3.08 & 5.17 & 5. 00 & 5. 92 \\
\hline & 4.00 & 3.58 & 2. 42 & 5. 75 & 8.75 & 5.67 & 2.00 & .42 & 3.08 & 5. 17 & 5.00 & 5.92 \\
\hline & 3.92 & & 2.67 & 5.92 & 8.50 & 5.50 & 2.00 & .42 & 3.08 & 5.17 & 5.00 & 5. 83 \\
\hline 30. & 3.92 & $\cdots$ & 2.92 & 6.00 & 8.50 & 5.33 & 2.00 & .33 & 3.08 & 5. 08 & 5.00 & 5.83 \\
\hline & 3 & ..... & 3. 25 & & 8. & & 1.92 & 33 & 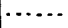 & 5. 08 & & 5.83 \\
\hline
\end{tabular}

March 9, 10, 11 and 12, waste gates opened; flashboards taken out. March 13, waste gates closed. April 6, waste gates opened. September 12, waste gates closed and flashboards put in. 
Spokane River at Dam of the Washington Water Power Company, Spokane, Washington, for 1894 .

\begin{tabular}{|c|c|c|c|c|c|c|c|c|c|c|c|c|}
\hline Day. & Jan. & Feb. & Mar. & Apr. & May. & June. & July. & Aug. & Sept. & Oct. & Nov. & Dec. \\
\hline 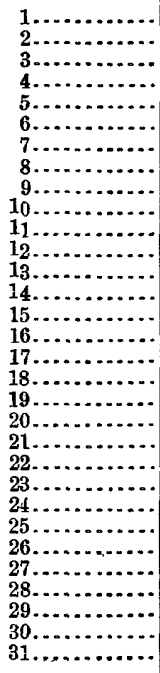 & $\begin{array}{l}5.75 \\
5.75 \\
5.67 \\
5.58 \\
5.50 \\
5.42 \\
5.33 \\
5.25 \\
5.17 \\
5.00 \\
4.83 \\
4.67 \\
4.67 \\
4.75 \\
5.17 \\
6.25 \\
7.00 \\
7.50 \\
7.58 \\
7.58 \\
7.50 \\
7.33 \\
7.00 \\
6.83 \\
6.67 \\
6.42 \\
6.25 \\
6.08 \\
6.00 \\
5.92 \\
5.83\end{array}$ & \begin{tabular}{|l|} 
5.67 \\
5.50 \\
5.33 \\
5.17 \\
5.00 \\
5.00 \\
4.92 \\
4.92 \\
4.83 \\
4.83 \\
4.75 \\
4.67 \\
4.50 \\
4.50 \\
4.50 \\
4.33 \\
4.33 \\
4.17 \\
4.00 \\
3.92 \\
3.83 \\
3.83 \\
3.83 \\
3.75 \\
3.75 \\
3.75 \\
3.75 \\
3.75 \\
$\ldots \ldots .$. \\
$\ldots \ldots .$. \\
$\ldots \ldots$
\end{tabular} & $\begin{array}{l}3.75 \\
3.75 \\
3.92 \\
3.92 \\
3.92 \\
3.92 \\
3.92 \\
4.00 \\
4.00 \\
4.00 \\
4.00 \\
4.00 \\
4.00 \\
4.08 \\
4.08 \\
4.08 \\
4.08 \\
4.17 \\
4.17 \\
4.33 \\
4.42 \\
4.58 \\
4.67 \\
4.67 \\
4.67 \\
4.75 \\
4.92 \\
5.08 \\
5.33 \\
5.67 \\
6.00\end{array}$ & $\begin{array}{r}6.50 \\
6.83 \\
7.00 \\
7.00 \\
7.08 \\
7.08 \\
7.08 \\
7.08 \\
7.08 \\
7.25 \\
7.25 \\
7.50 \\
7.83 \\
8.17 \\
8.50 \\
8.83 \\
8.92 \\
8.92 \\
8.83 \\
8.75 \\
8.67 \\
8.67 \\
8.67 \\
8.83 \\
9.08 \\
9.42 \\
9.58 \\
9.92 \\
10.08 \\
10.08 \\
\cdots \ldots . .\end{array}$ & $\begin{array}{r}10.08 \\
10.00 \\
9.83 \\
9.75 \\
9.58 \\
9.50 \\
9.50 \\
9.42 \\
9.42 \\
9.42 \\
9.33 \\
9.25 \\
9.25 \\
9.17 \\
9.08 \\
9.08 \\
9.33 \\
9.58 \\
9.92 \\
10.08 \\
10.17 \\
10.33 \\
10.50 \\
10.75 \\
11.00 \\
11.33 \\
11.67 \\
12.00 \\
12.17 \\
12.17 \\
12.42\end{array}$ & $\begin{array}{r}12.33 \\
12.33 \\
12.33 \\
12.25 \\
12.25 \\
12.25 \\
12.08 \\
11.92 \\
11.67 \\
11.33 \\
10.00 \\
9.83 \\
9.67 \\
9.42 \\
9.17 \\
9.00 \\
8.83 \\
8.75 \\
8.67 \\
8.50 \\
8.33 \\
8.25 \\
8.08 \\
7.92 \\
7.75 \\
7.58 \\
7.42 \\
7.25 \\
7.08 \\
6.92 \\
\cdots . . .\end{array}$ & $\begin{array}{l}6.75 \\
6.67 \\
6.50 \\
6.33 \\
6.17 \\
6.00 \\
5.92 \\
5.75 \\
5.58 \\
5.42 \\
5.33 \\
5.25 \\
5.17 \\
5.08 \\
4.92 \\
4.83 \\
4.67 \\
4.50 \\
4.33 \\
4.25 \\
4.08 \\
3.92 \\
3.83 \\
3.75 \\
3.75 \\
3.67 \\
3.67 \\
3.58 \\
3.58 \\
3.50 \\
3.50\end{array}$ & $\begin{array}{l}3.42 \\
3.33 \\
3.25 \\
3.25 \\
3.17 \\
3.08 \\
3.08 \\
3.00 \\
2.92 \\
2.83 \\
2.75 \\
2.67 \\
2.58 \\
2.50 \\
2.42 \\
2.33 \\
2.25 \\
2.17 \\
2.08 \\
2.00 \\
1.92 \\
1.83 \\
1.75 \\
1.75 \\
1.67 \\
1.58 \\
1.58 \\
1.58 \\
1.58 \\
1.50 \\
1.50\end{array}$ & $\begin{array}{l}1.50 \\
1.42 \\
1.42 \\
1.42 \\
1.42 \\
1.42 \\
1.33 \\
1.33 \\
1.33 \\
1.33 \\
1.33 \\
1.33 \\
1.25 \\
1.25 \\
1.25 \\
1.25 \\
1.25 \\
1.25 \\
1.25 \\
1.25 \\
1.25 \\
1.25 \\
1.25 \\
1.25 \\
1.25 \\
1.25 \\
1.25 \\
1.25 \\
1.25 \\
1.25 \\
\ldots . . .\end{array}$ & $\begin{array}{l}1.25 \\
1.25 \\
1.25 \\
1.25 \\
1.25 \\
1.25 \\
1.25 \\
1.25 \\
1.25 \\
1.83 \\
1.83 \\
1.83 \\
1.83 \\
1.75 \\
1.75 \\
1.75 \\
1.75 \\
1.75 \\
1.67 \\
1.67 \\
1.67 \\
1.67 \\
1.67 \\
1.67 \\
1.67 \\
1.83 \\
2.00 \\
2.17 \\
2.25 \\
2.33 \\
2.33 \\
1.9\end{array}$ & \begin{tabular}{|l}
2.42 \\
2.50 \\
2.58 \\
2.67 \\
2.67 \\
2.75 \\
2.75 \\
2.83 \\
2.92 \\
3.00 \\
3.17 \\
3.25 \\
3.25 \\
3.25 \\
3.25 \\
3.25 \\
3.25 \\
3.25 \\
3.17 \\
3.08 \\
3.00 \\
3.00 \\
3.00 \\
3.00 \\
3.08 \\
3.08 \\
3.08 \\
3.17 \\
3.25 \\
3.42 \\
$\ldots . .2$ \\
$\cdots$
\end{tabular} & $\begin{array}{l}3.50 \\
3.58 \\
3.58 \\
3.58 \\
3.58 \\
3.58 \\
3.50 \\
3.50 \\
3.50 \\
3.50 \\
3.50 \\
3.50 \\
3.33 \\
3.33 \\
3.25 \\
3.17 \\
3.08 \\
3.08 \\
3.00 \\
3.00 \\
2.92 \\
2.92 \\
2.83 \\
2.83 \\
2.75 \\
2.75 \\
2.75 \\
2.67 \\
2.58 \\
2.50 \\
2.50 \\
35\end{array}$ \\
\hline
\end{tabular}

April 4, waste gates opened. Flashboards were carried out in high water of April and May. Ootober 10, waste gates closed.

Spokane River at dam of the Washington Water Power Company, Spokane, Washington, for 1895 .

\begin{tabular}{|c|c|c|c|c|c|c|c|c|c|c|c|c|}
\hline D & Jan. & Feb. & Mar. & Apr. & May. & Jnne. & July. & Aug. & Sept. & Oct. & Nov. & Dec. \\
\hline $\begin{array}{r}1 \ldots \\
2 \ldots \\
3 \ldots \\
4 \ldots \\
5 \ldots \\
6 \ldots \\
7 \ldots \\
8 \ldots \\
9 \ldots \\
10 \ldots \\
11 \ldots \\
12 \ldots \\
13 \ldots \\
14 \ldots \\
15 \ldots \\
16 \ldots \\
17 \ldots \\
18 \ldots \\
19 \ldots \\
20 \ldots \\
21 \ldots \\
22 \ldots \\
23 \ldots \\
24 \ldots \\
25 \ldots \\
26 \ldots \\
27 \ldots \\
28 \ldots \\
29 . . \\
30 \ldots \\
31 \ldots\end{array}$ & $\begin{array}{l}2.42 \\
2.42 \\
2.33 \\
2.33 \\
2.25 \\
2.25 \\
2.25 \\
2.25 \\
2.25 \\
2.33 \\
2.33 \\
2.42 \\
2.67 \\
3.08 \\
3.50 \\
4.00 \\
4.33 \\
4.33 \\
4.33 \\
4.33 \\
4.42 \\
4.25 \\
4.25 \\
4.17 \\
4.17 \\
4.08 \\
4.00 \\
3.83 \\
3.67 \\
3.67 \\
3.58\end{array}$ & $\begin{array}{l}3.50 \\
3.42 \\
3.42 \\
3.25 \\
3.17 \\
3.17 \\
3.08 \\
3.00 \\
2.92 \\
2.83 \\
2.83 \\
2.75 \\
2.75 \\
2.67 \\
2.58 \\
2.58 \\
2.58 \\
2.58 \\
2.50 \\
2.50 \\
2.50 \\
2.50 \\
2.67 \\
3.17 \\
3.58 \\
4.00 \\
4.33 \\
4.67\end{array}$ & $\begin{array}{l}4.83 \\
4.83 \\
4.92 \\
5.00 \\
5.08 \\
5.08 \\
5.00 \\
4.83 \\
4.83 \\
4.75 \\
4.67 \\
4.50 \\
4.50 \\
4.42 \\
4.42 \\
4.42 \\
4.25 \\
4.17 \\
4.17 \\
4.00 \\
4.00 \\
3.92 \\
3.83 \\
3.75 \\
3.75 \\
3.75 \\
3.75 \\
3.67 \\
3.67 \\
3.67 \\
4.00\end{array}$ & $\begin{array}{l}4.17 \\
4.33 \\
4.42 \\
4.50 \\
4.67 \\
4.67 \\
4.75 \\
4.75 \\
4.75 \\
4.83 \\
4.92 \\
5.00 \\
5.00 \\
5.17 \\
5.25 \\
5.33 \\
5.50 \\
5.67 \\
5.67 \\
5.75 \\
5.75 \\
5.75 \\
5.83 \\
5.83 \\
6.00 \\
6.17 \\
6.50 \\
6.67 \\
6.92 \\
7.00\end{array}$ & \begin{tabular}{|l|}
7.00 \\
7.17 \\
7.17 \\
7.25 \\
7.25 \\
7.33 \\
7.50 \\
7.50 \\
7.58 \\
7.67 \\
7.67 \\
7.50 \\
7.33 \\
7.00 \\
7.00
\end{tabular} & $\begin{array}{l}6.00 \\
6.00 \\
5.83 \\
5.75 \\
5.67 \\
5.58 \\
5.58 \\
5.50 \\
5.33 \\
5.00 \\
5.00 \\
5.00 \\
5.00 \\
5.00 \\
4.67 \\
4.50 \\
4.33 \\
4.25 \\
4.17 \\
4.08 \\
4.00 \\
3.83 \\
3.83 \\
3.67 \\
3.58 \\
3.50 \\
3.50 \\
3.50 \\
3.50 \\
3.50\end{array}$ & $\begin{array}{l}3.33 \\
3.25 \\
3.00 \\
3.00 \\
3.00 \\
3.00 \\
2.83 \\
2.83 \\
2.75 \\
2.67 \\
2.67 \\
2.58 \\
2.58 \\
2.50 \\
2.50 \\
2.42 \\
2.42 \\
2.42 \\
2.33 \\
2.33 \\
2.25 \\
2.25 \\
2.17 \\
2.08 \\
2.00 \\
2.00 \\
2.00 \\
2.00 \\
1.92 \\
1.83 \\
1.75\end{array}$ & $\begin{array}{r}1.75 \\
1.75 \\
1.75 \\
1.75 \\
1.67 \\
1.67 \\
1.58 \\
1.58 \\
1.58 \\
1.58 \\
1.58 \\
1.50 \\
1.50 \\
1.42 \\
1.42 \\
1.42 \\
1.42 \\
1.33 \\
1.25 \\
.1 .17 \\
1.08 \\
1.00 \\
.00 \\
.00 \\
.00 \\
.00 \\
.00 \\
2.75 \\
.2 .75 \\
2.75 \\
2.75\end{array}$ & $\begin{array}{l}2.75 \\
2.75 \\
2.75 \\
2.75 \\
2.83 \\
2.83 \\
2.83 \\
2.92 \\
3.00 \\
3.00 \\
3.00 \\
3.08 \\
3.17 \\
3.00 \\
3.00 \\
3.00 \\
3.00 \\
3.00 \\
3.00 \\
3.00 \\
3.00 \\
3.00 \\
3.00 \\
3.00 \\
3.00 \\
3.00 \\
3.00 \\
3.00 \\
3.00 \\
3.00\end{array}$ & $\begin{array}{l}3.00 \\
3.00 \\
3.00 \\
3.00 \\
3.00 \\
3.00 \\
3.00 \\
3.00 \\
3.00 \\
3.00 \\
3.00 \\
3.00 \\
3.00 \\
3.00 \\
3.00 \\
3.00 \\
3.00 \\
3.00 \\
3.00 \\
3.00 \\
3.00 \\
3.00 \\
3.00 \\
3.00 \\
2.83 \\
2.83 \\
2.67 \\
2.67 \\
2.83 \\
3.00 \\
\text { 3. } 00\end{array}$ & $\begin{array}{l}3.00 \\
3.00 \\
3.00 \\
3.00 \\
3.00 \\
3.08 \\
3.08 \\
3.17 \\
3.17 \\
3.17 \\
3.17 \\
3.17 \\
3.17 \\
3.17 \\
3.17 \\
3.17 \\
3.17 \\
3.17 \\
3.17 \\
3.17 \\
3.17 \\
3.17 \\
3.17 \\
3.17 \\
3.17 \\
3.17 \\
3.25 \\
3.33 \\
3.33 \\
3.33 \\
\ldots . . .\end{array}$ & $\begin{array}{l}3.50 \\
3.50 \\
3.50 \\
3.50 \\
3.50 \\
3.50 \\
3.50 \\
3.50 \\
3.50 \\
3.50 \\
3.50 \\
3.58 \\
3.58 \\
\mathbf{3 . 6 7} \\
3.67 \\
3.67 \\
3.67 \\
3.67 \\
3.67 \\
3.67 \\
3.67 \\
3.67 \\
3.67 \\
3.67 \\
3.67 \\
3.67 \\
3.67 \\
3.67 \\
3.75 \\
3.75 \\
3.75\end{array}$ \\
\hline
\end{tabular}

August 23, waste gates opened. Angnst 25, flashboards put in. August 28, one waste gate closed. September 5, other waste gate closed. 
Spokane River at Spokane, Washington, for 1896.

\begin{tabular}{|c|c|c|c|c|c|c|c|c|c|c|c|c|}
\hline Day. & Jan. & Feb. & Mar. & Apr. & May. & June. & July. & Aug. & Sept. & Oet. & Nov. & Dec. \\
\hline 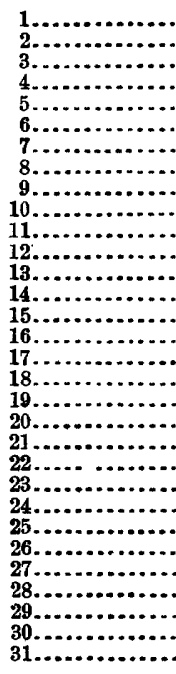 & $\begin{array}{l}\text { 3. } 75 \\
\text { 3. } 75 \\
3.75 \\
3.75 \\
\text { 3. } 83 \\
3.83 \\
3.83 \\
3.83 \\
3.92 \\
3.92 \\
3.92 \\
\text { 3. } 92 \\
4.00 \\
4.00 \\
4.00 \\
4.00 \\
4.00 \\
4.00 \\
4.17 \\
4.17 \\
4.33 \\
4.42 \\
4.50 \\
4.50 \\
4.67 \\
4.67 \\
5.00 \\
5.00 \\
5.00 \\
5.00 \\
5.00\end{array}$ & \begin{tabular}{|l}
$\mathbf{5 . 0 0}$ \\
$\mathbf{5 . 0 0}$ \\
$\mathbf{5 . 0 0}$ \\
$\mathbf{5 . 0 0}$ \\
$\mathbf{5 . 0 0}$ \\
$\mathbf{5 . 0 0}$ \\
$\mathbf{5 . 0 0}$ \\
$\mathbf{5 . 0 0}$ \\
$\mathbf{5 . 0 0}$ \\
$\mathbf{5 . 0 0}$ \\
$\mathbf{5 . 0 0}$ \\
$\mathbf{5 . 0 0}$ \\
$\mathbf{5 . 0 0}$ \\
$\mathbf{5 . 0 0}$ \\
$\mathbf{5 . 0 0}$ \\
$\mathbf{5 .} 00$ \\
$\mathbf{5 . 1 7}$ \\
$\mathbf{5 . 1 7}$ \\
$\mathbf{5 . 1 7}$ \\
$\mathbf{5 . 3 3}$ \\
$\mathbf{5 . 3 3}$ \\
$\mathbf{5 . 3 3}$ \\
$\mathbf{5 . 3 3}$ \\
$\mathbf{5 . 3 3}$ \\
$\mathbf{5 . 4 2}$ \\
$\mathbf{5 . 4 2}$ \\
$\mathbf{5 . 4 2}$ \\
$\mathbf{5 . 8 3}$ \\
$\mathbf{6 . 3 3}$ \\
$\cdots . .$. \\
$\cdots \ldots .$.
\end{tabular} & $\begin{array}{l}6.33 \\
6.17 \\
6.17 \\
6.00 \\
6.00 \\
6.00 \\
6.00 \\
6.00 \\
6.00 \\
6.00 \\
6.00 \\
6.00 \\
6.00 \\
6.00 \\
6.00 \\
6.00 \\
5.83 \\
5.83 \\
5.67 \\
5.67 \\
5.67 \\
5.83 \\
6.00 \\
6.33 \\
7.00 \\
7.00 \\
7.33 \\
7.50 \\
7.50 \\
7.67 \\
7.67\end{array}$ & \begin{tabular}{|l|}
7.67 \\
7.50 \\
7.50 \\
7.50 \\
7.33 \\
7.17 \\
6.83 \\
6.83 \\
6.67 \\
6.67 \\
6.67 \\
6.67 \\
6.75 \\
6.92 \\
6.92 \\
7.33 \\
7.50 \\
7.58 \\
7.58 \\
7.58 \\
7.50 \\
7.33 \\
7.17 \\
7.08 \\
7.00 \\
7.00 \\
7.08 \\
7.17 \\
7.17 \\
7.25 \\
$\cdots . .$.
\end{tabular} & $\begin{array}{l}7.33 \\
7.42 \\
7.42 \\
7.50 \\
7.50 \\
7.58 \\
7.58 \\
7.67 \\
7.67 \\
7.75 \\
7.75 \\
7.75 \\
7.75 \\
7.75 \\
7.75 \\
7.75 \\
7.75 \\
7.67 \\
7.67 \\
7.58 \\
7.50 \\
7.50 \\
7.50 \\
7.50 \\
7.58 \\
7.58 \\
7.67 \\
7.75 \\
7.83 \\
8.00 \\
8.17\end{array}$ & $\begin{array}{l}8.42 \\
8.67 \\
8.67 \\
8.75 \\
8.75 \\
8.83 \\
8.92 \\
8.83 \\
8.75 \\
8.75 \\
8.75 \\
8.75 \\
8.67 \\
8.67 \\
8.58 \\
8.58 \\
8.58 \\
8.58 \\
8.58 \\
8.58 \\
8.50 \\
8.50 \\
8.50 \\
8.50 \\
8.50 \\
8.42 \\
8.33 \\
8.33 \\
8.25 \\
8.17 \\
\ldots \ldots . .\end{array}$ & \begin{tabular}{|l} 
8. 00 \\
7.83 \\
7.67 \\
7.50 \\
7.33 \\
7.08 \\
6.93 \\
6.58 \\
6.33 \\
6.17 \\
6.00 \\
5.83 \\
5.67 \\
5.50 \\
5.25 \\
5.00 \\
4.75 \\
4.58 \\
4.42 \\
4.25 \\
4.17 \\
4.00 \\
4.00 \\
3.83 \\
3.67 \\
3.50 \\
3.42 \\
3.42 \\
3.42 \\
3.33 \\
3.33
\end{tabular} & $\begin{array}{l}3.25 \\
3.25 \\
3.17 \\
3.08 \\
3.00 \\
3.00 \\
2.92 \\
2.83 \\
2.83 \\
2.75 \\
2.75 \\
2.67 \\
2.67 \\
2.58 \\
2.58 \\
2.50 \\
2.42 \\
2.42 \\
2.33 \\
2.33 \\
2.25 \\
2.17 \\
2.17 \\
2.08 \\
2.08 \\
2.08 \\
2.08 \\
2.08 \\
2.00 \\
1.92 \\
1.92\end{array}$ & $\begin{array}{l}1.83 \\
1.83 \\
1.83 \\
1.75 \\
1.75 \\
1.75 \\
1.67 \\
1.67 \\
1.67 \\
1.67 \\
1.67 \\
1.58 \\
1.58 \\
1.58 \\
1.58 \\
1.58 \\
1.58 \\
1.58 \\
1.58 \\
1.58 \\
1.58 \\
1.58 \\
1.50 \\
1.50 \\
1.50 \\
1.50 \\
1.50 \\
1.50 \\
1.42 \\
1.42 \\
\ldots . . .\end{array}$ & $\begin{array}{l}1.42 \\
1.42 \\
1.33 \\
1.33 \\
1.33 \\
1.33 \\
1.33 \\
1.33 \\
1.25 \\
1.25 \\
1.25 \\
1.25 \\
1.25 \\
1.25 \\
1.25 \\
1.25 \\
1.25 \\
1.25 \\
1.25 \\
1.25 \\
1.25 \\
1.17 \\
1.17 \\
1.17 \\
a 1.4 \\
1.45 \\
1.45 \\
1.40 \\
1.40 \\
1.40 \\
1.40\end{array}$ & $\begin{array}{l}\mathbf{1 . 4 0} \\
1.50 \\
1.50 \\
\mathbf{1 . 5 0} \\
\mathbf{1 . 5 5} \\
\mathbf{1 . 5 5} \\
\mathbf{1 . 6 0} \\
1.60 \\
1.65 \\
\mathbf{1 . 6 5} \\
\mathbf{1 . 7 0} \\
\mathbf{1 . 8 0} \\
\mathbf{1 . 8 0} \\
\mathbf{1 . 8 0} \\
\mathbf{2 . 1 0} \\
\mathbf{3 . 1 0} \\
\mathbf{4 . 3 5} \\
\mathbf{5 . 3 0} \\
\mathbf{6 . 0 0} \\
\mathbf{6 . 3 0} \\
\mathbf{6 . 3 0} \\
\mathbf{6 . 3 5} \\
\mathbf{6 . 3 5} \\
\mathbf{6 . 2 0} \\
\mathbf{6 . 2 0} \\
\mathbf{6 . 0 0} \\
\mathbf{5 . 6 0} \\
\mathbf{5 . 5 0} \\
\mathbf{5 . 2 0} \\
\mathbf{5 . 0 0} \\
\cdots \ldots . .\end{array}$ & $\begin{array}{l}4.70 \\
4.60 \\
4.60 \\
4.70 \\
5.20 \\
5.50 \\
5.60 \\
5.85 \\
6.10 \\
6.30 \\
6.50 \\
6.60 \\
6.90 \\
7.20 \\
7.50 \\
7.60 \\
7.60 \\
7.50 \\
7.40 \\
7.20 \\
7.10 \\
7.00 \\
6.70 \\
6.60 \\
6.60 \\
6.20 \\
6.10 \\
6.00 \\
5.80 \\
5.60 \\
5.50\end{array}$ \\
\hline
\end{tabular}

$a$ New gage rod.

Umatilla River at Gibbon, Oregon.

\begin{tabular}{|c|c|c|c|c|c|c|c|c|c|c|c|c|c|}
\hline Day. & July. & Ang. & Sept. & Oct. & Nov. & Dec. & Day. & Jnly. & Ang. & Sept. & Oct. & Nov. & Dec. \\
\hline $\begin{array}{c}1 \ldots \\
2 \ldots \\
3 \ldots \\
4 \ldots \\
5 \ldots \\
6 \ldots \\
7 \ldots \\
8 \ldots \\
9 \ldots \\
10 \ldots \\
11 \ldots \\
12 \ldots \\
13 \ldots \\
14 \ldots \\
15 \ldots \\
16 \ldots\end{array}$ & & $\begin{array}{l}0.80 \\
.75 \\
.75 \\
.90 \\
.85 \\
.85 \\
.85 \\
.85 \\
.85 \\
.85 \\
.85 \\
.85 \\
.85 \\
.85 \\
.85 \\
.80\end{array}$ & $\begin{array}{l}0.80 \\
.80 \\
.80 \\
.80 \\
.80 \\
.80 \\
.80 \\
.80 \\
.80 \\
.80 \\
.90 \\
.90 \\
.85 \\
.85 \\
.85 \\
.85\end{array}$ & $\begin{array}{l}0.80 \\
.80 \\
.80 \\
.80 \\
.80 \\
.80 \\
.80 \\
.80 \\
.80 \\
.90 \\
.90 \\
.85 \\
.85 \\
.85 \\
.85 \\
.80\end{array}$ & $\begin{array}{l}1.00 \\
1.00 \\
1.00 \\
1.10 \\
1.10 \\
1.00 \\
1.00 \\
2.30 \\
1.90 \\
2.20 \\
1.90 \\
1.70 \\
1.70 \\
3.20 \\
4.10 \\
4.00\end{array}$ & $\begin{array}{l}1.60 \\
1.60 \\
1.60 \\
2.05 \\
2.45 \\
2.40 \\
2.30 \\
2.20 \\
2.20 \\
2.30 \\
2.35 \\
3.90 \\
3.80 \\
3.20 \\
2.80 \\
2.55\end{array}$ & $\begin{array}{l}17 \ldots \ldots \\
18 \ldots \ldots \\
19 \ldots \\
20 \ldots \\
21 \ldots \\
22 \ldots \\
23 \ldots \\
24 \ldots \\
26 \ldots \ldots \\
27 \ldots \ldots \\
28 \ldots \\
30 \ldots \ldots \\
31 \ldots \ldots\end{array}$ & \begin{tabular}{c}
$\ldots \ldots$. \\
$\ldots \ldots \ldots$ \\
\hdashline$\ldots \ldots$ \\
\hdashline$\ldots \ldots$ \\
\hdashline 0.85 \\
.90 \\
.85 \\
.85 \\
.85 \\
.85 \\
.85 \\
.85 \\
.80 \\
.80
\end{tabular} & $\begin{array}{l}0.80 \\
.80 \\
.80 \\
.80 \\
.80- \\
.80 \\
.80 \\
.80 \\
.80 \\
.80 \\
.80 \\
.80 \\
.80 \\
.80 \\
.90\end{array}$ & $\begin{array}{r}0.80 \\
.80 \\
.80 \\
.80 \\
.80 \\
.80 \\
.80 \\
.80 \\
.80 \\
.80 \\
.80 \\
.80 \\
.80 \\
.80\end{array}$ & $\begin{array}{r}0.80 \\
.85 \\
.85 \\
.85 \\
.85 \\
.85 \\
.80 \\
.80 \\
.80 \\
.80 \\
.85 \\
.85 \\
.85 \\
.90 \\
1.15\end{array}$ & $\begin{array}{l}4.00 \\
3.80 \\
3.45 \\
3.00 \\
2.70 \\
2.45 \\
2.30 \\
2.10 \\
2.05 \\
1.95 \\
1.80 \\
1.75 \\
1.65 \\
1.60 \\
\cdots . . .\end{array}$ & \begin{tabular}{c}
2.40 \\
2.30 \\
2.25 \\
2.20 \\
2.10 \\
$\dddot{2.05}$ \\
2.00 \\
1.90 \\
\hdashline 1.90 \\
1.85 \\
$\dddot{1.80}$ \\
$\ldots \ldots$
\end{tabular} \\
\hline
\end{tabular}


Sacramento River at Redbluff, California.

\begin{tabular}{|c|c|c|c|c|c|c|c|c|c|c|c|c|}
\hline D & Jan. & Feb. & Mar. & Apr. & May. & June. & July. & Aug. & Sept. & Oet. & Nov. & Dec. \\
\hline 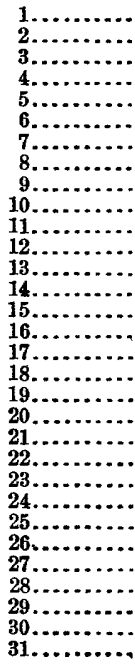 & $\begin{array}{r}1.00 \\
1.00 \\
1.00 \\
1.00 \\
1.00 \\
1.00 \\
1.00 \\
1.00 \\
1.00 \\
1.00 \\
1.00 \\
1.00 \\
1.00 \\
3.20 \\
7.20 \\
11.60 \\
23.50 \\
23.00 \\
22.30 \\
20.90 \\
22.40 \\
19.00 \\
14.80 \\
13.20 \\
19.60 \\
16.00 \\
24.10 \\
19.90 \\
16.10 \\
12.00 \\
9.40\end{array}$ & $\begin{array}{r}10.70 \\
8.90 \\
7.90 \\
6.90 \\
6.20 \\
5.90 \\
\mathbf{5 . 2 0} \\
4.90 \\
4.70 \\
4.60 \\
4.20 \\
4.10 \\
4.00 \\
3.80 \\
3.80 \\
3.70 \\
3.40 \\
3.20 \\
3.10 \\
3.00 \\
\mathbf{2 . 9 0} \\
\mathbf{2 . 9 0} \\
3.90 \\
\mathbf{2 . 7 0} \\
\mathbf{2 . 7 0} \\
\mathbf{2 . 7 0} \\
\mathbf{2 . 7 0} \\
\mathbf{2 . 8 0} \\
\mathbf{2 . 8 0} \\
\ldots . . . \\
\ldots . . . .\end{array}$ & $\begin{array}{r}3.40 \\
3.40 \\
3.30 \\
3.00 \\
3.10 \\
3.80 \\
5.50 \\
5.80 \\
6.20 \\
5.40 \\
4.90 \\
4.40 \\
4.40 \\
4.40 \\
4.20 \\
4.00 \\
4.00 \\
3.90 \\
3.90 \\
4.00 \\
6.60 \\
7.30 \\
\mathbf{9 . 9 0} \\
11.90 \\
\mathbf{1 3 .} 10 \\
13.60 \\
\mathbf{1 5 . 5 0} \\
\mathbf{1 3 . 0 0} \\
10.40 \\
8.50 \\
\mathbf{8 . 2 0}\end{array}$ & $\begin{array}{r}7.40 \\
\mathbf{6 . 9 0} \\
\mathbf{6 . 3 0} \\
\mathbf{6 .} 00 \\
\mathbf{5 . 8 0} \\
\mathbf{7 . 2 0} \\
\mathbf{9 .} 00 \\
8.50 \\
\mathbf{7 . 1 0} \\
\mathbf{7 .} 30 \\
\mathbf{6 . 9 0} \\
\mathbf{6 . 4 0} \\
\mathbf{6 . 1 0} \\
\mathbf{7 . 2 0} \\
\mathbf{6 . 5 0} \\
\mathbf{6 . 1 0} \\
\mathbf{5 . 7 0} \\
\mathbf{5 . 4 0} \\
\mathbf{5 . 5 0} \\
\mathbf{5 . 2 0} \\
\mathbf{5 . 1 0} \\
\mathbf{5 . 2 0} \\
\mathbf{5 . 5 0} \\
\mathbf{1 3 . 7 0} \\
\mathbf{1 5 . 3 0} \\
\mathbf{1 3 . 5 0} \\
\mathbf{1 2 . 7 0} \\
\mathbf{1 1 . 4 0} \\
\mathbf{9 . 5 0} \\
\mathbf{9 . 2 0}\end{array}$ & $\begin{array}{r}9.70 \\
9.20 \\
9.10 \\
16.90 \\
15.30 \\
12.60 \\
10.90 \\
9.80 \\
9.10 \\
8.40 \\
11.50 \\
9.80 \\
8.80 \\
8.10 \\
7.60 \\
7.20 \\
\mathbf{6 .} 80 \\
\mathbf{6 . 5 0} \\
\mathbf{6} .20 \\
\mathbf{5 . 9 0} \\
\mathbf{5 . 8 0} \\
8.30 \\
\mathbf{8 . 1 0} \\
7.40 \\
\mathbf{6 . 6 0} \\
\mathbf{6 . 9 0} \\
\mathbf{6 . 8 0} \\
6.90 \\
\mathbf{6 . 9 0} \\
\mathbf{6 . 8 0} \\
\mathbf{6 . 5 0}\end{array}$ & $\begin{array}{l}6.10 \\
5.90 \\
5.90 \\
5.80 \\
5.80 \\
5.80 \\
5.50 \\
5.40 \\
5.20 \\
4.80 \\
4.40 \\
4.20 \\
4.20 \\
4.20 \\
4.20 \\
4.00 \\
3.90 \\
3.80 \\
3.60 \\
3.40 \\
3.30 \\
3.20 \\
3.10 \\
2.90 \\
2.90 \\
2.80 \\
2.70 \\
2.60 \\
2.50 \\
2.50\end{array}$ & $\begin{array}{r}2.40 \\
2.30 \\
2.30 \\
2.20 \\
2.10 \\
1.90 \\
1.90 \\
1.80 \\
1.70 \\
1.70 \\
1.70 \\
1.70 \\
1.60 \\
1.60 \\
1.60 \\
1.50 \\
1.50 \\
1.40 \\
1.30 \\
1.30 \\
1.30 \\
1.20 \\
1.20 \\
1.10 \\
1.10 \\
1.10 \\
1.00 \\
1.00 \\
.90 \\
.90 \\
. .80\end{array}$ & $\begin{array}{l}0.70 \\
.70 \\
.70 \\
.60 \\
.60 \\
.60 \\
.50 \\
.50 \\
.50 \\
.50 \\
.50 \\
.50 \\
.40 \\
.40 \\
.40 \\
.40 \\
.40 \\
.40 \\
.40 \\
.40 \\
.40 \\
.40 \\
.40 \\
.40 \\
.40 \\
.40 \\
.40 \\
.40 \\
.30 \\
.30 \\
.30\end{array}$ & $\begin{array}{l}0.40 \\
.40 \\
.40 \\
.30 \\
.30 \\
.30 \\
.30 \\
.30 \\
.30 \\
.30 \\
.30 \\
.30 \\
.30 \\
.30 \\
.30 \\
.30 \\
.30 \\
.30 \\
.30 \\
.60 \\
.60 \\
.60 \\
.50 \\
.50 \\
.50 \\
.40 \\
.40 \\
.40 \\
.40 \\
.40 \\
. . .5\end{array}$ & $\begin{array}{r}\mathbf{0 . 4 0} \\
.40 \\
.40 \\
.40 \\
.40 \\
.30 \\
.30 \\
.30 \\
.30 \\
.30 \\
.30 \\
.30 \\
.30 \\
.30 \\
.30 \\
.30 \\
.30 \\
.30 \\
.30 \\
.30 \\
.30 \\
.30 \\
.30 \\
.30 \\
.30 \\
.30 \\
\mathbf{3 . 3 0} \\
.90 \\
.70 \\
.70 \\
.60\end{array}$ & $\begin{array}{r}0.80 \\
.70 \\
.60 \\
.50 \\
.50 \\
.50 \\
.30 \\
.40 \\
.80 \\
.80 \\
.80 \\
.60 \\
.60 \\
.60 \\
.60 \\
1.20 \\
6.60 \\
5.20 \\
3.00 \\
3.40 \\
2.80 \\
2.90 \\
6.70 \\
12.50 \\
7.60 \\
3.90 \\
.3 .20 \\
2.70 \\
3.30 \\
3.30 \\
\ldots \ldots . .\end{array}$ & $\begin{array}{r}3.60 \\
4.50 \\
4.80 \\
4.00 \\
3.90 \\
4.20 \\
4.20 \\
4.00 \\
4.00 \\
4.00 \\
4.60 \\
4.80 \\
6.20 \\
10.60 \\
20.60 \\
14.40 \\
9.70 \\
9.20 \\
8.30 \\
7.30 \\
7.10 \\
7.10 \\
7.60 \\
9.40 \\
8.20 \\
10.40 \\
11.60 \\
16.40 \\
15.20 \\
10.80 \\
9.40\end{array}$ \\
\hline
\end{tabular}

Sacramento River at Jellys Ferry, California.

\begin{tabular}{|c|c|c|c|c|c|c|c|c|c|c|c|c|}
\hline Day & Jan. & eb. & Mar. & pr. & May. & June. & July. & Aug & Sept & Oet & Nov. & Dec \\
\hline $\begin{array}{l}25 . \\
26 . \\
27 . \\
28 . \\
290 .\end{array}$ & \begin{tabular}{|l}
5.70 \\
5.70 \\
5.70 \\
5.70 \\
5.70 \\
5.70 \\
5.70 \\
5.70 \\
5.70 \\
5.70 \\
5.70 \\
5.70 \\
5.90
\end{tabular} & \begin{tabular}{|c}
13.60 \\
12.90 \\
11.90 \\
11.20 \\
10.60 \\
10.10 \\
9.80 \\
9.40 \\
9.10 \\
9.00 \\
8.70 \\
8.60 \\
8.30 \\
8.20 \\
8.10 \\
7.90 \\
7.80 \\
7.70 \\
7.60 \\
7.50 \\
7.50 \\
77.40 \\
7.40 \\
77.30 \\
7.30 \\
7.50 \\
7.90 \\
7.90 \\
\end{tabular} & \begin{tabular}{|r}
7.90 \\
7.80 \\
7.90 \\
77.70 \\
7.70 \\
8.10 \\
9.60 \\
10.30 \\
10.60 \\
9.70 \\
9.30 \\
9.00 \\
8.90 \\
8.80 \\
8.50 \\
8.50 \\
8.40 \\
8.30 \\
8.20 \\
8.80 \\
10.60 \\
11.80 \\
14.10 \\
16.30 \\
17.00 \\
17.80 \\
19.10 \\
16.40 \\
14.60 \\
13.30 \\
12.20 \\
\end{tabular} & $\begin{array}{r}10.50 \\
10.10 \\
9.80 \\
9.60 \\
9.60 \\
9.60 \\
9.30 \\
9.40 \\
9.40 \\
1.00 \\
18.50 \\
16.90 \\
15.80 \\
14.20 \\
13.10 \\
12.90 \\
13.50\end{array}$ & $\begin{array}{l}12.90 \\
13.30 \\
13.40 \\
21.20 \\
19.00 \\
16.50 \\
15.10 \\
14.00 \\
13.10 \\
12.40 \\
14.50 \\
13.70 \\
12.90 \\
12.40 \\
1.90\end{array}$ & $\begin{array}{r}10.30 \\
10.30 \\
10.10 \\
10.10 \\
10.10 \\
10.00 \\
9.80 \\
9.70 \\
9.40 \\
9.20 \\
8.90 \\
8.80 \\
8.60 \\
8.60 \\
8.40 \\
8.30 \\
8.20 \\
8.00 \\
8.00 \\
7.80 \\
7.70 \\
7.60 \\
7.50 \\
7.40 \\
7.30 \\
7.20 \\
7.10 \\
7.10 \\
7.00 \\
7.00\end{array}$ & $\begin{array}{l}90 \\
80 \\
70 \\
70 \\
60 \\
60 \\
60 \\
50 \\
50 \\
50 \\
40 \\
40 \\
40 \\
30 \\
30 \\
30\end{array}$ & $\begin{array}{l}\mathbf{5 . 9 0} \\
\mathbf{5 . 9 0} \\
\mathbf{5 . 9 0} \\
\mathbf{5 . 8 0} \\
\mathbf{5 . 8 0} \\
\mathbf{5 . 8 0} \\
\mathbf{5 . 8 0} \\
\mathbf{5 . 8 0} \\
\mathbf{5 . 8 0} \\
\mathbf{5 . 7 0} \\
\mathbf{5 . 7 0} \\
\mathbf{5 . 7 0} \\
\mathbf{5 . 7 0} \\
\mathbf{5 . 7 0} \\
\mathbf{5 . 7 0} \\
\mathbf{5 . 7 0} \\
\mathbf{5 . 7 0} \\
\mathbf{5 . 7 0} \\
\mathbf{5 . 7 0} \\
\mathbf{5 . 7 0} \\
\mathbf{5 . 6 0} \\
\mathbf{5 . 6 0} \\
\mathbf{5 . 6 0} \\
\mathbf{5 . 6 0} \\
\mathbf{5 . 6 0} \\
\mathbf{5 . 6 0} \\
\mathbf{5 . 7 0}\end{array}$ & \begin{tabular}{|}
$\mathbf{5 . 5 0}$ \\
$\mathbf{5 . 5 0}$ \\
$\mathbf{5 . 5 0}$ \\
$\mathbf{5 . 5 0}$ \\
$\mathbf{5 . 5 0}$ \\
5.70 \\
$\mathbf{5 . 7 0}$ \\
$\mathbf{5 . 7 0}$ \\
5.70 \\
$\mathbf{5 . 7 0}$ \\
$\mathbf{5 . 7 0}$ \\
5.70 \\
5.60 \\
$\mathbf{5 . 6 0}$ \\
$\mathbf{5 . 6 0}$ \\
$\mathbf{5 . 6 0}$
\end{tabular} & $\begin{array}{l}5.60 \\
5.60 \\
5.60 \\
5.60 \\
5.60 \\
5.60 \\
5.50 \\
5.50 \\
5.50 \\
5.50 \\
5.50\end{array}$ & $\begin{array}{l}5.90 \\
5.80 \\
5.80 \\
5.70 \\
5.70 \\
.70 \\
5.70 \\
5.70 \\
6.10 \\
5.90 \\
.90\end{array}$ & $\begin{array}{r}6.80 \\
6.90 \\
7.00 \\
7.00 \\
7.00 \\
7.10 \\
7.10 \\
7.00 \\
6.90 \\
6.80 \\
6.70 \\
7.20 \\
11.20\end{array}$ \\
\hline
\end{tabular}


Stanislaus River at Oakdale, 'California.

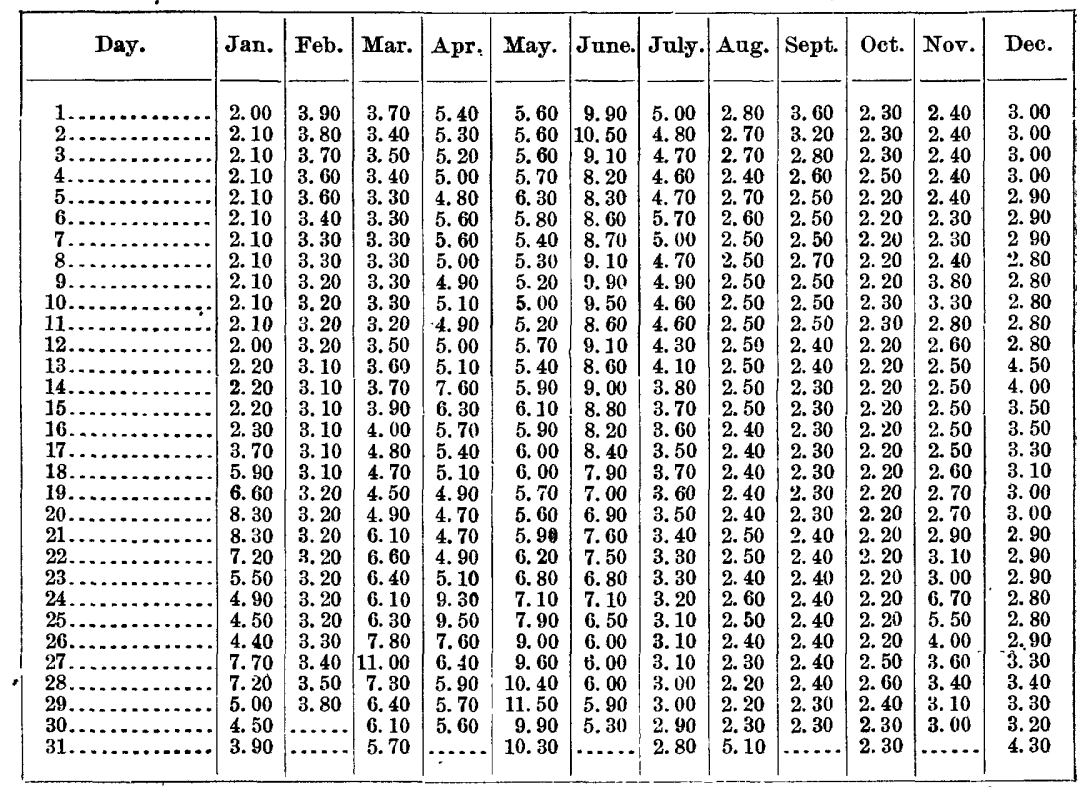

Tuolumne River at Lagrange, California.

\begin{tabular}{|c|c|c|c|c|c|c|c|c|c|c|c|}
\hline Day. & Jan. & Feb. & Apr. & May. & June. & July. & Ang. & Sept. & Oet. & Nov. & Dec. \\
\hline 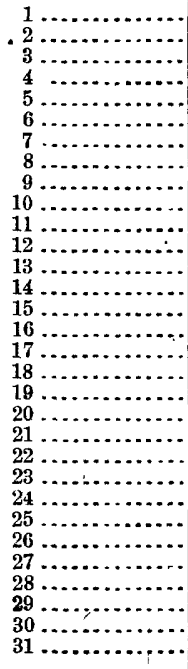 & $\begin{array}{l}4.50 \\
4.50 \\
4.50 \\
4.50 \\
4.50 \\
4.50 \\
4.50 \\
4.50 \\
4.50 \\
4.50 \\
4.50 \\
4.50 \\
4.50 \\
4.50 \\
4.50 \\
4.90 \\
5.60 \\
8.50 \\
7.20 \\
8.30 \\
8.10 \\
7.30 \\
7.00 \\
6.70 \\
6.10 \\
5.60 \\
8.90 \\
7.60 \\
7.50 \\
7.10 \\
6.40\end{array}$ & $\begin{array}{l}5.80 \\
5.80 \\
5.60 \\
5.50 \\
5.50 \\
5.40 \\
5.40 \\
5.30 \\
5.30 \\
5.30 \\
5.30 \\
5.30 \\
5.30 \\
5.30 \\
5.30 \\
5.30 \\
5.30 \\
5.30 \\
5.30 \\
5.30 \\
5.30 \\
5.30 \\
5.30 \\
5.30 \\
5.30 \\
5.30 \\
5.30 \\
5.30 \\
5.30 \\
\ldots \ldots . . \\
\cdots\end{array}$ & 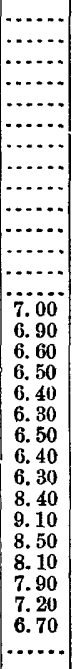 & $\begin{array}{r}6.40 \\
6.50 \\
6.70 \\
6.90 \\
7.00 \\
\mathbf{6 . 8 0} \\
6.70 \\
\mathbf{6 . 7 0} \\
\mathbf{6 . 7 0} \\
\mathbf{6 . 6 0} \\
\mathbf{6 . 7 0} \\
\mathbf{6 . 8 0} \\
\mathbf{6 . 6 0} \\
\mathbf{6 . 5 0} \\
\mathbf{6 . 6 0} \\
\mathbf{6 . 4 0} \\
\mathbf{6 . 4 0} \\
\mathbf{6 . 6 0} \\
\mathbf{6 . 3 0} \\
\mathbf{6 . 5 0} \\
6.80 \\
\mathbf{7 . 0 0} \\
\mathbf{6 . 9 0} \\
\mathbf{7 . 1 0} \\
\mathbf{7 . 8 0} \\
\mathbf{8 . 5 0} \\
\mathbf{8 . 9 0} \\
\mathbf{9 . 2 0} \\
\mathbf{1 0 . 2 0} \\
\mathbf{9 . 6 0} \\
\mathbf{9 . 6 0} \\
\end{array}$ & $\begin{array}{l}9.30 \\
9.30 \\
9.60 \\
9.20 \\
9.30 \\
9.20 \\
9.60 \\
9.20 \\
9.60 \\
9.20 \\
9.20 \\
9.20 \\
9.40 \\
9.20 \\
9.50 \\
9.50 \\
9.20 \\
9.20 \\
9.10 \\
9.80 \\
8.50 \\
8.60 \\
8.60 \\
8.10 \\
8.20 \\
8.00 \\
7.90 \\
7.90 \\
8.10 \\
7.60 \\
\cdots . .2\end{array}$ & $\begin{array}{l}7.40 \\
7.20 \\
7.10 \\
7.20 \\
7.70 \\
7.70 \\
7.50 \\
7.30 \\
7.40 \\
7.40 \\
7.40 \\
7.10 \\
6.80 \\
6.50 \\
6.30 \\
6.30 \\
6.40 \\
6.20 \\
6.10 \\
5.90 \\
5.90 \\
5.80 \\
5.70 \\
5.60 \\
\mathbf{5 . 4 0} \\
\mathbf{5 . 7 0} \\
\mathbf{5 . 6 0} \\
\mathbf{5 . 4 0} \\
\mathbf{5 . 3 0} \\
\mathbf{5 . 2 0} \\
\mathbf{5 . 1 0}\end{array}$ & $\begin{array}{l}5.00 \\
5.00 \\
5.00 \\
4.90 \\
4.80 \\
4.80 \\
4.70 \\
4.70 \\
4.60 \\
4.50 \\
4.50 \\
4.50 \\
4.50 \\
4.50 \\
4.50 \\
4.50 \\
4.40 \\
4.40 \\
4.40 \\
4.50 \\
4.50 \\
4.50 \\
4.50 \\
4.40 \\
4.40 \\
4.40 \\
4.30 \\
4.30 \\
4.30 \\
4.30 \\
6.50\end{array}$ & $\begin{array}{l}5.70 \\
5.20 \\
5.00 \\
4.90 \\
4.80 \\
4.70 \\
5.20 \\
5.10 \\
4.80 \\
4.70 \\
4.60 \\
4.50 \\
4.50 \\
4.40 \\
4.30 \\
4.20 \\
4.20 \\
4.20 \\
4.10 \\
4.10 \\
4.30 \\
4.30 \\
4.50 \\
4.50 \\
4.40 \\
4.30 \\
4.30 \\
4.30 \\
4.30 \\
4.20 \\
\cdots . . .\end{array}$ & $\begin{array}{l}4.20 \\
4.20 \\
4.10 \\
4.10 \\
4.10 \\
4.10 \\
4.00 \\
4.00 \\
4.60 \\
4.00 \\
4.00 \\
4.00 \\
4.00 \\
3.90 \\
3.90 \\
3.90 \\
3.90 \\
3.90 \\
3.90 \\
3.90 \\
3.90 \\
3.90 \\
3.90 \\
3.90 \\
3.90 \\
3.90 \\
4.20 \\
4.50 \\
4.40 \\
4.40 \\
4.40\end{array}$ & 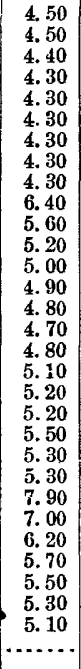 & $\begin{array}{l}\text { 5. } 10 \\
5.20 \\
5.20 \\
5.20 \\
5.20 \\
5.10 \\
5.10 \\
5.00 \\
5.00 \\
5.00 \\
5.00 \\
5.00 \\
6.80 \\
6.10 \\
5.70 \\
5.60 \\
5.40 \\
5.20 \\
5.10 \\
5.10 \\
5.10 \\
5.00 \\
5.00 \\
5.00 \\
4.90 \\
4.90 \\
5.10 \\
5.40 \\
5.50 \\
5.30 \\
5.70\end{array}$ \\
\hline
\end{tabular}


Tuolumne River at Modesto, California.

\begin{tabular}{|c|c|c|c|c|c|c|c|c|c|c|c|c|}
\hline Day. & Jan. & Feb. & Mar. & Apr. & May. & June. & July. & Aug. & Sept. & Oct. & Nov. & Dec. \\
\hline & 3.80 & 6.30 & 6.00 & 7.50 & 7.80 & 16.40 & 10.30 & 5,30 & 7.40 & 3. 70 & 380 & 5.20 \\
\hline & 3.80 & 6.10 & 5. 60 & 7.10 & 7.80 & 16.90 & 9.90 & 5.00 & $\begin{array}{l}7.40 \\
6.00\end{array}$ & $\begin{array}{l}0.60 \\
3.60\end{array}$ & $\begin{array}{l}\text { 5. } 80 \\
\text { 3. } 80\end{array}$ & $\begin{array}{l}0.20 \\
5.10\end{array}$ \\
\hline & $\begin{array}{l}0.70 \\
3.70\end{array}$ & 5.90 & 5.60 & 7.20 & 7.70 & 17.00 & 950 & 4. 80 & 5. 30 & 3.60 & 3. 80 & 5.00 \\
\hline & 3.60 & 5.80 & 5. 30 & 7.10 & 7.90 & 14.80 & 9.60 & 4.80 & 4.90 & 3.50 & 3. 80 & 5. 10 \\
\hline & 3. 60 & 5. 70 . & 5.20 & 6.90 & 8.10 & 13. 80 & 9.50 & 4. 90 & 4.80 & 3. 50 & 3.70 & 5.00 \\
\hline & 3.60 & 5. 50 & 5.10 & 6.70 & 8.70 & 14.30 & 12.50 & 4.90 & 4.40 & 3. 50 & 3.70 & 4. 90 \\
\hline & 3. 70 & 5. 30 & 5. 20 & 7. 20 & 7. 80 & 15.30 & 10.70 & 4. 70 & 4.30 & 3. 50 & 3. 70 & 4. 80 \\
\hline & 3.70 & 5. 30 & 5.30 & 6.80 & 7.50 & 17. 10 & 10.00 & 4.70 & 4. 80 & 3.30 & 3.70 & 4. 70 \\
\hline & 3. 70 & 5. 30 & 5.30 & 6.70 & 7.40 & 17.60 & 10.10 & 4. 60 & 4.70 & 3.30 & 3. 80 & 4. 60 \\
\hline 10. & 3. 70 & 5. 10 & 5.40 & 7.20 & 7.10 & 17.60 & 10.30 & 4. 50 & 4.60 & 3. 30 & 3.80 & 4. 70 \\
\hline 11. & 3. 70 & 5. 30 & 5. 60 & 7.20 & 7.00 & 16.30 & 9.90 & 4.60 & 4. 40 & 3.30 & 7. 20 & 4. 70 \\
\hline 12. & 3.80 & 5. 10 & 5. 80 & 6.70 & 7.70 & 16.80 & 10.50 & 4.50 & 4.30 & 3. 30 & 5. 70 & 4.60 \\
\hline 13. & 3.90 & 5.10 & 6. 00 & 6.80 & 7. 40 & 16. 80 & 9.70 & 4. 50 & 4.10 & 3.20 & 5.20 & 4. 60 \\
\hline 14. & 3.90 & 5. 20 & 6.30 & 7.70 & 7.70 & 16. 80 & 9.30 & 4. 40 & 3.90 & 3.20 & 4. 70 & 8.00 \\
\hline 15. & 4.30 & 5.00 & 6. 60 & 10.20 & 8.20 & 16. 70 & 8.80 & 4.30 & 3.90 & 3. 10 & 4. 50 & 6.40 \\
\hline 16. & 4. 10 & 5. 00 & 6.60 & 8.00 & 8.50 & 16. 70 & 8. 30 & 4. 20 & 3.90 & 3. 10 & 4. 40 & 6. 30 \\
\hline 17. & 4.40 & 5.00 & 6. 60 & 7.40 & 8.10 & 16. 60 & 8.20 & 4. 20 & 3.70 & 3.00 & 4.40 & 5. 50 \\
\hline 18. & 10.80 & 5. 20 & 6. 40 & 6.80 & 8.40 & 16.70 & 8.00 & 4. 10 & 3.70 & 3.00 & 4. 40 & 5. $\mathbf{3 0}$ \\
\hline 19. & 15.30 & 5. 30 & 6. 40 & 6.70 & 8. 30 & 15.00 & 7.90 & 4. 10 & 3.70 & 3.00 & 4. 70 & 5.20 \\
\hline 20. & 9.20 & 5. 30 & 6. 60 & 6. 70 & 7.70 & 13.90 & 7.40 & 4. 10 & 3.60 & 3. 00 & 4. 90 & 4. 80 \\
\hline 21. & 15. 20 & 5. 40 & 7.80 & 6.40 & 7.70 & 13.70 & 7.20 & 4. 10 & 3.50 & 3. 20 & 4. 90 & 4.60 \\
\hline 22 & 12.80 & 5.40 & 7.50 & 6.50 & 8.50 & 13.80 & 7.00 & 4.00 & 3.70 & 3.20 & 5. 40 & 4. 60 \\
\hline 23 . & 10.30 & 5. 10 & 8.00 & 7.00 & 8.50 & 13.30 & 6.80 & 4. 00 & 3.80 & 3.20 & 5. 30 & 4. 40 \\
\hline 24. & 8. 10 & 5. 10 & 8.20 & 6.80 & 9.00 & 13.50 & 6.70 & 4. 00 & 3. 80 & 3.20 & 7.00 & 4. 30 \\
\hline & 6. 90 & 5.00 & 8.00 & 17.70 & 10.40 & 13.50 & 6. 40 & 4.00 & 4.00 & 3. 20 & 11. 70 & 4. 30 \\
\hline & 7.00 & 5. 40 & 8.40 & 11. 70 & 11.90 & 12.30 & 6. 30 & 4. 00 & 3. 90 & 3.20 & 8.70 & 4. 30 \\
\hline & 9.30 & 5. 50 & 15.10 & 10. 70 & 13.40 & 11.70 & 6. 70 & 4. 00 & 3. 80 & 3.40 & 6. 50 & 4. 70 \\
\hline & 16.70 & 5.50 & 11.90 & 9.70 & 14.70 & 11. 50 & 6.50 & 3.90 & 3.80 & 4.20 & 5. 70 & 4.90 \\
\hline & 9.90 & 5.80 & 9.50 & 8.30 & 16.70 & 11.70 & 6.10 & 3. 80 & 3. 80 & 4.00 & 5.50 & 5.80 \\
\hline & 8. 30 & $\ldots$ & 8.40 & 8.40 & 17.60 & 11.00 & 5. 80 & 3.80 & 3.70 & 3. 80 & 5. 30 & 5. 50 \\
\hline 31. & 6.80 & $\cdots$ & 8.00 & .... & 16. 10 & ...... & 5. 60 & 4. 70 & ....... & 3.80 & ...... & 6. 00 \\
\hline
\end{tabular}

San Joaquin River at Herndon, California.

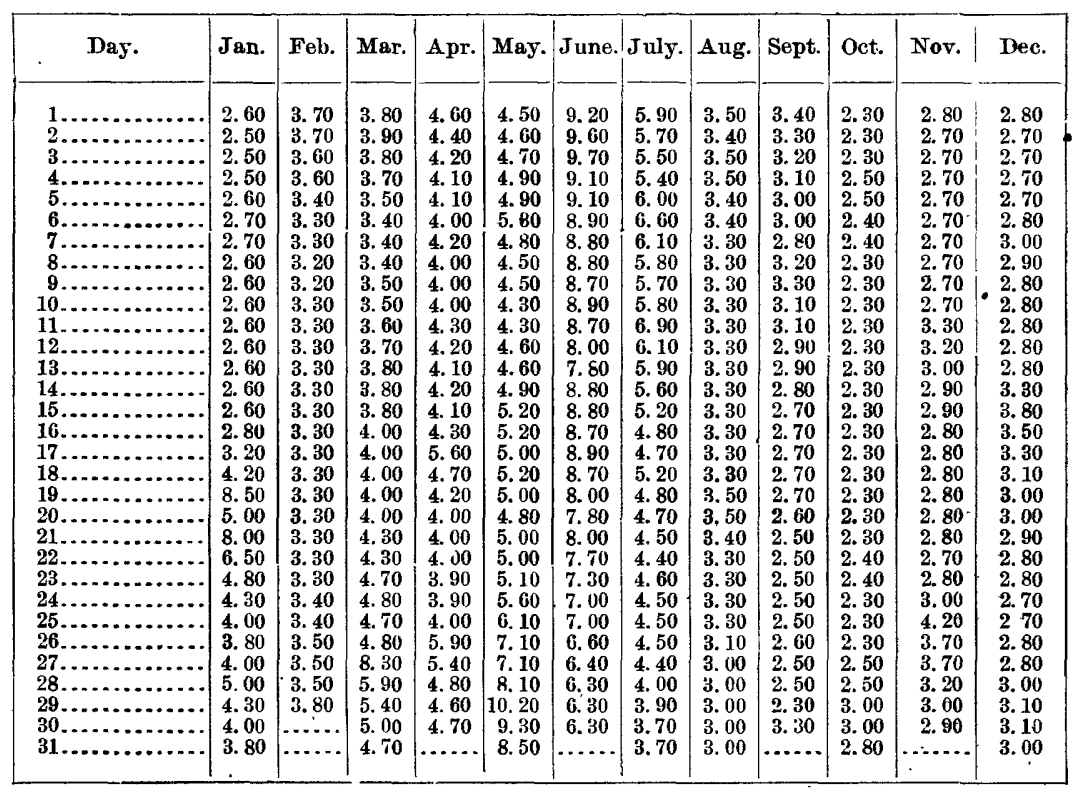


Kings River at Red Mountain, California.

\begin{tabular}{|c|c|c|c|c|c|c|c|c|c|c|c|c|}
\hline I & Jan. & reb. & Mar. & Apr. & May. & June. & July. & Aug. & Sept. & Oet. & Nov. & Dee. \\
\hline 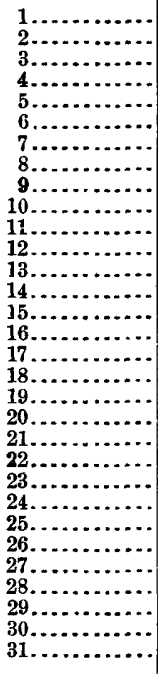 & $\begin{array}{l}4.00 \\
4.00 \\
4.00 \\
4.00 \\
4.00 \\
4.00 \\
4.00 \\
4.00 \\
4.00 \\
4.00 \\
4.00 \\
4.00 \\
4.00 \\
4.00 \\
4.00 \\
4.30 \\
4.90 \\
9.20 \\
7.00 \\
6.40 \\
9.80 \\
7.10 \\
6.40 \\
5.90 \\
5.70 \\
5.70 \\
5.50 \\
6.00 \\
5.70 \\
5.40 \\
5.30\end{array}$ & \begin{tabular}{|l|}
$\mathbf{5 . 3 0}$ \\
$\mathbf{5 . 1 0}$ \\
$\mathbf{5 . 1 0}$ \\
$\mathbf{5 . 1 0}$ \\
$\mathbf{5 . 0 0}$ \\
$\mathbf{4 . 9 0}$ \\
$\mathbf{4 . 9 0}$ \\
$\mathbf{4 . 9 0}$ \\
$\mathbf{4 . 9 0}$ \\
4.80 \\
$\mathbf{4 . 8 0}$ \\
$\mathbf{4 . 8 0}$ \\
$\mathbf{4} .80$ \\
4.80 \\
4.80 \\
$\mathbf{4 . 9 0}$ \\
$\mathbf{4 . 9 0}$ \\
$\mathbf{4 . 9 0}$ \\
$\mathbf{4 . 9 0}$ \\
$\mathbf{5 . 0 0}$ \\
$\mathbf{5 . 0 0}$ \\
4.90 \\
4.90 \\
$\mathbf{4 . 9 0}$ \\
$\mathbf{5 . 1 0}$ \\
$\mathbf{5 . 1 0}$ \\
$\mathbf{5 . 1 0}$ \\
$\mathbf{5 . 5 0}$ \\
$\mathbf{5 . 5 0}$
\end{tabular} & $\begin{array}{l}\text { 5.30 } \\
\mathbf{5 . 1 0} \\
\mathbf{5 . 3 0} \\
\mathbf{5 . 3 0} \\
\mathbf{5 . 3 0} \\
\mathbf{5 . 3 0} \\
\mathbf{5 . 2 0} \\
5.30 \\
5.40 \\
\mathbf{5 . 5 0} \\
\mathbf{5 . 7 0} \\
\mathbf{5 . 9 0} \\
\mathbf{6 .} 00 \\
\mathbf{6 . 0 0} \\
5.90 \\
\mathbf{5 . 8 0} \\
\mathbf{5 . 8 0} \\
6.00 \\
6.00 \\
6.30 \\
6.40 \\
6.40 \\
6.70 \\
6.90 \\
8.80 \\
8.00 \\
7.20 \\
6.50 \\
6.00 \\
5.50 \\
5.00\end{array}$ & $\begin{array}{l}5.00 \\
5.20 \\
5.30 \\
5.40 \\
5.50 \\
6.00 \\
6.50 \\
7.00 \\
7.50 \\
8.00 \\
7.00 \\
6.40 \\
6.90 \\
7.50 \\
7.10 \\
6.00 \\
5.00 \\
4.00 \\
4.50 \\
5.00 \\
5.00 \\
5.00 \\
5.50 \\
6.00 \\
6.50 \\
8.00 \\
8.00 \\
7.50 \\
6.00 \\
6.00 \\
\ldots . . .\end{array}$ & $\begin{array}{l}6.00 \\
6.00 \\
6.00 \\
7.00 \\
7.00 \\
8.00 \\
8.00 \\
7.00 \\
6.00 \\
5.50 \\
5.50 \\
5.50 \\
5.50 \\
7.00 \\
7.00 \\
8.00 \\
8.00 \\
8.00 \\
8.00 \\
8.00 \\
8.00 \\
8.00 \\
8.00 \\
8.00 \\
9.00 \\
10.00 \\
11.00 \\
12.00 \\
12.00 \\
11.00 \\
10.00\end{array}$ & $\begin{array}{r}10.40 \\
10.40 \\
10.40 \\
10.40 \\
10.40 \\
10.40 \\
10.40 \\
10.40 \\
10.40 \\
11.40 \\
11.40 \\
10.90 \\
10.70 \\
10.50 \\
10.60 \\
10.80 \\
10.90 \\
11.00 \\
10.70 \\
10.40 \\
10.10 \\
9.80 \\
9.50 \\
9.20 \\
8.90 \\
8.80 \\
8.80 \\
8.80 \\
8.50 \\
8.80 \\
\ldots \ldots . .\end{array}$ & $\begin{array}{l}7.90 \\
7.60 \\
8.00 \\
8.40 \\
8.70 \\
8.70 \\
8.70 \\
8.70 \\
8.60 \\
8.60 \\
8.60 \\
8.20 \\
7.80 \\
7.30 \\
7.20 \\
7.10 \\
7.00 \\
6.90 \\
6.70 \\
6.50 \\
6.50 \\
8.50 \\
7.50 \\
7.00 \\
6.50 \\
6.30 \\
6.20 \\
6.00 \\
5.80 \\
5.60 \\
5.60\end{array}$ & $\begin{array}{l}5.60 \\
5.50 \\
5.50 \\
5.30 \\
5.20 \\
5.10 \\
5.00 \\
5.00 \\
4.90 \\
4.90 \\
4.90 \\
4.80 \\
4.80 \\
4.80 \\
4.80 \\
4.80 \\
4.80 \\
4.90 \\
4.90 \\
5.00 \\
5.00 \\
5.00 \\
4.80 \\
4.80 \\
4.70 \\
4.70 \\
4.70 \\
4.60 \\
4.60 \\
4.60 \\
4.50\end{array}$ & $\begin{array}{l}4.50 \\
4.40 \\
4.40 \\
4.40 \\
4.40 \\
4.50 \\
4.50 \\
4.50 \\
4.50 \\
4.50 \\
4.50 \\
4.40 \\
4.30 \\
4.30 \\
4.20 \\
4.10 \\
4.10 \\
4.10 \\
4.00 \\
4.00 \\
4.00 \\
4.10 \\
4.20 \\
4.20 \\
4.20 \\
4.10 \\
4.10 \\
4.10 \\
4.00 \\
4.00\end{array}$ & $\begin{array}{l}3.90 \\
3.90 \\
3.90 \\
3.90 \\
3.90 \\
3.80 \\
3.80 \\
3.80 \\
3.80 \\
3.80 \\
3.80 \\
3.80 \\
3.80 \\
3.80 \\
3.80 \\
3.80 \\
3.80 \\
3.80 \\
3.80 \\
3.80 \\
3.80 \\
3.80 \\
3.80 \\
3.80 \\
3.80 \\
3.90 \\
4.30 \\
4.30 \\
4.30 \\
4.30 \\
4.30\end{array}$ & $\begin{array}{l}4.20 \\
4.20 \\
4.20 \\
4.10 \\
4.10 \\
4.10 \\
4.00 \\
4.00 \\
4.30 \\
4.60 \\
4.90 \\
4.60 \\
4.20 \\
4.40 \\
4.30 \\
4.30 \\
4.20 \\
4.10 \\
4.10 \\
4.10 \\
4.10 \\
4.10 \\
4.60 \\
5.00 \\
5.40 \\
4.80 \\
4.60 \\
4.40 \\
4.20 \\
4.90 \\
\cdots . . .\end{array}$ & $\begin{array}{l}4.30 \\
4.30 \\
4.30 \\
4.30 \\
4.30 \\
4.30 \\
4.30 \\
4.30 \\
4.30 \\
4.30 \\
4.30 \\
4.30 \\
4.20 \\
4.20 \\
4.30 \\
4.40 \\
4.40 \\
4.40 \\
4.30 \\
4.20 \\
4.20 \\
4.20 \\
4.20 \\
4.20 \\
4.20 \\
4.20 \\
4.20 \\
4.20 \\
4.20 \\
4.30 \\
4.30\end{array}$ \\
\hline
\end{tabular}

Figures in italies are estimated from observations taken at Kingsburg, on the same river, and from other rod readings at Red Mountain.

Kings River at Kingsburg, California.

\begin{tabular}{|c|c|c|c|c|c|c|c|c|c|c|c|c|}
\hline Day. & Jan. & Feb. & Mar. & Apr. & May. & June. & Juls. & Aug. & Sept. & Oct. & Nov. & Dec. \\
\hline 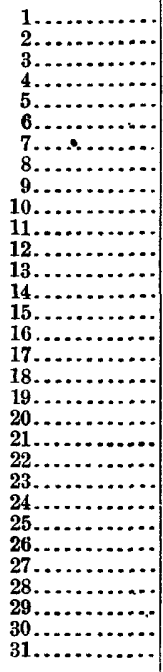 & $\begin{array}{l}2.20 \\
2.20 \\
2.20 \\
2.20 \\
2.20 \\
2.20 \\
2.20 \\
2.20 \\
2.20 \\
2.20 \\
2.10 \\
2.10 \\
2.10 \\
2.20 \\
2.20 \\
2.20 \\
3.30 \\
5.40 \\
8.70 \\
6.50 \\
8.00 \\
7.70 \\
6.50 \\
6.20 \\
5.90 \\
5.90 \\
5.80 \\
6.10 \\
6.10 \\
5.80 \\
5.50\end{array}$ & $\begin{array}{l}\text { 5. } 20 \\
5.10 \\
5.00 \\
4.80 \\
4.60 \\
4.60 \\
4.50 \\
4.20 \\
3.80 \\
3.70 \\
3.60 \\
3.50 \\
3.30 \\
3.10 \\
2.80 \\
2.80 \\
2.70 \\
3.00 \\
3.00 \\
3.00 \\
2.90 \\
2.90 \\
2.60 \\
2.40 \\
2.50 \\
2.50 \\
2.50 \\
2.60 \\
\text { 3. } 00 \\
\ldots . . . \\
\cdots . .-\end{array}$ & $\begin{array}{l}4.10 \\
4.30 \\
5.10 \\
5.10 \\
5.10 \\
4.60 \\
3.60 \\
3.10 \\
2.90 \\
2.80 \\
2.90 \\
3.10 \\
3.50 \\
3.60 \\
3.80 \\
3.60 \\
3.20 \\
3.30 \\
3.70 \\
3.70 \\
4.20 \\
4.50 \\
4.50 \\
5.00 \\
5.80 \\
5.50 \\
8.00 \\
6.30 \\
5.80 \\
5.20 \\
4.80\end{array}$ & $\begin{array}{l}4.30 \\
4.10 \\
4.00 \\
3.90 \\
3.80 \\
3.30 \\
3.10 \\
3.00 \\
3.00 \\
6.00 \\
5.70 \\
4.60 \\
4.00 \\
3.00 \\
6.10 \\
4.60 \\
3.70 \\
3.10 \\
3.00 \\
4.30 \\
4.20 \\
4.00 \\
3.40 \\
\text { 3. 30 } \\
5.00 \\
7.20 \\
6.60 \\
5.70 \\
4.70 \\
4.80 \\
\ldots \ldots . .\end{array}$ & $\begin{array}{r}4.00 \\
4.40 \\
4.80 \\
5.40 \\
5.90 \\
6.50 \\
5.90 \\
5.00 \\
4.60 \\
4.20 \\
3.70 \\
4.00 \\
4.70 \\
5.30 \\
6.20 \\
6.30 \\
5.80 \\
6.20 \\
6.20 \\
5.90 \\
6.20 \\
6.40 \\
6.10 \\
6.60 \\
7.40 \\
8.40 \\
9.30 \\
10.30 \\
11.10 \\
10.20 \\
9.80 \\
\end{array}$ & $\begin{array}{r}10.30 \\
10.30 \\
9.90 \\
9.30 \\
9.30 \\
9.30 \\
9.60 \\
10.30 \\
10.50 \\
10.70 \\
9.90 \\
10.00 \\
9.90 \\
9.60 \\
9.50 \\
9.30 \\
9.80 \\
9.30 \\
8.70 \\
8.40 \\
8.50 \\
8.00 \\
7.70 \\
7.20 \\
7.00 \\
6.80 \\
6.70 \\
6.70 \\
6.70 \\
6.50 \\
\ldots . . .\end{array}$ & $\begin{array}{l}6.50 \\
6.00 \\
6.10 \\
6.00 \\
6.70 \\
7.10 \\
6.70 \\
6.50 \\
6.30 \\
6.40 \\
6.70 \\
6.50 \\
6.30 \\
6.10 \\
5.90 \\
5.50 \\
5.50 \\
5.60 \\
5.60 \\
5.00 \\
4.70 \\
4.60 \\
4.30 \\
6.40 \\
5.70 \\
5.40 \\
4.90 \\
4.30 \\
\text { 3. } 90 \\
3.70 \\
\text { 3. } 60\end{array}$ & $\begin{array}{l}3.50 \\
3.30 \\
3.30 \\
3.30 \\
3.30 \\
3.30 \\
3.20 \\
3.20 \\
3.20 \\
3.20 \\
3.30 \\
3.60 \\
3.80 \\
3.80 \\
3.80 \\
3.70 \\
3.70 \\
3.70 \\
3.80 \\
3.90 \\
3.80 \\
3.80 \\
3.70 \\
3.70 \\
3.70 \\
3.70 \\
3.70 \\
3.70 \\
3.70 \\
3.70 \\
3.70 \\
3.70\end{array}$ & \begin{tabular}{|l|}
3.70 \\
3.70 \\
3.70 \\
3.70 \\
3.70 \\
3.70 \\
3.70 \\
3.70 \\
3.80 \\
3.80 \\
3.70 \\
3.80 \\
3.70 \\
3.60 \\
3.60 \\
3.50 \\
3.50 \\
3.50 \\
3.50 \\
3.50 \\
3.50 \\
3.50 \\
3.40 \\
3.40 \\
3.40 \\
3.40 \\
4.10 \\
4.30 \\
4.30 \\
4.30 \\
$\ldots . .$. \\
\end{tabular} & $\begin{array}{l}4.40 \\
4.50 \\
4.50 \\
4.50 \\
4.50 \\
4.60 \\
4.60 \\
4.60 \\
4.50 \\
4.50 \\
4.50 \\
4.50 \\
4.60 \\
4.50 \\
4.50 \\
4.50 \\
4.30 \\
3.80 \\
3.30 \\
3.30 \\
3.30 \\
3.30 \\
3.10 \\
3.10 \\
3.00 \\
3.00 \\
3.50 \\
4.00 \\
4.50 \\
4.40 \\
4.40\end{array}$ & $\begin{array}{r}4.40 \\
4.30 \\
4.30 \\
4.20 \\
4.00 \\
3.80 \\
3.80 \\
3.70 \\
3.70 \\
3.60 \\
4.00 \\
4.90 \\
4.50 \\
4.30 \\
4.00 \\
3.90 \\
3.70 \\
3.50 \\
3.50 \\
3.70 \\
3.70 \\
3.60 \\
3.70 \\
3.80 \\
4.70 \\
5.30 \\
4.70 \\
4.60 \\
4.50 \\
4.40 \\
\ldots \ldots . .\end{array}$ & $\begin{array}{l}4.40 \\
4.50 \\
4.50 \\
4.50 \\
4.50 \\
4.50 \\
4.50 \\
4.40 \\
4.30 \\
4.30 \\
4.40 \\
4.50 \\
4.30 \\
4.80 \\
3.50 \\
3.20 \\
3.30 \\
3.70 \\
4.00 \\
4.00 \\
4.10 \\
4.10 \\
4.00 \\
4.00 \\
3.80 \\
3.80 \\
3.90 \\
4.20 \\
4.40 \\
4.30 \\
4.20\end{array}$ \\
\hline
\end{tabular}


Santa Ana River at Warmsprings, California.

\begin{tabular}{|c|c|c|c|c|c|c|}
\hline Day. & July. & Ang. & Sept. & Oet. & Nov. & Dec. \\
\hline 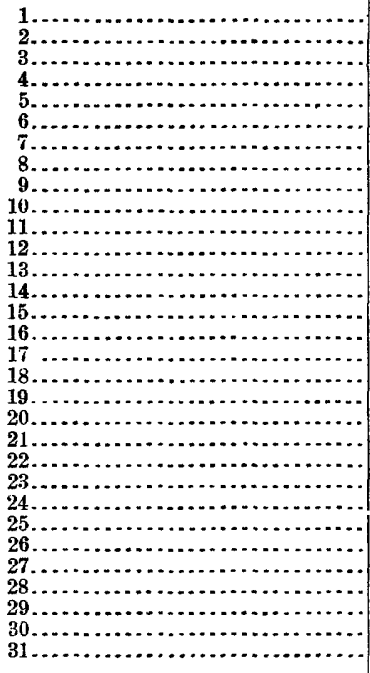 & $\begin{array}{l}14.50 \\
14.50 \\
15.00 \\
15.00 \\
15.00 \\
15.00 \\
14.00 \\
14.00 \\
15.50 \\
15.70 \\
15.50 \\
16.30 \\
16.50 \\
14.50 \\
15.50 \\
15.00 \\
16.00 \\
16.00 \\
16.00 \\
16.00 \\
16.00 \\
16.50 \\
16.50 \\
16.50 \\
15.50 \\
14.00 \\
15.00 \\
15.00 \\
16.50 \\
15.70 \\
16.20\end{array}$ & $\begin{array}{r}16.20 \\
15.30 \\
16.00 \\
15.50 \\
15.70 \\
15.30 \\
16.70 \\
15.30 \\
15.30 \\
15.30 \\
15.50 \\
15.20 \\
15.50 \\
15.20 \\
15.20 \\
\text { a 15. } 20 \\
13.20 \\
13.00 \\
16.00 \\
14.00 \\
14.20 \\
14.30 \\
12.00 \\
12.20 \\
12.70 \\
13.20 \\
13.20 \\
14.30 \\
16.00 \\
16.00 \\
14.20\end{array}$ & $\begin{array}{l}16.00 \\
16.20 \\
16.00 \\
14.50 \\
14.50 \\
12.50 \\
15.50 \\
15.50 \\
15.30 \\
15.20 \\
11.00 \\
11.30 \\
13.50 \\
15.00 \\
15.00 \\
16.00 \\
16.50 \\
16.30 \\
16.00 \\
15.50 \\
14.50 \\
14.30 \\
13.50 \\
13.30 \\
14.00 \\
14.70 \\
13.00 \\
13.50 \\
14.00 \\
15.00 \\
\ldots . . . .\end{array}$ & $\begin{array}{r}15.50 \\
16.50 \\
15.30 \\
15.00 \\
13.70 \\
13.50 \\
14.50 \\
14.50 \\
14.00 \\
15.00 \\
15.50 \\
15.00 \\
14.00 \\
14.00 \\
14.20 \\
13.00 \\
14.00 \\
14.30 \\
14.30 \\
14.30 \\
12.70 \\
13.50 \\
13.30 \\
13.00 \\
13.00 \\
13.00 \\
a 21.00 \\
14.00 \\
14.00 \\
14.00 \\
14.00\end{array}$ & $\begin{array}{r}12.00 \\
12.00 \\
12.00 \\
12.00 \\
12.00 \\
12.00 \\
11.50 \\
11.50 \\
11.50 \\
11.50 \\
11.50 \\
11.70 \\
11.50 \\
11.50 \\
11.50 \\
11.50 \\
11.50 \\
11.50 \\
11.50 \\
11.50 \\
11.70 \\
11.80 \\
12.00 \\
12.00 \\
14.30 \\
12.50 \\
12.50 \\
12.30 \\
12.30 \\
12.30 \\
\cdots \ldots . .\end{array}$ & $\begin{array}{l}12.00 \\
12.00 \\
12.30 \\
12.30 \\
12.30 \\
12.30 \\
12.30 \\
12.30 \\
12.30 \\
12.30 \\
12.30 \\
12.30 \\
12.30 \\
12.30 \\
12.30 \\
12.50 \\
12.50 \\
12.50 \\
12.50 \\
12.50 \\
12.50 \\
12.50 \\
12.20 \\
12.20 \\
12.30 \\
12.00 \\
12.00 \\
13.00 \\
13.00 \\
12.70 \\
12.30\end{array}$ \\
\hline
\end{tabular}

$a$ On August 16 the discharge gates of Bear Valley Dam were temporarily closed on account of a storm, and on Oetober 27 ther were closed for the season. Discharge of Santa Ana Canal is not included in the above statement.

\section{CURRENT METERS RATED IN 1896.}

No. 16. Large Haskell meter. Property of B. M. Hall, Atlanta, Ga. Last rated July 14, 1896, and returned to B. M. Hall July 17, 1896.

No. 25. Small Haskell meter. Returned to office December 12, 1895; rated July 27, 1896, by A. P. Davis at the Kensington station, Md.; sent to Prof. A. L. Lamb, Port Deposit, Md., November 12, 1896.

No. 27. Small Haskell meter. Rated June 27, 1896, by A. P. Davis at Kensington station, Md, sent to Oskar Huber, Spokane, Wash., October $20,1896$.

No. 63. Large Price electric meter. Rated by J. B. Lippincott and C. C. Babb June 18, 1896, atrating station, Kern County, Cal.; transferred by J.B. Lippincott to C. C. Babb and used by him during sum. mer of 1896; last rated December 10, 1896, by C. C. Babb at Kensington station, $\mathrm{Md}$.

No. 67. Large Price electric meter. Purchased July 2, 1896; rated July 14; sent to W. Richins, A rizona, July 27,1896; was damaged in shipment and returned to this office for repairs August 26, 1896; repaired and rated September 25, at Kensington, Md. Sent to J. B. Lippincott, Los Angeles, Cal. Sepember 26, 1896.

No. 68. Large Price electric meter. Purchased July 2, 1896; rated July 28, at Kensington station, Md., and used during the season of 1896 by E. G. Paul.

No. 69. Large Price electric meter. Purchased July 20,1896; roted July 28, 1896, by E. G. Paul at Kensington station, Md., and sent August 26,1896, to W. Richins for use in Queen Creek, Ariz.

No. 70. Large Price electric meter. Received December 15, 1896, to replace No. 62, large Price electric meter, which was destroyed by fire while in charge of a transportation company. Rated December 15, 1896, by E. G. Paul at Kensington station, Md.

No. 91. Small Price electric meter. Purchased November 12, 1896; rated December 10, 1896, at Kensington station, Md., by C. C. Babb and E. G. Panl. 
Rating tables made in 1896 for current meters.

\begin{tabular}{|c|c|c|c|c|c|c|c|c|c|c|c|}
\hline \multirow{2}{*}{$\begin{array}{l}\text { Revolutions } \\
\text { per second. }\end{array}$} & \multicolumn{11}{|c|}{ Velocities in feet per second. } \\
\hline & No. 16. & $\begin{array}{l}\text { No. } 25 \\
\text { H.P. }\end{array}$ & $\begin{array}{l}\text { No. } 25 \\
\text { L.P. }\end{array}$ & $\begin{array}{l}\text { No. } 27 \\
\text { H. P. }\end{array}$ & $\begin{array}{l}\text { No. } 27 \\
\text { L. P. }\end{array}$ & No. 63. & No. 67 . & No. 68. & No. 69. & No. 70. & No.91. \\
\hline 0.00. & 0.27 & 0.67 & 1.01 & 0.63 & 0.70 & 0.40 & 0.25 & 0.20 & 0.25 & 0.20 & 0.10 \\
\hline $.10 .$. & .33 & .73 & 1.12 & .70 & .83 & .63 & .45 & .51 & .53 & .35 & .25 \\
\hline $.20 .$. & .42 & .80 & 1.23 & .76 & .96 & .91 & .72 & .81 & .81 & .65 & .51 \\
\hline .30. & .49 & .86 & 1.35 & .82 & 1. 09 & 1.19 & 1.00 & 1.12 & 1.09 & .97 & .77 \\
\hline .40. & .57 & .93 & 1.46 & .90 & 1.27 & 1.47 & 1. 30 & 1.42 & 1. 37 & 1.26 & 1.03 \\
\hline $.50 \ldots$ & .69 & 1.03 & 1.58 & .99 & 1.44 & 1.75 & 1.60 & 1.73 & 1.65 & 1. 55 & 1. 29 \\
\hline .60. & .80 & 1. 13 & 1.72 & 1.07 & 1.61 & 2.03 & 1.90 & 2.04 & 1.93 & 1.85 & 1.55 \\
\hline .70. & .92 & 1.23 & 1.87 & 1. 15 & 1.79 & 2.31 & 2.19 & 2.36 & 2.21 & 2. 14 & 1. 81 \\
\hline .80. & 1.04 & 1.34 & 2.01 & 1.25 & 1.98 & 2.59 & 2.48 & 2.67 & 2.49 & 2.44 & 2.07 \\
\hline $.90 .$. & 1.16 & 1.44 & 2.16 & 1.33 & 2.19 & 2.87 & 2.77 & 2.98 & 2.77 & 2.73 & 2.33 \\
\hline 1.00. & 1.28 & 1.55 & 2.33 & 1.42 & 2.40 & 3.15 & 3.06 & 3.29 & 3.04 & 3.03 & 2.59 \\
\hline 1. 10. & 1.40 & 1.65 & 2.50 & 1.51 & 2.62 & 3.43 & 3.35 & 3.59 & 3.32 & 3. 33 & 2.85 \\
\hline 1.20. & 1.52 & 1.75 & 2.68 & 1.60 & 2.83 & 3. 71 & 3. 64 & 3.90 & 3.60 & 3.62 & 3.11 \\
\hline 1.30. & 1.64 & 1.85 & 2.86 & 1.69 & 3.04 & 3.99 & 3.93 & 4.19 & 3.88 & 3.92 & 3. 37 \\
\hline 1.40. & 1.76 & 1.95 & 3.03 & 1. 77 & 3.25 & 4.27 & 4.23 & 4.48 & 4.16 & 4.22 & 3.63 \\
\hline $1.50 \ldots$ & 1.88 & 2.05 & 3.19 & 1. 86 & 3.46 & 4. 55 & 4.53 & 4.77 & 4.44 & 4.51 & 3.00 \\
\hline 1.60. & 2.00 & 2.15 & 3.36 & 1.97 & 3.67 & 4.83 & 4.82 & 5.06 & 4. 72 & 4.81 & 4. 16 \\
\hline 1.70. & 2. $1 \hat{2}$ & 2.26 & 3.54 & 2.07 & 3.88 & 5.11 & 5.11 & 5. 36 & 5.00 & 5.11 & 4.42 \\
\hline 1.80. & 2.24 & 2.36 & 3.72 & 2.18 & 4.09 & 5. 39 & 5.40 & 5.65 & 5. 28 & 5.40 & 4.68 \\
\hline $1.90 \ldots$ & 2.36 & 2.48 & 3.90 & 2.29 & 4. 30 & 5.67 & 5.70 & 5.94 & 5.56 & 5. 70 & 4.94 \\
\hline 2.00. & 2.48 & 2.59 & 4.07 & 2.39 & 4.51 & 5.95 & 6.00 & 6.23 & 5.84 & 6.00 & 5. 20 \\
\hline $2.10 \ldots$ & 2.60 & 2. 71 & 4. 24 & 2.49 & 4.72 & 6. 23 & 6.29 & 6. 52 & 6.12 & 6. 29 & ร. 63 \\
\hline 2.20. & 2.72 & 2.83 & 4.42 & 2.60 & 4.93 & 6.51 & 6.58 & 6.81 & 6.40 & 6.58 & 6.07 \\
\hline 2.30. & 2.84 & 2.95 & 4. 59 & 2.71 & 5.14 & 6.79 & 6.87 & 7.10 & 6. 68 & 6.88 & 6.50 \\
\hline 2.40. & 2.96 & 3.07 & 4. 77 & 2.81 & 5.35 & 7.07 & 7.17 & 7.39 & 6.96 & 7.17 & 7. 60 \\
\hline 2.50 & 3.08 & 3. 19 & 4. 95 & 2.92 & 5.56 & 7.35 & 7.47 & 7.68 & 7.24 & 7.46 & 8.80 \\
\hline 2.60. & 3.20 & 3.31 & 5.13 & 3.02 & 5.77 & 7.63 & 7.77 & 7.97 & 7.52 & 7.76 & 10. 30 \\
\hline $2.70 \ldots$ & 3,32 & 3.43 & 5. 31 & 3. 12 & 5.98 & 7. 91 & 8.06 & 8.26 & 7.80 & 8.05 & 11.60 \\
\hline $2.80 .$. & 3.44 & 3.55 & 5.49 & 3. 23 & 6.18 & 8.19 & 8.36 & 8.55 & 8.08 & 8.34 & \\
\hline 2.90 & 3.56 & 3. 67 & 5.67 & 3.34 & 6.39 & 8.47 & 8.65 & 8.85 & 8.37 & 8.63 & \\
\hline $3.00 \ldots$ & 3. 68 & 3.79 & 5.85 & 3.44 & 6.61 & 8.75 & 8.95 & 9.13 & 8.65 & 8.92 & \\
\hline 3.10. & 3.80 & 3.91 & 6.02 & 3.56 & 6.82 & 9.03 & 9.24 & 9.42 & 8.94 & 9.21 & \\
\hline $3.20 .$. & 3.92 & 4.03 & 6. 20 & 3.66 & 7.03 & 9.31 & 9.53 & 9.71 & 9. 22 & 9.50 & \\
\hline $3.30 \ldots$ & 4.04 & 4.15 & 6. 38 & 3.77 & 7.24 & 9.59 & 9.82 & 10.00 & 9.51 & 9.80 & \\
\hline $3.40 .$. & 4. 16 & 4.26 & $6.5 b$ & 3. 88 & 7.45 & 9.87 & 10.11 & 10.29 & 9.80 & 10.10 & \\
\hline 3.50. & 4.28 & 4. 38 & 6.74 & 3.98 & 7.66 & 10.15 & 10.41 & 10.59 & 10.10 & 10.39 & \\
\hline $3.60 \ldots$ & 4.40 & 4.50 & 6.92 & 4.09 & 7.87 & 10.43 & 10.70 & 10. 89 & & 10.68 & \\
\hline $3.70 .$. & 4. 52 & 4.62 & 7.09 & 4. 19 & 8.08 & 10.71 & 10.99 & & & 10.98 & \\
\hline 3. $80 \ldots$ & 4.64 & 4. 74 & 7.27 & 4. 30 & & 10.99 & 11.28 & & & 11. 28 & \\
\hline $3.90 \ldots$ & 4.76 & 4.86 & 7.44 & 4.40 & $\cdots$ & 11. 27 & 11.57 & & & 11.57 & \\
\hline 4.00. & 4.88 & 4.98 & 7.61 & 4.51 & & 11.55 & 11.87 & & & 11.86 & \\
\hline $4.10 \ldots$ & 5. 00 & 5. 10 & 7.79 & 4. 62 & & 11.83 & 12.17 & & & & \\
\hline 4.20. & 5.12 & 5.22 & 7.97 & 4. 73 & & 12.11 & 12.46 & & & & \\
\hline 4. $30 .$. & 5.24 & 5.34 & 8.14 & 4.83 & & 12.39 & 12.76 & & & & \\
\hline 4.40. & 5.36 & 5.46 & 8. 31 & 4. 93 & & 12.67 & 13.05 & & & & \\
\hline $4.50 \ldots$ & 5.48 & 5.58 & 8.49 & 5.04 & & 12.95 & 13. 35 & & & & \\
\hline 4. 60. & 5.60 & 5. 70 & 8.67 & 5. 14 & & 13.23 & & & & & \\
\hline $4.70 \ldots$ & 5. 72 & 5.81 & 8.85 & 5.24 & ........ & 13.51 & & & & & \\
\hline 4.80. & 5.84 & 5.92 & 9.03 & 5. 34 & & 13. 79 & & & & & \\
\hline $4.90 \ldots$ & 5.96 & 6.04 & ....... & 5.45 & $\ldots \ldots$ & 14.07 & & & & & \\
\hline $5.00 \ldots$ & 6. 08 & 6.16 & ( & 5. 55 & 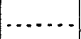 & 14.35 & & & & & \\
\hline
\end{tabular}




\section{DRAINAGE AREA MEASUREMENTS. ${ }^{1}$}

American Fork near American Fork, Utah . ........................... 66

Animas River at Durango, Colorado............................... 812

Arkansas River, East Fork, near Leadville, Colorado . . . . . . . . . . . . . . . . . 44

Arkansas River, Lake Fork, near Leadville, Colorado . . . . . . . . . . . . . . . . . . . 21

Arkansas River, Tennessee Fork....................................... 44

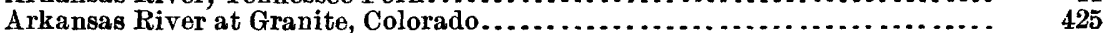

Arkansas River at Salida (above South Fork) . . . . . . . . . . . . . . . . . . . . 1, 160

Arkansas River at Canyon City, Colorado ................................. 3,060

Arkansas River at Swallows, Colorado............................ 4,300

Arkansas River at Pueblo, Colorado................................... 4, 600

Arkansas River at La Junta, Colorado (including Fountain Creek drainage,

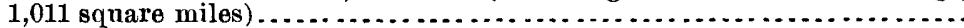

Arkansas River at railroad bridge above Holly, Colorado (including Animas

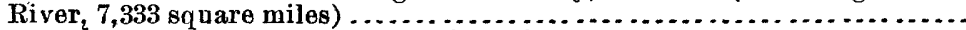

Arkansas River at Colorado-Kansas State line... . . . . . . . . . . . . . . . . . . .

Arkansas River at Hutchinson, Kansas . . . . . . . . . . . . . . . . . . . . . . . . . .

Bear Creek at Morrison, Colorado . . . . . . . . . . . . . $\ldots \ldots \ldots \ldots \ldots \ldots \ldots \ldots \ldots \ldots \ldots$

Bear River at Battle Creek, Idaho. . . . . . . . . . . . . . . . . . . . . . . . . . .

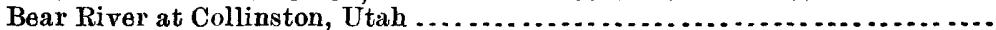

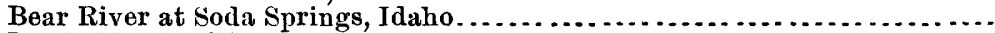

Black Warrior River at Tuscaloosa, Alabama . . . . . . . . . . . . . . . . . . . . . .

Blacks Fork, 3 miles west of Granger, Wyoming . . . . . . . . . . . . . . . . . . .

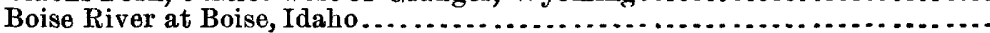

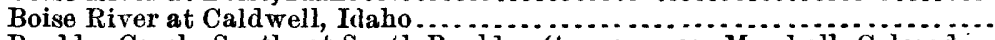

Boulder Creek, South, at South Boulder Canyon, near Marshall, Colorado. .

Boulder Creek, North, at Boulder, Colorado . . . . . . . . . . . . . . . . . . . . .

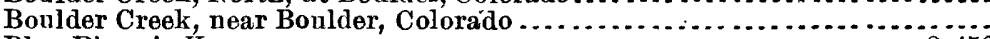

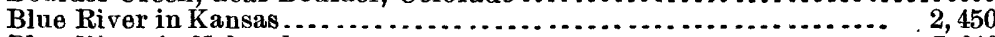

Blue River in Nebraska . . . . . . . . . . . . . . . . . . . . . . . . . . . 7,040

Broad River at Gaffney, South Carolina. . . . . . . . . . . . . . . . . . . . . . . . .

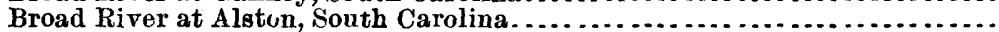

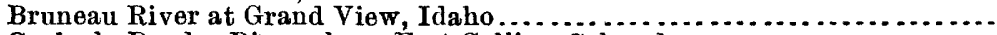

Cache la Poudre River above Fort Collins, Colorado ........................

Caliente Creek:

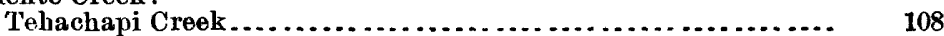

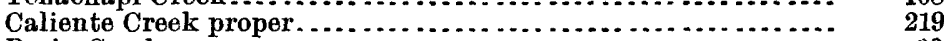

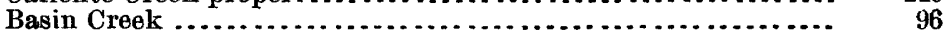

Canadian River in Kansas. ............................... 5,110

Canadian River in New Mexico ..................................

Canadian River in Texas ..................................... 18,620

Cape Fear River at Fajetteville, North Carolina . .......................

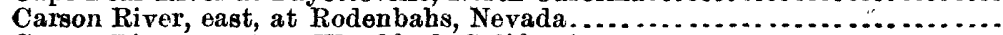

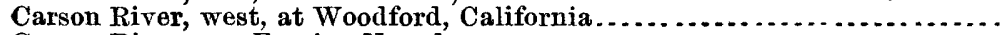

Carson River near Empire, Nevada....................................

Catawba River at Catawba, South Carolina . . . . . . . . . . . . . . . . . . . . . .

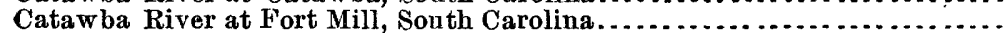

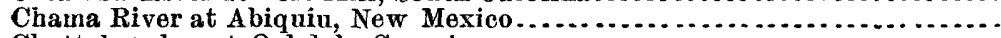

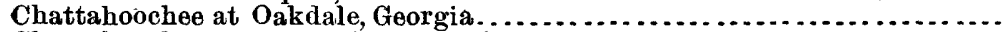

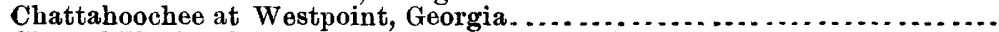

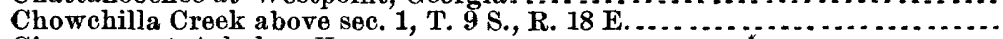

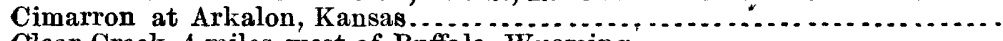

Clear Creek, 4 miles west of Buffalo, Wyoming, $\ldots \ldots \ldots \ldots \ldots \ldots$

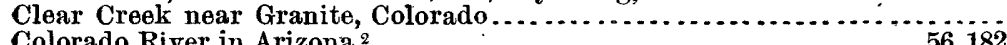

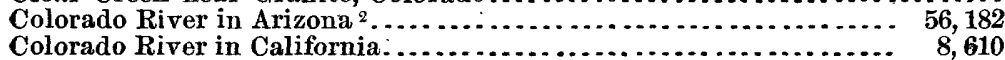

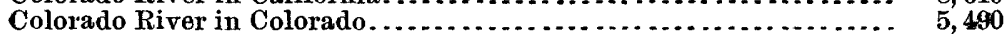

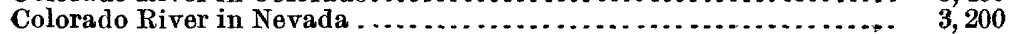

Colorado River in New Mexico . . . . . . . . . . . . . . . . . . . . . . . . . . . . 19,000

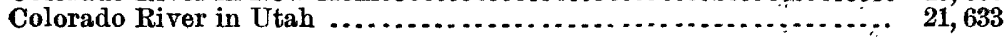

12,200

23,500

24,600

34,000

170

4,500

6,000

3,940

4, 900

1, 747

2,450

3,360

125

179

102

9,490

1,435

4,609

1,800

1,060

3,492

2, 987

2,300

1, 560

3,300

268

5,200

118

72

I A number of the drainage areas not given in this table are to be found on pages 107 to 110 of the Eleventh Annual Report. Part II.

2 Thirteenth Ann. Rept., Part III, p. 33; area in square miles of principal drainagé basins, by States. 
Square miles.

Colorado River at Yuma, Arizona............................... 225, 049

Columbia River in Oregon ${ }^{1} \ldots \ldots \ldots \ldots \ldots \ldots \ldots \ldots \ldots \ldots \ldots \ldots \ldots \ldots \ldots \ldots \ldots \ldots \ldots \ldots \ldots \ldots$
Columbia River in Idaho ${ }^{1} \ldots \ldots \ldots \ldots \ldots \ldots \ldots \ldots \ldots$

Columbia River in Montana................................ 25,000

Columbia River in Washington ............................ 26, 160

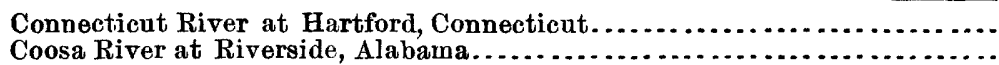

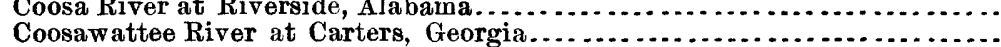

Cottonwood Creek, South Fork, near Buena Vista, Colorado ..............

Cottonwood Creek, Middle Fork, near Buena Vista, Colorado . . . . . . . . . . . .

Dan River at Clarksville, Virginia. . . . . . . . . . . . . . . . . . . . . . . . . . . . . .

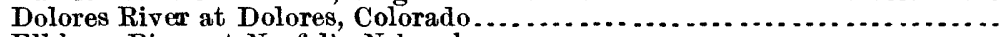

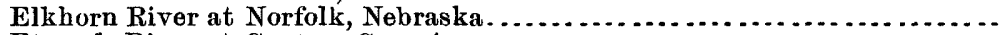

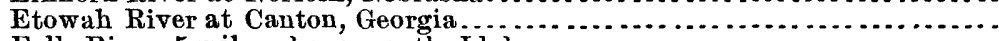

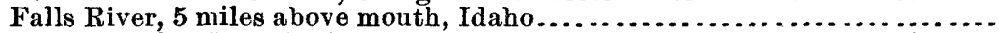

Fort Tejon Creek, including Cuddy Creek and Castac Lake (upper basin, 56 ).

French Broad River at Asheville, North Carolina. . . . . . . . . . . . . . . . . . . .

Frenchman River at Wauneta, Nebraska . . . . . . . . . . . . . . . . . . . . . .

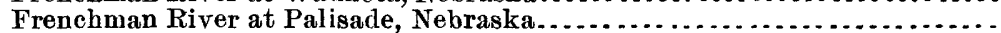

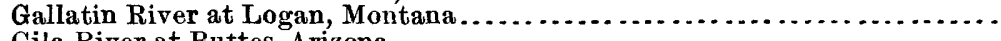

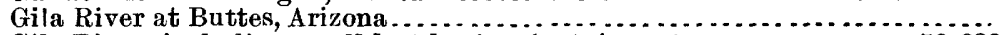

Gila River, including small lost basins, in Arizona ${ }^{2} \ldots \ldots \ldots \ldots \ldots \ldots .56,838$

Gila River, including small lost basins, in New Mexico............ 14, 300

Goose Creek, Big, at Sheridan, Wyoming, below mouth of Little Goose Creek. Goose Creek, Little, at Sheridan, Wyoming .

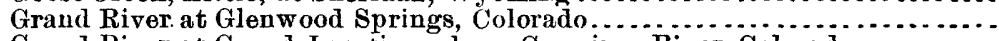

Grand River at Grand Junction, above Gunnison River, Colorado...........

Grand River at Grand Junction, below Gunnison River (including, above

Uncompahgre, 497 square $\cdot m i l e s$, and Gunnison, at mouth, 7,935 square

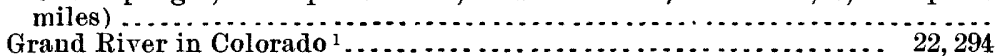

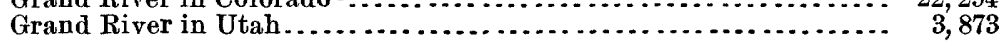

Great Basin in California ${ }^{1} \ldots \ldots \ldots \ldots \ldots \ldots \ldots \ldots \ldots \ldots \ldots \ldots \ldots \ldots . \ldots \ldots, 240$

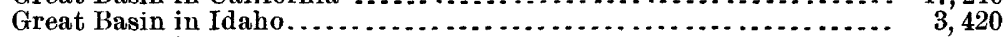

Great Basin in Nevada ................................... 102, 220

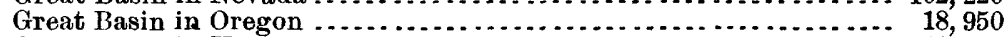

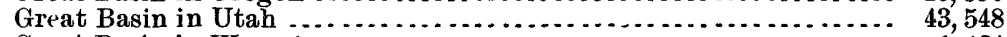

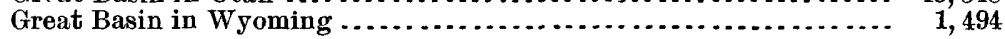

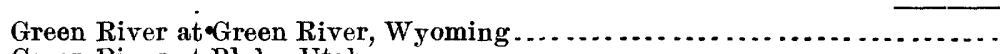

\begin{tabular}{l} 
Green River at Blake, Utah $\ldots \ldots \ldots \ldots \ldots$ \\
Green River in Colorado ${ }^{1} \ldots \ldots \ldots \ldots \ldots$ \\
\hline
\end{tabular}

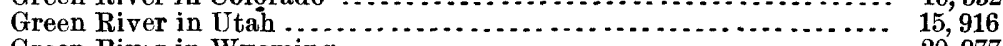

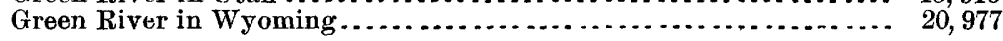

Greenbrier River at Alderson, West Virginia . . . . . . . . . . . . . . . . . . . . .

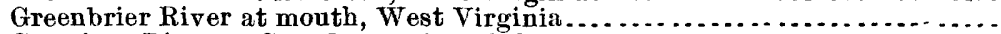

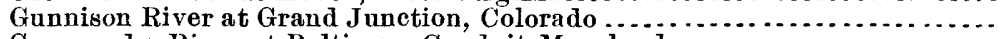

Gunpow der River at Baltimore Conduit, Maryland. . . . . . $\ldots \ldots \ldots \ldots \ldots \ldots \ldots$

Henry Fork, 1 mile above month of Falls River, Idaho ... . . . . . . . . . . . . .

Hiwassee River at Murphy, North Carolina ...........................

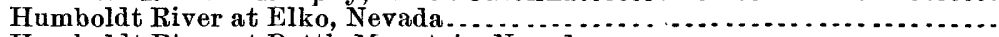

Humboldt River at Battle Mountain, Nevada . . . . . . . . . . . . . . . . . . . . . . .

Humboldt River at Golconda, Nevada . . . . . . . . . . . . . . . . . . . . . . . .

Humboldt River (including Grassy Valley) at Oreana, Nevada . . . . . . . . . .

Humboldt River, South Fork, at Masons Ranch, Nevada . . . . . . . . . . . . . .

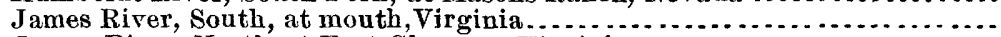

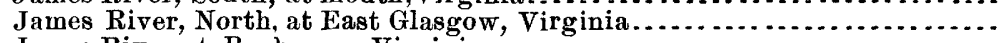

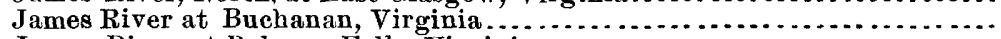

James River at Balcony Falls, Virginia . . . . . . . . . . . . . . . . . . . . . .

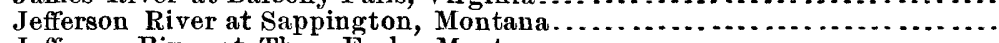

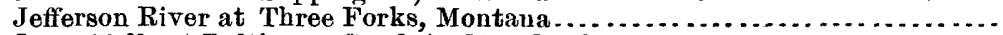

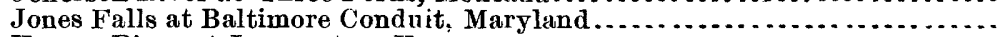

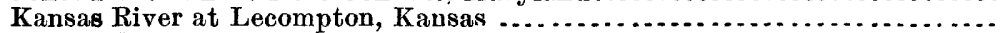

Kansas River at Lawrence, Kansag

59,040

10,234

6, 850

532

28

37

3, 798

562

2, 474

573

594

93

987

989

1, 032

1,620

13,750

71, 138

320

128

5, 838

8,644

16,579

26,167

216,872

7,450

38,200

47,225

1,344

1, 575

7, 935

294

931

410

2,840

7,800

10,780

14, 967

536

116

831

2, 058

3,027

8,984

9,400

59,841

1 Thirteenth Ann. Rept., Part III, p. 33 ; area in square miles of principal drainage basins, by States. 
Kansas River in Kansas.

Square miles.

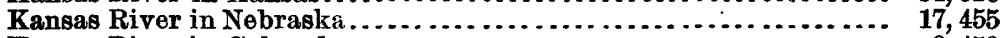

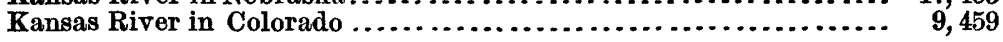

Kaweah River above sec. 3, T. 18 S., R. 27 E., California.................

Kern River at first point of measurement above Bakersfield, California, including-

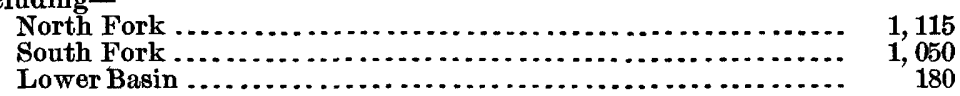

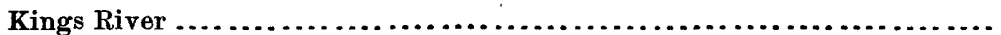

Laramie River above Woods Landing, Wyoming (in Colorado, 343) ........

Laramie Rivor at Uva, Wyoming (including in Colorado, 428).

Laramie River at mouth at Fort Laramie (not including 500 square miles of

lost drainage to James Lake, Cooper Lake, etc.) . .......................

Logan River at Logan, Utah...............

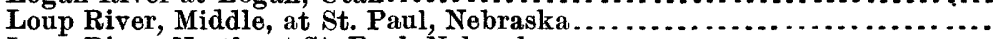

Loup River, North, at St. Paul, Nebraska . . . . . . . . . . . . . . . . . . . . . .

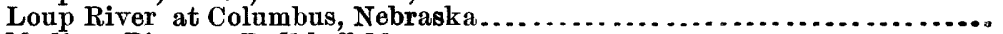

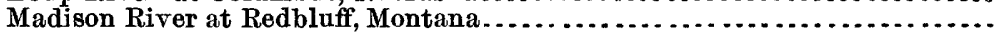

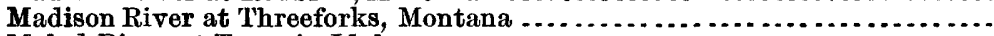

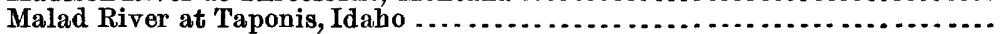

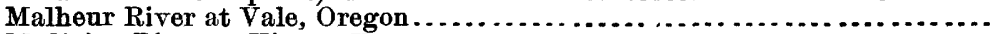

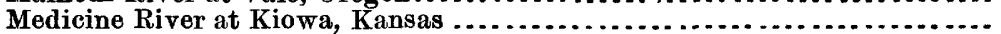

Merced River above Merced Falls, California ............................

Middle Creek near Bozeman, Montana.................................

Mississippi River at St. Paul, Minnesota (Minnesota River, 16,350) .........

Missouri River at Townsend, Montana................................

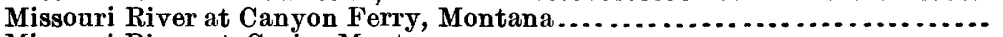

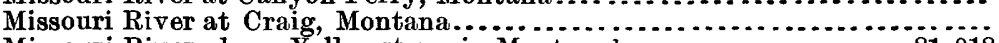

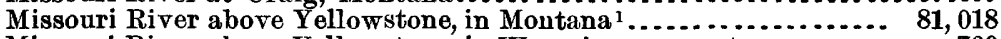

Missouri River above Yellowstone, in Wyoming ...................

Monocacy River at Frederick, Maryland .............................

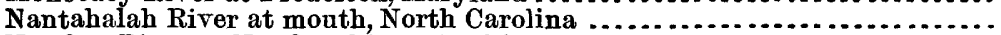

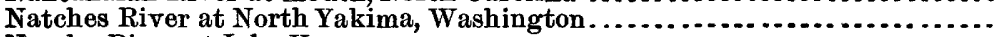

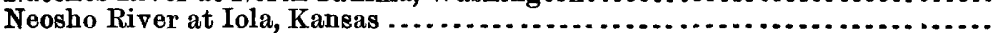

Neuse River at Selma, North Carolina. . . . . . . . . . . . . . . . . . . . . . . . . . . . .

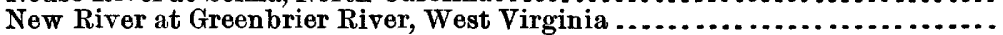

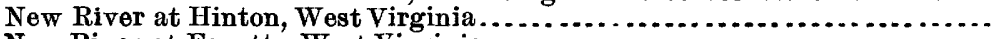

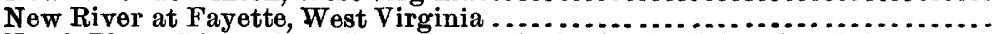

North Platte River above Sweetwater (including in Colorado, 1696) ........

North Platte River above Douglas, Wyoming ............................

North Platte River above Orin Junction, Wyoming $\ldots \ldots \ldots \ldots \ldots \ldots \ldots \ldots \ldots \ldots$

North Platte River above Fort Laramie, W yoming . . . . . . . . . . . . . . . . . . .

North Platte River below Laramie River, Wyoming . . . . . . . . . . . . . . . . . .

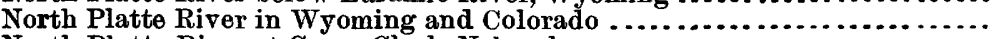

North Platte River at Camp Clark, Nebraska . . . . . . . . . . . . . . . . . . . . . . . . .

North Platte River to North Platte, Nebraska ...........................

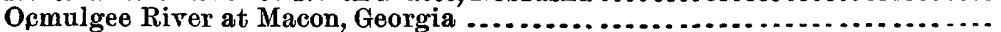

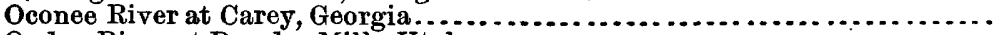

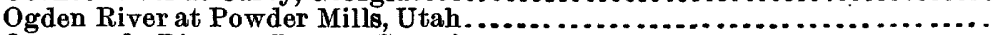

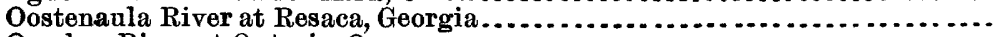

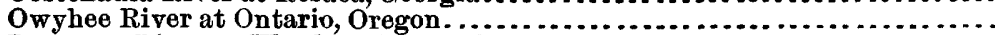

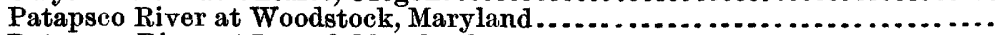

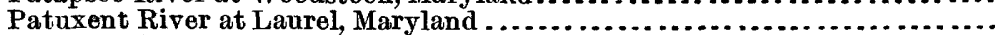

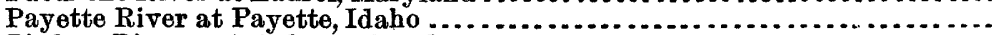

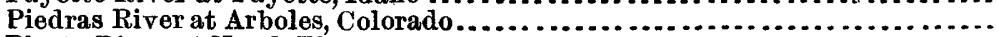

Platte River at North Platte:

North Platte River................................... 28,517

South Platte River......................................... 23, 294

Platte River at Columbus, Nebraska ............................. $\begin{aligned} & 51,811 \\ & 56,867\end{aligned}$

Platte River, North and South, in Colorado ${ }^{1} \ldots \ldots \ldots \ldots \ldots \ldots \ldots \ldots \ldots \ldots \ldots 2,230$

Platte River in Nebraska................................. 10,850

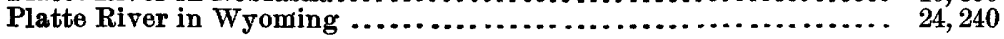

2,345

1,742

435

3, 179

4, 076

218

6,849

4, 024

13,542

2, 085

2,402

2, 190

$\mathbf{9}, 900$

1, 300

1, 076

55

36, 085

14,500

15,036

17,615

81,778

665

193

1, 000

3, 670

1, 175

4, 523

5,600

6,200

7, 668

14,255

14,828

16,416

20,492

23,643

24,225

28,517

2,425

1, 346

360

1, $\mathbf{5} 27$

9, 875

251

137

3, 565

650

57,320

Thirteenth Ann. Rept., Part III, p. 33; area in square miles of principal drainage basins, by States. IRR $11 \longrightarrow 7$ 
Portneuf River at McCammon, Idaho............................ 500

Potomac River, North Branch, above Bloomington, Maryland . ............ 293

Potomac River, North Branch, at Piedmont, West Virginia (including Savage

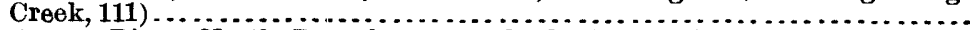

Potomac River, North Branch, at Cumberland, Maryland (including New Creek, at mouth, at Keyser, West Virginia, 56)........................

Potomac River, North Branch, at junction with South Branch (including Patterson Creek at Burlington, below Mill Creek, 155, and at mouth, 279).....

Potomac River, South Branch, at junction with North Fork (including at Franklin, 188)

Potomac River, South Branch, including North Fork (including North Fork

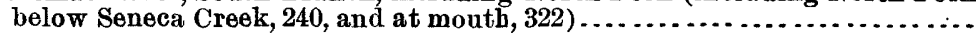

Potomac River, South Branch, at Moorefield, West Virginia (including Mili Creek at mouth, 101)

Potomac River,'South Branch, below Moorefield (including South Fork at Fort Seybert, West Virginia, 155, and at mouth at Moorefield, 301) ........

Potomac River, South Branch, at Romney, West Virginia..................

Potomac Ríver, South Branch, at United States Geological Survey station at railway bridge, between Romney and Springfield, West Virginia..........

Potomac River, South Branch, at mouth . . . . . . . . . . . . . . . . . . . . . .

Potomac Riverat junction of North and South branches..................

Potomac River above Great Cacapon River (including Little Cacapon River, 117)

Potomac River below Great Cacapon River (including Great Cacapon above

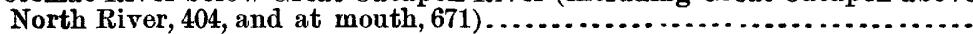

Potomac River at Hanoock, West Virginia $\ldots \ldots \ldots$

Potomac River at Williamsport, Maryland (including Warm Spring Creek at mouth, 16; Conoloway Creek, 125; Sleepy Creek, 146; Licking Creek, 195; Back Creek, 288, and Conocheague Creek, 579)

Potomac River at Harpers Ferry, West Virginia (including Opequon Creek, 335; Antietam Creek, 305).

Potomac River below Harpers Ferry, West Virginia (including Shenandoah River, 3,009)

Potomac River at Weverton, Maryland .

Potomac River at Point of Rocks, Maryland . . . . . . . . . . . . . . . . . . . . .

Potomac River at Edwards Ferry, below Goose Creek (including Monocacy River at mouth, 941, and Goose Creek, 384).

Potomac River at Great Falls, Maryland (including Seneca Creek, 132)

Potomac River at Chain Bridge, District of Columbia . . . . . . . . . . . . . . . . . . .

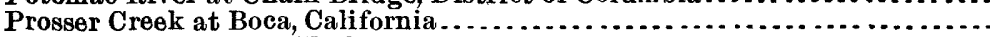

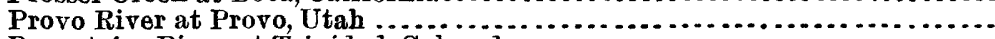

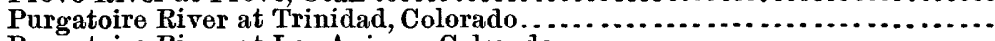

Purgatoire River at Las Animas, Colorado . . . . . . . . . . . . . . . . . . . . . .

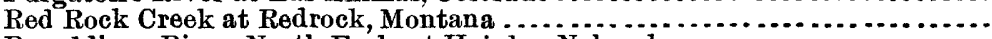

Republican River, North Fork, at Haigler, Nebraska . . . . . . . . . . . . . . . . .

Republican River at Superior, Nebraska . . . . . . . . . . . . . . . . . . . . . . .

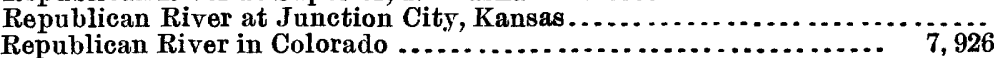

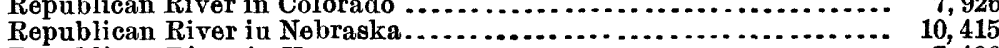

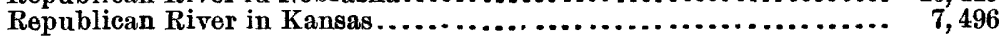

Rio Grande at Del Norte, Colorado...............................

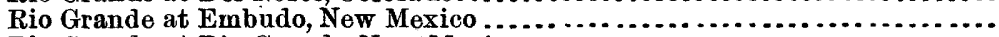

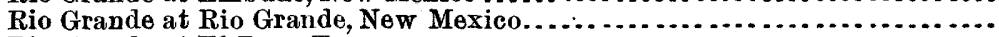

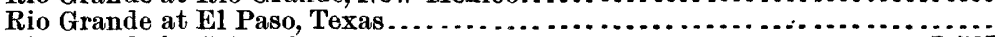

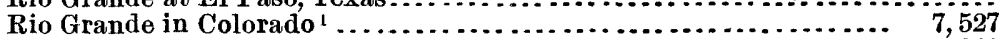

Rio Grande in New Mexico (including Pecos River) ............... 72,380

Rio Grande in Texas ....................................... 80,550

Roanoke Rirer at Roanoke, Virginia..............................

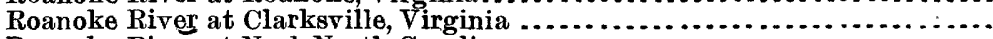

Roanoke River at Neal, North Carolina . . . . . . . . . . . . . . . . . . . . . . . . .

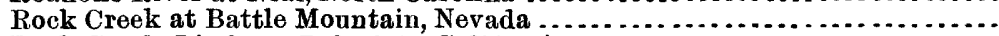

Rock Creek, Little, at Palmdale, California . . . . . . . . . . . . . . . . . . . . . . . .

Sacramento River at Jellys Ferry, California. . . . . . . . . . . . . . . . . . . . . .

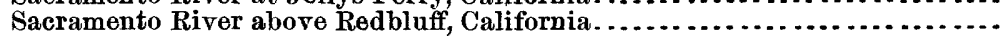


Sacramento and San Joaquin rivers in California

Square miles.

Sacramento River.

63,020

655

Salt River below Tonto Creek, Arizona . . . . . . . . . . . . . . . . . . . . . . . . . . . . .

Salt River in canyon 20 miles above Verde . . . . . . . . . . . . . . . . . . . . . . . . .

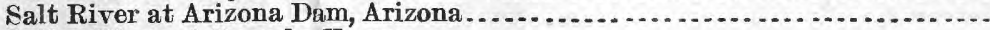

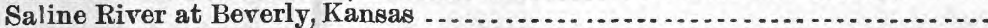

Saline River at mouth, Kansas . . . . . . . . . . . . . . . . . . . . . . . . . . . .

Saluda River at Waterloo, South Carolina.

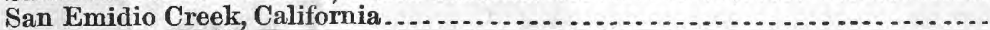

San Gabriel River in canyon above Azusa, California . . . . . . . . . . . . . . . . .

San Joaquin River above Hamptonville, California . . . . . . . . . . . . . . . . . . .

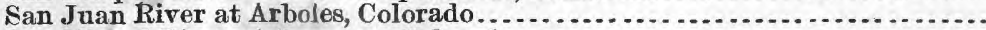

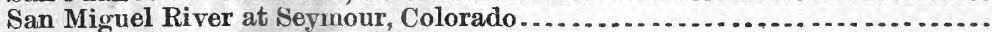

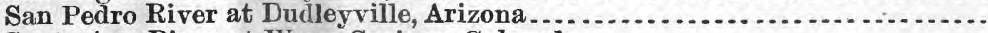

Santa Ana River at Wai m Springs, Colorado ... . . . . . . . . . . . . . . . . . . .

Savannah River below T.ocky River, at Calhoun Falls, South Carolina, above

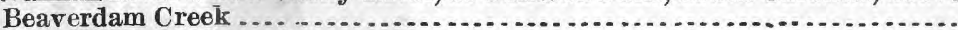

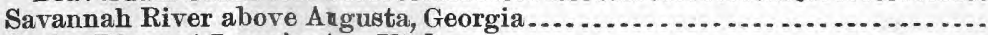

Sevier River at Leamington, Utah

Shenandoah River (Nortb Fork) at Brocks Gap, Virginia. . . . . . . . . . . . . . ...

Shenandoah River (North Fork) at Mount Jackson, Virginia ..............

Shenandoah River (North Fork) at Riverton, Virginia . . . .

Shenandoah River (South Fork) at Port Republic, Virginia (including Mid-

dle River to junction with North River, 363 ; North River to junction with

Middle River, 418 ; to juction with South River, 804; South River to junc-

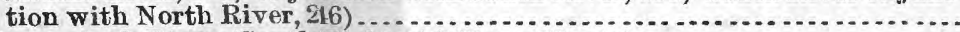

Shenandoah River (South Fork) at Milnes, Virginia... . . . . . . . . . . . . .

Shenandoah River (South Fork) at Overall, Virginia . . . . . . . . . . . . . . . . . .

Shenandoah River (So th fork) at mouth at Riverton, Virgivia............

Shenandoah River at Riverton, Virginla (including North Fork, 1, 037, and

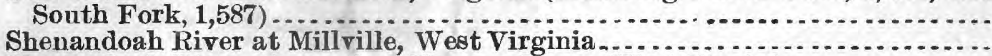

Shenandoah River at mouth at Harpers Ferry, West Virginia..............

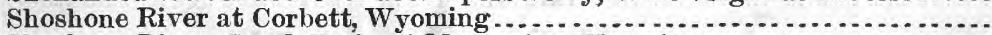

Shoshone River, South Fork, at Marquette, Wyoming .....................

Smoky Hill River at Ellsworth, Kansas (including in Colorado 1,533, in Kansas 6,447) ...

Smoky Hill River below Ellsworth, exclusive of Saline and Solomon rivers.

Smoky Hill River at mouth at Junction City, Kansas . . . . . . . . . . . . . . . . .

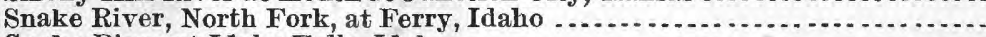

Snake River at Idaho Falls, Idaho ... . . . . . . . . . . . . . . . . . . . . . . . .

Snake River at Montgomery Ferry, Idaho $\ldots \ldots \ldots \ldots$
Snake River in Idaho ${ }^{1} \ldots \ldots$

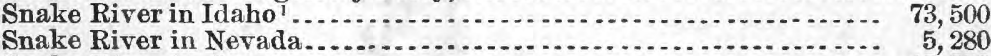

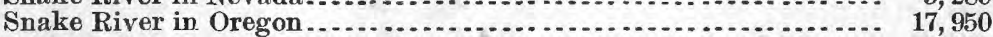

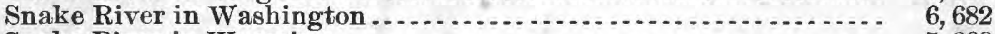

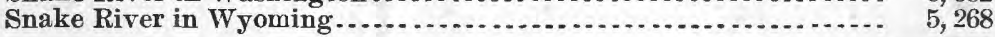

Solomon River at Beloit, Kansas ..................................

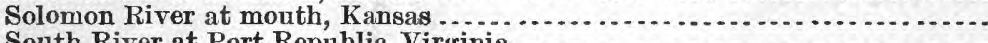

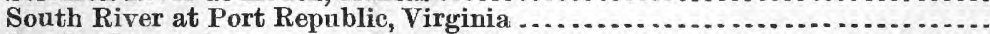

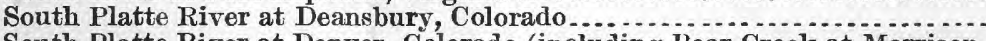

South Platte River at Denver, Colorado (including Bear Creek at Morrison,

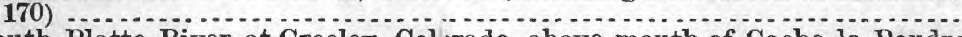

South Platte River at Greeley, Colurado, above mouth of Cache la Poudre

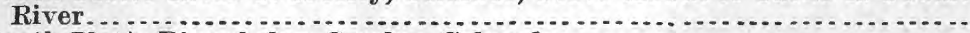

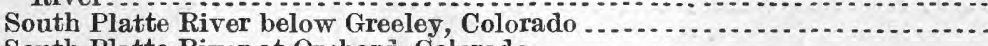

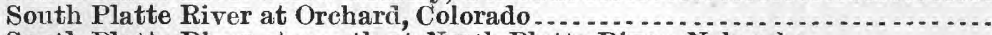

South Platte River at mouth at North Platte River, Nebraska . . . . . . . . . . .

Spanish Fork near Spanish Fork, Utah . . . . . . . . . . . . . . . . . . . . . . . . . . .

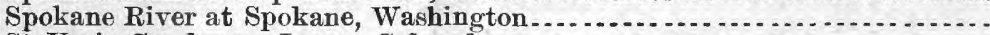

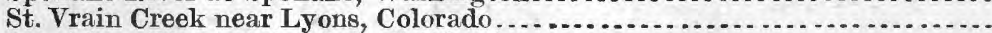

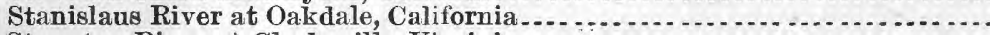

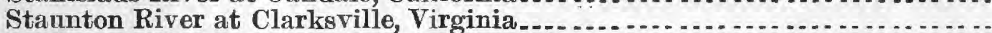

5,539

6,882

1, 050

2, 600

3,840

7,110

9,575

12,260

23,294

670

4, 005

209

1, 051

3,546

1,175

1Thirteenth Ann. Rept., Part III, p. 33; area in square miles of principal drainage basins, by States. 
Sweetwater River (Wyoming) at mouth............................ 2,929

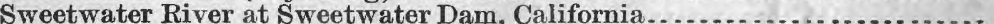

Tar River at Tarboro, North Carolina.... . . . . . . . ...................... 2, 290

Tejon House Creek at Tejon Ranch House, Kern County, California......... 17

Tennessee River, Little, at Judson, North Carolina ....................... 682

Teton River at Wilford, Idaho ...................................... 967

Thompson Creek near Loveland, Colorado . . . . . . . . . . . . . . . . . . . . . . . . 305

Tongue River at Miles, Montana.................................... 3,875

Truckee (Little) River at Boca, California................................. 186

Truckee River at Boca, California...................... . . . . . ... 887

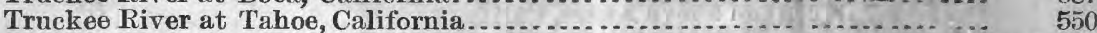

Truckee River near Essex, Nevada. . . . . . . . . . . . . . . . . . . . . . . . . . . . . . . . 991

Truckes River near Langhtons, Nevada ............................... 1, 054

Truckee River near Vista, Nevada. . . . . . . . . . . . . . . . . . . . . . . . . . . . 1, 519

Tuckaseegee River 3 miles above Bryson, North Carolina ................... 609

Tule River above Portersville, California ........................... 437

Tuolume River above Roberts Ferry, California, at foothils ........... 1, 501

Tuolumne River at Morlesto ....................................... 1, 635

Twin Lake Creek below Twinlakes, Colorado........................... 102

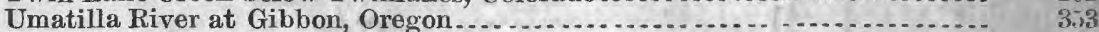

Umatilla River at Pendleton, Oregon ................................. 640

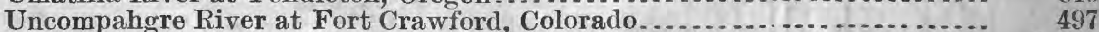

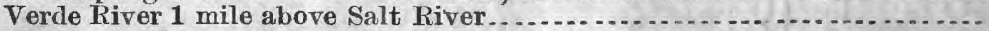

Verdigris River at Independence, Kansas . . . . . . . . . . . . . . . . . . . . . . .

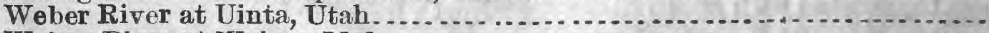

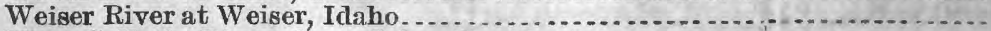

West Gallatin River above Salesville, Montana..........................

White River at White River City, Colorado ............................

Wood River at Hailey, Idaho .........................

Wood River, Little, at Taponis, Idaho ... . . . . . . . . . . . . . . . . . . . . .

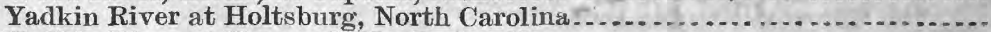

Yadkin River at Norwood, North Carolina. . . . . . . . . . . . . . . . . . . . . . .

Yakima River at Union Gap, Washington (of this, Tiaton, 330) ............

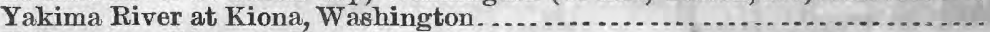

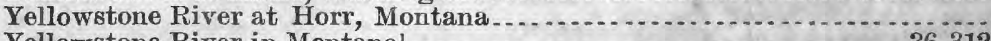

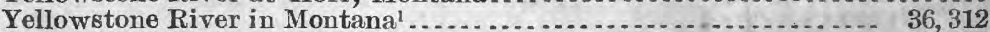

Yellowstone River in North Dakota. ............................... ${ }_{250}$

Yellowstone River in Wyoming..................... 33, 121

6,000

3. 067

1,600

1. 670

860

1, 773 ,

ars

1, 270

3, 399

4, 614

3,300

5,230

2, 700

69,683

IThirteenth Ann. Rept., Part III, p. 33; area in square miles of principal drainage basins, by States. 